Aesthetics

and subjectivity

\title{
from Kant to Nietzsche
}

Second edition A N D R E W B O W I E 


\section{Aesthetics and subjectivity}

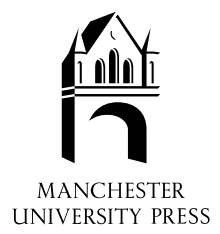


Andrew Bowie - 9781526137234

Downloaded from manchesterhive.com at $04 / 26 / 2023$ 11:57:42AM via free access 


\section{ANDREW BOWIE \\ Aesthetics and subjectivity: \\ from Kant to Nietzsche}

Second edition

\section{Manchester University Press \\ Manchester and New York}

Distributed exclusively in the USA by Palgrave 
Copyright (C) Andrew Bowie 1990, 2003

The right of Andrew Bowie to be identified as the author of this work has been asserted by him in accordance with the Copyright, Designs and Patents Act 1988.

First edition published 1990 by Manchester University Press Paperback edition published 1993

This edition published 2003 by

Manchester University Press

Oxford Road, Manchester M13 9NR, UK

and Room 400, 175 Fifth Avenue, New York, NY 10010, USA

www.manchesteruniversitypress.co.uk

Distributed exclusively in the USA by

Palgrave, 175 Fifth Avenue, New York, NY 10010, USA

Distributed exclusively in Canada by

UBC Press, University of British Columbia, 2029 West Mall, Vancouver, BC, Cananda V6T 1 Z2

British Library Cataloguing-in-Publication Data

A catalogue record for this book is available from the British Library

Library of Congress Cataloging-in-Publication Data applied for

ISBN 0719057388 paperback

This edition first published 2003

$\begin{array}{llllllllllllllllll}10 & 09 & 08 & 07 & 06 & 05 & 04 & 03 & 10 & 9 & 8 & 7 & 6 & 5 & 4 & 3 & 2 & 1\end{array}$

Typeset in 10.5/12pt Ehrhardt

by Servis Filmsetting Ltd, Manchester

Printed in Great Britain

by Bookcraft (Bath) Ltd, Midsomer Norton 


\section{CONTENTS}

Preface to the second edition

page vii

Introduction $\quad 1$

Aesthetics and modernity 1

Aesthetics and 'postmodernity' 8

1 Modern philosophy and the emergence of aesthetic theory: Kant 16

$\begin{array}{ll}\text { Self-consciousness, knowledge and freedom } & 16\end{array}$

$\begin{array}{lr}\text { The unity of the subject } & 19\end{array}$

$\begin{array}{ll}\text { The unification of nature } & 24\end{array}$

The purpose of beauty $\quad 32$

The limits of beauty 41

2 German Idealism and early German Romanticism 49

Thinking the infinite 49

A 'new mythology' $\quad 55$

Early Romantic 'new mythology' 63

3 Reflections on the subject: Fichte, Hölderlin and Novalis 69

$\begin{array}{ll}\text { Self and Other } & 69\end{array}$

$\begin{array}{ll}\text { Fichte } & 70\end{array}$

Hölderlin $\quad 82$

$\begin{array}{lr}\text { Novalis } & 88\end{array}$

4 Schelling: art as the 'organ of philosophy' 102

$\begin{array}{ll}\text { Nature and philosophy } & 102\end{array}$

$\begin{array}{ll}\text { The development of consciousness } & 108\end{array}$

$\begin{array}{ll}\text { Intuition and concept } & 113\end{array}$

$\begin{array}{ll}\text { The 'organ of philosophy' } & 117\end{array}$

$\begin{array}{ll}\text { Mythology, art and modernity } & 124\end{array}$

$\begin{array}{ll}\text { Mythology, language and being } & 127\end{array}$

5 Hegel: the beginning of aesthetic theory and the end of art $\quad 140$

$\begin{array}{ll}\text { Which Hegel? } & 140\end{array}$

$\begin{array}{lr}\text { Self-recognition } & 147\end{array}$

Music and the Idea $\quad 152$

$\begin{array}{ll}\text { Language, consciousness and being } & 160\end{array}$

$\begin{array}{ll}\text { The Idea as sensuous appearance } & 166\end{array}$

$\begin{array}{ll}\text { The prose of the modern world } & 175\end{array}$

$\begin{array}{ll}\text { Philosophy and art after Hegel } & 178\end{array}$ 
6 Schleiermacher: art and interpretation 183

Linguistic turns 183

The 'art of disagreement' 185

Immediate self-consciousness 196

Art as free production: 'individual' and 'identical' activity 200

Hermeneutics as art 203

Literature and the 'musical' 209

7 Music, language and literature 221

Language and music 221

Hegel and Romanticism: music, logos and feeling 227

The 'presence' of music 238

'Infinite reflection' and music 245

8 Nietzsche and the fate of Romantic thought 258

The old and the new Nietzsches 258

Schopenhauer: music as metaphysics $\quad 261$

Marx, mythology and art 270

Art, myth and music in 'The Birth of Tragedy' 275

Myth, music and language $\quad 284$

The illusion of truth 289

Music and metaphysics 292

Aesthetics, 'interpretation' and subjectivity 300

$\begin{array}{ll}\text { Conclusion } & 312\end{array}$

Appendix: the so-called 'Oldest System Programme of German 334

Idealism' (1796)

References 336

Index $\quad 342$ 


\section{PREFACE TO THE SECOND EDITION}

I began writing the original version of this book some fifteen years ago. This new version retains the basic structure of the first edition, but contains hardly a single sentence which is the same as in that edition. I have in fact totally rewritten the book, extending the scope and detail of the arguments, taking in issues which have become philosophically alive in the interim, and incorporating the results of more recent historical research. The main change of focus of the book is in relation to the development in America of sophisticated work in pragmatism and neo-Hegelianism, which draws both on the traditions investigated in this book and on resources from recent analytical philosophy. Though I obviously would say this, I do think any reader of the first edition would profit considerably from reading (and, preferably, buying!) the new edition. The previous edition was generally well received by the reviewers, but one or two rightly commented on its stylistic failings. Writing for an English-speaking audience about the German philosophical tradition is never going to be easy, and I do not claim to have produced a work of grace and elegance, but the new version will, I hope, find more favour for the way it is written than the old version. It will also hopefully find favour because it now says much more effectively what I was striving to say in the first edition.

The new presentation, clarification, and refinement of my ideas, and my revised accounts of the thinkers have greatly benefited from talking to: Alison Ainley, Karl Ameriks, Jay Bernstein, Arnfinn Bø-Rygg, Susan Bowles, Rüdiger Bubner, Tony Cascardi, Maíre Davies, Peter Dews, Manfred Frank, Roger Frie, Neil Gascoigne, Steve Giles, Kristin Gjesdal, Lydia Goehr, Stephen Hinton, Robert Holub, Stephen Houlgate, Nick Jardine, Peter Osborne, Robert Pippin, Richard Potter, Stephen Prickett, Jonathan Rée, Kiernan Ryan, Martin Swales, Robert Vilain, Nick Walker, among many others. Invitations to talk at many British universities, and at the universities of Berkeley, Copenhagen, Cornell, Drew, Oslo, Stanford, Stockholm, and Turku, and the National Endowment for the Humanities Summer School on German Romanticism at Colorado State, offered invaluable opportunities to try out my ideas on new audiences. An Alexander von Humboldt Fellowship and British Academy research leave some time ago now still form the basis of much of my work. During the time of writing I have moved from the Philosophy Department at Anglia Polytechnic University in Cambridge, whose students persuaded me by their acuity and enthusiasm that it was possible and worthwhile to communicate this difficult tradition to them, to Royal Holloway, University of London, where I hope my attempts to spread the word about German philosophy in a welcoming and supportive German department will meet with success. There are signs that the long-term decline of German studies might be partly arrested if a Romantic combination of different literary, cultural, historical and philosophical 
approaches became the norm. Those parts of the subject that wish to resist the instrumentalisation of modern languages that results from most Western governments' perception of universities as merely training places for the workforce must surely take this route. Germany is, after all - even though the contemporary state of the humanities would not suggest it - the main source of nearly all the major recent theoretical directions in the humanities.

As always I would like to thank my parents, who are invariably hugely supportive, and Liz Bradbury, who is my sternest and most amusing critic and indispensable companion. 


\section{INTRODUCTION}

\section{Aesthetics and modernity}

In recent years it has become apparent that many questions which first became manifest during the emergence of philosophical aesthetics at the end of the eighteenth century play a decisive role both in mainstream philosophy and in literary theory. The critiques of the idea that the world is 'ready-made' by Hilary Putnam and other pragmatically oriented thinkers, the concomitant attention by Nelson Goodman, Richard Rorty and others to the 'world-making' aspects of language, the related moves in the philosophy of language on the part of Donald Davidson and others towards holistic accounts of meaning, and the orientation in post-structuralism towards the undecidable aspects of interpretation all involve structures of thought which developed as part of the history of aesthetics. While some of these thinkers explicitly refer to the tradition to be examined in the present book, others have been notably unconcerned about many of their most significant precursors. In order to help overcome this underestimation of the role of aesthetics the present book will focus on some of the main accounts of the human subject and on the conceptions of art and language which emerge within the Kantian and post-Kantian history of aesthetics. My aim is both to rectify a series of misapprehensions about the history of modern thought which have become the prevailing orthodoxy in some areas of the humanities, and to develop plausible versions of some of the disregarded and misunderstood arguments in that history.

In 1796 a German politico-philosophical manifesto, whose author seems to have been either Hegel or Schelling (but may have been Hölderlin), proclaims the 'highest act of reason' as an 'aesthetic act'. Philosophical reflections on beauty and art have, of course, been around in Western thought since Plato, and Platonic ideas clearly influenced Hegel, Schelling and Hölderlin, but it is only around the middle of the eighteenth century in Europe that the notion of a distinct area of philosophy called 'aesthetics' develops. Between the end of the eighteenth and the end of the nineteenth century the relationship between art and the rest of philosophy undergoes a radical transformation, a transformation that is connected, as we shall see, to vital changes in both the production and reception of music. The ways in which this transformation relates to the development of some of the major directions in modern philosophy will form the focus of my investigation.

Modern philosophy begins when the generally accepted basis upon which the world is interpreted ceases to be a deity whose pattern is assumed to have already been imprinted into the universe. The new philosophical task is therefore for human reason to establish its own legitimacy as the ground of truth. This transformation is prepared in the seventeenth century when Descartes 
makes the 'I think' the main point of certainty upon which philosophy can build, but Descartes still relies upon God to guarantee the connection of ourselves to the order of the universe. Towards the end of the eighteenth century Immanuel Kant aims, in the light of Descartes's arguments about self-consciousness, to describe the shared structures of our subjective consciousness which are the 'condition of possibility' of objective knowledge, and he tries to do so without having recourse to a divinity who guarantees the order of the world. For Kant the only certainty philosophy can provide is grounded in ourselves, not in something outside ourselves. However, in order to establish more substantial links between the external world of nature and the internal world of self-consciousness, he subsequently becomes concerned with what makes us appreciate and create beauty. The reasons for Kant's turn to aesthetics will form the main starting point of the present book.

The new focus of philosophy on subjectivity established by Kant accompanies the complex and contradictory changes wrought by 'modernity': the rapid expansion of capitalism, the emergence of modern individualism, the growing success of scientific method in manipulating nature for human ends, the decline of traditional, theologically legitimated authorities, and the appearance, together with aesthetics as a branch of philosophy, of 'aesthetic autonomy', the idea that works of art entail freely produced rules which do not apply to any other natural object or human product. From being a part of philosophy concerned with the senses, and not necessarily with beauty - the word derives from the Greek 'aisthánesthai', 'perceive sensuously' - the new subject of 'aesthetics' now focuses on the significance of natural beauty and of art. Reflection on aesthetics does not, though, just involve a revival of Plato's thoughts about beauty as the symbol of the good. The crucial new departure lies in the way aesthetics is connected to the emergence of subjectivity as the central issue in modern philosophy, and this is where the relevance of this topic to contemporary concerns becomes apparent.

Much recent theory in the humanities has regarded the human subject as being 'subverted' by its failure to provide a stable ground for philosophy, because, for example, of its dependence on language or on the unconscious. The point is, however, that such ideas are not the radically new insights as which they have often been presented. Related ideas already play a central role in some of the reflections upon subjectivity which immediately follow Kant and are implicit in some of Kant's own arguments. Furthermore, these theories from the early modern period can actually be shown both to have helped initiate the ideas which inform current debates and, at times, to be superior to many current theories. The important fact about these theories in the present context is that they regard the experience of natural and artistic beauty and the fact of aesthetic production as vital to the understanding of self-consciousness. The ability to apprehend something as beautiful and the ability to make something beautiful, as well as the ability to create new meanings without following fixed rules are seen as involving aspects of the self which cannot be theorised in terms of the 
self's becoming transparent to itself as its own object of knowledge. The ways in which self-objectification seems to have inherent limits are an essential factor in the most significant of these theories of aesthetics.

Even more importantly for the story I wish to tell here, the reflections which lead to these models of the subject are frequently connected to the form of art most distant from representation of the object world: music. Towards the end of the eighteenth century 'absolute music', music without a verbal text, becomes increasingly vital in musical praxis, in philosophical reflection upon the significance of art, and as a means of understanding the self. The analogies between the changes in the understanding of the non-representational form of music for philosophy, and the development of theories, in the later Wittgenstein, the work of Heidegger, and post-structuralism, which reject the model of language as the representation of the pre-existing ideas of the subject, or as the representation of pre-existing objects in the world are not fortuitous. The role of music as the most symptomatic art form in this period will, then, be central to my argument because music exemplifies how our self-understanding can never be fully achieved by discursive articulation. If all we are can be stated in words, why does our being also need to be articulated in music, as every known human culture seems to suggest?

The often hyperbolic importance attributed to art towards the end of the eighteenth century evidently has its roots in the decline of theology and the disintegration of theologically legitimated social orders. As Marx put it in the Communist Manifesto, 'All that is solid melts into air', and the new orders of things cannot claim the same kind of authority as that provided by tradition and theology. The loss of a nature whose meaning is assumed to be inherent within it and whose structure is divinely guaranteed leads to a search for other sources of meaning and orientation. The new experience of nature as beautiful per se, rather than as a manifestation of the deity, and the new awareness of the fact that human beings can create aesthetic products whose interrelating parts are significant in ways which natural science cannot explain are essential to this search. These new aspects of modernity are, though, open to a wide variety of interpretations. Once it is clear that whatever coherence there is in the world, including in ourselves, can no longer be assumed to be underwritten by God, the relationship between the human and the natural becomes a serious problem. The task set for itself by the philosophy of the time is the creation of a coherent world with whatever natural capacities we possess and whatever innovatory capacities we can develop. Interrogation of the nature of subjectivity is therefore a reflection upon what these capacities are and how they relate to nature in ourselves and outside ourselves. This reflection is notably double-edged: the enthusiasm generated by liberation from theological constraints can easily give way to a suspicion of the resultant freedom and to the sense that the universe is inherently meaningless, because whatever meaning there is can only be a 'merely human' projection. The move that can be traced from early German Idealism to Schopenhauer can be seen somewhat schematically - German Idealism being a 
far more complex phenomenon than is often realised - as a move between these two opposed responses, responses which will later both occur in the work of Nietzsche. Despite their opposition, both responses to modernity attach considerable significance to art, either as that which provides images of what the world could look like if we were to realise our freedom and thus establish an appropriate relationship to the rest of nature, or as the sole remaining means of creating illusions which will enable us to face a meaningless existence. These positions are not necessarily wholly opposed: they both share a suspicion that the dominance of quantifying forms of rationality as the increasingly exclusive principle of modern life is part of what gives rise to the crises of meaning in modernity.

At the moment when philosophy becomes concerned in a rigorous manner with scientific method and the de-mythologising capacity of natural science, it also becomes concerned in novel ways with what is excluded by science. Nothing in the sciences provides a sense of the existential meaning nature can have for the individual subject. The point of science is the production of general laws which subsume individual cases and enable the manipulation and control of nature. In consequence, nature seen with the eyes of modern science can begin for many people to look like a machine which is being responded to in mechanical ways. Along with this suspicion of the possible effects of the new sciences goes the awareness that the growing domination of capitalist forms of exchange leads to nature being regarded in terms of the profit which can be extracted from it. One of the key ideas in the new subject of aesthetics is precisely that what makes an object beautiful has nothing to do with its usefulness or its exchange value. Even though artworks clearly do become commodities, neither their use-value nor their value as commodities can constitute them as works of art. The potency of aesthetic theory lies not least in its attempts to explore the implications of this special status.

Schelling states in 1800 that demanding usefulness from art 'is only possible in an age which locates the highest efforts of the human spirit in economic discoveries'. Given his admiration for the early Schelling, it is therefore no coincidence that many of Marx's insights into the social and cultural effects of capitalism have their roots in aesthetics. Marx's critique of the commodity form, for example, gains much of its force from the idea of the object which cannot be represented by anything else: the work of art. The idea of an intrinsic value in things which is independent of their exchange value is echoed in aesthetic theory's suspicion of the idea that nature is merely an object which is subsumable under general scientific rules, rather than something worthy of contemplation for its own sake. The process of rationalisation which leads to the penetration of rule-bound and quantifying procedures into all areas of science, administration and exchange is, then, both the irreplaceable foundation of the advances of modernity and the source of major uncertainties. Philosophical aesthetics responds to this process by providing a reminder that there are other ways of seeing nature and human activity, apart from the instrumental views offered by the sciences and commerce. The central new idea is that the beauty 
of nature need not have an ulterior function and can be its own purpose. Analogously, the rules of an art are seen as the self-legitimating products of human freedom, not as the result of the instrumental attempt to grasp objective necessities or natural regularities. Not surprisingly, in the light of the contemporary ecological crisis, the questions raised by aesthetics have come to seem more and more significant today. This renewed concentration on what can be learned from aesthetics makes it clear that there are no necessary grounds for assuming that concern with aesthetics should, as it often has been in the modern period, be connected to a rejection of rationality. Instead, art and the understanding of art can enable what has been repressed by a limited conception of reason to be articulated. The awareness of the danger of such repression which has, I believe, been one of the main sources of the appeal of post-structuralist critiques of 'metaphysics' that I discuss below and in coming chapters - is already apparent in the work of two of the founding figures of aesthetics: Alexander Baumgarten and J.G. Hamann.

Baumgarten's Aesthetica (Part 1 published in 1750, Part 2 in 1758: see Baumgarten 1988), and Hamann's Aesthetica in nuce (1762), begin to suggest what is at stake in the emergence of aesthetics as an independent branch of philosophy. Despite their obvious differences, Baumgarten and Hamann share a concern with the failure of the rationalist traditions of the eighteenth century to do justice to the immediacy of the individual's sensuous relationship to the world which is part of aesthetic pleasure. Philosophy based on the Cartesian ideal of 'clear and distinct ideas' finds art a problem because art lives from its particularity, which is not reducible to conceptual generalisation and so does not rely on clear ideas. Hartmut Scheible has suggested of Baumgarten that his life and work were 'formed by the short historical moment where it is possible, still safeguarded by an unshaken religious world picture, to devote one's attention uninhibitedly to the single empirical phenomenon' (Scheible 1984 p. 77). Aesthetic theory from Kant onwards, in contrast, often searches for the whole into which the single phenomenon can fit, once theological certainties have been abandoned, and this search is related to the other ways in which modernity attempts to make the world cohere, from the political to the scientific.

Both Baumgarten and Hamann are still able to celebrate the multiplicity of sensuous particularity, because each particular has its meaning in a whole which is divinely guaranteed. For Baumgarten the rules of art are simply different from the rules of logic. The natural sciences demand Cartesian intensive clarity', the analytical reduction of complexity to simple constitutive elements. Art demands 'extensive clarity', which allows ever greater differentiation into particularity. However, revaluing sensuous particularity and giving it primacy in one branch of philosophy poses instructive problems. How does one grasp the particular philosophically without abolishing its value as particular?

Since Parmenides Western philosophy had been suspicious of the unreliability of the world of sensuous particularity and had tried to transcend it by gaining access to an intelligible world of general essences; by the end of the eighteenth 
century the methods of modern science had come to seem by far the most appropriate way of achieving this. The very beginning of modern aesthetics therefore raises the question of the truth which may be attached to individual perceptions. Baumgarten reveals the incompatibility between a conception of truth based on sensuous particularity and a metaphysical world order, but this does not become a problem for him. The endless multiplicity of the particular and individual is an occasion of celebration, pointing to an infinity of meaning not, as it will often do subsequently, to a meaningless randomness. Baumgarten values aesthetic truth as the Wahrscheinliche, that which appears as true, even if it cannot finally be proved to be true, whereas the sciences can only ever claim truth for what is clear and distinct. The problem with the sciences is, then, that they exclude most of the content of what Edmund Husserl will later term the 'life-world', the untheorised horizon of our everyday experience, from any kind of truth. Baumgarten regards empirical perception as an inherent part of the truth of our relationship to the world, which is why he dignifies aesthetics with a constitutive role in philosophy. The question of the meaningfulness of this world does not arise, because our aesthetic pleasure in it suffices to fill the role played by metaphysics, even when the principle of the aesthetic - the particular - hints at problems to come. What happens - for Baumgarten this is evidently unthinkable - if there is no centre from which to organise the endless multiplicity, if this particular pleasurable moment has no connection with any other?

However little else he may share with Baumgarten, Hamann does share the wish to celebrate the endless multiplicity of the world. This wish is also grounded in theology: 'The unity of the creator reflects itself in the dialect of his works; - in all of them One note of unmeasurable height and depth!' (Hamann 1967 p. 114). Hamann's reliance on the word of God is based on an acute new kind of awareness of our dependence on language. Language for Hamann involves an endless process of translation 'from a language of angels into a human language, that is, thoughts into words, - things into names, images into signs' (p. 109), which never results in total communication between one person and another. Significantly, he thinks that the oldest language is music, and the coincidence of signifier and signified, the moment of identity, of 'representational' adequacy of what we say or write about the world to the world, is not his philosophical ideal. If this adequacy were to be achieved, it would prevent language's celebration of the exuberant fullness of existence, a celebration which is the basis of Hamann's conception of aesthetics. The signifying chain can therefore be celebrated for its endless differentiality precisely because it can never come to an end. The reasons for the more critical accent of Aesthetica in nuce are what makes Hamann a more modern figure than Baumgarten. Unlike Baumgarten, Hamann emphatically does not wish to integrate aesthetics into a narrowly Enlightenment conception of Reason.

Hamann insists, above all, upon the primacy of the image, of sensuous thinking, over its subsumption into generalised abstractions: 'Nature works through senses and passions. How can someone feel who mutilates its tools?' (p. 116). 
The 'Muse', poetry, 'will dare to cleanse the natural use of the senses from the unnatural use of abstractions' (p. 117). Reason is itself - and this is his decisive thought, which links him to later thinkers in the Romantic and post-Romantic traditions - dependent upon language. Language is never wholly separable from sensuousness, so it can never be said to be the pure articulation of truth. As such, Hamann claims, in a manner close to Rousseau, that 'Poetry [Poesie] is the mother tongue of humankind' and that 'Senses and passions speak and understand nothing but images' (p. 107). This empiricist-derived priority - Locke was a major influence - is directed against rationalist assumptions about the ultimate foundation of reason in mathematics, in an abstract 'intelligible' realm. For Hamann, then, scientific abstraction and reason are themselves constituted in particular historically-developed languages, which, along with their undoubted 'intelligible' aspect, have an ineliminable sensuous element.

The mathematically based 'Enlightenment' consequently depends upon a much more fundamental enlightenment: 'Let there be light! with this begins the sensation [Empfindung] of the presence of things' (p. 107). Despite his relation to empiricism, Hamann's proclamation here is actually closer to the later Heidegger's hermeneutic insistence upon the Lichtung des Seins, the 'clearing of being', which is the never fully articulable basis of the specific sciences, and which Heidegger sees as being manifest to us in language as the 'house of being'. Without the prior 'opening up' or 'disclosure' of being, thus without an inherent connectedness of ourselves to a meaningful world which is prior to any attempt to theorise such a connection, any account of a more specific cognitive relationship to being has no basis. Apprehending something as something, Heidegger argues, requires a structure more fundamental than is required to be able to quantify it or subsume it under an identifying rule or law once it has become manifest to us. Hamann's very particular, subversive notion of 'enlightenment' demands the inclusion of every aspect of sensuous contact with being in his philosophical project: hence the importance to him of the aesthetic in a conception of reason which is not to be based on exclusion of the particular.

In a strange way Hamann's thinking is therefore both behind and ahead of its time. His attachment to Locke's empiricism locates him in the past and, like Baumgarten, he holds on to a theological position which prevents the world from threatening to fall apart into its particularity. However, some of what he says, as the link to Heidegger already suggests, evidently prefigures the treatment of philosophy and language in post-structuralism: think, for example, of post-structuralism's insistence upon the 'materiality' of the signifier, or of Derrida's concentration on metaphor in philosophical texts as a way of questioning the dualisms of sensuous and intelligible in Western philosophy, as well as of his claim that communication relies on temporalised chains of signifiers that can never be completed. Hamann's ambivalent status is actually common to many of the thinkers to be considered in what follows, quite a few of whom were substantially influenced by him. Some of what these thinkers say belongs to theological and other ways of arguing that we may feel sure we have gone 
beyond; at the same time, significant parts of their work now seem to have a striking relevance to contemporary thought. At a time when suspicion of 'Enlightenment' reason is rife, it is surely important to look again at earlier versions of that suspicion to be found in the history of aesthetic theory.

\section{Aesthetics and 'postmodernity'}

In some quarters the recent critical debate about the nature of 'modernity' has led to the announcement that the whole 'project' of modernity has been discredited, and that we have consequently moved into a 'postmodern' era. According to such arguments, the modern era was established upon the 'principle of subjectivity'. The modern era is therefore characterised by the subject's domination of the object world which is achieved by reducing it to general concepts and by manipulating it technologically. Both those who rely on the idea of the postmodern and Jürgen Habermas - though he rejects the idea of the 'postmodern' - regard this principle as now having shown itself to be fatally flawed. They do so, however, for instructively different reasons. The former wish to question the idea of universal rationality, whereas Habermas wishes to sustain the universalising demands of rationality associated with modernity via a turn to intersubjective communication, rather than via reflection upon the nature of individual subjectivity. One of my main aims in what follows will be to show that subjectivity in modern philosophy is conceived of in more complex ways than have usually been acknowledged in many of the debates over modernity. The history of subjectivity in modern philosophy still current in these debates involves certain thinkers, usually Kant, Hegel and Nietzsche, to the exclusion of others. The ideas of Schelling, Schleiermacher, and the early Romantics, Friedrich Schlegel and Novalis, who are central figures in the present book, rarely appear in a serious form in these debates. However, philosophers like these, for whom aesthetics is a central concern, often advanced arguments as to why reason cannot ground itself in subjectivity that are closely related to contemporary arguments. At the same time, however, they also show why it is a mistake for philosophy to relegate subjectivity to being merely a function of something else, such as language, ideology, history, or the unconscious.

The story of modernity told by the proponents of the 'postmodern condition', like Jean-François Lyotard, has its roots in the work of Heidegger, and the power of Heidegger's ideas is also evident in the way they have influenced many contemporary theories of modernity. However, Heidegger's most notable arguments have themselves roots in the work of German Idealist and early Romantic thinkers, and Heidegger's thinking from the 1930s onwards is, significantly, never far away from questions concerning art. ${ }^{1}$ What links him to these thinkers from the early modern era is his questioning of the assumption that truth is adequately defined in terms of propositional assertion of 'what is the case', whether because God made it like that or because that is the way it is, independently of how we apprehend it. Heidegger himself makes too little 
of this link to the past, preferring to tell a story which highlights his unique role in reminding us of the 'question of being'. Western philosophy, he claims, has sought the truth about 'being' (Sein), by asking what being is or seeking an explanation of the fact that it is. Being consequently comes to be regarded as an object to be grasped by scientific theories, technological manipulation, or theology. The Neuzeit - the term Heidegger uses for what we have termed modernity - begins with Descartes, when the 'certainty of all being and all truth is founded on the self-consciousness of the single ego: ego cogito ergo sum'. Philosophy consequently becomes an expression of the 'subjectification' of being, in which everything is regarded in terms of its relation to our consciousness. Furthermore, the rise of the idea - now familiar from claims about 'artificial intelligence' - that consciousness can be explained in the same terms as the rest of nature is therefore also regarded as itself the product of the subject's growing control of nature. For Heidegger this sense of the subject's power is already adumbrated in German Idealism. German Idealism tries to prove that subject and object are identical, so that the way we think about the world and the world itself are inseparable, because the world is in fact a subject thinking itself. From Descartes, to Hegel's claim that 'the substance is subject', to Husserl's search for the 'principle of all principles', Heidegger maintains, the 'concern (Sache) of philosophy . . . is subjectivity' (Heidegger 1988 p. 70). Heidegger, then, poses the crucial question as to whether a philosophy based on subjectivity can establish a place from which it could really answer the question of how thinking and being relate. What is the relationship between the being of thinking, and the thinking of being?

In Heidegger's story - which is followed in certain respects by his pupil Hans-Georg Gadamer in Truth and Method - the emergence of aesthetics as a distinct part of philosophy is also itself part of the process of subjectification: beauty becomes solely a matter of subjective feeling, of 'taste'. Artworks are consequently reduced to the subjective contingencies of their reception, and an aesthetics based on subjectivity therefore has no way of articulating the truth in works of art. Moreover, near the beginning of the nineteenth century, Heidegger claims, the era of the production of great art itself comes to an end, because art can no longer aspire to be true in any emphatic sense, truth having come to be predominantly defined in terms of a subject's capacity for objectifying the world in science. This, for Heidegger, is the real import of Hegel's announcement of the 'end of art' as a form of truth in his Aesthetics that we will examine in Chapter 5.

However, it has often been noted that Heidegger conspicuously excludes music from the realm of great art in such reflections. ${ }^{2}$ Seen from a less philosophical and literary perspective, this exclusion can seem very strange. The fact that Beethoven's music is exactly contemporaneous with the supposed end of art already begins to suggest an alternative story of modernity which invalidates some of Heidegger's more emphatic claims. Because he uncritically adopts Hegel's view of music and Nietzsche's critique of Wagner, Heidegger is forced 
to see the growth of the importance of music in modernity as grounded in an attitude to art based just upon feeling 'which has been left to itself', and he links this to the notion that modern culture is the result of a decline from something greater. Music, he claims, lacks the seriousness of earlier art, and only 'great poetry and thinking' provide the kind of truth about existence which Heidegger demands. ${ }^{3}$ Anyone familiar with modern music must find this position highly questionable. The idea that the production - and even the reception - of music is based solely on feelings in the narrow sense is untenable: we would not even hear music as music if that were the case. The important consideration here is that the failure of certain positions in modern philosophy to take sufficient account of music often offers clues to why these positions are indefensibly reductionist in their understanding of modernity. This reductionism results from their inadequate response to music's complex relationship to verbal language. The non-representational, non-conceptual 'language' of music is seen by the early Romantics - who are one of the crucial exceptions to this reduction as enabling us better to understand aspects of ourselves which are not reducible to what can be objectively known and which are not to be written off as being merely inchoate feelings. At the same time, some aspects of music do also seem to be present in verbal language, and, as Schleiermacher suggests, this potentially introduces an aesthetic component, based, for example, on the particular rhythm and melody of utterances by a writer or speaker, into all communication. The 'subjectless' view of language characteristic of the later Heidegger and post-structuralism, which is summed up in Heidegger's remark that 'Language speaks. Man speaks to the extent to which he corresponds to language' (Heidegger 1959 pp. 32-3), simply does not offer sufficient resources for an adequate account of these issues.

Heidegger's inadequate account of subjectivity is, however, accompanied by a questioning of science and technology which is in certain respects more enlightening. For Heidegger the modern objectification of nature as a system of regularities which it is science's task to discover is only one way of interpreting how nature presents itself to us. Nature can, for instance, also be said to present itself to us through works of art: the attention to art as the counterpart of modern forms of rationalisation in Baumgarten and Hamann already suggested a model for this. Heidegger himself famously gives a role to certain forms of art as the 'happening of truth' because they 'disclose' being, without what is disclosed becoming, in the manner of natural science, merely a classifiable entity for a subject. One of Van Gogh's paintings of a pair of shoes is, he claims, 'the opening up of that which the material, the pair of peasant shoes is in truth. This entity steps out into the unhiddenness of its being', so that there is a 'happening of truth at work' in the painting (Heidegger 1960 p. 30), because it discloses the world in which the shoes possess their meaning (see Bowie 1997 chapter 7 for an extended discussion of this topic). Despite the questionable philosophical story about the subject which is attached to it (and the questionable choice of example), much of the substance of Heidegger's view of art echoes reflections 
on art which derive from the worries about rationalisation in post-Kantian philosophy.

The story Heidegger tells about subjectivity has, of course, spawned a number of imitations. In Lyotard's version of Heidegger's story the 'grand narratives' of Enlightenment that emerge in Kant and German Idealism, the stories in which (subjective) Reason was to free us from enslavement to nature and ourselves, have been wholly discredited. Lyotard equates reason with a dominating subjectivity, a will-to-power, without any acknowledgement of how complex the notion of subjectivity actually is in modern philosophy. In this perspective the modern insight into the dependence of consciousness on language, the so-called 'linguistic turn', is read as a major factor in the discrediting of subjectivity as the principle of modern philosophy. For Heidegger the dominance of the subject is subverted by our always already being located in languages which we do not invent and which we require in order to articulate our world. In the wake of Heidegger the very notion that subjectivity is a central issue in philosophy therefore gives way to the idea that subjectivity is an 'effect' of the 'discourses' or 'texts' in which we are located. Lyotard himself then adds a Nietzschean element to Heidegger's story, by claiming that the postmodern world is characterised by a multiplicity of agonistically competing and incommensurable 'language games', none of which can claim any ultimate legitimation for its way of articulating the world.

This story already begins to look questionable, however, when it is realised that the 'linguistic turn' takes place much earlier than is usually recognised. The origins of modern hermeneutics, the characteristic feature of which is the refusal to give any world view absolute validity, because of the need to interpret such views in particular languages, lie clearly in the period of Lyotard's supposedly totalising 'grand narratives' at the end of the eighteenth century. Hamann's critique of Kant in 1784 is based upon Kant's failure to see the necessary role of particular natural languages in the constitution of the 'categories' that are the condition of objective knowledge. In Schleiermacher's work after 1805, and in early Romantic reflections upon music and language, similar claims are used to give a view of subjectivity, language and art which can put into question many contemporary theories of language and literature. The real problem for the post-Heideggerian theorists of modernity is, therefore, the one-dimensional view of subjectivity upon which they base their diagnosis of the present.

The postmodern philosophical critics of modernity also largely ignore some very obvious features of modernity. The link between the centrality of the subject in modern philosophy and the dominance of science and technology over nature is, for example, hardly an immediate one. The emergence of modern aesthetics and its concern with aspects of subjectivity which are incompatible with wholesale rationalisation, such as the production and understanding of music as a new means of articulating feelings and moods, make this clear. The crucial fact is, of course, that scientific method and bureaucratic rationalisation actually attempt to exclude the individual subject in the name of 'objectivity', of 
what Thomas Nagel has termed 'The View from Nowhere'. This objectivity can indeed, as Kant argued, be said to depend upon its 'other', the subject: there would be no way of even understanding the term object without its counterpart. But the real question is the following: what sort of 'object' is the subject which is attempting to grasp reality objectively? Is it merely an individual will-based urge to control its other? Unravelling the complexities of this question as it appears in Kantian and post-Kantian philosophy will be one of the main tasks of the present book.

This question has, interestingly, re-emerged with a vengeance in the contemporary analytical philosophy of mind, where it has become the focus of serious worries about the scientistic direction of contemporary analytical philosophy. ${ }^{4}$ Nagel, who is himself in other respects a rather crude realist, suggests, in the manner of the thinkers we shall be examining, that:

One limit encountered by the pursuit of objectivity appears when it turns back on the self and tries to encompass subjectivity in its conception of the real. The recalcitrance of this material to objective understanding requires both a modification of the form of objectivity and a recognition that it cannot by itself provide a complete picture of the world, or a complete stance toward it. (Nagel 1986 p. 6$)^{5}$

The subjects that will emerge from the story told here will, then, not just be the objectifying tyrants of Heideggerian thinking because, as Fichte already shows in the 1790 s, the subject's capacity for objectivity reaches a limit when confronted with itself. - The fact that aesthetic theory is often concerned, for example, with the way in which the aesthetic object affects the subject without the subject wishing to determine the object suggests one implication of this view of subjectivity. Neither will the subjects be slaves to language: the capacity for situated linguistic innovation of the kind associated with the 'poetic' will be fundamental to the subject as conceived by some of the thinkers to be considered here.

It is, I want to argue, more apt to tell the story of modernity in terms both of the increase of control over nature, based upon the objectifying procedures of the sciences, and of the simultaneous emergence and repression of new individual attributes of human beings. These factors are complexly intertwined. Modernity evidently gives rise to greater possibilities for subjective freedom in all areas. This is immediately apparent in aesthetic production, where the diversity of means of expression and resources for new meaning increase, even if what is produced may not always attain to wider significance. At the same time, the scientific, organisational and technological factors which facilitate these possibilities can, as Max Weber argued, lead to an ever deeper feeling of the ultimate senselessness of that freedom and to irrational attempts to counter that senselessness. Modernity both creates space for the proliferation of individual meaning and tends to destroy the sense that such meaning really matters in the face of the dominant goals of society. The most important work in philosophical aesthetics attempts to confront the paradoxes involved in unifying the poten- 
tial for individual meaning that results from the decline of theology with the requirement that meaning should attain some kind of general validity.

Many 'postmodern' accounts of the subject in modernity are, even though they highlight important issues, often disturbingly narrow, relying on an overselective history of philosophy. Their appeal seems to lie in the attempt to diagnose the cause of the many symptoms of unease in contemporary Western culture, and they are, as such, vital indicators of real concerns. However, it is crucial to arrive at the best diagnosis of the sources of these concerns. The attempts to theorise the history of self-consciousness that appear in the philosophy I shall be considering also offer ways of understanding contemporary pathologies, from which everyone potentially suffers. The question is, then, which story of modernity is most plausible? Here there are no easy answers. All large-scale philosophical stories entail serious methodological problems. One should not, for example, just look for the history of an already constituted principle of subjectivity: that just pre-empts the issue in the manner of Heidegger making Descartes the supposed source of the essence of modern philosophy. Neither is one looking at anything objectively identifiable: the obvious fact about subjects in the sense at issue here is that they do not appear in the world. ${ }^{6}$ What is perhaps most worrying in thinkers like Lyotard is that they seem to regard subjectivity itself as the pathology underlying modernity. From this viewpoint, as Manfred Frank has argued, it becomes hard to see why one should bother to attempt to retrace the ways in which the supposed principle of modernity becomes pathological, because it ceases to be clear who or what suffers from it. Without an account of subjectivity that also acknowledges the desperately fragile and divided nature of individual human subjects the whole picture becomes distorted. Furthermore, such theories are often merely regressive, relying too heavily on some of the most questionable thinkers of modernity, like Nietzsche and Heidegger. In this sense, the portentous announcements of the radically new era beyond subjectivity that are present in the earlier Foucault and in Lyotard have more to do with repression of, than with a serious desire to engage with a past we cannot simply escape.

In this context it is notable that towards the end of his life Foucault himself, who had been one of the main sources of the idea of the death of the subject, became concerned with an 'aesthetics of existence' and with the invention of 'new forms of subjectivity' - something which, of course, already requires an inventor that would itself seem to have to be some kind of subject. In an interview in 1983 Foucault suggests that the 'transformation of one's self by one's own knowledge is, I think, something rather close to the aesthetic experience' (Foucault 1988 p. 14), and in 1984 he states: 'I do indeed believe that there is no sovereign, founding subject, a universal form of subject to be found everywhere . . . I believe, on the contrary, that the subject is constituted through practices of subjectification, or, in a more autonomous way, through practices of liberation' (1988 p. 50). If the subject can be constituted by 'liberation' there must, though, be some way in which one can conceive of what a free subject might be. 
Establishing this was one of the key tasks of aesthetic theory from Kant onwards, which - like Foucault - did not regard the subject as 'sovereign', and it is remarkable how far Foucault seemed to move back towards this tradition in his later period. ${ }^{7}$ Given this shift of perspective by a thinker noted for his earlier antagonism to subjectivity, the issues of aesthetics and subjectivity seem ripe for a re-examination.

The story I wish to tell is primarily motivated by recent theoretical debates, and not simply by interest in the history of philosophy. This explains the perhaps eccentric emphasis on certain thinkers and the omission of others. The most obvious of these is Schiller, who has anyway so far attracted much more scholarly attention in English than Schelling, Schleiermacher and the early Romantics, and whose account of the subject, though it was a major influence on some of these thinkers, is not as sophisticated as what they developed from it. I have not been able seriously to consider questions of influence, and the social and political importance, for their own time, of the ideas I consider is necessarily a secondary consideration. The relevant texts are often so complex that it is actually hard to know what effect they would have had. The first task in relation to these texts is, I believe, to attempt to understand them as responses to certain key philosophical and socio-political problems that still concern people now. Some texts to which I devote considerable space had not yet been published at the time they were written and were only heard as lectures or read as transcriptions of lectures; if they were published, they were not always widely read. The complexities of the route which leads, for instance, from Schelling and Schleiermacher, via Feuerbach, to Marx, or which leads from Schelling, via Schopenhauer, to Nietzsche - and beyond - are too great and still too little researched to allow one to make many really decisive assertions about influence. Any such assertions would also have to confront the fact that many relevant documents may have been lost forever.

Despite these problems, it is very clear that the existing stories in the English-speaking world about German Idealism, Romanticism and hermeneutics are still in need of substantial revision, as is the assessment on both Left and Right of the status of aesthetic theory in philosophy then and now. This revision will also require a different view of the origins and validity of many positions in contemporary literary theory and analytical philosophy. I have for this reason often given a substantial amount of exposition of little known arguments: without a serious engagement with these arguments some of the dead-ends of recent theory seem bound to recur. The story, of course, does not stop where I do. In a book published in 1997 I traced a route 'From Romanticism to Critical Theory' (Bowie 1997) which gives detailed accounts of the relationship of Heidegger, Benjamin, Adorno and others to the tradition at issue here. The two volumes do, I hope, complement each other, and the present revision takes some account of the research done for the later book. 


\section{Notes}

1 For a detailed demonstration of this, see Bowie (1997).

2 Rüdiger Safranski tells the story of Heidegger in 1944 hearing Schubert's last sonata in B flat and claiming: 'We cannot do that in philosophy' (Safranski 1998 p. 371).

3 There are also clear indications elsewhere in Heidegger's later philosophy that he finds music more important than he does in the work on Nietzsche in the 1940s I am considering here. On Heidegger and music, see Bowie 1999.

4 See, in particular, Frank 1990, for a detailed engagement with the links between the hermeneutic and analytical traditions.

5 Sadly, my hopes in the first edition of this book that Nagel's work would help establish a dialogue between the traditions has, in the case of Nagel himself, in contrast to Rorty, McDowell, Putnam, Brandom and others, led only to ill-informed invective against a misleadingly characterised 'hermeneutics' (see Nagel 1995).

6 I do not, then, in the manner of Strawson and others, equate personhood with subjectivity.

7 See also Peter Dews, 'The Return of the Subject in late Foucault', Radical Philosophy 51, Spring 1989, pp. 37-41. 


\section{Modern philosophy and the emergence of aesthetic theory: \\ Kant}

\section{Self-consciousness, knowledge and freedom}

The importance attributed to aesthetic questions in recent philosophy becomes easier to grasp if one considers the reasons for the emergence of modern aesthetic theory. Kant's main work on aesthetics, the 'third Critique', the Critique of Judgement (Cf) (1790), forms part of his response to unresolved questions which emerge from his Critique of Pure Reason (CPR) (1781) and Critique of Practical Reason (1787). ${ }^{1}$ In order to understand the significance of the $C F$ one needs therefore to begin by looking at the first two Kantian Critiques. ${ }^{2}$ The essential problem they entail, which formed the focus of reactions to Kant's work at the time, lies in establishing how the deterministic natural world, whose mechanisms are becoming more and more accessible to the methods of the natural sciences, relates to the world in which we understand ourselves as autonomous beings. The third Critique tries to suggest ways of bridging the divide between these apparently separate worlds by giving an account of natural and artistic beauty, relating beauty to natural teleology, the purposiveness of individual organisms and the possible purposiveness of nature as a whole. In doing so, however, it threatens to undermine essential tenets of the first two Critiques. The third Critique is not least significant because of the ways in which it informs subsequent attempts in German Idealism to integrate Kant's philosophy into an overall system, some of which give a major role to aesthetics. The $C \mathcal{F}$ has, furthermore, become increasingly important in contemporary discussions of Kant's work, appealing on the one hand to those who wish to question the perception of Kant's enterprise as merely an exercise in legitimating the natural sciences, and on the other to those who see the need to extend the scope of epistemology if it is not to founder on the problems that become apparent in the first two Critiques.

Dieter Henrich regards the crux of Kant's epistemology as the justification of 'forms of cognition from the form and nature of self-consciousness' (Henrich 1982 p. 176). The philosophical problem is therefore how the form and nature of self-consciousness are to be described. Descartes had famously located the certainty which warded off the scepticism generated by the unreliability of empirical perception in the thought that even if I doubt everything I think I 
know about the world I must exist qua doubter. Doubting the thought of my existence involved an undeniable existential relationship of the doubter to the thought that is being doubted, which therefore provided a minimal point of certainty: I must at least exist as a thinking being. However, Descartes provided very little else by this argument, and he needed God to establish the bridge back to a reliably knowable world outside self-consciousness. Kant tries to extend the certainty about the world to be derived from self-consciousness without using this theological support. How, though, can subjectivity be its own foundation? How can subjectivity itself give rise to objective certainty without relying upon the 'dogmatic' assumption of a pre-existing objectivity of the world of nature which the arguments of David Hume about the contingency of our knowledge of causal connections had rendered untenable for Kant?

Kant argues that any linking of perceptions from the multiplicity of what is given to us from the world in 'intuition' (the term is discussed below) requires a synthesis, which creates identity from inherent difference. Given that we arguably never receive the same input of perceptual data at any two moments of our lives, and yet are able to establish testable laws of nature, repeatable knowledge must, Kant maintains, rely on identities provided by the continuity of selfconsciousness. What we know by synthesis therefore cannot be wholly derived from our receptive, sensuous experience of nature, which, without the forms of identity involved in synthesis, just consists in endless particularity. The crucial issue therefore becomes the nature of the identity of that which synthesises, the identity of the 'subject'. As Henrich shows (pp. 179-83), Kant shifts Descartes's emphasis on the existence of self-consciousness, which is only ever certain at the moment of its reflection upon itself, on to the relationship of the thinker to every thought that the thinker could have, believing that if one can show that this relationship itself requires a binding identity there will then be a way of maintaining a cohesion in philosophy which is not derived from an external source. The ability to describe the rules which allow one to move from one case of 'I think' to the next thus becomes decisive. Unless philosophy can give an account of the ways in which self-consciousness is sustained between different cases of 'I think', it will be forced to abandon the only certain foundation it can now have.

Kant is insistent, then, that we can only know the world as it appears to us via the constitutive a priori 'categories' of subjectivity which synthesise intuitions into cognisable forms. The world as an object of truth is therefore actively constituted by the structures of the consciousness we have of it, which means that we cannot know how the world is 'in itself'. Instead of cognition following the object, the object comes to depend upon the subject's constitution of it $a s$ an object by giving it a repeatable identity in a predicative judgement. However, Kant's investment in the distinction between appearances and things in themselves has a further motivation, which does not just derive from his epistemological arguments: the distinction is meant to establish the role of freedom in his philosophy.

Nature, for the Kant of the first Critique, is generally defined 'formally', as 
that which is bound by necessary laws constituted by the subject. If the laws of nature were properties of the object world 'in itself' they would also apply to ourselves in every respect. In such a view the world, including ourselves, would become a deterministic machine and human responsibility would be an illusion. As a follower of Rousseau and the Enlightenment Kant is, though, most concerned with the fact that human agency involves the ability to follow moral imperatives which cannot be explained in terms of causal laws. His idea is that if I too am subject to the division between how things appear to consciousness and how they are in themselves, my action can be subject to causality as an event in the world of appearing nature but free as something in itself. The will which determines my action is thus inaccessible to cognition - if cognition is understood in Kant's sense of the synthesising of appearances by categories and concepts - in the same way as the thing in itself is inaccessible. We only have cognitive access to effects of the will, not to the will itself. As a consequence, we seem to exist in two distinct realms. We are determined by the laws of nature as sensuous beings in the realm of appearance, even though these are laws which are 'given to nature' by us. At the same time we are also free agents, who can be held to account for our deeds by other free agents.

Such a division raises fundamental problems with regard both to the access we have to our own capacity for cognition and to the capacity of our will to transcend determination by natural laws. The basic question here is: what sort of 'object' is this subject when it wishes to 'intuit' itself? The difficulty is actually very simple: what Kant wants to describe is not empirical, and what is not empirical is, for Kant, not a possible object of knowledge, in the sense of that which is arrived at by the application of concepts to intuitions, at all. ${ }^{3}$ However, Kant is convinced that we have sufficient warranted knowledge available to us Newton's laws, for example - to be able to attempt to see $m$ hy that knowledge is warrantable, without invoking theology or unquestioningly accepting that science represents a pre-existing reality. There can be no doubt about the truth of the propositions of mathematics and their capacity to generate potentially valid scientific knowledge. Neither does Kant doubt the existence of binding moral imperatives. What he wishes to establish is what in us makes them possible.

It should already be clear, however, that the explanation of what makes cognition and morality possible will have to be achieved in Kant's theory at the expense of everything individual in the subject. The point is to establish what is necessarily the case for everyone who counts as a rational being, now that theological foundations can no longer be invoked. What, though, of the new insistence by Baumgarten and Hamann on the value of the particularity of individual experience of the world we observed in the Introduction, which is essential to aesthetic theory? At the beginning of the main text of the second edition of the $C P R$ (B p. 36), the 'Transcendental Aesthetic', Kant adds a footnote about Baumgarten's new use of the word 'aesthetics' to designate the 'critique of taste'. He suggests that the attempt to bring judgements of beauty into philos- 
ophy will be in vain because such judgements are always based on empirical rules which cannot have the binding force of the rules of a science. In the $C P R$ Kant prefers to reserve the use of the word aesthetic for its old meaning of the 'science of all principles of sensuousness' (B p. 35, A p. 21). By the time of the CF, however, Kant will have overtly taken on more of what motivates Baumgarten's conception (in his unpublished 'Reflections' he had already concerned himself more seriously with aesthetics in the new sense). He writes a complex philosophy of aesthetic judgements which aims to establish links between nature in itself and the freedom of rational beings. The reasons for his desire to establish these links relate to difficulties that become apparent in the first Critique.

\section{The unity of the subject}

The success of Kant's philosophy depends upon the legitimacy of certain distinctions. However, these distinctions turn out to be hard to sustain. The 'transcendental aesthetic', the account of the conditions of perception, relies, for example, on the difference between 'empirical intuition', and the concepts which judge it. English-language philosophy has come to use the technical term 'intuition' to translate the German word Anschanung, which plays a decisive role in the philosophy of the period. The word has several senses, which are not always clear. In everyday usage anschauen simply means to 'look at', but in philosophy it is often used to designate various kinds of contact between a subject and its 'other'. Kant regards intuition as the most immediate form of relation of the subject's cognition to an object, which 'only takes place to the extent that the object is given to us' ( $C P R$ B p. 33, A p. 19), and he equates this with anything being 'real'. As he puts it in the 'Postulates of Empirical Thought':

existence has ... to do ... only with the question: whether such a thing is given to us in such a way that the perception of the thing could always precede the concept. For the concept's going before the perception means only its mere possibility; but perception, which gives the material to the concept, is the only character of reality. (B p. 273, A p. 225$)^{4}$

This lays the ground for the distinction between appearances, objects as given to us, and things in themselves, which are not given to us as appearances. The question is how 'inside' and 'outside' relate to each other, and here everything turns on the status of 'intuition'.

A basic example of intuition is the sensation of the hardness of a piece of rock. However, Kant separates empirical intuitions like this from what he terms 'pure intuition', which is concerned with 'extension and form' of the object and is the foundation of geometry. 'Pure intuition' refers to the framework within which we necessarily apprehend things. There are two forms of pure intuition: space and time. We cannot conceive of an object of the senses which would exist in a non-spatial and non-temporal form: we can never apprehend all of an object 
at once, even though we assume that all the object's aspects do exist at a particular time. We can, though, conceive of functions of thinking which are nonspatial and non-temporal, namely those aspects of thought which are wholly non-empirical, including the categories. Categories are forms of judgement, of synthesis, which are universally applicable to anything that can count as an object (which must be one or many, of a certain size, standing in a certain relationship to other objects, caused by something, etc.). To apprehend a sequence of events as causal, for example, one requires the particular form of synthesis that is designated by the category of causality. One can see $b$ follow $a$ in time (on the basis of the continuity of the I between the two events), but one cannot see the necessity of this occurrence that makes it a causal occurrence rather than a random connection of contingently related events. Categories, then, make possible the syntheses of intuitions which turn intuitions into reliable cognitions. Cognition has two distinct sources: 'receptive' sensuousness, which provides intuitions, and 'spontaneous' understanding, which can think objects as objects of knowledge by applying iterable categories, such as causality, and concepts to different intuitions.

The first stage of making coherence out of the multiplicity of sensuous intuitions is the effect of Einbildungskraft ('imagination'). Imagination is the power to organise images (Bilder) given in intuition into what Kant terms 'schemata' which can then be subsumed under categories and concepts. In version ' $A$ ' of the $C P R$ the 'imagination' both produces associations which cohere into something cognisable and is able to reproduce intuitions without the object of intuition being present. The imagination in this version seems both productive and receptive, which already suggests an ambiguity concerning the fundamental distinction between intuitions and concepts. Productivity is a function of the understanding, which is necessary for any synthesis of intuitions; receptivity is the characteristic of sensuousness. In the 'B' version of the CPR (1787) Kant changes the role of the imagination, in order to sustain the boundary between what we contribute to the world's intelligibility and what the world contributes, by subordinating the reproductive imagination to the functioning of the categories of the understanding. He therefore planned (but did not actually do so) to remove the famous description of the imagination as a 'blind but indispensable function of the soul without which we would have no knowledge' (B p. 103, A p. 78) and replace it with the assertion that all synthesis is based on the understanding. ${ }^{5}$ The problems lurking in the idea of a boundary between spontaneity and receptivity become most apparent in the decisive part of Kant's account of the structure of our subjectivity, the attempt of the I to describe itself. ${ }^{6}$ It is this account, the 'transcendental deduction of the categories', 7 which will have a major effect on German Idealism and early Romanticism, and thus upon the history of aesthetics.

The problem is that the highest principle of philosophy, the I, cannot be available as an 'intuition' in the sense Kant has so far employed in the $C P R$, for the following reasons. In order to overcome the key problem in Descartes Kant 
introduces the idea that 'The I think must be able to accompany all my representations' (B p. 132). This idea Kant terms the 'synthetic unity of self-consciousness', because different moments of consciousness are unified by the essential principle. He opposes this synthetic unity to Descartes's cogito, the 'analytical unity of self-consciousness', in which the thinking and the being of the subject are immediately identical, albeit only at the moment when I doubt my existence. The immediate 'Cartesian' consciousness of myself which can accompany each thought, and which makes it $m y$ thought, does not link this particular 'I think' to any other case of 'I think'. It is only through a synthesis of different moments of such consciousness that I can become aware of the identity of my own self-consciousness across time and can have a principle of unity for my representations. Synthesis is dependent upon an act of 'spontaneity' (B p. 133): it is 'self-caused', rather than being caused by something else. If it were the result of something else the task would then be to ground that something else in something else as its cause, and so on, either until one found a first cause, or ad infinitum, in which case the synthesis would never happen, and selfconsciousness - and knowledge - would become impossible.

The spontaneity in question therefore cannot itself be part of sensuousness, because everything sensuous is part of the causally determined world given in intuition. What is given in sensuous intuition is synthesised by the understanding, according to the rules of judgement - the categories - whose employment Kant is trying to legitimate in the transcendental deduction. This means, though, that the identity of consciousness actually seems to depend upon the multiplicity of intuitions which it synthesises according to the prior rules of the understanding. In the later-added passage on the 'refutation of idealism' Kant claims that 'even our inner experience, which was indubitable for Cartesius, is only possible under the presupposition of external experience' (B p. 275). However, Kant also claims in the Deduction that the synthesising process itself is what enables me to identify myself: 'only via the fact that I can grasp the manfold [of representations] in one consciousness do I call them all $m y$ representations, for otherwise I would have a self which is as differently multi-coloured as I have ideas of which I am conscious' (B p. 134). The problem is, of course, that there is no explanation here of what makes these representations mine. The identity of the I must, however, surely be established independently of the endless difference of what is given to it. The I, in Kant's terms, is a set of linked cognitive rules that process intuitions into the unity of experience and has a 'synthetic unity'. But what sort of awareness do I have of this self, according to Kant?

Kant's answer seems to boil down to the fact that access to our self-awareness can only be of the same order as our intuition of objects: 'Not the consciousness of the determining, but rather only that of the self that can be determined, i.e. of my inner intuition (to the extent to which its manifold can be connected in accordance with the general condition of the unity of apperception in thinking), is the object' (B p. 407). But this leads to much the same 
problem as the one just mentioned. The synthetic unity of the I is not guaranteed by our empirical perception because it is self-caused, although Kant claims empirical perception is a necessary condition of it. Kant talks of the 'pure synthesis of the understanding which is the a priori foundation of the empirical synthesis' (B p. 140) - meaning the I which, even though I am not aware of it in the sequence of differing experiences, must be there to accompany my experiences, thus making them mine - but then does not give any further account of this pure spontaneity. Manfred Frank points out what is lacking in Kant's account: 'In order to be aware of its own appearance (in time) the simple being of self-consciousness must always already be pre-supposed - otherwise it is as if the self-awareness were to lose its eye' (Frank 1985 p. 39), because it would not have any grounds for seeing representations in time as $i$ ts representations. This consciousness must already exist in some way if it is to be amare of the series of intuitions which constitute its appearance as object to itself as its omn intuitions.

Kant acknowledges this problem, but does nothing to solve it:

I am conscious of myself in general in the synthesis of the multiplicity of representations, therefore in the synthetic original unity of apperception, not as I appear to myself, neither as I am in myself, but only to the extent that $\mathrm{I}$ am. This representation is thinking, not intuition. (CPR B p. 157)

The I must, then, have some kind of ontological status which is not exhausted by what can be said about it as appearance in 'inner sense'. However, nothing can be said about the manner of its existence because it cannot be given in intuition, which, as we saw, is Kant's criterion for objective existence. Kant later expends considerable effort in refuting the argument that the $I$ is to be regarded as any kind of persistent 'substance' to which attributes are attached, but does not get any further with the basic problem. ${ }^{8}$ His problem is acutely summed up by Novalis in 1796: ' $\mathrm{I}$ is basically nothing - Everything has to be given to it - But it is to it alone that something can be given and the given only becomes something [i.e. an object in Kant's sense] via the I' (Novalis 1978 p. 185). How, though, is the I given to itself? As Novalis puts it: 'Can I look for a schema for myself, if I am that which schematises?' (p. 162).

If I wish to know myself I need to use the condition of knowing itself, which is self-caused, but I can only use it in relation to my appearing self, to the representations I have of myself and the world, which Kant insists I do not create. Self-consciousness is not ultimately a cognition of oneself, because cognition is only possible in relation to what is given as appearance. Even for Kant, then, 'reflecting' upon oneself as an object, splitting oneself into subject and object, cannot give a complete account of the nature of self-consciousness. A full 'cognition' of oneself would have to be an intuition of something which is wholly in the realm of the 'intelligible', the realm of spontaneity. This would entail a selfcaused intuition of the self-caused synthesiser of intuitions, which requires an 'intellectual intuition'. Kant rejects this notion as applied to the self because it 
contradicts his argument that intuitions without concepts are blind and concepts without intuitions are empty, which means that the identity of the I depends upon its having intuitions given to it to synthesise. Kant sees intellectual intuition as the mode of awareness of a deity which creates the objects of its thought, rather than relying on input from outside itself for the material of its thought. Frank sums up Kant's objection to intellectual intuition as follows: 'The whole deduction of the possibility of an objective cognition would become redundant if Kant were to have assumed an intelligence which creates its object from its own powers' (Frank 1985 p. 43). The intelligence Kant thinks he can describe constitutes the objective truth, not the existence, of the object, via the use of its synthetic powers in relation to the appearing spatio-temporal world. However, the truth of the subject is not adequately articulated in such an account. The highest cognitive point of philosophy cannot know itself, even if it must assume the necessity of its own existence. In the Paralogisms Kant claims that the 'The proposition, I think, or I exist as thinking is an empirical proposition'. ${ }^{9}$ Empirical propositions require intuitions if what they express is to be real, but what kind of intuition does the I have of itself? In a remark whose implications will, as we shall see, be echoed and expanded in key aspects of Romantic philosophy, such as the work of Schlegel and Schleiermacher, Kant claims in the Prolegomena to Any Future Metaphysics that the 'representation of apperception, the I', is in fact 'nothing more than the feeling of an existence without the least concept and only a representation of that to which all thinking relates' (Kant 1989 p. 106; p. 136 of original edition). As we shall see in relation to the $C \mathcal{F}$, this invocation of 'feeling', which probably has its source in Rousseau's 'Profession of Faith of a Savoyard Vicar' from Émile, will come to have very significant ramifications.

Kant faces a serious problem in using self-consciousness as the highest principle of epistemology, and the same problem recurs in his moral philosophy. When considering the reasons for the separation of law-bound appearances and freedom, we saw that there can be no evidence of freedom, because it cannot be an object of the understanding identifiable by a concept. Freedom is, though, the centre of Kant's enterprise. He talks of a 'causality through freedom' which brings about new states of the world via our ideas of what should be the case. Reason, the 'capacity for purposes', therefore realises something which cannot be empirical - freedom - in the world. But how are we to know this? We shall see in a moment how Kant's aesthetics later tries to respond to the difficulties inherent in the appeal to non-empirical freedom. Reason itself is in one sense 'infinite', because it cannot be determined by anything finite we know about the world. It 'shows a spontaneity so pure that it goes far beyond everything with which sensuousness can provide it' (Kant 1974 BA p. 109). The recurrence of the term 'spontaneity' here is important, because it links the problem of our free will to the problem of describing the existence of our cognitive self-consciousness in a manner which, via Fichte, will be crucial for the whole of German Idealism.

Kant is, as it were, in the position of Moses in Schoenberg's Moses und Aron: 
the most fundamental part of his philosophy cannot be explained, just as Moses is faced with the unenviable task of persuading people to believe in a God they cannot see. As Kant puts it:

For we can explain nothing except what we can reduce to laws whose object is given in some possible experience. But freedom is a mere idea, whose objective reality cannot be shown either according to laws of nature and thus also not in any possible experience. (BA p. 120)

Our will can seem to be motivated by natural drives, which are explicable in terms of the understanding because they are based on biological laws. However, the will can also be seen as motivated by something in the realm of the intelligible, which cannot be explained. We can exercise our rational will in order to bring about changes in the world which are guided by ideas of what ought to be the case and which would not occur if all were ruled by natural determinism. Kant thinks the fact that we can act in ways which are not in accordance with our appetites and which are in accordance with our moral duty reveals a potential for universally binding laws that point to a higher purpose in existence. In his moral philosophy Kant is, however, left once again with the problem that the fundamental principle cannot be articulated. As he does in the first Critique, Kant more than once invokes the spectre of 'intellectual intuition'. Discussing the categorical imperative - 'Act only according to that maxim through which you can at the same time mill that it becomes a universal lam' (BA p. 52) - he says that our consciousness of the imperative is a 'fact of reason'. Evidence for this fact would, though, require access to freedom, which would entail 'an intellectual intuition which one cannot in any way accept here' (A p. 57). In both the theoretical and the practical parts of his philosophy, then, Kant leaves a gap where the articulation of the highest principle should be located.

\section{The unification of nature}

The first two Critiques leave open how the new focus of philosophy, the subject, could give a grounding explanation of its activities in a non-circular, nontheological fashion. Furthermore, Kant's account of the understanding only explains how it is that we can generate a potentially infinite number of laws of nature. When it comes to the question of whether these laws constitute an overall system Kant claims we can only assert that they do so as a 'regulative idea' of reason. He thus acknowledges the need for the idea of the universe as a unified totality if the products of the understanding are not to be a merely random series of laws, but he is aware that this does not prove in any way that the universe is in fact such a totality. But without such proof there is no philosophical answer to the questions of $m h y$ we are able to synthesise intuitions in a law-bound manner, or $w h y$ we have a capacity for choosing to act contrary to natural inclination. Has this latter ability a goal which is linked to the ways in which we see nature functioning, or is it completely independent of what we know about nature? 
It would seem strange if there were no connection at all, because, for Kant, our cognitive faculty and our freedom are both self-caused and yet can have effects on the natural world. If one cannot find a way beyond this account of our relationship to internal and external nature the consequences can look disturbing. Is our capacity for reason, for example, as Hobbes and La Rochefoucauld had argued, actually just a form of self-interest based on self-preservation? This is, of course, not just a theoretical problem. Kant's separation of the spheres of epistemology and ethics into different aspects of ourselves mirrors the ways in which the spheres of science and technology become separated from the sphere of law and morality in modernity. As the new forms of cognitive relationship to nature produce the ability to manipulate nature to an ever greater extent, nature comes to be seen in terms of regulation and classification, making it primarily into an object which the subject determines. Grounding decisions about what ought to be done with the limitless capacity of the understanding to generate new knowledge thus becomes an unavoidable problem because the understanding itself can offer no indications concerning the desirability of using what it can produce.

The last thing Kant would have wanted are the concrete consequences of the division between what will become reduced in much of the philosophy of the nineteenth and twentieth centuries to 'fact' and 'value'. What he sought were ways of unifying understanding and reason without falling back into 'dogmatic' rationalism. The actual historical result of the divisions Kant reveals can be seen in terms of the separation of a world of cold scientific objectivity from a world lacking in ethical certainty and intrinsic meaning. However, this separation suggests a way of understanding the importance of a further dimension of modernity, namely the complex and diverse development of modern art, and the development of new philosophical ways of understanding art. Art is often seen as the location where those aspects of the self and the world, which are excluded by the dominant processes of modernity, can find their articulation. It is for this reason that the emergence of aesthetics is an important disruptive factor in the development of modern philosophy. Baumgarten and Hamann had already suggested why: aesthetics attends to that which is not reducible to scientific cognition and is yet undeniably a part of our world. Despite the strictures of the first Critique, Kant himself acknowledges the vital importance of this aspect of philosophy, even at the cost of putting some of his most fundamental ideas into question.

The $C \mathcal{F}$ attempts to show that the kind of consensus achievable via the compelling results of the activity of the understanding may be present, at least as a possible aim, in realms in which the understanding has no right of legislation. The need for the results of activity of the understanding to be integrated into the aims of reason becomes urgent when it becomes clear that the rules of the understanding can be applied to anything at all, because traditional constraints on what can or should be known no longer apply. It is no coincidence that attention to the Faust story in this period is so widespread. Divorced from any sense 
of the possibility of its goals being integrated into a meaningful whole, the sort of science which reduces nature to being a rule-governed machine can be seen as leading to what Horkheimer and Adorno will term a 'dialectic of enlightenment', in which liberation from theological tutelage creates new forms of imprisonment, despite the massive increase in technical control over nature. Kant's text may seem initially far from such wider concerns, but attention to the detail of his conception can show that it is not.

The basis of the third Critique, the 'pomer of judgement' - Urteilskraft - is the synthesising capacity which connects the particular to the general. This can happen in two ways. The first is when the understanding subsumes the particular intuition under a general category or concept. Kant terms this 'determinant' or 'determining' judgement. The law exists prior to the individual case and is to be applied to it. It is, though, anything but self-evident how one decides which law is applicable in any empirical case without getting into a regress of laws for the application of laws. Kant tried to address this regress in the $C P R$ via the schema, which creates empirical equivalents of a priori categories: perceivable existence at all times is, for example, the schema of necessity. The schema also makes concepts sensuous by immediately apprehending different parts of the otherwise inherently particular sensuous manifold as coherent entities of the same kind. In order to see the chihuahua and the Great Dane both as dogs, for example, one needs something which reduces their empirical difference to what can be subsumed into a general concept. The schema therefore connects the two sources of knowledge, sensibility and understanding. The problem of judgement underlying the notion of the schema, which Kant addresses in the $C \mathcal{F}$ in the account of the second kind of judgement, 'reflective judgement', will turn out to be one of the most durable in modern philosophy (see Bowie 1997). As we saw with regard to the understanding's relation to reason, no amount of applying determining judgement to nature will demonstrate that the multiplicity to which it applies has any kind of ultimate unity. The establishing of natural laws cannot in any way guarantee a systematic relationship between such laws, because that would require a higher principle which demonstrated that they fitted together. A principle of this kind would reintroduce 'dogmatic' metaphysics because it would cross the boundary of sensuous and intelligible by presupposing that nature is inherently intelligible. How are we to know that those parts of nature for which we as yet have no laws are subject to laws at all?

All the laws of nature we think we possess are based on intuitions which fill concepts, thus on what Kant means by 'reality', in the particular sense we examined above, and reality is contingent. We therefore have no cognitive grounds in Kant's terms for regarding nature as a unified system. In order to confront this problem Kant introduces the second form of judgement, 'reflective' judgement, whose task is to move from the particular to the general. This entails presupposing a higher principle inherent in nature, as otherwise nature becomes merely a 'labyrinth of the multiplicity of possible particular laws' ( $C 7$ p. 26). 
However, the principle is a necessary fiction, which assumes that nature in fact does function in a purposive way, 'as if an understanding contained the basis of the unity of the multiplicity of its empirical laws' (B p. XVIII, A p. XXVI). Kant underpins this position with the crucial claim that natural products are on the one hand mechanical, in terms of the laws which apply to them, but, on the other hand, when seen as 'systems, e.g. formations of crystals, all sorts of forms of flowers, or in the inner construction of plants and animals' they behave 'technically, i.e. at the same time as art' (p. 30). 'Art' here has the Greek sense of 'techne', the capacity to produce in a purposive way. Natural products appear to contain an 'idea' which makes them take the form they do, in the way an artist can realise an idea by making a work of art. It is as if the whole of an organism preceded the parts which we can analyse in the terms of the understanding: ' $A n$ organised product of nature is that in which everything is an end and on the other hand also a means. Nothing in it is in vain, pointless, or to be attributed to a blind mechanism of nature' (B p. 296, A p. 292). Kant here adverts to forms of intelligibility which scientific knowledge can never make accessible and which are fundamental to our comprehension of our own existence.

Although the problems addressed here begin as epistemological ones, it is important to see how they also lead in other directions which are central to the issues of this book. Kant's claims about organisms, for example, echo the second Critique's concern that rational beings should always be ends in themselves, and not just the means for the ends of other beings. If rational beings were solely means and not also ends the result would be a regress in which each being is just the means for another being, and so on, which would mean no being had any purpose in itself at all. The idea of the natural organism, in which nature is purposeful in a manner which scientific knowledge cannot grasp, relates to an analogous problem. In the $C P R$ Kant claims that the regress which results from each natural thing simply being conditioned by another thing that had in turn been conditioned by something else, etc., can be circumvented. While reason must postulate the "unconditioned ... in all things in themselves for everything conditioned, so that the series of conditions should thus become complete'

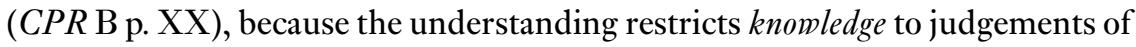
appearances, rather than trying to gain access to 'things in themselves', the contradiction of seeking what the contemporary writer and critic of Kant, F.H. Jacobi, termed 'conditions of the unconditioned' can be avoided. ${ }^{10}$ However, the subject's very ability to stop the regress in judging anything particular (which is the function of schematism) seems, as Fichte would soon argue, to give the subject itself the 'unconditioned' role in determining how nature is to be understood. This seems to be the case even though the ultimate nature of 'things in themselves' involves the whole grounding dimension of nature which is not accessible to our knowledge and though the nature of this subject is opaque in certain key respects.

A significant worry therefore arises here with regard to the status of the subject, which we shall later encounter in Schelling's criticisms of Fichte, and 
which now plays a role in contemporary ecological debates. When the subject analyses an organism it usually destroys the integrity of what it analyses. The plant which has become an object of scientific dissection can cease to exist as an organism because it has been taken apart. There is, of course, no inherent limit to how far such destruction can go, as the twentieth century has taught us. Furthermore, scientific analysis of nature cannot make nature into a coherent whole because of the regress we have just encountered. Nature seems therefore to be threatened in two ways, both of which have to do with the lack of a holistic conception of its relationship to our cognitive activity. On the one hand, the cognitive approach to nature threatens to disintegrate it into the endless multiplicity which results from the analytical functioning of the understanding. On the other, for the understanding to do its work, nature must be reduced to general principles based on the subject's capacity for abstraction. These principles enable the workings of nature to be predicted and controlled more effectively, but they have no necessary concern with the particularity of their object, and each principle's relationship to all the others is of no concern to the functioning of the understanding itself. The need to establish holistic conceptions that result from awareness of the limitations of cognition is what connects ideas about the organism to theories of society. The highest goals of a society, it is argued, can only be legitimate if they sustain the individual integrity of all their members of as ends in themselves, even as they are incorporated into the whole of society. Analogies between Kant's approaches to the organism and theories of the social world will play a major role in Idealist aesthetics, and the tension between science's need for analysis if knowledge is to increase, and the desire for an integrated relationship with the natural world which is often at odds with such analysis is crucial to the thought of this period, most notably in the work of Schelling and Goethe.

If Kant had continued to work solely within the terms of the $C P R$ the idea of an object as an integral organism would not have become a major philosophical issue. One reason it does has to do with the conception of the subject in that text. As we have seen, in the $C P R$ the world did not just consist in chains of endless random differences precisely because the subject is supposed to establish its own identity by the way in which it synthesises the 'sensuous manifold' into something reliably knowable. At this level the later Heidegger's critique of Kantian philosophy's 'subjectification of being', the making of the truth of being into a function of the subject, seems appropriate in certain respects. However, the purpose of the subject's syntheses could not be established in the terms of the understanding. The 'spontaneity' which produced the syntheses takes one beyond the syntheses to the founding principle of our capacity for cognition. If we were to have philosophical access to this principle we would know the value of knowledge. The second Critique introduced a further spontaneity, which again pointed, beyond anything we can know, to a higher purpose than can be described in terms of a nature legislated by the understanding: why is it that we can contradict natural causation in ourselves in the name of 'ideas' and 
of the moral law? The third Critique tries to link this sense of the higher purpose of rational beings to the sensuously based experience of both natural and artistic beauty. Such experience is grounded in the pleasure derived from contemplating how each part of the object contributes to the whole of that object without losing its own value - how each part can be both a means and an end in itself. This contemplation involves both intuition of the object and a relationship to the object which is not based upon reducing the intuition to what it has in common with other intuitions. The coherence of a particular object cannot be reduced to a general law of how things cohere and thus depends upon the integrity of that object in relation to the subject's capacity for judgement.

In Schopenhauer's words, the $C \mathcal{F}$ consists of the attempt at the 'baroque unification of cognition of the beautiful with cognition of the purposiveness of natural bodies in one capacity for cognition' (Schopenhauer 1986 p. 711), namely judgement. Judgement, Kant asserts, is to function here 'according to the principle of the appropriateness of nature to our capacity for cognition' ( $C \mathcal{F}$ B p. XLII, A p. XXXIX). This appropriateness cannot be definitively legitimated in philosophy, because proof of it would again entail access to the nonappearing intelligible realm. What is required is an account of why there is a congruence between the way we necessarily synthesise intuitions via the understanding and some immanent coherence of nature that gives pleasure in aesthetic judgement. Although Kant generally rejects positive answers to this question, he is aware that without some more developed position than that offered in the $C P R$ his philosophy cannot achieve its final aims. Why exactly, then, does the aesthetic become so important in this context?

Aesthetic judgement connects the capacity for cognition and the 'capacity for desire' (which is how Kant describes the will) by judging which objects give pleasure and which do not. The ground of aesthetic judgement is therefore the distinction between feelings of pleasure and non-pleasure. This division is, though, not based on a cognition of the object - which entails the subsuming of it under a rule - but precisely upon whether the object gives the subject pleasure or not. The object of the judgement is therefore not conceptual: it cannot be classified in terms of an identifying rule. What is in question is the 'immediate' feeling of the subject which does not rely on a 'mediated' synthesis of intuitions of the object. ${ }^{11}$ Even in his writings well before the first Critique, Kant thinks it vital to stress that 'sensation' differs from 'feeling'. The former is the sensuous material of cognition which is subsumed under the categories and classified by concepts, and Kant sometimes prefers to term it 'appearance'; the latter is the result of the relation of the object to the subject's pleasure or displeasure. Taste itself 'is not a mere [receptive] sensation but what arises from sensations which have been compared' (Kant 1996 p. 100): it therefore involves judgement, the activity of the understanding. Now this might seem to make aesthetic judgement 'merely subjective' in the manner referred to by Gadamer in Truth and Method, where he talks of Kant's 'subjectification' of aesthetics. However, Kant's argument cannot be seen in this manner, not least because he 
will come to regard the role of feeling as ineliminable even from the theory of knowledge. Objectivity itself, he argues in the $C \mathcal{F}$, must have some kind of ground in feeling. Although we now derive no pleasure from the synthesising activity of the understanding: 'in its time there must have been some, and only because the most common experience would not be possible without it did it gradually mix with simple cognition and was no longer particularly noticed any more' (CF B p. XL, A p. XXXVIII). The activity of cognition therefore has a history in which it is motivated by pleasure, and pleasure is not something within the cognitive control of the subject.

It is not surprising, then, as we shall see in a moment, that the pleasure occasioned by the aesthetic object derives precisely from its allowing our cognitive faculties to play in a manner that does not entail determination by concepts, thus suggesting that the ground of cognition is an activity which, like play, can take place for its own sake. In a further striking claim, Kant even maintains that a 'common sense', of the kind 'required for the universal communicability of a feeling', is 'the necessary condition of the universal communicability of our cognition' (B p. 66). ${ }^{12} \mathrm{He}$ connects this idea by analogy to music, for which he otherwise does not have a great deal of philosophical time, talking of the 'tuning/attunement' (Stimmung) of the cognitive powers, which is differently 'proportioned', depending on the object in question, and which 'can only be determined by feeling (not by concepts)' (B p. 66). Whether this 'common sense' is a 'constitutive principle of the possibility of experience' or only a 'regulative principle', for creating 'a common sense for higher purposes' (B p. 68), Kant leaves open. However, he does insist that we necessarily presuppose such a norm if we wish to make valid claims about taste, because such claims depend upon a difference between mere contingent individual pleasure and what we think everyone ought to be able to agree about. As he already claimed in 1769-70: 'Contemplation of beauty is a judgement, and not a pleasure' (Kant 1996 p. 109). Kant therefore seems to suggest both that our most fundamental relationship to the world and each other is at the level of the immediate 'feeling' which is the basis of the postulate of a 'common sense', and that there is a purposive sense in our cognitive capacity which goes beyond its subsumption of intuitions under rules. Given that he also suggests that our representation of ourselves is 'nothing more than the feeling of an existence without the least concept', the role of the non-conceptual aspects of the subject in central parts of his philosophy becomes inescapable, and this is why the aesthetic becomes so significant.

There is, however, an important potential danger here, which lies in how one understands the nature of feeling. If feeling is regarded as grounded in libido, for example, cognition can easily come to be seen as essentially motivated by interest in domination, because feeling is conceived of as a lack that must be overcome by bringing the lacking object under the subject's power. The object thus becomes a source of self-aggrandisement for the subject: as we shall see in Chapter 8, Nietzsche will later draw such a consequence when he claims that 
the intelligible basis of ourselves is in fact the 'will to power', and Heidegger will stylise such views into a verdict on subjectivity as a whole in Western metaphysics. Kant, on the other hand, is concerned with a kind of pleasure which cannot be reduced to self-interest because its significance lies, as the notion of the 'common sense' suggests, in its potential for being universally shared across social divisions, without it leading to an appropriative relationship to the object. In this sense the aesthetic relates to Kantian 'reason'. Although aesthetic pleasure is based on the capacity for desire, which does link it to volition, the crucial fact about the will for Kant is, as we saw, that rational beings can desire against their natural inclinations in the name of a higher purpose. The feeling of pleasure upon which judgement is founded derives from the sense of a harmony in nature which the understanding, that activity of the subject which can be seen as in some sense dominating the object, cannot establish. When I see a green meadow the understanding can invoke Newton to tell me about green as a property of refracted light, but I can also derive immediate pleasure from its green aspect. The pleasure in question, Kant repeatedly insists, is solely that of the subject, and provides no knowledge of the object: the capacity to evoke a harmonious play of the (subjective) faculties is not a property of the object because the basis of this judgement is not necessitated in the way judgements based on categories and concepts (as rules for identifying objects) are. This pleasure must, though, involve some sort of effect of the object on the subject (not just any object will occasion it, although the context in which the object is encountered can be decisive), rather than a rule-bound synthesis by the subject, and it does not rely on the subject appropriating the object. Kant's limitation of aesthetic pleasure to the subjective does seem, therefore, to conceal a more extended sense of how subject and object relate, which is linked to the nature of the will.

Despite his repeated insistence upon the subjective basis of aesthetic pleasure Kant himself returns on occasion to the idea that nature may give an indication that 'it contains in itself some basis, a law-bound correspondence of its products to our pleasure which is independent of all interest' (CF B p. 169, A p. 167 ), and he talks of a 'code through which nature talks to us figuratively in its beautiful forms' (B p. 170, A p. 168). Given our own status as part of nature, the idea of a deeper harmony between ourselves and nature is tempting, especially as Kant sees the fact of agreement over judgements of taste as testifying to the possibility of the sensus communis. Saying something is beautiful involves, as we have seen, a judgement which can command general assent that perhaps results - and Kant is emphatic about the 'perhaps' - from 'what can be regarded as the supersensuous substrate of mankind' (B p. 237, A p. 234). For the Kant of the $C P R$, remember, nature was almost exclusively that which appears to us in terms of necessary laws, not what will become the living nature of German Idealism and Romanticism. The laws sustained their general validity because they were based on the very conditions of our thinking intelligibly at all, which did not have to be tested every time to see if they were inherent in the endless 
multiplicity of things. The $C \mathcal{f}$ begins to undermine this view of nature by its attention to the other ways in which the subject can relate to it. The idea of the postulated 'supersensuous substrate', in which freedom and necessity harmonise, relies on the possibility, based on feeling, of the 'subjective purposiveness of nature for the power of judgement' (B p. 237, A p. 234). What Kant is looking for, then, is an indication of the role of the freedom of rational beings in the system of nature.

\section{The purpose of beauty}

Kant's attempts to come to terms with the 'supersensuous substrate' of the subject's relationship to the object threaten to invalidate the boundary between law-bound nature and the autonomy of rational beings which was essential to the $C P R$. However, a philosophy which aspires to grounding our place in the world via the principle of subjectivity clearly must do more than describe the subject's ability to legislate nature in terms of regularities and to prescribe ethical limitations to itself. Kant's problem in extending his conception is that he would seem to need a positive account of the spontaneity involved both in our knowledge and in our ethical self-determination. If he wished to establish a purpose in nature as a whole, any such postulate would have to include ourselves in the purpose of nature, which would make our spontaneity an aspect of nature in itself, our purposive productivity being of the same kind as that which results in natural organisms. ${ }^{13}$ Kant's aim, then, is to link the harmony manifest in aesthetic apprehension of natural objects with the idea of natural teleology, thereby revealing the ultimate connection of nature as a whole to the ways in which we think about it and relate to it. He remains ambiguous as to whether such a link is merely a regulative idea or could actually be constitutive, tending more to the former conception. Subsequent thinkers, like Schelling, will, as we shall see, try to make the link constitutive. Such a strong conception of natural teleology may be hard to defend, but what interests Kant in this question will not simply go away, even if one rejects teleology out of hand as inappropriate for the explanation of natural products.

What is most significant here is the fact that in aesthetic contemplation of nature the object can be said to affect us, instead of our determining it in terms of general rules. This does seems to point, as Hartmut Scheible indicates, in the wake of Adorno, to a 'mimetic' side to the imagination which suggests we can indeed in some sense harmonise with nature. We do not in this case wish to order nature in any other way than it is already constituted when we feel the disinterested pleasure it gives to us by appearing to be formed in terms of ideas. We can, furthermore, be said to have a kind of access to a possible non-deterministic aspect of nature, via our freedom to initiate new states of affairs, according to ideas of what we would wish to be the case and of what we ought to do to fulfil our duty. Neither of these can be said to be causally determined, and they cannot be derived from observation of the empirical world. Whether 
this access is any more substantial than that we cannot know. Neither do we know how it connects to nature as an object of cognition. The tendency in the Enlightenment is to see ourselves as the potential 'lord and master of nature', thereby ignoring the question of what, so to speak, nature might 'think' of this. Given our own inability, for Kant, to give a definitive account of what our thinking $i$, beyond its being a capacity for synthesis, and given that thinking itself relies on natural processes (while not being reducible to them), this is not a mystical question. The fact is that in these terms our capacity for manipulation of nature depends on something which is as cognitively inaccessible to us as nature is in itself.

Kant's attention in the $C f$ to trying to link our cognitive and ethical capacities via a third way of relating to the world also points to his awareness of the dangers that may result if we cannot make such a link. In Western history, attention to natural beauty for its own sake generally arises at the moment when nature ceases to be perceived as predominantly a threat to human survival. Those areas of society which develop their powers of technological control over nature, to the point where they can come to more real than mythical terms with it, can also become concerned with nature's beauty and integrity. ${ }^{14}$ It is at this point, therefore, that nature's value in itself as the object of contemplation can emerge: the secularisation of nature, which had previously been seen in terms of its manifestation of the magnificence of God, is inextricably linked to this development. At the same time, however, the question also arises as to whether modern science's continuing destruction of the mythical and the theological elements in our relationship to nature means that the role played by myth and theology will be wholly abolished. From Schelling and the early Romantics, to Marx, Nietzsche, Weber, Heidegger, and beyond this will be a central issue both in aesthetic theory and in theories of modernity - the tendency of certain aspects of ecological movements towards mythical ways of thinking is, for example, not fortuitous. The key issue here is the nature of the imagination.

For Kant, as we saw, aesthetic judgements rely upon the fact that the object is received in the subject in terms of 'feeling' and in terms of a harmonious play of understanding and imagination. We already began to see, though, how Kant gets into difficulties in the first version of the $C P R$ with the imagination, as the faculty which links sensuousness and understanding. Scheible argues that the problem of the relationship between differing ways in which we relate to the natural world is bound to persist because Kant 'brought the imagination temporarily into play as a capacity whose function exhausted itself in providing for the understanding' (Scheible 1984 p. 118), thereby restricting the relationship to one of classification. For something to become an object of knowledge the subsumption by the imagination in schematism must be added to by a further subsumption, in which the understanding discovers, as Scheible puts it, 'the one characteristic which allows a replacement of the intuitive synthesis of the imagination by the abstract unity of the conceptual system' (p. 120). The object becomes reduced to the terms of the subject via which, in Kant's account, it 
becomes an object at all. The working of understanding can consequently be regarded both as entailing a possible repression of my particular imaginative relationship to the object and as failing to integrate its products into a systematic whole.

However, in the $C \mathcal{F}$, as we have seen, Kant highlights the importance of what the imagination does which cannot be subsumed under categories and concepts. He acknowledges that in cognition the imagination is limited by the 'compulsion of the understanding' - a fact that was already implied by the objections of Baumgarten and Hamann to theories which fail to take account of pleasure in the particularity of perception. The term 'compulsion' points to its counterpart: freedom. In the aesthetic relationship to the object: 'the imagination is free, beyond every attunement to the concept, yet uncontrivedly, to provide extensive undeveloped material for the understanding, to which the latter paid no attention in its concept' (CF B p. 198, A pp. 195-6). Kant makes it clear both that the sciences can exclude vital aspects of ourselves from their view of nature, and that these aspects must be accounted for in other than cognitive terms. The freedom of the imagination not to be bound by existing concepts enlivens the powers of cognition, enabling them to develop further, thus suggesting the possible purpose of these powers in human self-development as part of the purpose of nature itself. Furthermore, Kant introduces a notion, freedom, which for him belongs in the realm of the supersensuous, into our sensuous relations to the world. The very fact that Kant now begins to use the term 'aesthetic ideas' suggests how far he has moved beyond key restrictions in the CPR. The 'Aesthetic' there provided the 'rules of sensuousness', which constituted the framework for the intuitions judged by the understanding. 'Ideas' were the basis of reason's attempts to unify the endless diversity of the products of the understanding into a whole, and could not be available to intuition because they would then have to be objects of the understanding. Ideas can, in contrast, now become aesthetic. Kant defines an aesthetic idea as: 'that representation of the imagination which gives much to think about, but without any determinate thought, i.e. concept being able to be adequate to it, which consequently no language can completely attain and make comprehensible' (CF B p. 193, A p. 190). This leads to a vital issue in my overall argument.

The perceived inadequacy of language to aesthetic ideas makes other thinkers, particularly the early Romantics and Schopenhauer, look for a 'language' which $i s$ adequate to such ideas. The language in question is, however, the conceptless language of music, to which some thinkers will even grant a higher philosophical status than to conceptual language. Although music is manifested in sensuous material, it does not necessarily represent anything and may in consequence be understood metaphorically as articulating or evoking what cannot be represented in the subject, namely the supersensuous basis of subjectivity which concepts cannot describe, where necessity and freedom are reconciled. Music's reliance on mathematical proportions, its links to biological rhythms in the body, and the relation of the feelings it can involve to the freedom inherent 
in non-conceptual judgements of taste already begin to suggest reasons for such claims.

Furthermore, the way music comes to be understood at this time also helps put into question the notion that verbal language itself primarily represents preexisting objects, rather than constituting what things are understood to be. Such questioning of the representational nature of language is already implicit in the fact that if, as Kant claims, our cognitive activity actually constitutes objects $a s$ objects in judgements, it cannot be said to imitate something that is already there. The move in conceptions of language away from the idea of representation involves a two-way process. The development, by Herder, Hamann and others, of the view of language as primarily 'disclosive' or 'constitutive', rather than representational, means that forms of articulation, like music, which are not understood as being linguistic if language is conceived of in representational terms, can be considered as linguistic if they disclose otherwise inaccessible aspects of the world. The 'language' of music can thus itself in turn come to change the way verbal language is understood. Here a further vital dimension of the notion of 'feeling' becomes apparent. As Anthony Cascardi suggests in relation to the CF: 'Feeling nonetheless remains cognitive in a deeper sense [than in the sense of 'cognitive' involved in the correspondence theory of truth as correct representation of the pre-existing object]; affect possesses what Heidegger would describe . . . as "world-disclosive" power' (Cascardi 1999 pp. 50-1). Critiques of metaphysics based on the rejection of the idea of language and concepts as primarily or inherently representational, which lead from their roots in the Romantic reactions to Kant, to Nietzsche, and to post-structuralism, are therefore importantly linked - despite the antagonism of many of those theories to role of the subject in modern philosophy - both to the 'feeling' of the subject and to the development of music and ideas about music towards the end of the eighteenth century.

Kant himself actually follows aspects of the Enlightenment tradition of understanding music and objects, by seeing music as a 'language of emotions' (C7 B p. 220, A p. 217). Music represents feelings, in much the same way as language supposedly represents ideas or objects. Johann Mattheson talked in 1739, for example, of how an 'Adagio indicates distress, a Lamento lamentation, a Lento relief, an Andante hope, an Affetuoso love, an Allegro comfort, a Presto eagerness, etc.' (ed. Strunk 1998 p. 699). However, Kant does not adopt this literalist conception in every respect. Music also communicates aesthetic ideas, but these are 'not concepts and determinate thoughts', though their purpose is solely to 'express the aesthetic idea of a coherent whole' (CF. B p 220, A p. 217), the pleasure in which is generated by the mathematical proportions upon which the whole is based. Because it just plays with feelings music is not the highest form of art. This status is confirmed by music's dependence on time and its inherent transience. What Kant sees as music's empty formalism will, however, soon become crucial to new conceptions of art. The divorce of music from representation is the vital step in the genesis of the notion of aesthetic autonomy, 
the idea that what is conveyed by the work of art could not be conveyed by anything else. ${ }^{15}$ This autonomy will entail a break with the direct connection proposed by Kant between aesthetic ideas and ethical ideas. Given his intention to link beauty and morality - beauty is a symbol of morality for Kant - his suspicion of music follows from the aims of the third Critique. However, even though the idea of aesthetic autonomy is not part of the argument of the $C \mathcal{F}$, the $C \mathcal{F}$ does help open up the possibility of its emergence.

Kant's aesthetic ideas are non-conceptual, but this does not exclude them from consideration in philosophy: instead, they 'strive towards something beyond the boundary of experience' (B p. 194, A p. 191) - 'experience' being understood here as the realm of possible true judgements of the understanding. He gives the example of how poets attempt to make the ideas of 'the invisible Being, the realm of the blessed, hell, eternity, the creation' (B p. 194, A p. 191) into sensuous images. Aesthetic ideas are the 'pendant' to ideas of reason, because the ideas of reason, such as goodness, cannot be manifested empirically. Soon after the writing of the $C \mathcal{F}$ Schiller will come to regard art as a means for the 'aesthetic education' of the people on the basis of his interpretation of Kant's conception: art is to make morality available through pleasure by sensuously conveying ideas, instead of trying to compel people in terms of abstract imperatives. Kant himself opposes 'the pure disinterested pleasure in the judgement of taste' (B p. 7, A p. 7) to a judgement based on an 'interest' in an ulterior purpose of the kind generated by sensuous appetites. To begin with, then, aesthetic pleasure does have an autonomous status of its own: it serves no other purpose beyond its own immediate existence, which, crucially, is not reducible to the ways it may subsequently be analysed in general terms. This view of the status of artistic beauty is, in different forms, essential to many versions of subsequent aesthetic theory. Aesthetic objects can be seen as irreplaceable and without price in terms of their aesthetic value, however much they may have a price in the marketplace which can make them useful for buying something else. Kant's role for the aesthetic is, though, more substantial than this.

Aesthetic pleasure must be able to be universally shared if the object is to be beautiful rather than the source of random individual stimulation. For a judgement of taste to be universalised it must therefore entail rules that enable it to be legitimated to others. Here we encounter one of the most productive and problematic tensions in Kant, which resonates throughout the subsequent history of aesthetics. In the Reflections of the 1760s Kant already points to the essential problem, when he notes that 'Beauty ought to be unsayable (müsse unaussprechlich sein). What we think we cannot always say' (Kant 1996 p. 62). The 'sayability' of anything in this sense depends upon shareable rules which enable it to be communicated. Beauty is supposed to be potentially universal, but seems to rely on something radically particular, that is resistant to subsumption under rules, namely the feeling of pleasure in the form of the object on the part of the individual as 'an irreducibly particular centre of affectivity' (Cascardi 1999 p. 48). Kant claims in reflections from the time of his writing of the $C F$ 
that: 'The general validity of pleasure [in beauty] and yet not via concepts but in intuition is what is difficult' (Kant 1996 p. 137). Intuitions, as we saw, are regarded by Kant as inherently particular: how, then, are they supposed to be the basis of something universal that is grasped in a judgement? The answer has to do with the question of feeling and its links to the possibility of a sensus communis.

Perhaps the best way to approach this issue is via the implicit role of verbal language, as the rule-based means of communicating the general truths which constitute the realm of the 'sayable', in the difficulty to which Kant refers. The early Romantic writer, Friedrich Schlegel, formulates this question most effectively in 1805 in relation to Kant's notion of the aesthetic idea. Schlegel was familiar with the work of Kant, and he takes up the indications we have observed concerning the nature of self-consciousness, such as the suggestion from the Prolegomena that the representation of the I is 'nothing more than the feeling of an existence without the least concept', linking it to music:

if feeling is the root of all consciousness, then the direction of language [towards cognition] has the essential deficit that it does not grasp and comprehend feeling deeply enough, only touches its surface ... However large the riches language offers us for our purpose, however much it can be developed and perfected as a means of representation and communication, this essential imperfection must be overcome in another manner, and communication and representation must be added to; and this happens through music which is, though, here to be regarded less as a representational art than as philosophical language, and really lies higher than mere art. (Schlegel 1964 p. 57)

In this perspective the very nature of language qua representational means of communication excludes it from being able to get in touch with the particularity of subjective existence grounded in 'feeling'. Even though feeling is nonconceptual it is, however, clearly not randomly subjective, because, as Schlegel suggests, the means via which it is grasped has claims to general validity: hence the idea that music is 'philosophical language'. The assertion that something is music rather than noise requires a judgement of taste. Such a judgement cannot be based on a concept of music: concepts can only identify the physical aspects of the piece, the pitches and durations of the sounds of which it consists, not establish whether the piece is music. Now it is evident that Kant would not have accepted the more unexpected consequences of this view of feeling. However, the idea that a vital dimension of the existence of the subject is only accessible as a non-conceptual feeling and that this feeling is best articulated by music is not inherently at odds with key aspects of Kant's conception. Subsequent thinkers, like Schlegel and Schleiermacher, will, in consequence, come to regard the exploration of the relationship between the conceptual, which can be understood via rules, and the non-conceptual, which cannot be understood via rules, as the crucial philosophical issue revealed in aesthetics. This theme will recur throughout the present book. 
The strange links between the new understanding of music and the concerns of Kant's philosophy become further apparent in the following. The initial way in which we constitute a world of rule-bound objects in the imagination is, as we saw, via schematism. In relation to the categories, Kant terms schemata 'nothing but determinations of time a priori according to rules' (CPR B p. 184, A p. 145). We need these determinations in order to be able to apprehend things in terms of the categories of, for example, causality, which relies on temporal succession; reality, which relies on presence at a specific time; necessity, which relies on presence at all times, etc. For these forms of apprehension to function one also, as we saw, requires the continuity of the subject between the different moments. This requirement is what leads Schlegel to his idea of the irreflexive sense of self given in 'feeling' - which, as Novalis puts it, 'cannot feel itself' that connects the differing moments without itself being accessible to cognitive determination. The point in the present context is that the schemata of time are also part of what is essential for hearing music as music, but, as we saw, establishing whether something is music also relies on a judgement of taste based on feeling that is not determined by rules. The role of musical rhythm, with its links to processes in nature, to somatic pleasure, and to the cognitive functioning of schematism, suggests a further way in which the borderline between the realms of nature and spontaneity begins to dissolve in perhaps more ways than Kant himself intends in the $C \mathcal{F}$.

The nature of this borderline plays a role in the question of how natural beauty relates to the beauty of art. Kant sees natural beauty, which pleases the subject without the need for a conceptual determination, as prior to the beauty of art. If the priority were reversed and artistic beauty were primary, the rules of art, as products of the subject, would be simply another way in which we 'give the law' to nature. As we shall see in a moment, Kant insists that in art it is the other way round: nature gives the law to us (via the genius), so that 'beautiful art is an art insofar as it at the same time appears to be nature' $(C 7 \mathrm{~B}$ p. $179, \mathrm{~A}$ p. 177). Art should be seen to be art but 'the purposiveness of its form must appear as free from all compulsion by arbitrary rules, as if it were simply a product of nature' (Kant 1996 p. 137). He goes on to claim, though, that 'Nature was beautiful if at the same time it looked like art' (p. 137), which is the basis of reflective judgement and the notion of the organism: in both cases the point is that the whole, the 'idea', precedes the parts. In the case of nature the argument leads to the question of whether the idea is to be understood theologically, as the intention of a creator, or as a productivity immanent in nature as a whole, of the kind soon to be suggested by Schelling. In the case of art the point is that the artist must not appear to have worked according to a pre-existing rule of the kind provided by the understanding for a scientist, which merely subsumes a particular under a concept. Instead, the artist should appear to have spontaneously produced something which has the self-sufficient coherence of a natural organism.

The natural organism's capacity to evoke pleasure is not limited to the plea- 
surable effect of its form upon the subject. Kant in fact insists that in natural beauty the 'existence' (Dasein) (B p. 167, A p. 165) of the object pleases, independently of any appeal to the senses or to any purpose of the object. The next stage of Kant's argument, where he introduces the notorious concept of 'genius', is perhaps the most instructive. Gadamer claims that genius is introduced into the $C 7$ simply in order to give works of art the same status as natural products. This would make the link between aesthetics and teleology constitutive, but Kant is often more circumspect than Gadamer's claim would imply. Kant describes genius as follows: 'Genius is the talent (gift of nature), which gives the rule to art. As talent, as an innate productive capacity of the artist, itself belongs to nature, one could also put the matter as follows: genius is the innate aptitude [ingenium] through which nature gives the rule to art' (B p. 181, A pp. 178-9). In the modern period rules in artistic creation are supposed to be the basis of individual, particular products. Particularity and rules are inherently opposed to each other, as we have seen. Kant's notion of genius is therefore also intended to explain 'the general validity of pleasure [in beauty] and yet not via concepts'. Kant argues (once more employing the musical metaphor of 'attunement') that judgements about artistic products cannot be based on rules derived from concepts:

Thus art [schöne Kunst] cannot devise the rule for itself, according to which it is to produce its product. As, nevertheless, a product can never be called art without a preceding rule, then nature must give the rule to art in the subject (and through the attunement $[$ Stimmung $]$ of the subject's capacities), i.e. art is only possible as a product of the genius. (CF B pp. 181-2, A p. 179)

The particular product's coming into existence therefore cannot be described theoretically: Kant claims that the genius cannot tell how the work came into existence, let alone tell anyone the rules which would enable them to emulate the genius. This seems appropriate, for example, in some areas of music, when Beethoven changes the canon of musical rules. One can describe this change retrospectively in terms of the new theoretical canon, but this will not explain what made Beethoven make the new moves, nor will the description enable any person who knows the old and the new rules to produce music as great as Beethoven's.

Here we again reach a point in Kant's philosophy where the essential principle is self-caused in ways which philosophy cannot articulate. Genius can be regarded as a 'spontaneity'. The difference from the spontaneity of understanding and of ethical self-determination is that this spontaneity produces empirical products, which are available to intuition and which themselves symbolise what belongs in the intelligible realm, and the spontaneity derives in Kant's terms from 'intelligible' nature. It is therefore not hard to see why the early Schelling will be tempted to see art as the 'organ of philosophy', precisely because it is supposed to make the highest point of philosophy available to intuition. If nature gives rules to the free productivity of the genius, the division of 
sensuous and intelligible is no longer absolute, and this threatens the whole edifice of Kant's previous philosophy. The artwork of the genius seems to be where the subject can intuit itself in terms of its spontaneous, intelligible capacities, and can therefore objectify itself, something which Kant had consistently denied was possible in relation to the capacity for knowledge, or in relation to freedom.

Kant's use of the notion of 'genius' is, though, significantly ambiguous. On the one hand, genius points to something that is constitutive of being an autonomous human being: the ability to express oneself in ways which cannot be prescribed in advance or reduced to someone else's way of expressing themselves. On the other hand, it is also a capacity limited to a few individuals. The fact that, for Kant, aesthetic products in which 'nature gives the rule' are only produced by a few people, Scheible maintains: 'is necessary in a society in which the potential for alienation has already grown so much that the ability to help nature express itself uninfluenced by pre-given rules and norms is already limited to a few individuals' (Scheible 1984 p. 127). The danger of this argument lies in its assumption that the self-expression of nature in the artist is necessarily repressed by social forms. This does not allow for the possibility that the positive 'natural' potential in subjectivity may actually only emerge via engagement with socially generated forms. The simple invocation of nature as the positive term in opposition to society as the negative misses the complexity of this relationship, in which ideas about the positive potential of nature themselves cannot be separated from social developments, as the historical emergence of the idea of self-legitimating natural beauty in the second half of the eighteenth century suggests. The real issue here is a dialectic which is inseparable from modern art. At the same time as the objective conditions for the liberation of individual subjectivity from myth, theology and tradition come into existence, not least via the technological application of the generalisable results of science, the conditions which contradict the potential of individual freedom are also produced, via the new division of labour and the forms of regulation it involves.

It is, then, not fortuitous that the association of art and madness, encountered in such figures as Lenz and Hölderlin, becomes a major issue in this period. Scheible suggests that the notion of genius reveals why, in modernity, the aesthetic of the autonomous work of art becomes more significant than the hopes for collective aesthetic production in a community. The fact is that modern societies often fail to create adequate space for forms of individual self-realisation which are not based upon appropriative interest. The availability of more and more aspects of the object world as commodities develops the consuming subject at the expense of the productive capacities of the subject. One can reread Hegel's account of 'Lordship and Bondage' in the Phenomenology of Mind in these terms. In modern capitalism it is more and more possible to be in the position of the consuming, unproductive lord and not to be in the position of the bondsman who can develop into the genius. In modernity art can express what is repressed by our cognitive and economic control of nature, and by our 
disciplining of ourselves under the division of labour. However, the increasing domination of our self-descriptions by the discoveries of the natural sciences and by the imperatives and pressures of the economic system make this articulation more and more problematic, as the ability of the advertising industry to assimilate avant-garde aesthetic production makes clear. The importance of music in subsequent aesthetic theory therefore lies not least in its link to the aspect of the subject which resists assimilation into the terms of the representable world. Because music is a predominantly non-representational medium, its rules seem in one sense to have no validity beyond the constitution of the medium itself, and this sustains music's autonomous status, as well as linking it to the unrepresentability of the subject. At the same time, the medium is not seen as devoid of meaning, for the reasons suggested above in relation to the development of non-representational conceptions of language. Furthermore, as we shall see, music's meanings are evidently affected by precisely the developments in modernity against which music often tries to react by avoiding representation. The more general movement of modern art away from representation - which occurs most obviously in visual art and which relates both to the attempt to avoid commodification and to the development of technological forms of representation such as photography - is, then, importantly prefigured in music, with philosophical consequences that will be central to the rest of the story being told here.

\section{The limits of beauty}

Much of the debate that results from Kant's re-thinking of our place within nature in relation to aesthetics revolves around whether there can be a reconciliation of our subjectivity with a nature which is both in us and outside us, and around the implications of this issue for the development of modern societies. Kant is fully aware that nature can appear, as it will do to Schopenhauer, Darwin and others, as an endless chain of eat or be eaten. ${ }^{16}$ He takes the example of plants, asking what they are there for, which leads into the chain of their existing for the animals that eat them, and those animals existing for the animals that eat them, etc. Only rational beings can attempt to transcend this situation, by seeing nature as a 'system of purposes', rather than as a 'purposeless mechanism'. This idea of nature leads Kant to the notions of happiness and of culture, the latter of which can be seen as the final purpose that 'one can attribute to nature with regard to the human species'. The need to discipline our natural inclinations in order to achieve the higher forms of culture means that there is a 'purposive striving of nature which makes us receptive to higher purposes than nature itself can provide' (CF B p. 394, A p. 390). The dual use of the word nature makes the problem clear.

The essential problem is that in Kantian terms we do not, and cannot, know what our nature is. Kant insists that our 'nature is not of the kind which will stop and be satisfied somewhere in the midst of belongings and enjoyment' (B 
p. 389, A p. 385), thus refusing to see the nature in us as solely appropriative and consequently determined by natural drives. This raises the question once more of the 'supersensuous substrate' in which conditioned nature and freedom can be reconciled. Kant's aim is to find a way of showing how the existence of rational beings who can transcend nature is the final purpose of creation, an idea he infers from the independence of moral self-determination from the chain of natural causality. Attempts of this kind to show the meaningfulness of existence in terms of human freedom will, as we shall see, be central to German Idealism. Once again it is important to remember that these issues are not merely abstract. The metaphysical ideas evidently relate to more concrete aspects of modernity, in which the relationship between science's capacity to reduce more and more of existence to deterministic explanation can come into conflict with the expression of human freedom in aesthetic production that can give meaning to individual existence.

For Kant both art and science are means for the development of reason. However, the way in which Kant attempts to explain them also reveals much about why the project of a self-legislating modernity will prove so problematic. In Kant's conception science alone can provide no orientation, even in terms of the relations between its own laws. It is, then, somewhat unexpected when Kant claims that the scientist is more important than the genius, even though he is excluded from being a genius by the fact that what he does can be reproduced by others following the rules he produces. This is because the scientist contributes to the 'ever-progressing greater completeness of cognitions and of all usefulness that depends on this' (B p. 184, A p. 182). Kant has, therefore, to depend here upon the aesthetically conceived postulate of reflective judgement, namely that there is a system that could notionally be completed inherent in what the scientist can only investigate in a piecemeal way - the postulates of reflective judgement were, remember, founded upon seeing nature as art. Nietzsche will attempt to revoke such postulates in The Birth of Tragedy (1871), claiming in the (unacknowledged) wake of Jacobi (see Bowie 1997 Chapter 1) that the principle of sufficient reason leads only to an endlessly regressing abyss of explanations with no cohering principle. Nietzsche thereby gives an even more emphatic role to aesthetics as a means of creating coherence in human existence, but for him the conviction that such coherence is a fiction is not, as it is for Kant, accompanied by the claim that it is a regulative idea which might also be constitutive. Kant's prioritisation of the scientist over the artist, which the early Nietzsche will invert, seeing science itself as just a kind of art, is historically explicable in terms of the importance of the public communicability of scientific knowledge and of its potential for reducing the misery of feudal life: scientific knowledge is seen by Kant in this respect as inherently emancipatory. Its potentially repressive side, which will sometimes be highlighted by Nietzsche, is, however, already present in what Kant has said in the Cf about the imagination's need for the possibility of escaping from the compulsion of the understanding.

Kant backs up his contention that science has prior importance by maintain- 
ing that art 'somehow stands still by having a limit beyond which it cannot go, which has also presumably been reached and cannot be further broadened' $(C 7 \mathrm{~B}$ p. 185, A p. 182). This reveals something crucial for subsequent thinkers. Kant is here not making a historical claim, because he evidently regards art as a static category, rather than, as it will already be for Schlegel and others, an essentially historical category. The genius, Kant argues, cannot be relied on, because his talent dies with him, and there is no saying when nature will endow someone with the talent to give the new rule to art. Because of the way in which he conceives of nature's relationship to art Kant does not make any significant link between sociohistorical conditions and the emergence of the major artist. His argument for the priority of the accumulation of knowledge in science over what art can communicate points, though, to the difficulties in the sphere of artistic production suggested earlier, which result from the speed of scientific and technological change. In modernity the relationship between the experience of the meaningful particularity of sensuous existence and the ways modern science explains the mechanisms of nature becomes increasingly distant. What is involved in this process can lead to the very notion of art being questioned. Questions about the status of art will famously emerge in Hegel's view of the 'end of art', and in Marx's puzzlement over the continuing appeal of ancient Greek art despite the scientific and technological developments in subsequent history. Hegel's and Marx's positions derive from the awareness of a crisis in art which results from the advance of science and of modern forms of rationalisation, an advance which depends on the continuing elimination of the kind of particularity characteristic of mythical stories in the name of what can be universally validated.

In Kant the assumption that art has a 'limit beyond which it cannot go' leads to a final important aspect of the $C \mathcal{F}$ - the 'sublime' - which must be considered before moving on in the next chapter to some of the reactions to Kant's philosophy in German Idealism and early Romanticism. The point of objects of natural beauty and art was that they made something 'infinite', reason, sensuously available as a symbol. If art has a limit, however, this means that the ways in which it can connect the sensuous and the intelligible are also limited. The sublime relates mainly to nature, though Kant does refer to some art works in connection with it. Whereas beauty relates to the form of the object, the sublime is concerned with what is unlimited or even formless, to the extent to which it makes us able to feel in ourselves 'a purposiveness which is completely independent of nature' (B p. 79). The sublime cannot, therefore, rely on the pleasure generated in judgement's sense of the purposiveness of the natural, or aesthetic object. As such, the sublime is only significant to the extent to which it reveals a purposiveness in ourselves with regard to our capacity to transcend nature by reason. The question remains, though, as Schelling and others will point out, whether a conception of self-determining nature which avoids Kantian dualism might not still be able to link such purposiveness in ourselves to the purposiveness which brings about natural forms.

The sublime has to do with things which are so big that they initially make 
us feel small. The bigness in question is not empirical because it depends upon an idea: 'something is sublime in comparison with mhich everything else is small' (B p. 84, A p. 83). This idea has to result from that which allows us to think beyond any quantity the senses could measure, so the idea is excluded from the understanding's capacity for rules. It does, though, involve intuition: 'Nature is sublime in those of its appearances the intuition of which carries the idea of its infinity within it' (B p. 93, A p. 92). What the sublime does, then, is to remind us of the limitations of our sensuous relationship to nature and actually to give us aesthetic pleasure via the initial lack of pleasure generated by this reminder itself: 'Something is sublime if it pleases immediately by its resistance to the interest of the senses' (B p. 115). The argument is dialectical: the sense of limitation entails the sense of its opposite, the fact that we also have a capacity for reason not limited by sensuousness. The 'mathematically sublime' is concerned with the aesthetic (thus subjective) apprehension of quantity and with the fact that, however large something in nature is, it is small in relation to the totality whose idea is provided for us by reason. The 'dynamically sublime' is experienced at those moments when we are confronted with natural powers which are beyond our power to control, such as lightning, volcanoes, hurricanes, the 'boundless ocean', but from a position of safety. This confrontation provokes in us a resistance which makes us wish to measure ourselves against nature because we realise that our moral feeling transcends anything we can experience in nature. Our capacity for reason can thus be heightened by the physical threat of nature. The potential of reason's non-finite relation to the laws of nature is, then, actually generated by the way in which nature reveals its irreducibility to our interests as sensuous beings in the experience of the sublime. The basis of Kant's argument will be central in post-Kantian philosophy. Because we feel our limits of our imagination we must also feel what is not limited in ourselves: otherwise we would have no way of being amare of a limit. Freedom emerges from a situation which seems empirically to be nothing but constraint.

In the sublime, therefore, the idea of the supersensuous emerges from the realisation that reason's attempts to grasp the totality are empirically unrepresentable. This realisation involves 'a feeling of the privation of the freedom of the imagination by the imagination itself' (B p. 117, A p. 116), the effect of which is to 'broaden the soul' (B p. 124, A p. 123) by taking one away from the finite sensuous world. Kant's argument takes a strange but significant turn when he cites the ban on images in Jewish theology as being the most sublime passage of the law book. He claims that the ban explains the enthusiasm for religion during the period of its institution. If the senses have nothing left as their object 'and the inextinguishable idea of morality still remains' (B p. 125, A p. 123), the idea's motivating force can be increased rather than diminished. Kant maintains that governments which surround their religion with sensuous representations therefore do so to limit the subject's capacity to 'extend his spiritual forces over the barriers which one has arbitrarily set up for him' (B p. 125, A p. 124), and thus to render him more passive. We saw above that in the first two Critiques 
Kant himself was in a situation rather analogous to that of Moses, trying to use the supersensuous, unrepresentable idea of freedom as the foundation of our place in the world. In the first two Critiques he was unable to do more than postulate what would have to be available to us in an 'intellectual intuition' if it were to provide a constitutive ground of our place within things.

The very fact that there is a third Critique is not least a result of the insufficiency of the first two Critiques with regard to the characterisation of the relationship between human freedom and the rest of nature. The crucial difference of the sublime from the beautiful is that in the sublime the 'unfathomability of the idea of freedom completely cuts off any positive representation of it' (B p. 125, A p. 124). In the aesthetic products of the genius ideas of reason were made sensuously available. The sublime does not represent ideas sensuously, because it reminds the imagination that the sensuous is not ultimately adequate to ideas anyway. On the one hand, then, Kant limits the significance of the sublime to the subjective realm, on the other the sublime would seem at the same time to suggest the limits of the beautiful, which is tied to the sensuous, even though Kant sees the pleasure in the sublime as merely negative and that in the beautiful as positive. This ambiguous position suggests why the status of the particular arts will become so significant in the reactions to Kant. Music, to which Kant himself does not grant an elevated aesthetic status, is, of course, the form of art which most immediately suggests transcendence of the sensuous. Although music is manifest as sensuous sound, it can only be music if one moves beyond the sensuous to its significance as ordered sound that cannot be described in physicalist or representational terms. ${ }^{17}$ As we have already seen, music in this period comes to be regarded as the highest form of art precisely because of its distance from representation. Furthermore, like the sublime, music's significance also lies in its relationship to feeling rather than concepts, which, in terms of Kant's arguments considered above, connects it to the most essential, 'immediate' aspect of the subject. We shall return to these issues in later chapters.

For the moment the main issue is the following. Kant's characterisation of the sublime in terms of the limitations of the sensuous and of the capacity for representation points to a decisive alternative in modern aesthetics, which has to do with the change in the status of music that takes place in Romanticism. The notion of the unrepresentability of the most essential aspect of our existence compels one to ask whether art can ultimately only sustain itself at the expense of any substantial relationship to the empirical content of social life, because the truth it can convey could otherwise be equally well or better communicated in other forms. What, for example, does the realist historical novel tell us that cannot be more reliably communicated by the right kind of narrative historiography? If art is to have a more committed function in the political life of the community the problem suggested in Kant's observations on using sensuousness as a political tool in religion arises, because the specific significance of art seems likely to become lost. Modern art is therefore located in an uneasy 
tension - of precisely the kind played out in Schoenberg's opera - between the 'aesthetic' demand for representation (Aaron) and the 'sublime' sense that representation ultimately destroys the real supersensuous point of the aesthetic (Moses), because ideas are fundamentally unrepresentable and only accessible via the negation of everything empirical. In Hegel this suspicion of the empirical will be part of what generates his claim that, because it is tied to the sensuous object, art has ceased to be a means of communicating the essential truth in modernity, this role having fallen to philosophy. On the other hand, art itself, particularly in the form of music, becomes increasingly wary of sensuous representation, and philosophy's own role clearly diminishes in the second half of the nineteenth century in the wake of the success of the natural sciences.

It is evident that these issues were very much part of the perception of the modern era in Kant's time. Hamann's theology relies, for example, upon the endless revelation of the divine mithin the sensuous. Kant, in contrast, rejects the suspicion that the power of the supersensuous involved in the 'merely negative representation of morality' might lead to the danger of what he terms Schmärmerei, roughly translatable as uncontrolled fantasy or religious 'enthusiasm'. Schmärmerei is described as a sort of madness which 'mishes to see something beyond every boundary of sensuousness, i.e. to dream in terms of principles (to rave with reason) [mit Vernunft rasen]' (C7 B p. 125, A p. 124). Unlike Hamann, Kant regards the fact that the sublime is only a reminder of the failure of representation to be adequate to the supersensuous as a reason not to spend one's time seeking something which is more than sensuous within the sensuous. The fact is, however, that early Romanticism will be based precisely on questions about how it is that the sensuous seems by its inherent incompleteness to point beyond the sensuous - in certain ways that was, of course, the point of the $C 7$ anyway. Given Kant's lack of success in convincingly establishing the nature of the division between the sensuous and the intelligible it is hardly surprising that some subsequent thinkers will try to revoke Kant's dualisms of appearances and things in themselves, of sensuous and intelligible, nature and freedom, often doing so in relation to music.

Art in the nineteenth century will itself tend to be located between two extremes. One is best embodied in the Naturalist attempt to collude with the advance of science, the other is exemplified by the rise of absolute music and the related development of the notion of 'pure poetry', an art devoid of representation, which is an idea Novalis will be one of the first to propose. Kant limits the sublime to nature, but as nature becomes more and more an object of scientific manipulation the attempt to reveal a non-sensuous truth not available to science often tends to be transferred into art. This truth is no longer representable in any other medium than the particular work of art concerned, which therefore becomes an attempt to say the unsayable. Furthermore, once the importance of language to these issues has been realised they will lead to essential questions that still concern philosophy today (on this see Bowie 1997).

The distinction of the beautiful and the sublime can also be regarded as the 
basis of the tension in post-Kantian philosophy between the desire for a 'new mythology' and the idea of the autonomy of the aesthetic work. The 'new mythology' - an idea developed in relation to Schiller's aesthetic letters - would sensuously present ideas of reason in order to communicate the advances brought about by autonomous subjectivity to all levels of society. It would thus integrate science and art into a unified collective project. In contrast, the idea of the superiority of the autonomous work of art over science and philosophy relies upon the conviction that the highest principle of philosophy is unrepresentable and must therefore be preserved from being used as a means for scientific and political ends. This distinction will occur in some form in all subsequent debates about aesthetics and politics, from Marx, to Lukács, to Brecht, to Adorno and beyond. It was the baroque honesty of Kant's attempt to ground truth in subjectivity which opened up the conceptual space for exploration of these issues in modernity. The further work of exploration is carried on at an often remarkable level of sophistication in German Idealism and early Romanticism, to which we now turn.

\section{Notes}

1 All page references to the three Critiques are, as is now standard, to the A and B versions of the Academy edition.

2 Kant wrote reflections on aesthetics from very early in his career onwards which suggest his awareness of the importance of the topic for his philosophy that culminates in the $C \mathcal{F}$.

3 Kant accepts that there is knowledge based on 'pure intuition' in mathematics, but knowledge of anything real has to involve perceptual input. See the discussion of 'real' below.

4 Kant employs this conception of 'reality' in order to refute the so-called ontological proof of God's existence.

5 See Makkreel 1990 pp. 28-9.

6 An influential recent attempt to suggest a way of obviating this problem is John McDowell's Mind and World, which argues, following Kant in a manner which echoes certain Romantic arguments (see Bowie 1996), that 'the deliverances of receptivity already draw on capacities that belong to spontaneity' (McDowell 1994 p. 41).

7 'Deduction' in the sense at issue here means 'legitimation of the use of'.

8 Dieter Henrich has recently argued that this may indeed be all that can be done with the problem.

9 My thanks to Manfred Frank for pointing this out to me.

10 I have dealt at some length with the implications of this important issue in Kant in relation to Jacobi, who most effectively highlighted the issue: see Bowie 1997 chapter 1. It would take too much space to outline the significance of Jacobi here.

11 What is at issue here also appears in debates in the analytical philosophy of mind over the nature of, for example, the immediate sensation of pain. The idea that there is in such cases an infallible, non-conceptual aspect to my self-knowledge - for which, as we shall see, the early Romantics and Schleiermacher used the term 'feeling' - is now widely accepted in the analytical philosophy of mind.

12 See Welsch 1996 pp. 490-5.

13 This is one way of understanding how Schelling sees nature in his 'philosophy of nature' (see Chapter 4). 
14 This is one reason why the emergence of aesthetics, as Terry Eagleton argues (1990), is linked to the rise of the bourgeoisie who break with the feudal world-picture. The Savoy peasant still thinks Baron de Saussure's desire to climb Mont Blanc is crazy, as Kant reports $(C 7 \mathrm{~B}$ p. 111).

15 On this see Neubauer 1986.

16 As Marx points out, this can be interpreted as a projection of the ruthless capitalism of the period on to nature, thereby again suggesting the danger of certain attempts to characterise nature 'in itself'.

17 It is worth remembering that, as Hamann suggested, something similar applies to language: the material of language is in itself not significant, and only becomes so via its relation to other material and to consciousness. Language is, though, generally regarded as, in some sense at least, inherently representational. 


\section{German Idealism and early German Romanticism}

\section{Thinking the infinite}

The immediate consequences from the 1790s onwards of the perceived failure of Kant's attempt to ground philosophy in the principle of subjectivity are apparent in two areas of philosophy which carry the broad names 'German Idealism', which is mainly associated with Fichte, Schelling and Hegel; and 'early Romanticism', which is mainly associated with Novalis, Friedrich Schlegel and (in some respects) Friedrich Schleiermacher. ${ }^{1}$ There are, as we shall see, crucial respects in which these two currents of thought can be distinguished. It would, though, be mistaken to regard either German Idealism or early Romanticism as unified philosophical schools, which is one reason why they have often, albeit mistakenly, been assimilated to each other. The problem is that, despite their differences, aspects of each are often found in the other: Schelling, for instance, can be seen as at times belonging both to Idealism and to Romanticism. A further complicating factor here is that 'early Romanticism' must be specified in this way to distinguish it from later reactionary doctrines which bear the name Romanticism in Germany. The term Romanticism is notoriously vague, and it is important to see that the early Romantics, who, after all, themselves established the term, can be characterised in a way which distinguishes them from later German Romanticism. What is at stake in this distinction is easily illustrated by the fact that the Nazis were not in the least bit fond of the early Romantics, who had a thoroughly cosmopolitan orientation and were therefore resistant to the nationalist assimilation of 'Romanticism' into Nazi ideology.

Peter Szondi maintains of German Idealism that 'One could say crudely that the philosophy of German Idealism tried to win back via the path of speculation what Kant's criticism had to renounce: the unity of subject and object, of mind and nature' (Szondi 1974 p. 221). 'German Idealism', which I shall generally refer to as 'Idealism' from now on, has little to do with the philosophical idealism represented by Berkeley's claim that 'being is perceiving'. Kant already objected to such idealism when he insisted upon the need in 'transcendental idealism' for intuitions given from outside the subject to fill the concepts of the understanding, if anything is to be considered as real - though his arguments 
were anything but decisive, as Salomon Maimon and others showed at the time (see Frank 1997). In the present context, however, the basic idea of transcendental idealism can be understood as follows: although objectivity depends upon a subject which constitutes an object as an object of truth in a judgement, the subject does not create the material which is judged to be the object at issue. The central question in Idealism is really, then, how what the subject does relates to the nature of which it is a part. This leads to questions both about the very fact that there is subjectivity at all, rather than there just being a world which does not come to know itself, and about the status of the forms of thinking in relation to forms in the rest of nature.

In the wake of Kant the philosophical unification of subject and object is seen as entailing the subject's explication of the identity between itself as autonomous, 'intelligible' subject, and itself as knowable object of intuition in the world of deterministic appearing nature. In other words, an explanation is required both of the relationship between the spontaneity of the subject and the materially instantiated activity of the rest of nature, including our own brains and the rest of our bodies, and of how we can know about this relationship. In 1784 Jacobi already asks how we can claim, as Kant does, that nature 'in itself' gives rise to appearances in the subject at all, if, as Kant also insists, nature in itself is topically separate from what we can know about it. Responses to these issues - which Kant began to develop in the $C F$ - need to offer ways of showing that the realms of necessity and freedom cannot ultimately be separate because the purposes which guide my free moral action are connected to the overall purpose of nature manifested in myself as natural being. One way in which the Idealists try to conceive of such a unification is in terms of each passing, sensuous, 'conditioned' moment of experience finding its truth in the totality, the 'unconditioned' or the 'absolute', which gives it its meaning, as opposed to its being simply another part of an endless, arbitrary sequence of what Jacobi termed 'conditioned conditions' (see Bowie 1997 Chapter 1). Jacobi's own arguments aim at revealing the inability of Enlightenment philosophy (which for him includes Kant) to ground the fact that the world is intelligible at all. He thinks such a philosophical grounding inherently impossible, which means he responds to the fact of the world's intelligibility by belief in a grounding deity. For the Idealists, who are, initially at least, opposed to established theology, the impetus behind the idea of the unconditioned is the replacement of theology in the name of human self-determination. Jacobi's arguments concerning the regress of conditioned conditions and the limits of rationalism are therefore regarded as likely to revive such theology. In consequence Idealist philosophy seeks an alternative account of our relationship to the unconditioned which does not have to rely upon something that transcends human reason. Idealism can, then, be seen as reflecting upon what it would mean for knowing and the value of knowing no longer to be separate, without the connection between the two involving reference to a deity. The fact that this reflection will lead in both philosophical and theological directions will be crucial to Idealism's relationship to aesthetics. 
A vital problem here is that if the finite, conditioned moments of existence are to become part of a unified, unconditioned totality they must in some way be 'negated', because they can never be sufficient in themselves. Such negation can, however, easily be construed as the mere abolition of the finite by the infinite, which renders the finite meaningless. This was why Jacobi thought modern science led inexorably to what he came to term 'nihilism', in which each explained condition is just the condition of another condition, and so on, in an infinite regress. In consequence, a philosophical account of the relationship between the finite and the infinite which comes to terms with the possibility of nihilism is a central aim of Idealism. One response of Idealist philosophy to Kant's dualism is, as a result of the controversy occasioned from 1783 onwards by Jacobi over whether Spinozism is necessarily a kind of atheism (see Bowie 1997 Chapter 1), a concern with Spinoza's monism, which obviates any final separation of subject and object.

Much of Idealist thought relies in varying ways on Spinoza's dictum that 'all determination is negation': something can only be what it is by its not being everything else, which defines what it is not. The subject's ability to move beyond anything specific, in order to establish the particular thing's identity in terms of its relations to other particulars and ultimately to the totality, is seen as demonstrating that mithin subjectivity there is a capacity which is unlimited, 'infinite', in a much more emphatic sense than Kant's account of reason would allow. Idealist philosophy tries to articulate what this ability to move beyond limitation means for philosophy, seeking ways of rethinking the relationship between the finite and the infinite without regressing into the dogmatic metaphysics which Kant had refuted. One of these ways is via the experience of the work of art, which is regarded, in the manner of the $C \mathcal{F}$, as an object whose individual parts are transcended into a greater whole, and which is therefore understood as offering a kind of insight which is inaccessible to philosophy. One of the other ways will be Hegel's attempt to create a system of thought which reconciles us to nihilism via philosophical insight into the inherent necessity of the abolition of every finite particular. The relationship between these two contrasting approaches, one of which suggests the limits of philosophy's ability to articulate the highest truths, the other of which regards art as merely a stage on the way to the real truth revealed by philosophy, is the basis of much of what this book has to say. Do art and philosophy have the same purpose, or can they be separated? What does this mean for philosophy and for art in modernity? The contrasts between the Romantics, Schelling and Hegel will have paradigmatic significance for these issues.

The essential thought behind the Romantic, as opposed to the Idealist, view of the post-Kantian situation was already present in the implications of Kant's notion of the sublime. The sublime resulted from the inability of thought to represent the infinite in the sensuous. At the same time the experience of the sublime aroused a sense of the infinite in the subject via the feeling of finitude it produced. In line with Kant, and in contrast to Idealism, early Romanticism 
acknowledges the ultimate philosophical inaccessibility of the absolute but, somewhat in the manner of Kant's Schwärmer, will not give up the endless attempt to grasp the infinite via the sensuous. The consequence of this endless failure is manifest in 'longing', a notion that plays a major role in both Romantic philosophy and art. Longing results from our inherent dissatisfaction with any claim to have attained the final truth via something in the transient empirical world. Friedrich Schlegel says of the sublime: 'The feeling of the sublime must naturally arise for everyone who has really abstracted. Whoever has really thought the infinite can never again think the finite' (Schlegel 19885 p. 98). The absolute therefore becomes unrepresentable: 'The impossibility of positively reaching the Highest by reflection leads to allegory' (p. 105), because allegory is the form in which what is represented is not what is meant. The result of the activity of the understanding can only be the dissection of reality; that which could reassemble it is only accessible via the sense of loss present in longing: 'The essence of philosophy consists in the longing for the infinite and the training of the understanding' (p. 99). It is important to note here, against a common image of Romanticism, that (as elsewhere) Schlegel clearly does not advocate a rejection of the results of modern science produced by the understanding, in favour of some kind of mystical longing, but rather seeks an integration of science into a more all-encompassing conception of reality. However, Schlegel's conception does not lead to Hegel's attempt to integrate all aspects of modern existence into a philosophical system which would show how the real has the same structure as thought. The question is whether the philosophical attempt to represent that which is unrepresentable is not mere selfdelusion.

As I shall show later, the Romantics' arguments have distinct echoes in aspects of post-structuralism: a philosophy of inherent incompleteness can be construed as a philosophy of deferral. It should be remembered, however - and this is what separates Romanticism from deconstruction - that if there were not some, perhaps inarticulable, sense of a lacking completion, the notion of deferral would be meaningless. Deferral means putting off for later, not abolishing. At the same time, the question remains - and it is still alive in contemporary philosophy, in the difference, for example, between Hilary Putnam and Richard Rorty - whether the Romantic regulative idea of truth as the goal of our spiritual life might not better be abandoned. Rorty argues, for example, that 'there is nothing that can plausibly be described as a goal of inquiry, although the desire for further justification, of course, serves as a motive of inquiry' (Rorty $1998 \mathrm{p}$. 38). In consequence 'truth' as a regulative idea is 'an ever-retreating goal, one that fades forever and forever when we move. It is not what common sense would call a goal. For it is neither something we might realise we had reached, nor something to which we might get closer' (p. 39). Putnam, in contrast, insists, like the Romantics, that: 'The very fact that we speak of our different conceptions as different conceptions of rationality posits a Grenzbegriff, a limit-concept of the ideal truth' (Putnam 1981 p. 216). 
Much depends here upon how the notion of truth is conceived. The significance of Romantic aesthetic thinking lies not least in its incorporation of a normative sense of the need to attend to all our relations to the world in terms of truth, rather than merely to our cognitive relation to the world of objects investigated by science. Manfred Frank's recent work (for example, 1997) has tended to interpret the Romantics rather too much in terms of their links to contemporary 'metaphysical realism', thereby restricting the interpretative perspective to epistemology. The Romantic notion of the absolute cannot, though, be straightforwardly assimilated to the metaphysical realist idea that the world independent of our finite, fallible knowledge of it is the true world, but involves instead an awareness that knowing things, as Stanley Cavell has suggested, is not the only way of relating to them. Even if we could know that our knowledge indeed corresponded to reality (assuming one could make sense of the idea of truth as correspondence of sentences or ideas to reality anyway), this would not solve the really important philosophical problems in modernity. Schlegel suggests why in the following remark: 'In truth you would be distressed if the whole world, as you demand, were for once seriously to become completely comprehensible' (Schlegel 19882 p. 240), and he observes elsewhere that 'If absolute truth were found then the business of spirit would be completed and it would have to cease to be, since it only exists in activity' (Schlegel 1991 p. 93). The idea of knowing everything, then, can just as easily lead to nihilism as can the scepticism which anyway inevitably results from metaphysical realism - if reality is pholly independent of what we think about it, nothing could ever confirm, in thought, that what we think is true of that reality. Both the idea of omniscience and radical scepticism involve the metaphysical realist assumption as a limiting 'absolute', but both, as Schlegel realises, fail to deal with the sort of relations to the world which give it meaning. ${ }^{2}$

The history of these ideas is an area of considerable complexity, and it is becoming clear that the stories told about the implications of Idealism and Romanticism are in need of considerable revision in the light of changing conceptions in contemporary philosophy. The standard story of German Idealism is that it is inaugurated by Fichte's radicalisation of Kant's turn to the subject, is continued in Schelling's System of Transcendental Idealism (STI) and 'identity philosophy', and culminates in Hegel's system. Hegel is then superseded by Marx, who turns Hegel's speculation into a new form of materialist philosophy. A logic of development is thereby suggested which is implicitly Hegelian: each stage of the process takes up the truth of the previous stage, refutes that stage's errors, and moves on to a higher stage. Views of history based on a necessary underlying continuity are, however, highly questionable: they follow Hegel in presupposing that what emerges from such conflicts is part of an inherent developing truth. It is, though, often the case that what has apparently been superseded has in fact merely been repressed, and will return later because it was never fully articulated. The recent attention in post-structuralism and elsewhere to the kind of questioning of the notion of truth characteristic of the work 
of Heidegger clearly has its roots in the unresolved issues of post-Kantian philosophy, rather than in a continuous philosophical development.

My contention is, therefore, that it is possible to tell a different story about the relationship of Idealism and early Romanticism to subsequent philosophy, which shows that very few of their concerns have really disappeared from the agenda of that philosophy. This is already evident if one looks at the role of aesthetic theory in the philosophy of the period with contemporary eyes. That Richard Rorty should now regard philosophy as a kind of literature, because he does not think it is possible for it to establish a privileged role in relation to other ways of articulating the world, is not fortuitous. Such a notion has nothing surprising about it for a Romantic thinker, and is not alien to Schelling's STI, which sees art as able to show what philosophy cannot say. It is, though, important to remember here that the major role given to art in German Idealism and early Romanticism is not just, as it is sometimes taken to be in the secondary literature, the result of exaggerated expectations about the ability to link the sensuous and the intelligible in a positive way, such that art directly symbolises the absolute. Often art is understood rather as 'deconstructing' the boundary between the intelligible and the sensuous, thus suggesting why philosophical attempts to define their respective roles in our constitution of the world are unlikely to succeed, and pointing to the need for other kinds of orientation in a post-theological world.

The increased importance of art in this period results, then, from the realisation that if collectively warranted truth were only available in the form of natural science based on empirical observation and the establishing of causal relations, we might be living in the nihilistic world of 'conditioned conditions' feared by Jacobi. The only truth in such a world results from the subsumption of natural phenomena under general laws, a subsumption which contemporary cognitive science now seeks, of course, to extend to the explanation of the subject's own thinking. The vital objection to conceptions like those now dominating cognitive science (and the public culture it has helped to create) is Kantian: what can cause something that is supposed to be exclusively causally explicable to apprehend itself as causal in the first place? Why does it bother? The whole point of transcendental philosophy is that there is a limit to the subject's capacity for self-objectification which forces philosophy beyond the conditioned world towards the exploration of what makes it possible to apprehend conditions as conditions, or, indeed, apprehend anything as anything at all. Whatever this is would seem therefore itself to be in some sense unconditioned, and thus inaccessible to knowledge of the kind we have of objects that are 'real', in Kant's sense of 'being given in intuition' - whence Kant's difficulties with the status of the I in the transcendental deduction.

Interpreting Idealism and Romanticism has been and is so difficult because it is still unclear what the demonstration that there must be an 'unconditioned' aspect of subjectivity actually means. The need for responses to the world which are based on more than what the sciences can explain in terms of deterministic 
laws is still relatively uncontentious in many areas of philosophy, but how far does this take one in terms of metaphysical commitments, for example, to the idea that nature in itself is inherently subjective, and thus 'unconditioned' in some respect? In the face of the ecological crisis we are perhaps less likely to reject such ideas out of hand and are perhaps even able to understand the arguments of philosophers of this period better than they understood themselves. ${ }^{3}$ Their attention to art is, in this perspective, not just a concern with the icing on the cake of a post-theological world. It is rather a response to the feeling, already present in Kant's account of the genius, that 'external' nature is not just a causally determined object and is part of ourselves as subjects in ways we cannot explain, and perhaps could not ever fully explain.

\section{A 'new mythology'}

Both Idealism and Romanticism are aware, as was the younger Marx, that the revelation of the hollowness of theology does not lead to the disappearance of the needs which gave rise to it. The 'opium of the people' does not just numb the capacity for resistance by obscuring the real nature of a humanly created oppressive reality, it also kills the pain of meaninglessness by making negativity part of something which can transcend it. Replacing dogmatic theology is one of the major tasks of modern German philosophy from Idealism to Feuerbach, to Marx, Nietzsche and beyond. The concern, in both Idealism and Romanticism, with mythology should therefore not be lightly associated with later reactionary appropriations of mythology in terms of the need for a return to primordial origins with which we have supposedly lost contact in modernity. The essential attribute of mythology in this context is rather its use of a story about particular beings to tell a general story about the meaning and nature of reality.

The so-called 'Oldest System Programme of German Idealism' (SP) (reprinted in the Appendix), is a manifesto for a new philosophy and exemplifies the spirit of early Idealism, not least with regard to mythology. It was written down in the hand of Hegel (in June 1796?), has some of the characteristics of the thought of the young Hegel, but many people would consider that it was probably written by Schelling. ${ }^{4}$ (Some consider neither to have been the actual author.) The impetus of the text was suggested in Szondi's remark at the beginning of this chapter: it wants to re-unify the world that has been split up by Kant's critique of traditional metaphysics, at the same time as reinforcing Kant's insistence on our capacity for self-determination. Beginning with Fichte, German Idealism attempts, for reasons explained more fully in the next chapter, to give a more emphatic role to the free activity of consciousness than is evident in Kant's cautious formulations. The $S P$ is a manifesto and has no pretensions to coherent argumentation. Despite this it makes certain major issues very clear.

The basis of the $S P$ is a radical notion of freedom: 'The first idea [Idee] is 
naturally the notion [Vorstellung] of myself as an absolutely free being'. As was evident in Kant, once theological 'dogmatism' ceases to define our place in the world the creation of our relation to the world becomes the task of rational beings. Whereas Kant did not wish to make absolute claims for the ego, because this would require intellectual intuition to back up claims about the selfdetermining nature of the I, the $S P$, in the light of Fichte's Wissenschaftslehre (Doctrine of Science), has no doubts about the absolute freedom of the intelligible self. When the SP asks: 'How must a world be constituted for a moral being?' the question is, significantly, posed from the side of the subject that will alter the world, rather than from the side of a world into which the subject should fit. The text then jumps abruptly to the demand for a 'new physics', suggesting how far such thinking is from simply adopting Kant's clear boundaries between the realm of the understanding and reason. Such a demand becomes comprehensible in the light of Schelling's and Goethe's conviction that nature, even within the realm of natural science, should not be just regarded as an object to be brought under causal laws. The immediately following abrupt attack on the state as a 'machine' that cannot correspond to the 'idea' of mankind is inseparable in such thinking from worries of the kind we saw in Chapter 1 about modern science's turning nature into a machine.

In Kant's philosophy, the antidote to the mechanistic view of nature was the conception that natural organisms could be understood as functioning as if they followed an 'idea', an idea being what makes an object into more than the sum of its observable attributes. Kant linked the organism to the artwork produced by the genius, thus linking natural teleology, the growth of the organism into its particular form, and artistic production of a whole which is more than the sum of its parts. The idea of the organism is fundamental to the $S P$ and is derived from Schiller's response to Kant in his Letters on the Aesthetic Education of Man (1795). Manfred Frank defines organisms as 'structures whose parts take part in the purpose of the whole and in such a way that the purpose is not external to them, but, rather, their own purpose' (Frank 1982 p. 188). In order for the state to become an organism the individual organisms in it must be united to form a greater organism: for this they need a purpose. A purpose justifies a new state of reality not in terms of what is known of reality up to now but in terms what reality ought to become. As Frank puts it: 'Whilst only understanding is necessary to comprehend the mechanical linking of states of matter . . . in order to perceive purposes one needs reason ... the capacity to turn plans into deeds . . . a purpose is not something given [gegeben], it is a task [aufgegeben]' (p. 158). Reason is a potential which we have in us, hence, as we saw, the idea that it is 'infinite', because it is not defined by the way things already are.

The culmination of the conception of the organism, even for Kant, is the idea that if nature as a whole is thought of as an organism there must be a purpose in nature which can be linked to human purposes and activities. Access to such a purpose would enable one to legitimate a form of social organisation which could bring us into harmony with what we are as part of nature. This purpose, 
it should be remembered, need not be seen as a controlling divinity: Kant does talk about nature's purposes, but this can be construed in terms of the idea that spontaneous self-determination is an inherent part of nature. On the other hand, if there is thought to be no purposiveness in nature in this wider sense, the consequence, as we shall see later in the work of Schopenhauer and Nietzsche, is a view of reality as a disintegrated series of warring forces that have no essential direction. The choice between what now seems to us an indefensibly optimistic metaphysical conception of the totality and what is in fact an equally metaphysical negative image of that totality increasingly seems a bad one, but what these conceptions articulate will not go away as long as ecological questions about the relation of human activity to the rest of nature are asked.

The $S P$ next introduces the 'idea that unites all ideas, the idea of Beauty, taken in the higher Platonic sense'. This follows from the reasons for Kant's introduction of the notion of reflective judgement and his connection of natural teleology to works of art. The idea of beauty is supposed to overcome the gap between laws of nature constituted via the understanding and what reason is to do with this endless diversity of particular laws. It is at this point that the $S P$ maintains that the 'highest act of reason' is an 'aesthetic act'. This aesthetic act would enable practical reason to integrate the truths produced by the understanding into a purposive whole. The work of art is purposively produced, via free human initiative; at the same time, it is accessible to the understanding because it is an object of 'intuition': you can see it, hear it, and so on. As such, it partakes of the two realms which Kant's first two critiques had sundered, and which he tried to unite in the third Critique. Manfred Frank suggests the consequence of this:

Even when I do not produce an aesthetic product, but enjoy one, I still must use my freedom. For nothing sensuously visible and reconstructable in thought is sufficient to impress the character of the aesthetic on an object of nature [i.e. the understanding cannot produce aesthetic judgements]. I must, in order to become aware of the freedom represented in the object, use my own freedom. (p. 158)

The aesthetic product thus becomes a utopian symbol of the realisation of freedom: in it we can see or hear an image of what the world could be like if freedom were realised in it. We can see it in this way precisely because of that aspect of self-consciousness whose basis cannot be articulated in concepts, if concepts are taken in Kant's sense as rules for identifying objects.

In the $S P$ an understanding of Kant's postulated 'kingdom of ends', which philosophy can only characterise in the abstract terms of a world where rational beings are 'ends in themselves', becomes available to intuition in the work of art. Bernhard Lypp has termed this view 'aesthetic absolutism', the 'invocation of the unity of absolute experience which rests upon the materiality of the aesthetic sense' (Lypp 1972 p. 13). Because the aesthetic product still remains, qua created object, in the realm of intuition, it is able to point to why the world of the senses is not radically separate from the intelligible world. What makes the 
work a work of art which gives aesthetic pleasure is our free judgement, which is independent of any instrumental relationship to the object. Without the sensuous art-object, though, we would have no access to how our freedom relates to the appearing world. This conception might sound as though it just makes into a certainty what Kant had been careful to leave in the realm of the 'as if'. However, even in Kant's terms there is more than just a determination of the object by the subject in aesthetic judgement. As Scheible puts it: 'Only where the subject for its part can be affected by the object, in the aesthetic judgement, can the object be really "known" [erkannt in the sense of "recognised"], as only here does it cease to be simply "determined" [bestimmt]' (Scheible 1984 p. 124). The transcendental subject's connection of intuitions in judgements of the understanding gives rise to a nature whose coherence depends solely upon the subject's determination of what can be connected within it according to fixed rules. The aesthetic sense, though, also involves a non-determining relationship to the object: the object affects us in ways about which the connection of phenomena in terms of rules can tell us nothing. The object can only affect us in this way because of our freedom, but that freedom is not evoked by every object and it depends upon a much more extensive ability of thought to integrate particulars into significant wholes than that required to subsume particulars under rules (though, as we have seen, the separation between these two kinds of judgement cannot be absolute).

As I suggested in Chapter 1, Kant's distinction between the beautiful and the sublime creates the conceptual space for issues of aesthetics and politics from this time onwards. The $S P$ 's view of beauty is not just based on the - often highly questionable - political import of organicist ideas. Another political dimension, which plays a decisive role in later debates over aesthetics and politics, is already apparent in the SP's scepticism about abstraction, in the sense of the lack of connection of general rules to what those rules might mean to individual human subjects in the life-world. Philosophy without aesthetic sense, the $S P$ claims, is based on the understanding's quantitative, rule-bound determination of objects, which ignores both their sensuous particularity and the ways in which they may meaningfully cohere with each other. In a philosophy without aesthetic sense even the imperatives of practical philosophy, the basis of concrete ethical life, involve the danger of abstraction, because they may, in their theoretical form, be either incomprehensible or lacking in any motivating force for most people. The philosophical exploration of the relationship between the sensuous and the intelligible is, then, closely connected to major political issues in modernity. How abstract imperatives are to be made accessible and desirable in the public sphere remains an inescapable political issue. Art's attachment to sensuousness therefore points to its political potential, but at the same time it gives rise to important suspicions. Kant's introduction of the sublime and his approval of the ban on images in Jewish theology imply that the sensuous world is really there to be overcome, because it blocks participation in our higher purpose, which is independent of anything given in sensuous nature. Suspicion 
of the sensuous will, as we shall see, also be important in Hegel's Aesthetics, where art constitutes a lower form of truth because of its dependence upon particular sensuous manifestation. Significantly, this suspicion will become a major target in Feuerbach's and Marx's attacks on Hegel, and in the later Nietzsche's attacks on philosophy's desire to escape the sensuous.

Kant himself was in the position of Schoenberg's Moses, trying to persuade people believe in a God - reason - which they could not see, and often doing so in a language largely devoid of aesthetic appeal. It is clear, though, as anyone teaching philosophy knows, that key abstract ideas can often best be explained by trying to tell stories about them. Derrida's work can, for example, be read in this perspective as revealing how such stories may not even be separable from the ideas at all: the metaphors on which philosophy lives are not a dispensable extra. As Hamann's conception of language as inseparable sensuous sign and intelligible meaning already suggested, the very idea of a boundary between the sensuous and the intelligible can be questioned, and Derrida's concern with deconstructing such oppositions is in this sense part of the Romantic tradition. Analogous ideas to Derrida's are, of course, legion in post-Kantian philosophy: the deconstruction of the sensuous/intelligible divide is prefigured, as we shall see, in Schelling's notion of 'absolute indifference', in which there cannot be an absolute division between the sensuous and intelligible because they are inseparable aspects of the same infinite continuum.

For radical Enlightenment thinkers, like the early Schelling, this too is a political issue. The disintegration of the theological world view brings with it a loss of collectively accepted concrete stories, of the kind which used to be present in mythology, that revealed supersensuous truth via the sensuous world. Works of art, which articulate collective concerns and retain something of the status attached to cult objects, are accordingly regarded as offering the possibility of re-uniting a world which the abstractions that govern the modern world have begun to pull apart. The modern destruction of mythical forms of coherence need not, of course, be interpreted just in terms of loss. Habermas considers the differentiation of cognitive, ethical and aesthetic spheres in modernity, which excludes authority-based myth from knowledge that is open to public debate as to its validity, to be a key to the advances made possible in modernity. However, this separation is already perceived by Idealist and Romantic thinkers as also involving dangers, which a new mythology would try to overcome by a new synthesis of art and science. The Idealist and the Romantic perception is of the need to find novel ways of linking individual ways of making sense, which have an inherent basis in sensuous intuition, both to the results of the activity of the understanding and to the purpose of these results as a means of gaining control over nature.

The vital factor here is the imagination. Novalis suggests in the light of Fichte, who takes up and extends the grounding role of the imagination in Kant, that 'All inner capacities and forces and all outer capacities and forces must be deduced from the productive imagination' (Novalis 1968 p. 413). If science 
depends on the production of schematised images which are processed by the understanding, this production can be seen as more fundamental than the one particular kind of synthesis achieved by the understanding. Indeed the imagination can be understood as what makes being intelligible, instead of remaining opaquely enclosed within itself. ${ }^{5}$ The structure of seeing something as something which is made possible by what Kant explains in terms of schematism need not result in determinate cognition, as the ability to create metaphors new ways of 'seeing as' - suggests. In this respect what the imagination produces seems to span both art and science. Taken a step further, nature's own productivity might seem not be essentially different from our own production of forms of coherence in our coming to know about nature: the new science adumbrated in the $S P$ would in this view reflect the conviction that the genesis of the natural 'product' and the genesis of the knowledge of the product are ultimately of the same order.

Such ideas have too often been written off as pure speculation. Interestingly, though, the kind of speculation linking aesthetic and scientific praxis characteristic of the Romantics and of Schelling's Naturphilosophie does produce a lot more than merely speculative scientific results. Thinkers like Rorty have argued that radical new theories initially take the form of metaphors, in that they are not meaningful in terms of the existing forms of linguistic usage, and the metaphors only become literalised when they are generally accepted and move into common usage. The fact that the discovery of ultraviolet, theories of the ice age, and electromagnetism, for instance, are all worked out as part of Romantic science suggests the plausibility of such views. Looking at the genesis of new science often reveals that it is not merely the result of new rule-based judgements based on detailed inference. Instead, imaginative leaps which reconfigure the whole area in question are required if the previously accepted picture is to be replaced. Recent speculative science also seems to confirm this. Stephen Hawking, whose enormously successful A Brief History of Time seems at times to breathe the air of Idealist and Romantic science, has remarked upon the extent to which he initially thinks in images rather than in mathematical formulae. The perception of science which develops later in the nineteenth century in Germany and which still dominates much of the contemporary public image of scientific practice is generally suspicious of such ideas. Significantly, though, they have returned in the light of the new physics and of the contemporary changes in the perception of science effected by Kuhn, Foucault and others, most of whom are demonstrably dependent upon ideas deriving from the period at issue here.

Whatever one may think of the validity of Idealist and Romantic ideas for the philosophy of science, they do also suggest how the relationship between the theoretical and the sensuous is a still barely understood dimension of the politics of modernity. With the emancipation from tradition of the subject's capacity to describe the world, the awareness becomes explicit that objects can be articulated in an indefinite number of ways, whether this is, for example, in high 
art or in advertising. Objects consequently lose any sense of possessing binding truth for a community, but gain the potential endlessly to be re-described and re-articulated, though this in turn carries with it the threat of mere arbitrariness. The understanding, on the other hand, seeks to process objects in such a way that they are reduced to being candidates for truth only in the respect in which they can be subsumed under explanatory laws. As we have just seen, for Kant and his successors, the source of both these approaches to objects is the imagination. The problem of induction, to which Kant responded with his notion of reflective judgement, suggests how this issue is inevitably bound up with both the cognitive and the aesthetic. Even the understanding can produce an indefinite number of possible laws for any phenomenon, and there seems to be no essential criterion which would guarantee which is the correct law: establishing this seems to rely on judgements of coherence with other explanations which are analogous to aesthetic judgements. When played out in the social world what is involved in the issue of the imagination again takes on a more political dimension, of the kind indicated by the $S P$. Although natural science relies for its validity claims upon the exclusion of individual imaginative articulations of the object, it does increasingly have recourse to the aesthetic when it comes to the attempt to communicate scientific claims to the rest of society.

This tension between the cognitive and the aesthetic relation to the object plays a vital role in the culture of modern capitalism, which, as Marx's theory of the commodity claims, leads to objects becoming involved in processes of abstraction not unlike the constitution of an object of science by the understanding. The object as exchange value is abstracted from all its sensuous particularity in order to make it exchangeable for any other commodity. This leads to the need for ways of restoring the role of sensuous particularity if the commodity's abstract status is not to diminish its desirability. Advertising's continual raids on the sphere of art can therefore be understood as seeking to give back an individual sensuous appeal to the object of exchange value. The object qua exchange value needs the adjunct of the aesthetic image for it to function as a desirable use value. Furthermore, the consumer's 'need' for the object may itself have initially been stimulated by the aesthetic images attached to the object, which are used to add a gloss to its status as, say, just another cancer-causing cigarette. $^{6}$

Aestheticisation of commodities creates an increasingly difficult situation for serious artists who have often responded to it with a revolt against sensuous beauty. Their need is to escape complicity with the adding of aesthetic pleasure to exchange values and thus to sustain the notion of art as being independent of appropriative interest and as a continuing challenge to established ways of seeing. This is one root of the emergence of avant-garde art, which tries to escape existing forms of communication and often makes no attempt to be sensuously pleasing. In this way the limits of representation suggested by the notion of the sublime become a matter of cultural politics. The development of the avant-garde can, in this respect, be seen as connected to the failure of the 
sort of hopes suggested in the $S P$. The synthesis of sensuous image and abstraction seems only to take place in a collective manner in contemporary societies in the sphere of advertising and in the manifestations of administered mass culture. It does not take place in a new political public sphere in which the aesthetic is an integrated part of culture that includes all dimensions of human existence. That such syntheses take place in this way in mass culture is, though, not something to be judged in moralising terms. The needs which are being catered for are real, however illusory their fulfilment may be. Modern societies are evidently intolerable to their members without some sensuous way of relating to the technological and economic developments within those societies, however much these actually depend upon the elimination of sensuous particularity. The increasing incursion of the admen into the world of science, technology and economics is an expression of people's ineliminable need for a re-integration of dimensions of modern existence whose internal development increasingly separates them from other dimensions of that existence.

When Habermas maintains that philosophy today might 'at least help to set in motion again the frozen interplay between the cognitive-instrumental, the moral-practical and the aesthetic-expressive, which is like a mobile that has become stubbornly entangled' (Habermas 1983 p. 26) he in fact echoes ideas from the $S P$, a text of which he is otherwise generally critical. The $S P$, then, points to coming dangers which result from the way Kant's separation of understanding and reason is manifested in the historical world. It is in this perspective that one has to understand the call for a 'new mythology' that would link the abstract and theoretical notions of philosophy and science to sensuous experience, in the form of images and stories. The mythology demanded is, remember, a mythology of reason: the point is that it is up to us to make it, in the same way as Kantian reason is the task we have as autonomous beings. The $S P$ concludes with the demand for a 'polytheism of the imagination and of art', and a 'mythology of reason', which would synthesise the potential released by science, art and critical philosophy in the manner that myths integrated the contradictions between nature and society in traditional cultures. As the $S P$ puts it: 'Before we make the Ideas aesthetic, i.e. mythological, they are of no interest to the people and on the other hand before mythology is reasonable the philosopher must be ashamed of it.' Such a view is echoed in Gramsci's conception of hegemony: if intellectual developments are not just to reinforce existing power structures, ways must be found of communicating those developments to the people, in order that they can make them effective in political emancipation. The synthesis of aesthetics and reason in the name of the radical democratic politics demanded by the $S P$ is consistent with the Idealist philosophical desire to reveal the higher unity in the diversity of the sensuous world and thus to prevent a disintegration of the world into merely instrumentalised particulars. This again points to the extent to which the philosophy of the period is inseparable from political and cultural concerns. The failure of the vision of the $S P$ will suggest the political failure of this version of the Idealist project. It also 
points to the difficulties that will be faced by Marxist theory in coming to terms with the significance of art in a world where the collective theological or mythical basis of society has disintegrated.

\section{Early Romantic 'nem mythology'}

The division that can be made between Idealist and Romantic thinking depends upon the extent to which each thinks it possible to restore unity to what the modern world increasingly separates. In the main the Idealist response to the divisions in modernity is to seek new philosophical foundations on the basis of the Cartesian and Kantian conception of the founding role of self-consciousness. For Idealism, what philosophy can analyse in the activity of consciousness is a higher form of the intelligibility present in nature, so that the task of philosophy is to show how our thinking is the key to the inherent intelligibility of things. The essence of the Romantic response, on the other hand, is a realisation that, while it must play a vital role in a modern conception of philosophy, the activity of consciousness is never fully transparent to itself. It can therefore never be finally incorporated into a philosophical system, because what we can consciously know of ourselves does not exhaust what we are (see Bowie 1997, Frank 1997). ${ }^{7}$

The initial proximity of the Idealist and Romantic conceptions is apparent in the fact that Friedrich Schlegel's Discourse on Mythology (1800) seems to rely upon a similar relationship between art and mythology to that indicated in the $S P$ and the $S T I$. However, Schlegel begins to break with a central assumption of the $S P$. He thereby already suggests reasons for the emergence of the notion of aesthetic autonomy, the modern idea that art is subject only to its own necessities. Aesthetic autonomy will become particularly significant in the light of the failure to synthesise the sensuous and the theoretical in an Idealist 'new mythology'. Karl Heinz Bohrer has claimed that: 'Schlegel's Discourse on Mythology is precisely "new" in that the "new mythology" which it announces, as opposed to the demand of the System Programme and to the aesthetics of the young Schelling, is expressly not "in the service of the Ideas", i.e. not a "mythology of reason"' (Bohrer 1983 pp. 56-7). The reason for this claim is that Schlegel's argument moves away from the emphasis seen in the $S P$ on revolutionary demands for new forms of communication in the political public sphere. The sense that human creativity is linked to a wider purpose in nature here already begins to give way to an ontology of spontaneous, non-teleological creativity of the kind later to be seen in Nietzsche's The Birth of Tragedy. Schlegel therefore loosens the links between aesthetics and ethical goals present in Idealism. Bohrer suggests - although in doing so he exaggerates the extent to which this is important in the Discourse and largely ignores the fact that in many respects the early Schlegel remains committed to the task of reason - that Schlegel initiates 'aesthetic reduction': 'the reduction of the time of the philosophy of history to an ecstatic moment' (p. 59). 
Such a reduction is apparent in Schlegel's notion of Witz ('wit'), the capacity to create random correspondences which suggest a unity of totally diverse phenomena, and thus of the whole world, in the manner of a myth. However, the crucial difference of wit from mythology is that it is characterised by randomness, suddenness and transience, rather than possessing an enduring meaninggiving function. Art thus begins to involve a temporality which no longer points beyond itself, exists only in the present of the engagement with the work, and is irreducible to anything else. The temporality in question here will, of course, be a central issue in modernist art: Proust's moment privilégié and Joyce's epiphany, for example, echo what Schlegel intends. The other aspect of art highlighted by Schlegel is allegory, which he understands as being a result of the impossibility of presenting a positive account of the absolute. Allegory points beyond itself and it is therefore not, as a symbol is, a sensuous embodiment of what it means. In this respect allegory is analogous to Kant's sublime. For Schlegel, then, one is left with the alternative between the evanescent transcending of the sensuous in wit and a failure to represent a transcendent unity in allegory, rather than with a way of seeing art as the sensuous manifestation of the infinite. The aptness of these ideas is already evident in the extent to which they become preoccupations of so much modernist art from this time onwards.

In the Athenäum Fragments Schlegel claims: 'A philosophy of art in general [der Poesie überhaupt $]$ would begin with the independence of the beautiful, with the proposition that it is separate from the true and the moral and should be separate from it and have the same rights as it' (Schlegel 19882 p. 129 Fragment 252). The culmination of this idea will be Nietzsche's contention in The Birth of Tragedy that the only justification of existence itself is as an 'aesthetic phenomenon', which is contemplated immediately for its own sake because it lacks any teleological justification. Schlegel, though, does still retain the idea that art can point beyond itself towards an unrepresentable absolute, which would, of course, connect it again to the cognitive and the ethical. This suggests an important division in modern thought, between early Romanticism and the more questionable tendencies which emerge from it, which have no concern with the ethical implications of the aesthetic. The problem of the modern artist is, Schlegel claims, that he must 'work out from the inside' and create 'every work like a new creation from nothing' ( 2 p. 201). Modern art lacks a 'centre', of the kind that mythology represented for ancient cultures, from which to derive collectively binding images and symbols. Schlegel insists that the new mythology cannot use 'the nearest and most lively aspects of the sensuous world' in the manner that Greek mythology did, and 'must on the contrary be formed out of the deepest depths of spirit [Geist]; it must be the most artificial of all works of art, for it is supposed to grasp all other arts within it, a new bed and container for the ancient eternal original source of poetry' (2 p. 201). The new mythology would link all forms of articulation, from the arts to the sciences, hence the need for it to be artificial, a synthesis in a new kind of culture of what modernity separates into differing spheres. 
The crucial difference of Schlegel's position here from that of the $S P$ resides in the fact that he sees Poesie as originating in the negation of the 'progress and laws of rationally thinking reason'. We should instead be plunged into 'the beautiful confusion of fantasy, into the original chaos of human nature, for which I know of no better symbol until now than the colourful swarm of the ancient Gods' (2 p. 204). Schlegel wishes to explore the creative potential in every particular sensuous object - if laws of reason are suspended anything can be the occasion of the operation of fantasy - which leads to an endless proliferation of possible articulations. This was, of course, the sort of thing which Kant warned against in his reflections on the sublime. The difference between Kant's concern that one should not to spend one's time seeking something which is more than sensuous within the sensuous, and Schlegel's Romantic position, is a precursor of some of the main divisions in the aesthetics of modernism. Modernist aesthetics is located between the tendency to abolish art altogether by revealing its ineradicable attachment to the sensuous, and the tendency to elevate art to a status possessed by no other aspect of the modern world on the basis of the idea that it is the only thing which can still take us beyond the sensuous, albeit in an essentially arbitrary manner. Schlegel's proximity to Hamann's Aesthetica in nuce is also evident here. Both regard mythology and art as deriving from the same source: an endless process of articulation at all levels of nature and of human activity: 'Mythology is . . . a work of art of nature . . . everything is relation and transformation, formed and reformed' (2 p. 204).

The further question in this context is how the subject relates to this process of 'relation and transformation', which functions in a manner which is not under the subject's control. Lévi-Strauss's structuralist account of mythology, whose analogies to aspects of Romantic thinking are apparent in its connecting of music to mythology and mythology to language, is seen by Paul Ricoeur as suggesting that mythology is 'a categorising system unconnected with a thinking subject ... homologous with nature; it may perhaps be nature' (Lévi-Strauss 1975 p. 11). Lévi-Strauss was influenced by C.G. Jung, who himself relied on elements of Schelling's early Naturphilosophie and later Philosophy of Mythology of the 1840s. Schelling suggests in 1841 that in mythology: 'the ideas (Vorstellungen) are products of a necessary process, or of natural consciousness which is left to its own devices, on which there is no influence of any free cause' (Schelling 1977 p. 250). Schlegel is similarly not concerned with the reflecting, intending subject at this point: the vital aspect is the surrender to the other, the loss of identity. This can be construed as a return to a more 'natural' way of being, as it often is in later Romantic theories and in subsequent reactionary politics, but it can also be interpreted in a perhaps less questionable manner. Unsurprisingly, given its links to Romanticism via Nietzsche, certain tendencies in post-structuralism parallel notions of a subjectless generation of difference of the kind suggested by Schlegel. The root of such conceptions of endless formation and reformation is the renewed interest in the myth of Dionysus that develops in the second half of the eighteenth century. Importantly for my 
overall argument, this interest becomes linked to the increased significance attached to music at this time.

Hamann had already prophetically linked Dionysus to art in Aesthetica in nuce (1762): 'Do not dare enter the metaphysics of the arts without being versed in the orgies and Eleusinian mysteries. But the senses are Ceres, and Bacchus the passions; - old foster parents of beautiful nature' (Hamann 1967 p. 111). In the essay On the Study of Greek Literature (1797), Schlegel says of Sophocles that he amalgamates 'the divine intoxication of Dionysus, and the deep sensitivity of Athene, and the quiet collectedness of Apollo' (Schlegel 19881 p. 107). Dionysus is seen here as the principle of disintegration which needs to be balanced by forces which give form to chaos. In Der kommende Gott (The Coming God) Manfred Frank explains how the figure of Dionysus plays an important role in both Idealist and Romantic thinking, making clear that the decisive aspect in the new understanding of this myth is the way in which it involves an inherent identity of opposites:

Dionysus is the God who does not have a high opinion of the principle of individuation, who drags everything into the frenzy, makes 'women into hyenas', tears down the barriers between the sexes, and in general manipulates the separate realms of being as he wishes, by on the one hand pulling them down into the whirlpool of undifferentiated identity, on the other, as the liberating God dedicated to progress and evolution, separating the realms of being anew and - in the literal sense of the word - differentiating them. Thus he participates both in the principle of unity and of separation. (Frank 1982 p. 20)

The vital point is, then, that creativity is inseparable from destruction, and this apparent paradox is at the root of the philosophy of this period.

The myth of Dionysus involves a version of the identity of identity and difference', which is central to the thought of the early Schelling, and to Hegel. The fascination of Dionysus has two related sources. The story of the god that is destroyed and reformed can be linked to the story of Christ, as it frequently was from the eighteenth century onwards, and it clearly also played a role in the initial formation of Christian mythology. At the same time a key problem in Idealist and Romantic thinking which they inherited from Greek philosophy how can the universe be shown to be both one and differentiated within itself? - appears in the sensuous form of the story of Dionysus. This link of unity and division is itself one way of interpreting the passion of the son of God in Christian mythology. ${ }^{8}$ Dionysus, then, becomes the ground of the intelligible world, which produces endless sensuous forms out of itself. For both Schopenhauer and the early Nietzsche this ground will be most readily accessible in music. ${ }^{9}$ The initial reasons for this conception are best outlined here: the topic will frequently recur later.

The link of music to Dionysus can be better understood if we consider Schlegel's view of 'Romantic art' in the famous fragment 116 of the Athenäum. Romantic art is 'still in a process of becoming; yes, that is its real essence, that it 
can eternally only become, can never be completed. It cannot be exhausted by any theory' and multiplies itself 'as if in an endless row of mirrors' (Schlegel 19882 pp. 114-15). Romantic art is thus analogous to Dionysus, the god who combines creation with destruction: each relation of the work to something else (in 'reflection', which can include theoretical explanation of the work) is negated in the next relation, but the overall process of relation between work and world cannot come to an end because there is always more to be said. Whereas the Idealist new mythology would constitute a binding together in collective forms of the products of modernity, this view involves the unleashing of a world of decentred diversity. The process of 'infinite reflection' in Romantic art is initially associated with music because of music's dependence upon the passing of time for the different moments of a piece of music to become linked in a significant manner. The condition of the identity even of the whole of a piece is the difference of its moments in time, a thought which is consistent with the Idealist conception of unity in diversity. However, what the musical whole signifies cannot be articulated in a definitive way: music is non-representational and seems, like Schlegel's allegory, to point beyond itself without our being able to say definitively what it is pointing to. It is this fact which makes music the form of art most likely to be associated with the failure to represent the absolute in a positive manner.

The relation of the idea of the 'new mythology' to music reveals a fundamental tension in modern art. Music inherently has the potential to sustain aesthetic autonomy via its non-representational character, and this is a major reason why it comes to be seen as a counter to the reduction of more and more of the world to scientific explanation. At the same time, however, music also becomes a major political issue, despite its distance from representation. Wagner will, for example, attempt to revive a nationalist mythology by creating music which moves beyond the existing formal norms and establishes new ways of integrating musical material. Wagner's project combines the political impetus of the $S P$, albeit in a distorted form, with the Romantic view of the liberation of the aesthetic from pre-given rules. The power of the resulting works, and their highly questionable subsequent reception and use as ideology, particularly by the Nazis, make it evident that aesthetic issues go to the very heart of modern politics. Analysing what is at stake in such aesthetic questions for the history of modern self-consciousness will be one of the major tasks of the chapters which follow.

The idea of the 'new mythology' has deeply worrying resonances in the light of the events of twentieth-century history: the most spectacular - albeit temporary - political success of a 'new mythology' was, of course, that of the Nazis. It is therefore no coincidence that Walter Benjamin, no stranger to the thinking of this period, attacked the Nazi use of mythology as the 'aestheticisation of politics'. It has also been argued that the failure of the Left to establish at least some aspects of a 'rational mythology' was a major factor in its inability to gain the kind of support required to defeat Hitler. Ernst Bloch and Benjamin were well aware of the irrationality of the mobilisation of energy via aesthetic means by 
the Nazis. Bloch, in particular, was also aware, however, that the ability to make 'ideas' - purposes which do not depend on the way the world already is - available in a 'sensuous' manner is vital to any political project, as is the ability to find ways to articulate what was previously articulated in the religious feeling of belonging to a greater whole.

The consequences for art of trying to come to terms with these conflicting demands can, of course, be catastrophic. Much of the tension in debates over aesthetics and politics in the twentieth can be related to the issues glimpsed in the $S P$ and the Discourse. Neither the demand to make ideas aesthetic, as a means of making them more accessible to people's experience and wishes, nor the simultaneous demand to sustain the claims of reason, is ever fulfilled in a manner that is adequate to the kind of technical and social changes that take place in advanced capitalist societies. The failure to fulfil these demands is one source of the world of projection which we now inhabit, where the marketing of images increasingly takes over from the attempt to deal with real socio-economic and cultural problems. The driving of art into the radical autonomy of the avant-garde is a reflex of this situation, but the difficulty such art faces is that refusal to share the communicative means of a society can only ever be temporary. Modernist art of the twentieth century is always eventually incorporated into the commodity-sphere: even Schoenberg can be made use of in an aesthetically insignificant manner for film music. In order to understand the further implications of the questions outlined here one must retrace the philosophical story of their emergence. Much of what is debated in this area today can be better understood by considering the conceptual resources generated in the reactions to Kantian philosophy in Idealism and Romanticism.

\section{Notes}

1 For a much more detailed and differentiated view see particularly Frank 1997.

2 In this sense the crucial implications of early Romanticism are in fact much closer to contemporary pragmatism than to metaphysical realism.

3 This idea is suggested by Kant in relation to Plato in the $C P R$, and is developed by Schleiermacher: see below Chapter 6 .

4 See, for example, Xaver Tilliette: 'Schelling als Verfasser des Systemprogramms?' in eds. Frank and Kurz 1975 pp. 193-211. Recent debate suggests both Hegel and Hölderlin as the author (see, e.g. Förster 1995, eds, Baur and Dahlstrom 1999).

5 Heidegger makes Kant's schematism chapter central to his reformulation of the question of being in his work in the 1920s.

6 Clearly the other vital aspect here is the employment of sexual images, but these are themselves aestheticised.

7 This is why so many accounts of Romanticism, which, like that of Isaiah Berlin, assimilate it to Idealism via the influence of Fichte are mistaken. I shall deal with this issue in more detail in the next chapter.

8 This is one of the reasons why the figure of Dionysus is so important for Hölderlin. The idea of unity in division also echoes the concept of the Trinity.

9 As we shall see in Chapter 8, it is Schelling who first makes this link. 


\section{Reflections on the subject: Fichte, Hölderlin and Novalis}

\section{Self and Other}

One of the most striking examples of the new concern with the nature of subjectivity in the eighteenth century is Rousseau's 'Scène lyrique', Pygmalion, in which the sculptor's creation, Galathée, comes to life and touches her creator, saying 'It's me.' Moving away, she touches a marble sculpture and says 'It's no longer me. . ' Finally, touching Pygmalion again, she sighs: 'Ah! Me once again ... , and he exclaims: 'it is you, you alone, I give you all my being; I shall no longer live except through you' (Rousseau 1776 pp. 32-4). Such a culmination of the subject's desire for self-recognition in the other already suggests the dangers that the desire brings with it. Is my whole being to disappear into the other? In what sense would I still be myself, if I have wholly gone over into my object? As the famous exchange in Act Two of Wagner's Tristan and Isolde (1859) suggests - Tristan: 'You are Tristan,/ I Isolde,/ no longer Tristan', Isolde: 'You are Isolde, / I Tristan,/ no longer Isolde', Both: 'Without naming,/ without dividing...' - the very sense of an individuated self may in fact be the obstacle to union with the other. However, the end of Wagner's music drama also implies that the ultimate union requires the death of the subject. Rousseau's text asks a further vital question: Is aesthetic creation a way out of the dilemma of self-knowledge or really just another version of it, because it makes one take the imaginary for the real? The precarious balance between subject and object is, as we are about to see, crucial to the issue of aesthetics and subjectivity in the early modern period.

A more sobering observation on the nature of the $I$ is suggested by Georg Christoph Lichtenberg (c. 1793). Along with the fact that Hamann already suggested inverting the cogito, sum in 1785 , this text makes evident how many accounts of the history of the 'decentring' of the subject need revising (the text is very likely the source of Nietzsche's critique of Cartesianism):

We become conscious of certain ideas that do not depend on us; others believe that we at least depend on ourselves; where is the border? We only know the existence of our sensations, ideas and thoughts. It thinks, one ought to say, as one says: it's thundering (es blitzt, literally 'it's flashing (lightning)'). To say cogito is already too much as 
soon as one translates it as I think. To assume the $I$, to postulate it, is a practical need. (Lichtenberg 1994 II p. 412)

Lichtenberg may well be thinking here of David Hume's notorious failure to find an I when he reflects on the content of his thoughts, but he is aware that some sense of the subject to whom those 'sensations, ideas and thoughts' pertain is required, even if it has to be an impersonal subject, an 'it'.

We already encountered some of the difficulties which emerge in the attempts of the I to understand itself in Kant's 'transcendental deduction'. During the later part of the eighteenth century, as the passages from Rousseau and Lichtenberg make clear, the nature of the self becomes a vital philosophical and cultural issue in many different areas of European society. Interestingly, the ways in which this issue are explored tend to produce very conflicting results. On the one hand, the I is often seen as what is required for the world to be intelligible at all, without which there would be nothing but opaque, inert being; on the other - and this can be the case even in theories which still give the I a central role in constituting the world's intelligibility - the I seems incapable of making itself intelligible to itself in any exhaustive way. This conflicting image of the $\mathrm{I}$ is evident in three of the most notable explorations of the nature of the I in German Idealism and early Romanticism: those of Fichte, Hölderlin, and Novalis, and the questions they raise remain central even to contemporary philosophy.

\section{Fichte}

Although he himself wrote little about aesthetics, the significance of J.G. Fichte (1762-1814), both for subsequent philosophy and for aesthetics in particular, can hardly be overestimated. The reasons for this have been suggested by his most influential recent interpreter, Dieter Henrich:

Fichte was the first to arrive at the conviction that all previous philosophy had remained at a distance from the life and self-consciousness of human-kind. It had had ontological categories dictated to it which were taken from the language in which we communicate about things, their qualities and their changes. With these categories philosophy had then investigated powers and capacities of the human soul. It was therefore fundamentally unable to reach the experiences of this soul, the processes of consciousness, the structure and flow of its experiences and thoughts. (Henrich 1987 p. 61)

Fichte radicalises the Kantian turn towards the subject, not just by making the world - at least the world as that which is intelligible - into a product of the ' $\mathrm{I}$ ', but also by an exploration of the structures of self-consciousness which reveals the irreducibility of self-consciousness to what can be said about the world of objects. It is this exploration which helps lead, via a very complex route (see Frank 1997), to Romanticism and beyond - even as far as to the contemporary philosophy of mind (see Frank 1991) - as well as to some of the most important ideas in aesthetic theory that we shall be looking at later. 
Günter Zöller has remarked in his valuable book on Fichte that 'As any reader of Fichte knows, criticizing him comes easy; the hard part is making him intelligible' (Zöller 1998 p. 6). One way of doing this is to look at Fichte in relation to an analogous account of the self from a famous subsequent account of subjectivity, Freud's thirty-first 'New Introductory Lecture', on the 'Dissection of the psychic personality'. Freud almost certainly did not know Fichte's work, but the influence of Fichte on Schopenhauer and on a range of other nineteenth-century thinkers with whose work Freud was acquainted meant that Fichte's ideas are likely to have had an indirect effect on how Freud thought about issues to do with the self. It is therefore not surprising that Freud's initial question in the lecture is reminiscent of some of the questions which Fichte poses for modern philosophy. In the lecture Freud develops the model of the psyche which divides the I into id, super-ego, and ego. The lecture concludes with the famous injunction that 'Wo Es war, soll Ich merden' (Where Id [It] was, Ego [I] should become). ${ }^{1}$ The aim of psychoanalysis, as Freud sees it, is to enable the I to integrate more of its basis in natural drives into itself and to become more independent of the super-ego, the locus of the imperatives internalised during socialisation which prevent it from developing in a healthy direction.

Freud assumes that the subject is divided within itself, and the crucial issue in his analysis is how the structure of the self can be described. The term 'dissection' has proto-medical connotations, and indicates Freud's aim of giving his analysis scientific status, even though, as we shall see, the issues involved are actually inherently metaphysical. Embarking on the 'dissection', he remarks that:

We want to make the I into the object of this investigation, our most personal [eigenstes] I. But can one do that? The I is after all the most essential subject, how should it become an object? Now, there is no doubt that one can do this. The I can take itself as object, treat itself like other objects, observe itself, criticise, do heaven knows what with itself. In doing so one part of the I opposes itself to the rest. The I is, therefore, splittable, it splits itself during many of its functions, at least temporarily. The pieces can unite themselves again afterwards. (Freud 1982 p. 497)

Given the struggles he has in establishing the structure of the self throughout his work Freud is remarkably relaxed about how his version of the dissection can be legitimated. If one looks a bit more carefully, the argument entails serious problems and points to a way of understanding what makes Fichte's arguments so significant.

Freud's first problematic assumption was implicit in Henrich's assessment of Fichte. Even though it is clear that the I can indeed in some sense split itself how else does one, for example, criticise one's own thoughts or actions to oneself? - can it look at itself in the same way as at any other object? Kant was unable to explain the 'transcendental unity of apperception', which must already be in existence for our multiple representations to be the representations of an ' $I$ '. He had to presuppose the existence of a grounding consciousness, 
despite his inability to say anything about it beyond describing the objectifiable results of its operations. The source of his problem was precisely that this consciousness does not have the status of an object: it is a 'spontaneity', and is thus the condition of its own activity, unlike objects, which are always conditioned by something else. This spontaneity is the prior condition of any attempt to know what the I- or anything else for that matter - really is. Freud is faced with an analogous difficulty to Kant, and this becomes apparent when he claims that, having split itself up into subject and object, the I can reunite itself afterwards.

The question is which 'piece' - Freud's own word - is able to do the reuniting. That which does the reuniting cannot be an object of the same order as the 'objective' 'pieces' it reunites. It has to include within itself both a subjective, spontaneous aspect, and an objective, knowable aspect, i.e. it must be that which does the dissecting, that which is dissected, and that which brings the pieces back together. What, then, is the principle that brings the pieces back together? The answer in Freud's terms seems to be the I of the theorist who undertakes the dissection of the object which is the self. But this is circular. The I has in this case to be defined as that which can reunite itself $a$ itself, having split itself into subject and object. However, the splitting in question, which Fichte refers to in terms of 'reflection', would seem to be the condition of knowing anything at all and thus of being able to theorise in the first place. How can one know something without there being an object that is other than oneself in some sense? But if that object is myself, how do I overcome the split I have introduced into myself and know it is $m e$ ?

Fichte had already described the structure of this issue as follows: 'for originally I am neither that which reflects, nor that which is reflected, and neither of the two is determined by the other, but I am rather both in their unity; I admittedly cannot think this unity, precisely because in thinking I sunder that which is reflected and that which reflects' (Fichte 1971 I p. 489). What, then, prevents consciousness disintegrating into the objective pieces into which it is split in the act of reflection? Without the unification of the pieces consciousness could never result in coherent experience of the kind required for reliable judgements, including, of course, about the I itself. Moreover, how can the I which does the looking at itself be shown to be the same as the I which is being looked at? This only seems possible on the basis of an initial, unanalysable presupposition that what we begin with is already a unified I, which was what Kant assumed in the transcendental unity of apperception. The point of the investigation, though, was to establish the structure of the I by analysing it, by 'self-reflection', which means that the unified I would have to be both the result and the ground of the analysis. Finally, it is unclear what brings about the act of reflexive splitting, the action of the I making itself into an object in order to analyse itself. Quite simply: why does it bother, rather than simply being whatever it is without reflecting? What is clear here is that something that makes itself into an object cannot initially be just an object, and it must therefore have a prior subjective status of some kind. The question is: what kind? 
Freud thinks knowledge of the psyche has to be of the same kind as other knowledge, if psychoanalysis is to count as a science. The complexities and confusions in his repeated reformulations of his theory seem not least a result of the assumption that he is engaged in an empirical investigation, when what is at issue necessarily depends upon prior assumptions which govern how empirical data are to be interpreted. These are, then, actually questions about transcendental philosophy in Kant's sense. One way of making the issues here clearer will be via the metaphor of self-objectification in a mirror. This, as the passage from Freud already suggests, tends to be how theories of self-consciousness are formulated. Henrich calls such a theory the 'reflection theory': 'this theory assumes a priori a subject that thinks. It then explains that this subject continually relates to itself. The theory further maintains that this relationship comes into existence via the subject's making itself into its own object. This capacity of becoming conscious of oneself via a reflexive act distinguishes human beings from animals' (Henrich 1982 p. 62). Fichte was probably the first person to realise just how problematic this theory is.

Fichte wishes to found philosophy upon the one 'condition' which must be absolute and immediately certain, which is therefore itself 'unconditioned'. For Descartes the I had played this foundational role, and Fichte begins with the need to establish a Cartesian foundation in a more decisive way than Kant had been able to in his demonstration of the necessary role in cognition of the synthetic unity of apperception. Kant's problem, Fichte believes, was that he had tried to see the I as a fact, a 'Tatsache', literally a 'deed-thing', which suggests it has the same status as any other fact. For Fichte the I must be an action, a 'Tathandlung', literally a 'deed-action', a word he concocts to try to express the idea that the 'fact' of the I cannot be understood as something objective. Furthermore, this 'action' is not of the same order as the rule-bound actions of the I when it synthesises empirical intuitions in cognition: 'As it should not be contained in those actions, which are all necessary . . . it must be an action of freedom' (Fichte 1971 I p. 71).

The highest act of philosophy for Fichte, which is what makes philosophy possible at all, has to be the spontaneous act of coming to think about thinking. His conception is essentially opposed to the idea that having thoughts might be conceived of as a causal process, occasioned by whatever affects one in the world and by the mechanisms of the brain. The 'dogmatist', by which he means the materialist philosopher, 'denies the independence of the I completely ... and makes it just a product of things, an accident of the world' (1971 I p. 431), so that the $I$ is part of a mechanical series of causes and effects. The crucial point about the I for Fichte is that the activity in question does not, as any effect of a cause does, lead endlessly to other effects, but can instead - think here of the capacity for memory or for decision preceding an action - 'go back into itself', and thus have a 'being for itself (1971 I pp. 458-9): 'I and activity which returns into itself are completely identical concepts' (1971 I p. 462). As Fichte suggests (and the adherents of the myth of artificial intelligence would do well to listen): 
'The mechanism cannot grasp itself, precisely because it is a mechanism. Only free consciousness can grasp itself' (1971 I p. 510). This grasping of itself cannot be the result of anything which determines the I to grasp itself, because that would require an account of what determines self-consciousness, and so on, either ad infinitum - in which case there would be no consciousness - or to a point which has to be assumed as the absolute origin, and this must, precisely, be the I itself: 'The Wissenschaftslehre descends from the last ground which it has to what is grounded; from the absolute to what is conditioned that is contained within it, to the real, true facts of consciousness' (1971 II p. 455). The impetus for this conception was already suggested by Kant, who claimed that the I of practical reason was capable of absolute beginning because it involved a 'causality through freedom' when it initiated an act for which there was no causal, conditioned necessity. As we saw in Chapter 1, the point of moral acts for Kant is that they can countermand the causal prompting of our instinctual nature.

How, though, do we give an account of the activity which is self-consciousness? Fichte's answer is simple: one cannot, if this means defining - and thus limiting - it like anything else in the causally governed world. This leads him to the polemical stance exemplified in his notorious claims that 'the philosophy one chooses depends on what sort of a person one is' (Fichte 1971 I p. 434), and that those who do not take on their freedom cannot comprehend his philosophy. The evidence for the essential act is the very act of philosophical thinking about the I itself, which is both theoretical and practical. Fichte:

if [philosophy] starts with the fact [i.e. with something that can be explained like any other object in the world] it places itself in the world of existence and finitude and will have difficulty finding a way from this world to the infinite and supersensuous; if it starts with an action it stands at precisely the point which links both worlds and from which they can be surveyed in one gaze. (1971 I p. 468)

Fichte insists on the primacy of action in order to get over the vital metaphysical problem of how a causally determined objective world can give rise to that which is subjective, is not causally determined, and is able to apprehend causal relations as causal in contrast to its own potential for self-determination. The 'infinite' in this passage refers to an action which cannot be defined as knowledge would be, not least because knowledge itself depends upon this activity.

Grounding philosophy in the I leads, then, to the realisation that the ground of philosophy cannot be an object of knowledge. The transcendental unity of apperception was the ground of the intelligibility of empirical consciousness for Kant because the coherence of experience across time required something which itself could not appear - that subsumed the endless diversity characteristic of sensuous appearance into identifying forms. Fichte insists on the necessary priority of what Kant admitted was required for experience in the following way: 'It is . . . the ground of explanation of all facts of empirical consciousness that before all positing in the I' - 'positing' can be understood via Kant's assertion that 
reality is 'the position of things ... in relation to perception' which precedes the ascription of a concept in a judgement (cit. Frank 1997 p. 673) - 'the I must itself previously be posited' (Fichte 1971 I p. 95). The difference from Kant is that Fichte refuses to accept that we have no access at all to this ground. For Fichte the access involves what he terms a 'thetic judgement', in which only the existence of something is posited, and nothing is predicated of that existence, so that in the case of the I: 'the place of the predicate is left empty to infinity for the possible determination of the I' (1971 I p. 116). Our access is therefore the result of the realisation that we have a capacity - freedom - which raises us above all of the rest of nature, and which has no ground other than itself. This realisation can only come about by each person doing the realising for themself: there can be no appeal to an objective fact $-i$.e. something which is referred to in a predicative statement - to establish the reality of freedom. A further way of seeing Fichte's conception, which has become important in recent philosophy, is, then, that this is a philosophy of praxis which prefigures aspects of pragmatism: 'We do not act because we know, rather we know because our vocation is to act; practical reason is the root of all reason' (1971 II p. 263). There can, then, be no empirical account of the initial act of thinking, as we are always already engaged in it, indeed already are it, when we wish to intuit it.

Fichte's epistemological problem is to find a way of describing an 'eye', or a 'look' - consciousness - that could 'see' itself seeing, which, of course, threatens a regress. It is the difficulty which emerges here that leads him to realise that the reflection model of consciousness, the model we saw in both Kant and in the passage from Freud, cannot grasp the undeniable fact of self-consciousness. The point of the reflection model, as was evident in Freud, is that it tries to make the I into something objectifiable. If the model fails to account for selfconsciousness it will mean that not all that pertains to knowledge can be characterised in the manner we characterise knowledge of objects. Consequently, any philosophy which claims to be able to begin with the explication of a foundational principle is doomed to failure, as is the claim that scientific knowledge can ultimately explain itself like any other aspect of the objective world. The failure of reflection thus gives an increased importance to aspects of consciousness that are not accessible to the understanding, and this will be one source for Schelling and the early Romantics, as it already had been for the Kant of the $C \mathcal{F}$, of attention to aesthetics, the realm in which what cannot be stated as verifiable fact may be articulated.

The only absolute for Fichte is the action of the I: all other kinds of knowledge are secondary. This does not, however, lead to an idealism like that of Berkeley. Fichte's argument derives instead from Kant's insistence on the transcendental unity of apperception: 'one is very wrong if one believes transcendental idealism denies the empirical reality of the world of the senses etc.: it just demonstrates the forms of knowledge in empirical reality and for this reason destroys the sense that they are self-sufficient and absolute' (1971 II p. 104). The ability to describe these forms entails the fact that the I must 
transcend them if it is to be able to reflect upon them, in the same way as the I in Freud had to be of a different order from that which it splits and reunites, while also being in some way the same as it. What this involves becomes most apparent in Fichte's account of 'reflection theory', which is most powerfully stated in the 'Attempt at a New Presentation of the Doctrine of Science' of 1797. As Manfred Frank has shown (Frank 1984), Fichte's account of self-consciousness here is not touched by most of the Heideggerian and post-structuralist attacks on 'the subject' as the ground of the 'subjectification of Being' in 'Western metaphysics'. Even though Fichte will not achieve a philosophically viable account of self-consciousness, his 'original insight' (Henrich) has remained significant even for the contemporary philosophy of mind.

Fichte begins by asking me, the reader, to ponder what I do when I think of myself as ' $I$ '. This thought is special because in it thinker and thought cannot be separate. The action of the I thinking about itself is an action upon itself. The I results, as we saw, from the return of thinking to itself: if it were always only thinking of an object there could, as Kant had shown, be no I, there would merely be unconnected, and thus opaque, empirical data. Fichte sees the problem of reflection as follows:

You are - conscious of your self, you say; accordingly you necessarily differentiate your thinking self [Ich] from the self that is thought in the thought of yourself. But in order for you to be able to do this, the thinking part of that thinking must be again the object of a higher thinking in order to be able to be an object of consciousness; and immediately you get a new subject which has again to be conscious of that which was being conscious of yourself. (Fichte 1971 I p. 526)

The result is one of those infinite series, of the kind ' $I$ know that you know that I know that you know' etc., where what is supposedly known disappears in the endless reflection. In Fichte's terms it is a case of ' $\mathrm{I}$ am conscious that I am conscious that I am conscious', etc. In the Vocation of Man (1800) he starkly presents the problem as follows: 'And do I really think or do I just think a thinking of thinking? What can stop speculation acting like this and continuing asking to infinity?' (1971 II p. 252). Clearly, consciousness cannot be explained in this manner.

The problem lies in differentiating the reflecting and the reflected ' $\mathrm{I}$ ' without losing the identity of reflecting and the reflected which is essential for there to be an I at all. Henrich puts it as follows: 'how can self-consciousness know that it knows itself if this knowledge is supposed to come about via an act of reflection? It is obvious that it cannot have this reflected knowledge without being able to lay claim to a preceding knowledge of itself' (Henrich 1982 p. 64). The nature of this 'knowledge' is a central problem in the development of Idealist and early Romantic philosophy. Even trying to find a word to characterise it which does not raise the problem of reflection involves difficulties. The 'knowledge' cannot be analogous to seeing oneself in a mirror: how would I know that I was seeing myself if I did not already 'know', before reflecting, that 
I was doing the looking? One can have certain knowledge of seeing an object that is actually one's own reflection without knowing that it is oneself. Rorty's objections in Philosophy and the Mirror of Nature (1979) to the mirror as the dominant metaphor for consciousness in Western philosophy do not, then, apply to this argument. The mirror metaphor actually just reveals the failure to objectify something whose existence is yet indubitable for the person seeing, namely their awareness that they are seeing at all, even if it they are mistaken about what they see.

Fichte is led to the demand for an immediate access to consciousness which does not entail any kind of splitting, and he will spend the rest of his philosophical life failing to give an adequate account of this immediate access and its grounding role in a philosophical system. As we shall see later, this immediate consciousness is often designated by the word 'feeling', for instance in Novalis's remark that 'Feeling cannot feel itself', precisely because having a feeling is not something that is initially objectifiable. The significance of 'feeling' for the thought of the period is not, pace many accounts of Romanticism, merely based on an increased attention to affectivity. For Fichte the subject qua object upon which the philosopher reflects must already be given in the original spontaneous act of self-consciousness, but it cannot be given as a knowable object. Consequently, the Kantian divisions of theoretical and practical, sensuous and intelligible, receptive and spontaneous, must result from a preceding unity in the I. According to Fichte the unity in question could only be denied if one were to deny the fact of self-consciousness, which is the very principle of thinking itself. This is what leads him to his most striking and problematic move, namely the extension of the notion of the I as spontaneity into being the principle of all reality, on the assumption that the intelligibility of things revealed in thought, and not their brute undifferentiated existence, constitutes their essential nature.

Fichte adopts the notion of 'intellectual intuition' in the attempt to grasp the decisive principle, but he characterises it in a different way from Kant, seeing it as 'that through which I know something because I do it' (Fichte 1971 I p. 463). Intellectual intuition involves both the act of thinking and the consciousness of that act: 'the consciousness of my thinking is not something which is just coincidental to my thinking, something which is added to it afterwards and thus linked to it, rather it is inseparable from it' (1971 I p. 527) - otherwise it is not clear what makes my thoughts mine, rather than random events in the universe. Zöller describes intellectual intuition as 'an inferred condition, grasped in philosophical thought by means of abstraction from what is empirical in consciousness and reflection on what remains after such abstraction' (Zöller 1998 p. 36). Self-consciousness is not subject to the division of theoretical and practical: the (practical) action of the I intuits itself as (theoretical) object of philosophy. The intellectual intuition which Kant rejects is, Fichte contends, analogous to the arguments of people who accept the ontological proof of God, who 'must regard the existence of God as a simple consequence of their thinking' (Fichte 1971 I p. 472). This kind of intuition would have to create a non-sensuous being, that 
of the 'thing in itself' (as opposed to our intuitions of it), by pure thinking. Fichte's intellectual intuition is not the creation of a non-sensuous existence via thinking, it is an intuition - in the sense of a direct apprehension, rather than a conceptual grasping - of the action of thinking itself. The problem it entails is manifest in the fact that Fichte soon becomes unsure whether the absolute I is immediately grasped in intellectual intuition (which as the term already suggests, entails a duality of the kind he is trying to avoid), or whether the absolute I is only an 'ideal' that is endlessly approached but never reached (see Frank 1997 pp. 744, 764). The notion of the intuition of the action of thinking leads to the apparently bizarre, but consistent idea in Fichte that the activity in question, which is both theoretical and practical, is the highest principle of reality. Why exactly should this be?

The highest principle cannot be located as part of an external world of nature, because without the activity of the I the very idea of a highest principle would not emerge from the causally determined appearing world. For Fichte nature's externality, the existence of a realm of objects, can itself only be, as it will be for Hegel, a product of thought: 'the consciousness of a thing outside us is absolutely nothing else than the product of our own capacity for thinking and ... we know nothing more of the thing than we precisely know about it, posit via our consciousness' (Fichte 1971 II p. 239). Because we always require the activity of the I for knowledge we only know even what is 'outside' us via what is 'inside' us: 'everything that occurs in consciousness is grounded, given, caused via the conditions of self-consciousness; and a basis of the same outside self-consciousness absolutely does not exist' (1971 I p. 477). If this sounds absurd it is worth trying to say anything about the world whilst subtracting the I which thinks what is to be said. It is important to note, though, that self-consciousness functions for Fichte within conditions that are given to it as part of what it is, and that it does not consciously make the world what it is. The resistance to objectification at the heart of self-consciousness means, as Zöller claims, that it is 'a being that has reason without being its own ground or reason' (Zöller 1998 p. 27), and does not mean that it is some kind of self-creating deity. In conscious experience any specific thought is filled by the intentional object of the thought, be it this computer in front of me or my inner speech at this moment. This, though, is not what is most important for Fichte, as it had not been for Kant. It is the ability to move intelligibly from one intentional object to something beyond it that reveals the 'productive imagination' as the real basis of self-consciousness. The imagination, which, as we saw, played such a central role in Kant, is the result of the fact that the I 'posits itself as finite and infinite at the same time' (Fichte 1971 I p. 215), finite when it limits itself to a determinate thought, and infinite when it moves beyond it.

Such movement requires freedom: nothing in the realm of necessity, the realm of the synthesising of intuitions into categories and concepts, could give rise to this aspect of imagination, because the very intelligibility of different syntheses of the same data depends upon it. Self-consciousness has a status 
which puts it above anything one can say about objectivity, because it is for Fichte, as indeed it was for Kant, the condition of objectivity. It must, as such, be both subject and object, both the production of knowledge and the ability to describe the forms of that production. This is why the notion of the being of the world independent of our thinking, the 'thing in itself', has no content for Fichte: 'the concept of a being that should occur, from a certain point of view, independently of thinking [Vorstellung], would yet have to be deduced from thinking, as it is only supposed to exist via thinking' (1971 I p. 500). We shall encounter another version of this position in Hegel. Fichte is insistent that 'One cannot abstract from the I' (1971 I p. 500): to do so would require the I to abstract from itself, which is absurd, because the abstraction would require an account of what does the abstracting, which must be the I itself.

What is puzzling about Fichte's view for most people is why we experience the world as external, hard, objective necessity if it is in fact our thinking which is the condition of the world's objectivity. Manfred Frank warns, though, against a hasty dismissal of Fichte's arguments:

If it is right that our feeling of being compelled [by the thing in itself as external to ourselves] presupposes as condition of its possibility the consciousness of our self, then we do not have the slightest right to reverse the sequence of the facts and to maintain that it is rather the thing (in itself) which determines our consciousness (including the consciousness of our receptivity). (Frank 1985 p. 80)

The reason that the I was the highest principle for Fichte lay, as we have seen, in a radicalisation of Kant's practical reason, of the capacity to move beyond causally determined compulsion, in making ethical decisions. For Kant this entailed a self-determining 'spontaneity'. Spontaneity was also the basis of $\operatorname{cog}$ nition, because the synthesising of sense data requires that which synthesises, which is not reducible to the data themselves. As this spontaneity formed the basis of both the first two Kantian Critiques, it seems clear to Fichte that the task of philosophy is to describe it, thus unifying the theoretical and the practical. Doing this, though, seems to involve attempting to make the fundamental free action into something which philosophy can objectify, and this gives rise again to the dilemma for theory that what can be objectified is solely what is causally determined as part of the appearing world.

This is the source of Fichte's insistence upon an 'intuitive' access to the action, which does not require a splitting into subject and object. However, the invocation of intuition also has the consequence that those unable to carry out the intuition are excluded from access to the highest point of philosophy. If one remembers Kant's remark in the Prolegomena that the representation of the I is 'nothing more than the feeling of an existence without the least concept' (Kant 1989 p. 106), and his subsequent attempt in the $C 7$ to arrive at an account of universality of feeling in (non-conceptual) aesthetic judgement, the importance of this issue becomes clear. Is the I ultimately to be something fully explicable, or is its very resistance to conceptual determination what is most important about 
it? As we shall see in Chapter 4, Schelling will suggest in 1800 that 'aesthetic intuition is precisely intellectual intuition which has become objective' (Schelling 1856-61 I/3 p. 625), which makes art into the 'organ of philosophy', because it shows via perceptible objects what philosophy cannot definitively explain. Furthermore, the revaluation of the non-semantic medium of music in this period points to the sense that the resources of philosophy may be inadequate to the subject's self-understanding in modernity.

Perhaps the most disturbing question for the transcendental philosopher in all this, which is vital for the development of Romanticism, is why the absolute I should objectify itself at all in an intelligible world which includes ourselves. Either the absolute, the unity of subject and object, remains enclosed in itself as an undividable unity, which means that there would be no manifest world and that any philosophical articulation is a priori impossible, or a division must take place within the absolute, which would enable philosophy to articulate it. Given that the highest thought for Fichte is that of the I, which is necessary for any articulation, there must in his terms be a way of articulating the absolute. The $I$ is, after all, specified in terms of its lack of limitation, because it inherently transcends the finitude of the particular. However, it seems that no description of the I can explain why there is limitation and finitude at all. Fichte tries to account for the split in the I that limits its infinite nature and makes things determinate by his obscure doctrine of the 'Anstoss' (usually translated as the 'check'). Fichte says the following regarding the source of the world of limitation and determination:

Now the activity of the I which goes out into infinity is to be checked at some point and driven back into itself; and, accordingly, the I is not to fill out infinity. That this happens, as a fact, simply cannot be inferred from the I . . but one can at least say that it has to happen if real consciousness is to be possible. (Fichte $1971 \mathrm{I}$ p. 275)

Having occurred, the 'check' gives rise to the demand for the I to fulfil its nature as infinite activity by overcoming finite limitation. This demand is manifest to us in moral action which countermands natural causality and therefore, as Kant suggested, may point to a goal beyond the finite. The check can only have its effect on something which is already active, but this still does nothing to explain the check in a non-circular manner, as more than an essentially arbitrary answer to a major philosophical problem. Fichte himself repeatedly admits that it cannot be explained in the theoretical part of his philosophy.

What emerges here is a further example of how at crucial junctures Fichte's philosophy leaves an inescapable opacity which soon leads thinkers initially attracted by his undoubted insights into the nature and role of self-consciousness to abandon his extreme version of the self-grounding Idealist project. Schelling, as we shall see in Chapter 4, will initially focus on the unconscious aspect of the activity of the $I$ in a manner which prefigures psychoanalysis, and he will in his late philosophy become obsessed with the problem of whether Idealist philosophy can overcome the contingency of the fact that there is a manifest world at all. There is, then, an essential tension in Idealist and 
Romantic thought which resides in the uneasy coexistence of the (Idealist) desire to be able to say what it is in thinking that is unlimited, with an accompanying (Romantic) sense of the impossibility of saying it, an impossibility which seems to make the philosophical enterprise of grasping the absolute itself questionable. The Romantic attachment to art can be seen as deriving from an awareness of the need to respond to this tension.

The tension is the source of a further, related philosophical problem. Fichte's account also gets into difficulties because the activity of his 'absolute I', the unlimited dynamic principle of reality, has, via the 'check', to generate an I and a non-I which are relative to each other. This means, though, that it becomes very hard to know in what sense the absolute principle could be referred to as an ' $\mathrm{I}$ ': seeing $\mathrm{A}$ as opposed to not-A is impossible if it has to happen solely in terms of A itself. The problem is echoed in Freud's difficulties in giving an account of the overall structure of the psyche, if all psychic energy is seen as deriving from the id, which is unconscious. For Freud the id splits itself and directs its own forces against itself by the incorporation of the limiting, selfpreserving ego and super-ego into the structure of itself. A grounding of this account requires the forces of the id to describe themselves, and this leads to another version of the problem of reflection. For the id to knom itself as the chaotic, drive-basis of the I, it would already require the division into itself and its non-chaotic other. However, this division means that the id itself cannot be represented because it must always already be split into the representer, the conscious ego, and what is supposed to be represented, the unconscious id - whence Freud's insistence that only representations of drives, not drives themselves can appear. Freud, it is worth remembering in the present context, almost invariably has recourse at some point to the aesthetic means of metaphor when attempting to describe the nature of the id. Faced with the analogous problem with the absolute I, which is, like the id, the source of all activity, Fichte is forced into the move whereby 'I oppose in the I a splittable I to a splittable non-I' (Fichte 1971 I p. 110). While it is possible to argue, as Fichte does, for the epistemological necessity of a prior subject without which the notion of an objective world makes no sense, calling the overall process ' $\mathrm{I}$ ' within which I and not-I become separate, is inconsistent, as Hölderlin will be one of the first to see.

Fichte's philosophy is a strange mixture of defensible transcendental arguments which apply to aspects of thinking that seem inescapable in any attempt to work out a viable account of the self, and, at the level of the absolute I, of highly questionable speculation derived from the inflation of the activity of thought into the spontaneous activity which constitutes the whole of intelligible being. The basic Idealist problem with which Fichte is faced is that if there is to be an absolute, an unconditioned basis of philosophy, any dependence of the highest principle on something else must be transcended. If self-consciousness is relative to its other, Fichte's project must fail because it introduces a moment of dependency that the whole project has to avoid if it is to achieve what Kant had failed to achieve. Fichte's attempt to give an account of free self-consciousness which does 
not reduce it to being an object is bravely revised and differentiated throughout his career, but it never overcomes the problems we have seen. The early Fichte's achievement should not be forgotten, though, as Henrich has argued. Fichte's real insight is that reflection upon the subject by the subject reveals a reality which will never exhaust itself in what could be known objectively. It is this insight that will make him so important to those engaged in aesthetic theory and aesthetic praxis.

\section{Hölderlin}

Some of the problems in Fichte's philosophy were quickly seen by the poet, Friedrich Hölderlin (1770-1843), who in his youth was friends with both Schelling and Hegel, and who was in contact with the intellectual circles which established both Idealism and Romanticism. ${ }^{2}$ The fact that an investment in understanding the I also becomes central to many areas of literature from this period onwards poses important questions about the relationship between art, aesthetics and the rest of philosophy. Can the literary text say what the philosophical text cannot, and what does this mean for that part of philosophy which itself seeks to explain the significance of art? Hölderlin is best known as a poet and novelist, but he also explores the question of the $\mathrm{I}$ in theoretical texts, and for a time he thought of devoting himself mainly to philosophy. Three theoretical texts, the last of whose remarkable use of syntax gives it claims to aesthetic status even as it relies predominantly on logical argument, present Hölderlin's prophetic insights in relation to the issues raised by Fichte: a letter to Hegel in January 1795, the untitled fragment usually called 'Judgement and Being' of $1794 / 5,{ }^{3}$ and the untitled essay which is often called 'On the Working of the Poetic Spirit'. In the letter Hölderlin questions Fichte's use of the I as the highest principle of philosophy:

his absolute I . . contains all reality; it is everything, and outside of it there is nothing; there is therefore no object for this absolute I, for otherwise the whole of reality would not be in it; but a consciousness without an object is unthinkable, and if I am this object myself, then as such I am necessarily limited, even if it only be in time, thus not absolute; thus there is no consciousness thinkable in the absolute I, as absolute I $[I c h] \mathrm{I}[i c h]$ have no consciousness, and to the extent to which I have no consciousness I am (for myself) nothing, therefore the absolute I is (for me) nothing. (in (eds) Frank and Kurz 1975 p. 124)

The highest principle can never appear in my reflection on anything, including myself, if it is taken as an object, because this would contradict its very essence as the absolute, which must be both subject and object. Access to the absolute I requires an intuition which is beyond any division of subject and object, but, crucially, this intuition cannot be conscious, because intentionality requires a split between subject and object for it to be consciousness of something. The idea that access to the highest and loss or transcendence of consciousness are 
inherently connected, which will later appear, via Schopenhauer's influence, ${ }^{4}$ in Wagner's Tristan and Isolde, therefore emerges logically for Hölderlin from the way he understands the structure of Fichte's account of the absolute I.

'Judgement and being' investigates the separation of subject and object, which it sees as united in 'intellectual intuition', though the problem with this notion of intuition is already apparent in the letter just cited: how can a philosophy be founded on the loss of all awareness? Hölderlin claims that the origin of consciousness entails an 'original separation' (the German word Urteil means both judgement, and, via the artificial separation of its two parts into ' $U r-T e i$ ', 'original-separation') of subject and object. His decisive innovation lies in his suggestion that subject and object therefore also presuppose a 'whole of which subject and object are the parts' (Hölderlin 1963 pp. 490-1). This whole cannot, for the reasons suggested in his letter to Hegel, be termed an absolute 'I'. He therefore terms the whole 'being'. Being cannot be incorporated into an account of the relationship of our thinking to the object-world, because it is the common ground of both our thinking and the object world. It therefore becomes 'transreflexive', not determinable in the way that a subject determines an object of knowledge, whether it be the subject as its own object or any object in the world. It is worth citing Hölderlin's argument at some length, as it also highlights the problem inherent in the reflection model of self-consciousness. For Hölderlin 'Being - expresses the link of subject and object . . . this being must not be confused with identity' (490-1). Identity, which is expressed in the statement 'I am I', necessarily entails non-identity, because what is supposed to be unified has been taken apart in the act of reflection involved in the attempt to state its unity. The very form of the proposition entails a difference between the $\mathrm{I}$ in the subject- and the $\mathrm{I}$ in the predicate-position. The real unity of the $\mathrm{I}$ is, then, not grasped by what can be said about it by analysing it into its components. Hölderlin continues: 'If I say: I am I, then the subject (I) and the object (I) are not unified in such a way that no separation can be undertaken without infringing upon the essence of that which is to be separated; on the contrary, the I is only possible via this separation of the I from the I' (pp. 490-1).

There can, then, be no analytical account, no Freudian 'dissection', of the I which would grasp the unity that precedes the act of reflexive splitting, even if, in order to be amare of itself as an I, it must reflect:

How can I say 'I' without self-consciousness? But how is self-consciousness possible? By my opposing myself to myself, by separating myself from myself, but despite this separation by recognising myself in the opposition as the same. But to what extent as the same? I can, I must ask the question in this way; for in another respect the ego is opposed to itself. Thus identity is not a unification of object and subject that happens absolutely, thus identity does not $=$ absolute being. (pp. 490-1)

Self-consciousness that seeks to know itself always involves a relationship, in which the terms are relative to each other (and so not absolute). This relationship has to be carried by an existential continuity which cannot be split up into 
the moments of the analysis of the I. 'Judgement', then, entails a relation which separates what it wants to unify in the act of attempting to demonstrate its unity. For Hölderlin the act of self-identification is subsequent to and depends upon the existential fact of being. Self-consciousness is not to be understood here as just 'mind' opposed to the world because it has its own source in a whole which cannot just consist in the objective being of what is given in sensuousness and is subject to causal laws. Hölderlin therefore regards reflective selfconsciousness as being unable to establish the unconditioned foundation that Fichte wanted to provide with the idea of the 'absolute I'. Frank sums up Hölderlin's insight as follows:

What is revolutionary about Hölderlin's reflection is that on the side of consciousness a [Kantian] primacy of intuition before the concept corresponds to the primacy of the real before the possible. For this reason absolute being can only be grasped by a limit-concept of cognition, intellectual intuition; because only immediate intuition concerns being; judgements, as cognitions mediated by concepts, must fail to grasp it. (Frank 1997 p. 725)

Being thus becomes an idea which orients our inquiries into what there is, but it can never be said to be fully present in any concept. However, this leaves a significant problem: how do we come to terms with the sense that our selfconsciousness is divided in itself, and yet must involve some kind of prior unity if such division is to be apprehended as division at all?

Hölderlin explores this problem in 'On the Working of the Poetic Spirit'. Many of of the implications of the idea of the dependence of subjectivity on an other that is beyond its control, from Schopenhauer (the 'will') to post-structuralism (Derrida's subject that is an 'effect of the general text'), are already contained in this essay. However, Hölderlin does not conclude, as much of this tradition does, that self-consciousness is consequently not a vital issue for philosophy. In 'On the Working of the Poetic Spirit' he shows the inadequacy of three basic attempts to give an account of the fact of self-consciousness. All three attempts end up splitting the $I$ in ways which make it impossible to sustain the undeniable necessity of self-consciousness. As we have seen, the requirement of the transcendental unity of apperception was for an I which was not 'as differently multi-coloured as I have ideas that I am conscious of' (Kant $C P R \mathrm{~B}$ p. 134). Without this unity nothing can be said about either the world of the subject or the world of the object, because the principle of their intelligibility would be lost. Hölderlin's question is how one is to sustain oneself as oneself in the endless changes with which one is confronted in the world, an idea which he sees as central to poetic production. It should already be clear, and this is a vital factor in the text, that Hölderlin does not limit his exploration to the cognitive dimension of the self, but seeks to incorporate the affective and other dimensions of the self as well. ${ }^{5}$ The aim of the 'Poetic Spirit' must be to 'remain present to itself in the various moods' (Hölderlin 1963 p. 515), rather than to lose itself in each moment. It must both avoid becoming 'something indeter- 
minable', and yet not 'disintegrate into an infinity of isolated moments' (p. $515) .^{6}$

Hölderlin poses the problem of the identity of the self in modernity in paradigmatic fashion. How does one avoid a fixed, dead identity that would be the result of total self-objectification, in which the point of being a living, moving self is lost? Total self-knowledge arguably obviates the point of our being at all. At the same time, how does one also avoid a complete loss of the self in the engagement with the diverse and conflicting moments of the other. How does one avoid simply being 'spoken' and determined by the language and cultural forms into which one is socialised and by the natural urges which are filtered through these forms? Hölderlin's attempted response to these undesirable alternatives is particularly significant because it sees aesthetic production as offering resources for dealing with them. He argues that the three attempts to analyse self-consciousness reveal an I which is 'in real contradiction with and for itself', because it can be grasped neither (1) as something known grasped by the knower; nor (2) as a knower grasped by the knower; nor (3) as known and knower grasped by knowing, or knowing grasped by the knower.

In (1) the difference of knower and known (I as subject and I as object) would have to be regarded as a 'deception ... which the I makes to itself as unity, in order to recognise its identity, but then the identity which it thereby recognises is also itself a deception, it does not recognise itself' (Hölderlin 1963 p. 517), an idea that is later echoed in Lacan's accounts of the I's self-misrecognition, and which recurs, as we shall see, in Novalis. Hölderlin, however, rejects this position and so does not take self-consciousness to be merely the form of narcissistic self-deception it is for Lacan.

In (2) the I assumes that it is inherently divided in itself: the very act of knowing presupposes the division of knower and known, which would also apply to the knowledge of oneself. This means, though, that the acts which the I performs cannot be identified as its own acts because it is already unable to identify itself as a unity: to which part of itself would the acts belong, to itself as knower or as known? If the I is inherently divided the division cannot itself be a result of the I, because the whole point is to establish the prior unity of the I. As such, 'the I is not that which is different from itself, rather it is its nature [which is different from itself], in which it behaves to itself as something driven' (p. 518), thereby becoming unable to account for why it is aware of itself as itself. The problem is much the same as the one we saw in Freud, where an initially unified I was required which splits itself up and is yet still there to reunite itself, having split itself up. A related Freudian problem that we have already examined in relation to Fichte also echoes Hölderlin's reflections. Because the id provides the energy for the ego, which is a result of the drives of the id being divided against themselves, it becomes impossible for the ego to know its real basis, the id: the ego requires the id for the activity of knowing in the first place. In other words, to what extent must I think of myself as driven by something, my 'nature', which is not my conscious self? If I know that I am driven by the other 
of nature in myself, have I not already transcended this natural basis? But if the very activity of knowing itself depends upon a basis in an other that is ineliminably part of me, I can never fully transcend that basis, though I may gain indirect insight into it. Schelling will suggest that this is what takes place in aesthetic production, which fully conscious awareness of all one is doing renders impossible.

In (3) the I wishes to see itself as identical with itself, as split into subject and object. To do this, however, again relies on a split in the I, which destroys the unity that is supposed to precede the reflection: for it to see itself as itself requires prior awareness of itself as not divided from itself (as 'Judgement and Being' suggested). The argument is another version of the arguments against 'reflection theory', and Hölderlin therefore denies that the I can represent its own essential nature: it is more than it can know in reflecting upon itself.

Hölderlin does not overcome this problem at an epistemological level, but instead sees the striving to grasp the unity of the $I$ in these three moves as leading to questions of 'poetic representation'. The internal reflection of the I leads it to the awareness of its dividedness once it tries to grasp itself as itself. Hölderlin therefore wishes to move beyond this division: 'It is a matter of the I not simply remaining in interaction with its subjective nature, from which it cannot abstract [by reflecting on itself] without negating itself' (p. 518). If it remains in this state it will be likely to resign and regress to the childhood state, where it 'was identical with the world', thus to what Lacan means by the 'imaginary'. In the imaginary the I only reflects itself and does not move beyond this form of self-identification. The other bad alternative for Hölderlin will be that the I will 'wear itself out in fruitless contradictions with itself' (p. 519). In order to get beyond this condition the I must exercise its free spontaneity in the choice of an external object, which will reveal its 'poetic individuality'. The external object qua object is separate from the I, but if the choice of the object is the result of the most fundamental act of the I, its free choice, a new form of relationship can result. In it the I escapes imprisonment within the imaginary by engagement with the freely chosen external object, but is also able to explore itself in new ways by abstracting from the object of its choice and reflecting upon itself via the object. The stress is now on the creative relationship to the object, not upon a cognitive relationship. This point is crucial. What is at issue in Hölderlin's vision is not an answer to epistemological scepticism about the external world, generated by a version of Cartesian doubt. Instead he is seeking a way in which self-conscious awareness does not just lead both to self-alienation and alienation from the rest of the world. On this basis he seeks to find ways in which we can live with the suspension between finitude and the often painful feeling, generated by our amareness of our relativity, that we are more than merely imprisoned in finitude.

'Poetic individuality' relates closely to the argument we saw in the $S P$ (it has, remember, been argued that Hölderlin was involved in the writing of the $S P$ ), in which the 'highest act of reason' was an 'aesthetic act'. Only the I, as free 
spontaneity, can apprehend nature aesthetically or produce aesthetic objects. The object here enables the subject to grasp what it would be like to achieve a harmonious existence, and prevents the division in self-consciousness leading merely to alienation. Because it recognises itself in the external world without surrendering itself, which it would do if it made itself dependent upon the desire to appropriate the object, the I can begin to realise how it need not repress its divided nature and can instead regard this nature as a source of ever-renewed possibility. The division, it is important to remember, came about by a free act, which moved the I beyond the imaginary stage into the complex world of selfconscious reflection. Hölderlin, then, wishes to make the dividedness of selfconsciousness part of its own creative potential. The I can strive to show in aesthetic production what it would be to overcome its dividedness, without regressing into an imaginary unity.

Hölderlin thinks language is crucial to this process, but he does not regard it as a necessarily repressive symbolic order which prevents my self-realisation. The 'vocation of life' is to 'edify oneself from original simplicity to the highest form' (p. 524), to move from unarticulated feeling to the differentiated articulation of one's being, and this is also the vocation of 'Poesie', perhaps best translated here as 'creative language'. From the 'original feeling (Empfindung)' of unity with which one begins in childhood, the poet has 'struggled upwards by conflicting attempts towards the tone (Ton), towards the highest pure form of that feeling and sees himself completely comprehended in his whole inner and outer life in that tone' (pp. 525-6). ${ }^{7}$ At this point 'he has an intuition of (ahndet) his language', a language which must not just be taken up from the existing symbolic order, but which must be made essentially his own on the basis of his 'feeling', even as it is also taken from the resources available to all. Inspiring and fruitful as this vision is - the links of such ideas of unity in division to an essential individual 'tone' which is yet universal offer, for example, a way of understanding the unique power of Beethoven's music - Hölderlin's own poetry will reflect the struggle that results when the objective political and social world makes it harder and harder to believe in the possibility of individuality harmonising with the universal. The poems more and more take on the attributes of autonomous art, of a refusal to communicate in generally accessible terms, via their growing complexity and resistance to interpretation, which is combined with a remarkable tragic and musical power. ${ }^{8}$ Hölderlin will, of course, be worn out in contradictions with both himself and the object world: he goes mad.

Although Hölderlin offers a vision of language which does not make it a 'prison house', but rather a source of vital possibilities for self-realisation, there is warning in his fate and that of not a few other artists of the age. He was evidently aware, as his letters show, that attempts creatively to explore the I which are not backed up by social and political advances will lead to a growing alienation of advanced aesthetic production from any effective social role. The need seen in the $S P$ to link the aesthetic and the political is a necessary part of Hölderlin's vision: his own aesthetic production, though, moves increasingly in 
the direction of an autonomy which threatens his work's communicability to others. As such, his work constitutes a prophetic index of the consequences of the failure to achieve a link between the aesthetic and the political in a nondestructive manner. This pattern is often characteristic of the fate of modern art.

\section{Novalis}

Friedrich von Hardenberg (1772-1801), who used the name Novalis in his published work, conceives his philosophy, like Hölderlin, in relation to Fichte. In the English-speaking world Novalis has until recently rarely been considered as a philosopher, being seen rather as the archetypally doomed Romantic poet. However, like so many of his contemporaries, he did not regard the boundaries between forms of theoretical and creative activity as fixed, and his philosophy is an integral part of a wider project which includes scientific and literary work. One of the main reasons why Novalis has become the focus of recent attention is that he asks questions about subjectivity which already involve issues relating to the 'subversion of the subject' which has become the theme of so much recent theoretical discussion. Claims in certain areas of contemporary European philosophy and literary theory to be saying something radically new should, then, be tempered by the evidence from Novalis and others that, from the very earliest stages of modern philosophy, the subject does not necessarily occupy the position of sovereign. I want here to give a brief account, partly based on the socalled 'Fichte-Studies' (FS) (1795-96), of Novalis's reflections upon subjectivity. These reflections, which only appeared in a philologically acceptable edition in the 1960s, ${ }^{9}$ again lead in the direction of aesthetics. Before considering the reflections it is worth citing one of Novalis's later fragments, in order to underline how the still dominant image of Romanticism, aspects of which are sometimes admittedly to be found in Novalis, is inadequate to the complexity of his thought: 'I am convinced that one achieves true revelations rather by cold, technical understanding and calm moral sense than by fantasy, which just seems to lead us into the realm of ghosts, this antipode of the true heaven' (Novalis 1978 p. 775).

The FS are not a coherently-argued sequential whole: they are a series of sometimes fragmentary reflections relating to Fichte's Wissenschaftslehre. The difficulty for the interpreter is that the problems they confront are repeatedly explored in differing vocabularies, and it is not always clear whether Novalis is simply making notes about what Fichte has said, or whether he is formulating his own position. What is clear is that he rejects certain key Fichtean ideas. His basic objection to Fichte is summed up in a later fragment: 'Fichte has, as it were, chosen the logical schema of science as the pattern of a real construction of humankind and the world' (Novalis 1978 p. 684), thereby, as Hölderlin suggested, inverting the relationship between being and thought. As the most important commentator on Novalis, Manfred Frank, puts it: 'According to 
Novalis the error of idealism consists in taking what reveals itself as the first in consciousness for what is first in itself' (Frank 1997 p. 823).

The problem with which Novalis begins is the same as concerned Kant, Fichte, and Hölderlin. How can the I that is supposed to be the highest principle of philosophy give an account of itself? The basis of the attempt would obviously seem to be the statement of identity, 'I am I'. However, Novalis, like Hölderlin at much the same time, shows that the very attempt to determine the nature of something by a statement of identity robs it of its essential nature:

The essence of identity can only be demonstrated in an pseudo-proposition [Scheinsatz]. We leave the identical in order to represent it - Either this only apparently happens - and we are persuaded to believe it by the imagination [Einbildungskraft] - what already Is happens - naturally via imaginary separation and unification - Or we represent it by its non-being, by something non-identical -a sign. (Novalis 1978 p. 8)

The basic point here is, as Hölderlin also argued, ${ }^{10}$ that the splitting of the $\mathrm{I}$ in reflection means its essential character is lost. The separation required for consciousness to be of something, including itself, contradicts the prior necessity that there be a unity for the I to be an I at all: 'There can be no more content in the proposition I am I than there is in the simple concept of the I' (p. 9). The first and the second $I$ in a proposition need a prior I to unite them, so that $I$ as 'content' and I as 'form' can be the same, but our awareness of this is reflexive, and so is only 'apparent', part of the I as 'form'. The I as 'form' comes after the existential fact, the 'content', required for the analysis to be possible in the first place. Designating myself as I by the signifier ' $\mathrm{I}$ ' means employing something external to me, the publicly used mark, to stand in for me. The signifier introduces a non-identity into myself in articulating my identity. As Hölderlin suggested, then, if I need to reflect on myself to know myself as myself it is already too late to know the essential ground of my self. Any result of such reflection will be a self-deception because what I know as the result of my reflection cannot be the same as what inaugurates the reflection in the first place. We

do not become aware [of the 'first action'], in consequence we feel it as not-free. Why we do not become aware of it [?] - because it first makes becoming aware possible, and consequently this [becoming aware] lies in its sphere - the action of becoming aware therefore cannot go out of its sphere and wish to comprehend the mothersphere [because it depends upon it, is within it, in the manner of subject and object in relation to Hölderlin's 'being']. (1978 p. 8)

As such the I cannot be transparent to itself, because this would require comprehension of what makes it aware in the first place. It cannot use itself to explain what makes it aware, because it would need to step outside itself to do so. This structure of the totality which cannot describe itself as a totality is vital to Romantic thought, and to much subsequent philosophy which rejects the idea that we can gain a 'view from nowhere'.

Novalis's initial question is, therefore: how do I represent myself to myself? 
The difficulty echoes the difficulty faced by theories of truth as the true representation of what there is in the world. What criterion does one use to compare the representation with the object, in order to see if it corresponds to it? To do this would require taking up a position outside the relationship between representation and object, which is impossible, because this would also involve a representation of the relationship, and so on, to infinity. Unlike the rest of being that can be 'real' in Kant's sense of being a possible object of perception, consciousness itself is 'a being outside being in being' (p. 10). This means it is an 'image', 'an image of being in being' (p. 10). Novalis invokes a 'Theory of representation or of non-being in being, in order that being is able to exist (daseyn) for itself in a certain manner' (p. 10). In existing in a certain manner, namely as image, as consciousness, being does not exist absolutely. ${ }^{11}$ Consciousness is therefore, a kind of relative non-being, like Sartre's 'néant' (as opposed to the absolute nothingness of ' $r i e n$ '): 'I is basically nothing - Everything has to be given to it - But it is to it alone that something can be given and the given only becomes something via the I' (p. 185). Novalis then relates the image to the linguistic sign via the notion of the schema - 'Every comprehensible sign ... must stand in a schematic relation to the signified' (p. 14) - and he links this to the problem of how different subjects can understand the same signs used by others (see Frank 1997 pp. 804-6 for the influence on this of Fichte's conception of language). The schema, as Kant argued in his account of the imagination, is the bridge that connects the receptive and the spontaneous sources of knowledge, the realms of necessity and freedom. In consequence, for Novalis, the sign can be a sign because those who use signs are 'necessarily free' (Novalis 1978 p. 14), the schema linking the necessary and the free. Language can only be understood as language via the assumption that the necessitated material events in which it is instantiated (moving airwaves, electric charges, etc.) are linked to the meaning intentions of a (free) subject. Communication is possible, then, via the 'as it mere, free contract' (p. 15) inherent in language. One accepts the objective, learned necessities entailed in communication with other language users at the same time as acknowledging their capacity as free agents to mean something only they can mean. ${ }^{12}$ Describing the position of the language user who relies in this way on schematism, Novalis suggests that in doing so they 'will, without realising it, have painted their own image before the mirror of reflection' and that 'the picture is painted in the position that it paints itself' (p. 15). They will, then, be schematising themselves, and this involves some crucial problems which go to the heart of Novalis's thinking, and are suggested in his question: 'Can I look for a schema for myself, if I am that which schematises?' (p. 162).

Let us take up some possible implications of Novalis's metaphor. Remembering that consciousness is an 'image of being in being', in a painting in which the painter includes himself or herself as painter of the picture within the picture, he or she reminds the viewer that without the painter there would be no painting. At the same time we actually see the painter not as creating subject, but as object, who appears as visible image mithin the painting, which is 
supposedly his or her own, thus inverting the real state of affairs. Something analogous applies to the use of the term 'I'. We all use this term in the same way to designate ourselves, even though the point of using 'I' is to differentiate myself from everything and everyone else, but at the same time my ability to use it relies on my unique pre-reflexive sense of self which Novalis will term 'feeling'. We can infer from the picture that the painter is theoretically necessary in bringing about the existence of the image we have before us, but we have no access to what generates the image, because we see the creator as object, thus as a result of reflection, with the concomitant inversion. To see the painter as subject we would have to become him or her as I, and thus be able to generate the painting ourselves.

A famous example of the sort of issues involved in a self-reflexive painting is Velasquez's Las Meninas. In Las Meninas there would have to be a mirror in the position where the viewer of the painting is located, which the painter would be looking at in order to see himself and to be able to paint his own reflection. In fact, though, one infers that in the position of the assumed mirror is the object that the painter is supposedly painting: the King and Queen of Spain. We infer this by the fact that the object the painter is supposed to be representing exists as empirical object, within the painting, as a faint reflection in a mirror that is represented on the farthest wall of the painting. The $\mathrm{I}-$ the painter - retreats into a position in which he cannot be represented as active I: he is dependent upon a reflection in a non-existent mirror. It is clear, though, that this does not mean that the I does not exist: the very sense of its absence points to its undeniable existence, not least as the ironic creator of a baffling aesthetic object. This coexistence of presence and absence evokes the world of Novalis's reflections upon the I, and hints at how they relate to aesthetics.

Taking up the image of the painting with regard to language, Novalis argues that the signifier ' $I$ ' is the 'Non-being' (p. 8) of the I because it attempts to represent the I. If the signifier is to represent the I to anyone else - if it is to be a signifier at all - it must be recognisable to another as designating the I, but therefore not being the I. It cannot express the being of the I, because it is merely an objective condition of the I being taken by another as an I. In order to understand the signifier, the other must already possess a pre-reflexive sense of self which is precisely not dependent upon the ability to use the objectively existing word I (how, as the problem of seeing oneself as oneself in a mirror suggests, would they know that the word can be used to refer to themself?). The identity of the signifier is, furthermore, constituted by its difference from every other signifier: 'In its place each [schema] is only what it is by the others' (p. 14). It is, as such, wholly determined by its relationship to everything else: ' $\mathrm{I}$ ' is not 'you', is not 'he', etc. However, the dependence of the signifier on its not being all other signifiers for its identity means that what determines it - all other signifiers also points to the opposite of determination. There seems to be a negative way of representing the absolute, in that the very relativity of the attempt to represent it makes us aware of what is being missed in the attempt. This happens, 
though, in a way which we cannot articulate: 'We feel ourselves as part and are precisely for that reason the whole' (p. 44). We could not feel only a part if we did not also have a feeling, but not a concept, of what it would be to be whole. This 'feeling' leads Novalis to a central idea in early Romanticism.

The idea is that philosophy sees things the wrong way round because it relies on reflection. ${ }^{13}$ Fichte's response to Kant began with reflection upon the activity which is required for reflection to take place at all: the action of the I. The I is, therefore, already subsequent to its basis: without some prior being of the I there would be no reason to reflect at all, because there would be nothing to reflect upon. Novalis terms this basis 'Gefühl', literally 'feeling', which refers to the pre-reflexive I whose being is more than can be thought, all thoughts depending on the structure of reflection. However, this entails a fundamental problem. If the I is to be represented as the highest principle of philosophy it must somehow give an account of its pre-reflexive basis. This basis, though, as we have seen from a similar argument in Hölderlin, is not available to reflection: 'Feeling cannot feel itself ... It can only be looked at in reflection - the spirit of feeling not there any more' (p. 18). Novalis insists that it is 'not possible to represent the pure form of feeling. It is only One and form and matter as composed [in the sense of made up of differing elements as in a 'judgement'] are not at all applicable to it' (p. 21). Reflection begins by an awareness of the difference of subject and object, a limitation of the self by another, even if that other is in fact itself. For Novalis, reflection puts what is derived, the I of conscious reflection, before what it is derived from, 'feeling', thereby inverting the real sequence. This can, though, lead a stage further, to a vital argument.

The metaphor of the mirror can help to explain the basic idea and how Novalis responds to it. In a reflection of myself in a mirror what is in fact my right eye will appear as my left eye. In order not to confuse the reflected image with my real being I must further reflect in my 'mind's eye' to invert what I see and arrive at a correct representation: 'The image is always the inverse of being. What is on the right on the person is on the left in the image' (p. 47). The 'correct' image, though, is an image created by a double reflection, not a form of direct access to reality. ${ }^{14}$ Frank suggests that for Novalis 'reflection can itself illuminate and thus correct the inverted relation of consciousness to being/reality by a further reflection upon the ordo inversus inscribed in it' (Frank 1997 p. 823). However, this does not mean that he falls into a pre-Kantian version of the claim to know reality in itself, beyond the conditions of consciousness: '[reflection] does not at any moment step over the critical boundaries of the immanence of consciousness and yet it has explained that not everything is in consciousness' (p. 823). If one applies this argument to the absolute I the result is the realisation that it is unrepresentable, because what is to be represented would itself have to do the representing at the same time (with the problems seen in the picture metaphor). Novalis says of freedom: 'All words, all concepts are derived from the object [Gegenstand, in the sense of that which "stands over against" its other] - [they are] objects - and they therefore cannot 
fix the object. Namelessness constitutes its very essence - for this reason every word must drive it away. It is non-word, non-concept. How should something make an echo which is only a voice?' (Novalis 1978 p. 110). If words are constituted differentially, no word could be absolute because of its dependence on all other words. In this sense words are objects: cognition of any object is, as we have seen, dependent upon what is not the object for the object to have a knowable identity. The argument points towards the realisation that philosophy cannot positively achieve its task of showing the identity of subject and object. For philosophy to grasp the real nature of the absolute it has to correct the inversion which results from putting reflection first. Marx's claim that Hegel's philosophy must be stood on its feet because it reverses the relationship between thought and social being contains an echo of the Romantic thinking at issue here. It seems likely, as Frank suggests, that there is a traceable route from these ideas, via the later Schelling, Schleiermacher, and Feuerbach to Marx. The question is then whether one gives up talk of the absolute, as Nietzsche will later do in his attempt to say farewell to metaphysics altogether.

Hegel's suggestion with regard to the absolute will be that it is present in the very principle of philosophical reflection. Everything is what it is via its relations to everything else. If it can be shown that the structures of thought exhaust the way things can be articulated, the absolute will be arrived at precisely by describing the necessities of thought. In this Idealist conception of the absolute, the forms of thought are shown to be the forms of being. Novalis, in contrast, maintains that 'the true philosophy could never be represented' (Novalis 1981 p. 557), because, as Frank puts it: 'Being is not completely resolved into the form of thought (the judgement). It [being] just (metaphorically speaking) separates a layer of itself from itself which is accessible to thought . . . Kant had already called existential "being" [that there is something] absolute positing, and the "is" of the predicative judgement [what it is said there is] relative positing' (Frank 1997 pp. 832-3). Hegel, as we shall see in Chapter 5, essentially assimilates the former to the latter, the 'real ground' to the 'cognitive ground', via his attempt to work out the logic of the 'speculative proposition'.

The link of the question of the absolute to aesthetics begins to become apparent when Novalis maintains that Poesie, in the sense of Poiesis, creation, 'represents the unrepresentable' (Novalis 1978 p. 840). Philosophy for Novalis is 'striving' to think an absolute 'basis' (Grund) that would allow its completion. However, 'If this were not given, if this concept contained an impossibility then the drive to philosophise would be an endless activity' (p. 180). The need that generates philosophy can, therefore, only be satisfied in relative terms, and thus never be satisfied. The free activity of my I is destined not to achieve the absolute because it would thereby cease to be itself, given that the drive to philosophise, which is itself the free act of the I, is based upon a 'voluntary renunciation of the absolute' (pp. 180-1), an acceptance that the condition of knowing is the acknowledgment of finitude. The 'ideal' can never be achieved because 'it would destroy itself. To have the effect of an ideal it must not stand in the sphere 
of common reality. The nobility of the I consists in free elevation above itself consequently the I can in a certain respect never be absolutely elevated' (p. 170). If the goal of philosophy were present there would be no need for philosophy to seek it. This is why Fichte's I, as the supposed absolute beginning, is just 'a Robinson [Crusoe] - a scientific [i.e. philosophical] fiction - to make more easy the presentation and development of the Wissenschaftslehre' (p. 645), but it is only a fiction, not the real principle of being.

These reflections lead Novalis to introduce a strikingly modern temporality into his argument: 'Time can never stop - we cannot think away time - for time is the condition of the thinking being - time only stops with thinking' (p. 180). He therefore, pre-empting Nietzsche, breaks with conceptions of philosophy which see it as concerned with the representation of the eternal. Elsewhere he talks of his 'conviction ... that precisely the old lament that everything is transient can become the most joyful of all thoughts' (p. 433). Such ideas also lead in the direction of a conception of art which, as I suggested in relation to Kant's sublime, regards the limitation of the finite as a way of attempting, always inadequately, to experience what is beyond the finite, the world of reflection. If philosophy is a striving for the absolute, the absolute cannot already be achieved in philosophy, even though philosophy presupposes the absolute as its aim; without it philosophy would not be philosophy: 'The unattainable cannot by its very character be thought of as being attained - it is, so to speak, just the ideal total expression of the whole sequence and therefore apparently the last member' (p. 643). As such: 'The absolute which is given to us can only be recognised negatively by acting and finding that what we are seeking is not reached by any action' (p. 181); in short, 'We everywhere seek the unconditioned (das Unbedingte), and always only find things' (p. 226). Novalis relies on the paradoxical principle, which will influence Adorno, that philosophy 'must be systemlessness brought into a system' (p. 200). In another formulation: 'There is no philosophy in concreto. Philosophy is, like the philosopher's stone - the squaring of the circle etc. - just a necessary task of scientists - the absolute ideal of science' (p. 623). The 'true philosophy' could not 'be represented' (p. 828) because representing an ideal does not make it real, for the reasons we have seen in the remarks on reflection.

Along with his consideration of why philosophy cannot come to an end, Novalis also repeatedly ponders, against Fichte, why philosophy should have a beginning: '(What is a beginning for at all? This unphilosophical or half-philosophical goal leads to all mistakes)' (Novalis 1968 p. 383). Elsewhere he claims 'There is no absolute beginning - it belongs in the category of imaginary thoughts' (Novalis 1978 p. 699), and 'Every real beginning is a 2nd moment. Everything which exists, appears, is and appears only under a presupposition' ( $\mathrm{p}$. 380). Consequently, 'All seeking for the First is nonsense - it is a regulative idea' (p. 164). The idea of a beginning is itself reflexive: something only becomes the beginning because of what follows it, so it is inaccessible to our thinking until it has ceased to be what it was. It can therefore only be an idea which motivates 
our search for truth. Instead of thinking that philosophy could represent the fundamental ground, the 'initial impulse' of freedom that Fichte posits as the absolute beginning, philosophy must, then, realise its own inherent failure to be complete. Philosophy 'teaches the relativity of all grounds and all qualities - the infinite multiplicity and unity of the construction of a thing etc.' (p. 616). At the same time, philosophy does not renounce the absolute completely, because the sense of lack generated by our sense of a missing unity is what makes us desire to know and act at all.

Philosophy which adopts such positions gravitates towards art, which 'represents the unrepresentable' (p. 840). Novalis relates his rejection of an absolute beginning to art: the 'beginning is already a late concept. The beginning arises later than the I, for this reason the I cannot have begun. From this we see that we are in the realm of art' (p. 485). The necessary failure to represent an absolute ground, in this case the I which occasions the beginning, leads to thinking about forms of representation which might incorporate this failure. This idea is the source of Novalis's Romantic notion of art. As we have seen, the I seems to be constitutionally lacking, but this very fact leads it beyond itself: 'I means that absolute which is to be known negatively - what is left after all abstraction What can only be known by action and what realises itself by eternal lack./Thus eternity is realised through time, despite the fact that time contradicts eternity' (p. 181). The relationship between our empirical self, and the feeling of a self which transcends temporality but which can only be a regulative idea is crucial to Novalis's conception of art:

Self-consciousness in the greater sense is a task - an ideal - it would be that state mithin which there was no movement in time ... In real self-consciousness we would just change - but without going any further ... we are not $I$ by inferences and indirectly - but immediately ... All our memories and events link to a mystical unity which we call I. (p. 672)

He therefore seeks a medium in which the sense of lack generated by the dissonance between the transient empirical self and the idea of an a-temporal prereflexive self can be meaningfully articulated. What is lacking would here be somehow gestured towards, without it being able to appear as itself. He realises that the idea is particularly appropriate to the temporal form of music. A piece of music makes most sense, is music in the most emphatic sense, if its temporality is overcome by our ability to link together its different moments into an integrated whole that seems to abolish time. The relationship between language and music is central to this conception.

Language for Novalis is, as we saw, constituted differentially. It is therefore marked by reflexivity, each of its elements lacking something which can only be completed by the other elements. Language relates to the question of the absolute, in that the need in the signifier for all other signifiers for it to be itself points to an unrepresentable totality: 'The whole of language is a postulate' (p. 347). The temporality of thinking means that the attempt at completion will be a 
continual movement from signifier to signifier. Something akin to différance, Derrida's idea of the deferral of signification as presence that is occasioned by the signifier's dependence on other signifiers is, then, already part of Romantic thinking. However, Novalis seeks to avoid the problem present in the notion of deferral, which must entail some kind of 'presence', of the kind evident in the non-inferential, pre-reflexive aspect of self-consciousness that enables experience to be possible, if the notion of what is lacking and thus deferred is to be felt as lacking at all. ${ }^{15}$ The alternative to this is, of course, a mere infinite regress of signs, with no account of why the regressing movement takes place at all.

Novalis describes music as a 'general language', an idea which we will encounter again in Schopenhauer; 'language' itself is, in turn, a 'musical ideasinstrument' (p. 597). In a famous fragment Novalis says philosophy is 'really homesickness, the drive to be at home everymhere' (p. 675). Our sense of the absolute as lack is clear in this: in order to feel homesick we must have a feeling, but not necessarily a clear concept, of what home would be. If one puts this remark into the context of one of his remarks about music a decisive link becomes apparent. Music, he claims, enables the mind to be 'for short moments in its earthly home', because the mind is 'indeterminately excited' (p. 517). In such excitement it does not seek to define its intentional object, and can thus be with itself in the non-objectifying manner suggested by the notion of 'feeling'. In the FS Novalis frequently formulates the problem of reflection in terms of the separation of the determiner from what it determines. Music is linked to 'feeling', which does not entail such a separation, because, as we saw, it 'cannot feel itself'.

On the one hand, Novalis regards music as inferior to painting, because animals can be said to possess music, whereas they could never see the beauty of a landscape, and the power of music's effect should therefore not be used as the measure of its 'intellectual rank' (p. 364). On the other, 'Musical relations really seem to me to be the fundamental relations of nature' (Novalis $1981 \mathrm{p}$. 528), and he asks 'Might musical relations be the source of all pleasure and unpleasure?' (Novalis 1978 p. 772). He elevates music above other art when he imagines an attempt to 'speak determinately through music', our language having lost its musical quality and become prosaic. The indeterminate excitement occasioned by music would then become a new determinate way of relating to what we are. For this to happen language 'must become song again' (p. 517). This idea can be understood via the fact that music reveals a lost past and a hoped-for future, based upon what lacks in the present. It contains a dynamic which integrates all dimensions of time, thereby in one sense fulfilling the demands of philosophy, as well as giving hope for an at least temporary affective reconciliation with our transience. In verbal language indeterminate propositions 'have something musical' and are able to give rise to 'philosophical fantasies - without expressing any determinate path of philosophical thinking' (Novalis 1968 p. 319). As we saw, Novalis refused to give freedom a name: music thus seems better able to express freedom, because of its conceptual indeterminacy. 
Music's lack at the level of concepts actually means it is able to evoke what Novalis requires for the 'Universal system of philosophy', which 'must be like time, A thread along which one can run through endless determinations' ( $p$. 201) and cannot be a 'positive system'. Like signifiers in language, notes depend upon other notes for their significance, attaining their full, but non-conceptual, sense in the overall temporal continuum of the piece. The idea of rhythm, to which Novalis, like Schlegel, attaches serious philosophical importance is essential to his conception. ${ }^{16}$ Novalis claims: 'All method is rhythm', and that 'Fichte did nothing but discover the rhythm of philosophy and express it verbalacoustically' (p. 544). Rhythm, like language, is a form of meaningful differentiation; a beat becomes itself by its relation to the other beats, in an analogous way to the way in which the I of reflection is dependent upon the not-I, the signifier on the other signifiers, the beginning upon what follows it. Rhythm is therefore a form of reflection. It is constituted temporally and is not semantically determinate, being the movement from one articulation to the next within a pattern. To regard a rhythm as a rhythm, though - and this is the vital point - requires a subject for which it is felt as a series of linked articulations, and not a random set of phenomena of the kind the empirical I would be subjected to were it not grounded in the pre-reflexive I. What is important, then, are forms of articulation which are not dependent on representation and are a means of finding new ways of understanding self-consciousness: 'The musician takes the essence of his art from himself - not even the least suspicion of imitation/representation (Nachahmung) can touch him' (p. 363). Novalis, then, does not, as Derrida tends to, for example, cease to pay attention to self-consciousness because of its resistance to representation. ${ }^{17}$ Instead he looks for new approaches to what philosophy seeks to do which do not exclude the interrogation of the subject. What, though, does this tell us about the wider issues which emerge from the Fichtean and Romantic reactions to Kant?

It might seem 'Romantic' in the bad old sense to suggest that philosophy's conceptual failure means it should delegate its most significant role in this period, that of articulating freedom, to the aesthetic form of music. Such hyperbole can, however, be made sense of, and offers a way of approaching some major philosophical issues that were first seen clearly in Romanticism. To conclude this chapter, let us, give a rough recapitulation of the philosophical choices that have emerged, in order to see how the issues ensue from these choices. Fichte's post-Kantian opposition to materialism was based on the claim that there is no way in which what is merely objective and mechanical can give rise to what is subjective and self-determining, which, following Kant, is the essential focus of a philosophy no longer reliant upon theological dogmatism. This led Fichte to an absolutisation of the I. The advantage of this absolutisation was that it seemed to obviate the problem of the thing in itself, which is now located within the I, albeit in a largely unexplained manner. If this approach were to work it would get over the problem of how objective causality could give rise to subjective representations, abolishing the divide between the objective and the 
subjective by making the former that which is derived from the self-division of the latter. In Fichte's view the philosopher's job was to overcome the finite limitations of anything particular in the name of what is the essence of our being, namely self-determining spontaneity. Lurking in this conception, though, was the sense that the activity of mind is driven by something which is prior to any kind of conscious control - this will be why Fichte's conception of the will becomes so important for Schopenhauer, who, of course, will link his conception of the will to music as the 'true philosophy' (see Chapter 8).

The aim of Fichte's system was, then, to find a new ground for philosophy and it attempted to do so by taking an Idealist route, albeit one in which there was a concern to avoid Berkeleyan problems. By establishing the foundations of philosophy in the I the pattern of reality was to be derived from the patterns of what the mind does, and reality was to be regarded as intelligible only through that activity. As we have seen, this strong Idealist programme came up against difficulties in relation to its founding principle. The Romantic problem lay therefore in giving an account of philosophy in which the undeniable fact that the I seems ineliminable from intelligible cognition (causal inputs alone giving no way of explaining the structure of seeing things $a$ s things) can go along with the ideas (a) that the ground of the self relates to a being to which the self has no cognitive access, and, consequently, (b) that being, including our own being, is always more than we can know about it.

Two initial interpretations of this position offer themselves. As we saw in Chapter 2, Frank thinks the essential Romantic position is akin to contemporary 'metaphysical realism'. In this view reality is assumed to be the way it is independently of our thinking about it, so that, at best, we can see truth as an 'endless approximation' to it. The real in this sense, though, seems to be truthdeterminate in the way the empirical world seems to be - it is just that we have not reached that truth yet. The problem with this kind of position, as we saw, is that it relies on the correspondence theory of truth, a theory which has proved very hard to defend (which Schlegel, for example, explicitly rejects and which Schleiermacher, like Davidson, suspects may be vacuous). ${ }^{18}$ Without a valid version of the correspondence theory which can show how it is that we are converging on the truth, without just presupposing that we are, metaphysical realism leads straight to an emphatic form of scepticism, because we could never know what it would be for our though to correspond to reality, precisely because reality is the way it is independently of thought.

The weaker version of the non-Idealist position, which is closer to Novalis's position and comes closer to certain versions of contemporary pragmatism, makes truth a regulative idea. This position admittedly involves a form of scepticism about any claims to know in a definitive manner - such claims would seem anyway to require the representationalist correspondence theory - but it derives its force precisely from a sense that truth-claims should always be open to revision, even though there is no guarantee that there is any ultimate truth at all - How would we know if there is? Truth effectively becomes a normative, rather 
than an epistemic matter. Both interpretations of the Romantic position lead to the idea that the best we can actually do is make our beliefs as coherent with each other as possible, which is an endless task, because each justified new piece of knowledge will require adjustments in the system. Novalis's claims that philosophy 'must be systemlessness brought into a system' and that philosophy is the - unrepresentable - 'absolute ideal of science' suggest what this entails. The move to aesthetics has here to do with the coexistence of the need for ways of understanding (and enjoying) the ways things can cohere beyond what we know about them with the realisation that, for us, knowledge could never be complete.

Frank claims that the 'primacy of being over consciousness' leads Hölderlin and the Romantics to a ground which can only be represented by 'the darkness of aesthetic representation' (Frank 1997 p. 753). This darkness can be suggested most evidently in music's combination of a meaningfulness generated by the relation of parts to a whole with its resistance to semantic determination. In Schelling's emphatic version of this position, considered in Chapter 4, art's combination of resistance to definitive interpretation with its enduring ability to reveal new aspects of being in a direct manner contrasts with the claim that progress in science is only ever particular and is simply the next step in an endless chain of determination. Art therefore takes on the privileged role in philosophy, giving access to a truth nothing else can. This view has plausible aspects, some of which I hope already to have indicated, but it also needs to be located in a historical argument relating to real works of art, and here things become much more difficult.

After an initial flowering of art from the 1790s onwards, particularly the music of Beethoven, which indeed seems to aspire to something no other form of articulation can achieve, art itself becomes increasingly problematic, to the point in the early twentieth century where the avant-garde puts the very notion of art into question. One of the many reasons for this questioning is the growing ability of science to explain and solve problems which were previously inexplicable and insoluble, a development which also continually reduces the role of philosophy in determining our place in things. Odo Marquard has seen this situation in terms of the move in nineteenth-century secularised thinking from aesthetics, the means of reconciling ourselves, at least in the realm of appearance, to the pain of a now irredeemable existence, to 'anaesthetics', as a scientifically produced means of really countering pain. The fate of aesthetics and notions of the subject in relation to the movement from aesthetics to anaesthetics will concern us in the chapters to come.

\section{Notes}

1 If, as one should, one translates 'Es' as 'It', the proximity of Freud to the tradition to which Lichtenberg (and, in his wake, Schelling and Nietzsche) belong becomes apparent. It should be clear from the context that I think the traditional, rather than the Lacanian reading, is apt here. Aspects of Lacan's theories will be apparent in some of the theories of the I in Romanticism, most notably in Schelling's 1833-34 Lectures On the 
History of Modern Philosophy (see Schelling 1994, Bowie 1993, Zizek 1996).

2 For the details of the reactions against Fichte, see Frank 1997.

3 It now seems clear that this text should be called 'Being and Judgement'. As I am working from an edition of the text which has the older title I shall use that one, even though the logic of Hölderlin's argument makes it clear why the new title better describes the order of the text.

4 It is clear that Fichte and Schelling both had a decisive influence on Schopenhauer's conception, despite his attacks on them. The idea of loss of self being a condition of access to the absolute is, of course, part of Buddhism, and Schopenhauer himself was influenced by Buddhism.

5 Like Friedrich Schlegel and the early Hegel, Hölderlin also considers this issue in relation to love, as the form of self-transcendence which can lead to a non-alienated sense of identity that does not lead to retreat from the world.

6 It seems clear from Hölderlin's presentation of this issue that Hegel derived many of his key ideas from these arguments.

7 The word 'Ton' usually means 'note', but it seems clear that it here relates to the 'moods' whose meaningful connection he sees as essential to the self's search for unity in division.

8 Something similar, as Adorno has argued, takes place in the late Beethoven's rejection and reconfiguring of musical convention in the light of his growing disappointment with the development of the society of his time.

9 How much the ideas they contain played a role in the history of philosophy is therefore an open question. As Frank 1997 shows, the criticisms of Fichte they contain were part of a widespread reaction against Fichte's idealism - which itself was hugely influential in many areas of intellectual life - on the part of many thinkers at the time, and the basis of the criticisms has reappeared in many forms in subsequent philosophy, some of which was at least indirectly influenced by Romantic thought (see Bowie 2000).

10 See Frank 1997 for details of the common sources of these arguments for Hölderlin and Novalis.

11 This pattern of thought recurs in an almost identical form in Schelling's critique of Descartes in the early 1830s (see Schelling 1994).

12 Frank points out, though, (Frank 1997 p. 808) that this does not explain how it is that one subject comes to interpret the physical manifestations of the other as signs in the first place.

13 See Manfred Frank and Gerhard Kurz: “ "Ordo inversus”. Zu einer Reflexionsfigur bei Novalis, Hölderlin, Kleist, und Kafka' in Geist und Zeichen Festschrift für Arthur Henkel, Heidelberg 1977 pp. 75-92.

14 The failure to see that this is therefore not a dogmatic claim has led critics of the later Schelling, who uses the same argument, to mistake this for a pre-critical position.

15 Derrida's exclusion of any vocabulary to do with feeling, and his over-concentration on the subjectless semiotic generation of difference derives, one suspects, from the Cartesian-Husserlian heritage which he seems to regard as the only significant philosophical history of subjectivity.

16 Discussing the origins of philosophy in the Orphic period in Greece, Schlegel claims that 'rhythm in this childhood of the human race is the only means of fixing thoughts and disseminating them' (Schlegel 19882 p. 16).

17 In recent texts Derrida has become more concerned with questions about the subject, but his Heidegger-inspired antipathy to the subject as the subject of 'Western metaphysics' is very evident in his earlier work. 
18 The essential objection to correspondence theories is, as Habermas argues, that 'we would "have to step out of language" with language itself . . Obviously we cannot compare the linguistic expression with a piece of uninterpreted or "naked" reality' (Habermas 1999 p. 247). Davidson sees the notion of correspondence as 'without content'. 


\section{Schelling: art as the 'organ of philosophy'}

\section{Nature and philosophy}

One of the great issues which divides thinkers in modernity is the status of 'nature'. If nature can no longer be said to have a theological basis, what determines how we are to understand what nature is? Kant's ambivalence with regard to 'nature' suggest why this issue creates so much controversy. On the one hand, nature 'in the formal sense' is simply that which functions in terms of necessary laws, and is therefore the object of natural science; on the other, in the form of organisms and as an object of beauty, nature appears to have purposes which cannot be explained by what we can know. Similarly, we ourselves are to be understood deterministically as natural objects, but are supposed to be selfdetermining as free beings. The question therefore arises as to whether there is a link between 'intelligible' human freedom and the possible purposiveness of nature. The temptation is then to make a direct link between the fact that we can see nature as beautiful and the fact that we can produce art objects which possess a similar status to beautiful nature. However, the possibility of this link only adds to the ambivalences to which Kant gives rise. Kant himself is insistent that beauty is purely a matter of the subject's feeling, which would seem to render any such link invalid, but at the same time, as we saw in his account of reflective judgement, he thinks that the very capacities which enable us to make judgements of beauty are also required for us to be able to communicate the results of apprehending nature objectively.

Where, though, do such capacities come from, given that we have developed as a part of the rest of nature? Fichte's response to this problem is, as we saw, to make the very ground of nature ultimately 'subjective', because an explanation of how a merely mechanical nature could give rise to self-determining subjectivity otherwise appears impossible. The problem with his account of the 'absolute I' was that the I seemed to have to be unconscious, if it was not to be relative to its intentional object and thus dependent in a manner which contradicts its absolute status. Furthermore, Fichte leaves open the question whether we can understand the I directly, through the awareness of our own unconditioned mental action in 'intellectual intuition', or whether the idea of grasping the absolute I is merely regulative, generated by the need of practical reason to go 
beyond the world of the understanding. Fichte also tends to regard nature merely as an undeveloped aspect of the highest principle, while at the same time suggesting that what drives the highest principle is itself not available to discursive philosophy. He consequently suggests that the conscious I has an unconscious basis, and it is this idea which will be crucial to Schelling's influential account of nature.

In parts of his philosophy before 1800 Schelling follows something like Fichte's position. Even in this period, though, he works on the idea that, because the activity of the I is in some sense 'natural', nature itself is not just dead objectivity which is to be given life by the I. A particularly acid comment by Schelling in a letter to Fichte (1801), not long before they broke off their correspondence, makes clear why he moves against Fichte's conception of the I's relationship to nature by this time:

I am thoroughly aware of how small an area of consciousness nature must fall into, according to your conception of it. For you nature has no speculative significance, only a teleological one. But are you really of the opinion, for example, that light is only there so that rational beings can also see each other when they talk to each other, and that air is there so that when they hear each other they can talk to each other? (ed. Schulz 1968 p. 140)

By 1806 he becomes even more critical of Fichte: 'in the last analysis what is the essence of [Fichte's] whole opinion of nature? It is this: that nature should be used . . . and that it is there for nothing more than to be used; his principle, according to which he looks at nature, is the economic-teleological principle' (Schelling I/7 p. 17).

It is not hard to see, even from these brief comments, how Schelling has claims to be one of the first serious ecological thinkers in modernity, and his ideas will be echoed by Heidegger, Horkheimer and Adorno, Ernst Bloch, Hans Jonas and many others, most of whom were influenced by him. His criticisms of Fichte suggest how philosophies of self-consciousness can come to be regarded, as Heidegger will regard them, as philosophies of 'subjectification' which reduce being solely to the measure of the human. ${ }^{1}$ Schelling's counter to Fichte, which impressed the young Karl Marx, is that the forces of the I as he conceives it are forces which depend on nature. Nature cannot in this sense be seen as simply being there to be dominated, because such domination could, as Horkheimer and Adorno will claim, lead to domination of ourselves as part of nature. As we have seen, a conception of nature of this kind tends in this period, to be connected to aesthetics. The genius as conceived by Kant had the law of its production given to it by nature, and Kant saw beauty as 'purposiveness without purpose' because it involved a disinterested relationship to the object, thereby possibly revealing something fundamental about our place in a teleologically constituted nature. Schelling's suspicion of Fichte is connected to this side of Kant's third Critique, but his philosophical alternative to Fichte faces a revealing problem, which is the source of many of the ambiguities in his work. 
For the philosophers who come after Kant and who regard Kant as having decisively changed the project of philosophy, a fall back into 'dogmatism', the assumption of an inherent pre-existing order of things which grounds philosophy, must be avoided. At the same time, Kant's separation of the knowable world of appearances from unknowable things in themselves left the notorious problem of how those things were supposed to cause representations, and was therefore seen as leading to an implausible dualism. During the 'Pantheism Controversy' initiated by Jacobi in 1783, the monist philosophy of Spinoza came to public attention and it seemed to many thinkers to offer a way of getting over Kantian dualisms because it claimed there could only be one substance, which was both extended and thinking. On the other hand, Spinoza's philosophy failed to answer vital questions regarding the emergence of the crucial anti-dogmatic notion of self-determining subjectivity. ${ }^{2}$ Schelling's early work is therefore an attempt to square the circle by reconciling Kant, seen through the eyes of Fichte, with Spinoza, by finding a route between 'Criticism' and 'Dogmatism', a project he characterises in terms of a reconciliation of Idealism and Realism, or of transcendental philosophy and Naturphilosophie. The main problem this involves is the primacy of the two approaches in relation to each other. Prioritising transcendental philosophy avoids dogmatism, but at the expense of rendering nature secondary to the I, and thus giving rise to Fichte's problems. Naturphilosophie gives an account of the I's ground in material nature, but seems to have to rely on dogmatic premises to do so - if nature can only be known under the conditions inherent in the I, what right does one have to suggest we have access to nature in itself?

The essential idea of Schelling's Naturphilosophie is that, in the same way as the I of self-consciousness is both active and yet can try to reflect upon itself as an object, nature is both actively 'productive' (in the sense of Spinoza's natura naturans) and is made up of objective 'products' (natura naturata). The understanding deals with transient 'products' and is consequently confined within the limits of determinate cognition; Naturphilosophie tries to theorise nature's 'productivity', without which there would be no products, and thus goes beyond what science can know, which is always particular and finite, to what is 'infinite'. This idea can be made to sound less implausible if one considers the fact that the material in which a living thing is instantiated is in one sense less significant than its 'idea', which constitutes what it is by combining matter into the particular kind of organism it is. The actual matter of the living thing is constantly being replaced, without the thing losing its identity - Schelling uses the metaphor of the eddies in a stream which are filled by different water but retain their shape. Science now tells us that once the 'idea' of an organism is in existence it is transmitted chemically in the form of DNA, but DNA does not explain the emergence of organisms in the first instance, and it is precisely the emergence and development of an articulated nature which then develops into living organisms that makes sense of the idea of nature as a 'productivity'. Schelling's idea is, then, to link the intelligibility of the matter in nature, which can become 
part of self-regulating organisms, with the self-determining nature of thought which can carry out purposes in the world. Without such a link it is, Schelling thinks, unclear how we could truly know anything about what there is, or affect the relationships between the elements of what there is. He therefore talks of nature's reaching its highest goal of 'becoming completely objective to itself' in man ... 'or more generally' in 'reason, through which nature first completely returns into itself, and through which it becomes manifest that nature is originally identical with what is known in us as intelligent and conscious' (I/3 p. 341).

Schelling attempts to address the identity of the processes of nature with the processes of thought in terms now more familiar from Freud. Nature is described as being 'unconsciously' productive, and 'mind' as being 'consciously' productive. Manfred Frank and Gerhard Kurz suggest that 'Freud and Schelling both presume that consciousness means becoming conscious, a fragile synthesis of voluntary and involuntary motivations, and that this consciousness, like existence in general, is only to be grasped as the memory of a dark, never fully recoverable basis' (ed. Frank and Kurz 1975 p. 41). This conception does not, however, make Schelling an irrationalist: 'Schelling's concept of reason is enlightened about itself. Reason is not the Other of nature, it is its - undeveloped - part' (p. 42). His essential argument can be interpreted as establishing a link between Fichte's notion of the 'thetic judgement', in which 'the place of the predicate is left empty to infinity for the possible determination of the I', and the notion of nature as subject, whose ever renewed and destroyed 'products' are the predicates of the productivity. Schelling attempts to envisage nature as subject via the following argument, which, as we shall see in Chapter 8 , recurs in an unacknowledged way in Schopenhauer: 'As the object is never absolute [unbedingt] then something per se non-objective must be posited in nature; this absolutely non-objective postulate is precisely the original productivity of nature' (Schelling I/3 p. 284). The I of Fichte's Wissenschaftslehre is seen as the highest principle of philosophy, and thus as the highest 'potential' (Potenz) of nature: without it nature would be 'blind'. However, the Wissenschaftslehre in Fichte's sense is only 'philosophy about philosophy' (I/4 p. 85). It is based upon the functioning of the I as already the highest principle, which attempts to 'intuit' itself.

Schelling comes to believe that the I in this sense presupposes a prior unconscious history in nature. In the essay 'On the True Concept of Naturphilosophie and the Correct Way to Solve its Problems' (1801), in which Schelling completes his move away from Fichte, he asserts that, in order to gain access to such a history, one needs to subtract the conscious attributes of the I. By abstracting from the conscious I one is then left with the 'purely objective' (I/4 p. 86), which gives the 'concept of the pure subject-object (= nature), only from this do I raise myself up to the subject-object of consciousness (= I)' (1/4 p. 86). The conscious I, then, emerges from unconscious, but not inert, nature. This happens because 'That pure subject-object is already determined for action by its 
nature (the contradiction it has in itself)' (I/4 p. 90). The contradiction in question is the dynamic principle of living nature, which forces things beyond themselves, so that their identity as living things is dependent upon becoming what they are not. This principle then develops to its highest point, the conscious I, which is the light with which nature reveals its inner nature to itself, as light reveals external nature. There is, though, now no absolute difference between inner and outer, so a Kantian dualism is avoided. As Wolfram Hogrebe has suggested, the problem is how this claim is to be established:

However plausible it initially seems to be that the world which has produced a knowing being (Wesen) has to be thought in such a way that the producing forces are in the last analysis also capable of such a result, it is still problematic that these forces are supposed to be of the same kind as what they have produced. (Hogrebe 1989 p. 54)

Despite many valiant attempts throughout his career, Schelling will not succeed in carrying out his metaphysical programme, as can be suggested by his remark in his late philosophy that 'our self-consciousness is not at all the consciousness of that nature which has passed through everything, it is precisely just our consciousness [. . . ] for the consciousness of man is not $=$ the consciousness of nature [. . .] Far from man and his activity making the world comprehensible, man himself is that which is most incomprehensible' (Schelling II/3 pp. 5-7). Despite the philosophical failure to carry out the programme, his attempts in his early work to do so open up vital new perspectives on a post-theological nature.

For Schelling natural products are limitations of an infinite productivity, 'intuitions' of nature by itself: each thing is what it is via its limiting of other things and via their limiting of it. The ultimate ground of the finite product is therefore a 'Dionysian' reality: 'The . . idea of something absolutely formless which cannot be represented anywhere as determinate material is nothing but the symbol of nature approaching the productivity. - The nearer it is to productivity the nearer it is to formlessness' (I/3 p. 298). The seeds of a Schopenhauerian or Nietzschean view of the foundation of nature are present here and the basic idea will be further developed by Schelling in his philosophy from 1809 onwards, which is the source of key ideas in Schopenhauer. Both Schopenhauer and Nietzsche associate the kind of view suggested by Schelling with the idea that the Will or Dionysus is the essential generative force in art. This force depends upon particular forms to be manifest, in a manner analogous to the way the productivity relates to its determinate products: like Freud's id the productivity cannot appear as itself. Unlike Schelling, though, Schopenhauer and Nietzsche tend to regard rational attempts to articulate the relationship between the ground of nature and its consequences as a form of selfdeception, because reason is seen as always subverted by a ground which escapes it, rather than being a necessary, though perhaps finally unfulfillable, task.

At this time Schelling himself tries to construct a philosophy which would 
be 'One uninterrupted row which ascends from the most simple in nature to the highest and most complex, the work of art' (I/4 p. 89), though he will later develop a much more conflictual vision of how differentiation in nature functions (see Bowie 1993 Chapter 5). His investment in a view of nature which considers the best way of understanding our place in it not to be located in natural science but rather in art has again become important in the light of contemporary concerns about science's relationship to the ecological crisis, and of growing concerns about scientism. John McDowell sees the problem with scientism as follows: 'When we ask the metaphysical question whether reality is what science can find out about, we cannot, without begging the question, restrict the materials for an answer to those that science can countenance' (McDowell 1998 p. 72). Schelling is one of the first to take up this metaphysical question in a serious way in modernity, and he does so in a manner which, despite many now obsolete features and untenable arguments, still has resonances for contemporary thought.

The crucial question posed by the Schelling of the $S T I$ is how art relates to philosophy, a question which has recently reappeared in post-structuralism and in aspects of pragmatism. In the contemporary reflections on this question the relationship between art and philosophy is often linked to a wider criticism of traditional metaphysics, which is understood as the attempt either to establish the ground of subjectivity or to use subjectivity as the ground of philosophy. This link is not overtly made at the end of the eighteenth century, but the tension between Idealist and Romantic conceptions suggests both how reflections on aesthetics can come to be linked to certain criticisms of metaphysics, and how the failure of the attempt to ground subjectivity leads to ideas echoed in contemporary thought. In the System Programme it was claimed that art and beauty manifest the connection between the sensuous and the intelligible, thereby making into a metaphysical certainty what Kant had cautiously postulated in the CF. This claim is the core of an Idealist view of aesthetics, contrasting with the Romantic view, in which, as we saw in Novalis, art only gives a negative sense of the absolute, thus putting a positive metaphysical account of the ground of subjectivity into question. Schelling's work is often uneasily located between Idealist and Romantic views, and this is particularly apparent in the $S T I$. Despite all its failings, this text does provide important arguments as to why a modern conception of reason should include an adequate account of aesthetics. In doing so it offers an alternative to Hegel's contentions, to be considered in Chapter 5, that modern philosophy has gone beyond art and that philosophy's main task now lies elsewhere. Adorno says of the contrast between Schelling and Hegel: 'When Schelling declared art to be the organ of philosophy he unintentionally admitted what the great Idealist speculation otherwise kept silent or, in the interests of its self-preservation, denied; accordingly Schelling also, as one knows, did not carry through his own thesis of identity [of subject and object] as relentlessly as Hegel' (Adorno 1973 p. 511). This contrast will frequently concern us in the coming chapters. 


\section{The development of consciousness}

The $1800 S T I$ attempts something which is characteristic of Kant's successors, but which Kant did not countenance. Kant's restriction of the idea of transcendental idealism to the a priori forms of intuition, space and time, in the Transcendental Aesthetic prevents him from assuming that transcendental idealism could form a complete philosophical system. From Fichte onwards the problem of the relationship between the passive side of intuition and the active side of concepts leads to the attempt to extend the notion of transcendental idealism to the whole of a philosophical system by giving a genetic account of both spontaneous and receptive sides of thinking (see Zöller 1998). The STI has an ambiguous relationship to Fichte's philosophy, which is occasioned by Schelling's attempt to reconcile Spinozism with Idealism: 'philosophy must set out either to make an intelligence out of nature or a nature out of intelligence' (Schelling I/3 p. 342). By the time of the essay 'On the True Concept of Naturphilosophie and the Correct Way to Solve its Problems' Schelling is emphatic that the Naturphilosophie he sees as lacking in Fichte is the prior philosophy because it describes the ascent of nature to the highest potential, the thinking I, which can then be considered in the 'transcendental philosophy' in the manner of the Wissenschaftslehre. The STI tries, somewhat unsatisfactorily and not always consistently, to keep Naturphilosophie and 'transcendental philosophy' on an equal footing. In the $S T I$ the 'transcendental philosophy' tries to explain how it is that 'life', the infinite 'productivity' of the Naturphilosophie, comes to the point of being able to think about itself at all, in 'a continuous becoming-object-to-itself of the subjective' (I/3 p. 345). This entails an account of how it is able to find a medium - art - in which it can objectify itself, given that it cannot have access to itself as an object of knowledge, all knowledge being relative to other knowledge. The 'transcendental philosophy' wishes to write what Schelling terms the history of the 'transcendental past' of the I, thus introducing the dimension absent from Kant, which helps open the path for the historicisation of philosophy in the nineteenth century. The fact that the self-conscious I comes to the point of tracing its own past means that the I must be able to become aware, through an act of freedom which is essential to it, of the necessities it depends upon for its own existence. This awareness cannot be derived from a reflexive splitting of the I into knower and known. As Hölderlin and Novalis showed, the I would then be unable to recognise itself in the stages which precede self-conscious reflection. Any such split must be grounded in a unity of some kind, and the problem is how one could have access to such a unity. Schelling tries to trace the development of the I in the way that the development of organisms in nature from lower to higher forms can be traced, by showing how the present developed form must have resulted from a previous less developed form, from the objective world of nature as 'only the original, still unconscious poetry of spirit' (I/3 p. 349), to aesthetic production. The STI begins with lower forms of spirit, which function in the self-organising - but 
not self-conscious - way that natural organisms do, and ascends to the higher forms, culminating in art, in which 'in the subjective, in consciousness itself, that at the same time conscious and unconscious activity can be shown' (I/3 p. 349).

The $S T I$ takes over the assumption, seen in Fichte, that the ' $I$ ' must be both subject and object, but questions exactly how this is the case. In his Munich lectures of (probably) 1833-34 (Schelling I/10; Schelling 1994), Schelling looks back at his own philosophical development in relation to Fichte and gives an account of the $S T I$ which sometimes makes more sense than the original text does - by this time Schelling is clearer about what he thinks is wrong in Fichte. Fichte had seen the 'imagination', in the Kantian sense of the faculty that produces schemata in the subject which are processed as objectivity by the understanding, as the source of our awareness of an 'external' world. His argument is strange but consistent. For Fichte the producer cannot see itself producing whilst in the act of production: 'Hence our firm conviction of the reality of the things outside us, and without any contribution on our part, because we do not become conscious of the capacity for their production' (Fichte 1971 I p. 234). Schelling is suspicious of the manner in which Fichte makes the act of the I into an absolute act which generates subject and object: 'With this self-positing: I am, the world begins for every individual, this act is in everyone the immediately eternal, timeless beginning both of themself and of the world' (Schelling I/10 p. 90). For the later Schelling this act has a history because the world of hard external necessity produced by the I is produced by something in the I which is not dependent upon its ' $w i l l$ ', but rather upon its (therefore unconscious) 'nature'.

The initial Fichtean move is, Schelling thinks, unquestionable as a way of getting beyond Spinozism, but is actually little more than an extension of Descartes: 'it is no doubt the case that the external world is only there for me in so far as I myself am there at the same time and am conscious of myself' (I/10 p. 93). The existence of the 'I think', however, does not warrant the assumption that everything else exists because I, as empirical, reflecting subject, exist: 'the already conscious I cannot in any way produce the world' (I/10 p. 93). The fact that my 'already conscious I' cannot produce the world does not mean that the pre-conscious 'I', the I of 'productivity' which has a history of 'unconscious' development leading to my particular conscious I, could not. For Schelling it is clear that one cannot use the reflexive I - the I that thinks about itself thinking - to get to the idea of the external world: 'Nothing, though, prevented one going back with this "I" which is now conscious within me to a moment where it was not yet conscious of itself, - nothing prevented one assuming a region beyond my now present consciousness and an activity which does not itself come but rather only comes via its result into consciousness' (I/10 p. 93). The result is what is seen by the individual I as the 'external' world, but it is not external in a Cartesian sense, because the very possibility of my thinking at all depends upon this activity, the self-contradicting, self-limiting aspect of nature: 'coming to consciousness and being limited is one and the same ... the limiting activity 
falls outside all consciousness ... Limitation must appear as independent of me because I can only look at my being-limited, never the activity by which it is posited' (I/3 p. 390). This activity is what I depend on to be and to think at all, so it cannot finally be separate from me, even though I have no cognitive access to it.

The 'imagination' itself therefore has a history: the actions which lead to its development can be traced by reflection upon the actions it now performs. This history starts from the lowest form of sensation, in which there is a difference between one thing and its other, and rises to the highest point of being able to reflect philosophically upon the relationship of thinking to what exists. Philosophy, argues Schelling, in a view usually attributed to Hegel's Phenomenology of Spirit, must be concerned with retracing the process that has led us to reflection upon this world that feels external to us, and yet of which we are a part. This makes philosophy into a history of what consciousness has become: 'this coming-to-itself that expresses itself in the I am presupposes a having-been-outside and -away from itself' (I/10 p. 94). Schelling stresses the fact that the I which is prior to my consciousness is not yet an individual, selfdetermining I; it only becomes this in each of us in the process of 'coming-toitself'. Most importantly in the present context, he attributes a central role to art as the means of proposing his argument.

Schelling links natural 'activity', which he sees as 'unconscious', with what the human will does in acting in the world: 'the activity by which the objective world is produced' should be 'originally identical with that which expresses itself in willing, and vice versa' ( $\mathrm{I} / 3$ p. 348). The difference is that the will involves conscious reflection upon what it produces - think of Marx's comment about the difference between a bee and even the most unskilled builder who builds a house. Schelling therefore seeks a medium in which the 'unconscious activity' of nature and the conscious activity of our thinking can be shown to have the same source. This medium cannot simply be located in conscious thinking about thinking, because the activity in question has to be connected to the unconscious activity of the rest of nature. The $S T I$ insists that the absolute must include both the development of nature and the process of reflection, hence the concern to unite Naturphilosophie and transcendental philosophy. Schelling's aim is to overcome the split between theoretical philosophy, in which nature is the apparently external world, and practical philosophy, in which the actions of the I in Fichte's sense are central.

Philosophy needs some form of 'intellectual intuition' to get beyond the difficulties in Kant's transcendental deduction, but this intuition cannot just consist in thought's immediate grasping of itself in the act of thinking. Philosophy has to include the activity of nature which leads to our being able to think at all. Schelling tries to show that consciousness itself involves both conscious, reflexive, and unconscious activity. This fact could not be revealed, as it is for Fichte, solely by reflection which relies on a 'direct inner intuition' (I/3 p. 350) of the activity of our thinking, because that can only be an intuition of 
what is conscious. In Schelling the subject is 'decentred', and needs to 'remember' what has gone on in nature that is continued in its reflection, but on a different level. The following passage from the Munich lectures makes the idea clearer: "having arrived, then, at the "I am", with which its individual life begins, [the I] no longer remembers the path which it has covered so far, for as it is only the end of this path which is consciousness, it (the now individual I) has covered the path unconsciously and without knowing it' (I/10 p. 94). Dieter Jähnig explains: 'As what is to be remembered is something that, before its being remembered, was never "in consciousness", then it obviously cannot be an act of memory that is only concerned with consciousness' (Jähnig 1966 p. 233).

Despite many confusing shifts throughout his philosophical career, Schelling always sees a tension in consciousness, which is not fully present to itself - hence his concentration in the $S T I$ on art as a non-conceptual means of understanding it. Consider the following from the Munich lectures, which takes up matters relating to reflection theory in a manner reminiscent of Hölderlin and Novalis: 'But the subject can never grasp itself $a s$ what it Is, for precisely in attracting itself [im sich Anziehen, which has the sense of 'putting on' what one is] it becomes an other, this is the basic contradiction, we can say the misfortune in all being' (Schelling I/10 p. 101). As soon as one has related oneself to the Other there must be a tension between what one was before this relation and the fact that one has now irrevocably become something different, in the manner of the move in Lacan from the imaginary to the symbolic. The interrogations of the concept of the subject in Western philosophy characteristic of some recent philosophy, particularly in certain areas of post-structuralism, evidently ignore the complexity of the concept of the subject in Schelling and the thinkers considered in Chapter 3. ${ }^{3}$

In the $S T I$ the lack of transparency of the subject to itself is linked to the notion of unconscious productivity, which can never be directly accessible in philosophical thinking. The productivity of nature is, as we saw, 'originally identical' with that of our will, so the products of nature 'will have to appear as products of an activity that is both conscious and unconscious' (I/3 p. 249). Nature appears both as produced in a manner analogous to the conscious, willed production of an object - in organisms which 'give the law to themselves' as we do in practical reason - and as a blind mechanism - when its deterministic laws are the object of inquiry. Nature is 'purposeful mithout being explicable in terms of purposes' (I/3 p. 249). The echo of Kant's third Critique, where beauty was 'purposiveness without a purpose', suggests how Schelling is attempting to link natural teleology and aesthetics in a more substantial manner than Kant had done. For Schelling we can understand nature as both organism and blind mechanism via an activity which is both conscious and unconscious: aesthetic activity. Philosophy's reliance on 'intellectual intuition', in the sense of thinking about thinking, is itself an active process, but it is directed inwards, not towards an object, 'whereas the production in art is directed outwards, in order to reflect the unconscious through products' (I/3 p. 351). Art therefore becomes 
the 'document' and 'organ' of philosophy, turning what otherwise must remain inaccessible to us into 'intuitable' objects.

The $S T I$ is a difficult work, not least because it tries to tell a story for which there can be no direct evidence. It extends the understanding of consciousness in ways which will never be reducible to an explanatory theory, thereby already pointing to a major strand of modern thought. The story is the story of consciousness, including what happens before self-consciousness. Odo Marquard has pointed out the analogies of this project to what Freud was to attempt one hundred years later in the Interpretation of Dreams (in eds Frank and Kurz 1975). Schelling and Freud share a model which entails both conscious and unconscious aspects. Both use the notion of 'drive' (what Schelling also calls 'activity') which is limited, 'repressed' (Schelling uses the term 'verdrängt'). Repression makes possible the development of the conscious mind, which is thereby divided within itself, cannot directly satisfy itself, and cannot have direct access to its own history. It 'only finds in its consciousness as it were the monuments, the memorials of that path, not the path itself' (I/10 pp. 94-5). The task of 'science' is therefore an 'anamnesis' of what precedes our conscious reflections. In Freud this takes place in order to reveal the repressed aspects of the self which lead to the neuroses of the present. In Schelling what is required is a way of coming to terms with the apparent division in the I between nature and reflection, receptivity and spontaneity, which he thinks can be transcended in the work of art. Without the process Schelling describes we would not be able to arrive at the point of reflection from which the story of the 'progressive history of self-consciousness' (I/3 p. 331) is told. The difference between Schelling and Hegel will be that Schelling, even at this early stage of his work, does not think philosophy, 'science', can grasp the absolute in a fully articulated manner. For Hegel this will entail a failure to carry out the 'exertion of the concept', the totalising philosophical reflection necessary to reveal the conceptual truth about art.

Schelling does not sustain the emphatic view of art of the $S T I$ in his later writings. However, as Hartmut Scheible puts it, Schelling's 'determined emphasis on intuition over the concept . . is historically necessary in order to counteract a process which Plessner has described as the "gradual separation of intuition and conceptualisation" in our dealing with nature' (Scheible $1984 \mathrm{p}$. 264). Schelling therefore plays an important role in establishing some of the bases for subsequent hermeneutic questioning of the scientistic claim that natural science represents the only kind of truth possible about nature. The separation of intuition from the conceptualisation of nature in modern natural science can also be seen in terms of the growing dominance of 'instrumental reason', which concentrates merely upon ways of manipulating nature for human purposes, without considering how this fits into a more holistic conception of our relationship to nature. In its extreme form the suspicion of the effects of natural science can go as far as the claim that there may be, as Wittgenstein will later maintain, 'nothing good or desirable about scientific knowledge' and 
that, if this is the case, 'humankind which strives after it is running into a trap' (Wittgenstein 1980 p. 56). Schelling does not ever countenance this degree of suspicion of scientific knowledge, but he does help open up alternative perspectives on understanding just what that knowledge is, of the kind echoed in the work of Nietzsche and Heidegger. Seen in this light, Schelling's philosophy can be linked to Adorno's attempt to trace the pathologies of modernity via reflection upon the subject's relationship to internal and external nature, in which reflection upon art also plays a major role. Schelling's history of the development of consciousness, then, contains the seeds of a questioning of aspects of modern science that will be vital to subsequent aesthetic theory. Let us now look in more detail at the $S T I$.

\section{Intuition and concept}

The main terminological difficulty in understanding the $S T I$ lies in its frequent use of words referring to self-consciousness, or the I, which have divergent meanings. The essential distinction, which we observed in Schelling's account of Fichte in the Munich lectures, is between the self-consciousness of ourselves as individuals, which is relative to the object world and tries to grasp itself by reflection, and the absolute ' $\mathrm{I}$ ' which cannot be grasped with the means of reflection (I shall generally employ 'I' to designate this usage). Schelling's use of the term 'I' for the latter is a residue of his attachment to Fichte. As it does in Fichte, the confusion this generates results from Schelling's using ' $I$ ', which suggests the need for a 'not-I' for its determinacy, and thus relies upon a structure entailing a relationship between two terms, to designate the absolute. This leads him to talk also of the 'absolute identity' or 'absolute indifference' of subject and object, and later, when he realises that even this implies a relationship of mutual dependency and determination, of 'being' as the trans-reflexive ground of the subject-object relationship, in something like the sense we observed in Hölderlin's 'Judgement and Being'.

The STI goes through the stages required to explain how an 'intelligent', in the sense of differentiated and organised, nature leads to differentiated and organised thinking and free action. The 'I' is termed an 'infinite activity', and the question is how it can be described as such. The problem with any totality is that it cannot describe itself $a s$ a totality, because the description would have to be external to the totality, thus rendering the totality incomplete. Because any limitation upon it from outside itself would prevent it being absolute the activity must be limited by itself: 'If you think of an infinitely producing activity spreading out without resistance then it will produce with infinite speed, its product is a being [i.e. it will all happen at once and be wholly unarticulated], not a becoming. The condition of all becoming is therefore limitation or the barrier [Schranke]' (Schelling I/3 p. 383). This limitation enables the totality to become manifest to itself in finite products. It sets a process in motion which, as Manfred Frank puts it, 'fulfils the demand for the infinity of the I (every 
barrier which is put up is crossed into infinity) and the demand for finitude (the process will never represent itself in what is not a finite product)' (Frank 1985 p. 90). This corresponds to the basic idea of the Naturphilosophie, where the productivity is essential, not the finite products, even though the two are inseparable.

Nature produces 'unconsciously'; our thinking about it, including about ourselves as part of nature, is conscious. The $S T I$, as we saw, wishes to assert that art is the medium in which the unity of these two sides can be understood, giving us a sense of our place in the whole of the kind Kant hints at but leaves in the realm of the 'as if' in the CF. Philosophy can only postulate the unity in intellectual intuition. Schelling's argument is much the same as in Fichte: 'intellectual intuition presupposes a capacity simultaneously to produce and intuit certain actions of mind, so that the object and the intuition itself are absolutely one .. . But this intellectual intuition is itself an absolutely free act, this intuition cannot therefore be demonstrated it can only be demanded' (Schelling I/3 pp. 369-70). The apparently external 'object world' is seen as part of the process of the I's attempt to 'intuit itself' (sich anschauen). In our reflection it appears to us as the Other, but in fact derives from the Same of which we are a part. We cannot 'know' this in the way that we can conceptualise objects of nature in natural science, hence the need for the postulate of intellectual intuition, for a non-conceptual means of understanding our relation to the Other that does not just understand it as a world of conceptualisable objects ontologically separate from us. The further need, though, is for a means which would enable us as free human beings to understand this postulate in a non-cognitive manner. This is why the analysis of art forms the conclusion of the STI.

In order to get to the point where art is able to offer this kind of understanding the $S T I$ goes through various stages of 'intuition', which are the I's attempts to 'see' itself without knowing at the outset that this is what it is doing: 'the I cannot simultaneously intuit and intuit itself as intuiting, thus also not as limiting' (I/3 p. 403). Only at the end of the process is the telos of the process apparent. The argument is basically Fichte's, but the attitude towards nature and the conception of the history of the 'I' are particular to Schelling. The first stage is 'sensation', an initial division within the ' $\mathrm{I}$ ', whereby difference arises at all within what was previously an undifferentiated One. ${ }^{4}$ After this comes 'productive intuition', in which the infinite activity produces finite products by further splitting itself, thus giving rise to the differentiated material world of nature. Following this comes the stage of 'organisms', living natural beings such as plants, which are 'symbols of the intelligence' (I/3 p. 490) because they are selfdetermining wholes whose life is their own self-production. The next stage, the act of 'absolute abstraction' (I/3 p. 524), takes one from the world of nature into the realm of consciousness and self-consciousness, and thus initiates a new sequence of development. This stage cannot take place in terms of the kind of necessity encountered in nature, the realm of 'theoretical philosophy', so that 'with regard to [the theoretical philosophy] only the absolute demand remains: 
such an action in the intelligence [i.e. absolute abstraction] should take place' (I/3 p. 524). The moment of theoretical opacity present here in the emergence of the spontaneity of the will plays a vital role in subsequent philosophy. From Schelling's own later work on human freedom, which still tries to come to terms with the contingency and lack of ground of human freedom, to Schopenhauer, Feuerbach, Marx, Nietzsche and others who espouse what Herbert Schnädelbach has termed 'speculative naturalism', the German Idealist insistence on self-determining freedom is often subordinated to the idea that freedom is grounded in something else, thereby, of course, threatening philosophy with a return to dogmatism. Habermas suggests the resultant 'danger of hypostasising the prius of Nature, Society and History into an in-itself and thus of falling back into a covert pre-critical thinking' (Habermas 1988 p. 47), indicating one reason why concern with the nature of human freedom has remained a live metaphysical issue ever since.

Schelling's basic problem in the $S T I$ is a version of what concerned Kant: how do concepts relate to what they are concepts of, without the answer generating another implausible dualism or an inarticulable monism? For Schelling, if one asks in a Cartesian manner how concepts located in an isolated consciousness can correspond to objects one has already created an insoluble problem: 'a philosophy which starts with consciousness [in the sense of the separation of subject and object] will therefore never be able to explain that correspondence, neither is it to be explained without original identity, whose principle necessarily lies beyond consciousness' (Schelling I/3 p. 506). Although Schelling employs the problematic notion of correspondence (see the discussion of correspondence theories in Chapter 3), what he, albeit only partially, begins to see here has played a major role in recent moves in analytical philosophy, where the attempt to join a mind conceived of in Cartesian terms and a world conceived of in materialist terms via the intermediary of 'sense-data' is increasingly rejected (see e.g. Putnam 1999).

The problem with sense-data theories is part of what gave rise to Kant's notion of schematism: given that, in sense-data terms, the patterns of data we encounter at different times can never be exactly the same, it becomes impossible to explain how we can see numerically different cases of the same type of object as the same. The notion of schematisation was intended to overcome this problem by providing an intermediary between sensuous particularity and abstract concept, what Schleiermacher will term a 'shiftable' image that can, for example, accommodate both the bonsai and the giant redwood as trees. As we saw, Kant regarded schematism as a 'hidden art in the depths of the human soul' - prompting Novalis's question 'Can I look for a schema for myself, if I am that which schematises?' For Schelling 'The schema ... is not an idea (Vorstellung) that is determined on all sides, but an intuition of the rule according to which a particular object can be produced' (I/3 p. 508). As 'intuition' - in the sense of immediate givenness - rather than as knowledge of a rule, the schema cannot itself be cognitively determined: for that, as Kant made clear, intuitions require 
concepts, rules for identifying objects. Schelling also suggests that language's capacity for using a finite number of words to determine an infinite number of possible things depends precisely upon this 'intuition of a rule' - otherwise it becomes impossible to know how one would learn to use a word without getting into a regress of rules for using a word. The activity involved in schematism cannot, then, be determined in the way that an object of cognition is determined: it is itself required for the determination - hence Novalis's question. We therefore cannot give a conceptual account of it on the basis of rules which would identify what it is. The impetus towards other forms of access, of the kind offered in aesthetic production, is apparent here. A further area of theoretical opacity gives rise to the next part of the argument.

Schelling links the self-determination of a natural organism to our capacity for self-determination. However, there is a major difference: 'Every plant is completely what it should be, what is free in it is necessary and what is necessary is free. Man is eternally a fragment' (I/3 p. 608). We are determined by inner division: consciousness cannot ground what motivates consciousness, because the motivation derives from the absolute ' $I$ '. In our conscious reflection this 'I' 'is to become aware of itself as producing unconsciously. This is impossible and only for this reason does the world appear to it as ... present without its action' (I/3 p. 537), as the 'thing in itself'. We are, though, able to change aspects of the object world and ourselves by actions based on our will, and Schelling links the 'absolute will' of the 'I' with our freedom. There can, however, be no intersubjectively available proof of our possession of selfdetermination: 'What that self-determination is, nobody can explain who does not know it via their own intuition' (I/3 p. 533). The argument is derived from Fichte's attempt to achieve what Kant had not, but now has a different basis and different implications. The implications are central to Schelling's thought and to understanding its difference from that of Hegel, and they point to a fundamental divide in modern philosophy.

Unlike Hegel, Schelling leaves the problem of how to understand freedom open. A dialectic of recognition, of the kind Hegel employs in the Phenomenology, that enables me to apprehend my own freedom by acknowledging the other as free subject, cannot, despite Hegel's claims, tell me what my freedom is. If another person demands that I should do something, they give me the choice of fulfilling the demand or not. This way the 'action is explained if it happens [as a product of my free-will], without its having to take place' (I/3 $\mathrm{p}$. 542). Whilst our consciousness of having a will does require the other as its object, in order for it to have some means of self-manifestation, this does not explain how we are aware of our will as self-determining. The problem is a version of the problem of reflection that Fichte discovered. I must already be prereflexively aware of what it is to exercise my freedom, otherwise there would be no way of understanding another person's demand that I exercise my freedom in relation to their appeal to my capacity for decision. How would I grasp what they are asking, given that freedom is not something that can appear in the world 
that I can learn to understand by observing the behaviour of other beings? Freedom therefore seems to involve an inherent resistance to intersubjectively agreed articulation of the kind required for theoretical knowledge, but this, for Schelling, is precisely its price. Hegel sees the truth of freedom as established by its reflection in the other, which constitutes what it is intersubjectively; even at this early stage in his career Schelling wonders whether the ultimate ground of what we are is really accessible to philosophy in this manner. The implication is that the being of the subject is not something that philosophy can exhaustively describe. The differences over aesthetics between Schelling and Hegel derive from this fundamental contrast.

\section{The 'organ of philosophy'}

How, then, does Schelling make art the culmination of the system? The STI aims at a view of nature in which our free actions can be in accordance with what happens in both external and internal nature. As we saw, Schelling used the model of the plant in order to suggest a unity of subject and object, freedom and necessity. Human knowledge, on the other hand, can never be completely unified: its very essence lies in its difference from, and simultaneous dependence on its object, which it tries to transcend by grasping the object in the concept. Concepts cannot, though, grasp the totality of an object: the development of science keeps on showing that there is another way of describing any object of inquiry. Furthermore, in the case of organic life, scientific analysis will tend to destroy what is analysed (think of the limits on what is ethically acceptable in certain forms of biological research). The basis of Idealist philosophy is inherent in these aspects of the metaphor of the plant: one can differentiate the reality of the organism into an indeterminate number of different moments, but those moments are also identical as parts of the organism. The identical whole cannot be without the different parts, and the parts cannot be what they are without the whole. The single moment is therefore dependent upon all the others for its ultimate identity. As we saw in the Naturphilosophie, 'productivity' takes precedence over the particular product. In the Fichte-influenced terms of the STI the question is therefore how the 'last foundation of the harmony of subjective and objective can become objective to the I itself' (I/3 p. 610). Schelling seeks a way of recognising, beyond the self-determination to be observed in natural organisms, how our individual productivity and our will can be revealed as being part of the same totality as these organisms, without our surrendering the capacity for self-determination.

The work of art in the $S T I$ is the 'organ' via which the unity of the theoretical and the practical can be shomn. Philosophy alone is not able to demonstrate this unity because 'the striving of intelligence to become conscious of its action as such continually fails', which is why 'the world becomes really objective for it' (I/3 pp. 536-7) and requires the endless task of scientific explanation. Art also cannot provide an articulated 'proof' of such unity, but the point of the 
argument is precisely to suggest the importance of what art can show that cannot be said by philosophy. The reasons for the significance of music in Romantic thought already begin to become apparent here, though Schelling does not make anything of this in the STI. Nature as blind purposiveness, the plant growing into its particular form, constitutes, in the terms of the $S T I$, one form of the unity of conscious and unconscious activity. The 'productivity' involved in the growth of the plant does not lead to a random product but to something articulated and organised. At the same time there is nothing in the plant or our cognitive accounts of what the plant is that would explain why the plant takes that particular form. ${ }^{5}$ If we are to get beyond this limitation on what we can know: 'One must therefore be able to show an intuition in the intelligence via which in one and the same appearance the $I$ is at the same time conscious and unconscious for itself' (I/3 p. 610). In the actions of my will I have awareness for myself of free activity, but this awareness cannot be demonstrated as an event in the appearing objective world. The medium in which philosophy is able to gain access to what otherwise would not be available to it has to include both the cognitive level of our consciousness of objects in the appearing world and the basis of that consciousness in what can never appear as itself.

Natural science for Schelling is an infinite task because each determination of what something is gives rise to new determinations, without there being any way of knowing that the task of determination could finally be completed. Art, in contrast, already shows how the two productivities coincide: the conscious intention of the artist to produce an object in the world coincides with the unconscious compulsion of the artist's genius. Art therefore need have no further purpose, because the finite human product embodies a purpose which cannot be cognitively grasped, but only intuited. This product involves something like the purposiveness of the organism. In contrast to the organism, though, the product involves the further aspect that its real purpose is manifest to those who understand the object as a work of art, thus as a combination of the subjective and the objective which overcomes the split between them.

Although Schelling clearly attributes a hyperbolic role to art in the $S T I$, his reasons for doing so remain significant for philosophy and aesthetic theory at the beginning of the twenty-first century. The question for Schelling is how one is to make any sense of the unity of subject and object from our perspective as finite, striving, divided individuals. Theoretical philosophy cannot articulate a way of overcoming our sense of division, because division, as we have seen, is inherent in the necessary incompleteness of cognition. Previous philosophy had, of course, answered such questions in terms of dogmatic theology. In certain respects Schelling's answer does involve a covert piece of theology: the work of art combines the apparent contingency of the particular object with the sense of the purposiveness of that object, and can therefore be construed as a metaphor for the world as a whole. What happens to exist may appear contingent in cognitive terms, because overcoming its contingency is an endless task, but it can be taken beyond contingency when apprehended teleologically. 
Schelling actually wishes to avoid a theological position, which is why art is burdened with such a vital role in our self-understanding and why the STI, even though we may now only be able to employ its resources metaphorically, is a vital document of modern aesthetic theory. Schelling's aim at this stage of his career is akin to what we observed in the 'System Programme', namely to make ideas sensuous. In order that the higher purposes of existence be comprehensible within society as a whole they must be manifest in a concrete, perceptible form. Art is, then, 'the generally acknowledged and undeniable objectivity of intellectual intuition. For aesthetic intuition is intellectual intuition which has become objective' (I/3 p. 626). Consequently, art 'always and continually documents anew what philosophy cannot represent externally, namely the unconscious in action and production and its original identity with the conscious' (I/3 pp. $627-8$ ). What is separate in nature (unconscious) and history (conscious) is united in art.

The process of nature in the $S T I$ begins unconsciously, but develops towards our conscious reflection. Art, on the other hand, begins with the artist's conscious reflections about what is to be produced, but it ends unconsciously because what is produced is not identical with the techniques and rules which were required to produce it: if it were, it would, as Kant suggested, be mere mechanically reproduced craft. The artwork can therefore represent the absolute, as the 'sole true and eternal organ and at the same time document of philosophy' (I/3 p. 627). This final move is clearly the one which is most troubling: the account of artistic creation as involving both conceptual thought and elements which are not reducible to the way they can be conceptualised is not at all implausible, but the elevation of this conception into the highest philosophical insight is harder to swallow. This can be interpreted in two ways. On the one hand, it can be seen as an Idealist claim to have directly overcome the lack brought about by the necessarily incomplete nature of scientific knowledge, but then the problem arises as to which works can be said to achieve this and why those works, and not others, achieve it. On the other hand, it can be interpreted as a Romantic reminder, of the kind suggested by Novalis, that we can only ever gain a sense of the absolute by our failure to grasp it, a fact manifest in our inability to give definitive accounts of the meanings of works of art, even as they may seem to embody a completion lacking elsewhere in life. Music offers a model for this latter interpretation: the meaningfulness of music relies precisely on the way in which it demands a level of conceptual thought to be explicitly understood, but it can at the same time always provoke new ways of being grasped. In this view the problem for the Idealist position in establishing which works of art can be said to fulfil the aim of philosophy is circumvented by the idea that it is precisely the works which continue to give new insight - and, of course, to give a necessary sense that each insight is only ever partial - which are philosophically significant.

The Idealist aspect of the STI's investment in the aesthetic becomes apparent when the development of history is seen in the same terms as the work of 
art. The results of conscious actions are rarely exactly what is intended, yet Schelling conceives of the overall process of history teleologically. History is, he claims, not merely haphazard, because its ground is 'productivity', the attempt of the 'I' to grasp itself which is manifest in the ever greater insight generated by the development of human thought and action. At the same time, it is clear from our experience of history that conscious reflection cannot reliably tell us where the history is leading. In a characteristic move, Schelling links the notion of genius to the notion of destiny: 'the power, which, via our free activity without our knowledge and even against our will, realises purposes which have not been imagined is called destiny' (I/3 p. 616); in turn, the inexplicable side of apparently free, self-determining aesthetic production, which adds an 'objective' (in the sense of unconscious and unintentional) aspect to the product, is 'genius'. The claim is evidently an attempt to skirt the consequences of removing divine Providence from history. If one can find a domain of human existence where the apparently inexplicable effects of our activity make sense, then the possibility of history being more than contingency remains open. The significance of an attenuated version of this sort of idea for Feuerbach and the early Marx should not be underestimated. If history is to be the fulfilment of our 'species being', then the goal will have to be achieved with powers given to us by our nature. Marx insists that we cannot as yet fully grasp these powers because their potential has been blocked by existing circumstances. In this respect the notion of genius can be seen as a mystified version of the attempts of post-Providential philosophies of history to make history intelligible. The most important of these attempts will, of course, be that of Hegel.

Problematic as the connection of destiny and genius is, the notion of genius itself does raise a series of significant issues. As we have seen, genius relates to the level of aesthetic production which can never be reduced to the results of technique. Ideas of this kind can be and evidently are ideologically abused. The argument should, though, first be understood historically: such views of art only became possible in the philosophical climate with which we are concerned here. This climate is influenced by two factors in modernity which are highlighted by aesthetic theory. Liberation from theological constraints releases many hitherto unknown or inaccessible capacities in the subject, and these often seem to give rise to a sense of endless potential. At the same time, this sense of the subject's potential is combined with the awareness of the subject's inability to be wholly transparent to itself. Schelling's idea of the genius combines these two factors. He insists that art manifests the unity of conscious and unconscious activity as part of the attempt to give access to aspects of self-consciousness which Kant could only remove to a realm to which philosophy has no access. Schelling is convinced that these aspects are accessible, albeit not with solely theoretical means, and faces the consequences of showing how this is the case.

In Aesthetic Theory Adorno suggests that the emergence of the term genius in philosophy at this time relates to what we have seen as the tension between the 'new mythology' and aesthetic autonomy, the tension between art being a 
new synthesis, which includes philosophy, of the different means of articulation of a community, and art saying what other means of articulation cannot say. Adorno maintains that the concept of genius becomes important at the moment when 'the character of the authentic and the obligatory, and the freedom of the emancipated individual move apart from each other. The concept of genius is the attempt to bring the two together by a piece of magic' (Adorno 1973 p. 254). Genius is, then, a false reconciliation of the universal and the individual. The 'authentic and the obligatory' corresponds at this moment in history to the new mythology: it implies that the products of the genius manifest a collectively binding sense of value in the work of art, of the kind seen in a theological culture. The 'emancipated individual' is, for Adorno, following Marx, in fact an illusion occasioned by the failure to see how emancipation in modern capitalism relies upon the destruction of freedom elsewhere in society. For Adorno the attempt to reconcile individual and universal in modernity almost invariably ends in the suppression of the individual, because the reconciliation always happens in terms of the universal. This is one of the reasons why he favours Schelling's ambivalences against Hegel's 'thesis of identity', which he thinks is actually a manifestation of the growing dominance of the commodity structure that destroys individuality in modern societies. Art for Adorno retreats into autonomy in order both to resist a false reconciliation of individual and univer$\mathrm{sal}$, and to prevent what has been repressed in this enforced reconciliation being forgotten. The 'genius is supposed to be the individual whose spontaneity coincides with the deed of the absolute subject' (p. 255), thereby, as it does in one interpretation of the $S T I$, playing the role of fulfilling the Idealist project in a proto-theological direction. However, this means that: 'In the concept of genius the idea of the creator is ceded with Idealistic hubris from the transcendental subject to the empirical subject, to the productive artist' (p. 255).

The 'untruth' of the notion of genius also lies for Adorno in the way that it obscures the fact that works of art are not real living organisms. Art is Schein, 'appearance', and neither a creation of the kind attributable to God, nor a reconciliation of self and nature of the kind which he believes would require a wholesale transformation of social relations and of the relations of society to nature that the present state of society renders almost unthinkable. Furthermore, the technical side of aesthetic production depends upon the preceding social labour of others, who established the forms within which the supposedly authentic, individual and autonomous artist works. However, Adorno actually retains vestiges of a conception of the reconciliation of nature and consciousness, of spontaneity and reflection from an Idealist position. The ambiguity in his position comes out in the following passage, which questions the use of the term genius by insisting upon the prior material and technical basis of artistic production: 'The whole Appassionata lies in the keyboard of every piano, the composer only has to get it out, and for that one admittedly needs Beethoven' (p. 403). Adorno's failure to give an even barely adequate account of what he means by the 'Beethoven' required to get the Appassionata out of the 
piano suggests why the notion of genius cannot be dismissed too lightly. It may not deserve the dignity Schelling affords it, but it does point, as Adorno also seems aware, to dimensions of subjectivity whose articulation is not reducible to the already available technical means in a particular society. Bach may adopt the Chaconne form from tradition, but what he does with it opens new musical horizons which are also horizons of the modern subject's possibilities for selfarticulation.

A more serious problem in the $S T I$ is that it characterises the work of art produced by the genius in terms of organic unity and self-containment, as an image of the realisation of Idealist philosophy's aim of overcoming contingency. From our contemporary perspective it seems clear that the Idealist version of aesthetics is less convincing than some of the ideas of Novalis and Schlegel. The Romantic conception of the unrepresentability of the absolute led to the idea that the work of art always points to its own incompleteness, while at the same time adverting to what is beyond it. This conception prefigures the characteristic sense in modernist art of a continual striving for something which is never really achieved, but which is the apparently inexhaustible motor of new aesthetic production. There are evident problems with the description in the STI of an art in which 'Every drive to produce stops with the completion of the product, all contradictions are negated, all puzzles solved' (I/3 p. 615). The description sounds exactly like a description of ideology, the reconciliation of real contradictions in an illusory form, or like the 'imaginary', in the sense seen in Hölderlin, where the I regressively ignores the necessity really to engage with the otherness of the object.

However, the issue is more complex than this. Hölderlin himself saw a way out of the imaginary in art, and this is clearly a vital dimension in serious consideration of art in modernity, including art which still, like that of Beethoven, works in terms of the resolution of contradiction. In considering the organic view of the work of art it is also important to remember that Schelling does not attribute a functional role to art. Like Kant, who insisted art be without 'interest', Schelling regards the demand that art be useful as 'only possible in an age which locates the highest efforts of the human spirit in economic discoveries' (I/3 p. 622). As Kant did in the CF, Schelling also shows a prophetic awareness of the dangers of modern rationalisation. Rationalisation will become, as we have seen, a central concern of Marx- and Weber-influenced theorists, like Adorno, who regard Marx's critique of commodity exchange, of the valuation of everything in terms of its equivalence to something else, as a vital issue in aesthetic theory. Schelling is already concerned to preserve a sphere of meaning that cannot be subsumed into the demands of scientific or economic rationality. In the $S T I$ the work of art's self-contained organic status means that its purpose lies within it and need not be sought elsewhere. This autotelic status of the aesthetic object will repeatedly, and in many contexts justifiably, be invoked from this period onwards as a means of countering the tendency of the modern world towards instrumental rationality and the devaluation of activities which are self- 
legitimating and not reducible to the instrumental purposes to which they can be put. Significantly, in this period, it is once again the music of Beethoven, particularly the late Beethoven, which seems most able to exemplify a selflegitimation which resists wholesale functionalisation.

The misuse of organic conceptions of art to legitimate reactionary political views has been a constant factor in political life since this period, and music is not exempt from such misuse. The reactionary notion of organicism subordinates urgent political and social conflicts which are directly or indirectly apparent in aesthetic works to the demand that we should contemplate the unity the human spirit is capable of achieving in artistic production. Critical and analytical approaches to art come in this view to be seen as infringing upon the integrity of the artistic totality, which takes on the status of a sacred object. In the twentieth century Brecht and others, with some justification, questioned the organic view of the work of art. However, there is, once again, another dimension to the argument, that can be illustrated by considering a passage from a letter by Hölderlin to his brother (1799), not long before the appearance of the $S T I$. In this he points to a view of aesthetics that is often underestimated in reflections on aesthetics and politics, particularly in the light of views of art which see it merely as ideology. The tension between the desire for a 'new mythology' and the emergence of aesthetic autonomy appears here once more.

Hölderlin's letter is a critique of German society, pointing to its narrowmindedness and lack of awareness of the need for a creative community if individuals are to flourish. Hölderlin sees some hope in the liberation suggested in Kantian philosophy, and in the growth of interest at the time in the political concerns of the community. This is, however, not enough. He goes on to attack the way the importance of art in public life is underestimated, which he sees as evident in the view of art as play or game (Spiel):

one took [art] as a game because it appears in the modest form of the game, and so also, reasonably enough, no other effect could result from it than that of the game, i.e. diversion [Zerstreuung, which also has the sense of dispersal], almost the exact opposite of its effect when it is present in its true nature. For then people compose themselves in it and it gives them peace, not empty but living peace, where all powers are alert and are only not recognised as active because of their inner harmony. It draws people closer and brings them together, not in the manner of the game, where they are only unified by the fact that they forget themselves and nobody's living particularity is able to appear. (Hölderlin 1963 p. 755)

Whereas philosophico-political education (it seems clear he is referring to Kant and Schiller) may unify people in the recognition of duty and the law, far more is needed if a real community is to be established. A sense of what he means is manifested in the organic unity of the work of art. One should, as this passage makes clear, think of this in terms of something like Beethoven's music, whose unity is achieved by facing the challenge of integrating the greatest diversity and contradiction, not by ideologically conjuring away contradictions. ${ }^{6}$ Hölderlin concludes his letter with a passionate political exclamation, which clearly relates 
to the nature of his own poetry, and is echoed by Beethoven, of the need to 'bring everything human in us and others into ever freer and profounder connection [Zusammenhang], be it in aesthetic [bildlich] representation or in the real world, and if the realm of darkness should break in with violence, then we will throw the pen under the table and go in God's name to where the need is greatest, and where we are most needed' (p. 757).

It seems to me mistaken to think that we have outgrown such a vision of aesthetics and politics because we are aware of the dangers of misusing the power of art to allow us to bring things 'into ever freer and profounder connection'. The political potential of the semantic resources which Hölderlin sees in art's manifestation of organic cohesion are those which Ernst Bloch, himself profoundly influenced by Schelling, will see as lacking in the Left's political armoury in the fight against fascism. It is, therefore, not clear that the organic implications of the aesthetic theory present in the $S T I$ are per se reactionary. Bloch's argument is that if the Nazis, however temporarily and deceptively, fulfilled real needs, there is no point in trying to ignore these needs. Modernity, as we saw in Chapter 2, results in a need to unify the results of the random proliferation of new knowledge with the contexts of meaning of everyday life and the unfulfilled hopes and desires of that life. Is this, then, merely a retreat into the imaginary? The stringency of Adorno's questioning of notions like genius and organic integration is based upon his claim, in the light of his experience of historical catastrophe, that such a unification is always a deception because it promises in art what is denied to people in reality. This leads him to the insistence upon aesthetic autonomy, in order to preserve a sphere of meaning which cannot be subsumed into the reified ways of making sense within modern societies. Within the sphere of autonomous art it is, though, noticeable that even he employs attenuated notions of organic coherence: without some sense of reconciliation of the antagonisms in a work the very possibility of meaning disappears for Adorno. It is, then, important to remember that in the Germany of the time of the STI organic notions had more the character of utopian hope than of selfdeception. Bloch's arguments in the 1930s are essentially concerned with how mythical thinking functions within modern societies. It is therefore vital to consider the STI's account of mythology in relation to the broader development of modernity.

\section{Mythology, art and modernity}

The final part of the STI examines the idea of the 'new mythology'. Philosophy, argues Schelling, is born originally of 'poetry' (Poesie), in the sense of creativity, poiesis. The 'completion' of philosophy would be its return to the 'general ocean of poetry'. Art brings our conscious reflection into harmony with what is given to us unconsciously by nature, by making ideas sensuous. It thereby performs an analogous role to that played by mythology in pre-modern cultures, which explained the otherwise inexplicable origin of a people in terms of a con- 
crete story. The return of 'science' - in the broadest sense of 'philosophy', which would include natural science - to 'poetry' would have to take place in terms of the kind of integrated world picture present in mythology. Such a mythology would reconcile the conflicting cognitive, ethical and aesthetic demands characteristic of modernity. As we saw in Chapter 2 and in the remarks at the end of the last section, any vision of this kind raises controversial questions. On the one hand, the achievements of modernity depend precisely upon analysis and specialisation, on the other hand, without some guiding sense of the aim of the proliferation of specialised practices and the technology-generated social changes which affect their lives, members of modern societies will tend to seek guidance in irrational forms.

Hegel sees it as the task of philosophy to achieve this integration of the spheres of modernity. The limitations of thinking in which 'intuition' still plays a central role, such as mythology and art, mean that mythology and art are incapable of offering an adequate response to the complexities of modern forms of knowledge and action. However, Hegel's vision for philosophy will depend upon his ability to integrate 'intuition' into his system, a problem most obviously manifest in his need to suggest that philosophy can articulate the essential truth about art because, unlike art, it is not tied to the particular. More recently, Habermas's accounts of modernity see the separation of the cognitive, moral and aesthetic spheres as precisely what enables the scientific, legal and artistic advances characteristic of modernity. Conflating validity claims from the different spheres leads in his view to the kind of irrationality characteristic of feudalism, which the Enlightenment justifiably sought to overcome. The spheres are also conflated in fascism, where criteria of public accountability become randomly decisionistic and aesthetic means are used in political manipulation. At the same time, as we saw in Chapter 2, Habermas is aware of a need to establish a more productive interplay between the spheres than is evident in the continuing tendency of modern technological and bureaucratic systems to colonise the life-world of ordinary people.

Not all of Schelling's position is rendered invalid by such objections. In the face of the disasters that have been produced by some forms of modern science, of the potential for future disaster created by new forms of technology, as well as of the coercive forms of integration characteristic of modern capitalism, the kind of re-examination of how we think about the relationship of cognitive, ethical and aesthetic spheres suggested by Habermas is an urgent task. If the aesthetic is the realm in modernity where any sense of the harmony of our reflexive and of our natural being might be sustained, it may yet offer resources that the growing separation of the spheres has tended to obscure. In the light of the history since Schelling it is clear why Habermas is wary of the idea of a new mythology: its effect in politics is rarely anything but catastrophic. However, Habermas tends to overplay the extent to which the spheres are inherently separate forms of communicative action. Much more scientific practice than he supposes is, for example, reliant on the characteristics of judgement seen in 
aesthetic judgement, as Kant's account in the $C \mathcal{F}$ suggested. Habermas's aim is to find new forms of orientation which would re-integrate these differing spheres of modernity, rather than allowing means-ends rationality to dominate. But this aim leads towards ideas that contradict the notion that they constitute separate spheres in the first place. These problems will clearly not go away, and it is important to attend to some more of the detail of how they become manifest in Schelling's work.

The crucial fact about Schelling's new mythology is that it has to be made, and it is the task of reason to make it. The mythology cannot be re-established from the past, and has nothing to do with later reactionary nationalist conceptions of mythology as a return to lost origins. The problem is that it would have to be produced collectively, and Schelling has no illusions about the difficulty of doing this in modern societies. He attaches great importance to the collective reception of art, frequently citing the links between the genesis of Greek tragedy and the fact that Athenian society was based on 'public freedom', not on the 'slavery of private life'. The holy, he argues, is constituted at the level of the community, not in the individual: 'A nation which has nothing holy . . . cannot have true tragedy' (I/6 p. 573), thereby establishing the terms of the debate over the possibility of tragedy in the modern world. The problem for modern art is that the collectively binding status of Greek tragedy as a norm for the community could not be achieved by an individual artist:

But how a new mythology, which cannot be the invention of the individual poet, but of a new people [Geschlecht - it should be remembered, in order to counter the apparent hint of racism, that the $S T I$ also argues for a cosmopolitan constitution to guarantee human freedom], which as it were represents One poet, can arise, is a problem whose solution can only be expected from the future fate of the world and the further course of history. (I/3 p. 629)

How could the individuality of a non-theologically based art ever gain collective public significance? In modern capitalism collective significance is increasingly the preserve of the mass media, which constitute a public sphere with little essential ethical content. If art is to retain ethical force it must have this collective significance: if it does not, any link between the aesthetic and the ethical is threatened. As we saw, the notion that the ethical and the aesthetic should be separated starts to emerge at the time Schelling writes the $S T I$, in the work of Friedrich Schlegel, and the increasing autonomy of art in the nineteenth century takes it away from a predominantly ethical vision of the kind encountered in Kant or Schiller.

What, then, does the artist who sees his or her task in terms of the need for a new mythology do in the meanwhile, in a society which seems to be moving further and further away from the integration sought in that mythology? This was precisely Hölderlin's problem, which suggests how intense the problem can become for the modern artist. Widely divergent responses are possible to this dilemma. The artist may have to give up art altogether, as Hölderlin suggested, 
in the name of political praxis, or, as Brecht and others will later do, try to make art itself into a form of political praxis. In the case of Wagner, art really is supposed to become the mythology of the present. Wagner attempts this, however, in an often regressive way. At the same time as taking on the challenge of the new aesthetic freedom more emphatically than virtually any other composer, he tries to resuscitate old national mythologies, ignoring the need for a new, cosmopolitan mythology. Those aspects of his music which were later to be effortlessly assimilated into the culture industry point to other dimensions of his regression. The complex but more than tenuous links between Wagner's music-drama and the use of aesthetic modes in Nazi political praxis suggest the dangers in his response, though the case of Wagner is clearly not exhausted by this dimension of his art. $^{7}$ The avant-garde artist, on the other hand, can produce an art which tries to remind a society of all it has repressed, by refusing to communicate in the terms of the dominant society, with the danger that he or she will either not be understood, or will simply be ignored. The fact is, as Schelling makes clear, that there is no individual solution to these dilemmas, though it would at the same time be a mistake to underestimate the extent to which aesthetic modernism does have substantial political and social effects.

\section{Mythology, language and being}

Schelling develops some of the $S T T$ 's ideas on art and mythology in the slightly later - 1802-3 - Philosophy of Art (PA), a text which is, however, much more obviously linked to Idealism than to Romanticism. The $P A$ argues that something vital is lost when the modern world ceases to be able to articulate meaning in the manner of mythology: 'The modern world begins when man tears himself away from nature, but as he has no other home he feels himself alone' (Schelling I/5 p. 427). Schelling tells a story that will recur in a similar form in Lukács's Theory of the Novel and in Benjamin's The Origins of the German Play of Mourning, and, implicitly, in certain aspects of Heidegger's philosophy. In this story myth-based cultures do not reach the level of reflection at which the images used to interpret reality are separated from other, abstract, articulations of reality. This separation is therefore seen as part of the loss of 'home' characteristic of modernity. Re-reading this aspect of Schelling's theory in the light of contemporary debates offers some interesting perspectives on questions of aesthetics, language and modernity.

The $P A$ argues that Greek mythology recounts the origins of the world in 'concrete' terms, in terms of stories and images. The stages expressed abstractly in the $S T T$ 's account of the I's attempt to 'intuit itself' are articulated in stories of the Gods, much in the same way as the work of art objectified intellectual intuition: 'all the possibilities located in that realm of ideas which is constructed in philosophy are exhausted [erschöpft] in Greek mythology' (I/5 p. 400). The crucial difference is that these stories are concrete and self-sufficient. Our modern interpretation of the figure of Jupiter in such stories as, in Schelling's 
terms, the linking of absolute power and absolute wisdom, has little to do with how the story functions within its own culture. It is not that the figure of the God 'means' something else which is more abstract: the Gods 'do not mean it, they are it' (I/5 p. 401). A gap between concrete image and what it represents does not exist in such a culture. (The concern here is not, one should add, with the historical or philosophical validity of this view, but rather with its implications for conceptions of language in the early modern period in Germany.) According to the $P A$ there is no need, with regard to this sort of myth, for philosophical reflection, because what we have come to see in terms of the idea and its objective embodiment are already united. The stories are 'of' the Gods in the 'subjective' and the 'objective' genitive: they come from the Gods and they are about the Gods. Schelling links this conception of myth to his notion of 'absolute identity'. Absolute identity does not divide sensuous and intelligible, because they only differ from each other in degree, as part of the same absolute continuum. Modernity, as we have seen, entails the growing divorce of individuals' subjective experience of the world from the way the objective world is explained in science. Schelling's questioning of this divorce becomes most significant for contemporary philosophy when language is introduced as part of the argument.

The beginnings of a characteristically modern conception of language were apparent in Novalis: the signifier, itself constituted in opposition to other signifiers, is a condition of the reduction of my particular intuition to a general meaning. In modernity, once the old philosophical division between particular and general becomes a concern in a new way because of the emancipation of subjectivity, language itself can begin to be experienced both as a constraint upon the individual subject and as essentially arbitrary. Using the general signifier for individual experience can therefore become a problem, as it clearly is for many modern artists. At the same time, however, poetic language must attain at least a minimal level of comprehensibility for others, if it is to be understood at all. In the $P A$ Schelling argues that 'In language we always only use general designations, even for the designation of the particular' (I/5 p. 408). He suggests that the universality implicit in Greek mythology's ability to 'imagine' (in the sense of making into an image) is therefore only available in modern art to those artists who by their individuality are able to create a 'closed circle of poetry [Poesie]' (I/5 p. 444), an individual mythology, from their limitation. As Peter Szondi points out, Proust, Kafka, Joyce (or, one might add, composers such as Mahler or Scheonberg) do achieve a canonical status by establishing an aesthetic world which has a coherence not derived from the dominant languages of their society. Schelling thus already indicates one of the grounds of autonomous art's refusal to communicate in the language of the rest of modern society, and, like Adorno after him, he points to the paradox that it is precisely this kind of art which will attain the most general significance. The idea is that articulations of true individuality can convey the possibility of non-coercive social integration. They can do so because they resist the ways in which modern 
rationalisation tends to restrict individuality, even as it in other respects creates conditions which would seem favourable to the development of individuality. In Chapter 3 we saw how Novalis speculated on music as a language which might communicate a sense of the freedom of the subject by escaping the generalising tendency of representational verbal language. It seems no coincidence that in early-modern German culture, where the political public sphere offers less space for real individuality than does much of the rest of Europe, the nonrepresentational form of music should therefore flourish more than elsewhere.

In the $P A$ the special status of 'classical' art, particularly Greek sculpture, is grounded in the fact that it embodies a unity of form and content which modern art cannot have for us. This unity is echoed in Schelling's account of myth, in which the form and content of Jupiter are the same. Schelling argues that 'Beauty is posited where the particular (the real) [in the sense of the sensuous particular object] is so appropriate to its concept that the concept, as infinite, enters into the finite and is contemplated in concreto' (I/5 p. 382). This links art to the unification of image and idea which is supposedly always already present in mythology: in art 'eternal ideas of reason' become 'objective as the souls of organic bodies' (I/5 p. 383). The sensuous object is able to communicate the absolute when the object does not require that which would explain it in other terms, scientific explanation leading into an endless chain of determinations of determinations. The same description could, of course, apply to a theological view of language, in which the 'eternal idea' is manifested in the concrete word, rather than the word being dependent on its relations to other words for its signification. ${ }^{8}$ Schelling demands that in art each thing should have a 'particular and free life. Only the understanding subordinates, in reason and in imagination [Einbildungskraft] all is free and moves in the same ether ... each for itself is also the whole' (I/5 p. 393). Art thus becomes a kind of language in which idea, word and thing are inseparably bound up with each other, rather than arbitrarily attached. The significance of the particular intuition is sustained, not for its particularity, but for its potential to show the totality in itself. This is the classic definition of the symbol, as opposed to allegory's arbitrary link between signifier and signified, and it highlights a crucial difference between Idealist and Romantic conceptions of art.

The insistence upon the function of art in conveying the general by the particular is characteristic of Schelling's argument in the $P A$. In modern science the effects of the separation of intuition and thought cannot be subtracted from our relation to the object. The object is constituted as an object by its separation from us, which we try to overcome in the general concept, thereby eliminating its particular way of appearing in intuition. One can here already begin to see the roots of Schelling's divergence from Hegel. Although he accepts Schelling's view of 'classical art' as vital in an earlier phase of the development of culture, Hegel sees the break-up, via reflection, of the unity between the particular object of intuition and the totality as part of the process of the absolute's becoming articulable in philosophy. Without this break-up thinking would 
remain tied to particularity and be unable to reach the level of universalisation which is the foundation of modern science and law, and, more generally, of philosophy's ability to grasp the truth of modernity.

Hegel's attitude to sensuous particularity exemplifies the dominant pattern of modernity. In each realm of science new developments are increasingly the result of the elimination of residual mythical elements. The ever greater abstraction from the particular to the general constitutes the world more and more as the object of a subject which has transcended sensuous particularity by subordinating it to general concepts whose value is established via their relation to other concepts within a system. An analogous process takes place with regard to the object considered as an exchange-value in the commodity market. Furthermore, the idea of exchange value as constituted by the object's relation to other commodities, rather than by its intrinsic value, is analogous to the conception that language consists of arbitrary signifiers which only gain their identity via their relations to other signifiers within a linguistic system, rather than by an essential relation to their referent. ${ }^{9}$ Worries about these forms of abstraction, which are linked to questions about the dominance by the subject of the Other, recur in varying ways in modern philosophy. ${ }^{10}$ Most recently it has been those thinkers who, like Heidegger, Foucault and Derrida, question the nature of the subject in 'Western metaphysics', which is seen as being manifested precisely in philosophical systems, the commodity system and in language as a system, who have revived interest in the questions first raised by the tradition at issue in the present book. A central concern for these thinkers is the language of poetry, conceived as a counter to the objectifying language associated with the idea that 'the Cartesian basic attitude knows in advance, or thinks it knows, that everything can be proven and grounded in an absolutely strict and pure manner' (Heidegger 1983 p. 30).

The later Heidegger's account of modern philosophy as the 'subjectification of being' leads to his attempts to find a different language for philosophy, which would circumvent the metaphysical tradition. Despite his reference to the ancient Greek past as a source of his project, this attention to language cannot be separated, either from the desire of modernist poets, such as Mallarmé, to 'purify the words of the tribe', or, indeed, from Romantic ideas about music current in the nineteenth century. The modern need to seek a language beyond objectifying representation and instrumentality relates to the kind of view of mythology at issue here. The crucial question is how this need is understood. It is now a commonplace that understanding subjectivity depends upon understanding the subject's relationship to language, but as I have tried to show, many accounts of the subject which rely on this view to criticise 'Western metaphysics' too often ignore the fact that thinkers like Novalis and Schelling do not necessarily see the subject as transparent to itself and thus as being in a position of Cartesian mastery.

In the $P A$ Schelling suggests the problem with language is that it is 'nothing but a continual schematisation' (Schelling I/5 p. 408), which points to the need 
for a 'higher language', of the kind present in Greek mythology, which is what is also demanded in 'absolute artistic representation' (I/5 p. 411). The conception of language suggested here can be formulated in the terms of the STI. Because words are collectively constituted and not the result of individual intention they are, on the one hand, 'products', results of 'unconscious productivity' that are manifest as real objects (sound waves, marks, etc.). On the other hand, they can only $b e$ words if they involve 'conscious productivity' that endows them with meaning in particular use - computers do not mean anything by the sentences they produce. Schelling claims nature is 'a poem which lies locked away in secret miraculous writing', so that 'through the sensuous world [of nature] meaning only shows as it does through words' (I/3 p. 628). On this view, the literal, established meanings of words can be seen as the result of making language into an objectified 'product', and metaphors make apparent the resistance of language to wholesale literalisation. To the artist, nature is 'only the ideal world appearing under continual limitations' (I/3 p. 628). In order to get beyond the limitations of the sensuous world, which are what give rise to science and philosophy's endless task, the artist has to reveal the unlimited in a limited product, and this connects metaphor's resistance to literalisation with the work of art's manifestation of productivity. To this extent Heidegger's extreme notion of a 'language of metaphysics' which supposedly dominates Western thought becomes otiose, because the possibility of escaping reified language is perennial, although it may become more difficult in particular societies, as more and more metaphors lose their disclosive power.

In Philosophy of Mythology (1842) Schelling returns to the question of why, in mythology, image and idea are not separate, claiming that this must be considered in relation to language. His awareness of the dependence of subjectivity upon a ground which is not accessible to reflection, apparent in the $S T T$ 's conception of genius, is here directly linked to language:

But what treasures of poetry lie hidden in language in itself, which the poet does not put into language, which he, so to speak, only lifts out of it as out of a treasure chamber, which he only persuades language to reveal. But is not every attribution of a name a personification, and if all languages think or expressly designate things which admit an opposition with differences of gender; if the German says der Himmel [sky], die Erde [earth]; der Raum [space], die Zeit [time]: how far is it from there to the expression of spiritual [geistig] concepts by male and female divinities. One is almost tempted to say: language itself is only faded mythology, in it is preserved in only abstract and formal differences what mythology preserves in still living and concrete differences. (II/1 p. 52)

The conception is not so far from Heidegger calling language the 'house of being' and from the contention that 'We do not only speak the language [which implies language is an object], we speak out of language' (Heidegger $1986 \mathrm{p}$. 254). Given Schelling's arguments about the lack of ultimate transparency of the subject to itself, it is clear, as it is elsewhere in his work (see Bowie 1993), that he does not fit into the Cartesian paradigm. 
The passage suggests, in a manner later echoed by Gadamer, that there is a metaphoric process at the very heart of language, which we cannot ever circumscribe by drawing a definitive line around what is literal and what is metaphorical. The traces of the sources of language that can never be reached in their original form are always necessarily part of the language we are using. The link to the aesthetic of conceptions of language in the Romantic traditions to which Schelling belongs also informs the genesis of the modern hermeneutic conception of language as constitutive of what we understand, rather than as a representation of a ready-made reality. In the hermeneutic conception there can be no position from which language could be definitively explained by philosophy or science. In order to understand the explanation itself we would still be reliant on the natural languages into which we have already been socialised and which are a horizon of our understanding that we can never definitively step beyond. This points to another way of seeing Schelling's awareness of the role of both conscious and unconscious 'production' in our self-understanding. The words we speak are not produced consciously, but we can still consciously intend to say something with them, even though what we say may escape our intentions or may go beyond what has been meant with these words before. Schelling is, of course, a major influence on Schleiermacher, the founder of modern hermeneutics (see Chapter 6).

Near the beginning of the $P A$ Schelling makes the apparently unmotivated statement, perhaps derived from Hamann's work, that 'Very few people reflect upon the fact that even the language in which they express themselves is the most complete work of art' (Schelling I/5 p. 358). The immediate reason for this is that language is 'the direct expression of an ideal - of knowledge, thought, feeling, will etc. - in something real, and, as such, a work of art' (I/5 p. 482). Language must exist as objective sensory phenomena, but it must also be intelligible. It is in this sense a manifestation of 'absolute identity'. As Hamann argued, language is not explicable in terms of a division of sensuous and intelligible: how would the sensuous be able to communicate thoughts if it belonged to a wholly different realm? In consequence: 'sensuous and non-sensuous are one here, what is most graspable becomes a sign for the most spiritual (Geistigste). Everything becomes an image of everything else and language itself becomes thereby a symbol of the identity of all things' (I/5 p. 484). Language is the necessary 'real' articulation of the 'ideal', but 'absolute identity' means that this difference is only ever relative: the ideal's dependence on the real is mirrored by the real's dependence on the ideal. This view of language moves in two divergent directions. On the one hand, as we saw, in the direction of a metaphysical, Idealist view of language, which, like the Classical conception of art, sees it as a symbol of the infinite in the sensuous; on the other, in the direction of the differential view already seen in Novalis, in which the elements of language gain their value via their shifting network of relations to other elements. In an echo of Gadamer's dictum that 'being that can be understood is language', Schelling, then, regards all intelligible reality as a 'primary speaking', because 
it is both knowable via 'ideal' conceptual discrimination and empirically manifested as differentiated 'real' matter.

The extension of the idea of language to include all forms of art becomes most fruitful when Schelling talks about music. ${ }^{11}$ In Schelling's account of the ascending continuum of the arts in the $P A$, which he sets out in terms of the relative roles of the ideal and the real within each art, music comes first, being the 'most closed of all arts' because it only articulates the movements of things and does not determine the things conceptually (I/5 p. 504). Music's status as the form of art most dependent upon time means it is 'the living which has entered death - the word spoken into finitude - which still becomes audible as sound' (I/5 p. 484). This is because, like all sound, music ceases to be, even as it becomes manifest. Music's essential relation to time - elsewhere Schelling says 'time is itself nothing but the totality appearing in opposition to the particular life of things' (I/ 6 p. 220) - is based on the fact that music's form is 'succession'. This necessarily connects music to self-consciousness, which makes possible the unity between successive moments of time: music's essence is rhythm, the 'imprinting of unity into multiplicity' (I/5 p. 492), 'the transformation of a succession which is in itself meaningless into a significant one' (I/5 p. 493). Schelling terms rhythm 'the music in music' (I/5 p. 494), because the structure of identity in difference is repeated both in melody's unifying different pitches into intelligible forms and in the unification in harmony of different pitches, from the overtones in a single note to the notes in a chord. The idea of 'the transformation of a succession which is in itself meaningless into a significant one' can, of course, be interpreted much more widely than just in relation to music: the same idea applies to the sequence of noises in a sentence, or the initially random flow of events in experience. In some respects the idea is actually definitive of what metaphysics is intended to explain.

Heidegger claims that the 'difference of a sensuous and a supersensuous world' is the basis of 'Western metaphysics' (Heidegger 1986 p. 101). It should by now be clear that there is no absolute distinction of this kind for Schelling, and that many assertions like those of Heidegger rely on a too restricted account of the history of modern philosophy. In the most extensive statement of the identity philosophy, the 'Würzburg System' (1804), Schelling, developing ideas from Spinoza, makes it clear why there cannot be any cognitive foundation for knowledge, in a way which can be related both to music and to differential views of language, in which each signifier relies for its identity upon its relations to other signifiers. Schelling says: 'No single being has the ground of its existence in itself' (Schelling I/6 p. 193): it must be relative to all other beings and thus within 'absolute identity', otherwise it could have no way of being itself. $\mathrm{He}$ claims that 'Every single being is determined by another single being, which in the same way is determined by another single being etc. into infinity' (I/6 p. 194). The aim is, once again, to unite the difference of the moments into a higher identity, in order to prevent a regress. There can, though, be no cognitive access 
to this higher identity, because knowing entails following the chain of determination. ${ }^{12}$ The problem here points to a major reason for the emergence of German Idealism.

Kant thought he could avoid this problem by restricting knowledge to what can come under the conditions of the subject's ability to synthesise, rather than attempting the impossible task of establishing the chains of determination of things in themselves. Kant's conception of the subject resulted in the need for the transcendental deduction, which is precisely the attempt to explain, in terms of the 'I that can accompany all my experiences', how meaningless succession becomes meaningful. The problems in the transcendental deduction led Fichte to absolutise the spontaneity of the subject in order to ensure its autonomy from the regress of determinations. At this stage of his thought Schelling relies upon a version of 'intellectual intuition' to overcome the Kantian separation between subject and things in themselves which goes much further than Fichte's notion:

The sameness of subject and object is not limited to the consciousness of myself; it is universal. The object of an intellectual intuition cannot therefore be an external sensuous object, but just as little can it be the empirical subject. For all objects of the same are just as limited and transitory as those of the external sense. Therefore only what is infinite, completely unlimited, what is affirmed by itself can be object of an intellectual intuition. (I/6 p. 154)

Both the $S T I$ and the $P A$ argued that the art object could unite the sensuous and the intelligible, the determined and the free, in specific objects, thus objectifying intellectual intuition, and making the artwork into a symbol of the absolute. In this sense, as the PA puts it, 'beauty is the absolute intuited in the real [das real ansgeschaute Absolute]' (I/5 p. 398). ${ }^{13}$

The positive account of the presence of the absolute in the symbol now seems implausible, compared with the Romantic position, but the logic of what leads Schelling to advance it reveals an important issue that still haunts contemporary philosophy. Put simply, what is in question is precisely how 'successions' can become meaningful, which can be seen in terms of Schelling's prefiguration of Gadamer's idea that 'being that can be understood is language'. The obvious way to make successions meaningful is to integrate them into a totality, and this is what Schelling intends with the extended notion of intellectual intuition just cited. Without some manner in which unification takes place, each particular thing can only be grounded in something else, and each moment of the subject's reflection upon itself leads to Fichte's question, 'Do I really think or do I just think a thinking of thinking?' Both lead to a regress, unless there is a ground within which the elements or moments are linked. Access to this ground has to be in some form of a direct 'intuition', as otherwise all the problems of regress will recur.

One of the reasons this pattern of thought has remained significant becomes apparent if it is seen in linguistic terms. In the same way as things are deter- 
mined by their relations to other things, the Saussurean signifier gains its determinacy by its relations to other signifiers in the langue. The problem with the notion of the langue, as with the notion of the ontological ground, is that it is either assumed somehow to exist without one being able to give a description of it, because it is not an entity like the entities of which it consists, or the attempt to grasp it leads into a regress. Furthermore, as Derrida has argued, if there are no ideas without their articulation in language, one can only have access to what is signified via the signifier's negative relationship to other signifiers, not by reference to a pre-existing signified. How do we say what a word means without just using other words to do so, given that we cannot just point to what we mean and be understood? We can, in these terms, therefore only work with chains of signifiers which are mutually dependent upon each other. Each element of language bears the trace of the other elements of the language, in a manner analogous to Schelling's remark that 'No single being has the ground of its existence in itself. For Derrida, the consequence is that full signification, the 'presence' of meaning is inherently deferred.

As I have argued in more detail elsewhere (see Bowie 1993 Chapter 4, and also Frank 1984, Dews 1987), the Derridean position conceives of meaning, as Ricoeur has contended, in purely differential, semiotic terms, which leads to a regress, on the basis of the unavailability of the totality of the langue. Derrida's 'disseminal' conception fails to give even a minimal account of semantics that would account for the what Gadamer intends with the claim that 'being that can be understood is language'. The basic point is that Derrida makes extravagant demands on the notions of truth and meaning, by understanding them as entailing the impossible 'presence' of signifier to signified. He offers no plausible way of accounting for the functioning of everyday communication, let alone of our ability to understand - albeit never with absolute certainty - highly complex new uses of language. In consequence Derrida seems in one respect as much a prisoner of the Cartesian heritage he wishes to deconstruct as Husserl. Schelling's conception of identity philosophy may be untenable as a positive claim about philosophy's access to the absolute (though in such negative formulations as 'the totality posits or intuits itself, by not-positing, not-intuiting the particular' (Schelling I/6 p. 198) we do gain some sense of how one might understand being's transcendence of what we can say about it). What Schelling does succeed in showing is that without some, perhaps inarticulable, grounding identity, of the kind that enables us to understand the connection of singular and general term in a judgement, even the idea of the dependence of the different signifiers on each other is unintelligible: apprehending difference as difference requires identity. In the arguments seen so far this has been seen in terms of a non-reflexive self-consciousness for which difference can be difference, and this is connected to the idea of the trans-reflexive status of being we considered in Hölderlin and Novalis.

Thinkers as diverse as Heidegger and Donald Davidson argue that the understanding of truth and meaning is prior to any attempt to analyse it (and is 
necessary for any such analysis anyway). Truth requires an intuitive understanding, which in the earlier Heidegger's case is a prior practical understanding of being that always subtends all our linguistic articulations of what there is. Davidson talks of our 'general and pre-analytic notion of truth' (Davidson 1984 p. 223) and of an 'intuitive grasp we have of the concept' (p. 267) in order to avoid a regressing definition of the definition of something which we have already to understand in order to understand the very idea of defining in the first place. Given the ineluctability of this intuitive grasp, which Derrida seems not to countenance, it becomes unclear how language in Derrida's view can ever really mean anything at all. ${ }^{14}$ Peter Dews maintains that this question involves similar difficulties to those Fichte showed to be present in the reflection theory of consciousness. Dews claims: 'Just as the regress of reflection renders the phenomenon of consciousness inexplicable, so - on Derrida's account - there could never be an emergence of meaning: there would be nothing but an unstoppable mediation of signs by other signs' (Dews 1987 p. 30). It is not enough, to regard signification, as Derrida does, as always deferred because, as Schleiermacher, for example, argues, the possibility of misunderstanding is omnipresent. If something is indefinitely deferred it cannot be said to happen at all, and, as such, cannot even be considered to be deferred. We would have no understanding that deferral takes place if we did not already have a pre-theoretical, pre-reflexive sense of what meaning is. This does not therefore mean that we have to assume there are 'meanings' in a Fregean sense, and is simply a demand that a theory which may convincingly show why such meanings are implausible entities also show how it is we make ourselves understood a lot of the time.

Derrida's contentions are mainly directed against philosophical attempts to ground truth and meaning in an absolute Cartesian manner: hence the notion of the presence of meaning that is undermined by the nature of the differential constitution of the sign. The idea of the deconstruction of presence is then, of course, extended by Derrida to the metaphysical tradition from Descartes to Hegel and to Husserl. He characterises this tradition as relying on the 'absolute desire to hear oneself speaking' (Derrida 1967 p. 115). This account of metaphysics presupposes precisely the reflexive model which Fichte revealed as flawed, and Derrida is justified in rejecting it as a possible absolute foundation. Hearing oneself speaking, however, only makes sense on the assumption that, in order to hear oneself $a s$ oneself, rather than hearing something objective which is, even though one may not know it, oneself, there must be a kind of self-awareness which is outside the reflexive subject-object relationship (cf. the arguments about reflection in Chapter 3). Derrida, as Frank has shown, never addresses this issue. Even Schelling's invocation of 'intellectual intuition', as what would transcend the reflexive split between subject and object, and would therefore overcome the problem of the regress of reflection, itself clearly fails to overcome a reflexive split between intuiter and intuited, as the very duality in the term suggests. In this sense Schelling does not succeed in solving the metaphysical problems he addresses with this version of 'self- 
presence'. In his negative characterisation of the absolute, in which 'the totality posits or intuits itself, by not-positing, not-intuiting the particular', he actually employs a structure of thought which is hardly different from Derrida's in his characterisation of différance. In neither case does the conception permit a systematic account of the ground of philosophy, though Schelling is aware of the need for more than the merely regressing position that results in Derrida, and this will lead him later to ontological reflections which have echoes in the ideas of Heidegger (see Bowie 1993).

In his later philosophy Schelling eventually abandons the Idealist attempt to transcend the contingency of being in a systematic philosophy, precisely because being cannot be understood in reflexive terms. In Chapter 5 I will look at Hegel's claim to have obviated the problems associated with intuition via an Idealist philosophy which relies wholly upon the structure of reflection. In such a philosophy there is therefore no 'question of being' and no difference between 'ontic' access to particular beings and the 'ontological' fact that all such access is secondary to the 'intuitive', immediate fact that being is disclosed at all, which can never be explained in ontic terms. The contrast between Hegel's attempt to absolutise reflection and Schelling's insights during his career into the resistance of the intuitive ground of thought to reflection offers an instructive model for investigating modern philosophy, in which aesthetic questions play an important role. Philosophers like Heidegger, Adorno and Derrida can, for example, also be seen as concerned with the consequences of the failure of reflection to articulate a complete system of philosophy. Philosophy for these thinkers, as it was for the early Schelling, is regarded as needing to enlist the services of art in order not to exclude dimensions of our being which cannot be reduced to cognitive terms. In the later Heidegger this involves an attention to poetic language which has similarities to the way Schelling understands art, language and mythology. ${ }^{15}$ Adorno, as we have seen, regards Hegel's view of art with deep suspicion, precisely because of its tendency to eliminate, as the process of modern rationalisation does, all that is particular and resistant to conceptual determination in the object. He therefore invokes works of radical aesthetic modernism in the name of a philosophy of 'non-identity', in which the irreducibility of objects to concepts plays a major role.

The later Schelling's criticisms of Hegel's dissolution of being into thinking, in which self-consciousness is able to give a complete conceptual account of itself via its relations to the world, can be seen as deriving from the same sources as his earlier attention to art. Schelling insists that human reason cannot explain its own existence, and therefore cannot encompass itself and its other within a system of philosophy. We cannot, he maintains, make sense of the manifest world by beginning with reason, but must begin with the contingency of being and try to make sense of it with our reason, which is only one aspect of being. The difference between Schelling and Hegel is in some respects paradigmatic. In the modern world there seem to be two dominant opposed tendencies in philosophy: on the one hand, because systematic scientific thought is able to give an 
explanatory account of more and more aspects of being, systematic philosophy increasingly dissolves into the sciences; on the other hand, the meaningfulness of our existence seems to depend upon forms of activity and thinking which reveal aspects of being, particularly those to do with self-consciousness, that cannot be dealt with in purely explanatory terms and which take philosophy in the direction of the 'world-disclosure' characteristic of art. Schelling himself attempts unsuccessfully to formulate a philosophy of theological revelation which would comprehend the contingency of being and make sense of our place within it. Interestingly, many subsequent thinkers, like Heidegger and Adorno, come back, in the light of the decline of theology, to the idea that art is the locus of kinds of self-understanding which are not accessible to the sciences. In this respect one of the vital aspects of modern thought is precisely the way in which needs previously catered for by theology can be catered for by forms like the aesthetic. The fraught history of aesthetics and politics since Schelling's time makes evident just what is at stake here, and the tension between Schelling's and Hegel's responses to the relationship between philosophy and art offers many resources for understanding that history.

\section{Notes}

1 Heidegger includes Schelling among the philosophers of subjectification, which is, given such utterances as the one just cited, even inappropriate for aspects of the younger Schelling, and is certainly inappropriate for the later Schelling (see Bowie 1993).

2 It is worth remembering here just how much the understanding of Spinoza's principle of determination as negation is echoed in contemporary thinking about the mind as a digital computer, and how much the objections in the philosophy of mind to this reductionist model echo the Idealist and Romantic response to Spinoza (see Bowie 1993).

3 When I asked Derrida about this tradition, in response to some remarks of his about the 'whole history of subjectivity', as exemplified by Descartes, Kant, Husserl, etc., his reply was that we 'would have to read Schelling together'. Slavoj Zizek has realised the extent to which Schelling prefigures Lacan in his recent work (see Zizek 1996).

4 Why this takes place will later become a major focus of Schelling's philosophy, and he never arrives at a satisfactory answer (see Bowie 1993).

5 As suggested above, DNA is not the modern scientific answer to such metaphysical questions, because the transmission of the form is not the same as the fact that forms emerge at all, instead of nature remaining a mere chaotic flux.

6 On Adorno's claims that Beethoven also moves in the direction of ideology, see Bowie 1999.

7 Seeing Wagner merely in these terms hands him over to precisely the people who ensure that the revelatory and progressive dimension of his work gets forgotten. This is an all too common failing of Left cultural politics.

8 On this issue in some detail see Bowie 1997, particularly with regard to Walter Benjamin.

9 Saussure often uses the analogy of language to money.

10 It is important to remember that without these processes much of what we cannot do without in the modern world becomes impossible. A wholesale rejection of them is not an option, though a critical awareness of how they can distort our relations to ourselves 
and the rest of the world is still a vital task. As I have already suggested above, the rise of digital technology is another of these processes.

11 The conclusion of the $P A$ suggests the power of the connections Schelling makes between the different languages of art: in an anticipation of Wagner's theory of music-drama, he reflects on the 'most complete combination of all arts ... which was the drama of antiquity' and wonders if opera, which is at present merely a 'caricature' of ancient drama, may become able to lead back to 'ancient drama combined with music and song' (Schelling I/5 p. 736).

12 The source of Schelling's awareness of this problem is Jacobi's account of Spinoza, where the answer to this regress is theological.

13 The $P A$ talks of 'fantasy', which is manifested in particular images but which also involves the freedom of the subject, as 'intellectual intuition in art' (I/5 p. 395).

14 See Wheeler 2000 for a more charitable view, which brings Derrida closer to Davidson.

15 As I show in Bowie 1997, Heidegger's essay on the origin of the work of art derives key conceptual structures from Schelling's philosophy of the 'Ages of the World', which Heidegger was studying around the time his essay is written. 


\title{
Hegel: the beginning of aesthetic theory and the end of art
}

\begin{abstract}
Which Hegel?
Hegel's work has come in recent years to exemplify many of the choices facing contemporary philosophy. The changed status of Hegel can, though, seem rather odd, given the labyrinthine nature of his texts, the huge divergences between his interpreters from his own time until today, and the fact that some of the philosophers who now invoke him come from an analytical tradition noted for its insistence on a clarity not always encountered in Hegel himself. Even contemporary interpreters range between those who still pursue his grand aims by trying to show how he offers a systematic answer to the major problems of modern philosophy, and those, like Robert Brandom and John McDowell, who are now mobilising his arguments in order to escape from some of the epistemological impasses in the analytical tradition. Most histories of German Idealism see Hegel as making the vital steps towards a complete systematic philosophy which goes beyond the limitations of Fichte and Schelling (for example, Kroner 1924). More recently it has been precisely this aim of completeness that makes Hegel the target of philosophers whose aim is to deconstruct such pretension, as part of the wider attempt to overcome 'Western metaphysics'. In the preceding chapters I have tried to show that there always has been something of a 'deconstructive' tendency in modern philosophy, though it is generally one which seeks to elaborate new conceptions of subjectivity, not completely to obviate the role of the subject. In the present context it is important to keep the focus on the significance of aesthetics in Hegel's work, but it will soon be obvious that, as we have seen in relation to the other thinkers we have looked at, this focus will force us into a broader interrogation of his philosophical claims. The issue with which we shall be concerned is how Hegel's account of art's relationship to philosophy is to be assessed, given its reliance on assumptions which go to the heart of his philosophical project.

Hegel's work on aesthetics has two main aspects. On the one hand, he produced the most influential systematic aesthetics of the nineteenth century; on the other, he announced the 'end of art' as an expression of the 'absolute' in modernity. Hegel wants to make philosophy into the complete articulation of
\end{abstract}


what he thinks is only incompletely expressed in art and religion, the preceding forms of 'absolute spirit'. ${ }^{1}$ What he means by this term can be understood in relation to the notion of 'objective spirit', the preceding, less developed form of Geist in his overall system. 'Objective spirit' is the realm which encompasses the history of attempts to overcome contradictions between the individual, the family and the state, which are manifest, for example, in forms of production and exchange, and in the legal forms which regulate the distribution and ownership of property. 'Absolute spirit', on the other hand, is supposedly not encumbered by the demands of technical and practical reason, and allows thought to be self-determining. The notion will become clearer when we see how Hegel creates a hierarchy of the arts on the basis of their increasing emancipation from the need for physical embodiment, the highest art being the most self-determining manifestation of Geist, namely literature (in the form of comedy). Interpreting exactly what Hegel means by his conception of philosophy is, though, far from easy. One of the major difficulties is that his work comes as a package: you cannot simply take bits of his system, because the whole point of his thought is that if you adopt one bit you are forced to adopt the rest, the two being inextricably connected. Objections to Hegel are consequently often rejected by Hegelians on the grounds that some other part of the system will be able to cater for the objection. The result is a situation where all you seem able to do is enter the textual labyrinth called 'Hegel', on the assumption that it has a centre. You cannot, of course, know in advance whether this is really the case, otherwise the enterprise would be pointless: you have to assume it as an act of faith. It is arguable that Hegel himself assumes such a centre and then sets about showing us the way to it.

Recent interpretations of Hegel have tended to focus on translating his apparently hyperbolic contentions into arguments which show that he can be part of contemporary philosophy, rather than the megalomaniac nineteenthcentury representative of what Adorno termed 'the stomach become spirit'. Whatever one makes of these interpretations, aspects of which I will touch on, it does seem clear that philosophy for Hegel, as it did for the other Idealists, is to show how thought and being are inseparable. This makes them 'identical' in Hegel's particular sense, so Kantian worries about knowledge being only of the way the world appears to us, rather than of things in themselves, are eliminated. The question is how this identity is to be revealed and what exactly is meant by the way it is revealed.

The early Schelling claimed that art overcame the division between thought and its ground by revealing how the 'unconscious productivity' of nature required for there to be thought at all could be combined with the 'conscious productivity' involved in philosophical and other reflection. Hegel confronts in a new way the problem of how mind and world relate. As Fichte argued, if the I is wholly opposed to something else - the world, the object, matter, things in themselves - Kant's problems must remain unsolved, leaving us with separate worlds of freedom and necessity. We have already considered various attempts 
to solve Kantian problems. Few of these are entirely free either of implausible idealism, of the kind present in Fichte's absolutising of the I, or of a reversion to dogmatism, as in certain aspects of Schelling's Naturphilosophie. Only the early Romantics offer a perspective which potentially skirts the traps of strong foundationalism. What is Hegel's contribution to these attempts?

Before dealing with some of the detail of Hegel's texts, let us briefly consider one of the contemporary approaches to Hegel. This should make the issues clearer and will suggest how the more immanent discussion which follows can be related to present-day debates. Terry Pinkard epitomises the deflationary interpretation of Hegel in the following description of what he thinks absolute spirit means: 'the human community comes to an awareness that it is in working out the internal requirements of its own reason-giving activity that it sets for itself what is to count for it as its absolute principles' (Pinkard 1994 p. 254). Like Robert Pippin, Pinkard, regards Hegel as the most important thinker of a selflegislating modernity. In this interpretation, Geist, the immanently developed thought of a community, is absolute because there is no longer any possibility of appealing to a transcendent authority. Communities must have recourse to intersubjective agreement without invoking anything outside what they can work out within their own institutions. Hegel's idea of thought's 'self-determination' need not, then, be read as though there were an immanent driving force of rationality, called Geist, which forces us to acknowledge that the 'real is the rational'. The idea instead designates the recognition on our part that, as Pippin puts it, 'we always require ... a narrative account of why we have come to regard some set of rules or a practice as authoritative' (Pippin 1999 p. 68). The idea of all-inclusive immanence therefore no longer forces one to invoke obscure notions like the 'self-determination of the concept', because what is at stake amounts to nothing more than the fact that legitimation of all kinds in modernity has to include reflection on the sources of our decisive notions in the concrete history of a human community. The task of philosophy is to show that there is a wider development of rationality in modernity, evident, for example, in things which have become impossible to justify, like slavery and the oppression of women. The impossibility of now finding an intersubjective justification for such things is the final court of appeal, but this court does not have transcendent authority, and its justifications can always be revised in terms of a better, more inclusive, narrative of legitimation.

Habermas has objected to this deflationary account of Hegel by pointing out that

Even from the point of view of a completely inclusive community there is an unmediated difference between the social world which we share intersubjectively and the objective world with which we are confronted and have to cope. Just as little can, secondly, the tension between what is valid 'for us' and what is valid 'in and for itself' be removed. What is rationally acceptable according to our lights is not necessarily the same as what is objectively true. (Habermas 1999 pp. 218-19) 
There is no way in which we can anticipate what the completion of knowledge could look like, because our knowledge is arrived at by explaining what we encounter contingently in nature and by arriving at fallible consensuses about what there is. The recent attempts to convert Hegel's conception of 'absolute knowledge' into an account of intersubjective agreement are consequently, Habermas claims, both inaccurate as an account of what Hegel could have meant and inadequate to the nature of 'post-metaphysical' rationality. The attempts are inaccurate because Hegel does lay claim to a completed philosophical knowledge at the end of the system, to a 'context of all contexts' (pp. 218-19), that is arrived at by explicating all the ways in which thought and its object can be in contradiction, and by then revealing how the contradictions are necessarily overcome. What is actually, in Hegel's own terms, objective spirit, in the sense of the contingent, socially-located historical manifestations of our conceptions, is, Habermas argues, taken by Hegel as absolute spirit. Hegel, then, ignores the genesis of spirit in specific historical contexts and therefore regards it as thought's own complete self-understanding. The attempts are inadequate to the 'post-metaphysical' situation because they conflate fallible rational acceptability in an all-inclusive community with the regulative idea of absolute validity. For Habermas this regulative idea arises from the 'cooperative search for truth' (p. 221) of historically contingent individuals who seek to transcend their contingency by extending the contexts of their knowledge, even though they can never know if anything is absolutely valid: 'nothing gives us the right to expect that we will have the last word' (p. 209). In short, the notion of consensus alone fails to take account of the absolute conception of truth which transcends all particular contexts. Habermas regards the absolute conception of truth as an essentially normative, not a substantive notion, and argues that Hegel lays claim to it in an invalid manner by blurring the line between absolute and objective spirit, in order to incorporate the transcendence of truth into his immanent conception.

How, then, do these conflicting approaches to Hegel relate to what we have investigated so far? As we saw in Chapter 3, the early Romantics' response to the question of absolute truth was precisely to make it a regulative idea which was a reminder of the finitude of our thought. This conception led them to link truth to art, because art manifested that reminder in a way which could always lead to new and unexpected insights, whereas systematic philosophy attempted to 'foreclose' something which, given our finitude, must necessarily remain open. Interpreting this position led to difficulties in deciding whether it entails a version of metaphysical realism, or whether the turn to the aesthetic was not rather a pointer both to the limitations of positions too concerned with epistemology at the expense of other relations to the world, and to the aporias of the correspondence theory of truth. Schlegel's comments that 'In truth you would be distressed if the whole world, as you demand, were for once seriously to become completely comprehensible' (Schlegel 19882 p. 240), and 'If absolute truth were found then the business of spirit would be completed and it would 
have to cease to be, since it only exists in activity' (Schlegel 1991 p. 93) suggested an aesthetic awareness that the very fallibility of our approaches to truth may actually be a way of avoiding nihilism. The awareness of fallibility sustains the motivating goal of attempting to create ever more inclusive forms of understanding. It is, though, possible from another point of view to regard this fallibility as itself leading to a sceptical nihilism, given that something which is endlessly deferred can be seen as irrelevant to what we actually do in the world. ${ }^{2}$ The basic difficulty here is suggested in Schlegel's assertion that 'For a positive criterion of truth the truth itself would have already to be present and be given which is therefore a contradiction' (Schlegel 1971 p. 58). If the criterion cannot be said to be present, does that mean that we have no ultimate way of claiming anything is true, or at least of understanding what we might mean by truth's transcendence of all finite justifications? This is the problem Hegel wishes to resolve and it still forms the focus of many of the major debates in contemporary philosophy.

It is clear that Hegel's position is a response to the sceptical option, and this pushes one in the direction of a decision on Habermas's contentions about what Hegel meant. Hegel responds to scepticism by arguing that the complete, 'nihilistic' destruction of all particular theories does not lead to a negative conclusion, but instead to the highest, most universal philosophical insight. This is because all theories have to begin by opposing some existing position, which they therefore need to negate for their own positive conclusions to emerge - no Galileo without Ptolemy. We can never begin with a foundational theory, because this leads to a regress of justifications, so we must establish the absolute justification at the end. As the conclusions of theories which negate previous theories are then themselves negated in turn, the question becomes whether there is a position which eventually overcomes this process by showing that there is nothing left to negate. For Hegel this position is precisely that of absolute spirit, because it makes us aware of the inherent relativity of particular truth claims, having immanently reconstructed all the possible forms in which things can be articulated. In one respect, then, he comes close to Kant's attempt to delineate the categories in the transcendental deduction, though his method is clearly different. Hegel thinks one can eventually map the relations between each form of thought because they all result from the contradictions in previous forms. The apparently foundational thought of 'being' is really only determinate in relation to 'nothing', and this results in the notion of 'something', a kind of being which both is and is not, because it is itself only relative to other somethings which it is not. This negation of the apparent foundation gives rise to an account of what sorts of something there can be, which are made determinate by their relations to the somethings they are not, and so on. ${ }^{3}$

The most basic contradiction would, of course, appear to be between thought and being. Hegel aims to show that this contradiction is overcome by the very fact that our thinking has to move beyond the indeterminate thought of being to become thought in any meaningful sense at all. The movement of thought 
through all the subsequent contradictions reveals that the initial thought is not foundational in the normal philosophical sense of being the basis which supports everything else. The initial thought is merely a passing stage in the real self-description of thought, and the movement is completed when the initial contradiction finally leads to a systematic account of all the relationships in thought. The essential idea therefore comes at the end, and the beginning is merely something indeterminate which must be overcome if thought is to have any determinate content. Whether this squares with Pinkard's 'communitarian' immanent reading is open to question. Pinkard says the absolute idea is 'the normative self-correcting structure of a rational form of modern "social space", and forms the "pure normative structure" of the patterns of reciprocal recognition that make up modern mind, Geist' (ed. Ameriks 2000 p. 177). While I think his reading offers a valuable way of keeping Hegelian issues alive with regard to the need for philosophy to sustain a public culture of the justification of norms, it tends, as Habermas's objection to the elision of absolute and objective spirit suggests, to obscure some of the more questionable ways Hegel has affected the course of modern philosophy. ${ }^{4}$

The basic question here is how one interprets Hegel's attempt to show that there is in principle nothing outside what can be articulated in a publicly accessible manner. In this view any form of articulation which appears to suggest the limits of philosophy's normatively governed justifications is therefore merely a form of 'immediacy' which can be overcome. It is, of course, in relation to art that this model is most controversial, because it leads to the claim that what is perhaps most characteristic of art in modernity, its 'infinite interpretability', is also precisely what diminishes its philosophical status. This is because art supposedly retains a moment of immediacy, via its being manifest in an empirical object or telling a concrete story about individuals, which philosophy transcends through its universality. At this point we encounter the crucial division within German Idealism. This division becomes paradigmatic for two major directions in modern philosophy, the one pointing to how the sciences take over from both art and philosophy in defining the nature of the modern world, the other questioning, in the name of art, the domination by the sciences of our relations to the world. The underlying question here is the one which has concerned us in the preceding chapters: how does what philosophy can say about art relate to what art may say that cannot be articulated by philosophy?

As we have already seen, aspects of this latter position are developed in the early Schelling. Even though he later no longer makes art central to philosophy, the questions which led him to his early position keep recurring in his work. Schelling's philosophy retains in varying ways, and continues to do so until his later work, a sense that the highest principle of philosophy is not conceptually accessible. Thought's immanent self-description of its own structure does not explain how it is that there is rational thought at all, let alone account for the fact that being is disclosed in the first place (see Bowie 1993). Schelling's conception has a significant influence on attempts to explore the sources and limits 
of rationality, which means he is important both for questionable irrationalists like Nietzsche, and for thinkers like Adorno, who seek to avoid a too narrow conception of rationality by adverting to what art can tell us that philosophy cannot. The 'space of reasons' for thinkers like Adorno does not, as neo-Hegelians like Pinkard and McDowell think it must, necessarily exhaust what is of normative significance. Were it to do so, art would become, as Habermas suggests it does, just one of the spheres of modern rationality, namely the one in which the modern world articulates the aesthetic-expressive dimension of human existence. The historical legitimation for this view is clear. As Hegel argues, art is indeed no longer the essential determinant in how the modern world understands itself: it cannot play the role tragedy did for a time in the Greek polis. However, this still leaves open the question of whether art is as reducible to philosophy as Hegel suggests. His argument relies, of course, upon the success of his wider philosophical project, and the extent to which the neo-Hegelian conception relies on this success will affect how we regard its role in contemporary philosophy.

Hegel claims to achieve what Schelling does not when he argues that: 'The deficit in Schelling's philosophy is that he places the point of the indifference of subjective and objective at the beginning; this identity is set up absolutely, without it being proven that this is the truth' (Hegel 1971 III p. 435). Hegel's claim is, then, that he can articulate the unity of subject and object - rather than postulate it, as Schelling does in the idea of 'intellectual intuition' - by explicating the initially indeterminate content of intellectual intuition. The System of Transcendental Idealism argues that intellectual intuition is manifested objectively in the artwork. For Hegel, it is merely a form of undeveloped 'immediacy' which philosophy must mediate by conceptualising it. How we are to read his claim is a classic example of the difficulty of deciding which Hegel we are understanding. On the one hand, the idea of beginning without any founding presupposition which cannot be articulated as part of the system seems to bring him into the fold of a pragmatic 'space of reasons' approach, for which philosophical issues are negotiated within communities without appeals to transcendence. On the other hand, the claim in the Logic that he can explicate, by the method described above, the overall structure of the truth which emerges from the splitting up of the immediate contact of thought and being seems to take us in the direction of the absolutisation of objective spirit. I do not wish to adjudicate on this matter here, and my real aim is to explore it in relation to Hegel's account of aesthetics. The difference we are concerned with is, then, between philosophies which retain the conviction that the Cartesian separation of thought and being is untenable, but which never fully square the circle by demonstrating how this is so in an immanent account of their relationship, and a philosophy which claims to have means to solve the problem by articulating it in a new kind of conceptuality. This difference, as the contrast between Idealist and Romantic aesthetics suggested, is often manifested in the differing approaches to art of such philosophies. 


\section{Self-recognition}

Hegel's position becomes easier to understand if one sees it in its historical context. The turn to the subject in modern philosophy parallels the development of modern individualism and is essential for the development of aesthetics. Habermas suggests the main contradiction involved in this development, which German Idealism tries to overcome: 'subjectivity reveals itself as a onesided principle. It admittedly possesses the unprecedented power to produce a development of subjective freedom and subjective reflection . . but it is not powerful enough to regenerate the religious power of unification via the medium of reason' (Habermas 1987 p. 31). Whereas religion and mythology give an a priori basis for action and reflection, which is necessarily shared by others, a culture grounded in the individual's freedom to think and act selfinterestedly must necessarily generate new kinds of conflict. Who or what legislates on the issue of whether my freedom is not your enslavement? The power of Hegel's philosophy lies, as Pinkard suggests, in its demonstration of the need for mutual recognition as the ground of normativity in the modern world. Habermas puts this as follows: 'The experience of self-consciousness ... results for Hegel from the experience of the interaction in which I learn to see myself with the eyes of the other subject ... only on the basis of mutual recognition does self-consciousness develop, which must be anchored in the reflection of my consciousness in the consciousness of another subject' (Habermas 1974 p. 789). Here we arrive once again at a familiar issue, which goes to the heart of the most ambitious side of Hegel's project and of its employment in contemporary philosophy: Hegel relies on the structure of reflection, about which some of the thinkers we have already considered raised serious doubts. Quite simply, is the reflection of my consciousness in the other a sufficient criterion of self-consciousness? Hegel's claim is that it is only as reflected in the other that I become myself. But in what sense am I myself if my self-knowledge derives from what is not myself? The assumption must be that my immediate consciousness, which must already be in existence if it is to be reflected, only becomes its true self via the other. However, this does not give me the criterion by which I know it is $m y s e l f$ that is being reflected. If the 'mirror' in question is a shared language, then self-reflection will come about via the ability to use the signifier I. As the recent philosophy of mind has shown, though, this form of self-knowledge relies upon a non-propositional form of self-knowledge if I am to know that the predicates ascribed to the referent of ' $\mathrm{I}$ ' are to be ascribed to myself. Dieter Henrich argues that 'Hegel conceives the unity of opposites only dialectically, in terms of what results from their opposition. However, the phenomenon of the Self requires that this unity be interpreted as original and primordial' (Henrich 1982 p. 52), otherwise the I loses the immediate basis of selfknowledge which is the prior condition of reflexive self-awareness.

Clearly Hegel's overall conception cannot be dismissed simply on the basis of this argument. The importance of his conception is evident when it is 
employed as a way of legitimating legal structures which rely on mutual acknowledgement for the establishing of rights, in relation to claims about myself which I must make to others in a common language, or in the experience of love, where self-transcendence comes about by the acknowledgement of the essential role of the other in who I become (though this may not be as harmonious as a Hegelian conception can suggest). However, as we saw, there are dimensions of self-knowledge which cannot be construed in terms of the reflexive structure, of the kind we examined in previous chapters in relation to the notion of 'feeling'. If this is right, Hegel's model could lead to an account of the modern subject which fails to come to terms with one of its most essential dimensions, namely its irreducible individuality. In the view which argues for the limits of the reflection model it is precisely the ontological gap between myself and the other inherent in the fact of immediate self-consciousness which gives rise to the need for new forms of articulation and expression. While these forms are intersubjectively constituted - Beethoven uses many of the musical conventions of his time - they can yet be employed in unique, individual ways.

Let us see, then, how Hegel arrives at his position. The Phenomenology of Spirit $(P G)(1807)$ is an account of the stages of this process of 'self-recognition in the other'. It follows the developmental model of the $S T I$, but aims to show how the structure of reflection can be revealed to be present in the development of thought from its lowest to its highest stages. In the $P G$ self-consciousness is revealed not to be the prior principle it is in Descartes or the early Fichte. Selfconsciousness can only come about for Hegel via that which it is not: another self-consciousness. Without the other, I would remain in a state of unreflecting immediacy, like an embryo that never becomes a person. In reflection I can come to realise that consciousness only develops via its relationship to what it shares with others. Self-consciousness therefore depends upon self-objectification, the negation of itself as subjective, inward 'Cartesian' awareness. Only by selfdivision, by relating both to the object world and to the thinking of others (which is therefore in one sense not $m y$ thinking) can I achieve real awareness of myself. Consciousness itself $i s$ precisely this process of division: it is what it is via what it is not. This argument can help explain why Hegel has begun to come back into recent Anglo-American philosophy. His conception is, for example, in some respects congruent with an 'externalist' semantics, for which 'the content of sentences (and, derivatively, the content of beliefs and other languagedependent psychological conditions) is at least partly dependent on the determination of the reference in the particular context ... of the terms used in the sentence, and that reference depends on factors that are external to the speaker's body and brain' (Putnam 1999 p. 119). In this way the intension of a term becomes dependent upon its extension, so the two are inseparably linked. I shall return to the issue of Hegel and language later, but for the time being it should be initially clear how his way of refusing to separate subject and object can be connected to some important current philosophical positions.

The structure of reflection, in which the subjective is only intelligible via the 
objective, and vice versa, is present in Hegel's philosophy at all levels. In Hegel's terms: 'the subjective is that which transforms itself into the objective and the objective is that which does not remain as it is but which rather makes itself subjective. One would have to show via the finite itself that it contains the contradiction in itself and makes itself infinite' (Hegel 1971 p. 435). The finite is the domain of Kant's understanding, which can only ever synthesise the contingent data of intuition, and cannot know things in themselves. In the Encyclopedia Hegel rejects Kant's dualism with the following argument: 'the designation of something as finite or limited contains the proof of the real presence of the infinite, of the unlimited . . . there can only be knowledge of the boundary insofar as the unbounded is immanent in consciousness.' (Hegel 1959 p. 84). Kant's amareness of the limits on scientific knowledge and of the need for ideas of reason to make our knowledge of nature cohere is therefore the proof that mithin thought there is more than limitation. The constant move beyond each theory to more inclusive theories means for Hegel that there is nothing in principle outside what can be thought, because the truth comes about through the continuing process of determination by negation, without which we merely have the opaque abstraction of being, about which nothing can be said. Dieter Henrich suggests that 'Hegel took the decisive step towards the thought of the 'absolute, which ... is Geist, by reaching the thought of something finite that is an other in relation to itself' (Henrich 1982 p. 155). The phrase 'constant change' captures the essence of Hegel's argument. Gadamer puts it very aptly: 'What remains, what is real, is namely the fact that everything continually disappears ... constancy is, then, no longer the simple opposite of disappearing, but rather is itself the truth of disappearance' (eds Fulda and Henrich $1973 \mathrm{p}$. $113)$.

Hegel characterises Kant's notion of the understanding as follows: 'If the determinations of thought are attached to a fixed opposition, i.e. if they are only of a finite kind, then they are inappropriate to the truth which is absolutely in and for itself. ... Thought which only produces finite determinations and moves within such is called understanding' (Hegel 1959 p. 58). Reason is therefore constituted by our awareness of this limitation, which, of course, requires the limitation for it to be able to emerge as its dialectical counterpart. Henrich usefully describes the basic structure here: 'The absolute is . . . the finite insofar as the finite is nothing but the continual process of self-negation [Sich-selbst-Aufheben which has Hegel's threefold sense of negate, preserve, and elevate]' (Henrich 1982 p. 160). Hegel wants to prove that finite appearance, properly understood, is in fact the essence of reality. The notion of a 'thing in itself' apart from appearances is therefore empty and actually requires a complex philosophical reflection for the notion to result at all: 'The thing in itself . . expresses the object inasfar as one abstracts from it everything that it is for consciousness, from all determination of feeling as well as all distinct thoughts of the object. It is easy to see what is left, - the total abstraction, total emptiness' (Hegel 1959 p. 69).

The $P G$ sums up how appearance can be essence: 'Appearance is the coming 
into being and passing away which itself does not come into being and pass away, but is rather in itself and constitutes the reality and movement of the life of truth' (Hegel 1970 p. 416). The example of the plant Hegel uses in the preface illustrates what he means. Each stage of the plant, from seed to flower and back, negates, destroys the previous stage, but without the previous stage the subsequent stage could not become itself. The sequence of appearances that makes up the plant is equally a sequence of disappearances. Hegel's philosophy presents the essence of the plant (reality) as being a process in which each stage destroys the previous stage but in which the whole of this process constitutes the 'concept' of the plant, rather than just a particular stage, such as the flower, where it might be thought to be most itself. Without all the stages which enable it, there would be no flowering of the plant, so any account of the developmental nature of reality must incorporate this kind of necessity. The same idea applies to philosophy: Kant needs Leibnizian metaphysics even as he refutes and transcends it, and Hegel needs Kant as the other of himself. There seems, of course, to be no need for philosophers after Hegel. This either means that he can be regarded as bringing philosophy to an end or that subsequent philosophers will merely echo his principles, which is why Hegel these days is read at the same time as the inaugurator of post-metaphysical thinking and as a metaphysical system-builder.

The overall movement Hegel describes is only explicable via that which it is not, so the 'Idea', the whole plant from its emergence to its decline, is not subject to the same movement as the series of appearances of the plant, but is yet inseparable from them. Hegel's thinking is in this respect, as Henrich puts it, 'a dynamised Platonism' (Henrich 1982 p. 190). It rejects the idea that there is truth in the transient, sensuous world which does not have to relate to its other, the eternal:

If . . the Idea should not have the value of truth because it is transcendent in relation to appearances, because no object in the sensuous world that corresponds to it can be given, this is a peculiar misunderstanding via which the Idea is not granted objective validity because it lacks that which constitutes appearance, the untrue being of the objective world. (Hegel 1969 II p. 463)

Hegel can, like Schelling, be seen as arguing that the contingency of the material in which something is instantiated means the essence of that thing is what gives it its dynamic structure and intelligibility, not its passing manifestations. In the present context, though, the decisive issue is the claim that appearance is the 'untrue being' of the objective world. What does this mean for Hegel's conception of art?

Hegel argues that art can only be the 'sensuous appearing of the Idea', and not, as it was in the $S T I$, the organ of philosophy. As the idea of 'dynamised Platonism' suggests, the goal of philosophy is the explication of the Idea, the 'true being' of the appearances of the objective world, so the Idea entails the move beyond the contingencies of particular things. The Idea is at the same time 
inseparable from the appearances in so far as they, via their necessary self-negation, are required for its self-manifestation. The aim of Hegel's Logic is to work out the inherent contradictions in the notions we need for thinking at all, in order to characterise all possible modes of thought independently of the particular empirical content they can articulate. This leads Hegel to the following statement, which has troubled his interpreters ever since: 'The logic is accordingly to be grasped as the system of pure reason, as the realm of pure thought. This realm is truth as it is without veil in and for itself. One can therefore say that this content is the representation [Darstellung] of God as he is in his eternal essence before the creation of nature and a finite mind' (1969 I p. 44).

The Logic is therefore a description of thought which has no need to go through the historical stages of thought's development in relation to nature. This means that the pure forms of thought must in some sense precede both nature and history. Hegel's conception can, it seems to me, only be productively understood in terms of his rejection of the idea that the empirical world can provide the criteria for truth. Most charitably, it can be seen as a version of the rejection of what Wilfrid Sellars calls, in a famous essay which refers to Hegel's criticism of immediacy on its first page, the 'myth of the given' (Sellars 1997), the idea that sense data are an immediate, non-inferential foundation of certainty which are the ultimate court of appeal in epistemological matters. What causes the real trouble in interpreting claims of the Logic is the relationship between the Phenomenology's closely argued and historically based account of the genesis of the essential modes of thinking through the working out of contradictions in the historical forms of thought in society, and the Logic's use of a similar method which does not refer to history as such and makes such claims as the one just cited. ${ }^{5}$ It is not evident to me that Hegel himself was wholly clear about this relationship. We are therefore left with a tension, which has influenced Hegel's effect on modern thought ever since, between his radically modern sense of thought as being reliant solely on our social practices and their intersubjective justification, rather than on some form of immediate empirical access to the truth, and his systematic urge, which points back to earlier forms of metaphysics as the expression of the universalia ante rem.

The Encyclopedia puts the division of philosophy in the following order: 'I Logic, the science of the Idea in and for itself, II Naturphilosophie as the science of the Idea in its being other, III Philosophy of Geist as the Idea which returns to itself from being other' (Hegel 1959 p. 51). Again, interpretation of this is controversial, but it seems clear that this is a form of Idealism which regards the truth as beyond the contingency of material nature and history, and, above all, as being accessible to a self-correcting mode of thinking of the kind explicated in the Logic. The Logic relies on the following assumption: so far in the history of thought every approach to truth has revealed itself to be relative and transitory. In consequence, the establishing of an account of the absolute cannot be another version of the attempt to fix an absolute principle, but will instead make relativity, the movement of negation, the source of the accessibility of the 
absolute. While the process of revealing this is temporal, what is arrived at when the Idea is reached is not: 'Geist appears necessarily in time . . it appears in time as long as it does not grasp its pure concept, i.e. does not abolish time' (Hegel 1970 p. 584). Nothing transient or empirical can lead us to the absolute, which is the final mediation of the initial immediate, opaque concept of being: 'Being has achieved the significance of truth when the idea is the unity of the concept and reality; it is then only what the Idea is. Finite things are finite inasmuch as they do not completely have the reality of their concept in themselves, but need other finite things to have it' (Hegel 1969 II p. 465).

The proximity to Schelling's identity philosophy is clear: this is a version of the Spinozist principle of the dependence of the particular upon other particulars ad infinitum for its identity. Spinoza argues that mathematical infinities, such as the infinity of points contained in a finite geometrical figure, which mutually define each other by their not being all the other points, are determinate, rather than indeterminate in the manner of an infinite number expressed by $n+1$. In an analogous manner, Hegel insists that the dependence of things on other things for their identity does not lead to an infinite regress and so to an absolute which it is beyond philosophy's capacity to articulate. Instead the absolute for Hegel is present in the movement of articulation itself. The infinite is the finite's self-negation, not an endless progression or regression. The geometrical points which become themselves via their fully determinable relations to the infinity of other points in the figure suggest the structure upon which he relies. The presentation of the Logic must, then, avoid any ultimate dependence upon aspects of the 'finite' empirical world, as these must, in order to be thinkable at all, be secondary to the universal structures of contradiction and resolution of contradiction that make up the Logic itself.

\section{Music and the Idea}

Hegel's Logic is 'abstract' because it has to be independent of anything 'concrete' in the empirical world. Characteristically, in Hegel's terms, this actually makes it concrete, because without abstraction the truth of the concrete cannot be revealed. The apparently most concrete, the sensuous immediacy of what is in front of you as you read this sentence, is indeed 'abstract'. It omits all the complex mediations required for you to be reading it at all, such as the universally employable indexical notions of 'this' and 'here' which enable us to concretise our thought. The thought with which the Logic is concerned is, Hegel maintains, what enables us to reach the truth of the sensuous world:

The elevation of thought above the sensuous, the transcendence of thought over the finite to the infinite, the leap which is made with the breaking off of the sequences of the sensuous into the super-sensuous, all this is thinking itself, this transcending is only thinking. Animals really do not make such a transition; they remain with sensuousness and intuition; this is why they have no religion. (Hegel 1959 p. 75) 
Art's necessary connection to sensuous particularity means it is still connected to the animal realm in a way that the highest forms of absolute spirit are not. However, the very attempt to understand the Logic already suggests an important problem. Thinking in pure thought must be intransitive: it must be thinking about thinking itself. If it were not, the infinite would, in order to be communicable, become dependent upon finite objects, in the manner of theological ideas which require symbols, or of Kant's 'aesthetic ideas'. These perceptible images stand for something non-concrete in the realm of ideas. Representing the infinite in thought gives rise to the difficulty that any representation limits what is to be represented, which was the whole point of Hegel's criticism of the immediacy of sensuous representation and of his stress on thought's ability to transcend the finite.

Kant's notion of the sublime offered one way of suggesting how one might 'represent' the infinite capacity of thought. In the experience of the sublime the subject negates anything sensuous precisely because of its orientation towards 'ideas of reason'. The impetus to do this is, though, occasioned by a feeling evoked by the sensuous. It is the failure of the sensuous to represent the infinite which constitutes our access to the infinite, an idea which, as we saw, is closely linked to the Romantic conception of the absolute. Hegel's attempt to transcend the sensuous is more ambitious, but gives rise to suggestive difficulties. The problem most often highlighted in the Logic by its critics concerns how the move is made from pure thought to nature. Schelling points to Hegel's problem in the Munich lectures cited in Chapter 4. The decision to think about thinking cannot be 'real [mirklich] thinking. Real thinking is that through which something opposed to thought is overcome. . . Hegel himself describes this movement by simple abstractions, like being, becoming, etc., as a movement in pure, i.e. unresisting ether' (Schelling I/10 p. 141). Schelling uses the example of poetry as an analogy to the problem facing a philosophy of pure thought. ${ }^{6}$ In what way are the moves in thought of the Logic 'real'? Poetry can, he maintains, represent a 'poetic soul in relation to and in conflict with reality. ... But poetry can also have poetry in general and in abstracto as its object - it can be poetry about poetry' (I/10 p. 141). He cites Romantic 'poetry about poetry', claiming that 'no one has held this poetry to be real poetry' (I/10 p. 141). The idea of such Romantic poetry is, as we shall see later, closely associated with music because of its negation of referentiality. Is there, then, really a difference between the Logic's status as thought about thought, and poetry about poetry?

This is not merely a rhetorical question based on a tendentious analogy: one of the ways in which Hegel himself attempts to explicate the movement of thought in the Logic is by the example of music, the form of art which is most obviously intransitive. Adorno often makes a link between Hegel's Logic and Beethoven's sonata movements, such as the first movement of the Eroica symphony. For Adorno the sonata movements which triumphantly resolve the contradictions they have set themselves in their opening material function in much the same way as the Logic. The point of the Logic is that it should resolve 
its own initial self-generated contradictions in order to create a self-comprehending totality. Music, on the other hand, does not necessarily aim to reveal the truth about the world, even though it may give deep insights into the nature of our being. Hegel's problem is that he must show how his self-contained construction can indeed become transitive. The complexities here are enormous, not least because of the very different ways in which the Logic is understood. However, it does seem clear that the Logic must be both self-contained and able to claim that it exhausts the ways in which the world can be articulated. This is, of course, what Hegel's opponents think leads to an unnecessary foreclosure of our possible forms of access to the world. The very fact that no serious scientist would feel the need to be aware of the Logic, even though there is no doubt that, as Schelling admits, many of its conceptual moves can be enormously productive, suggests that its status may have more in common with a work of art than its advocates would like to admit.

Hegel links musical harmony to his notion of the 'concept' in more than one place in his work. For Hegel, as we saw, the 'concept' includes the whole process of which it is the concept: the process and the concept are identical in his specific sense of being unable to be what they are without each other. The concept 'tree' therefore does not refer to the transient empirical object I have in front of me, nor is it my thinking of a tree when I cannot see one. Instead, the concept includes all the stages of the tree that have preceded what I might now see (as well as what will succeed these stages), and their reflection in my and others' thinking. Without the process of thought the object could never be revealed; indeed, the object itself would, as Kant argued, never be a determinate object at all. Seeing a tree 'as' a tree involves a consciousness that sets itself against its object, but in opposing itself to the object it also relates itself to it: the thought without the object would be empty. The concept in Hegel's sense obviously cannot be thought by an individual subject all at once, but if the concept is to be 'of' the object it must take us beyond the contingency and temporally determined nature of the particular. Hence, as we saw, his rejection of the immediate as a locus of truth, and his requirement that thinking be 'speculative', in the sense of refusing to take finite propositions about reality as definitively true. The ultimate result is that the highest concepts must be the most abstract and thus able to include all concrete particulars within themselves. How does Hegel himself link his view of the concept to music?

Hegel's argument refers to diatonic music, music in a specific key, which, though it may leave this key, will return to it. In the section of the Logic on 'elective affinities' (where he links music and chemistry) music is an analogy for his whole method: 'the single note [like the empirical phenomenon] has its sense only in the ... connection with another note and with the sequence of other notes. . . The single note is the tonic of a system but just as much again a member in the system of every other tonic' (Hegel 1969 I p. 421). The note C only becomes $\mathrm{C}$ by its negative relation to other notes in the key system: it is not $\mathrm{B}$ or $\mathrm{D}$, etc. $\mathrm{C}$ can be the tonic of the key of $\mathrm{C}$ if it relates to the other notes as 
that which defines their harmonic role, but it can just as easily be the dominant of $F$ or the subdominant of $G$ if these are the defining note of the key. Hegel sees an analogy of this kind of relationality to the way in which elements such as carbon or hydrogen are combined in compounds. His view of how notes gain their identity is, of course, also analogous to the way a differential view of language characterises the signifier, which gains its identity via its relations to other signifiers, not by anything substantial within itself. In this conception music is therefore 'reflexive' in the sense we have seen: each element becomes what it is by needing the other elements to be itself.

In the Aesthetics Hegel links his idea of the 'concept' to the major triad, the basic unit of consonance in diatonic music (for example, the chord CEG). The triad expresses the 'concept of harmony in its simplest form, indeed [it expresses] the very nature of the concept. For we have a totality of different notes before us which shows this difference just as much as undisturbed unity' (Hegel $1965 \mathrm{II}$ p. 296). The unity of the differing notes is a higher form of articulation than the single note's existence in isolation, an isolation which in fact does not allow it to be determinate at all. Something can only be a note, rather than a pitched frequency, via our capacity to hear it as related to other pitches. Just repeating $C$ would be rather like saying 'Hari Krishna'; adding E and G combines difference and identity to make something whose relations establish a whole series of possibilities for transitions to new combinations of notes. The significance of diatonic music is, then, not only based on consonance, in the same way as advance in the sciences is not just based on agreement. Hegel regards musical dissonance, of the kind introduced by the dominant seventh (B flat in the key of $\mathrm{C}$ ), as creating tension that must be resolved, and this forms the crucial link to the argument of the Logic.

Dissonance, Hegel asserts:

constitutes the real depth of notes [Tönen] in that it also progresses to essential oppositions and is not afraid of their severity and disunity. For the true concept is admittedly unity in itself but this subjectivity negates [Hegel uses the verb aufheben with its triple meaning of negation, preservation and raising up] itself as ideal transparent unity into its opposite, into objectivity, indeed it is as the simply ideal itself only a one-sidedness and particularity . . . and only truly subjectivity when it goes into this opposition and overcomes and dissolves it (II p. 297).

The next stage, then, has to be the resolution of dissonance: 'Only this movement, as the return of identity to itself, is the Truth' (II p. 297). The analogy between music and the overall movement of the Logic is very evident here. The famous C sharp in the opening bars of the Eroica Symphony could, for example, be understood as 'subjectivity', in the form of the self-contained unity of the tonality of E flat, 'negating itself' into what is opposed to itself in order to articulate its own content more fully. The symphonic sonata movement finally resolves this tension by revealing its goal some 400 bars later when the $C$ sharp recurs in the context which retrospectively gives its initial occurrence its true 
significance. In consequence, rather than having remained 'one-sidedly' within itself, the home key with which the movement eventually concludes has become deepened via its relations to what temporarily 'dissolved' it. Seen from the end of the movement, the 'negativity' of the famous dissonance thus becomes a way of expressing unity at a higher level.

The Logic expresses the same idea for philosophy: ultimately thought and its object are in harmony, but this can only be revealed by their opposition and reconciliation. In the same way as all the notes of the chromatic scale may appear in a symphony at some point, and at the end will become part of the path to the re-establishing of the tonic key, the divisions involved in conceptual thinking are integrated into the teleology of the Idea, a sort of ultimate harmonic resolution. The power of this conception can perhaps best be experienced in a huge symphony like Bruckner's Eighth, where the sheer length and complexity of the path to the final resolution make that resolution into the major tonic key so overwhelming. In such a conclusion all the preceding tensions become retrospectively oriented to their goal. The beginning and the end in music of this kind require each other, as they do in the Logic: only at these two points is the identity of subject and object complete. In the first they are united in an unarticulated, 'immediate' manner, which has to be split up to make it manifest, in the second in a fully articulated manner, once the process of contradiction resulting from the initial immediacy has been resolved by showing how it must be mediated. From an 'immediate' $E$ flat in the opening chord of the Eroica, which only makes sense because it creates the expectation that more must happen to give it its sense, one moves to the end of the movement, where the contradictions that can give the initial chord its sense have been laid out and resolved.

The 'symphony' of the Logic culminates like this:

The identity of the Idea with itself is one with the process [of differentiation]; the thought which frees reality [Wirklichkeit in the sense of empirical reality] from the appearance of purposeless changeability and transfigures it into the Idea, must not imagine this truth of reality as dead tranquillity, as just an image, dull without drive or movement . . . the Idea, for the sake of freedom which the concept achieves in it, also contains the hardest contradiction in itself; its tranquillity consists in the sureness and certainty with which it eternally creates contradiction and eternally overcomes it. (Hegel 1969 II pp. 467-8)

A theme emerges, disappears, reappears in changed form, and is finally made part of a wider movement, which ends such that the varying themes, which contrasted with and replaced each other, are seen as belonging together. A symphony does this, though, for a listener who hears the elements and feels their effect as a coherent unity. The symphony can consequently manifest affective coherence by making sense of what may initially feel merely negative.

It is not surprising therefore that in the nineteenth century the symphony began to take on many of the roles formerly filled by religious observance, offering an affective replacement for theological forms of meaning. ${ }^{7}$ What func- 
tion can the Logic be seen as fulfilling in this respect? As we saw, Hegel does refer to the Logic in theological terms, as offering the 'representation [Darstellung] of God as he is in his eternal essence', but who the 'listener' is supposed to be in relation to the Logic is unclear. Schelling says that the Idea at the end of the Logic is: 'subject and object, conscious of itself, as the ideal and the real, which thus has no need any more to become real any more and in any other way than it already is' (Schelling I/10 p. 152). This interpretation reinforces the sense that the Logic is self-contained like a symphony, which need represent no more than itself - though it can reveal new aspects of existence if employed in the right contexts by specific empirical individuals. ${ }^{8}$ That, however, is not the ultimate intention of Hegel's text.

What seems questionable is that in the Logic the Idea really does seem to develop immanently in the manner of a theme in an organically integrated piece of music, where the material posited at the beginning develops a whole world out of itself. This is, of course, at the same time also part of the fascination of reading Hegel's amazing text. Schelling, however, questions why, if the development is completely immanent, the Idea needs to prove itself by, as Hegel puts it, 'releasing itself as nature' from itself: 'But for whom should the Idea prove itself? For itself? But it is that which is certain of itself . . and knows in advance that it will not be destroyed in being other; for the Idea this struggle would be completely pointless. Should it, then, prove itself for a Third, for a spectator? But where is the spectator?' (I/10 p. 153). The essential problem is that Hegel's avoidance of a founding presupposition seems to require the conjuring away of the contingency of being, in order to allow it to be integrated into the overall system: hence the claims that being and nothing are identical, and that the Idea releases itself as - therefore non-contingent - nature at the end of the Logic. On the one hand, what Hegel is trying to do can rightly be seen as vital, as it also is for Schelling, to the project of getting away from Cartesian hang-ups about thought's contact with the world, by making it clear that such contact is inescapably part of what thought is. On the other, it is not clear that the aim of obviating scepticism-inducing splits between subject and object necessarily leads to the idea that thought can therefore use its immanent self-description to obviate contingency.

Schelling again makes the main objection clear:

In Hegel's philosophy the beginning relates to what follows as a simple nothing, as a lack, an emptiness, which is filled and is admittedly felt as emptiness, but there is in this as little to overcome as there is in filling an empty vessel; it all happens quite peacefully - there is no opposition between being and nothing, they don't do anything to each other. (I/10 p. 137)

The tensions and contradictions of reality in Hegel already have within them their resolution because they can be comprehended in thought, and, as such, follow the necessities of the infinite movement of thought. Again, the parallel to music can help here: the dissonance in a symphony already has the telos of its 
resolution within it, as does Hegel's division of being and nothing. Hegel's recourse to music as part of the attempt to represent the movement of the Logic can, then, be seen as pointing to the crucial difficulty in his philosophy. Hegel himself takes a medium, music, which allows reconciliation in the realm of art, thus in what he regards as the realm of the sensuous manifestation of the idea, as a metaphor for the reconciliation required for the articulation of the absolute in philosophy. However, music does not positively assert what this resolution is, leaving a freedom to explore never finally determinable possibilities. This is one of the key sources of its central role in the culture of modernity. In this respect what music can be seen as gesturing towards is akin to the Romantic absolute, which serves as a reminder of the inescapable imperfection of our conceptions and as a spur to further exploration. ${ }^{9}$

We come here to a vital issue which affects this whole debate. Music's importance for aesthetics from this period onwards relates to its existential significance as a means of making at least temporary sense of finitude - hence its taking up of some of the roles of a declining theology. Music's existential significance depends in many ways upon its ability to articulate feelings. Crucially, though, music is also connected to conceptual developments, as the links of Beethoven's music to the Logic suggest. This combination of a unique ability to articulate feelings and a connection to concepts is the source of music's cultural significance. If we are to understand music, rather than merely remain at the level of unarticulated feeling in relation to it, we must therefore incorporate affective and conceptual dimensions into our understanding in ways which are not reducible to each other, and this clearly cannot be achieved from the conceptual side alone. However much we rely on the ability of words to articulate music's significance, this does not exhaust the ways in which a piece of music can be understood - think of the way, for example, that dance or the use of images can illuminate dimensions of music which words cannot. In existential terms, the aim of the Logic would seem to be to enable us to become reconciled with the ultimately transient nature of all determinate things, including ourselves. The reconciliation is achieved via thought's ability to grasp the necessity of that transience if the world is to develop, and this leads to a non-transient, dynamic system of linked concepts. However, despite Hegel's claim that the Idea 'contains the hardest contradiction in itself', it seems strange that he takes no account of the idea that the 'hardest contradiction' also includes the affective dimensions of thinking existence. What does the 'tranquillity' Hegel thinks is part of the Idea's overcoming of contradiction consist in for any real person trying to come to terms with the necessity of transience? The question here is whether music involves dimensions which are never reducible to what philosophy can say about them, and, if it does, what importance is attached to these dimensions.

Hegel himself sees music, like other art forms, as only part of the prelude to the fully transparent and articulated concept of philosophy. Because music is 'completely abstract' (Hegel 1965 II p. 261), in the sense that it does not repre- 
sent things in the world, but only expresses 'subjective inwardness', it forms a subordinate stage in the realisation of the absolute. It is therefore also a lower form of art than representational forms like drama, which connect directly to conflicts in the social world. Implicit in this conception of music is the Logic's purported demonstration that being can become transparent to itself via conceptual articulation. The simple consequence is that philosophy would seem to have to be able to give the true account of what music is.

Once again we here come up against the question of how Hegel is to be understood. Take, for example, the history of modern music. At each stage of musical development there is a revision of the canon of the acceptable, which changes what can count as music. One way of understanding a Hegelian view of this would be that revisions in the theory of harmony, for example, are intrinsic to the 'concept' of harmony that is revealed at the end as the Idea of harmony. Each turn away from the established canon is contained in the Idea that emerges at the end of the process, which philosophy can articulate. What status does such a description actually have? It is clear that descriptions of this kind can indeed grasp the logic of complex musical changes in a manner which shows the often remarkable interconnectedness of apparently disparate phenomena. The development of Western harmonic thinking makes considerable sense in terms of music's increasing ability to incorporate contradiction into itself, though the advent of atonal music makes the matter more complex. However, such theoretical descriptions cannot deal with the relation of music to the dimension which Anthony Cascardi sees as decisive in Kantian aesthetics, namely its attention to 'the specific element in subjectivity that is "incapable of becoming an element of cognition"' (Cascardi 1999 p. 17) because it cannot be articulated by using the same words as everyone else. As we saw, Cascardi argued that the implication of the Critique of Fudgement was that: 'Feeling nonetheless remains cognitive in a deeper sense [than in the sense of 'cognitive' involved in the correspondence theory of truth as correct representation of the pre-existing object]; affect possesses what Heidegger would describe . . . as "worlddisclosive" power' (Cascardi 1999 pp. 50-1).

Hegel's view of music can, despite its vital insights into the development of modern art, be seen as lacking an adequate account of this world-disclosive dimension. His conception necessarily underplays the importance of a medium which does not per se represent conceptual ideas, but which is not devoid of meaning. I understand meaning in the present context as what is conveyed by any form of articulation that can disclose the world in ways which affect the conduct and understanding of life. If meaning is what can be understood, then music is meaningful by the very fact of its being music rather than noise. Hegel sees music as limited to 'subjective inwardness': I shall try to show in more detail below and in Chapter 7 that he therefore fails to exhaust the significance of music as a way of exploring both modern self-consciousness and the world. Although music cannot be said to possess general semantic content of the kind present in verbal language, ${ }^{10}$ the affective and other dimensions it reveals are not 
merely subjective and inward, as the way music and history influence each other can suggest. In order to be able to grasp why this is the case, an account of subjectivity must countenance dimensions which Hegel's excludes or regards as of relative insignificance. Despite his undoubted insights, Hegel's insufficiency in relation to music is, then, part of his more general problem with adequately theorising self-consciousness, and thus with his aesthetic theory.

\section{Language, consciousness and being}

However much artists rely both on material that is established by their predecessors and contemporaries, and upon a pre-existing natural language, aesthetic innovation in modernity is also inseparable from individual self-consciousness. How, then, does language relate to aesthetics in the present context? As Charles Taylor has contended, from around the 1770s onwards, language moves from being understood as the symbolic means of representing pre-existing ideas and of representing already-constituted objects in the world, to being understood as 'constitutive' or 'expressive' of what becomes intelligible to us. In this latter view language reveals aspects of the world and ourselves which could not even be assumed already to exist before their articulation in language. The vital consequence is that forms of articulation which are not understood as linguistic if language is conceived of exclusively in representational terms can come to be considered as linguistic if they disclose otherwise inaccessible aspects of the world and ourselves. Language involves a tension between its capacity for schematising universalisation - what Taylor calls its 'designative' function, which makes it the essential tool of scientific research - and the fact that individuals can come to feel this very generality as an obstacle to what they wish to say, which leads to the new awareness in this period of its expressive and constitutive functions. ${ }^{11}$ As I have tried to show, this is one of the main sources both of the changed views of music and literature, and of the changed artistic practices which emerge at this time.

In the Phenomenology Hegel claims that language is the 'existence (Dasein) of Geist' (Hegel 1970 p. 478), which helps suggest why his communitarian interpreters think he is so vital to contemporary debate. Elsewhere he already points to the roots of one of the divisions between semantic and hermeneutic approaches to language which is so important to philosophy today. In the Encyclopedia he says of language:

Because language is the work [Werk] of thought nothing can be said in it which is not general. What I only mean is mine, belongs to me as this particular individual; but if language only expresses the general I cannot say what I mean. And the unsayable, emotion, feeling [i.e. that which is particular to me qua individual] is not the most excellent, the most true but rather the most insignificant, most untrue . . . if I say ' $\mathrm{I}$ ' I mean myself as this person that excludes all others; but what I say, i.e. I, is what everyone is; I that excludes all others. (Hegel 1959 p. 56) 
Hegel's play on the word 'meinen', which means 'to mean', what is 'mine', and 'to be of the opinion', contrasts - in Platonic fashion - 'opinion', as uncommunicable private feeling, with 'truth', which is publicly available in the language of a community. Thoughts are therefore candidates for acceptance or rejection in a community in a way feelings clearly are not until they attain a public form as utterances or performances that can be responded to positively or negatively. Hegel's position is very close to Wittgenstein's rejection of the notion of a private language which can only be understood by one person. The idea is that a language could not intelligible as a language in such terms, being precisely something which is intersubjective and exists in the world rather than in the head. What Hegel maintains here is congruent with the interpretation of him, by Pinkard, Brandom and others, as a philosopher concerned with the public cashing-in of discursive commitments.

Hegel's argument depends on the main structuring principle of his philosophy, the 'identity of identity and difference'. The 'sign', the 'signifier' 'I', can only attain its meaning via its general application as a universal indexical, but it is thereby also able to designate my particular I. Once again, however, this raises the question of reflection that we considered above. Hegel's disqualification of 'feeling', the 'unsayable', relies on the assumption that what consciousness is is fully explicable via the general structure of its reflection in the other of language, so that my self-knowledge is necessarily propositional and thus of the same order as others' propositional knowledge of me. To be myself in these terms, as Ernst Tugendhat (for example, Tugendhat 1979) has argued, entails knowledge on my part that another person could use any of my first person ascriptions in the second or third person and the proposition would still refer to me. In much the same way as, for Hegel, I can only be myself via the structure of self-recognition in the other, which is the basic structure of Geist, in the semantic model of self-knowledge, which Hegel already adumbrates, language takes over the role of Geist by being what permits me to individuate myself from other world objects, including other subjects. What, though, apart from my own prior, non-reflexive acquaintance with what it is to be self-conscious - which is the basis of what makes language meaningful articulation, rather than just a sequence of noises - could 'cause' me in the first place to judge that a material event occasioned by a physical object in the world is a linguistic sign produced by another subject?

There seems here, as the Romantics argued via the notion of feeling, to be a prior dimension of self-consciousness which is necessary for the very ability to understand and use the term 'I'. The mere ability to perform the social act of saying 'I' does not have to involve this pre-reflexive dimension of my awareness. It is this dimension, though, which makes this performance more than something a robot can do and therefore opens up the complex realm of ethical interaction, where the alterity of the other is a fundamental challenge, rather than just a moment of my self-reflection. Now it is generally accepted that, as Hegel argues, the truth about anything in the world must be propositional. ${ }^{12}$ This also 
means, though, that such truth is essentially fallible, for the reasons Hegel gives in his account of the limitations of the understanding. All specific knowledge claims can, in the light of new knowledge, be re-classified under a new concept, or be described in a new vocabulary. However, self-knowledge of the kind at issue here does not have this status. Manfred Frank suggests why: 'Knowledge of myself as of myself does not depend on classification. But it also does not depend on identification [on ascribing a mental predicate to a singular term which stands for myself, so that 'I know that I $\phi$ '] - for how should I identify an object which could not be anything but myself?' (Frank 1991 p. 407). Frank also argues that: 'If self-consciousness cannot be characterised by the as-structure then it may no longer be described as conceptual knowledge (every concept relates indirectly - by virtue of an attribute which is common to many objects - to a content of thinking)' (1986 p. 34). It is precisely this irreducible dimension of self-consciousness which is not articulated by the words common to everybody that points to one of the reasons why aesthetics can play a disruptive role in Hegel's attempt to establish an all-inclusive philosophical system.

As we have seen, the truth of consciousness for Hegel lies in its articulation in the concept, not in the pre-reflexive moment of immediacy. For those recent philosophers, from Sellars to Brandom, who follow Hegel, the appeal of his position lies in the resources it offers for countering empiricism as the philosophy which tries to invoke a non-inferential, foundational access by the subject to the world, thus for countering the 'myth of the given'. The point about the Romantic objection to the reflexive account of self-consciousness is not, though, that there is some kind of foundational immediacy in what comes from the world as sense data, but that even our undoubted practical contact with things is only intelligible at all because of a non-inferential dimension of our selfconscious being. This dimension can only be made publicly manifest in forms such as music and gesture, which are not fully articulable by our propositional descriptions. Schleiermacher, as we shall see in Chapter 6, gives gesture a central role in his understanding of 'immediate self-consciousness', and he links gesture closely to music.

Friedrich Schlegel makes clear the difference of the Romantic position from empiricism in his Transcendentalphilosophie (which Hegel may well have heard as lectures in 1801). In doing so he shows that the alternative to Hegel need not be a return to scepticism-inducing empiricist assumptions: 'One has always regarded it as the greatest difficulty to get from consciousness to reality (Daseyn). But in our view this difficulty does not exist. Consciousness and reality appear here as the connected parts (Glieder) of a whole' (Schlegel 1991 p. 74). The disagreement lies in how philosophy relates to the understanding of this whole. The Romantics regard the sense of incompleteness generated both by our feeling of being's transcendence of what we can know and by our awareness of our own being's transcendence of our knowledge as the source of our continuing attempts to articulate in new ways. Elsewhere Schlegel argues, in much the same way as Hegel later will, against a strict Kantian separation of what is given 
to us from the world and what the world gives to us. This is the further main source of his conception of the whole as the necessary link between consciousness and reality:

The grasping of what is given demands spontaneity, one's own exertion, own activity. The smaller is the quantity of spontaneity which the grasping of the appearance demands, the more the appearance appears. There are no absolute maxima on either side; without any spontaneity there is no receptivity: and if all receptivity stopped, then the appearance would cease to be appearance and become a concept, for pure spontaneity. (Schlegel 19885 p. 170)

Appearance can, of course, never do this, so a philosophical system can neither seek a foundation by explicating the mind-world/spontaneity-receptivity relationship at the beginning, nor overcome their difference by fully explicating the structures of their interaction at the end. The difference from Hegel therefore lies in the way this position leads to the aesthetic as the location of affective and other ways of being which philosophy (and science) cannot definitively explain, and which require other modes of articulation.

The kind of thinking at issue here occurs in modern philosophy among those thinkers who are concerned that exclusive focus on a cognitive account of subjectivity omits key ontological questions. Sartre argues against the Hegelian position, for example, that 'the very being of consciousness, being independent of cognition, pre-exists its truth ... consciousness was there before being known' (Sartre 1943 p. 284). He illustrates the limitations of the reflexive cognitive model by the example of pleasure. Pleasure 'is not a representation, it is a concrete, full and absolute event ... The pleasure is the being of the consciousness (of) itself and the consciousness (of) itself is the law of being of pleasure' (p. 21). ${ }^{13}$ The development of the concept of pleasure which, as Hegel suggests, comes about by opposing it to its other and by its articulation in the language of a community, has as its precondition the existence of pleasure, which is not reducible to its subsequent conceptualisation, precisely because it can always be redescribed. Neither, of course, is pleasure conceived of in empiricist terms: its crucial attribute is its meaningfulness to the subject, not a meaningless immediacy. How, then, does this differ from Hegel?

The truth of consciousness emerges for Hegel via its reflection in universal structures. Self-consciousness is formed in social interaction, in a totality within which it can ultimately be subsumed. Because I need your consciousness to realise my own, the truth of consciousness is part of the universal structure of Geist which negates individual subjectivity, even though it at the same time realises itself through that subjectivity. Consciousness is thus implicitly already part of a self-reflecting whole, Geist, which our common capacity for thought can reveal. This idea recurs in the recent post-Wittgensteinian appropriations of Hegel, in which language, as Hegel suggests, is the 'existence of Geist'. In many respects this is a convincing approach to thinking about subjectivity in modernity. However, the question this approach raises with regard to aesthetics concerns the 
status of utterances or performances whose particularity, as Schelling argued, can take on a universal significance. This universality does not, though, result from art's being characterised by general concepts. The vital issue is rather how art can be of great significance in the most varied social and historical contexts. Instead of particularity being what is to be overcome by the concept it here becomes the source of a kind of significance which has its omn form of universality. The problem in Hegel's aesthetic theory is basically that the truth of a work of art emerges most completely via its conceptual articulation, which therefore leads one beyond the art work towards philosophy. As we shall see, the hierarchy of arts in his system, in which the art closest to philosophy, literature, is given the highest status, depends on this priority of philosophy before art.

The power of Hegel's position is evident in its importance for such disciplines as the sociology of literature. Here we encounter a familiar dilemma in modern thought, which is echoed in sociological debates over whether individual actors determine social developments or whether the structures within which they act are the real determinants. Hartmut Scheible sees Hegel's achievement as making possible the analysis of the historical development of art as part of the overall development of modernity. The price for this historicisation of the truth of art is, though, that 'with the turn towards an aesthetics of conceptually fixed content the individual subject ceases to play a role in the constitution of aesthetic truth' (Scheible 1984 p. 290). Art becomes comprehensible to an unprecedented extent, but the result is both a reduction of the semantic potential of art and a repression of the subject's individuality in the name of a general understanding of the work's relationship to its context and to the development of art as a whole. The ensuing tension in aesthetic theory, which still determines contemporary debate, lies between theorists who wish to sustain the notion of art's irreducible semantic potential, and theorists who wish to subordinate art to other forms of understanding, such as philosophy, history, or the analysis of ideology. ${ }^{14} \mathrm{~A}$ recent version of something like Hegel's position is represented by Arthur Danto (though, as we shall see, Danto is in some respects closer to the Romantics): 'For when art attains the level of self-consciousness it has come to attain in our era, the distinction between art and philosophy becomes as problematic as the distinction between reality and art. And the degree to which the appreciation of art becomes a matter of applied philosophy can hardly be overestimated' (Danto 1983 pp. 1-2).

One of the reasons for questioning Hegel's position can be illustrated by the following example. In The Man Who Mistook His Wife For a Hat Oliver Sacks describes the case of a patient he calls the 'autistic artist'. Sacks's patient moves from almost total non-communication to being able to express himself in drawings of natural objects which have a peculiar intensity. He does not learn to communicate linguistically. Sacks says of the autistic:

Lacking, or indisposed to, the general [they] seem to compose their world entirely of particulars. Thus they live, not in a universe, but in what William James called a 'multiverse', of innumerable, exact and passionately intense particulars. It is a mode of 
mind at the opposite extreme from the generalising, the scientific, but still 'real', equally real, in a quite different way. (Sacks 1986 pp. 218-19)

Looked at in Hegelian terms, the truth of an autistic world is simply that of particularity which has not been transcended in order to reach its concept. The patient is caught in the particular via their lack of a means of general articulation. Sacks's point is that there are lessons to be learned from his patient. The 'autistic artist' escapes dominant attitudes to the natural world which are increasingly the norm in the Western world: he does not, so to speak, 'subjectify being'. He relates to the particular plant, animal, or whatever, in an intense manner which 'normal' people lack, and his means of communicating his experience is images, rather than intrinsically general words.

I do not wish to argue here that mental pathologies can lead to profane revelation, but given the grave difficulties in adequately identifying and understanding many such pathologies, an openness to the possibilities they may also offer is essential. If one accepts the fact that these pathologies need not just be the unsolved problems of medical and social engineering, one must take seriously the reality that Sacks refers to, the existential reality of a self-consciousness which can never be wholly transcended into a universal structure. To take an example closer to Hegel: artistic creation and mental pathology are often closely linked in the nineteenth century. Hölderlin - once Hegel's friend, and, as we saw, a philosopher of some considerable talent - eventually became mentally ill. Before descending into madness he produced poems which use language in a manner that still challenges us today. Hölderlin's experience is characteristic of some of the most significant modern artists: they exhibit an almost unbearable tension between individual consciousness and the collectively acknowledged systems of signs in which that consciousness tries to express itself. ${ }^{15}$ Hegel's view of language, and of aesthetics, tends to underplay this tension in the name of language as the general means of articulation. Vital as accommodation to the language of a community is, there is always a risk that such accommodation can add to the repressions within that community. This is why individual attempts to break out of existing communicative resources are at the same time both dangerous and necessary, and this needs to be reflected in any philosophical account of the relationship between the individual and the universal. $^{16}$

Paradoxically, as anyone who has read much Hegel will be more than aware, Hegel's own relationship with language is hardly an illustration of his theoretical point. Not the least fascination of Hegel's philosophy is the struggle for articulation carried on within his texts. It is clear, for example, that the Phenomenology's significance is not exhausted by the philosophical arguments which can be extracted from it. The dimension opened up by Hegel's innovative philosophical language cannot just be said to be an inferior manifestation of what his work communicates. Clearly, if his philosophy did conclusively obviate the philosophical questions which dominate the Western tradition by resolving the problem of the absolute, the manner of its presentation would be a secondary 
consideration. The real historical effects of the Phenomenology have, though, more often depended upon the imaginative impulses conveyed by such passages as 'Lordship and Bondage', or the reflections on Antigone, than upon the grand systematic conclusions. The further point here is that the boundaries between philosophical and aesthetic texts are not fixed. As I have tried to suggest with regard to the Logic, the power of Hegel's work may in certain respects have much to do with its aesthetic dimension that is not simply a function of the propositions of which it consists.

This somewhat heretical view of Hegel may help us come to terms with an important element of thinking about language in this period. It is no coincidence that other thinkers in Germany are increasingly concerned, like Novalis, with the 'unsayable', and this has for too long been understood as though it were solely a mystical Romantic quirk. However, this notion is important for its reminder that general resources of articulation always exist in a potential tension with the non-propositional aspects of the subject's self-consciousness. The subject is here not just regarded in terms of its being transparent to the general linguistic community, or, to cite the post-structuralist version of the same issue, in terms of its being an 'effect' of the textual mirrors in which it reflects itself. Consciousness, as we have already seen in Schelling and the Romantics, is instead seen as having a ground which can never be fully transparent to it and this gives rise to the individual need for expressive resources which cannot be resolved into philosophical explanation. Thinking about these issues is, as we have seen, linked to theoretical reflection on language, to aesthetic theory, and to music. Hegel's attempt to resolve the contradictions in the principle of subjectivity (its 'one-sidedness', as Habermas put it), and his announcing the 'end of art', coincide with the emergence of the kind of reflection upon language described by Taylor, and with the related flowering of musical creativity. These changes constitute a constellation which will concern us in the coming chapters. Although Hegel inaugurates vital dimensions of historical reflection upon aesthetics, he at the same time does not countenance that some of the positions he develops may be inadequate to the phenomena they are supposed to grasp. Let us therefore now turn directly to the Aesthetics.

\section{The Idea as sensuous appearance}

Hegel famously defines beauty as the 'sensuous appearing of the Idea' (Hegel 1965 I p. 117). Qualifying beauty as the 'sensuous' appearing of the Idea puts beauty into an inferior position to philosophy: its reliance on the sensuous brings with it a dependence upon what is transient. Even artworks, which, because of their status as manifestations of Geist, are not subject to the same natural iron law of disappearance as living objects possessing natural beauty, cannot sever all connection with the transient. Only the Idea, speculative philosophy in Hegel's sense, can do this. The problem here is again how such a claim is to be understood. On the one hand, Hegel's claim prefigures Max Weber's 
account of the processes of rationalisation in the modern world, in which modern societies are increasingly determined by universal forms of thinking and organisation which must exclude the particularist kinds of thought necessary for art; on the other, if 'philosophy' is understood in an emphatic sense and fails in its task of overcoming contingency, Hegel's model needs to be questioned in the light of other ways of thinking about the art/philosophy relationship. The detail of Hegel's argument does, though, lead at the same time to some very important insights.

Hegel insists, against Kant, that beauty in works of art has a higher status than natural beauty. This might seem strange, given the apparent temporal priority of natural beauty before artistic beauty. However, Hegel can actually be understood here as revealing something vital about aesthetics and modernity. His intention is, as always, to undermine any kind of reliance on 'immediacy'. He therefore denies that there is immediate pleasurable contemplation of the natural world - 'natural beauty appears only as a reflex of beauty which belongs to Geist' (Hegel 1965 I p. 14) - and the history of Western art tends to back up his suspicion. Before the modern period the beauty of landscapes was generally not a central focus of pictorial art, the landscape serving either as mere background or as a source of symbols of God's creation. The idea that nature can be beautiful per se simply does not square, for example, with the widespread sentiment in Europe prior to the second half of the eighteenth century that mountains involving dangers to human beings are ugly. The appreciation of wild nature for its own sake is indeed predominantly a modern phenomenon, and landscape painting first emerges in a major way in a country, Holland, which is itself largely a result of human control of nature.

There is, however, another dimension to this important historical point. Hegel's argument relies on the idea that nature is seen by pre-modern societies as a threat to be overcome in the interests of self-preservation, so that the appreciation of natural beauty is linked to the subject's control over nature. The danger here is that the relationship can be understood in a one-sided manner, so that it is only to the extent that mind develops in relation to the ability to control nature that the beauty of nature can emerge. The problem in questioning this one-sided relationship is that one seems to have to rely on a dogmatic conception, of the kind suggested in Kant's invocation of a 'code through which nature talks to us figuratively in its beautiful forms' (CF B p. 170, A p. 168), to do so. On the other hand, the complete refusal to countenance the idea that a certain stage of the development of human thought might render it open to the new resources of a nature which was previously seen as divine creation and as a threat beyond our control repeats precisely the problem Kant was concerned with in the Critique of Fudgement. How do we avoid a wholesale split of our subjective being from nature? While it is clear that there is a socially mediated history of the appreciation of natural and artistic beauty, this does not mean that natural beauty is wholly accounted for by what we know of the history of its emergence. 
In Aesthetic Theory Adorno approvingly cites Verlaine's line, 'la mer est plus belle que les cathédrales' (Adorno 1973 p. 103) to suggest the complex dialectic involved in this issue. In order to be aware of the superior beauty of the sea one might first need cathedrals, a result of Geist, but for Gothic cathedrals one also needed the forms of trees for Geist to mediate. As Adorno puts it: 'That the beauty of nature is resistant to determination by mind misleads [Hegel] into a short-circuit in which he devalues that in art which is not mind qua intention' (p. 407). For Adorno 'intention' is the basis of instrumental reason, the control of things for human purposes. Natural beauty is therefore a manifestation of a non-instrumental relationship to nature, a result of contemplating nature as appearance: 'Like the experience of art, aesthetic experience of nature is an experience of images' (p. 407), that is, not of something concrete that is to be worked upon or conceptualised. Adorno's contention suggests why this question can lead to a central issue in contemporary philosophy.

When Rorty claims that we must give up the idea that we are answerable to anything other than ourselves, because the alternative is the kind of obeisance to a mythical something beyond ourselves characteristic of the ChristianPlatonic heritage, he limits his argument to the cognitive and ethical spheres. His aim is to say farewell to 'representational thinking', in which thought is to correspond to the 'ready-made' world, and to abandon the search for absolute ethical certainties. Rorty thereby wishes to avoid what he sees as an unproductive 'public' pursuit, as a 'project of social cooperation', of what he thinks belongs in the sphere of 'private transcendence', namely 'projects of individual self-development' of the kind associated with aesthetic experience. However, this leaves too little space for the idea that learning to be answerable to nature for its own sake, rather than just for the sake of our own survival, may, as Heidegger and Adorno suggest, involve a dimension of thinking which itself is able to question some of the effects of the Christian-Platonic metaphysical tradition.

One point of the change in modern thinking which results in the emergence of aesthetic theory is precisely the acknowledgement of the need to attend to the resources opened up by the awareness of nature's value for its own sake. This awareness plays a vital role in the genesis of new kinds of aesthetic awareness and, as it already did for Schelling, in the genesis of ecological thinking. The emergence of concern with natural beauty is indeed, as Hegel claims, a historical stage in the development of Geist, but this concern can also be understood both as suggesting the limits of subjective reason and as a warning about the dominant developments in the modern history of Geist, of the kind that lead to ecological devastation. The underlying question here is whether what previously belonged to 'religion' as a public 'project of social cooperation', must, in the light of secularisation and rationalisation, now be located in the private domain. Any positive claims about the significance of natural beauty evidently do run the risk of mere dogmatism if they are seen as substantiating a strong teleological view. However, the wholesale exclusion of what can be learned from 
the appreciation of natural beauty leads to an even more questionable impoverishment of thought that itself has evident public, political effects.

Adorno suggests how one might conceive of a position which steers clear of the danger of invalid teleology and yet sustains the question of a more than cognitive link to nature when he claims: 'We are really no longer ourselves a piece of nature at the moment when we notice, when we recognise that we are a piece of nature', so that 'what transcends nature is nature which has become aware of itself' (Adorno 1996 pp. 154-5). This recognition is closely linked to the significance of natural beauty as a means of appreciating what is beyond a merely narcissistic relation to the objective world. The question is to what extent Hegel's conception of the relationship between natural and artistic beauty entails the kind of one-sidedness suggested by Adorno. This takes us to the heart of Hegel's conception.

It is not, of course, that Hegel ignores the significance of what we can learn from aesthetic experience. In the Aesthetics he states, underlining his view of the primacy of artistic over natural beauty, that: 'Far from being simple appearance the appearances of art should be seen as possessing the higher reality and the truer existence in relation to normal reality' (Hegel 1965 I p. 20). Unlike immediate empirical reality, which appears to offer the most obvious source of truth, but which requires universal forms of thought to be intelligible at all, artistic appearance 'points through itself to something spiritual [Geistiges]' (I p. 20). A picture of a mountain involves more 'mediation' than just looking at a mountain in a natural landscape: think, for example, of a Caspar David Friedrich painting, which will probably not have been painted from a direct apprehension of the mountain, but rather as a subjective projection in a studio. The painting can then change how we are able to appreciate real landscapes. However, even this does not reach the level of abstraction which Hegel sees as vital to modernity.

The central issue emerges when Hegel makes the famous claim that art, because it is 'limited to a distinct content', no longer 'fulfils our highest need'. 'Thought and reflection have overtaken [überflügelt in the sense of 'flown over'] art' (I p. 21). The arguments of the Logic about the need to transcend the particular and the transient are therefore carried over into a historical assessment of art's relationship to philosophy. The higher truth, as we saw, belongs to other modes of reflection, culminating in Hegel's system:

The constitution of reflection of our contemporary life makes it necessary, both in relation to the will and in relation to judgement, to establish general view-points and accordingly to regulate the particular, so that general forms, laws, duties, rights, maxims are valid as the bases of determination and are the principle rulers.... The science of art is thus in our time much more necessary than in times in which art for itself as art provided complete satisfaction. (I p. 21)

Aesthetic theory emerges for Hegel at the time when aesthetic praxis is no longer central to the articulation of truth. Earlier in the chapter I cited Danto's comment about the contemporary art scene, that 'the degree to which the 
appreciation of art becomes a matter of applied philosophy can hardly be overestimated'. For Danto, though, this state of affairs also leads to questions about the borderlines between philosophy and art, art having become increasingly self-reflexive - in this respect at least he comes closer to Adorno and the Romantics than to Hegel. Yet again the difficulty lies in interpreting the appropriate context for Hegel's remarks.

There is no doubt that the establishment of the modern cultural spheres of natural science and law depends in important respects on overcoming the particular and on setting up universal ways of thinking that can be applied to particular cases. The fight against feudalism and the arbitrary authority of the Church in the name of universal rights and scientific objectivity are inescapable necessities in modernity. However, it is clear that the process of modern rationalisation has not led to the triumph of philosophy, if philosophy is conceived of as the discipline which is to bring about the unification of the ever more specialised spheres of human practice. ${ }^{17}$ The real nature of modernity consists in the takeover of the tasks of philosophy by the natural sciences, and there is no doubt that this has major effects upon the nature and significance of art. Hegel sees one side of this, but he does not ponder the consequences of the failure of philosophy to integrate the differing spheres of practice. As Heidegger suggests, Hegel's claims for philosophy actually lead in the direction of the subordination of philosophy to the sciences, as part of the fate of 'Western metaphysics'. The unifying function which Habermas refers to as 'the religious power of unification via the medium of reason' is not fulfilled by philosophy, of a Hegelian or any other kind. Neither, of course, does modern art achieve this unification, despite the hopes suggested in the $S T I$ and, as we shall see in Chapter 8, by Nietzsche in The Birth of Tragedy. How, then, is Hegel to be understood here? The answer to this question will affect how one conceives of the task of modern philosophy and how one understands the role of art in modernity.

The contemporary renewal of interest in Hegel relates not least to fears about scientism and to the desire for philosophy to make a contribution to the problems which arise from reflection on what Habermas describes as the 'colonisation of the life-world' by the systematic forms of modern life. Hegel is seen as making major contributions to our understanding of the roots of these issues and to a holistic view which may affect how we confront them. Accepting that Hegel is right in arguing that art cannot be the determining form of Geist in the modern world, what of its relation to philosophy in the historical account of the development of the arts in the Aesthetics? It is against this background that judgements on Hegel's contemporary significance must be made.

Hegel frequently supports his arguments about the subordinate role of the art in modernity with the example of the superiority of Christianity over Pagan, and particularly Greek, religion. Hegel is obviously a monotheist. Greek art, which represents the Gods in human form, has as its content 'the unity of human and divine nature, a unity which precisely because it is immediate and in 
itself can be adequately manifested in an immediate and sensuous manner' (Hegel $1965 \mathrm{I} \mathrm{p}$. 86). The knowledge of the unity of human and divine, which requires reflection, cannot, for Hegel, be present in a concrete object like a sculpture. We have already seen a version of this argument in Schelling. Classical art (by which Hegel usually means Greek sculpture) expresses a stage of the development of Geist in which the sensuous and Geist are unified: the material ceases to be just a block of marble and is formed by Geist into a sensuous manifestation of itself. The advance in Christianity is that God 'should be known as Geist and in Geist. His element of existence is thereby essentially inner knowledge and not the external natural form via which he will only be representable immediately and not according to the whole depth of his concept' (I p. 79). This argument should also be familiar.

Hegel echoes Kant's evaluation of the ban on images in Jewish theology in his account of how the sublime arises from awareness of the limits of sensuous manifestations of the supersensuous. The highest points of Kant's philosophy were only ever regulative ideas, which led him to the notion of 'aesthetic ideas' as a means of concretising what were otherwise abstractions. Hegel's claim that philosophy can transcend sensuous representation means that for him the idea of the 'new mythology', which would make ideas aesthetic for modern societies, is merely another form of 'immediacy' which the 'general forms' that regulate modern societies render inadequate. The difficulty here lies in how the relationship between sensuous phenomena and abstract thought is conceived, and in the ways this understanding affects modern culture. As we saw, Baumgarten's concern with sensuous particularity is a result of fears about those aspects of the Enlightenment which threatened to neglect the particular dimension of human experience manifest in sensuous perception. His aim was to suggest how the sensuous particular could be seen as part of a meaningful whole without being subsumed into cognitive abstractions. Hamann linked the concern with sensuous particularity to the nature of language, which is always both sensuous and intelligible. His ideas have been echoed in Derrida's argument in the essay 'White Mythology' (Derrida 1972) that a certain understanding of the divide between sensuousness and pure thought has dominated Western philosophy and that the divide can be deconstructed by attention to the ineliminable role of metaphor in philosophical discourse. ${ }^{18}$ That this issue has been a constant problem in Western culture is evident from the following famous example.

The origin of what Freud calls the 'Moses religion' is, as Kant already argued, linked to the ban on images, which means one is compelled to honour a God one cannot see. For Freud this meant a 'subordination of sensuous perception to an idea that is to be called abstract, a triumph of spirituality [Geistigkeit] over sensuality, strictly speaking a renunciation of a drive with its psychologically necessary consequences' (Freud 19829 p. 559). Renouncing a drive is likely to give rise to neurosis, a form of the return of the repressed. Schelling already described the development of the ' $\mathrm{I}$ ' in the $S T I$ and in the Munich lectures in terms of drives and repression. The link of the constitution of subjectivity to 
the effects of what has to be repressed for the subject to sustain its identity is a constant theme from Romanticism until the present. Genevieve Lloyd (1984) points out that this 'triumph of spirituality' is attached by Freud to a story which has patriarchal implications. The subordination of the sensuous results from the privileging of the father, whose invisible role in the production of children is given primacy over the visible role of the mother. This privileging requires an inference that an act committed nine months before the appearance of the child is the vital act - hence Freud's interpretation of the genesis of abstraction. The same kind of story is told at the end of Oresteia, when the new law of the city is legitimated against the matrilineal law of the country in terms of the myth of the decisive role of the father in procreation. The achievements of Geist often seem, then, to be brought about at the expense of the female. In Greek culture the contradictions involved in the establishing of new law were enacted in a cultural form which helped that culture cope with the most devastating kinds of necessity. We now see via the development of feminism the extent to which even this aesthetic response to repression involves a further kind of repression. Despite this, the aesthetic representation still tries to articulate the pain involved in facing contradictions in ways which a philosophical account of the founding of a new system of law cannot.

Hegel's view that one can regard the painful overcoming of contradiction with tranquillity once one has understood its necessity failed, as we saw, to offer any real means of dealing with the affective dimensions of our transience and fragility. Such tranquillity can, therefore, only be achieved by means of repression. Although Hegel is aware of the pain involved in the development of reason and in our awareness of our facticity, this does not seem to lead him to ask whether the dynamic of rationalisation might sometimes demand too high a price, for which other aspects of culture must compensate. This compensatory function cannot be eliminated or replaced by the advances of philosophy, science or law. If this is the case, art may sometimes indeed be what fulfils our highest need in a post-theological culture, because it links the affective, the ethical and the cognitive in ways which philosophy and other cultural forms cannot. Art can no longer do so in the way tragedy did for the polis, but that does not mean that art can be surpassed in the way Hegel suggests. The idea that aesthetic awareness is ultimately just a prelude to something higher may make sense as part of a historical story in which the move from myth and religion to science and philosophy is seen as what characterises modernity. Only if Hegel's totalising philosophical aims were realised, though, would this story be able to exhaust the relationship between the cognitive, the ethical and the aesthetic. There is not space to do justice to it here, but it is clear that a feminist account of aesthetics can provide important new insights into these questions.

Hegel's account of the development of art shows the considerable strengths and undeniable weaknesses of his aesthetics. There are three stages of the development of art: the Symbolic, the Classical, and the Romantic. In 'Symbolic' art the meaning of the work and the form used to express it are not essentially 
related. Hegel regards mythological stories about abstract themes like life and death as symbolic. Abstract ideas are expressed via what can be observed in nature: the observation of how plants die and grow again the next year is used as a story to explain death and the continuation of life in general. This way of thinking always involves the disadvantage of beginning with the concrete - for example, a particular story like the myth of Persephone - which is not adequate to express a general truth because it is tied to the other meanings of the specific story. The Sphinx is for Hegel the 'Symbol of the Symbolic itself' (Hegel 1965 I p. 352): human Geist attempts to emerge from the animal realm but does not fully succeed because the animal body remains. The body is, of course, linked to a female torso. Hegel's interpretation of this fact, like Freud's story of monotheism, omits the dimension of the history of patriarchy in which the lower forms of mind are associated with the female, as does his account of Antigone in the Phenomenology. Feminist interpretations have changed our perspective on such issues, and Hegel's method of classifying forms of art in a developmental history fails to take account of the way in which the interpretation of symbols can never be fully controlled. Symbols for Hegel retain something which is not clearly articulated (a word that repeatedly recurs in the Aesthetics), and must, as such, be transcended into literal concepts by philosophy. At the same time as Hegel is presenting this account of symbol, as we shall see in Chapter 6, Schleiermacher develops a view of interpretation which will undermine it by pointing to the ways in which interpretation can never be complete.

The next stage of art in Hegel's scheme, the 'Classical', unites 'meaning and corporeality' (I p. 418): 'only the externality of man is capable of revealing the spiritual in a sensuous manner' (I p. 419). Greek sculpture based on the human form is an expression of the Ideal, though it is still tied to particularity. The Romantic stage is founded in the idea of incarnation: God becomes flesh and so undergoes the pain of self-division. There is here an echo of the statement at the end of the Logic, that the already constituted Idea 'freely releases' itself into nature (Hegel $1970 \mathrm{II}$ p. 573). In Christian culture - which is largely what Hegel means by 'Romantic art' - this self-division is expressed in the story of Christ's crucifixion, which is followed by God's return to himself in the resurrection and the ascension. Romantic art expresses the 'return into self' in a concrete story, and so still remains at the level of the image. The Christian story, though, is dynamic in a way that Greek sculpture's 'noble simplicity and quiet greatness' (Winckelmann) in the face of pain and death is not. For Hegel, Romantic art 'sublates', in the threefold sense of negates, preserves and elevates, the divisions that Greek art only contemplates. It can be argued, therefore, that Hegel's philosophy is a transformation of Protestant theology which raises it above its residual attachment to the particular story, such as the incarnation and the crucifixion, in order to reveal the universality it contains.

The Protestant dimension becomes most clearly apparent when Hegel discusses death in the Aesthetics. In Romantic art death 
is only a dying off of the natural soul and of finite subjectivity, a dying off which only relates negatively to that which is itself negative, which sublates that which is vain [das Nichtige, i.e. the transient] and thereby mediates the liberation of Geist from its finitude and division, as well as mediating the reconciliation of the subject with the absolute. (Hegel 1965 I p. 504)

Greek art's existence in an objective sensuous form reflected a view of life as the external existence of the body. Death was, correspondingly, mere negativity. In contrast: 'In the Romantic world view death means negativity, i.e. the negation of the negative, and therefore turns just as much into the affirmative, as the resurrection of Geist out of its simple naturalness and inappropriate finitude' (I p. 504). In a characteristic example of the identity of identity and difference, death is therefore the life of Geist. To what extent are such arguments simply theological? Hegel's arguments about death in the Aesthetics involve the same attitude towards sensuous existence as did those of the Logic: it is only the overcoming of the sensuous that constitutes its truth. If one is able to adopt a Platonic attitude to the truth, as the ultimate aim of philosophy, Hegel's transformation of religious ideas can be integrated into a meaning-giving picture. The question for subsequent philosophy is whether even this attenuated and dynamised form of Platonism can really 'answer our highest need', or whether it simply represses vital dimensions of finite human existence.

Hegel's discussion of the 'end of art' now takes on another aspect. For Hegel, Classical art's combination of the spiritual and the natural is the 'completion of the realm of beauty. There cannot be anything, and nothing can become, more beautiful' (I p. 498). Consequently, in Romantic art, 'beauty in its most appropriate form and its most apt content is no longer the ultimate aim' (I p. 499). Instead of trying to reflect the truth externally, which would burden it with transience, Romantic art, as a more developed manifestation of Geist, shows that the truth of thought is independent of contingency and externality. It therefore does not really matter which external content is used in Romantic art: even the most prosaic objects of daily life can be used, because the truth depends on their being overcome. At moments like this the power of Hegel's conception is evident. His theory even has considerable predictive power when considered in relation to modernist art of the kind that culminates with Duchamp's readymades. Hegel also refers to music in this context. Music's content is not specific and it is, as such, the 'key-note' of Romanticism. Its task is 'not to echo objectivity itself [i.e. represent external objects], but rather to echo the way in which the inner self is moved in itself according to its subjectivity and inner soul [i.e. represent feelings]' (II p. 261). Music does not engage with objectivity in the manner that conceptual thought does, and so has no essential effect on social reality, but it is a more developed form of Geist than visual art. Further implications of this position will be discussed in Chapter 7 .

Hegel argues, then, that the modern period has moved beyond art: 'we get as the final point of the Romantic per se the randomness of the external and the internal and a falling apart of these two sides, via which art negates itself [ sich 
aufhebt] and shows the necessity for consciousness to appropriate higher forms for the grasping of truth than art is able to offer' (I p. 509). The higher forms are evidently those of philosophy, in the wide sense we have seen, which includes the natural sciences and the law. Instead of being concerned with the actual results of the Idea's move into the pain and division of natural existence, which are dealt with in the particular sciences, philosophy's task is to show how this form of the existence of the Idea can be transcended.

\section{The prose of the modern world}

What, then, does this imply for the theory of art? The art form that most evidently relates to Hegel's account of why Romantic art 'negates itself' is the novel, the form which, as Mikhail Bakhtin will suggest, can encompass all other literary forms, and which is therefore able to speak of any aspect of existence. ${ }^{19}$ What is lacking in the novel is therefore an essential relationship between form and content. The content in a novel, which is necessarily particular because it is the story of specific individuals, cannot, for Hegel, be what is most essential. The content shows by its arbitrariness that art no longer articulates the essential truth of the modern world. A novel can be the story of anyone, and that story only becomes significant to the extent that the individual is transcended into a more general significance. ${ }^{20}$ On the other hand, it is precisely the ability of the novel to include any aspect of modernity, however contingent, that has made it so vital to our self-descriptions. The novel keeps horizons open by its very resistance to 'closure', something reflected in the often apparently insurmountable difficulties of many of the most significant novelists in bringing their work to a satisfactory conclusion. ${ }^{21} \mathrm{~A}$ similar point could be made, of course, about the move away from the triumphant tonal conclusion in Western classical music. In Hegel's terms, overcoming the contingency of modern life that is evident in the novel is the task of philosophy. But the question arises once again: what if philosophy cannot fulfil this task?

Hegel is predominantly employed these days to support the argument that philosophy should renounce foundational claims and should instead explore the normative commitments within communities which make possible the truths which determine our world. However, even if this is an apt way to describe what Hegel is doing, it is by no means clear that such exploration is mainly the preserve of philosophy. Indeed, Rorty has argued that it may well be that the historical exploration of our normative commitments which is one of the great innovations of Hegel's work may now better be carried out by the novel. The novel's ability to countenance the sort of contingency which keeps our moral imagination alive, rather than trying to reduce morality to systematic philosophical form, is in this sense more apt to the ethical complexities of the modern world. In certain areas of human life, such as the sphere of the development of the kind of human feelings which can lead to a more humane world, the novel, rather than moral philosophy, also tends to have had a more widespread impact 
on society. ${ }^{22}$ Here we encounter another of those puzzles concerning how Hegel is best interpreted.

Consider the following constellation. During the period in which philosophy reaches the stage where Hegel claims the absolute, the reconciliation of our thinking and the world, can be explicated, reality has at the same time become 'prosaic'. For Hegel the novel 'in the modern sense presupposes a reality which has been sorted out into prose' (II p. 452). In a typical reversal, the novel becomes concerned with how the 'poetry' of life - usually in the form of an idealistic hero - attempts to reassert its rights against the prose of daily existence. For Hegel this can end tragically or comically. The hero may founder on the contradictions between his ideals and the reality in which he tries to realise them, or he may, as often in Goethe, become reconciled with the social order by insight into its necessities. As an empirical point about the German novel of Hegel's time this is pretty apt. However, what is important for our understanding of Hegel - and this is not just explicable by his conservatism in his later years - is why he is happy to juxtapose the highest reconciliation in philosophy with his claim that life in bourgeois society has become prosaic.

Interpretations of Hegel are, as I suggested at the outset, often divided between those which seek to make him part of a 'post-metaphysical' reorientation of philosophy and those which seek to sustain his emphatic role as the great systematic resolver of the problems of philosophy. The sorting out of reality into prose is closely analogous to Weber's description of the 'disenchantment' inherent in modern rationalisation, where real technical and organisational means take over from imaginary ones, at the price of the loss of an immanently meaningful world. The absolute Idea thus seems from this perspective to be open to two interpretations. In the first, the realisation that the erstwhile metaphysical hopes of philosophy have been overcome by a method which obviates the need to seek foundations leaves us to get on with what we already successfully doing while also demanding explicit public forms of legitimation. In the second, philosophy recognises the superiority of the forces which take over the modern world - in the manner suggested by the later Heidegger's interpretation of the history of Western metaphysics which culminates with Nietzsche as the history of the subjectification of being. To what extent does the former position shade over into the latter and is there an alternative approach to philosophy's role? Clearly it would be absurd to attempt to challenge the superiority of the natural sciences as the most effective means of controlling and explaining the workings of the natural world. What can be challenged, as we have seen, is the exclusive orientation to the sciences as the source of legitimacy: as Weber insists, the sciences are not self-legitimating. In this sense it seems appropriate to consider whether Hegel's aesthetics does not suggest a weakness in the general direction of his philosophy that cannot be skirted by his antimetaphysical interpreters.

A notorious passage on what Hegel refers to as the 'novelistic' - that clash of novel hero and reality paradigmatically present in the figure of Don 
Quixote - highlights the problem. Hegel considers the hero who confronts the world with his ideals: 'These battles are, in the modern world, nothing but the years of apprenticeship' - he uses the word Lehrjahre, echoing Goethe's Wilhelm Meister - they are 'the education of the individual via existing reality and they gain their meaning thereby'. The education of the hero leads to the following:

Finally he usually does get his girl and some job or other, marries and becomes a Philistine like all the others; the wife runs the household, children inevitably arrive, the adored woman who initially was the Only one, the Angel, looks roughly like everyone else, the job involves work and unpleasantness, the marriage the burden of domestic life, and so it is that one ends up with the hangover that everyone else has. (I p. 568)

How does Hegel reconcile the resigned cynicism of this passage with the claims of his system, and what does this tell us about interpreting the absolute Idea?

The danger of slipping from the first to the second interpretation is apparent in passages like the following. In the conclusion of the Philosophy of History Hegel claims that 'Philosophy moves to contemplation through its antipathy to the movements of direct passions in reality. ... Only the realisation that what has happened and happens daily not only does not happen without God but is also essentially His own work can reconcile Geist with world history and reality' (Hegel 1961 p. 605). The ease with which one can slip from a position which, as the Phenomenology does, shows how our thought is necessarily connected to the real and must be immanently justified within social practices, to one in which this connection implies a questionable reconciliation with the status quo is very evident here. It is a commonplace of the Western tradition that philosophy should transcend the 'direct passions', but the history of the novel, which relies precisely upon such passions, should give pause for thought. Both the stubborn refusal of the novel, with its constant incorporation of new perspectives, to disappear from the repertoire of means we use to make sense of the modern world, and the diminishing importance of the products of academic philosophy as a means of orientation in modern societies suggest that at least one of the versions of Hegel's attempts to transcend contingency may be invalid.

In his Theory of the Novel, written in the face of the outbreak of the First World War, Georg Lukács completely changes the interpretation of the way in which modern reality has become 'prosaic'. For Lukács, who follows ideas from Friedrich Schlegel and Kierkegaard, the prosaic development of reality and the sense of the individual's alienation that are graphically expressed by Hegel in the passage on the novel's hero, are not adequately confronted by claiming that a philosophical understanding of modernity can explain how they come about. What Hegel regards as the 'randomness of the external and the internal and a falling apart of these two sides', Lukács interprets as the expression of 
'transcendental homelessness'. The novel becomes the locus of the search for meaning in a post-theological culture. This search for meaning in a world where the significance of individual existence is subordinated to the universal is not something that can be coped with by philosophical insight into how it could not be otherwise. The First World War made it rather difficult to argue that the most philosophy was capable of was 'reconciling Geist with world history and reality', rather than making it possible to oppose historical reality in the name of future hope.

Lukács also follows the Romantic claim that the subject can never gain the self-transparency which Hegel sees as achieved in his philosophy. 'Irony', the central term in Lukács's theory, which he borrows from Schlegel, is the expression of the ultimate failure of the subject to find a truth which would make it at home in the world. The subject's failure to be transparent to itself within a wider totality is therefore constitutive of the novel form, which makes it the most characteristic form for modernity. ${ }^{23}$ Lukács's argument echoes the assertion in Schelling's Philosophy of Art that in the modern era 'Poesie' is 'only possible for whoever can create for himself a mythology, a closed circle of Poesie out of his very limitations' (Schelling I/5 p. 444). His position also echoes the Romantic account of the failure of the reflection model of subjectivity. Instead of the work of art being transcended into something higher, as it is in Hegel, the novel becomes a response to the subject's failure to attain a stable sense of its place in the world. This leads to a further difficult interpretive issue. The choice we saw in the Introduction between regarding modernity as the onset of nihilism and regarding it as the opening up of endless new possibilities is echoed in the differing assessments by Lukács's and Mikhail Bakhtin of the significance of the novel. Lukács's pessimistic assessment of the rise of the novel is not echoed by Bakhtin, who instead focuses both on the way the novel can give a voice to those in a society who are excluded from the dominant languages of that society and on the plurality of world-disclosure this makes possible. Bakhtin thus offers a different way of examining the novel's role in modernity which does not involve the kind of apocalyptism present in Lukács, and which does not give philosophy the kind of privileged role it has in Hegel. What does this mean, then, for the relationship between philosophy, art and aesthetics in modernity?

\section{Philosophy and art after Hegel}

The predictive force of Hegel's view of the novel is undeniable, and lends plausibility to significant parts of the rest of his argument about the nature of modernity. The incorporation into the novel of random material from daily life, such as newspaper cuttings or unedited historical documents, and the attention to more and more of the basic functions of life, of the kind present, for example, in Joyce's Ulysses, is characteristic of the modernist novel and is congruent with Hegel's verdict on the arbitrariness of the relation between 
form and content in 'Romantic' art. The arbitrariness in the novel is, significantly, often balanced, as it is in Ulysses, by the employment of residual elements from mythology as a means of sustaining a coherence that the material otherwise lacks. There is, though, a tension in relation to such phenomena between opposed conceptions of modernity, which is also apparent in the differing readings of Hegel's philosophy we have examined. Adorno claims in Aesthetic Theory that:

Hegel's philosophy fails in relation to the beautiful: because he equates reason and reality ... he hypostasises the structuring of all being by subjectivity as the absolute. He regards the non-identical solely as a fetter on subjectivity, instead of determining the experience of non-identity as the telos of the aesthetic subject, as its emancipation. (Adorno 1973 p. 119)

For Adorno, even though it is unequivocally to be welcomed, the weakening of the hold of tradition in modernity in fact results mainly in new kinds of constraint, despite the new possibilities for freedom. One evident manifestation of this situation is that the means to alleviate suffering, and material and cultural deprivation are constantly being augmented, but the forms of social organisation and the cultural resources which would make them generally effective do not develop in the same manner, and even regress into barbarism. A further manifestation is the fact that those aspects of the subject which resist being subsumed into generality and which are vital to the genesis of modern art become relatively insignificant in relation to the demands of modern societies for what can be measured, counted and administrated.

From Adorno's perspective, Hegel conspires with this situation because, as Habermas also argues, he makes contingently developed objective spirit into absolute spirit. One must, though, again be careful to differentiate here. Hegel's argument about the novel is important because it offers a way of understanding both why it is that the material of modern art has less and less substantial connection to the main tendencies of modern societies, and why modern art is confronted with the problem of trying to incorporate abstractions of the kind inimical to the particularity upon which art had previously relied. ${ }^{24}$ This does not necessarily mean, however, that Hegel's interpretation of the situation is wholly adequate. Hegel, as we saw, thinks philosophy can overcome divisions between the ever more specialised spheres of social life in a way art cannot. It is arguable, though, that the main kinds of 'unification' that characterise modernity are, as Adorno claims, the industrialisation of scientific knowledge, the dominance of the commodity form, including in the culture industry, and the spread of bureaucratic standardisation across the globe, none of which need of its own accord add to human self-determination. ${ }^{25}$ Given the decisive role of these developments, which encroach upon virtually every sphere of modern life, the question is what relationship philosophy should have to them and what relationship philosophy should have to what they exclude. Once again Hegel's role in deciding this is ambiguous, given his simultaneous desire both to grasp the 
reality of an ever more systematised and differentiated world, and to restore the kind of unity which religion had previously made possible.

The questions generated by Hegel's arguments about the end of art are now perhaps best understood as cultural questions concerning the results of the separation of spheres of human activity. One of the most informative facts here is that the most significant artistic production in modernity - Mahler, Kafka, Proust, Schœnberg, Klee, Charlie Parker - is on the one hand radically individual and particular, and on the other seems to derive a universal significance from precisely this individuality and particularity. It is not clear that Hegel's view of art can really help us to understand such a relationship between the universal and the particular. Hegel's attempt at a philosophical explication of the overcoming of the particular by general forms of truth offers a profound diagnosis of the nature of modernity, but it also contains an important unintended warning about the possible negative consequences of that overcoming. I shall return to the issues raised by Hegel in the Conclusion, when I examine them in relation to Rorty's separation of 'public' and 'private' in the understanding of modern culture. Rorty regards the 'public', problem-solving resources of natural science and 'projects of social cooperation' as rightly becoming separate from 'private' projects of self-development in modernity. The tensions this entails already appear in the work of Hegel's contemporary and rival Friedrich Schleiermacher. It is to him that we now turn for an account of philosophy and art which proposes a serious alternative to Hegel. Schleiermacher tries to understand the relationship between the universal and the particular in ways which are now being echoed in contemporary philosophy. As we shall see, he comes closer in some respects to what contemporary Hegelians want from Hegel than Hegel himself.

\section{Notes}

1 As neither Mind nor Spirit is wholly apt for translating the term Geist, the former being too reminiscent of 'the philosophy of mind', the latter having too many quasi-religious connotations, I shall sometimes use the German word and try to make clearer in the context what is meant.

2 This can also be read in a more positive direction, in which scepticism ceases to be a concern because of our undoubted ability to cope with the world and find better ways of doing so. This is Rorty's position, which shares much with the deflationary reading of Hegel, not least because of his influence on it.

3 This is not exactly how Hegel argues this progression but I think McTaggart is right to suggest that 'something' has to follow 'being' and 'nothing'.

4 This is partly because Pinkard tends to base his position predominantly on the Phenomenology.

5 The Logic does involve historical references, but they are not to be understood in the same way as those in the Phenomenology, which, albeit generally indirectly, refer to major historical events and texts. 
6 It should be clear that what is at issue here is not the fact that logical forms of thinking must involve a specific empirical content, which, in terms of the usual idea of logic is clearly false, but rather Hegel's conviction that a complete self-defining system can be shown to map the very structure of the real.

7 The change in the nature of concerts and in the behaviour of listeners, in which the music becomes the more exclusive focus of attention, points to this.

8 Lenin, for example, thought the Logic of great value for his thinking.

9 Clearly not all music can be construed in these terms, but the most revealing music of Hegel's period seems to sustain its power for reasons of precisely this kind.

10 Though even this raises questions: whether a word really can be said to possess a general meaning in the manner demanded by many semantic theories is still highly contentious, as I suggested in Chapter 4 in relation to Derrida.

11 Taylor argues that the constitutive function is more essential to language, and this is in line, for example, with Rorty's contention that thinking of language solely in terms of representation gives rise to many of the intractable dilemmas in the analytical philosophy of language. Rorty thinks the development of 'semantical metalanguage', in which we can 'say things like, "It is also called ' $Y$ ', but for your purposes you should describe it as X"' (Rorty 1999 p. 65) is the deciding factor in what may be called a language. The fact that there can be music about music, for example in Mahler's ironic employment of musical material, suggests that music can function as a kind of metalanguage to those who understand music. The point is that there is no definitive way of determining the extension of the concept of language.

12 This admittedly ignores the idea that works of art can, in a non-semantic sense, be true, by being 'right' in their own particular world-disclosing manner, which I explore in Bowie 1997.

13 This issue recurs in the philosophy of mind in the debate about pain: why is it nonsense to say 'I thought you had that pain yesterday, not me?'

14 In Germany today these positions are paradigmatically represented by Karl Heinz Bohrer and Peter Bürger.

15 He did, of course, produce poetry after he became mad. The poetry is strangely conventional and lacks the disturbing imaginative power of his late work before his breakdown.

16 This issue has recurred in the differences between Gadamer and Habermas, and Gadamer and Frank, with Gadamer playing the role of Hegel.

17 This is well suggested by Weber's account of the increasing specialisation and loss of general perspectives in modern life in 'Science as Vocation'.

18 As we saw, Schelling's identity philosophy does not straightforwardly fit this pattern.

19 The argument also works in certain respects for Western music's incorporation of more and more that was previously noise, at the risk of ceasing to be music at all for most listeners.

20 This point, as Lukács realised, has to do with why the novel very rarely has major historical figures as its central characters, and why, when it does, the novel in question rarely succeeds.

21 This includes both authors, like Kafka, who seem not to be able to conclude at all, and authors like Zola, Thomas Mann and others, who take over mythical apocalyptic forms of ending which are inadequate to the material which precedes the end (see Bowie 1979).

22 In certain respects Rorty therefore echoes the arguments of the 'System Programme'. 
23 Frank has explored this idea via the motif of the 'endless journey' (Frank 1979, 1995).

24 This issue is superbly dealt with in Heinz Schlaffer's account of Goethe's use of allegory in Faust Part II (Schlaffer 1981). The link between the need to deal in abstraction - such as issues to do with the development of capital - and the increasing use of allegorical or mythological elements is the key here (see also Bowie 1979).

25 This is not to say that these aspects of modernity are somehow to be rejected: that would be impossible and absurd. The question is what means are available for countering their effects, given their irresistible power in many areas. 


\section{Schleiermacher: art and interpretation}

\section{Linguistic turns}

The recent growth of interest in German Idealist and Romantic philosophy has tended to focus on Fichte and Hegel, and, to a lesser extent, on Schelling. However, given the philosophical motivation for the new attention to the thought of this period, it is actually rather strange that its main focus has not been the work of F.D.E. Schleiermacher (1768-1834). The contingent reasons for the neglect of Schleiermacher are, admittedly, quite simple. Schleiermacher's theological work, as the major Protestant theologian of the nineteenth century, has largely determined his reputation, and he did not produce definitive versions of his major philosophical works, which have consequently often been misunderstood or underestimated. Furthermore, only a few works had been translated into English until quite recently, and some of the most significant works still await translation. However, despite his lack of influence on mainstream philosophy, Schleiermacher is of major philosophical importance, being the first to combine the sort of ideas concerning the mind-world relationship we have encountered in the early Romantics, Schelling and Hegel with sustained attention to the role of language in philosophy. Most people are aware that Schleiermacher formulated the first modern account of hermeneutics, but too few people seem aware that this was only part of a wider philosophical project, some of which has now turned out to prefigure central ideas of key thinkers in contemporary philosophy, such as Brandom, Davidson, Gadamer, Habermas, McDowell and Rorty. The appearance of Gadamer in this list might appear surprising, given his critical account of Schleiermacher in Truth and Method, but it will become evident in what follows that Gadamer's flawed interpretation is a further source of the failure to appreciate Schleiermacher's real significance. ${ }^{1}$ It should perhaps be stated at the outset that I shall not be dealing with Schleiermacher's theology. Most of his philosophy can stand without his theology, and this was something upon which Schleiermacher himself often insisted. ${ }^{2}$

A brief consideration of influential changes in the analytical philosophy of language since Quine can begin to make it clear both why Schleiermacher deserves more attention than he has yet received in the English-speaking world, 
and why the tradition to which he belongs deserves far more credit for its insights than its sometimes rather crude reception has granted it. The guiding theme of the changes in the philosophy of language has been the move away from the idea that philosophy could establish definitive ways of explaining meaning and truth by giving an account of how words connect to pre-existing determinate things in the world. Such accounts, as Rorty has suggested, essentially repeat many of the moves by which earlier philosophy had attempted to explain how concepts represent reality. The history of the analytical philosophy of language, which was not least a consequence of the empiricist rejection of Hegelianism by Russell and others, moves from the attempt to ground meaning in the single word's relation to some kind of 'given' in the world (early Wittgenstein and Russell), to the invocation of grounding 'observation sentences' whose meaning is given by the means by which they could be verified (logical positivism), to a concern with language as a whole as rule-governed human practice (speech act theory), and finally to a - in certain respects Hegelian - holism which no longer sees language and the world as separable (Sellars, Quine and Davidson). This last move is what brings about the new relationship between the analytical or post-analytical tradition, and the hermeneutic tradition of Heidegger and Gadamer. As we shall see, it is also what connects contemporary philosophy to Schleiermacher, who is already very explicit in his rejection of the attempt to understand language in terms of isolated words or sentences, in his rejection of the immediate givenness of things, and in his insistence on the ineliminability of context and background knowledge in all understanding.

The underlying pattern in the history of analytical philosophy is the move from the conviction that the world provides a 'given' which is the immediate foundation of certainty in questions of truth and meaning, to the realisation, as Wilfrid Sellars argued, that such a given is in fact a 'myth', because all awareness that can lead to knowledge must be linguistically structured and thus necessarily involves mediation by the language in which it is articulated. As Samuel Wheeler has suggested, what links Davidson and Derrida, for example, is the rejection of the idea of a grounding 'presence' of thing to thought which enables elements of language to be correlated with determinate elements of the world (see Wheeler 2000). Once it is admitted that direct access to a pre-existing determinate reality cannot be established, because that notion itself is a myth, many of the reasons that had previously led to the rejection of the German Idealist and hermeneutic traditions dissolve, and issues seen as relating mainly to aesthetics again become significant. The focus of philosophy returns, therefore, to the concerns of the Kantian and post-Kantian traditions, which concentrated on the role of thought and language as constitutive of what we take the truth about the world to be, rather than as simply mirroring the truth of what is already supposedly there in an intelligible form. Kant himself saw the 'conditions of possibility' of knowledge as being the necessary categorial operations of our consciousness. Schleiermacher's essential move is to argue, while providing 
an account of self-consciousness which is still significant for the philosophy of mind, that these conditions depend on language, and that languages change with history. There is therefore no timeless structure to the way knowledge is organised. Schleiermacher, then, does not assume that language mirrors some kind of given. He consequently opens up, in a more developed way than anyone in the first half of the nineteenth century, some of the most important space in which contemporary philosophy is played out.

Habermas has characterised the move in the direction of what he terms 'post-metaphysical thinking' as involving the claim that 'world-constituting capacities are transferred from transcendental subjectivity to grammatical structures' (Habermas 1988 p. 15). This is precisely what Schleiermacher also claims, albeit with certain provisos we shall encounter later. Because grammatical structures are historically contingent, there is no extra-historical location from which to establish the relationship between language and what there is, not least because any investigation of this kind has itself to be carried out in a historically developed natural language. In case anyone is in doubt about just how close contemporary philosophy comes to Schleiermacher, consider the following. Schleiermacher pre-empts by well over 100 years the essential move in Quine's critique of logical positivism. He rejects the analytic/synthetic distinction for the same kind of reasons as Quine himself in his rejection of the 'dogmas of empiricism':

The difference between analytical and synthetic judgements is a fluid one, of which we take no account. The same judgement (ice melts) can be an analytical one if the coming into being and disappearance via certain conditions of temperature are already taken up into the concept of ice, and a synthetic one, if they are not yet taken up. ... This difference therefore just expresses a different state of the formation of concepts. (Schleiermacher 1839 p. 563)

For Schleiermacher, 'No real concept can be constituted to the point of complete knowledge' (p. 195), precisely because any changes in a concept's place in a web of concepts will bring about a re-assigning of the role of that concept, so this process cannot be said to have a conclusion. Given his anticipation of one of the defining arguments in the formation of contemporary philosophy, Schleiermacher's work is clearly in need of revaluation. Furthermore, the way in which he prefigures so many contemporary issues suggests something important about the relationship between philosophy and the natural sciences. This relationship is shifting back for many of the most important contemporary philosophers in directions that were already mapped out in Romantic philosophy.

\section{The 'art of disagreement'}

Schleiermacher is not the only philosopher of his time to advert to the vital role of language in philosophy, though he does work out the implications of the linguistic turn more thoroughly than anyone else. ${ }^{3}$ The argument that transcendental 
philosophy relies on the prior existence of natural languages had already been proposed by Hamann as early as 1784, in his critique of Kant, the 'Metacritique on the Purism of Reason'. Like many philosophers today, Hamann - and, in his wake, Schleiermacher - thinks Kant's separation of the sensuous and the conceptual, the receptive and the spontaneous cannot be strictly upheld. Hamann maintains in his own inimitable manner that our need for language means that a notion of philosophy based on a priori forms of cognition lacks a decisive dimension. His argument is worth quoting at length, because its baroque form is also part of its content:

So another main question remains: how the capacity of thinking is possible? - The capacity to think right and left, before and mithout, with and beyond experience? One needs no deduction to prove the genealogical priority of language before the seven holy functions of logical propositions and conclusions and their heraldry . . the whole capacity to think depends on language . . Sounds and letters are therefore a priori forms in which nothing which belongs to the sensation or to the concept is met and are the true aesthetic [in the sense of having to do with sensation] elements of all human cognition and reason ... Words therefore have an aesthetic and logical capacity. As visible and audible objects they belong with their elements to sensuousness and intuition, but in terms of the spirit of their employment and their meaning they belong to the understanding and to concepts. Consequently words are both pure and empirical intuitions as well as pure and empirical concepts: empirical because the sensation of sight or sound is affected by them, pure in so far as their significance is not determined by anything which belongs to those sensations . . . Meaning and its determination results ... from the linking of a sign [Wortzeichen], which is a priori arbitrary and indifferent, but a posteriori necessary and indispensable, with the intuition of the object itself, and via this repeated connection the concept itself is communicated to, impressed and embodied into the understanding as much by the sign as by the intuition itself. (Hamann 1967 pp. 224-6)

Language 'deconstructs' the opposition of the empirical and the a priori because it is itself both sensuous and intelligible, and therefore puts in question the division between receptivity and spontaneity which was essential for Kant's foundational aim of mapping the forms which make knowledge possible. (Hamann also, incidentally, deconstructs the opposition between spoken and written language, refusing to give priority to the voice over written communication.) As we saw in the Introduction, Hamann arrives at this position through his desire to celebrate the endless diversity of God's universe.

The crucial consequence of these arguments about language and philosophy for Hamann is that it becomes impossible to sustain the idea that philosophy can wholly escape from its location within a historically specific language. The idea of a 'general philosophical language' is therefore an idealist illusion. Languages are necessarily bound to the contingencies of their particular historical development, in which abstract and sensuous moments cannot be definitively separated. This fact should, however, he maintains, not be regarded as a problem. Instead, the historical specificity of languages should be seen as an opportunity, because 
it augments the possibilities for world disclosure. (The contrast between conceptions which see modernity as leading to nihilism and confusion, and those which see it as an opening of horizons is once more in evidence here.) It is in relation to ideas like Hamann's (and, as Charles Taylor (Taylor 1985) has argued, those of Herder) that thinking about language in this period becomes linked to the development of aesthetics. If, as we have seen, language comes to be understood as 'constitutive', rather than merely 'designative', it becomes part of a whole series of means by which we engage in 'world-making', rather than the means of reflecting a ready-made world.

The danger of this position is that it can be used to advocate a thoroughgoing linguistic relativism, of the kind that is present in some contemporary literary theory, in which the boundaries between linguistic communities become uncrossable. One of the most productive aspects of Schleiermacher's work is its rejection of naive versions of relativism and its insistence on truth and objectivity. At the same time, he gives full weight to the fact that the problems involved both in communication within languages and translation between them deeply affect the nature of the philosophical enterprise, rendering it inherently impossible to complete in the manner demanded by traditional metaphysics.

Although he is perhaps best known as the theologian who affirms, in the famous text On Religion (1799), written in response to his friend Friedrich Schlegel's 'educated despising' of religion, that religion's 'essence is neither thought nor action, but intuition and feeling' (Schleiermacher n.d. p. 53), Schleiermacher the philosopher is in certain respects an Aristotelian. He begins his Aesthetics, for example, by insisting that 'praxis has always been something earlier than theory' (Schleiermacher 1842 p. 1). Somewhat surprisingly, given Schleiermacher's status as a Protestant theologian concerned with 'intuition and feeling', the nearest equivalent to some of his most significant contentions can be found in Marxist thinkers such as Bakhtin, and in Sartre, as well as in post-Wittgensteinian thinkers like Davidson. This is because he regards language as a form of action, being probably the first person to use the term 'speech act' (Schleiermacher 1977 p. 80): 'What we call thought as a whole is an activity . . . such that everyone can act by designating in the same way' (Schleiermacher 1990 p. 256). He also comes close to these thinkers because of his suspicion of philosophical attempts to arrive at an absolute conception of reality. It is here that he most obviously belongs on the Romantic side of the Romanticism/Idealism divide, and differs from Hegel in particular.

For Hegel, once the transience of systems of thought is philosophically understood it is possible to overcome the differences of the various systems in the absolute Idea. His Logic is therefore a self-contained 'science of knowledge', which can articulate itself through the immanent contradictions in thought that give it its dynamic quality. The idea that such a conception can both be wholly immanent and yet provide a complete description of itself was also the source of Schelling's and others' doubts about how such a system could become transitive. In contrast to what we encounter in Schleiermacher's Dialectic, disagreement in 
Hegel's system is ultimately revealed to be part of a higher agreement which emerges when the final contradiction turns into the absolute Idea. Everything particular is therefore only particular because it is mediated by the universal. Nothing can stand on its own as irreducibly particular: it must become itself via the concept, which articulates its truth. For this reason art, which is tied to particularity, is a lower form of Geist than philosophy, and is transcended when it is philosophically understood. However, Spinoza's idea of determination by negation need not be employed in the way Hegel employs it. It is possible to accept that there are no non-relational properties, and that everything we know is therefore mediated by its relations to other things, without thinking that this insight can be cashed out into a wholly articulated system which abolishes the contingency of being. The dialectic can be infinite, because we can always end up changing our thought in the light of new tasks by rejecting previous ways of thinking. What distinguishes Hegel's Idealist from the Romantic philosophy of Schlegel, Novalis and Schleiermacher is his desire exhaustively to systematise the way in which this improvement occurs and to give it a teleological significance.

Schleiermacher, then, has no time for the idea that there can be total or final agreement, even the agreement generated out of the universality and necessity of disagreement. In the Aesthetics he argues, for example, that the connection of aesthetics to the rest of philosophy means one would require a generally agreed system of philosophy to be able to establish aesthetics on a firm foundation: 'But this would mean deferring the matter to infinity' (Schleiermacher 1842 p. 48). Philosophical systems can dominate an era, but they pass away and are replaced by competing systems. There is for Schleiermacher, though, no system which could fully encompass this insight and transcend it. Like the early Romantics, with whom he had close, but sometimes critical contact, Schleiermacher denies philosophy's ability to provide an absolute conception of reality: 'Absolute, Highest Unity, Identity of the Ideal and the Real are schemata' (Schleiermacher 1988 p. 67), and therefore not 'real concepts', so 'The idea of absolute being as the identity of concept and object is . . not knowledge' (Schleiermacher 1839 p. 87). He also invokes the 'Opposition of the universal and the particular. The latter that which cannot be purely represented in thought, the former that which cannot be purely given in being' (Schleiermacher 1986 p. 68). Consequently, the scheme/content distinction, the distinction 'between what language contributes to the object and what the world contributes' (Rorty 1999 p. 108) plays no real role in his thought. ${ }^{5}$ In the 1833 Introduction to the Dialectic he therefore maintains that 'we must be satisfied with arbitrary beginnings in all areas of knowledge' (Schleiermacher 1988 p. 149), and, echoing Friedrich Schlegel, that 'beginning in the middle is unavoidable' (p. 104). His orientation towards praxis means that, instead of seeking foundations of knowledge in the manner of Kant and Fichte, the Dialectic sets itself the following task: 'instead of setting up a science of knowledge in the hope that one can thereby put an end to disagreement it is now a question of setting up a doctrine 
of the art [Kunstlehre] of disagreement in the hope that one can thereby arrive at common bases for knowledge' (Schleiermacher 1942 p. 43). The difficulty of translating the word Kunstlehre underlines why Schleiermacher's position, despite its acknowledgement of the dialectical nature of thought, stays outside a Hegelian model.

The word Kunst in Schleiermacher varies in meaning, as it does in many of his contemporaries, between the older sense of technique or craft, and the newer sense that refers specifically to 'art' as the product of human freedom, which is the object of aesthetics. The ambiguity in the meaning of Kunst is the key to some of Schleiermacher's most far-reaching claims. He argues in the hermeneutics that interpretation is itself also an 'art'. What he means is that interpretation relies upon the ability to use rules, as one does in a craft, but that the application of rules cannot be made completely rule-bound, on pain of a regress of rules for rules. Kant had already suggested this point when he described judgement's role in the use of cognitive rules as follows:

If judgement wanted to show universally how one is to subsume under these rules, i.e. distinguish whether something belongs under the rule or not, this could only happen via a further rule. But because this is a rule it requires once more an instruction by judgement, and thus it is shown to be the case that the understanding is admittedly capable of being instructed and equipped by rules, but that judgement is a particular talent which cannot be given by instruction but can only be practised. (CPR B p. 172, A p. 133)

This argument, which is usually associated with Wittgenstein, has proved vital in the work of Brandom and others, who insist on the practical dimension of communication, which cannot be reduced to the following of linguistic rules. Brandom characterises 'Wittgenstein's Regress Argument' as follows: 'The rule [of language] says how to do one thing correctly only on the assumption that one can do something else correctly, namely apply the rule' (Brandom $1994 \mathrm{pp}$. 21). If a regress of rules for rules is to be avoided, one has to assume that the subject possesses an active capacity for judgement, the application of rules, on the basis both of background knowledge which can never be fully reduced to rules, and of individual initiative. These enable the use of an existing rule and, significantly for Schleiermacher's conception of aesthetics, make possible the creation of new usage not based on existing rules. Schleiermacher: 'We call art ... every compound product in which we are aware of general rules, whose application cannot in the particular case be again brought under rules' (Schleiermacher, cited Rössler 1990 pp. 232-3, from Short Account of Theological Study). The result of this crucial methodological point is, then, a link between what is involved in the everyday understanding and use of language, and aesthetic judgement. Aesthetic judgement also relies on rules but cannot be determined by rules, or 'mechanised', as Schleiermacher puts it.

Even though his hermeneutics 'rests on the fact of the non-understanding of discourse', Schleiermacher, like Habermas and Davidson, insists upon the need 
for a counterfactual notion of possible agreement in communication with others. Davidson says of his method of interpretation that it is not designed to eliminate disagreement, nor can it; its purpose is to make meaningful disagreement possible, and this depends entirely on a foundation - some foundation - in agreement' (Davidson 1984 pp. 196-7). For Davidson it is not even clear that we would be employing language at all if there were no foundation of our communication in agreement; hence his advocacy of the 'principle of charity', which insists that one should presume that most of what people say is true, if circumstances can be envisaged in which it would be right to say what they do. How far, though, does undecidability of interpretation go, once the assumption is made that there are no grounding rules for interpretation based on the relation between language and the given? It might seem that there is only an endless potential for disagreement. Schleiermacher maintains, however, that: 'Disagreement per se presupposes the acknowledgement of the sameness of an object, as well as there being the relationship of thinking to being at all' (Schleiermacher 1988 p. 132). The real difference, then, is between using a language to try to communicate at all, and not using anything that can be recognised as a language; it is not a difference between utterly irreconcilable uses of language.

This issue has played an often exaggerated role in some aspects of recent French post-structuralist thinking. The argument suggested by Schleiermacher is that misunderstanding is in principle potentially omnipresent because experience cannot be an unmediated guide to truth and meaning, and has to be shared via language. The perennial possibility of misunderstanding does not, however, obviate the possibility of agreement on what is held to be true, even though what is held as true is always fallible. A notorious example of the difficulty here can make the significance of Schleiermacher's position evident. Jean-François Lyotard claims the argument of the French fascist, Robert Faurisson, that, because all the direct witnesses of the gas chambers of Auschwitz are dead, one cannot prove that there really were any gas chambers used for mass murder, is irrefutable, even though he, Lyotard, patently does not believe it himself. Lyotard's position seems to me rather like being unsure if the light is on when the fridge door is shut. If one gets in the fridge, closes the door, and suffocates, there is no proof, because there is no testimony; but, of course, even if one survives and gets out there is no guarantee that anyone will believe one's testimony to the presence or absence of the light. In this case the only possible refutation of Faurisson's view seems for Lyotard to be dependent upon precisely what the critiques of empiricist theories of meaning show to be impossible, namely the 'presence' of the truth on the basis of some unmediated contact with it. The truth is clearly absent in so far as the victims die, but if it is inherently absent anyway, because this notion of presence is useless for an account of truth and meaning, one is simply looking at the matter in the wrong way.

The result of this situation for Lyotard is a 'différend', what Kant terms a 'Widerstreit', an argument which is irreconcilable because the opponents are 
arguing from incommensurable premises. Lyotard's argument, however, as I try to show in more detail elsewhere (Bowie 1997 Chapter 5; see also Frank 1988), relies upon the notion that languages can be incommensurable because they consist of 'rules' of a 'regime of discourse', regimes being constitutionally irreducible to each other. If it were true that understanding relied primarily on rules, Lyotard might have a point, but the whole impetus of the arguments of Schleiermacher, and Davidson, is precisely that a conception of language as essentially rule-bound is not defensible, for the reasons already seen by Kant in his claims about judgement. Rules alone lead to a regress and thus to the impossibility of a language ever functioning as a means of communication at all. As Frank has shown (Frank 1988), it is impossible even to claim there is a disagreement if there is complete incommensurability. In order to realise that the assumptions of one participant in a dialogue are incommensurable with those of another, an intuitive understanding of truth based on an awareness of what it is to take something as true has to be presupposed.

The dispute between Faurisson and those who accept that people were gassed in unimaginable numbers at Auschwitz is about how people died, not about irreconcilable regimes of discourse. ${ }^{6}$ It may be unlikely that Faurisson would change his mind about Auschwitz, but it is mistaken to maintain that, because he lives in a different discourse regime, he could in principle never come to see he was wrong. After all, history is full of examples of such radical changes of mind, which would be impossible if there were no conceivable transition from one set of ways of talking to another. It is, furthermore, possible to have held radically opposed views to one's present views and yet understand why one could have held them. Given that Schleiermacher thinks we are always in contact with reality, even though we may radically differ as to how we interpret it, he sees this as an issue of praxis: 'if we take [the] relation of thought to being away: then there is no conflict, but as long as thought only remains purely in itself, there is only difference' (Schleiermacher 1988 p. 134). Once one enjoins communication one is already necessarily involved in issues of truth and justification. An argument is not an argument if a priori it cannot be settled, and even knowing that there is a differend requires more than can be thought of in terms of two wholly incommensurable regimes of discourse. In which regime could the ability to claim there was a différend be located? ${ }^{7}$ Schleiermacher accepts that any conceptual agreement cannot lay claim to absolute certainty (cf. his claims about the 'complete concept'), because being transcends what we can say about it, but meaningful disagreement must involve a relationship based on some - perhaps minimal - identity concerning what is at issue. Brandom suggests a more plausible conception of incommensurability when he argues that vocabularies which are 'not intertranslatable, and not evaluable as alternative means to a common end, tools adapted to some one purpose from outside them both' are in one sense incommensurable. However it 'does not follow . . that they are incommensurable in the sense that "there is no way to bring them together at the level of theory"' (Brandom 2000 p. 179). The point is that the 
same person could use two such vocabularies, for example as a neurologist working on the brain chemistry of trauma, and as a parent concerned about the mental health of their child resulting from the child's traumatic experiences which could be helped by the right kind of therapy.

One of the more puzzling aspects of contemporary debates about language and truth is that positions which acknowledge that the myth of the given is indeed a myth seem to end up with wholly divergent ideas about what consequences this has for the future direction of philosophy. Wheeler argues that the divergences relate to how far a philosopher thinks 'philosophical notions . . . infect the rest of culture' (Wheeler 2000 p. 71). Lyotard's concentration on the supposedly aporetic consequences of the lack of a given contrasts very sharply indeed with Davidson's and Rorty's claims that the end of this particular philosophical search can liberate one from fruitless attempts to make meaning and truth into something based on 'presence', and instead can enable one to see vocabularies, as Rorty does, as tools for achieving what we want. Lyotard (and, as we saw, Derrida) seem to make demands for what is basically Cartesian certainty about meaning and truth. The result is that if presence cannot be achieved one is left with a situation in which someone like Faurisson has to be taken seriously, even though the same justification as Faurisson offers for his contentions could be used to deny the truth of absolutely anything that happened in the past. ${ }^{8}$ This then leads Lyotard to a general judgement on the nature of postmodernity as the age which is characterised by irresolvable differences which it is repressive to try to overcome.

Scepticism may be irrefutable by argument, but the approach to scepticism shared by Schleiermacher and some contemporary pragmatists regards this as immaterial, because we cannot avoid the activities of taking as true and justifying to others, being communicating agents, rather than isolated Cartesian spectators. Schleiermacher claims that in real situations, where there never is absolute certainty, and where we must constantly make practical decisions, 'a real willing is always the ground of conditioned thought which relates to an action; and here the real value of thinking is its agreement with what is thought. I do not wish to think the whole object, but only that aspect of the object which relates to my action' (Schleiermacher 1942 p. 330). There may be an indeterminate number of possible aspects of the object which are not countenanced by my thinking at a particular time, but the fact that actions can be successful means that this need play no role in most of my encounters with the world. The positive side of a sceptical attitude for Schleiermacher is instead that it can remind one of the constant possibility of improving knowledge: the 'presupposition of the possibility of error in all knowledge, this knowledge that knowledge is neither complete, nor will ever be complete, does not damage the belief in the idea of knowledge, but just provokes criticism' (Schleiermacher 1839 p. 32). This leads him to a fallibilism of the kind familiar in Habermas and others.

Schleiermacher insists that 'behind the difference of separate knowledge we 
must necessarily presuppose a universal identity, and by this we hold firm to the idea of the purity of knowledge, even if we cannot show an object in which it manifests itself' (p. 69). His claim is, then, that this is a normative issue: the presupposition that there is something to be right about is something we share by the very fact that we exchange speech acts. Even if much communication is actually strategic, it still relies upon a shared assumption at some level that what is said could be justified: without this assumption, even strategic deception, which relies on making someone take something as true which is not, makes no sense.

What is not clear in Schleiermacher is the extent to which this 'universal identity' relies on the realist presupposition of a 'world which is objective and independent of our descriptions' (Habermas 1999 p. 249). Habermas, who has recently argued for the necessity of a realist presupposition of this kind, also claims, though, that it is a merely formal assumption of argumentative praxis, for much the same reasons as Schleiermacher. Rorty, in contrast, cites Davidson, who contends that the notion of the 'objective world' that is independent of what we think about it 'derives from the idea of correspondence, and this is an idea without content' (Rorty 1998 p. 161). The goal of an objective world 'is neither something we might realise we had reached, nor something to which we might get closer' (p. 39). Schleiermacher does hold on to the notion of some kind of absolute, but, as we have seen, he thinks we can never maintain that our claims to knowledge correspond to it. The absolute is, then, a regulative idea. It cannot be based on the certain existence of what it invokes - the 'view from nowhere' - because this would entail the claim to know what the search for knowledge alone could find out, and this search cannot be completed. As such, the apparent realist assumptions play a normative, not an ontological role in his thinking (see Bowie 2001). What we can hope to achieve is Rortian 'more justification', though we seek this because of an inescapable need to get it right that is generated by the awareness of the fallibility of our conceptions.

The conflicting responses to the farewell to an epistemological ground exemplified by Lyotard and Davidson suggest another division in conceptions of modernity. Those, like Lyotard, who see modernity as the pursuit of a totalitarian absolute conception, regard modernity as a 'narrative of legitimation' which represses the plurality of differing conceptions characteristic of the 'post-modern'. In contrast, those, like Habermas, who concentrate on the advances made possible by modernity's demands for strong forms of universalising justification in science and law, deny the very validity of the term postmodern. Schleiermacher evidently belongs in many respects on Habermas's side, as a representative of the 'philosophical discourse of modernity', but there is a dimension of his thought, developed in opposition to Hegel, which both separates him from Habermas's position and makes him immune to Lyotard's strictures on the repression of difference that supposedly defines modernity. 


\section{Schleiermacher talks of a}

further element whereby the area of knowledge is limited, by virtue of which in thought everyone is different from everyone else. This is the individual [das Individuelle]. To the extent that there is some of this everywhere no act will completely correspond to the Idea of knowledge [in the sense of the 'complete concept'] until after this element has been eliminated. And this can be only indirectly solved if the totality of the Individual as such, i.e. with its foundations, is known, and with this we have a completely endless task. (Schleiermacher 1942 p. 131)

Schleiermacher's notion of individuality might be seen as entailing the kind of scepticism involved in Lyotard's arguments, or some kind of wild subjectivism. However, his argument has in fact to do with the inescapability of communicative action in the genesis of objectivity. One side of this issue has to do with the world of objects which Schleiermacher talks of in terms of the 'organic function', the receptive side of our relationship to the world. Given the fallible nature of all knowledge of the world and the fact that all communicating agents learn their language both via a completely different set of causal encounters with the world and via the different situations in which they learn the use of words, the need to interpret what the other is saying is inevitable. One can never be sure that others are using a word in exactly the same way as oneself, because the background knowledge that gives the word its meaning for a speaker is never fully accessible. Davidson, who also sees the issue in similar terms, stressing the idea that we have to assume individuals have an idiolect because of their differing causal histories, tends, though, to give the causal effects of 'objects and events' too exclusive a role in bringing about what people think words mean. While accepting the fact of differing causal stories, Schleiermacher's point is that the individual subject can also actively bring about a re-assignment of terms, which is then taken up by others by understanding a term in a new way. Our very ability actively to arrive at an understanding of another's nonstandard usage suggests that we are not just caused to assign meanings in terms of perceived truth-conditions, and can actively engage in the genesis of new usage.

For Schleiermacher the aim of publicly accountable knowledge is to diminish destructive social conflicts generated by our irreducible otherness to each other. He does not, however, adopt anything resembling Lyotard's SocialDarwinist image of the 'agonistic' essence of social life, where consensus is always likely to be suspected of involving some kind of coercion. At the same time, though, he is concerned with the contingency of the individual, which it can (but need not) make sense to eliminate when seeking to achieve technical purposes, but which it does not make sense to eliminate when responding ethically to other people. Schleiermacher's concern with aesthetic issues, even in his Dialectic, makes it clear that he does not think the overcoming of difference in scientific knowledge for the sake of the community is the necessarily prior activity in terms of which everything else is to be judged. 
In the Aesthetics Schleiermacher distinguishes between 'identical activities' and 'individual activities', which is his version of what Rorty sees in terms of 'public' and 'private' (cf. the end of Chapter 5). In philosophy thinking is 'identical', it aims to articulate universals: 'But if we now look at thinking in reality, then everyone thinks here in a specific language, and there is already a difference in this; so that in general we posit thought as identical, but at the same time we posit that it is different in reality' (Schleiermacher 1842 p. 51). No two people think exactly alike, even if they use the same words. This judgement itself requires identical thinking, because it involves a universal claim. Its content, though, is the reality of individual difference, which no statement can articulate, precisely because it must employ general terms. Other communicative resources, of the kind encountered in aesthetic forms, are therefore necessary for access to individuality. The relationship between the universal and the particular, which was crucial to Hegel and which is at the heart of major divergences in modern thought, once again leads towards the aesthetic.

In Leibniz's classically metaphysical conception the particular was revealed as being deducible from the general: 'in the least substance eyes as piercing as those of God could read the whole sequence of the things of the universe' (quoted in Frank 1986 p. 110). For Hegel 'being' only comes to itself via its determination into particularity, but the particularity is precisely a moment of the coming to itself of the universal, which is its truth. Schleiermacher, on the other hand, argues that the individual, which is not just another term for the particular that can be determined by a general term, is not reducible to a concept that exhausts its truth (see Frank 1977). The certainty that Hegel wishes to establish derives from his presupposing that, however contradictory the movement of Geist in particularity appears to be, it is in fact the self-manifestation of the absolute. For Schleiermacher such a position would only be tenable if one already had 'the general construction of all knowledge in which all individual thinking is included/negated [aufgeht]'. Without such a system there is no way of saying whether there is any thought which 'is excluded from all influence of the individual' (Schleiermacher 1942 p. 134). In the same way as the 'absolute unity of being' (p. 224) is 'not a concept any more' (1988 p. 31), and therefore becomes a regulative idea that motivates the search for better descriptions, 'the individual' becomes a normative limit on our certainty that we have understood the other, a reminder that there may always be something that escapes our ability to articulate it in general terms. In relation to the contemporary versions of Hegel, Schleiermacher offers a reminder that a community which 'sets for itself what is to count for it as its absolute principles' (Pinkard 1994 p. 254) always brings with it the danger that it will exclude and repress members of that community who cannot subordinate themselves to this kind of universality. This danger is what motivates Schleiermacher to insist that his dialectic requires the complement of a hermeneutics that will be able to give a proper role to the individual. In order to see more exactly why this is the case we need to consider Schleiermacher's account of self-consciousness. 


\section{Immediate self-consciousness}

Schleiermacher develops his account from the difficulties we encountered in Kant. As we saw in Chapter 1, Kant's problem was the relationship between the reflecting and the reflected self, the I as subject and the I as its own object. The identity of the subject could not be grounded in empirical consciousness: 'the empirical consciousness which accompanies my representations is in itself dispersed and has no relation to the identity of the subject'. Given that experience does not consist of a chaos of empirical representations there must therefore be a subject which creates a unity between those representations, otherwise 'I would have a self which is as multicoloured and multiple as the representations that I am conscious of having' (CPR B p. 133), or, no self at all. Kant admits the need to posit the existence of the grounding I, whose knowable identity must, like all knowledge, then be synthesised from its different intuitions. However, he insists we have no cognitive access to the I that is the condition of synthesis: it is 'nothing more than the feeling of an existence without the least concept'. What makes knowledge possible is the categorial apparatus of our thinking; this, though, is based on 'the synthetic unity of apperception', which is 'the highest point to which one must attach all use of the understanding, even the whole of logic' (CPR B p. 134). Identity therefore depends upon the unifying of different intuitions in judgement. The problem is that this unity seems to rely on the multiplicity given in receptivity, even though he also argues that there must be a prior ground which makes the unification possible. This was a crucial part of what led to the questioning of Kant's version of the 'unity of apperception' by Fichte.

For Schleiermacher consciousness is grounded in the fact that our very being consists in the 'linking of different moments' (Schleiermacher 1942 p. 272). The reason this linking cannot depend on consciousness as cognition, thus on acts of spontaneity, is precisely that cognition itself depends upon the differentiation of moments that are then synthesised. ${ }^{9}$ There must therefore be an immediate ground which is prior to the differentiation upon which mediated cognition depends. Cognition is necessarily temporal, because it is made up of a succession of intuitions, the linking of the intuitions must transcend this temporality, so that which makes the linking possible cannot be known, yet must exist as its necessary connecting ground. ${ }^{10}$

Schleiermacher distinguishes between Kant's synthetic 'I', and what he terms 'immediate self-consciousness': 'If we look at the single I as something which persists in the developments, thus as something constant and the same in a sequence of time, then the self-consciousness of this identity is only something derived' (Schleiermacher 1842 p. 68). Whereas conscious, reflexive identity relies upon inferences linking different experiences, the individual, immediate $\mathrm{I}$ is the basis of any act of inference: its awareness therefore does not rely on inference to know that $i$ is thinking what is thought. It is the 'activity of the individual [Einzelne] as such in its difference' (p. 69). The difference here is 
between $m y$ individual, existential self-consciousness and all other self-consciousnesses. Schleiermacher's question concerns how one can provide an account of the linking of the different moments of consciousness while avoiding the problems we saw in Kant and the thinkers who follow the reflection model. That model gives no way of explaining how I can apprehend my experiences as my own.

Schleiermacher tries to explain the structure of self-consciousness in terms of 'thought', which is our being receptively affected by the world in, for example, scientific observation, and 'will', our ability to affect the world. When there is a transition from one to the other, from our observing an object to our acting on the object, there must be something which remains the same, otherwise the continuity of self-consciousness necessary for the world to be intelligible becomes inexplicable. Furthermore, receptivity is never wholly passive and activity always involves some degree of passivity:

If at one moment the whole of life is not posited then this is a defect to which a supplement must be added. In the activity of thinking the consciousness of the object is also posited, in the act of will the consciousness of resistance is also posited; one moment completes the other. The transition of both moments into each other must include the positing of the other, that is, it must be posited as pure immediate selfconsciousness. (Schleiermacher 1942 pp. 286-7)

Schleiermacher's account of the subject also relies on what he terms the 'transcendent basis', which is beyond all particular acts of cognition:

as thinkers we are only in the single act [of thought]; but as beings we are the unity of all single acts and moments. Progression is only the transition from one moment to the next. This therefore takes place through our being, the living unity of the succession of the acts of thought. The transcendent basis of thought, in which the principles of linkage are contained, is nothing but our own transcendent basis as thinking being. (Schleiermacher 1942 p. 274)

In consequence, 'The transcendent basis must now indeed be the same basis of the being which affects us as of the being which is our own activity' (p. 275). What must be the case with regard to being, if different things are to be predicated of the same object and for revised judgements about an object to be possible is, therefore, the case for self-consciousness as well: the two are structurally linked by their transcendence of what can be known about them.

Schleiermacher sometimes terms immediate - in the sense of non-reflexive - self-consciousness, as Novalis does, Gefühl, 'feeling'. 'Feeling', as Novalis also argued, cannot feel itself, it is 'different from reflected self-consciousness - I, which only states the identity of the subject in the difference of the moments and thus depends upon a synthesis of the moments which is necessarily mediated' (Schleiermacher 1942 p. 288). The I that we can retrospectively synthesise as having 'accompanied all our representations', which can remember my childhood experiences as $m y$ childhood experiences depends upon an existential continuity of the self which cannot be present to consciousness: 'Feeling and 
the principle of combination [by which he means judgement] are One. For selfconsciousness comes between each moment, because otherwise the acts would be indistinguishable' (Schleiermacher $1990 \mathrm{p}$. 71). In a note added to this part of the Ethics he suggests, echoing Kant in the C7, 'If one goes a step further then all action as combination [i.e. all judgement] is grounded in feeling' (p. 73).

In Being and Nothingness Sartre echoes what Schleiermacher intends by the notion of feeling: 'Consciousness is not a particular mode of cognition, called intimate sense or self-cognition, it is the transphenomenal dimension of the being of the subject. . . . Self-consciousness is not double. If one wants to avoid infinite regress it has to be an immediate and non-cognitive relation of self to self' (Sartre 1943 pp. 17-19). For Schleiermacher we are dependent on the mode of being of our self-consciousness in ways which we could not definitively explain, and this gives rise to the need constantly to interpret oneself and others. Self-consciousness' reflection upon itself is always subsequent both to the fact of its own existence and to the existential fact that there is anything at all rather than nothing.

Schleiermacher explains the nature of the self further in relation to our prereflexive experience: 'We have no idea of the I [in the sense of reflected, synthesised self-consciousness] without reflection. This only gradually develops in human beings after their physical life has already begun' (Schleiermacher 1942 p. 291). The means via which this self-reflection comes about are in part linguistic. Becoming a reflexive 'I' does entail, as Lacan puts it, the 'defiling' of the subject by the signifier. A moment of disruptive non-identity, using an external term that others use, is required for the emergence of my identity. For Schleiermacher, though (and likewise, Novalis), this does not mean that the I is simply a function of the general signifier that comes to denote it. If the I were merely such a function, the individual, existential fact of consciousness would be reduced to the general means of articulation, the signifier. Because Schleiermacher's I does not have cognitive access to its own ground, it is reducible neither to what it is at any particular moment, nor to its reflection in the signifier as the other of itself.

We have repeatedly seen that the mirror or the signifier, i.e. the object side of the relation, cannot provide a criterion of self-identification: this requires something which is not objectifiable. Linguistic articulation is a necessary condition of self-consciousness, but it is not a sufficient one. Understanding words as meaningful requires more than the possibility of a linguistic response that seems to indicate understanding: how do we ever come to understand the words of others as meaningful noises at all? Schleiermacher shows that what is required in an adequate theory of self-consciousness and communication is a way of distinguishing what one must be familiar with to understand what it means for a self-conscious individual to say 'I', from what is required for the noise 'I' to be produced via a rule-governed mechanism. There is, then, an inherent non-identity between immediate self-consciousness, and any general means of signification, and this will be the basis of Schleiermacher's philosophical evaluation of both aesthetics and hermeneutics. 
This conception of the I is where Schleiermacher most obviously diverges from contemporary thinkers who resemble him in other respects. Is immediate self-consciousness, though, not precisely a kind of 'presence', of the kind Schleiermacher excludes with regard to knowledge of the world via his linguistic turn? The answer to this is clearly no. Schleiermacher insists that: 'There are no thoughts without discourse $[R e d e]$. . . one cannot think without words' (Schleiermacher 1977 p. 77), so no mental items can be invoked which would ground meaning without the necessity of language. However, if immediate selfconsciousness does not entail a version of presence, and yet is not to be equated with determinate thought, how are we to say anything about it?

This is, of course, the crux of the matter. Descartes says that I can know nothing more fully than my own mind, which therefore involves the grounding form of presence: 'I think, I am'. As we have already, immediate selfconsciousness does not rely on a propositional form of knowledge. 'Feeling' need not be verbally determinate. Frank summed up this issue as follows, as we saw in the last chapter: 'Knowledge of myself as of myself does not depend on classification. But it also does not depend on identification [on ascribing a mental predicate to a singular term which stands for myself, so that 'I know that I '’] - for how should I identify an object which could not be anything but myself?' (Frank 1991 p. 407). 'Feeling' may begin in one sense to look suspiciously like an 'I know not what', and this is certainly the case if one assumes that all that is accessible to us must be propositional. However, feeling also seems to play an ineliminable epistemological role even in fallible determinate knowledge - in so far as I must be infallibly aware, at the moment I think I know something via my perception of the world, that I think it is the case, otherwise the possibility of self-correction becomes incomprehensible. Furthermore, feeling is for Schleiermacher the basis of forms of awareness and articulation which cannot be reduced to the terms in which we can verbally articulate them. The link to music is vital here.

Although the notion of feeling is perhaps the most problematic aspect of Schleiermacher's thought, it does point to a dimension which is sometimes neglected or glossed over in much philosophical discussion of language. We evidently must rely, for example, on verbal language to extend our understanding of music; at the same time, though, music itself can change our understanding of verbal utterances. The interplay of metaphorical and literal usage in articulating an understanding of music points to what is at issue here. Samuel Wheeler has argued that when Davidson claims the only meaning a metaphor has is its 'literal' meaning, what is meant is that most of what language does need not be thought of in terms of bi-conditional statements which define literal meaning, such that "'Snow is white" is true if snow is white.' Instead metaphors, like music, matter because of what they make us notice, because of the world-disclosing capacity that they share with music. The employment of metaphor in trying better to understand music suggests why what music says is never reducible even to our best ways of verbally articulating it. We are rarely, if ever, 
wholly happy with a metaphor, even though it may disclose aspects of a piece of music that other descriptions cannot. ${ }^{11}$ The fact that even in a verbal utterance the musical can be more significant than the semantic is too often forgotten in discussions of music and language. However problematic the notion of immediate self-consciousness may seem, then, it at least keeps open ways of thinking about meaning and intelligibility which are not reducible to what can be said in words, and this is, of course, vital to aesthetic theory.

\section{Art as free production: 'individual' and 'identical' activity}

So far I have tried to show how Schleiermacher initiates a 'linguistic turn', but not in the manner that dominated so much analytical philosophy until the advent of the holism of Quine and Davidson. Communication depends for him, as it does for Habermas and Davidson, primarily on the preparedness and ability of individual language users to overcome differences by reaching agreement or understanding, despite the lack of determining rules. Perhaps the most vital part of his conception, however, which differentiates Schleiermacher even from these contemporary theories of communicative action, is his focus both on linguistic change, and on those aspects of our self-conscious being which are not adequately articulated by what can be said about them. With regard to the former, Schleiermacher characterises the shifts in linguistic usage that redefine our relationships to the world and each other in a way which relies on human subjects as potential initiators, rather than just passive objects, of the worlds articulated in language. At the same time his position does not underestimate the extent to which people can be objects of language: 'A simple appropriation of thoughts which have already been laid down in language is not an activity of reason, and if we assume someone whose whole thinking is nothing more than those thoughts, then that person is hardly a person at all (Schleiermacher 1990 p. 264). 'Poetic' usage, creative initiatives in language are, then, not a special case, or deviations from a norm, but are instead inseparable from the very nature of language. It is for this reason that Schleiermacher's hermeneutics is so closely linked to aesthetics.

Probably the first explicit formulation of the 'hermeneutic circle', the attempt to understand the part of a text or utterance via the whole, and the whole via the parts, derives from the application of Schelling's philosophy to the question of interpretation. In 1808 Friedrich Ast, a pupil of Schelling, suggested a method for understanding texts from the past based on a questionable appropriation of Schelling's identity philosophy. For Ast, we are able to reproduce the thought of the past through its essential identity with our own thinking. He uses the idea of the organism to ground understanding in the idea that each thought can be understood as part of an intelligible whole:

The basis of all understanding and cognition is finding the spirit of the whole from the single part and grasping the single part via the whole ... each is only posited with and by the other, just as the whole cannot be thought without the single part as a 
member of it and the single part cannot be thought without the whole, the sphere in which it lives. (in Gadamer and Boehm 1976 p. 116)

Schleiermacher was clearly influenced by Schelling, and he already begins work on hermeneutics in 1805 . The conclusions he reaches are, though, very different from the Idealist ones reached by Ast. The irreducibility of individuality that derives from immediate self-consciousness means that the part could only relate to the 'spirit of the whole' via an unprovable postulate that the whole is the other and complement of itself. Grasping the relationship of part and whole, which Schleiermacher sees as essential to understanding, is therefore a task that cannot be completed because of the contingencies of our access to what is relevant for a particular case. Context is constitutively boundless, even though we can make pragmatic decisions about what needs to be taken into account in particular interpretations. Schleiermacher also questions Ast's position by insisting upon the inextricable involvement of thought with particular languages, there being, as there was not for Hamann, no 'general philosophical language' which would reconcile the differences of the particular languages. Importantly, he associates this argument with aesthetics.

Schleiermacher, as we saw, regards all the operations of our thinking as involving 'art'. He characterises art, in the narrower sense of the object of aesthetics, as 'free production . . . of the same functions which also occur in the bound [gebunden] activity of humankind' (Schleiermacher 1842 p. 375). Everyday understanding and aesthetic judgement are linked by the fact that they both rely on rules, but these rules rely on application, which cannot itself be bounded by rules. He now establishes a link between everyday understanding, aesthetic judgement, and the production of art, all of which involve differing degrees of the rule-bound and the spontaneous. Instead of setting up definitive boundaries between art and non-art, Schleiermacher sees the possibility of transitions from one to the other in any sphere of activity. This allows the too often unreflectively reiterated question of 'What makes something a work of art?' to be circumvented by the idea that art is a perennial possibility in any form of meaningful articulation. Whether something is art will then depend upon the relation between bound and free production in a community, a relationship which is not historically stable. If I write the handbook for a nuclear power station, for example, I am bound by the purpose of not having the station function like Three Mile Island or Chernobyl. No one will be terribly concerned whether the free productive act of writing the handbook has a value in itself for me because of my pride in my ability to construct beautiful sentences. To this extent my handbook would, in Schleiermacher's terms, be unlikely to be a work of art, even though some sentences in it may achieve aesthetic status by offering possibilities beyond the function of the handbook. In other circumstances, however, it is conceivable that parts of such a text could be transported into a novel in a manner which was essential to the novel's aesthetic achievement. 
In a further example of the role of a 'private'/'public' divide, Schleiermacher maintains that art is an 'individual activity', whereas a technical handbook would involve 'identical activity', and 'artistic activity belongs . . . to those human activities which ... presuppose the individual in its difference from the other' (p. 61). Art and individuality are inextricably connected: the wider question here is how the divide between the public and the private manifests itself in the culture of modernity. Hegel's Aesthetics failed to give a convincing account of how the very individuality of art, of the kind encountered, say, in Kafka, could give rise to an enduring universal significance. Because aesthetic productivity is linked to 'immediate self-consciousness', a notion for which Hegel had no time at all, the results of aesthetic production cannot, for Schleiermacher, be subsumed into an identical meaning for everyone: "not a trace of knowledge arises yet out of all thinking in poetry [Poesie with the sense of poiesis, productivity], it only expresses the truth of the single consciousness' (p. 66). What, though, makes this the 'truth' of that consciousness, without this meaning that there are simply as many individual 'truths' as there are poems by individuals? Knowledge, as belief that can be justified to others, makes claims to universality and so negates the individual in favour of general propositions which can be accepted in a linguistic community. The creative text allows for the unfolding of something which need not be reduced to what can be affirmed or denied by a community: think, for example, of the way in which some of the most effective metaphors may elude any kind of truth determinacy or general agreement as to their import. However, the point about individuality characteristic of art is that it can still take on collective significance. This apparent contradiction leads to the core of Schleiermacher's thinking.

The never to be realised, counter-factual goal of Schleiermacher's philosophy is the 'individual-universal'. What Schleiermacher intends is in certain ways close to what was implied by the connection of freedom and beauty in the System Programme. Unlike the Idealists, however, Schleiermacher does not assume there could be an ultimate reconciliation of individual and universal. In that case, what status does this goal have? As will be clear from the present context, this is not just an issue concerning truth conceived of as the regulative idea of the overcoming of particularity by universality, of the kind we considered in relation to the question of knowledge and the absolute in Schleiermacher. It is rather a question about the wider goals of a culture which would allow for difference and individuality, while demanding that such difference and individuality be open to discussion as to its legitimacy. Art is significant, therefore, because of its constant testing of the relationship between the 'identical' and the 'individual'. In this perspective the history of art comes to be seen as the manifestation of the attempt to combine individuality and universality in free human production. Art is, then, not simply the individual, 'private' counterpart to identical 'public' science and philosophy. It is both a reminder of what can be excluded by science and philosophy, and can disclose new ways of looking at things which affect science and philosophy. 
Because his aesthetics relies upon the idea of a productivity which plays a role in all forms of human activity Schleiermacher bypasses the dichotomy between an aesthetics of reception and an aesthetics of production: 'Because beauty is produced via human activity more than by anything else the production and reception of it are the same. Productivity and receptivity are only different in degree' (Schleiermacher 1984 pp. 3-4). This leads him to the following piece of apparent Romantic hyperbole: 'all people who make a work of art their own in some way are to be regarded as artists' (p. 178). If something is to be music at all the listener must hear it as music. There is no rule for deciding the status of a sequence of noises, so the listener must be involved in the same kind of 'production' as the composer, because they must transcend anything that can be learned mechanically, albeit to a much lesser degree than the composer. This ability is, of course, and this is the crucial point, also exercised all the time in the everyday understanding of and production of novel utterances. Robert Brandom has suggested, admittedly associating his view with Hegel, what is at stake in a view like that of Schleiermacher:

What matters about us morally, and so ultimately, politically is . . the capacity of each of us as discursive creatures to say things that no-one else has ever said, things furthermore that would never have been said if we did not say them. It is our capacity to transform the vocabularies in which we live and move and have our being (Brandom 2000 p. 178).

In such a perspective the role of the aesthetic is not, though, as it was for Hegel, merely to be the prelude to a higher form of truth. The aesthetic becomes instead the locus of debates over what matters to people which should not be dominated by the manipulations either of a levelling, commodity-determined culture, or by scientism. At one end of the notional scale of human production for Schleiermacher is 'science', in the sense of agreed objective knowledge; at the other end is art, free productivity for its own sake. This difference is, though, only ever relative and is only possible because of 'what matters most about us', namely our ability to do things in new ways even within the most 'identical' practices.

\section{Hermeneutics as art}

For Schleiermacher the necessity for interpretation of the utterances of others is always present, and not just necessary in special cases. The 'stricter practice' of the 'art' of hermeneutics therefore presumes that 'misunderstanding results as a matter of course and that understanding has to be desired and sought at every point' (Schleiermacher 1977 p. 92). In the same way as knowledge could only ever aim to eliminate the individual element, without there being a position from which the elimination could definitively be verified, the hermeneutic circle results from the fact that 'each person is . . . a location in which a given language forms itself in an individual [eigentümlich] way', but it also results from 
the fact that 'their discourse is only to be understood via the totality of language'. These two quantities, the individual and the totality are, as we have seen, not reducible to each other. Because language results from 'speech acts' (p. 80) the speech act is inherently individual: it is your or my act at a particular time in a specific situation. Uttering the same words is not the same speech act. As such, speech acts 'cannot be subordinated to calculation', they cannot be 'mechanised' in terms of grammar because interpretation relies on never fully available contexts. The idea of the totality of a language, the langue in Saussure's terms, is itself just a regulative idea: 'No language is totally available to us, not even one's own mother-tongue' (p. 84). There are, furthermore, no linguistic 'rules ... that would carry the certainty of their application within them' (p. 81).

It is clear, of course, that there are plenty of linguistic rules and constraints, without which we could not communicate, but this idea only captures one dimension of language, the public norms without which intelligibility would never be possible at all. The point is that these norms also can be transcended by individual usage. The individual can come into play in any situation in life where new usage makes something new possible, offers a solution to a problem, or just rebels against the established way of saying something, and this is why Schleiermacher does not confine the notion of art to the more narrowly aesthetic sphere. He introduces the notion of art in order to suggest how the individual, disclosive dimension of language is always an issue in interpretation: 'The full business of hermeneutics is to be regarded as a work of art, but not as if carrying it out finished in a work of art, rather in such a way that the activity only has the character of art because with the rules the application is not given as well i.e. cannot be mechanised' (p. 81). The level of language which might seem 'mechanisable', reducible to a set of rules that explains its functioning, Schleiermacher terms the 'grammatical'. The grammatical corresponds to the notional totality of the linguistic system, the sum of the norms which govern intelligible linguistic practice. However, such a totality is infinite 'because every element is . . . determinable by the other elements' (p. 80). We can only understand one speech act via a whole series of others that form its background of intelligibility, and this applies to these acts in turn.

We encountered the structure of this argument in a different context in Schelling's 'Würzburg System', and in Hegel. In the light of Jacobi's arguments about Spinoza, Schelling saw scientific investigation as infinite because 'Every single being is determined by another single being, which in the same way is determined by another single being etc. into infinity.' That was why he argued that, because it does not primarily depend upon concepts, which lead to chains of determination, the work of art can attain a kind of immediate meaning that is inaccessible to science. As we saw, Derrida's demonstration that a single signifier could never generate the presence of a meaning echoes Schelling's ideas on the deferral of presence in his identity philosophy. On one level Schleiermacher himself prefigures Derrida: because each linguistic element is affected by its context it would only be fully determinable if one were able to specify all con- 
ceivable contexts in advance. However, Schleiermacher is interested in the successful praxis of communication, rather than, as Derrida sometimes seems to be, in the consequences of the ultimately aporetic claim that there can be no Cartesian certainty about meaning.

The fact that language is manifest in the form of speech acts leads Schleiermacher in important new directions. Language users are individuals, and the existence of language is therefore dependent upon their contingent acts: 'language must individualise itself. Otherwise it can only be thought of as a capacity but not really exist' (pp. 363-4). Humboldt makes the same point: '[language] has nowhere, not even in writing, a permanent home, its so to speak dead part must always be reproduced anew in thought' (Humboldt 1973 p. 57). The individuality that Kant reserved for the genius in art, who established new rules via aesthetic production, is carried over into all areas of linguistic usage and thus into all areas of human activity. Without the ability to move from the grammatical level to the level of context and application which is not bounded by rules, we could neither understand nor communicate in an effective manner. ${ }^{12}$ Now this may really sound like Romantic hyperbole, but much the same point has been made by Brandom:

Every use of a vocabulary, every application of a concept in making a claim, both is answerable to norms implicit in communal practice - its public dimension, apart from which it cannot mean anything (though it can cause something) - and transforms those norms by its novelty - its private dimension, apart from which it does not formulate a belief, plan, or purpose worth expressing. (Brandom 2000 p. 178)

Once one moves away from theories of language which seek to explain it solely in terms of how it represents bits of the world, towards a conception of it as a public activity through which individuals can realise themselves and their wishes, this whole dimension of Schleiermacher's thought turns out to offer significant resources for contemporary revisions of the idea of the philosophy of language, let alone, of course, for revision of the history of the philosophy of language.

Presenting Schleiermacher in these terms may seem rather odd, given his reputation for subjectivism, apparently demonstrated in remarks like this: 'We cannot know whether the other person hears and sees just as we do' (Schleiermacher 1942 p. 371). The insistence of Rorty and others on the fact that there can be nothing sub-propositional, so that the 'space of reasons' is inherently linguistic and intersubjective, relegates the dimension of immediate self-consciousness to meaninglessness, because it cannot result in the cashing in of a public validity claim. The aesthetic dimension of what Schleiermacher intends is, though, not touched by this kind of claim. His contention is that knowledge of 'whether the other person hears and sees just as we do' requires the assumption that there is an identity in the way we schematise our perceptions. We must make this assumption, which can never be definitively confirmed, precisely because there is no shared given, only the contingencies of 
what each individual receives in 'organic affection' and schematises with a shared language which is acquired in conjunction with those contingencies. As such, thoughts, as propositionally articulated publicly shareable objects of agreement or disagreement, must be distinguished from what does not reach the level of propositionality.

At the level of propositions Schleiermacher claims that even if what another person sees as the Bild, the 'image' of the colour, may be different from what I see: 'This can never be established, but this does not matter if the object is only the same one that I have and the other person describes the same actions in relation to the object as I describe' (p. 373). He does, however, insist on the contingency vital for his claims about art by claiming that 'all communication about external objects is a constant continuation of the test as to whether all people construct identically' (p. 373). There is, then, no guarantee that any series of classified observations can be generally grounded in certain knowledge that is common to all people. This leads him to a vital hermeneutic point:

The identity in the construction of thought as the element of knowledge is only manifest in language [i.e. the system of iterable signifiers with which we communicate]. But there is no general language, therefore there is also no general identity of construction. Thus this characteristic is not realised and will not be realised. All attempts to reach a general language are failures; for the agreement about a general language is itself subordinated to particular languages. (p. 374)

He also prefigures Habermas's Heidegger-derived insistence that theory is grounded in the background consensuses of the language of the life-world:

Language never begins to form itself through science, but via general communication/exchange (Verkehr); science [Wissenschaft, which includes the sense of 'philosophy'] comes to this only later, and only brings an expansion, not a new creation, in language. As science often takes the direction of beginning from the beginning, it must choose new expressions for new thoughts. Forming new root words would be of no help because these would in turn have to be explained by already existing ones. (p. 511)

In a typical example of his misapprehension of what Schleiermacher argues, Gadamer attributes this point to Wittgenstein (Gadamer and Boehm $1976 \mathrm{p}$. 323). Just as Wittgenstein moved away from the idea in logical empiricism of a logically purified language, Schleiermacher argues in the wake of Hamann that if one wished to be able to articulate the correspondence of subject and object, language and reality, one would have to presuppose an 'absolutely general language. But there is no means of producing such a language . . . For language is not always susceptible to construction and remains connected to the area of nature' (Schleiermacher 1942 p. 379). Natural languages both arise in and are transmitted through the contingencies of the sensuous world and they are the basis of our primary understanding of the world. Any subsequent attempt to arrive at a general language therefore cannot be shown to have wholly obviated the forms of understanding which first make the world intelligible to us at all. 
How, then, is it that differing organisms can come successfully to employ the same communicative forms? Part of Schleiermacher's explanation involves his most controversial and misunderstood term: 'divination'. Since Dilthey in particular, 'divination' in Schleiermacher has been assumed to mean 'Einfühlung', 'feeling one's way into' another person's thoughts via their utterances. This notion has rightly been attacked as psychologistic, and has helped produce some truly awful literary criticism. Even from the arguments seen so far it is evident that Schleiermacher could not have meant anything of the kind. Furthermore, he did not use the term Einfühlung. His own explanation of 'divination' uses the telling example of children's initial language acquisition. Language cannot be language without universals: in communication 'Everyone seeks to fix the universal image for themselves and others' (p. 373). The problem here, which we already encountered with regard to reflective judgement in Kant, is how a general schema is to be applied to a potentially infinite series of different objects which are supposed in some way to be the same, for example, a tree.

The obvious answer would seem to be comparison of different cases, but this does not solve the problem because one would need a first tree, an idea that is the basis for the subsequent series of comparisons. A tree can only be said to be a tree via the discriminations made possible by language, and language relies on the structure of one thing being determined by another. Unless one has already presupposed that the first object really is a tree no series of comparisons will definitively establish its identity, because they will lead to an infinite regress. ${ }^{13}$ Schleiermacher argues that everyone finds themself in the situation of requiring this presupposition when they acquire their first language, but that the presupposition cannot be firmly grounded. There is no 'given' upon which one can build, the given itself is constituted from the interaction of private experience and the public use of language, and the two sides can never be clearly divided from one another, private experience always being in some measure organised in public forms, and public language in real usage always being in some measure affected by 'private' interpretation. Any attempt at understanding another's utterance therefore involves a hiatus between the use of general signifiers and the particular individual's understanding. For children this is particularly the case:

They do not yet have language, rather they are looking for it, but they also do not yet know the activity of thinking because there is no thinking without words: on what side do they begin [i.e. by comparison or 'divination']? They have not yet got any points of comparison but they only gradually acquire them as the basis of an unexpectedly quickly developing comparative procedure; but how do they fix the first thing? (Schleiermacher 1977 p. 326).

The answer is 'divination'. But what does the term mean?

Divination involves 'production', 'creation' (Erzeugung). Children have what Schleiermacher calls an 'inner mobility towards creation on their own part' ( $\mathrm{p}$. 327 ), which goes along with a 'directedness towards the reception of others'. He 
uses the same terms in relation to art. There is no absolute point from which the process of language acquisition can be said to begin, but given that we do successfully acquire language, we must infer that individuals can make revisable, ungrounded guesses at their 'first thing'. Davidson suggests, in the same vein, that our understanding of the use of words in context relies on forming of what he terms 'passing theories'. These have the same function as reflective judgement, moving from the particular to the general without a guiding rule, and 'there are no rules for arriving at passing theories, no rules in any strict sense, as opposed to rough maxims and methodological generalities' (Lepore $1986 \mathrm{p}$. 446). Acquisition of language therefore can be neither the accommodation of consciousness to a system of pre-existing signifiers in which it mirrors to itself what it is, nor the means for the direct apprehension of given ready-made objects, nor a merely solipsistic individual giving of meanings to signs. All use of language must be potentially creative because it can bring about a revision of how a term is used. Divination is, then, a necessary component of our everyday, and always incomplete, rule-governed praxis of understanding each other and the world. When I say something which is understood by another, or understand something they have said, this does not mean that I simply reproduce the words used with an identical sense. It means instead that, as Frank has put it, I 'carry out another articulation of the same linguistic chain' (Frank 1986 p. 123). Because it is the articulation of a different individual in a different set of circumstances there is always an aspect which is different, even when the 'same' sentence is used. ${ }^{14}$

Schleiermacher's expression of admiration for children's powers of language acquisition shows how his conception also involves an ethical dimension: 'it seems to me that we only smile at the wrong uses that children make of the elements of language they have acquired - which they not infrequently make only via too much logical consistency - in order to console ourselves for or revenge ourselves on this preponderance of an energy which we ourselves no longer possess' (Schleiermacher 1977 p. 327). Children create new forms of language in their attempt to produce communication with others. At the same time, every time we ourselves fail to understand or make ourselves understood, some aspect of this 'energy' must come into play if we are to overcome our misunderstanding. I have elsewhere termed the result of this situation the 'hermeneutic imperative' (Bowie 1997). Individual lives are a never completed series of attempts to grasp, via divination and comparison, the meanings of others (and, of course, by reflecting on one's own past utterances, one's own meanings). The acquisition of 'grammar' is vital in this, but 'the more the soul already possesses, its receptivity becomes more sluggish in its movements, so that even in the most lively soul, precisely because each in its individual being is the non-being of the others, it is the case that non-understanding will never completely dissolve' (Schleiermacher 1977 p. 328). One has therefore to live with the contingency of understanding, even as one cannot escape trying to achieve understanding. Schleiermacher's conception of understanding and interpretation is, then, in 
many respects a precursor of contemporary moves towards normative accounts of meaning in terms of discursive practices which are not susceptible to the kind of rule-bound explanation sought by the founders of analytical philosophy. A further dimension of Schleiermacher's exploration of language, which played virtually no role in the dominant accounts in the analytical tradition and which is still often underplayed in contemporary philosophy, must now be examined.

\section{Literature and the 'musical'}

One of the deciding factors that makes Davidson's account of interpretation more plausible than that of Lyotard is that it is hard to see how one could assert that two people are communicating in a language at all if they did not share some kind of presupposed intuitive understanding of truth. Faurisson is making a claim about how something can be legitimated, not denying the possibility of truth in relation to Auschwitz. Sequences of noises only become meaningful signifiers via some kind of shared world that we understand in terms of claims that something is or is not the case, even if we disagree totally about some fundamental aspects of that world. Something analogous would seem to be the case for music: what makes organised sound into music is a consensus on the part of a community of listeners that the sound conveys something intelligible and organised, even though it may have no directly assignable semantic content. In this sense music plays the role of a kind of 'vocabulary' for that community by disclosing aspects of, for example, its affective world to it. The bridge between language and music can, in a rather loose but useful way, be understood via the idea of metaphor. A metaphorical utterance of the kind present in a radical modernist poem may in many cases not be comprehensible in terms of its truth conditions, but may yet be world-disclosive in some significant respect. Music can be seen in this perspective as part of a historically shifting continuum of means of articulation which goes from 'identical' scientific language, via differing degrees of metaphor, to 'individual' musical forms. It is along such a notional continuum - whose divisions are neither stable nor in any way definitive - that the issue of 'literary' language in modernity is located.

The 'poetic', in the sense of the creative and the 'literary', has remained a contentious issue throughout modernity, with some theories granting literary usage a radically special aesthetic status, and others denying that there is any such thing as literature at all because literary texts are just texts like any others. The genesis of the disciplines of 'literary criticism' in the English-speaking world and of 'literary hermeneutics' in the German-speaking world - whose roots can be traced to this period - highlights the degree of contention surrounding the question of literature. ${ }^{15}$ From what we have seen so far, Schleiermacher's basic intuition about the nature of interpretation suggests something important about the battles carried out in these disciplines over the understanding of literary texts. If the task of interpretation is to find agreed rules of meaning, or certainty about what a particular text means, why do so 
many people in the modern period spend so much time in never finally resolved institutionalised conflict over utterances of others, 'literary' or not? Why are there no 'definitive' interpretations of major literary, or, for that matter, any other significant texts?

As we have seen, this situation can be thought of either, as those like Lyotard and others influenced by Heidegger do, as pointing to some major crisis in modernity to which philosophy has privileged access, or, as it is for Davidson and Rorty, as the source of a new awareness of the different uses of differing kinds of discourse that leads to the need for a constant renegotiation of how our communicative practices are to be justified. Adhering to the idea that this constant need for renegotiation should be regarded as the sign of a universal crisis requires one to take philosophy too seriously, making an often rather restricted version of the history of philosophy, as it largely is for Heidegger, the key to history as such. It is only by a much more wide-ranging and specific analysis of the justificatory and expressive practices of modern societies that the effects of the putative end of universal forms of understanding can be adequately assessed. The best way to achieve this is by what Brandom described as the constant interplay of the public and private aspects of our communicative practices. Schleiermacher offers interesting possibilities here. By centring his attention on the presence of an individual, private aspect even in the most apparently rulebound activity, Schleiermacher is able to claim that 'everywhere, including the realm of science [meaning natural science], there is a free play of thoughts which is a preparation for artistic production' (Schleiermacher 1977 p. 180) - 'artistic' relating once more to the fact that rules do not apply themselves and therefore demand divinatory application. Post-empiricist theories of science now stress that any new theory can be considered to be a metaphor, which may be false in terms of the present web of explanations, but which can become literalised and true if the theory is accepted. Once the assumption, generated by the myth of the given, that there are bits of the world that make our sentences true comes to be regarded as unintelligible, a strict line between the literal and the metaphorical gives way to a conception in which the purposes of particular uses of language are the central issue.

The rejection of definitive divisions between the metaphorical and the literal is what leads Schleiermacher to claim there is no absolute difference between 'aesthetic' and other aspects of linguistic usage. Although Poesie is bildlich, concerned with intuition and particular images, it is present in some degree even in the most rule- and observation-bound activities: 'The more distinct the laws of a form are, the more empty is the production of individuality. In this way the individual life is opposed to what is mechanised. But the relation of the two varies in different texts. The individual never completely recedes' (p. 191). To this extent science and art 'cannot possibly be totally opposed to each other' ( $p$. 194). There will always be a tension between the notional general status of any signifying system and its particular use in practice, which can never be 'mechanised'. Schleiermacher opposes 'poetry', creative, literary production to 
'prose', which includes any discursive practice, including in the natural sciences: the 'general hermeneutic difference between poetry and prose is that in the former the singular wishes to have its particular value as such, in the latter it only wishes it in the whole, in relation to the main thought' (p. 140). The Dichter (in the sense of any 'creative writer') most directly confronts this issue, but its implications are inherent in all linguistic usage. This conception is, then, in line with contemporary pragmatist ideas about the need to see scientific practice in terms of its differing 'discursive commitments' to those involved in 'poetry', not in terms of its inherent superiority to or radically different nature from other forms of human practice.

What rarely plays a central role in contemporary conceptions, however, is the following vital aspect of language, to which Hamann, Schlegel and Novalis also adverted. ${ }^{16}$ Schleiermacher sees language as having both a 'logical' and a 'musical' aspect. It is the latter which is essential for Poesie. The 'productivity' that leads to articulation in language is internal: 'but it only becomes external via the note $[T o n]$. This is analogous to the musical element and in the use of language we always get an impression of this musical element' (Schleiermacher 1842 p. 633). The musical element in language is evidently not directly semantic, but the fact that the illocutionary force of any utterance can be changed by its tone, emphasis and rhythm means that in actual communication what an utterance does is not adequately understood in terms of its supposed semantic content. Schleiermacher connects the significance of the 'musical' to his arguments about immediate self-consciousness, suggesting one way of countering objections to this notion. Habermas has reportedly claimed in conversation that immediate self-consciousness is really just a residue of our animal past, which can only become significant when its intersubjective consequences are articulated in language. However, this view underestimates the way in which human culture is unthinkable without forms of articulation which cannot be reduced to the ways in which we try to understand them in language, but which have their own kind of 'meaning', the meaning which Novalis and Schelling found, for example, in rhythm. ${ }^{17}$ In a further move, which makes this difficult concept more plausible, Schleiermacher connects the kind of meaning which is implied by the 'musical' to immediate self-consciousness.

I suggested in Chapter 5 that 'any form of articulation that can disclose the world in ways which affect the conduct and understanding of life' can be regarded as possessing meaning, and that 'if meaning is what can be understood, then music is meaningful by the very fact of its being music rather than noise'. For Schleiermacher the meaningfulness of music depends on the fact that the human mind has a strong tendency 'to be able to represent itself purely in its mobility, apart from everything logical' (p. 400) - by 'logical' he means 'propositional' and truth-determinate. Musical production makes us 'conscious of the mobility of human self-consciousness' (p. 395), a mobility which affects the nature of our being in the world, and which can in turn be affected by music. To the extent to which this mobility has little or no semantically determinate 
content and must be experienced in interaction with a work of art, it can become a criterion of aesthetic autonomy. What Schleiermacher intends here therefore also prefigures the emergence of forms of modern art which rely precisely upon their freedom from representation. This move away from representationalism leads to a further vital idea. As we shall see in more detail in Chapter 7, the establishment of music as an increasingly central aspect of modern culture is linked to the emergence of the modern notion of literature. ${ }^{18}$ Why this might be the case becomes evident from Schleiermacher's arguments concerning language and style.

The identification of a 'literary style' depends, in a broad sense, upon the 'rhythm' of an author's sentences, which depends upon characteristic recurrences of linguistic elements and the manner of their combination. The 'logical' is that which is open to examination as to its truth status. This status must have to do with general criteria of judgement which, though articulated in particular utterances, should transcend the particularity of the individual utterance. Even in the realm of the 'logical', 'a sentence can as an expression in language be completely adequately formed in terms of its logical constitution, but it offends us because it does not satisfy the musical' (p. 635). This piece of apparent aestheticism is in fact better understood in terms of the pragmatist claim that language can be a tool in more than one way. The ability persuasively and effectively to communicate a claim can be as important to its success as is its 'logical' soundness. The questioning of the borderline between rhetoric and truth that we will see in an extreme form in Nietzsche is dealt with here in a much less melodramatic and in some respects more enlightening manner.

Oliver Sacks cites the case of the aphasiacs who, while being incapable of understanding distinct words as such, were able to unmask a lying speech by President Reagan by understanding the way in which language was being used on the musical level. Sacks says of aphasiacs that one has the feeling 'that one cannot lie to an aphasiac' because they 'have an infallible ear for every vocal nuance, the tone, the rhythm, the cadences, the music, the subtlest modulations, inflections, intonations, which can give - or remove - verisimilitude to or from a man's voice' (Sacks 1986 p. 78). Most of the audience of aphasiacs laughed at Reagan's speech. The real sense of the 'musical' was lacking in what Reagan said: 'it was . . . above all, the false tones and cadences of the voice, which rang false for these wordless but immensely sensitive patients' (p. 78). What is at stake in Schleiermacher's connection of aesthetics and language therefore reveals a political dimension in the need to attend to the musical. The fact that it requires a shared mental pathology to make it collectively obvious that Reagan made mendacious speeches reveals the extent to which the particular sensibility to the use of language highlighted by Schleiermacher and Sacks is easily lost, or may never even develop. The concrete political implications of this are anything but easy to pin down, but the role of the aesthetic here does involve a clear warning that the kind of philosophy which concerns itself solely with the propositional dimension of language will fail to address other dimensions of lan- 
guage which are inseparable from the culture of truth in a community. Because he takes individuality so seriously Schleiermacher sees non-violent communication between individuals as an imperative. In this way a theory of language inseparable from aesthetics adumbrates a political theory whose aim is to establish a situation in which individuality can articulate itself without overriding the individuality of others.

Art in this perspective is concerned with articulating what 'identical' thinking can repress because of its orientation towards fixing a world of stable objects for technical and scientific purposes. If philosophy is conceived of as the discipline which is to arrive at general truths, the difficulty of writing philosophy which does justice to the aesthetic dimension is obvious, and is part of what gives rise to controversies over the relationship between the literal, and the aesthetic and rhetorical dimensions of texts. The Dichter for Schleiermacher has to 'provide something that cannot really be given by language, for language only ever provides the general' (Schleiermacher 1842 p. 639). A flower in a botanical handbook (Schleiermacher's example) must be described via a schema that applies to others of the species. This is generally no problem in pragmatic terms: one recognises the flower if the schema fits, though the explanation of how this is possible faces all the difficulties we encountered in the questions of judgement and language acquisition. A flower in a literary or other aesthetic context - this applies also to painting: think of Van Gogh's sunflowers - is not to be seen as a token of a type. If the depiction of a flower in a botanical handbook is judged to have aesthetic value this will not depend upon the image being able to be used as a means of identifying the flower in a field. Neither will a 'poetic' evocation of a flower be judged by whether it enables you to recognise and pick the flower on a summer's day. For Schleiermacher 'the poet . . . is concerned with the truth and complete determinacy of the singular' (p. 639). The 'truth' of the singular cannot be the truth of thinking whose aim is identification and classification, but this raises the crucial question of what sort of truth it can be.

The answer to this question leads to another dimension of the relationship between the public and the private. The very possibility of language depends upon iteration: the public aspect of language relies on repeatability of signifiers as a necessary condition of intelligibility. However, one of the concerns about language that emerges with Romanticism is that the iterability of words can become a too exclusive means of fixing a world which is in fact dynamic. The 'private' dimension of the subject that resists being reduced to sameness therefore requires language to function in a different manner. Schleiermacher argues that one of the things that can occur in literature is that 'the changing, floating, purely transient aspect of the state of mind [Gemütsstimmung] should be presented [zur Anschauung gebracht]' (p. 640) against the fixity of the signifier. Music has an obvious advantage here: it can convey dynamic states of mind because, even though the Western chromatic scale consists only of twelve notes, music's significance is the result of the relations between these notes, the representational function of music being subordinate in a manner which it is not in 
everyday language. ${ }^{19}$ The same chord or musical phrase can function in an indefinite number of ways and thus can articulate something singular - think of Adorno's example of the disruptive use of the $\mathrm{C}$ major chord in the atonal context of Berg's Wozzeck. Language, Schleiermacher claims, is 'simply irrational in relation to the singular' (p. 643) because one of the primary pragmatic functions of language is to reduce difference to identity. ${ }^{20}$

Iterability and singularity are, then, opposed, yet meaning requires both if language is to work in real contexts. Thought requires language if it is to be determinate, and even the realisation of our particular desires requires the articulation in general terms of what we desire. Such articulation, though, obviously involves more than the simple schematisation required for identifying entities in the world. What I want may very often not exist in the world, so I am hardly able to identify it by representing it in an already constituted language. The key factor in Schleiermacher's response to this issue is again the 'musical'. The musical 'consists of nothing but transitions . . . by virtue of this, language is capable of directly representing the changeable in spiritual being' (p. 642). This is, incidentally, another case where music can be regarded in a manner analogous to Hegel's Logic, which, as we saw, tried to characterise the pure movement of thought. The movement between signifiers or statements is what gives rise for Schleiermacher to Poesie. The effect generated by such movement is not, though, merely indeterminate, as the very specific nature of poetry makes clear. The semantic potential of a poem or piece of literary prose is connected to the level of its specific formal organisation, which is constituted by the movement between the signifiers as it is read.

For Schleiermacher the possibility of creativity 'is already originally in language, but admittedly it is only the poetic where it appears' (p. 643). At the point at which language realises potential for new significance it is necessarily individual. Originality cannot, though, consist in the endless production of new words, because of language's reliance on iterability. What counts is the combination of the words in new configurations, which is, of course, where language again comes close to music, by relying on rhythmic and other aspects of articulation that are not directly semantic and come about through the different combinations of the same elements. Schleiermacher maintains that 'there can be no concept of a style' (Schleiermacher 1977 p. 172), that is, a rule for identifying a style, because what is at issue does not emerge in terms of a semantic analysis of a text, relying rather on individual combination of the same elements as are used by others. We can derive semantic consequences from the organisation of words and their rhythmic and phonic interaction, but the effect of such organisation is not primarily semantic, and constitutes a vital aspect of the aesthetic effect of a text. Such organisation has evident analogies to the way in which the harmonic and melodic organisation of the notes, the rhythms and the tone colours in music generate its disclosive effects. The important fact about 'style' in this sense is that its irreducibility to concepts does not derive from the words of the text themselves, which are the same as those used by others, but results instead 
from the gaps between the words that make the new relationships between them possible, thus via what cannot be said in the text (on this see Frank 1989). This is how individual style relates to what Schleiermacher maintains about immediate self-consciousness, which is not accessible via specific acts of thought, but only via what grounds their connection. He also connects these ideas to the way music is an expression of immediate self-consciousness: 'just as the infinity of combination of articulated sounds belongs to human thought being able to appear in language, so the manifold of measured (gemessen) sounds represents the whole manifold of movements of self-consciousness, to the extent that they are not ideas, but real states of life' (Schleiermacher 1842 p. 394).

The analogies at issue here between language and music, like the analogies we saw in Hegel between music and the concept, depend on the idea that all determinacy is relational. The following remark can thus apply both to a word and to a musical note: 'in its single appearance the word is isolated; its determinacy does not result from itself but from its surroundings . . . The complete unity of the word would be its explanation and this is as little present as the complete explanation of objects' (Schleiermacher 1977 p. 106). A word is understood 'via its being together with the words that surround it' (p. 116), thus involving, as it does for Derrida, a constitutive lack that must be completed by the words in the rest of the signifying chain, in the same way as the notes in a piece of music require the other notes to become music. Hegel ultimately wishes to resolve these differences into the fully articulated identity of the absolute idea. Derrida, in contrast, can in this respect be understood as adverting to the musical aspect of language, which inherently defers 'presence' in the sense employed above. Adorno's remark in 'On the Present Relationship between Philosophy and Music' that 'As a sphinx [music] makes a fool of the spectator by continually promising meanings - and even intermittently granting meanings - which are for it in fact only, in the truest sense of the word, means towards the death of meaning, and in which [meanings] it for that reason never exhausts itself' (pp. 154-5) points to a way in which Derrida's ideas might be profitably explored in relation to music. Derrida's conception (and at times Adorno's) becomes problematic because he offers too few plausible ways of considering how language can be a successful means of communication. Schleiermacher accepts that there is an essential asymmetry between the semantic desire to regulate linguistic practice in the name of transparency of meaning, and the potential inherent in any utterance for an individual user of the language to mean something different by it which may only become apparent in terms of the revelation of more and more contexts of that language user's world. He does not, though, fetishise deferral of meaning in the way Derrida sometimes seems to, because, as we have seen, he does not conceive of meaning in Cartesian terms.

Hamann deconstructed the intelligible/sensuous divide by showing that language must involve both. Again something analogous applies to music, which Hamann suggested was the 'oldest language' (Hamann 1967 p. 224), and this can suggest an interesting further way of understanding Schleiermacher's 
insights. In The Order of Things Michel Foucault claims that 'The threshold between Classicism and modernity ... has been definitively crossed when words cease to intersect with representations and to provide a spontaneous grid for the knowledge of things. At the beginning of the nineteenth century they rediscovered their ancient enigmatic clarity' (Foucault 1970 p. 304). By 'Classicism' Foucault therefore means the era when words and representations intersect; modernity supposedly abolishes firm frameworks for knowledge because the world ceases to be ready-made, once the constitutive role of language becomes apparent. The move away from representational functions gives language an autonomy which Foucault thinks is the source of much of the most significant writing in modernity. The idea of a divorce of language and representations is related to what Schleiermacher, writing at the time when words rediscover their 'clarity', discusses in terms of the irreducibility of words to concepts, which is linked to his ideas about Poesie and music.

What seems of most interest in the present context is the historical fact that at this time in the early nineteenth century in Germany the judgement becomes more and more current that music without words is the highest form of art, at the same time as conceptions of language move away from ideas like the divine origin and thus put in question straightforwardly representational views. The change in the relative status of language and music often relates, as we have seen, to the new understanding of subjectivity in the thought of the period. The decisive fact about the music that develops at this time is precisely that the notes can be heard independently of any link to representation of the objective world. I should perhaps stress here, in order to avoid any misunderstanding, that the way the notes in the music of a particular society come to be ordered $i$ clearly bound up with ideological issues: composers do not compose in a vacuum. However, the ordering of notes is never wholly comprehensible in terms of the way that ordering is socially determined, because it does not just represent some other form of social practice or structure. Music is inherently able to sustain a degree of aesthetic autonomy, however minimally this may be the case in particular examples. The frequently misused idea of music as a universal language is, then, not merely a piece of ideology. In contrast to the incomprehensibility of unfamiliar natural verbal languages, unfamiliar music can make considerable sense, for example of the kind present in gestures or looks used while communicating with someone who does not share your language.

Foucault claims that attention to the consequences of the shift in language away from representation only really emerges later in the nineteenth century with Nietzsche. In the light of the arguments concerning music that we have seen so far, this is evidently not the case. The emergence of the idea of language as existing for its own sake, for example in Novalis's reflections on poetry, is inseparable in Germany from thinking about music aesthetics. The interesting question is exactly why music takes on this new status, and this has to do with the new ideas about language, and with the changing relationship between the affective and the conceptual in modern life. For Schleiermacher, as we have 
seen, the boundary between the musical and the conceptual is never absolute. Wittgenstein echoes this idea when he claims that:

Understanding a sentence in language is much more related to understanding a theme in music than one thinks ... Why should the strength and tempo move in just this line? One wants to say: 'Because I know what that all means'. But what does it mean? I couldn't say. In 'explanation' I could compare it with something else that has the same rhythm (I mean the same line). (One says: 'Can't you see, that is as if an inference were being made' or 'That is, so to speak, a parenthesis' etc. How does one ground such comparisons? - There are different kinds of groundings.). (Wittgenstein 1982 pp. 226-7)

Oliver Sacks's aphasiacs also indicated how the rhythmic and the musical play an essential and never fully determinable role in language use. What all these approaches share is a conviction that the claim to establish an authoritative philosophical language, of the kind promised but not delivered by the sort of system proposed by Hegel, risks repressing vital aspects of our subjective being in the world. These are manifest precisely in aspects of communication that are still often underplayed in modern philosophical approaches to language. If one thinks of language as communicative action, rather than representation, it seems evident that the dimension of subjectivity Schleiermacher thinks of in terms of immediate self-consciousness can play a significant role in thinking about language. What is at issue here with regard to the relationship between subjectivity and non-verbal forms of articulation is not susceptible to criticisms of the kind made against the myth of a pre-linguistic presence as the supposed ground of epistemology. Rorty is rightly wary of the sub-propositional 'given' when it is used as a means of grounding semantics, but this is not what is at issue here, as Wittgenstein's comments suggest.

The crucial point is that while language is undoubtedly a necessary condition of the claim to understand anything about music, the fact that language itself seems to have roots in the musical means that it cannot exhaustively characterise the content of what it has itself to rely on. This leads back to an idea encountered in earlier chapters. Rhythm is, as we saw, constituted in terms of iterable differences - the same beat only becomes the same beat via the occurrence of the next 'same' beat. Saussure argued that the signifier can only become a signifier, rather than an arbitrary noise or mark, via both its iterability and its difference from other signifiers, which means that it essentially relies on 'rhythm'. As I have shown elsewhere (see Bowie 2001), this idea is explored by Friedrich Schlegel in relation to Kant's theory of the schema, which Schleiermacher understands as the basis of the ability to use the same words to refer to an indefinite number of different things. What is clear, then, is that the rhythmic and the musical are not contingent additions to language, and that their relationships to each other can shift in different kinds of articulation - this issue will be explored in more detail in Chapters 7 and 8.

Schleiermacher's awareness of the broader significance of these issues is 
highlighted when, discussing music in the Aesthetics, he uses 'divination' in relation to the virtuoso's playing of the work of a composer: 'in performance there is always something which cannot be represented either by signs or words and which has to be found by divination. The composite marks which are supposed to represent the idea [of the whole piece] are largely laughable' (Schleiermacher 1984 p. 75). Virtuosity is simply mechanical and could effectively be computerised 'if one were to invent a complete system of marks for all the nuances that a note is capable of . . . finally one would be left, apart from reading correctly, just with the exactitude of touch and of the rests' (pp. 75-6). Recent technology has, of course, made this possible, as it has made possible forms of translation and interpretation in relation to circumscribed kinds of language use. Schleiermacher's point is that the mechanical playing of a piece does not constitute it as aesthetically important music, in the same way as successful interpretation of a text cannot be wholly 'mechanised'. This example points to a much broader issue, with which we can conclude this chapter.

There is no doubt that the development of modern science depends upon the elimination of much of the 'musical' aspect of communication and what it reveals to us. The growing contemporary importance of the levels of communication explored by Schleiermacher is a result of the awareness of the negative consequences of this elimination, even though he is clearly aware of its value in the appropriate 'identical' contexts. Oskar Negt has suggested how, around Schleiermacher's time, 'human possibilities were superior to what was technically available'. ${ }^{21}$ These days, of course, the opposite is increasingly the case: the technical means have massively increased, but what individuals can do with them has not always kept pace. Much contemporary Western culture simply relies on new technical means to dress up what is in fact an impoverished repetition of exhausted cultural forms. How this fact is connected to the relationship between the public and the private is one of the vital questions in contemporary culture. Many of the responses to this question rely on Heidegger's story of modernity as the era of the technological dominance of being by the subject, a story which I have been concerned to question by the alternative story being offered here. Schleiermacher's account of the subject acknowledges the potential for the subject to function in terms of domination if it relies wholly on 'identical' activities, but he also suggests ways in which we can still think about subjectivity that offer alternatives to Heidegger's story. As we have seen, philosophers like Brandom and Rorty use similar arguments to Schleiermacher as a way of questioning scientism. But where they tend to diverge from him, and come closer to Heidegger, is in relation to the issues of subjectivity and non-verbal forms of articulation explored in this chapter. It is here that Schleiermacher still has much to offer, though much work still needs to be done to develop convincing versions of his conception. The gravitation towards music in nineteenth-century Germany by many philosophers concerned with aesthetics will concern us further in the following chapters. Nineteenth-century philosophical aesthetics increasingly focuses on music as 
the paradigm for all art, and the resultant arguments have a role to play in a variety of contemporary debates.

\section{Notes}

1 I have edited and translated Schleiermacher's 'Hermeneutics and Criticism' and Other Texts (Schleiermacher 1998).

2 This is very apparent in the following remark from the Dialectic: 'Except for the fact that the divinity is, as transcendent being, the principle of all being, and as transcendent idea is the formal principle of all knowledge, there is nothing to say about it in the realm of knowledge. Everything else is just bombast or the interference of the religious, which, because it does not belong here, must have damaging effects' (Schleiermacher $1839 \mathrm{p}$. 328).

3 Wilhelm von Humboldt also proposes some vital philosophical arguments concerning the centrality of language, but he does so in a philosophical programme which is less significant than Schleiermacher's.

4 The last part of this passage makes clear Hamann's debt to Locke, and is not the most convincing part of his account of language, as the objections of Sellars and others against a given based on sense data make clear.

5 In a paper on this issue ('Schleiermacher and Post-Metaphysical Thinking', forthcoming) I suggest that, even though Schleiermacher invokes arguments involving such distinctions, they actually do not have a real effect on how he sees knowledge working in the world.

6 The trial in 2000 involving the mendacious historian David Irving in Britain showed that there is little trouble in establishing the falsity of such views as those of Faurisson, on the basis of expert testimony and documentary evidence.

7 Davidson's 'On the Very Idea of a Conceptual Scheme' shows in more detail what Lyotard seems not to appreciate. Peter Dews has suggested that Lyotard's position confuses validity claims, which can be part of any language game, and language games themselves.

8 Furthermore, as soon as he makes a counterclaim, Faurisson is involved in the contradiction of claiming validity on a basis he denies to others. If there is no testimony that what he claims did not happen did happen, there cannot be any reliable testimony that it did not.

9 Cf. Hölderlin's play on the notion of ' $U r$-teil' as 'original separation/differentiation'.

10 This is why Kant has to make spontaneity something which is outside of the sensuous world of cause and effect and temporal succession, thus giving rise to the question how spontaneity can be said to have causal effects.

11 Think also of the ways conductors try to communicate how they think music 'should go' by metaphors, gestures and all sorts of other kinds of non-verbal signals.

12 Schleiermacher refers to this aspect of interpretation as 'technical' or 'psychological'. in which 'language with its determining power disappears and only appears as the organ of the person, in the service of their individuality' (Schleiermacher 1977 p. 171).

13 Wheeler's account of Derrida's objections to Husserl's essentialism involves the same point: 'an item's being of a given kind cannot be fully present, since to take it as of a kind is to take it together with past and future repetitions' (Wheeler 2000 p. 82).

14 Frank tends to underestimate the degree to which we are in certain respects indeed 'spoken' by the habits involved in the languages we have acquired: the level of creativity 
in language use seems to me to be located more in our ability to make sense of utterances in ever new contingent contexts.

15 The ever more apparent crisis in traditional literary disciplines and the rise of 'theory' indicate how much these disciplines are a historical product which is now being transformed into something else in the light of new forms of communication and other social changes.

16 As we shall see later, Wittgenstein is the exception in the twentieth-century tradition that emerges from analytical philosophy.

17 The justifiable objection that nothing in the world is reducible to what we say about it, and that the idea of this irreducibility being significant therefore rests on a mistaken conception of language, does not invalidate what I propose here.

18 See also Neubauer 1986, Bowie 1997.

19 It is, as Rorty has argued, not useful to claim that language has no representational function. The point is that it cannot be shown to represent a ready-made world, so that it is always also at some level constitutive of what it represents, but this does not mean that we do not use it representationally. The fact that music can also perform representational functions suggests how, once again, the boundaries are not fixed here.

20 Clearly there is a sense in which language does involve singularity all the time, people regularly saying sentences which have never been said before, but social communication in the name of the coordination and effecting of actions primarily relies on the reduction of singularity.

21 The rapid development of musical instruments during the nineteenth century is part of this situation. 


\section{Music, language and literature}

\section{Language and music}

The divergent interpretations of the relationship between music and language in modernity are inseparable from the main divergences between philosophical conceptions of language. The attempt to explain language in representational terms in the empiricist tradition that eventually leads to analytical philosophy, and the understanding of language as a form of social action and as constitutive of the world we inhabit in the hermeneutic tradition give rise to very different conceptions of music. One paradigmatic contrast has emerged in the preceding chapters, which can somewhat crudely be summarised as follows. On the one hand, music can be regarded as a deficient means of articulation in relation to the kind of possibilities for manipulating and changing the world for human purposes offered by verbal language. On the other, it can itself be regarded as a kind of language - which can even be privileged above verbal language - because it reveals aspects of being in the world that verbal language is unable to reveal. Schleiermacher's work offered a pragmatic way of understanding different forms of articulation which did not require a wholesale split between verbal language and music; it also elucidated important differences between the way each can come to be employed and can affect its recipient. From this perspective the - hyperbolic - alternative of regarding music as 'higher' than verbal language for metaphysical reasons makes sense in relation to a specific historical situation, namely one in which the universalising nature of verbal language is felt to be inadequate to the individual experience of the modern subject. In this view the practice of musical production and reception offers a universally accessible means of articulation which at the same time involves the subject in an individual manner. Musical 'vocabularies' can therefore serve vital purposes in disclosing affective and other significance that other forms of articulation and communication may not be able to disclose.

The very fact that this is a specifically modern conception suggests how vital it is for philosophical views of this issue to get away from the idea that there could be a definitive theory of how the semantic relates to the musical in language, or of the difference between verbal language and music. Such a theory would require a representationalist account of what each 'really is', and 
this would simply repeat the problems associated with such positions in explaining why such supposed 'entities' as music keep changing so radically. What is needed instead is a conception which takes account of the interaction between what gets talked about as music and what gets talked about as language, these being quantities which are never wholly separable anyway. This interaction has generally been seen, for example by Hegelians like Carl Dahlhaus, in terms of the effects of conceptual shifts upon the nature of musical composition. What is significant in the period we have focused on so far is that music itself helps to change conceptions of language. It obviously does so for a conceptual reason: language ceases to be regarded in purely representational terms during this period. However - and this is the vital point the roots of this conceptual shift themselves also have to do with the effect of new kinds of autonomous music. Dahlhaus himself reveals the importance of the rise of the idea of 'absolute music'. He tends, though, to underplay the ways in which this idea also results from what is achieved by new musical practices. These practices are not solely the result of conceptual reflection and arise rather from the more intuitive, expressive and pragmatic aspects of musical composition and performance.

Hamann's and others' idea that the first language was music was itself a manifestation of a dissatisfaction with the idea of language as representation of a ready-made world. Once one becomes aware of the world-disclosive nature of language it becomes possible to understand language in the wider sense of comprehensible articulation that I proposed in the preceding chapters. This makes it possible to understand music as language without falling into the trap - sometimes encountered in 'new musicology' - of trying to reduce music's content to a verbal equivalent. ${ }^{1}$ How, though, are we to establish a useful contemporary, but historically informed, way of approaching the interaction between the verbal and the musical which does not lead to the kind of rigid divisions that, until recently, have made the analytical tradition so incapable of saying anything much about music that really matters?

The links I have tried to establish between Romantic thought and contemporary pragmatism can be of further help here. A vital part of Rorty's conception of language is suggested by his question: 'At what point in biological evolution did organisms stop just coping with reality and start representing it?' His answer is: 'Maybe they never did start representing it' (Rorty 1999 p. 269). In consequence: 'there was no decisive moment at which language stopped being a series of reactions to the stimuli provided by the behaviour of other humans and started to be an instrument for expressing beliefs' (p. 74). At this level there can be no fundamental difference between, say, rhythmically based verbal or proto-verbal communication, and something which can eventually become aesthetically significant music (though we can question Rorty's - and others' - impoverished notion of subjectivity as mere reaction for doing too little to account for the genesis of significant new reactions). The sort of line Rorty (who never discusses music) would probably draw between verbal lan- 
guage and music relies on the idea of the development of 'semantical metalanguage', in which language use is distinguished from mere instinctual expression by the fact that we can 'say things like, "It is also called ' $\mathrm{Y}$ ', but for your purposes you should describe it as X"' (Rorty 1999 p. 65). One of the key functions of verbal language is, then, to enable language-users to pick out different aspects of objects for human purposes, which entails the ability to use metalanguage.

Music, in contrast, can really only pick out aspects of the world of objects in a very limited manner, such as when it functions as a signal for getting up because it is morning. Music may, however, be able to function in something like a metalinguistic manner at affective and other levels. The simplest kind of instance of this is ironic music, that metalinguistically says: 'I am not saying what I seem to be saying.' An example of this would be Mahler's march music, which takes up a kind of music often positively associated with the military and undermines its military connotations. Like verbal irony, musical irony clearly relies on a whole series of contextual assumptions which are not fixed and which change historically, but once one accepts that meaning is never atomistic, but always contextual, music can function in an analogous manner to words in this respect. Beethoven's String Quartet op. 135 functions in a metalinguistic manner in its last movement by using closely related musical material both to ask an apparently serious question about life and death, and to answer it in an essentially humorous manner. Beethoven wrote the words 'Must it be?' and 'It must be' above the music at these points, but they need not determine a listener's way of hearing how the very differing kinds of music comment on each other. The listener may, furthermore, not be able to state verbally what they think this relation between the question and answer means. Indeed, they may not even be explicitly aware of the relationship between the musical material of the introductory question and the answer, but they can still gain a sense of meaning from the piece's change of mood. The relation of mood to meaning is also vital in poetry, where the interaction of metaphors can create an affective climate which cannot be inferred from what the poem says propositionally, and which relies on the phonic and rhythmic organisation of the words as much as on their meaning. A perhaps rather over-used example of this would be Goethe's 'Über allen Gipfeln ist Ruh', where the semantic content is minimal but the sound patterns and cadences of the verse both amplify the semantic content and take it into the realm of something like music.

The underlying issue here is the way in which patterns of identity and difference, which are not semantically determinate, but which are world-disclosive, are shared by musical and linguistic forms. These patterns will function differently in different practices, but they rely on an interaction of two dimensions of articulation. The increased attention to the musical qualities of words occasioned by the great Romantic composers in some nineteenth-century poets, like Mallarmé, is one example of such cross-fertilisation, as are aspects of Beethoven's declamatory and rhetorical styles in relation to the dramas that impressed him. How, then, do these issues relate to Foucault's contention we 
saw in Chapter 6, that a major change took place in language at the beginning of the nineteenth century?

Foucault regarded this change as marking the threshold between 'Classicism', in which words and representations intersect, and modernity, where language is no longer tied to representation. His argument is oriented towards the developments in literature which lead to 'poésie pure' and to the possibility that 'language may sometimes arise for its own sake in an act of writing that designates nothing other than itself' (Foucault 1970 p. 304). Foucault's version of the story of theoretical reflection about language omits any mention of Hamann, Schleiermacher or Humboldt: his examples are Grimm, Bopp and, somewhat oddly, as we shall see later, Friedrich Schlegel. His claim is that: 'Literature is the contestation of philology (of which it is nevertheless the twin figure: it leads language back from grammar to the naked power of speech)' (p. 300). The argument is dialectical: 'Literature' comes into existence because of the emergence of the other of itself, the science of language. In the same passage Foucault relates Mallarmé's poetry to this process, characteristically giving language the attributes of subjectivity: 'To the Nietzschean question "Who is speaking?" Mallarmé replies - and constantly reverts to that reply - by saying that what is speaking is, in its solitude, in its fragile vibration, in its nothingness, the word itself - not the meaning of the word but its enigmatic and precarious being' (p. 305). Foucault's account could also apply to the relationship between language and music: a word minus the kind of meaning that semantics tries to provide in terms of truth conditions can play a similar role to a note in a piece of music. Lévi-Strauss talks of musicality as 'Language minus meaning', and Novalis and Schopenhauer think of music as a 'universal language'. Music overcomes the lack of a philosophical master vocabulary by communicating, in a manner that transcends what can be said in the vocabulary of specific natural languages, ways of being which are universal; at the same time, music is a highly specific form of articulation. However, the question with regard to Foucault's claims is what the 'being' of the word/note says if it does not relate to the subject that is threatened by loneliness and fragility. Foucault seems just to be committing a strange version of the pathetic fallacy, with the word taking the place of some aspect of nature onto which human feelings are projected.

In The Order of Things Foucault's structuralist exclusion of the subject relies upon a Heideggerian account of subjectivity as the locus of domination of the other, of the kind that has already been seen to be inadequate to the thought of this period. If one looks at the development of the idea of literature in German philosophy in the early Romantic period in relation to music perspectives on these issues become available which offer an account of subjectivity of the kind lacking in so much recent theory (see also Bowie 1997). Foucault concentrates on that aspect of thinking about language which fits his thesis about the emerging new 'episteme', the fact that language itself becomes an object for science and therefore generates a counterpart, 'Literature', which cannot be such an 
object. It is, however, evident from what we have seen in Hamann and his successors that a different view of language also emerges in the theory of the time, most notably in Schleiermacher. According to this view it is impossible for language to be wholly transparent to a science of language, because there can be no 'general language' that would be able to be the master code for the other languages. Part of the reason for this is that the 'musical' is an ineliminable aspect of language: it can be foregrounded so that the semantic aspect of language almost wholly recedes, coming close to what Foucault suggests in answer to the question of who is speaking. The problem is how one interprets this move away from the semantic to language without 'meaning'.

Despite the central role of music in this area it is a topic that Foucault, like Heidegger (and unlike Mallarmé), hardly ever seriously discusses in print, even though he was very interested in it. He is not alone in this reticence. The difficulty of engaging with music on more than an analytical level, which has fuelled much recent debate in musicology, is an index of precisely what is at issue here in the relationship between music and language. The fact that even musical performers are often signally inept at enlightening others in words about music which they can perform in a revelatory manner further underlines the tensions in the understanding of this relationship. These tensions are germane to the issue of subjectivity and language. As I have tried to show, the peculiar nature of music can help reveal the weaknesses of conceptions of subjectivity which are limited to the idea that what can be communicated is ultimately propositional - good conductors can, for example, by the use of all kinds of gestures and expressions, get music to go the way they want without using any words at all. ${ }^{2}$ Music's relationship to non-verbal forms of communication and expression has led to it being neglected both in some recent theories of language, and in the history of aesthetics, where left-wing theorists, with some exceptions, such as Ernst Bloch and Adorno, have often either attempted to reduce it to its ideological context or have regarded it as, in Thomas Mann's phrase, 'politically suspect'. Music's non-representational character too often leads to it being disqualified as something which has not attained the seriousness of real thinking and praxis.

An admittedly questionable passage from Freud - do 'primitive languages' really have no grammar? - can suggest why the language of music may be regarded as suspect in this way: 'All the linguistic means via which the finer relations of thought are expressed, the conjunctions and prepositions, the changes of declination and conjugation, lapse, because the means of representing them are lacking; as in a primitive language without a grammar, only the raw material of thought is expressed' (Freud 19821 p. 462). Freud is actually referring not to music, but to dreams. The applicability of his description to wordless music connects the 'royal road to the unconscious' to what makes articulating the relationship between language and music such a problem. ${ }^{3}$ In much the same way as Wittgenstein claimed that the structures of the understanding of music and of language were not wholly separate, Freud's comment can be used to suggest 
how the working of self-consciousness also relates to what music and dreams can do that the more 'grammatical' aspects of thought cannot.

One of the decisive issues in the present book has been how consideration of the theme of aesthetics and subjectivity offers ways of understanding the role of the natural sciences in modern culture. It seems no coincidence that music itself embodies precisely the kind of relationship between what is accessible to scientific explanation and what is not that is decisive for understanding that role. On the one hand, the basis of Western music, the diatonic scale, relies on mathematically expressible proportions, which derive, in certain respects at least, from what is also the foundation of the modern scientific world view. On the other hand, music, especially in the modern period, takes on a vital role in relation to the emotions. It moves from being regarded in the first half of the eighteenth century as merely a manner of representing already familiar feelings, to being seen in the second half of that century, and in much of the nineteenth century and since, as being able to give rise to and articulate new kinds of feeling (see Bowie 2001a). The openness of music to the new technologies makes it clear that production and reception of music engage the understanding, Kant's faculty for rules. At another level, music also engages the individual subject in ways which relate to the notion of 'feeling' as developed by Novalis, Schlegel, Schleiermacher and others. Feeling can never be represented as such, but is a motor for finding means of articulation which are not prey to the inadequacy of verbal language to certain fundamental aspects of our being.

Music can be understood, therefore, as functioning in terms of a kind of dialectical 'identity of opposites'. The mathematical and the affective are both essential to it, but the former is universal, the latter individual. The temptation to use music as the means of access to an absolute which would overcome the division between the mathematically explicable, deterministic nature manifest in the rules of the harmonic series, and the world of affect is apparent in this combination of extremes. ${ }^{4}$ Such a means of supposedly revealing the absolute does not, of course, result in a representation or a philosophical articulation of how necessity and freedom can be reconciled. This theoretical lack can be understood both as a source of the power of music and as a reason why music can come to be regarded as socially and politically dangerous. Instead of offering a rationally comprehensible account of our place within things, music is regarded as suspending or transcending discriminations that are inherent in the identifying nature of conceptual thought. Such extreme claims about music in modernity also suggest a strange possible consequence for philosophy. If truth is supposed to be inherent in the word, anything which suggests that the word is no longer fully adequate as the expression of the highest truth opens up a very different path for philosophy. This idea may be hyperbolic, but it does suggest a kind of power which music seems able to exercise that verbal language may not. How this power is exercised and understood is, though, what makes music a political as well as a philosophical issue. The history of music's role as a social and political practice in the modern period is often related to the fact that its 
distance from determinate representation can make it an adjunct of forms of social deception, from the use of music in Nazi rallies, to use in modern advertising. At the same time, however, music can also be a means of resisting the thinking behind such deceptions, via its ability - which depends precisely on its non-representational nature - to refuse to follow established patterns of sensemaking and articulation in a society. These complex issues can be explored if we look further at some of the ways in which music has been understood in German philosophy.

\section{Hegel and Romanticism: music, logos and feeling}

In contrast to the late eighteenth- and nineteenth-century thinkers who give music a central role - such as the early Romantics, Schopenhauer and Nietzsche - Hegel, as we have seen, regards music, like the rest of the arts, as a subordinate manifestation of truth. In order to put Hegel's response to music in an appropriate context we need now to take a further look at changes in the understanding of music in Hegel's time. Carl Dahlhaus explores these changes in Die Idee der absoluten Musik (The Idea of Absolute Music). At the end of the eighteenth century the idea of the superiority of music with a vocal text increasingly loses currency and 'conceptless instrumental music - and precisely because of and not despite its lack of concepts - was elevated to a language above verbal language' (Dahlhaus and Zimmermann 1978 p. 179). The preceding conceptions of music had Platonist roots. Music consisted of Harmonia, Rhythmos and Logos: 'By Harmonia one understood regulated, rational relations of notes brought into a system, by Rhythmos, the temporal order of music . . . and by Logos, language as the expression of human reason' (Dahlhaus 1978 p. 14). Importantly, composers, such as Haydn, had already begun to undermine this conception in praxis, before the move away from the conception began to be articulated theoretically. ${ }^{5}$

The idea of Logos, whether in the form of a liturgical text or of the words of a song, is still basic to Hegel's conception. Although he evidently enjoyed music, Hegel did not regard it as being particularly important. His remarks in the Aesthetics are in some respects most notable for how they epitomise a view of music which plays a role in much subsequent aesthetic theory, particularly in the Marxist tradition. Hegel's description of music is also echoed in Foucault's Mallarmé-derived notion of the 'act of writing that designates nothing other than itself'. Hegel, though, draws the opposite conclusion to Foucault about the value of such an act. In the section of the Aesthetics on 'Independent Music', music without words, Hegel claims: 'Subjective inwardness constitutes the principle of music. But the most inward part of the concrete self is subjectivity as such, not determined by any firm content and for this reason not compelled to move in this or that direction, rather resting in unbounded freedom solely upon itself' (Hegel 1965 II p. 320). The structure of the argument should be familiar from what was said about the Logic in Chapter 5: 'subjectivity as such' 
is analogous to being at the beginning of the Logic, which must come to itself by self-division. Only when being has been articulated in the concept can it become being for itself as the absolute. Subjectivity is likewise only able to realise itself via engagement with objectivity, as the structure of reflection in the other makes clear. The principle of music may be 'subjective inwardness', but this requires its objective other to be externalised as sound and thus to be music at all. Music's insufficiency lies for Hegel in the fact that the externalisation in question takes place in the transient medium of sound.

For Hegel, purely 'musical music' has to free itself from the 'determinacy of the word'. However, instrumental, wordless, music will only really appeal to the expert, who will enjoy it because he can compare the music he hears with 'rules and laws he is familiar with' (II p. 322). There is, then, little sense here that music may in some circumstances be able to 'say' what other means of articulation are unable to. The expert will try to find 'more distinct ideas and a more familiar content' in the music, and 'In this respect music becomes symbolic for him, but in attempting to grasp the meaning he is faced with puzzling tasks which rush quickly past, which are not always amenable to being deciphered and are capable in fact of the most various interpretations' (II p. 322). Statements like this make it clear how Adorno's objections to Hegel's neglect of the 'non-identical' in art derive not least from Hegel's failure to see more in the problem of understanding music than a deficit on the part of music itself. Furthermore, being 'capable of the most various interpretations' is probably the most apt description of significant literature in modernity - indeed, as Schleiermacher indicated, it is also a possibility for any kind of text. Given its interpretative history, Hegel's own work can hardly be said to be very different, in this respect at least, from music. Part of what he means is, of course, simply based on the transience of the playing and hearing of music, as opposed, say, to the physical persistence of a text or a painting. However, pieces of music are actually idealisable in much the same way as texts are. I can keep the patterns of the movement of a symphony in mind, or if I have the right sort of training, of a piece of improvisation, in much the same way as I can that of a novel, and in listening to one or reading the other in real time I am subject to temporality in much the same way. ${ }^{6}$ As such, it would seem that much of Hegel's objection comes down to the fact that music is not representational and referential in the manner of verbal language. Hegel's objection epitomises the way conceptions of language begin to diverge in this period.

This divergence is evident in the fact that Hegel's conception of the musical note is echoed in Mallarmé's idea of the pord's 'fragile vibration': it is 'an expression which precisely by the fact that it is externality immediately makes itself disappear again' (II p. 262). ${ }^{7}$ The body which vibrates to produce the note is negated in its static state, but returns to this state once the note has passed. The body persists where the note does not, although it is displaced when it vibrates - the body functions here rather like being in the Logic, which only becomes something when it is negated. Consequently the body's truth lies, not in its inert 
facticity, but in the movement that takes it beyond itself, propelling it into a relationship with itself as at one moment present in one place, and at another in another place. In order to sustain a radical difference between music and Logos, Hegel has to argue that the ideal content of a word can survive this 'fragile vibration' in the same way as the Idea overcomes the transience of its objective embodiments. This is precisely what Foucault denies is the case for what he sees as philosophically the most significant development of language in modernity. For Hegel the note 'admittedly also does have a content, yet not one in the sense of the visual arts or literature; for what it lacks is precisely objective formation [Sichausgestalten], whether it be formation into forms of real external appearances or into the objectivity of spiritual intuitions and ideas' (II p. 261). The note lacks both persistent objective existence and persistent ideal existence. A painting or the ideas about the external social world in a novel do not suffer from this kind of lack, though they are tied to the particular in a way music is not. The implication has to be that the philosophical word can overcome even the deficit inherent in artistic forms which engage with the particulars of the world. Philosophy can achieve the 'presence' those particulars lack because of their dependence on other particulars.

Music, then, fails to reach the level which is the result of the real interaction of subject and object whose structures philosophy articulates, and so remains just 'subjective'. We shall see later that Hegel makes the same point against what he understands to be Schlegel's conception of irony, because it also fails to test itself against the real resistance of the objective world. In irony everything is 'produced by the subjectivity of the ego' (I p. 72) and can therefore just as easily be destroyed by it. Whereas the plastic arts 'take up the forms of a broad, multiple world of objects into themselves', the note is 'completely abstract' (II p. 261). However, Hegel's own claims are strangely analogous to the objections Schelling made against the intransitivity of the end of the Logic, where, as Schelling put it, the Idea 'has no need any more to become real any more and in any other way than it already is' (Schelling I/10 p. 152). Hegel thinks that the Idea is able to reveal itself as what can overcome the contingency of being by 'releasing itself into nature'; it seems once again, though, that its actual status may in some respects be closer to music. What is most convincing about Hegel's conception is the way in which it conveys the inherent dynamic of thinking, in a manner akin to a Beethoven symphony. Evidently such a claim only deals with one dimension of what Hegel achieves, but, as we saw, if his claim that the Logic exhaustively reveals the ways in which being can be articulated turns out to be unjustifiable, the ultimately representational aim of his philosophy remains unfulfilled, even though the internal dynamics of his thought still have much to offer. The questions about Hegel's ultimate aim necessarily affect the viability of his conception of language, and thence his conception of music. The way Hegel relates text and music can suggest how.

For Hegel the text which accompanies music or which music accompanies 'gives certain ideas and thereby tears consciousness away from that more 
dreamy element of feeling without ideas' (Hegel 1965 II p. 306). - He does insist, though, that the music must also retain its own autonomy and not just be there in the service of a content dictated by the text. He considers philosophical comprehension of the conceptual work of the understanding to be the route to the absolute. Music can never attain this higher status because it is an expression of 'feeling' (Empfindung). 'Feeling' is 'immediate' because it does not separate the subject that intuits from the object of intuition, as we do in conceptual thinking by ascribing differing predicates to an external object in terms of its inferential relations to other objects. Like a dream, feeling is wholly within the subject. However, something is missing here. Music can clearly change my way of relating to the world of objects by offering new affective, rhythmic and other resources, of a kind that are also available in poetic usage, but not necessarily in more instrumental uses of language. Hegel's view therefore fails to be adequate to the phenomenon in question. If language is not conceived of representationally the borderline Hegel sets up here ceases to be plausible because music and language can each achieve similar, or perhaps at times even identical effects. The interaction between music and the subject is not ultimately reducible to what can be verifiably said about it, thus to an account from the objective, conceptualisable side of the relationship, of the kind sought in some kinds of music psychology. At the same time, it is not the case that the effects of this interaction merely remain in 'that more dreamy element of feeling without ideas'. This would only be the case if feelings were wholly indeterminate. In that case, there could only be one feeling, rather than the endless gradations of which feeling actually consists. The role of music as a social practice that is evident, for example, in music therapy, which brings people back into contact with the social world, or in the way we can apprehend 'musical ideas' and deepen our comprehension of them both in words and in the practice of listening or playing, makes it clear that drawing strict lines here obscures some important aspects of the relationship between verbal language and music. One could say, then, that Hegel's account is 'insufficiently dialectical'. For Hegel feeling, as something immediate, can only reach its truth when it is comprehended in the concept. The question is whether feeling is reducible to what can be said about it. On some occasions feeling may only become itself by being expressed in words, on others feeling may only be accessible to a non-verbal form of articulation.

The difficult problem here is exactly how the immediate aspect of feeling relates to forms of music production and reception which are themselves conceptually mediated but can never be definitively described in verbal terms. The neo-Hegelian version of the rejection of immediacy was, as we have seen, directed against the idea that something directly given from the world could determine the truth or falsity of what we say. I argued in Chapter 6 that the argument against immediacy would seem to apply to the inferential nature of knowledge of the world, but not to the immediate awareness that the Romantics termed 'feeling'. In an essay on Sellars, Robert Brandom makes a distinction between differential responses to a stimulus, of the kind a photocell can make 
into relation to red and non-red things, and the conscious perception of something as red, which relies on knowing the 'inferential significance of applying the concept red ... that it is coloured, not a prime number, and so on' (www.ditext.com/brandom). A note can be registered in terms of differential response to its frequency, but, as Hegel argues, it only becomes a note via its relation to a series of contexts, in which it is defined by its function. However, even this function is accessible in one respect to a complex differential response of the kind a computer can perform in relation to a score. What makes the note into part of a piece of music is, though, not adequately grasped either by the idea that we know the inferential significance of saying that it is such because it relates to other notes in a rule-governed manner, or even by the idea that we know it is music because we grasp the conceptual content of the term music can that content really be arrived at by music's being sound, not being painting, etc.?

It is at this level that the immediacy of feeling still seems to play a decisive role, of the kind Cascardi rightly regards as essential to the aesthetic. Stanley Cavell suggests another way of considering immediacy in this connection, when he claims that 'It is essential in making an aesthetic judgement that at some point we be prepared to say in its support: don't you see, don't you hear, don't you dig? .. . Because if you do not see something, mithout explanation, then there is nothing further to discuss' (Cavell 1976 p. 93). The content of what is at issue here is neither exhausted by the justifiable application of the term music, nor by all we can come to know about the sounds in question. This kind of excess of the affective and other content of music over what is accessible to concepts highlights the limitations of a Hegelian approach to music's relationship to selfconsciousness.

Now it is clear that feelings are themselves historically mediated, and that what counts as music changes with history. This does not, though, affect the argument that a Hegelian model lacks the essential dimension which the Romantics discuss in terms of their notion of feeling. ${ }^{8}$ The point about feeling is that it is not reducible to the ways it comes to be understood because it need not become conceptual, being susceptible to kinds of articulation which do not involve the generality of verbal language. The significance of this idea depends on understanding the historical circumstances in which it originates. The rise of the idea of musical autonomy and the concomitant emergence of non-representational conceptions of language suggest ways in which more diverse and differentiated forms of world disclosure emerge in modernity via the decline of dogmatic metaphysics. This decline is, once more, two-edged. It opens new horizons, but it also gives rise to a sense that something important has been lost, namely a way of sometimes being in harmony with the rest of the world, despite all the pain and division encountered in it. It is therefore not surprising that the idea of a non-representational, non-conceptual medium being able both to communicate the irreducibility of self-consciousness to the increasingly differentiated results of the activity of the understanding and to offer an 
affective reconciliation with transience occurs to more than one thinker in this period. Music's distinctive ability to convey aspects of our being in the world appears as a crucial compensation in the face of scientific accounts of what we are which become harder and harder to integrate with our experience of the inherently feeling-imbued life-world and our existential sense of transience.

This attribution of philosophical import to music in Hegel's time also relates to music's apparent proximity to 'nature', another concept which involves an often highly problematic - sense of immediacy. Nature should be understood here, not in Kant's 'formal' sense, as what is bound by necessary laws, but in the sense of the Critique of Fudgement, as what is not reducible to being understood in conceptual terms. Nature in this sense is most obviously manifest in pain and pleasure. ${ }^{9}$ Hegel relates music to 'primitive' expressions, such as birdsong or wordless cries, but, as Schleiermacher also insists, for something to be music it requires more than what is present in non-human nature: 'For neither the expression of a momentary sensation by a . . . speechless natural sound, nor speaking which approaches song are music, but are only the transition to it' (Schleiermacher 1931 p. 369). Music for Schleiermacher is only music if it conveys 'movements of self-consciousness, to the extent that they are not ideas [i.e. not propositional], but real states of life' (p. 394). It is this status in between the 'natural' and the conceptual which makes music so instructive in the present context.

What music conveys is neither wholly mediated in the manner of inferentially articulated conceptual knowledge, nor immediate in the manner of raw feeling, and this can also, as we saw in Schleiermacher, be said of certain aspects of language. For Hegel the transformation of natural sounds into music is analogous to the representation in a painting of a natural scene which reveals more than would be revealed by the contemplation of the scene unmediated by art. Music, like the concept which reveals the higher truth of sensuous immediacy, has to bring 'feelings into determinate relations of notes' and to 'take the natural expression out of its wildness, its raw state, and moderate it' (Hegel 1965 II p. 273). What, though, does philosophy then do with music, apart from try to suggest the need to overcome it in turn, in the name of the higher forms of articulation upon which conceptual knowledge of the objective natural and social world relies? It is this kind of Aufhebung which certain thinkers in this period think music resists.

E.T.A. Hoffmann's famous review of Beethoven's Fifth Symphony in 1810 offers an extravagant version of what I mean: 'Music opens up an unknown realm to man; a world that has nothing in common with the surrounding external world of the senses and in which he leaves behind all feelings which are determinable by concepts in order to devote himself to the unsayable' (Dahlhaus and Zimmermann 1978 p. 197). Hoffmann, it should be remembered, is both a talented composer, and a more than competent musicologist, as the rest of his review of Beethoven's symphony shows. Both Hegel and Hoffmann share a metaphysical suspicion of the 'external world of the senses'. 
Their reasons for the suspicion are, though, very different. For Hegel the truth of music is eminently sayable in the form of philosophy, which is able to grasp the truth of the outer world of the senses while overcoming its transience. As we saw, he maintained that the 'Unsayable, emotion, feeling is not the most excellent, the most true, but rather the most insignificant, most untrue' (Hegel 1959 p. 56). For Hoffmann music can, in contrast, articulate the 'unsayable', which is not representable in language and is based on 'feeling'. Writing about Beethoven's Op. 70 piano trios in 1813, he suggests that 'in the midst of this unlocked realm of spirits the delighted soul listens to the unknown language and understands all the most secret intimations by which it is seized' (Hoffmann 1988 p. 121). The difficulty of sustaining this position becomes apparent, however, in his essay 'Old and New Church Music' (1814). Hoffmann here presents the conception of music as the means of access to a realm beyond the sensuous, not, as he did in the Beethoven pieces, in terms of wordless instrumental music that is free of the compulsion to relate to what words may say, but instead in terms of the church music tradition deriving from Palestrina, which relies on the setting of liturgical texts. The inconsistency between these positions could only be overcome by making a substantial link between what is 'said' by wordless music and the content of liturgy.

Hoffmann's defensible point is that music discloses dimensions of self and world which verbal language alone cannot disclose. He gets into difficulty because he makes the same sort of metaphysical claim about music as Hegel makes about philosophy. The instructive problem for Hegel lies in the connection between language and music. He actually describes music in a way which many contemporary thinkers would now see as applying to verbal language: 'the notes are in themselves a totality of differences, which can divide themselves and combine themselves into the most multiple kinds of direct consonances, essential oppositions, contradictions and mediations' (Hegel 1965 II p. 273). We have already considered the idea of the totality in which the determinacy of each element depends on the other elements in the analogies of music to the Logic. The same conception recurs, no doubt via the influence of the thought of the Idealist and Romantic traditions, in Saussure's account of language. Schleiermacher had already shown that there are no concepts independent of linguistic articulation. Saussure drives home the point that, instead of pre-existing ideas being represented by words, the opposite is in fact the case. Ideas themselves depend on the differential articulation of the material of the signifier, whether the signifier be material marks or moving sound waves. The specific material itself is not central to the constitution of meaning: it is the relationships between the elements that count, not anything inherent in the particular element. ${ }^{10}$ Because meaning is independent of the specific form of existence of the signifier, it seems that it must be grounded in 'nothing', the difference between signifiers, and this opens up a vital connection to music. When enjoined to 'taste the difference' between $a$ and $b$, one can only first taste $a$ and then $b$ : this therefore entails what is itself neither $a$ nor $b$. The subject would seem to 
be located here, as the locus of the relations between linguistic, musical, gustatory and other moments. In order to be such a locus the subject requires the existential continuity Schleiermacher termed 'immediate self-consciousness'.

The structuralist view of differentiality derived from Saussure has come to be seen as a way of questioning the metaphysical division between thought and the manifestation of thought in language, and such questioning is common to various traditions in contemporary philosophy, connecting such differing thinkers as Derrida and Sellars (see Wheeler 2000). Schelling's version of the issue of differentiality already pointed the way, as we saw in the comparison with Derrida in Chapter 4, to contemporary questioning of metaphysics (while also suggesting problems in some of that questioning). How, then, does Hegel fit into these versions of the idea of language, music and difference? For Hegel, the relationship between determinate content in music and the text which can make it determinate seems to reduce the former to the latter, as the former has, at best, a deficient kind of determinacy. Is this account, though, able to do justice, for example, to those Schumann songs, such as the last song of Dichterliebe, in which, after the words cease, the solo piano comments on what has been said? Were the text to continue at this point, the power of what the song 'says' would be greatly diminished. Particularly in the final song of the cycle the piano postlude comes at precisely the point when words are no longer any use for the evocation of what the poet is enduring. ${ }^{11}$ How, then, can we give an account which does justice both to Hegel's insights and to the dimensions he neglects?

What links language and music here is the fact that linguistic elements can only become meaning-bearers within the context of other elements, and notes can only become musical notes, rather than physical frequencies, within the context of other notes and in relation to a subject that hears them as music. ${ }^{12}$ In both cases forms of articulation which can enable us to understand more of and orient ourselves in the world, and which can therefore themselves be understood, share a related structure. Furthermore, each form is able to do things the other cannot, and some aspects of each seem to be very closely analogous to aspects of the other. Rorty talks of people like himself 'for whom language is a tool rather than a medium, and for whom a concept is just the regular use of a mark or noise' (Rorty 1991 p. 126). In these too reductive terms there can actually be no essential difference between music and language, the one being able to be a tool for what the other may not achieve, and both relying on repeated use of certain noises. Leaving apart the question of what makes it clear that a mark or noise is really being used to mean something, which seems to demand an account of the subject, the fact is that Rorty's approach does too little to elucidate why the relationship between music and language became such an issue at the time of the emergence of the non-representationalist conceptions of language upon which his pragmatism relies.

For Hegel to argue as he does, at least in relation to music, he has to think of verbal language in representationalist terms, not as a tool in the pragmatist sense, and this, I think, is another of the reasons why he cannot be co-opted as 
readily into their canon as some of his contemporary pragmatist advocates would wish. Schleiermacher's hermeneutics, in contrast, regarded the aspects of language that are resistant to conceptual articulation, not as contingent additions, but as constitutive elements of language. His attention to the 'musical', language as sound and dynamic transition, was based upon his understanding of immediate self-consciousness. The epistemological justification of this notion becomes apparent when considering Hegel's account of the relationship of language to the I. The problem with the way Hegel discussed the signifier ' $\mathrm{I}$ ' in the Encyclopedia was, as we saw, that the structure of reflection failed to give a criterion of self-identification for the I. As Derrida has suggested, and here he moves close to the critiques of reflection we have examined from Fichte onwards, the determinacy of the linguistic mirror in which I reflect myself - the signifier ' $\mathrm{I}$ ' - itself depends on its relations to other signifiers, which means that it can never be finally determinate. As Frank points out, Derrida seems to offer no account of self-consciousness at all, rather than seeking an alternative to the model of self-presence via reflection in the other of language. In order to escape precisely this problem Schleiermacher insists, as we saw, that there is a noninferential dimension of the self which is not fully articulable by our propositional descriptions, without which inferentially based self-identification can make no sense. The inaccessibility of this aspect of ourselves to what can be determinately said - truth-determinate utterances relying on sharable inferential grounds - leads Schleiermacher to the idea that it is only by circumventing language's universalising function that 'feeling' and individuality could be made manifest. What Schleiermacher means by the 'musical' is an aspect of this kind of use of language, which articulates or shows something beyond what can be determinately said. When looked at in this perspective Hegel's description of music as a 'totality of differences' means music is actually less easy to distinguish from language than he claims.

The central issue here is what deconstruction, in the wake of Heidegger, calls the 'metaphysics of presence'. What the term refers to is exemplified in Hegel's account of language in the Phenomenology. Derrida has characterised what he means by metaphysics as 'presence' in terms of reflexive 'hearing oneself speaking', and this idea is apparent in Hegel's comments, in which the individual subject hears itself speaking via the language it shares with other subjects. Hegel describes language as

the existence of Geist. It is self-consciousness which is for others, which is immediately present as such and is as this general [self-consciousness]. It is the self which separates itself from itself, which as pure I = I becomes objective to itself, receives itself in this objectivity equally as this self, as it flows together directly with the others and is their self-consciousness. (Hegel 1970 pp. 478-9)

The equivalence between the individual self and others comes about via the assumption of semantic symmetry between self and other. However, it is precisely the notion of meaning as something universally shared by, and identical 
between, self and other which falls prey to the questions concerning meaning that we considered in Schleiermacher. He held such a notion of meaning to be a regulative idea for interpretation of and interaction with the other, not something whose existence could be assumed or proved. Furthermore, Hegel's account also fails to deal with the fact that the self-presence which is supposed to result from becoming other through language cannot anyway be attained in these terms. For the reasons we have just seen, if one is to be able to designate oneself with the signifier I, one cannot rely solely on the shared, general structure of Geist manifested in language.

Friedrich Schlegel's claims about the relationship between music and feeling cited in Chapter 1 show one way of connecting this issue to music:

Now if feeling is the root of all consciousness, then the direction of language [towards cognition] has the essential deficit that it does not grasp and comprehend feeling deeply enough, only touches its surface ... However large the riches language offers us for our purpose, however much it can be developed and perfected as a means of representation and communication, this essential imperfection must be overcome in another manner, and communication and representation must be added to; and this happens through music. (Schlegel 1964 p. 57)

'Representation and communication' do not, Schlegel argues, do justice to the source of what matters to us and thus to our capacity to ascribe and receive significance. This 'root of all consciousness', the ground of significant differentiation, cannot be articulated propositionally: 'Feeling and wishing often go far beyond thinking.' Schlegel therefore talks of 'music as inspiration, as the language of feeling'. The claim that music is 'the only universal language' (p. 57) need, then, not be merely hyperbolic, if one accepts the notion of a language as a form of articulation which can be understood, rather than just as a means of representing the world. Furthermore, the emergence of philosophical concern with the idea of literature at this time, as Schlegel goes on to suggest, is inseparable from music: 'The higher language as well should be music; here literature is the link which connects music and language' (p. 58). Literature connects the two because, like music, it cannot be understood as such in representational terms - as Schleiermacher argued, there can be no concept of a style - and therefore depends on aspects of self-consciousness which are not reducible to reflective, propositional awareness of the kind that are apparent in our understanding of rhythm, tone, mood, and so on. As we have seen, there is always a musical aspect to any use of language, and this aspect is not reducible to language's referential functions.

Importantly, the sort of questions raised by Schlegel can lead in both metaphysical and non-metaphysical directions. They lead in a metaphysical direction if music is taken, as it is by Hoffmann, to furnish what philosophy cannot, namely the means of reconciling freedom and necessity that is manifested by music's saying the unsayable. Hegel's metaphysical argument is, in contrast, that philosophy has to overcome the sensuous, the finite, including the finite in 
music and language, in order to arrive at the realisation of the inherent lack in the finite that takes one from understanding to reason. Against Hegel it can be argued, however, that 'absolute music' may in one sense be able to overcome the finitude inherent in language more effectively than language as Hegel conceives it. What interests many of those who seek positive metaphysical content for music is the fact that music is not bound to the objective world in the way that even the most abstract verbal language is. The word 'abstraction', of course, itself has roots in very concrete human activities. Hegel's attempt to suggest how these roots can ultimately be overcome relies on the idea that everything sensuous negates itself, leading eventually to the point where there is nothing left to negate. Verbal language, though, seems inherently resistant to such a conception.

The metaphysical aim of the purism seen in Hegel is encountered in a variety of guises in modernity. If one assumes language is bound up with the world of objects in a way that makes it impossible for language fully to transcend that world, other less representationally bound forms can be seen as taking on a new significance. Around the beginning of the twentieth century these kinds of ideas are often connected with a 'language crisis', such as the one described in Hofmannsthal's 'A Letter' (1902). Here the arbitrariness of the signifier is understood as threatening to separate language from the world, so that the subject loses any stable contact with the world. Another manifestation of this kind of idea is the desire to express philosophy in a logically purified language that is encountered in the early stages of analytical philosophy. This involves an attempt to transcend the perceived contingent limitations of natural languages. ${ }^{13}$ In Soul and Form (1910), which formulates an aim akin to that of Idealist metaphysics, Georg Lukács similarly wishes to overcome contingency by seeking to 'arrive where everything becomes necessary because everything expresses the essence of man, nothing but that, completely and without residue - where everything becomes symbolic, where everything, as in music, is only what it means and means only what it is' (Lukács 1971 p. 23). Because the relationships in music are internal, music is not bound to the contingent objective world in the ways that verbal forms are. What is sought in a theory like this is precisely a means of establishing a kind of metaphysical presence which the arbitrariness and differential constitution of the signifier make inaccessible to verbal language. The problem is that such theories have to give positive metaphysical content to music by stating in what music's transcendence of concepts consists. The more promising option is to employ music to show how concepts themselves involve elements that are inseparable from music and so cannot wholly absorb the significance of music into themselves. This latter conception points to the non-metaphysical alternative suggested in Schlegel's remarks linking music and the idea of literature, and in Wittgenstein's remarks cited in Chapter 6.

The issue of music here raises an interesting possibility for investigating differing interpretations of the current understanding of the effects of the 
centrality of language on the perception of the tasks of philosophy. In Chapter 6 I contrasted the responses of Lyotard, Davidson and Rorty to the situation in which a grounding of meaning in 'presence' is renounced. Wheeler suggested that the difference in response depended on how far a philosopher thinks 'philosophical notions . . . infect the rest of culture' (Wheeler 2000 p. 71). The nonmetaphysical interpretation of the issues raised by Schlegel results from the idea that regarding the representational aspect of language as merely one limited part of a much larger picture of language as human practice opens up new possibilities and loosens boundaries between language and music. ${ }^{14}$ We have seen in previous chapters how Rorty's contemporary claim that philosophy is really a kind of literature is based on the rejection of the 'metaphysics of presence' involved in representationalist conceptions of language. The consequences for Rorty are a refusal to privilege the kind of discourse philosophy has traditionally been assumed to be, and a concomitant attention to the relationship between the public and private significance of different types of discourse. It is here that some of the most emphatic manifestations of the differing responses to the perceived end of the representationalist paradigm have emerged. Music can, for example, provide an occasion to consider the viability of some influential claims in post-structuralism.

\section{The 'presence' of music}

The extreme response to the consequences of a rejection of representationalism is very apparent in Paul de Man's essay on Derrida, 'The Rhetoric of Blindness'. De Man describes the 'metaphysics of presence' as 'a tradition that defines Western thought in its entirety: the conception of all negativity (non-being) as absence and hence the possibility of an appropriation or a re-appropriation of being (in the form of truth, of authenticity, of nature, etc.) as presence' (de Man 1983 p. 114). De Man's extravagant comment means that the whole of Western thought is supposedly infected by a problem which is really characteristic only of certain kinds of philosophy. His claim is, for example, probably applicable to Hegel's conception of language as the general means via which the individual subject appropriates itself. 'Presence' in Hegel is supposed to be guaranteed by the structure of reflection, by the recognition of the discourse of the other as constitutive of the truth of the self. Both de Man and Derrida think that this conception can be deconstructed, but, as we shall see, they ignore the fact that a non-reflexive model of the self, of the kind we have repeatedly encountered, cannot be characterised in terms of self-presence.

Somewhat ironically, however, de Man's own totalisation of 'Western thought' already deconstructs itself when, in the same essay, he interprets Rousseau, presumably a key representative of 'Western thought', as revoking the notion of presence in certain aspects of his view of music. De Man shows that, in the Essay on the Origin of Languages (1762), Rousseau was aware of the differential constitution of music and its relationship to language: 'With 
remarkable foresight, Rousseau describes music as a pure system of relations that at no point depends on the substantive assertions of presence, be it as a sensation or as a consciousness' (p. 128). What makes a musical sign a sign is, he argues, neither its iterability nor its reference to a 'state of consciousness':

Music does not imitate, for its referent is the negation of its very substance, the sound. Rousseau states this in a remarkable sentence... . 'It is one of the main advantages of the musician to be able to paint things that one could not hear, whereas it is impossible for the painter to represent things you cannot see; and the greatest feat of an art which operates only by movement is to be able to convey by movement the very image of repose' (translation of Rousseau amended). (1983 p. 130)

De Man wishes, then, in opposition to Derrida's interpretation of Rousseau in Of Grammatology (1967), to show that Rousseau's connecting of music and language involves a deconstruction of the 'metaphysics of presence': 'Like music, language is a diachronic system, of relationships ... The structural characteristics of language are exactly the same as those attributed to music: the misleading synchronism of the visual perception which creates a false illusion of presence has to be replaced by a succession of discontinuous moments' (p. 131). De Man's dramatisation of his contentions cannot conceal the fact that what is at issue is basically another version of the attack on the 'myth of the given'. He understands the given in terms of the optical metaphor which takes meaning to be the re-appropriation of something which is already supposedly present as itself. His conception is therefore in line with the pragmatist critique of the idea that our language could be said to latch on to something already given as such in the world. Similar questioning of this idea in Romantic thought allowed music to play a role in re-thinking our understanding of language. It is, though, not clear that one can simply equate verbal language and music, even though there may be no final way of determining the division between the two, not least because neither can be thought of as a natural kind.

The real question here has to do with the consequences that are drawn from the historical changes in the understanding of music in relation to language, and this is where the assessment of modern philosophy's significance for the rest of culture divides responses to the rejection of representationalism. De Man says of Rousseau's conception of the musical sign:

the musical structure obeys an entirely different principle from that of structures resting on a 'full' sign, regardless of whether the sign refers to sensation or a state of consciousness. Not being grounded in any substance, the musical sign can never have any assurance of existence. It can never be identical with itself or with prospective repetitions of itself ... the identities of physics have no bearing on the mode of being of a sign that is, by definition, unaffected by sensory attributes. (pp. 128-9)

The 'full' sign is presumably the sign according to the empiricist conception, in which the sameness of a sensation or state of consciousness is the condition of meaning. This conception was, of course, already rejected by Kant, because intuitions cannot be strictly identical, identity depending instead on judgements 
according to the rules of the understanding, which can always be misapplied in real situations. In the wake of Kant, a hermeneutic conception like that of Schleiermacher rejects the empiricist conception because of the inferential nature of all understanding of utterances. The question in relation to de Man is why he attaches such polemical weight to music's non-representational character when the basic argument can also be used to suggest that thinking of verbal language primarily in representational terms is simply a mistake, albeit one that did dominate a significant part of the history of Western philosophy until the Romantics.

One important aspect of de Man's contentions, which helps explain his stance, is his strong conception of aesthetic autonomy. The relationship between music and language means that 'What is here called language . . . differs entirely from an instrumental means of communication' (p. 131). Art thus becomes constituted by lack of 'plenitude': it cannot be understood in terms of a metaphysics of presence. Presence is linked to instrumentality in a move which has been decisive for the history of aesthetic theory. In contrast to the conceptions encountered in Kant and some of the German Idealists, independence from the sensuous no longer points to the unrepresentable supersensuous, it now points instead to the end of 'metaphysics'.

De Man's conception echoes a whole series of Romantic-influenced accounts of the relationship of art to philosophy, of the kind we have touched on in previous chapters, such as those of Adorno, the later Heidegger, and thinkers like Lyotard. In some respects all these thinkers tend to arrive at a very similar and questionable position. The validity of this position depends first of all upon there being a substantive historical link between the history of metaphysics and the dominance of instrumental reason, so that what happens 'on the ground' can be traced to an origin in a particular, totalised account of the nature of thought. The associated further pre-condition of the validity of the position is the claim that the link between metaphysics and instrumental reason is to be made in terms of the subject as that which has to dominate the other in the name of self-preservation.

However, De Man's own assertion that there is a 'tradition that defines Western thought in its entirety' seems to rely precisely upon the notion of presence that he opposes in the same argument. The claim that all Western thought is defined by the search for presence presupposes what one can only term the presence of all of Western thought if it is to be valid. However, that presupposition offends against de Man's other claims about language and presence, which must allow for an indeterminately diverse number of interpretations of the tradition of Western thought. His way of characterising that tradition is, then, based on the kind of 'appropriation' that de Man himself wishes to reject as metaphysical. As we have seen, though, this totalising - Heideggerian - account of the history of conceptions of the subject is anyway misleading and one-sided. A further problem here is that just because philosophers may often have thought in terms of their texts appropriating the truth, their actual effects on culture 
need not be understood in those terms, nor need the culture in question have functioned in terms of the way philosophers thought. ${ }^{15}$ The contextualist view I have been proposing suggests the need for a much more differentiated historical picture, which does not assimilate history to a disputed history of philosophy. If this view is accepted, the kind of importance attached both to art's non-instrumental character and to the history of metaphysics must be reconsidered. Music provides an ideal illustration of the need for this kind of approach, precisely because, as de Man shows, it helps us to ask vital questions about the nature of language in relation to metaphysics and aesthetics. At the same time, it is important to remember that music is also a form of human practice which signifies in a whole variety of ways that are not directly connected to philosophical questions.

De Man's own account of the relationship of music to language is so extreme that his insights into these questions get obscured. The notorious problems associated with the notion of identity can suggest why. If signs have to be physically embodied they may indeed, as he suggests, never be 'identical', in one strict sense of the word. This was part of the reason why Kant insisted that identifications were instead a result of the activity of judgement, not of the manifold of intuitions given to the subject. Given that judgement itself relies on sensuously instantiated language, thus on signs, there is clearly a problem in explaining how it is, for example, that the occurrence of one speech act can have the same meaning as the same sequence of words being uttered at another time. This has led to a whole series of deconstructive arguments which aim to show that the interpretation of speech acts is perhaps undecidable in the last analysis. At the same time, as Derrida has acknowledged, the iterability without which language could not function at all as language, must involve some idealisation of the sign that allows it to be identifiable with another occurrence of the 'same' sign at a different time. While ruling out any sense in which language is to be understood in representational terms, thus as providing a 'full sign' which would allow definitive interpretation, this does allow at least some basis for being able to argue about the meaning of a word in a context, even if there is no foundational meaning of the word in terms of which its occurrences can be understood.

De Man is, then, for the reasons we have just seen, aware that the identity of a sign cannot be based on its materiality, but what criterion does he have for talking about 'music' at all, if there is no sense in which musical signs can be identical? His claim is based on the fact that musical signs do not have an assignable referent, and are not to be identified in terms of their physical frequencies, as the idea that musical movement is able to convey its opposite suggested. The same can, though, be the case for the use of verbal language. If the notion of correspondence to a ready-made reality is rejected, language comes to be regarded as a tool for a whole variety of purposes, including, as we have seen, saying things that are meaningless in terms of existing expectations about the meaning of a word. ${ }^{16}$ The dramatic consequences de Man wishes to draw from this fact 
depend upon the initial assumption that there is a totalising story to be told about 'Western thought', in which language has only ever been understood in representational terms. These consequences are also a result of the fact that he pays little attention to language as a social practice which can play cognitive, expressive and a series of other roles. In the case of music there must be, however minimally, some kind of identity between differing cases of this form of articulation, which would enable them to be regarded as musical signs, even if this identity is historically shifting. ${ }^{17}$ This identity can, for example, be thought of in terms of shifting social consensuses about the appropriate employment of such terms as music. The further point here is one we encountered in relation to Schelling and Derrida: difference must relate to something which remains the same, for it to be difference at all. Schleiermacher's notion of immediate selfconsciousness, which he claimed was essential to thinking about music, provided the existential continuity without which the sustaining and changing of social patterns of difference and identity becomes incomprehensible. The further epistemological point about immediate self-consciousness was that it avoided the problems of reflection.

Now both De Man and Derrida reject, in much the same way, the reflection model of consciousness. Derrida does this, however, in a manner which leads him at one point to what Manfred Frank aptly refers to as a 'Hoffmann-esque Nachtstück'. At his most pessimistic, Hoffmann's worries about self-knowledge, of the kind encountered in the figure of the Doppelgänger, mean that the split within the self required to arrive at self-knowledge provides no ontological stability for the self: the other does not reflect a familiar self back to one. For Hoffmann music is able to provide a way out of this dilemma because it revealed a way in which self-consciousness could harmonise with itself while engaging with something beyond itself. ${ }^{18}$ We have already seen why the idea of selfreflection in the signifier could not explain self-consciousness. Derrida characterises consciousness' relationship to language with the metaphor of a mirror without a tain, which reflects an uncontrollable alterity back to it - precisely the Romantic nightmare encountered in the figure of the Doppelgänger. The unstable other which does not reflect one back to oneself is what de Man characterises by the notion of the empty sign which precludes full presence. Derrida moves from the tain-less mirror to the paranoid fantasy that 'A language preceded my presence to myself . . . a sentence was waiting for "you", is looking at you, is watching over you' (cited in Frank 1989 p. 807) as a description of subjectivity's relationship to language. The problem is that in his desire to avoid any sense that the subject could be 'self-present' - that would raise all the problems of reflection - Derrida transfers attributes of self-consciousness into language. His language that waits, looks and watches leaves one with the problem of how differential articulations can wait, look, pay attention, let alone hear something as music. The idea that these may be attributes of a consciousness that cannot be theorised in terms of reflection seems, as Frank shows (p. 811), not to have occurred to him. One cannot characterise subjectivity in terms of self-presence, 
but that does not obviate the need to acknowledge its role in the functioning of systems of articulation.

The subject in this sense is what allowed differences to become manifest as differences, and thus to make meaning possible. As Brandom argued, the mere registering of difference is, to use Schleiermacher's term, 'mechanisable': a computer can do it. What is at issue here is the difference between the mere registering of $x$ as not $y$, and the registering of $x$ as conceptually or affectively significant to someone, the latter being the reason for developing more varied ways of carrying out the former. Why would we bother to distinguish things in ever more complex ways if they did not already matter to us? As we saw, Kant claimed that although cognition is now divorced from pleasure it must previously have been connected to pleasure, 'and only because the most common experience would not be possible without it did it gradually mix with simple cognition and was no longer particularly noticed any more' $(C \mathcal{F}, \mathrm{B}$ p. XL, A p. XXXVIII). The same kind of synthesis as is required to make a cognitive judgement is required to hear different notes as part of a piece of music, thus suggesting links between the cognitive and the affective. Frank suggests that the 'musicality' of language in poetry, which depends upon the rhythm and sound of language, cannot be adequately explained by the assumption that there are two dimensions of language, the poetic and the referential: 'For if according to Saussure - a language only consists of differences, and if, furthermore, the differences are unsayable, then one can justifiably claim that the unsayable is the ground of the sayable' (Frank 1984 p. 601). The significance of the repetition of a word in a text is, for example, not inherent in the word repeated, but rather in the transition from the same, to the different, back to the 'same' which now has a different significance. That difference is a difference to a subject that exists between the two moments of articulation and can invest their connection with a feeling of significance. The same kind of feeling is at issue in the repetitions in music. The subject's relationship to language is, then, 'unsayable', because saying something meaningful always also depends upon what cannot appear in the saying itself, namely what connects the temporalised, differentiated moments of the utterance and can form and develop itself via those connections.

Far from the musical sign being most apt as a means of deconstructing the notion of constitutive, instrumental subjectivity, then, it points instead to dimensions of communication which make possible a different understanding of subjectivity and language in modernity. Oliver Sacks tells how recognising aphasia can be very difficult, to the point where a computerised voice synthesiser is required, because the patient would otherwise use all sorts of extraverbal affective cues to understand what is being said: 'With the most sensitive patients, it was only with such grossly artificial mechanical speech ... that one could be wholly sure of their aphasia' (Sacks 1986 p. 77). These patients have, tragically, lost something essential, but 
something has come in its stead, has been immensely enhanced, so that - at least with emotionally laden utterance - the meaning may be fully grasped even when every word is missed. This, in our species, Homo loquens, seems almost an inversion of the usual order of things: an inversion, and perhaps a reversion too, to something more primitive and elemental. (p. 77)

Despite tending towards that side of Rousseau which invokes a pre-lapsarian state, which Derrida unmasks in Of Grammatology, Sacks does suggest ways in which language in modernity can involve more than just the primary repression associated with the insertion into the symbolic order that is suggested in Derrida's remarks about 'the sentence'. The mechanisable side of language can involve a repression of other vital dimensions of experience, but music can help to articulate these dimensions, and this points to a very different account of the subject's relationship to language.

In Proust's The Captive the narrator says the following on hearing the Vinteuil sextet:

And, just as certain creatures are the last surviving testimony to a form of life which nature has discarded, I wondered whether music might not be the unique example of what might have been - if the invention of language, the formation of words, the analysis of ideas had not intervened - the means of communication between souls. It is like a possibility that has come to nothing; humanity has developed along other lines, those of spoken and written language. (Proust 1981 p. 260)

The pre-lapsarianism in this passage can obscure its real significance, which lies in what it says about the perception of language in the modern period. The passage echoes ideas originating in Romanticism. Novalis, for example, ponders the idea of a 'purely poetic language' with no determinate meaning, and claims that for the poet 'words are not universal signs - they are notes - magic words which move beautiful groups around themselves . . . for the poet language is never too poor but always too universal' (Novalis 1978 p. 322). Similarly, the Symbolists attempt to write 'absolute poetry' as a means of renewing a language which is seen as increasingly subject to the instrumental imperatives linked to the universalisation required for modern rationality. The metaphysical idea of music as a form of language which achieves something that is lost to representational verbal language has been a powerful impetus behind many aspects of aesthetic modernism. The important thing is to understand this idea in a sufficiently differentiated manner.

If music is understood in relation to de Man's idea that all Western thought has been the failed attempt at 'an appropriation or a re-appropriation of being', it can easily come to be regarded as a straightforward substitute for that philosophical failure. This then connects music to other ways of attempting to deconstruct the metaphysics of presence. The problem is that de Man himself relies on just as absolute a claim for his account of the significance of music as what he opposes, as if the history of metaphysics were really a unified entity, rather than a never fully denominable collection of texts and their past and future 
interpretations and effects. An even bigger problem, perhaps, is de Man's account of the subject, which ignores the questions regarding its relation to music of the kind that have concerned us in the present book. It consequently becomes difficult in his terms to understand why music should have been the source of some of the greatest cultural triumphs in the modern period. Now it $i s$ arguable that instrumental language, the language of the 'subject', in the sense of that which dominates its other, does increasingly colonise many forms of modern social existence, as Heidegger, Adorno and others claim. What such an argument can too easily exclude, however, are the aspects of subjectivity which have nothing to do with domination.

It is here that the potential of music for helping to sustain a different conception of the subject becomes crucial. If the subject is not transparent to itself, as the arguments for immediate self-consciousness suggest, it is inherently divided between what it is and what it knows of itself, and these can never finally coincide. The history of the subject in modernity can therefore also be characterised in terms of the subject's attempts to come to terms with the divisions which constitute it, as Hölderlin already suggested. One way of trying to get beyond such divisions is precisely to resist the ways in which language can become reifying, by seeking to circumvent its instrumental use, and the desire to do this is a crucial aspect of modernism in all the arts. This desire is one of the main sources of the idea of aesthetic autonomy, which is inseparable from the changes in the status of music at the end of the eighteenth century, and from the connection of these changes to the rise of the modern conception of literature. A decisive aspect of this conception is that the reduction of language to semantic determinacy is viewed with suspicion, as being capable of obscuring aspects of the world which other forms of articulation can reveal. This is why the 'birth of deconstruction out of the spirit of music' suggested by de Man's remarks can be linked to early Romantic philosophy, which sometimes talks of music in ways which are analogous to the way deconstruction discusses texts. In this perspective the remarkable popularity of deconstructive reading, especially in the United States, can be seen in some respects as a further aspect of the history of Romantic thought. The main differences between these recent approaches and Romantic thought lie, though, in their account of subjectivity and in the assumptions about the consequences of the rejection of representationalism for conceptions of modernity. Here we need to take another look at aspects of Romantic thought in relation to recent theory.

\section{'Infinite reflection' and music}

In the section of the Aesthetics on 'Irony' Hegel argues that Friedrich Schlegel's notion of irony is a result of Schlegel's Fichtean notion of the I, which posits the non-I: 'What is is only through the I and what is through me can just as much be destroyed by me again', so that everything is regarded as 'produced by the subjectivity of the I' (Hegel 1965 I p. 72). The echoes of Hegel's remarks 
about music should be clear: music was limited because it is subjectivity 'resting in unbounded freedom solely upon itself'. The freedom of Fichte's I is, Hegel maintains, completely abstract, and this, he thinks, leads Schlegel to the idea of living one's existence 'artistically'. All that the I produces is solely 'appearance', lacking the 'seriousness' that results from engagement with objective social reality. This lack of seriousness means any product of the subject can easily be dissolved by an all-consuming irony. There is, therefore, for Schlegel, no investment in subjectivity transcending itself into the objectivity which would result from the encounter with other subjects. These claims mirror Hegel's concern that music fails to objectify itself either in the form of determinate objects or ideas. The puzzling side of music without a text for the expert, which made it 'capable ... of the most various interpretations', is, then, also present in Hegel's negative view of the implications of early German Romantic notions of irony. Hegel, of course, sounds here more than a little like many contemporary critics of deconstruction.

Subsequent research (see the discussion of Benjamin below, and Frank 1997) has made it clear how far Schlegel had already moved away from his attachment to Fichte by 1796, so Hegel's judgement is already problematic in this respect. Schlegel's irony is really a result of his rejection of the Idealist assumption that philosophy could constitute a complete system based on the positing of the subject. Romantic irony requires the negation of an assertion, but, unlike the familiar rhetorical trope, not in favour of a determinate contrary assertion, because that assertion will be equally partial and incomplete. Hegel actually adopts a similar idea in his notion of the negation of the negation, but at the end of his system he abolishes the ironic, transient status of its particular elements in the way we have seen. It should be apparent from the difference between the two why Schlegel might come to seem close to aspects of post-structuralism. Post-structuralism adopts many Hegelian moves devoted to the avoidance of giving the subject a grounding status, but rejects the closure of the system (see Frank 1984). A consideration of a few aspects of how early Romanticism regarded music can help to clarify the relationship between the attention to undecidability of meaning in Romanticism and in deconstruction, and can also help to assess the aptness of Hegel's judgement on Schlegel's position.

Schlegel makes it evident how important he finds music in the 'Literary Notes' (1798): 'beauty (harmony) is the essence of music, the highest of all arts. It is the most general [art]. Every art has musical principles and when it is completed it becomes itself music. This is even true of philosophy and thus also, of course, of literature [Poesie], perhaps also of life. Love is music - it is something higher than art (Schlegel 1980 p. 151). This piece of apparently shameless hyperbole can make sense if we look at some more early Romantic claims. Novalis thought music allowed the mind to be 'for short moments in its earthly home' because we are 'indeterminately excited by it', and it is the understanding of this indeterminacy that is decisive. For Hegel indeterminacy is ultimately just the failure to carry out the work of the concept to the point where indeter- 
minacy is revealed as being determinable. Romantic philosophy resists this kind of closure via such notions as irony. Music can indeed, then, as Hegel's comments suggest, be seen as being closely related to irony. How the resistance to closure is assessed depends upon assumptions about the status and role of philosophy. Anything determinate we say about significant music is, for example, always also tinged with the sense that there is a necessary mismatch between it and the music. This can either mean that one concurs with Hegel's position by regarding that mismatch as having to do with music's inadequacy, or it can mean that music is important precisely for either its transcendence of or revelations of the limits of the sayable. A version of the difference between an Idealist and a Romantic conception emerges again at this point, and we need now further to elucidate the Romantic conception.

The key secondary work for the difficult question of the Romantics' relationship to Fichte remains Walter Benjamin's The Concept of Art-Critique in German Romanticism (1919). Although subsequent research has revealed much about the early Romantics that Benjamin could not have known, his approach is still valid in many of its essential outlines. Benjamin, like Hegel, regards Fichte as the figure behind the development of early Romantic thinking. Fichte's insistence upon the irreducibility of the subject to objectivity led, as we saw, to a new kind of exploration of the nature of consciousness. A valid conception of Romanticism, for Benjamin, depends on how one approaches the possibilities of this exploration, and the question of 'reflection', the splitting into related aspects that mediate each other, is central to such a conception.

We have seen how Fichte and the Romantics raised the problem of the regress of reflection that lies in the attempt of consciousness to ground itself as the cognitive principle of philosophy. In order to escape this regress Fichte posits a grounding immediacy of consciousness which does not depend upon reflection: 'Thus Fichte is looking for and finds an attitude of mind in which self-consciousness is already immediately present and does not need first to be summoned by a reflection which is in principle endless' (Benjamin $1980 \mathrm{I}, 1 \mathrm{p}$. 26). The essential principle of Fichte's consciousness is the free action, the Tathandlung (literally 'deed-action'). This ground of reflection cannot itself be available to reflection because it is required for the reflection to take place at all: it must remain unconscious, and therefore external to reflection. For Benjamin the concept of the unconscious is associated with the irrationalist tradition that arises from Schopenhauer's appropriation of Fichte, which leads, as we shall see in Chapter 8, to a monolithic conception of the significance of art as the means for coping with an underlying reality which is otherwise irredeemable. The relation of the early Romantics to such a conception is anything but straightforward, because the immediacy of 'feeling' cannot be reduced to reflection, which seems to keep them in a position similar to that of Fichte. The question is, though, whether any conception can do without the function performed by some kind of immediacy, even though that immediacy plays its role in thoroughly mediated contexts. For the Romantics, feeling and reflection have to 
accompany each other: difference presupposes identity if it is to be known as difference. Use of the notion of feeling therefore does not entail a dogmatic claim about the nature of reality in itself: reflection in Novalis did not 'at any moment step over the critical boundaries of the immanence of consciousness and yet it has explained that not everything is in consciousness' (Frank $1997 \mathrm{p}$. 823). Feeling 'can only be looked at in reflection - the spirit of feeling not there any more' (Novalis 1978 p. 18), but that does not mean it does not exist. The simple point of feeling in this respect is that it points to why these things come to matter at all, which is not explicable in terms of reflection. Why does reflection keep taking place in new ways?

Benjamin does not deal with this aspect of Romantic thought. His main concern is to suggest that the endlessness of reflection need not be regarded, as, for example, Jacobi thought it did, as leading to a regress into the abyss. Understanding reflection as leading to an empty infinite regress, whereby selfconsciousness could never result because the series 'I think I think I . . . etc.' carries on ad infinitum is, Benjamin claims, not the only way to understand reflection. The Romantics, he argues, regard the process of reflection as involving the potential for endless articulation, and thus as being something 'fulfilled'. Hamann had already suggested that the endless reflection inherent in different languages should be regarded in terms of a celebration of the multiplicity of God's creation. For the Romantics, in Benjamin's reading, articulation does not require an immediate foundational point from which to develop. There is, therefore, no 'action' of the kind Fichte regards as the necessary origin of thought, which thought itself cannot articulate. For the Romantics, Benjamin claims, 'reflection is logically prior . . . Only with reflection does the thought emerge that is reflected upon' (p. 39). His claim echoes Hegel, for whom the immediate always already requires the other of itself, determinate thoughts always being necessarily split by reflection, the beginning only being the beginning because of what follows it. Benjamin, though, wants to point to the - nonHegelian - sense in Romanticism that there is no ultimate goal of articulation beyond an endless diversity of reflection, which connects nature, art and language, without the need for an initial self-presence of the subject in terms of which this takes place. In this he echoes Hamann, for whom a grounding of articulation would diminish the capacity to celebrate the divine. Hamann considered music to be the oldest language.

In the light of the argument so far, it is not surprising that Derrida's account of différance resembles Benjamin's arguments about Romanticism. Derrida states in Positions that 'différance is not preceded by the originary and undivided unity' (Derrida 1972a p. 17). The subject is secondary to the movement of differance: 'the subject ... depends upon the system of differences and on the movement of différance . . . it is not present and above all not self-present before différance ... it only constitutes itself by dividing itself' (p. 41). The rejection of the myth of the given is applied to the subject itself so that it has the same status as all aspects of the world, which are only ever accessible in terms of their 
mediation in a language. Such arguments show the problem of any attempt to make the subject a self-present, transparent foundation for knowledge, of the kind sought by the Idealist tradition from Descartes onwards. They are, though, not adequate in relation to the non-reflexive conceptions of the self offered by Novalis and Schleiermacher. For these thinkers the infinity of reflection results from the subject's simultaneous lack of self-presence and need to come to terms with this lack. This means the subject is not an absolute point of beginning, but it does not mean that it is only constituted by dividing itself. The sense of lack that leads to reflection requires something which is already in some way itself for that lack to be felt as its lack. The point is that this account of reflection gives a way of understanding what moves the subject beyond itself into 'endless reflection', something which becomes impossible to understand in merely differential, reflexive terms. Benjamin maintains that Schlegel and Novalis understand 'the endlessness of reflection as a fulfilled endlessness of connection [Zusammenhang, which also means 'context']: in it everything is supposed [to connect] in an endlessly multiple manner' (Benjamin $1980 \mathrm{I}, 1$ p. 26), but this does not explain why this endless multiple connectivity could matter to the subject.

Schlegel ponders the question of foundations in philosophy and suggests:

In relation to every concept and every proof one can again ask for a concept and a proof of the same [concept and proof]. For this reason philosophy must begin in the middle like the epic poem, and it is impossible to present it and add to it piece by piece in such a way that the First would be completely founded and explained for itself from the very start. (Schlegel 1963 p. 518)

This passage denies a foundational status to anything like Fichte's I. The Fichtean I can never be grounded in a stable manner: as Derrida argues, it will always be dependent on an unstable other that prevents it being able to be an absolute foundation. However, one must be careful how this argument is related to conceptions of the I. It is too easy to slip from the rejection of the I as selfpresent absolute foundation to an account which excludes ineliminable aspects of self-consciousness altogether and leads merely to randomised difference. Winfried Menninghaus, who links his interpretation of the Romantics to both Derrida and Benjamin, characterises endless reflection as follows: 'one can say that the whole "Being" of endless reflection consists, as a totality of relation, in the reflectings of all its parts: a decentered continuum of centres of reflection' (Menninghaus 1987 p. 47). Unfortunately this does not make sense. For something to be a 'centre of reflection' it must have a periphery which is not itself. The critique of reflection theory makes it clear that such a centre of reflection would have already to be non-reflexively self-aware for it to be able to define itself against its other and thus to be a centre, as opposed to being merely a moment, of reflection at all. If the 'decentred' centres (?) (elsewhere Menninghaus talks of difference becoming a '(non-absolute) Absolute' (p. 87)) cannot, in some sense, be centres and not peripheries, it is impossible even to 
use the notion of a centre. There would in this case be no difference between centre and periphery, no difference between the computer and the self-conscious being. Menninghaus's interpretation of early Romanticism, and at times Benjamin's, simply relies on another version of the untenable reflection model, which, in the haste to escape any foundational role for subjectivity, ends up with no way of accounting for ineliminable features of awareness and self-awareness. A non-reflexive conception of individual self-consciousness like Schleiermacher's is able to allow that philosophy could not found itself from the very start. This is why he argues for a dialectic which tries to facilitate the admittedly endless, but socially necessary, attempt to come to terms with difference without repressing individuality.

Despite these difficulties, Benjamin's account of endless reflection can be productively linked to both a theory of the work of art and a theory of language which point forward to many of the issues of contemporary literary theory. Benjamin's account comes close to Schelling's contention that artworks can be endlessly interpreted 'as if they contained an infinity of intentions, whereby one can never say whether this infinity lay in the artist himself or just in the work of art' (Schelling I/3 p. 620). The similarity is a result of the fact that in both artworks are seen as not having the status of objects of knowledge. The System of Transcendental Idealism, though, saw art as combining the conscious and the unconscious, and as involving a moment in which time is abolished, so that all reflection ceases. This made it into the 'organ' of an Idealist philosophy, in which identity of subject and object was documented.$^{19}$ Romantic philosophy does not rely on this identity, and instead sees our only sense of this identity in the incompleteness we feel in relation to our ability to grasp art. The artwork is therefore inherently temporal, consisting in a potentially endless series of 'reflections'. These reflections occur both in those who engage with the work and via the work's relations to other works of art from the past and the future. Something like what is meant here is the theme of Borges's story about the nineteenth-century author of Don Quixote, Pierre Menard, whose text is richer than that of its original creator, Cervantes, even though the texts are actually identical. In the famous Athenäum Fragment 116, Schlegel claims that the 'romantic work of literature [Dichtart] is still in a process of becoming; yes, that is its real essence, that it can eternally only become, can never be completed. It cannot be exhausted by any theory' and that the work of art multiplies itself 'as if in an endless row of mirrors' (Schlegel 19882 p. 115). The 'mirrors' can, then, be individuals who can never exhaust the work of art, but who also contribute to its mode of existence as a dynamic entity, and they can also be other works which change its significance, in the way that Don Quixote is, for example, changed by its reflection in the work of Symbolist poets.

These issues relate closely to the changing status of music in Schlegel's period, and this also helps generate the kind of conception of literature we observed in Foucault. In Positions Derrida emphasises how important the emergence of the notion of 'literarity', which he sees mainly as an achievement of 
Russian Formalism, is to his project of deconstruction. The notion of literarity enables one, he argues, to avoid reducing texts to thematic, sociologistic, historicist, or psychologistic readings, and it forces one to see how philosophical writing is never 'purely philosophical' because of the metaphorical excess which language always generates. How, though, does this awareness of the nature of literary discourse emerge historically? The fact is that without the growth in the importance of instrumental music the theories being discussed here are much less easy to account for. Consider Schlegel's initiation of the notion of a 'musical idea' in the Athenäum Fragments:

Many people find it strange and ridiculous if musicians talk about the thoughts in their compositions; and often it can happen that one sees that they have more thoughts in their music than about it. But those who have a sense for the wonderful affinities of all arts and sciences will at least not look at the matter from the flat viewpoint of so-called naturalness, according to which music is only supposed to be the language of feeling, they will in fact not find it per se impossible that there is a certain tendency of all pure instrumental music towards philosophy. Must pure instrumental music not create a text for itself? and is the theme in it not as developed, confirmed, varied and contrasted as the object of meditation in a sequence of philosophical ideas? (Schlegel 19882 p. 155)

This passage, more than almost any other, gives the lie to the notion of Romanticism as being initiated by an obsession with the expression of feeling. It is not that Schlegel would deny the importance of feeling, but the elevated role he gives to music makes it clear that his real interest is in new forms of articulation which integrate different cognitive and affective aspects of existence, not in 'self-expression'. Schlegel's polemic is directed in favour of the kind of conception of language we have been concerned with, not of a 'Fichtean' sense of self. Like Schleiermacher (and Hölderlin, Novalis and Schelling), Schlegel simply does not fit into the reductive Heideggerian story of Western metaphysics that regards it as linked to the domination of the subject. The Romantic view does not rely on a representational conception of language, and at the same time keeps a plausible role for the subject in understanding vital aspects of modernity. One key to these positions is precisely music.

Pure instrumental music's 'tendency towards philosophy' derives from the non-representational aspect of wordless music: both philosophy and music are concerned with what transcends the sayable. This transcendence creates a further link with the notion of literature which develops at this time. As we have seen, the notion of the unsayable need not be conceived of mystically: it relates to what happens in language that is not explicable solely in semantic terms, and was suggested in Wittgenstein's remarks about understanding a poem. Schlegel's idea becomes clearer when he refers to Kant's repetition of ideas as making his texts 'musical enough', and to Kant's 'musical repetition of the same theme'. The point is that saying the same thing repeatedly changes the effect of what is said, a fact which is crucial in music. When Beethoven repeats a phrase over and over in the Scherzo of the String Quartet Op. 135, he reveals the way 
that musical repetition can easily tip over into something bordering on madness. The same device which structures much of Beethoven's work can, then, also threaten its very existence as meaningful articulation. Once again the crucial factor here is the subject who apprehends the disturbance to a received order, even though what can be said about this disturbance differs from the actual experience of hearing the piece. This kind of possibility also occurs in texts which come to be regarded as literary because their effects do not derive from their propositional content. It is not, though, as Foucault and De Man implied, that language therefore becomes something purely self-referential, in the form of Literature, the dialectical other of the science of language. Their idea relies too heavily on the claim that language in modernity is tied to instrumentality and that a wholly other sense of language is therefore required to escape this situation. Schlegel's conception is directed rather towards the importance of creating differing forms of articulation which may overcome the limitations of other forms. Strangely, it was Schlegel whom Foucault saw as one of the initiators of the science of language in this period. There is no doubt that Schlegel did help initiate modern linguistics - and modern philology - but he did so as part of a vision which evidently includes the issues that Foucault raises. The main problem with Foucault's account is that it makes the genesis of the notion of literary autonomy too separate from the aesthetic praxis of the time.

Foucault claims the separation of literary language from the 'discourse of ideas' is the key change in this period. This claim becomes more plausible if it is connected to music in the way suggested here, but the sort of separation Foucault intends misses much of the point of the Romantic position. Music is inextricably linked to the emergence both of aesthetic autonomy and of the modern idea of literature. Schlegel says of the novel: 'The method of the novel is that of instrumental music. In the novel even the characters may be treated as arbitrarily as music treats its theme' (Schlegel 1980 p. 146). In the famous review of Goethe's Wilhelm Meister Schlegel claims that: 'The second book begins by repeating musically the results of the first', and that 'This harmony of dissonances is even more beautiful than the music with which the first book ended.' The changed perception of the non-representational character of wordless music becomes a model for the other arts. This is what lies behind Benjamin's claims about Romanticism and reflection: music generates significance by the internal, non-semantic relationships between its elements, though these also relate beyond themselves to the social and historical world. Similarly, instead of characters and events being the 'final purpose' of the novel, such that the novel would be seen as 'representing' a world in the way, say, a newspaper or history book can be said to do, Goethe's novel is a book 'which one can only learn to understand out of itself', which is an internally self-reflecting structure (Schlegel 19882 pp. 159-61). Any part of the text will have a different significance if seen in relation to any other part. This does not preclude reading such a text in representational terms - one can reflect the text in the history of its time - but that is only one kind of reflection. If this kind becomes exclusive, 
one loses precisely what makes the text into what Benjamin calls a 'medium of reflection'.

In his Monologue (1798), Novalis writes of the 'musical spirit of language' in similar terms to the way Schlegel characterises the novel and music. At the same time he makes a further link to mathematics. For both Novalis and Schlegel the idea of representation becomes secondary to the idea of languages as possibilities for world making. This is what makes possible the link between music and literature:

One can only be amazed at the ridiculous mistake, that people think they speak for the sake of things ... If one could only make people understand that with language it is as with mathematical formulae - They constitute their own world - They only play with themselves, express nothing but their wonderful nature, and this is why they are so expressive - precisely for this reason does the strange game of relations of things reflect itself in them. Only via their freedom are they members of nature. (Novalis 1978 p. 438)

The reconciliation of nature and freedom sought by Idealist philosophy is seen not in terms of the establishment of a closed philosophical system, but in the endless creation of new relations between things which give rise to new revelations that is possible in literature and music.

Romanticism has too often been understood as merely revelling in indeterminacy. At this time the sense of liberation via the escape from established kinds of determinacy clearly does dominate the Romantic conception. However, it is important to remember that the freedom from determinacy inherent in music does not turn out to be an arbitrary freedom. The reflection of the 'game of relations of things' in music is, of course, one of the keys to understanding the move of music, from the attempts of Classical and Romantic symphonies to achieve something like the aims of Idealist philosophy in works which integrate more and more diverse material into a harmonically end-directed form, to free atonality, which employs relations that are not given in the harmonic system and which leads to deep problems concerning how to organise works and concerning their reception. Similar issues inform the development of literature that leads to Mallarmé and beyond, in which the gain in freedom generated by the move away from representation also generates ever greater problems in deciding how to write texts that live up to the most stringent aesthetic demands. These inner-aesthetic questions are also an indication of extra-aesthetic matters.

Modernity simultaneously generates systematic determination of more and more areas of life, and the awareness that such determination involves inescapable repressions. The reception of art can therefore turn into a battle between those wishing to fix signification, be it by historical research, attention to the life of the artist, computer analysis of texts, scores, and so on, and those, like the Romantics, who see such an enterprise as inimical to the nature of art, and demand attention to the capacity of art for generating ever new significances. ${ }^{20}$ 
Deconstruction would, in this view, be another version of Romanticism's questioning of the legitimacy of an Enlightenment which sees its task as the conceptual ordering of reality. The danger of this side of 'Romanticism' can lie in the tendency to elevate the demonstration of the limitations of determination to being the main aim of philosophical thought. The concern to avoid 'foreclosure', to avoid arresting the 'play' of différance, must, though, at some point be legitimated as part of the practice of cultural communication and expression. The legitimacy of the work of the early Romantics derived from their showing up the possible repressive effects both of the new attempts to establish a foundational philosophy and of the growing separation of the sciences from the lifeworld. In order to achieve this they needed not only to show how difference could not always be foreclosed, but also to show why this mattered in terms of a new conception of the subject's role in an increasingly system-determined world.

The contemporary return of related ideas derives both from the perceived failure of foundational accounts of language and meaning in philosophy, accounts which derived from the - sometimes too - close links that develop between analytical philosophy and the natural sciences, and from the much more general tendency in modernity for there to be a movement from times where the desire for order prevails, to times where order is perceived as stifling. This latter point is not an abstract one: an effective critical approach to these issues needs to look at how they have manifested themselves in differing historical circumstances. As we shall see in Chapter 8, for example, similar ideas to those of the Romantics are mobilised to very different ends by Nietzsche. Similarly, the debates about the function of art in the face of fascism, which led Walter Benjamin to adopt reductive views for reasons of political responsibility in the later $1930 \mathrm{~s}$, make it clear that these issues are primarily to do with how the real cultural world functions in relation to the challenges of modernity. At some point the 'infinite reflection' in the work of art has to be seen in relation to real people who engage with that work: lives are not lived merely by deferral and art is not ultimately a theoretical matter.

In the case of the Romantics, the historical shift that leads to the conceptions of language and music at issue here involves a motivation that does not derive solely from texts and works themselves. The difficulty of telling this kind of history is that it involves the history of something which is in one sense always absent, namely 'the subject' - remember Schelling's remarks on the fact that the subject can only find 'the monuments, the memorials of that path, not the path itself' of its own history. The connections between the rise of the idea of absolute music and the emergence of radical ideas about art and interpretation of the kind suggested in the notion of infinite reflection are 'monuments' of this history, but they do not give direct access to it. At the moment when, as is most evident in Fichte, subjectivity becomes the principle concern of philosophy, potentials of the subject which were previously unarticulated emerge, along with the realisation of the potential boundlessness of what this may mean. The 
theory and praxis of music is the area in which this realisation is most evidently manifested, precisely because music offers a series of monuments which speak in a way verbal monuments can only do when they move towards music in the form of radical literature.

Connecting this kind of history to other histories involves problems that have rarely been satisfactorily dealt with in the history of ideas. The history of subjectivity articulated in aesthetic theory cannot be told in solely conceptual terms. What aesthetics can say theoretically relies on what is shown and disclosed in a non-theoretical manner in the arts. This does not mean that we therefore descend into meaningless immediacy, but the nature of aesthetic understanding is such that at some point conceptually expressed justifications come to an end and one has to invoke what can only be shown, which may therefore elude one's interlocutors. Whereas in the sphere of knowledge this preparedness to countenance an end to justifications will rightly be considered a dereliction of the duty of justification, in aesthetics this preparedness is germane to the very discipline. Furthermore, it is not that aesthetic issues therefore cease to connect to the politico-social world. This is apparent if one considers that the growth in the importance of music in the German-speaking public sphere in the early nineteenth century is connected to the failure to develop a politically effective public sphere. Much of the energy of Beethoven's music, for example, derives from his admiration for the French Revolution, and to his subsequent feeling of political impotence in the Restoration period. Rudolf Bahro has suggested that the political energy which sometimes appears in Fichte's best writings on freedom, and which elsewhere in Europe led to transformative political action, is only really articulated in Germany in Beethoven's music (Bahro 1979). At the same time, the works of art which help document the history of the subject in Germany should not merely be regarded as the results of a repression. The point of the theory of infinite reflection is that genuine works will continue to signify in a multitude of other ways. Beethoven still speaks so uniquely to those prepared to listen because he suggests a way of being to which we may still aspire, even if history has taught us that it may never be possible to achieve. ${ }^{21}$

The fact remains that many of the questions considered so far in philosophical terms do here also become political questions, and their subsequent history is linked to the unhappy history of Germany in modern Europe. On the one hand, music's growing importance can be associated with political impotence and the concomitant development of 'inwardness', which is evident in Hegel's claim that music involves the subject indulging itself in an abstract freedom which never has to engage with the public political sphere. The failure of this public sphere in modern Germany until after the Second World War is a major source of the disasters of Germany's history, and the contribution to that history of thinking which believes it is possible to withdraw from public action into inwardness cannot be ignored. On the other hand, music can also sustain a potential for new articulation which can provide both individual and collective 
motivations. This is itself a political matter, as the considerable role of music in revolutions from the French revolution to the liberation of South Africa from apartheid suggests. Furthermore, music, unlike the other arts of the time apart from drama, involves collective reception of the kind Schelling regarded as so vital to Greek tragedy. In an anticipation of Wagner, Schelling concludes the Philosophy of Art with reflections on the 'most complete combination of all arts ... which was the drama of antiquity' and wonders if opera, which is merely a 'caricature' of ancient drama, may become able to lead back to 'ancient drama combined with music and song' (Schelling I/5 p. 736). Aspects of Romantic thinking and the music of this period suggest, then, that there is more to the politics of culture than can be encompassed within a Hegelian framework, but these aspects are fraught with danger as well as offering new cultural potential. The case of Wagner will further bring out the inherent tensions between the Hegelian and Romantic conceptions. These tensions will be particularly apparent in Nietzsche's reactions to Wagner and in the exploitation of Wagner by the Nazis.

The route which can be traced from the changes in the understanding of language associated with music at the very beginning of modernity, via Schopenhauer, Nietzsche and Heidegger, to post-structuralism, needs, then, to be examined in this perspective. Debates about the political implications of deconstruction have much to gain from studying this aspect of aesthetic theory, not least because the missing dimension of the subject that is not simply an 'effect of the general text' (Derrida) cannot be ignored in such theory. Despite the repeated references to postmodernity in these debates, they are in many respects being carried on in the terms largely established in the Romantic period of modern philosophy. Given the fraught history of the politics of this aspect of German philosophy, a historical awareness of the sources of these theoretical issues is vital if old mistakes are not to be repeated.

\section{Notes}

1 I am thinking of the kind of analysis which reduces Schumann's Frauenliebe und Leben to its place in an ideology of gender in the nineteenth century. The text of the cycle may belong in such a context, but to hear the music solely in these terms is not to hear it at all.

2 Clearly this is only going to be successful against a background of verbal communication which enables the conductor to play such a role, but what is at issue here are the aspects of communication which go beyond what can be said. Why otherwise do the conductors not just tell the orchestra what to do verbally?

3 It is perhaps worth mentioning here that Davidson regards metaphor as the 'dreamwork of language'.

4 Such an account of music would, of course, fail to take into account the fact that the division of the harmonic series is not natural, because it is historically changeable. The fact that the changes can be expressed in mathematical proportions does not explain why, for example, there is a move from untempered to tempered scale.

5 The composers clearly did so in ways that were also influenced by philosophical, literary 
and other ideas, but the mistake is to think that what they did and the effect of what they did is solely the result of such influences. The point of the early Romantics' insights here, as we shall see, is that they were the first to see that music itself could affect conceptual thinking.

6 The same can even be said of the way we apprehend a painting.

7 Mallarmé was familiar with some of Hegel's work.

8 The ambiguity here between the epistemological use of the term to designate immediate self-consciousness, and the use to designate feeling as affect can be resolved by the fact that both fit Novalis's dictum that 'feeling cannot feel itself'. The point is that neither is inherently propositional.

9 It is worth remembering here that the analytical philosophy of mind has often focused on facts like pain, which are inadequately discussed in terms of propositional knowledge.

10 This, of course, connects Saussure's ideas to contemporary inferentialism.

11 It is often claimed that Schumann does not fully grasp Heine's irony, which would indeed seem to be the case. However, he can also be understood as revealing the source of that irony by revealing the pain and longing that lies beneath it.

12 In Brandom's terms the subject is understood as that which can grasp the conceptual content of an articulation, rather than being what is merely the locus of a differential response. As I suggested above, this does not seem enough to account for what is at issue in music.

13 This attempt, of course, ignores Schleiermacher's hermeneutic point that understanding the purified language relies on first having learned a natural language via which the world is intelligible at all.

14 Representation therefore is just understood as a one-to-one correlation of a word to a thing, without any sense that the essence of the thing is adequately conveyed by the word.

15 There is a way of making such connections, and of using aspects of 'objective spirit' to understand deeper historical trends, but the methods for doing this are still essentially contested, so making totalising judgements in terms of them is problematic.

16 On this see Davidson's 'A Nice Derangement of Epitaphs' in Lepore 1984.

17 Wittgenstein's notion of family resemblance can suggest what is meant here.

18 See Charles Lewis (1985): 'Kant and E.T.A. Hoffmann: "The Sandman”', in Ideas and Production 3, pp. 28-43. The structure is rather like the one observed in relation to Hölderlin's idea of poetic representation in Chapter 3.

19 This is only one way of reading Schelling's text: I suggested some provisos in Chapter 4.

20 It should not be forgotten here that Schlegel can be considered the founder of serious literary history, but that is only part of his wider aims.

21 Adorno's difficulties in finishing his work on Beethoven seem to relate to the sense that his admiration of Beethoven's work, and his conviction that Beethoven is yet symptomatic of the wider failure of German culture cannot easily co-exist. 


\section{Nietzsche and the fate of Romantic thought}

\section{The old and the nem Nietzsches}

The alternatives in some of the most controversial debates in recent philosophy often come down to whether what is at issue is in essence a Hegelian, or a Nietzschean position. The differences between Habermas and Rorty, or between the early Derrida and Davidson, for example, can be seen in these terms. These figures are all what Habermas would call 'post metaphysical' thinkers. However, despite their renunciation of the idea of a foundational 'first philosophy', Habermas and Davidson wish to sustain a universalist conception of rationality; Rorty and Derrida, in contrast, think that such a conception is a residue of a philosophical past which it is time to leave behind. Significantly, the former do not give a decisive role to the aesthetic, whereas the latter do, and it is not fortuitous that what is at stake in both positions can be elucidated in terms of the interpretation of Nietzsche. Whereas, for Habermas, Nietzsche is part of a questionable tradition of critiques of modern rationality, Rorty thinks he offers the possibility of escaping from many tired philosophical obsessions via his rejection of representationalism and the correspondence theory of truth, and via his concern with self-creation. At the same time he contends that Nietzsche must also be regarded with caution because he was not a democrat, and had no investment in what to Rorty matters most, namely the reduction of human cruelty and suffering. For post-structuralists this latter worry seems to play virtually no role in their view of Nietzsche. He is instead an epochal figure who, along with Heidegger, has decisively changed the face of philosophy in ways which go to the heart of Western civilisation. This more dramatised view relies in some respects on the sort of questionable assumptions about the history and the effects of Western philosophy that we encountered in de Man in Chapter 7. Adorno warns in Negative Dialectics that 'The new beginning at a supposed zero-point is the mask of strenuous forgetting' (Adorno 1975 p. 79). One of the problems with the 'new Nietzsche' is that this kind of amnesia can be seen to be present in some of his recent reception.

Two approaches to Nietzsche suggest how this might be the case. The first has formed the focus of recent work by Robert Holub, who shows that Nietzsche was reliant at times on contemporary ideas that even his most fervent admirers 
would have great trouble taking seriously, and that he used questionable secondary sources for his knowledge of many of the thinkers in modern German philosophy whom he was so eager to dismiss. ${ }^{1}$ The second approach will inform this chapter, namely the revelation of the extent to which the structures of thought upon which Nietzsche relies are part of a Romantic aesthetic tradition which requires a more adequate presentation than it has generally received. Furthermore, especially in relation to the questions at issue in the present book, some of the positions of the Romantics are arguably superior to those of Nietzsche. This is not least because the Romantics see themselves as offering a corrective to some of the reductions in Enlightenment thinking, rather than a totalising critique of rationality.

Many advocates of the 'new Nietzsche' might object, however, that such an approach misses the point. If Nietzsche's thought is as revolutionary as they suggest, then measuring it by standards developed from a tradition he aims to overcome makes it impossible to appreciate just how different he is from what preceded him. Rorty has suggested one problem with this position in Contingency, Irony and Solidarity, namely that by making his attitude to his philosophical predecessors into a unified overall story Nietzsche puts himself in the position where one has to 'claim that none of the descriptions that applied to them applies to you', so that one is 'acting as if a redescription of one's predecessors got one in touch with a power other than oneself', such as the 'Will to Power' (Rorty 1989 p. 107). When Nietzsche does this, Rorty argues, he rejoins the metaphysics he opposes with other aspects of his thought, and is therefore open to the same kind of objections as are appropriate in relation to anyone in the tradition of strong foundationalist thinking. The aptness of this criticism will become apparent when we consider some of Nietzsche's responses to the questions we have been concerned with so far.

However, there is a further objection to the approach I shall adopt which has more force, and which involves a central issue in contemporary debate. Nietzsche is, like Rorty, in many respects a performative thinker. What matters are the effects of what he says, language for Nietzsche not being primarily a means of representation, but rather, as it already was for Schleiermacher, a form of social action. The evident fact that Nietzsche makes inconsistent statements about so many questions cannot, in this perspective, be a reason for accusing him of an inconsistency which invalidates his 'position'. This is because he is not aiming at a set of coherent true statements about the matters which concern him, but rather at performative effects on those who think that seeking for true statements about topics like, precisely, 'the Truth', should be the aim of philosophy. For Nietzsche these people are involved in a practice they would be better off giving up, and persuading people to do something different, as any politician knows, is more likely to be a question of rhetoric than of consistent counterargument.

The standard way of countering this stance is to argue that it involves a performative contradiction. By employing language in this manner, one is relying 
on the fact that one holds it to be true that such people should give up their pursuit of philosophical goals. One is therefore covertly relying on truth at the same time as arguing for its subordination to something else. This argument will in some contexts be a valid objection to some of the Nietzschean strategy. However, the argument starts to look less generally compelling when it is considered in relation to aesthetic questions, where the issue of rhetoric becomes more complex.

As Gadamer has shown, the concern with the 'Wahrscheinliche', both with what appears to be true and with the 'truth of appearances', which is initiated by Aristotle's attention to rhetoric as the complement of analytic and dialectic, comes in the eighteenth century to form a link between Baumgarten's new concept of aesthetics and the discipline of hermeneutics. Even though, Gadamer suggests, 'the tradition of rhetoric broke off particularly thoroughly' in eighteenth-century Germany, 'it remained effective in an unrecognised way in the realm both of aesthetics and of hermeneutics' (Gadamer 1986 p. 111). If Nietzsche's texts are themselves regarded as literary works, and their rhetoric is therefore a part of their aesthetic status, their argument-based, truth-claiming aspect need only be one of their aspects, and may not be the most significant. What a novelist may argue to be true in a novel, for example, might not be what makes their work significant, because the arguments are only presented by one voice in a polyphony of different voices with different rhetorical effects. Such a conception of language and art is, as we have seen, already part of Romanticism. Schlegel's conception of irony puts in question the assumption that texts must inherently be about truth in a limited, propositional sense. Look, for example, at the essay 'On Incomprehensibility', where Schlegel at one point plays with the strategy of claiming that what he is saying is not ironic, a claim which it seems impossible to make in the context of a text about irony, because the very making of the claim ironically undermines it.

The core problem for the interpreter of Nietzsche is that performative effects are not something which can be inferred directly from a text. ${ }^{2}$ The effects themselves will depend upon the recipients of the text, and the identifying of performative intentions depends on the vagaries of interpretation. At the same time, it is clear that Nietzsche has had performative effects, and did have performative intentions. One cannot, for example, make him into a liberal democrat, even if some of his ideas may be usable in some contexts for democratic purposes (as they were for the pre-First World War German Social Democrats). In the light of the effects of anti-democratic thinking on German history, these intentions can hardly be said to be unproblematic. The question is, therefore: were such texts likely to achieve things one would find desirable, and are they likely to do so now? Some of the decisions on this question must be made in terms of Nietzsche's historical role, and here one has a right to be vigilant. However, it does seem evident, especially from his reception by Derrida, Nehamas, Rorty and others, that Nietzsche's texts also offer other resources of a different kind. Given his relationship to the tradition at issue in this book, my question will be 
whether these resources really offer so much more than those established by some of his predecessors.

One response to the sort of issues raised by Nietzsche's texts is the - explicitly Hegelian - argument by Robert Brandom that the shared social practice of fulfilling the commitments entailed in any discursive claim made to another person has priority over the preferences and desires which give rise to what one may claim. While an advocate of a Nietzschean stance may wish to give priority to the literary, strategic and ironic aspects of Nietzsche's texts, in Brandom's view this prioritisation cannot be self-legitimating and itself needs justification. Brandom's rationalism suggests the kind of challenge which any defence of a Nietzschean strategy must face, and similar views have also been advanced by Habermas and others. What this comes down to is in many respects a decision on the very status of theorising in philosophy. Brandom's claim is that theory cannot just be performative, and that the traditional goals of philosophical legitimation can therefore still be pursued. They can, moreover, be pursued in a form, which, pace Nietzsche, does not rely on a representationalist conception of truth and regards language as social practice. However, the goals do entail the kind of universal claims that Nietzsche (in some respects, at least) opposes.

Nietzsche, like post-structuralists such as Lyotard, would regard this position as involving a different version of the closure inherent in the pursuit of metaphysical aims, and therefore as blocking access to what may be outside existing kinds of legitimation. Concern with the possibility that philosophy has been the source of a repressive failure to engage with the radically 'other' is, of course, the reason for the extensive attention to the aesthetic in such thinkers. The question which is decisive for the present book, is, therefore, the relationship of aesthetics to rationality. The Idealist hopes for an integration of the subject into a new conception of nature that would harmonise what we know with what we should do give way later in the nineteenth century to a much more fraught view of the subject in relation to the ideas of reason and nature. In order to see why, we need to consider certain aspects of the anti-Idealism of Schopenhauer and Marx, before turning in more detail to Nietzsche's own texts.

\section{Schopenhauer: music as metaphysics}

The importance of Arthur Schopenhauer (1788-1860) for the work of the early Nietzsche is well established. However, it is not clear that Nietzsche's rejection of Schopenhauer in his later work means that he in fact rejected all of the elements of Schopenhauer's thought which had been central to his early texts. Schopenhauer is an interestingly symptomatic thinker in the present context, because he gives a decisive role to music in his philosophy, but at the same time adopts a largely rationalist conception of natural science. He also combines an anti-Idealist naturalism, which would only become influential, well after he published his main work in 1818, in the wake of Darwin and Wagner in the 
1850 s, with a Platonism that is the basis of his investment in art. Schopenhauer's main animus is directed against any attempt, like that of Hegel, to suggest that history can be understood teleologically, as the locus of the realisation of reason. Instead he regards history as the zoology of the species mankind. The reasons that this view leads Schopenhauer to lend such importance to art take one to the core of some of the philosophical questions raised by aesthetics in modernity. Schelling regarded art as a means of coming to terms with the cognitively inaccessible motivating forces upon which reason is founded, and this gave art an active role in the subject's self-understanding. Schopenhauer, in contrast, regards art as the only means of temporarily escaping the fundamentally futile nature of reality. Art's essential role is therefore not to enlighten us about ourselves, in order to make possible new ways of dealing with the world, but rather to enable us to escape what we already intuitively know about the irredeemable nature of what we are. This latter position will be what eventually leads Nietzsche to reject Schopenhauer, but Nietzsche's subsequent ideas retain some of the problems of what he rejects.

Like Fichte, Schelling and Hegel, Schopenhauer develops his philosophy by questioning Kant's division of the world into phenomena and noumena. He differs from Kant over the notion of the world 'in itself', to which he thinks we have direct intuitive access. The world's 'most inner essence, its kernel, the thing itself' he terms 'according to the most immediate of its manifestations: Will' (Schopenhauer 1986 I p. 67). The Will is the real ground of the world which science can only cognitively apprehend as appearance. Like Freud's id, the Will therefore cannot appear as itself and has to be accessible to us in a different manner from what can be known. What is meant by the Will can be understood via our own body: 'The parts of the body must . . completely correspond to the main desires via which the Will manifests itself, must be the visible expression of these desires: teeth, gullet and intestine are objectified hunger; the genitalia the objectified sex drive; grasping hands, swift feet correspond to the already more indirect striving of the Will which they represent' (I p. 168). This conception provides a metaphysical, rather than a biological, answer to the question why the body is constituted in the way it is. The phenomenal world is grounded in the Will, which objectifies itself in different ways in all of nature, but never appears as itself.

This conception should already be familiar. Schopenhauer relies, without admitting it, on Schelling's assumption that: 'As the object is never absolute then something per se non-objective must be posited in nature; this absolutely non-objective postulate is precisely the original productivity of nature' (Schelling I/3 p. 284). The Will, Schopenhauer claims, is apparent to us in that kind of consciousness in which each person 'recognises his own individuality [Individuum] in an essential way, immediately, without any form, even that of subject and object . . . as the knower and the known here coincide' (Schopenhauer 1986 I pp. 172-3). His claim echoes what is intended by the notion of 'intellectual intuition' that Schelling developed in relation to Fichte. 
Schelling's argument was that if an implausible idealism is to be avoided, Fichte's version of intellectual intuition, in which 'If I think an external object then the thought is different from the object, but if I think myself then subject and object are one' (cited Schelling I/6 p. 154), must extend beyond the empirical subject to a grounding identity between subjectivity and objectivity. This identity is the condition of overcoming the Kantian division between appearances and things in themselves. My thoughts of my empirical self qua object are in fact just as transient, just as much part of the realm of appearance, as those of external objects, which means they cannot grasp the 'absolute identity' required to overcome the subject-object division. Schopenhauer avoids the term 'the absolute', but his notion of the Will has the same function as the absolute in the structure of the argument. ${ }^{3}$

The key problem, then, is the status of the Will, which is only available to us in an 'intuition', in which the subject-object split is immediately overcome. Schopenhauer's Will is inherently at odds with itself: 'the Will in itself . . . is an endless striving' (Schopenhauer 1986 I p. 240). Its self-objectifications continually seek to overcome each other in order to sustain their identity, but in the case of any particular thing this identity is bound ultimately to be destroyed: the only real identity is therefore the absolute identity of the Will. The structure of this conception is again very close to structures encountered in German Idealism. Schelling says of time, for example, that it 'is itself nothing but the totality appearing in opposition to the particular life of things' (I/6 p. 220), and much the same can be said of the Will. The difference is that the destruction of the particular by the Will is not seen by Schopenhauer as enabling us to comprehend the absolute by the revelation of the necessary structure of developmental change. For Schopenhauer any transcendence of the incessant battle for self-preservation in which appearing nature consists is only temporary and does not lead to a progressive development. Each particular being owes its existence to its negation of other parts of existence, but there is no sense in which this means that the highest aspect of existence is, as it is for Kant and the Idealists, the rational, thinking subject. ${ }^{4}$ Knowledge is essentially the means for preserving the individual, so the principle of subjectivity is self-preservation, not a capacity for self-transcending reason which can lead us to a moral goal. This post-Hobbesian vision leaves open the question of why the subject should then preserve itself at all, which Hobbes had answered in terms of the self-justifying capacity for continuing self-gratification. Unable to tolerate the consequences of such a view, which just promises a life of endlessly renewed dissatisfaction, of the kind inherent in the very nature of the Will, Schopenhauer seeks a way of transcending the Will that is based on aesthetic contemplation.

Schopenhauer's conception of art derives from Kant's notion that aesthetic contemplation is a pleasure free of appropriative interest. In order to reinforce the idea that this pleasure is not based upon the continually renewed need to overcome dissatisfaction, Schopenhauer combines Kant's notion with a Platonist metaphysics. Both the thing in itself and the Platonic Idea testify for 
Schopenhauer to the limitations of the time-bound phenomenal world. We can only transcend these limitations by separating our cognition from its motivation by the Will. To do this one must lose the sense of one's individual, Will-determined existence, and lose oneself in contemplation of the object, becoming a 'pure, will-less, painless, timeless subject of cognition' (Schopenhauer 1986 I p. 257). This idea has an important consequence for his account of the subject, and it is here that the real source of Schopenhauer's link between pessimism and aesthetics becomes apparent. Only by losing ourselves as individual, sensuously receptive subjects can we achieve a state which is not dominated by the transient world of pain and pleasure. Aesthetic contemplation dissolves the opposition of subject and object, not in order to give the intelligible subject the ultimate role in the constitution of the truth, as is arguably the case in Fichte and Hegel, but rather to overcome the individuated, desiring subject. The artistic genius is therefore characterised by a capacity for objectivity which allows him (in Schopenhauer one can advisedly say 'him') to apprehend the essence of things, their Idea, without being distracted by any relation to the object generated by passing wishes or desires. ${ }^{5}$

For Schopenhauer the art object's aesthetic status therefore primarily derives from its universality. His aesthetic theory becomes an inverted Platonism. Whereas, in the Republic at least, Plato attacks art for only representing the single object, not the Idea, Schopenhauer maintains that art is the only locus in which the Idea can be represented. The higher truth becomes attainable by eliminating the illusion that the subject could relate to a world which means something essential to it qua sensuous subject. This elimination requires a relationship to things of disinterested contemplation which takes one beyond the temporal world. Schopenhauer therefore does not regard the historical development of the subject as part of a larger, philosophically intelligible story. He 'cannot help seeing the same thing in all history, like in a kaleidoscope one always sees at every turn the same things in other configurations' ( $\mathrm{V}$ p. 526). As such, there are clear metaphysical answers to the questions of human existence: finite human existence is ultimately vain because of its basis in the Will. In consequence, art, at least temporarily, makes insight into the essential nature of our existence tolerable.

Schopenhauer attempts to demonstrate the metaphysical truth of art in his account of music, giving music the kind of privileged philosophical role it had at times for the early Romantics. As we saw in Hegel, the idea of the truth of art is threatened in modernity by new kinds of legitimation in the scientific, legal and political spheres, which rely on excluding particularity from the realm of the highest truth. Schopenhauer, though, sees the highest truth as still located in art, not in science, law or philosophy. Despite his Platonic (and Buddhist) antagonism to the Will-driven sensuous subject, Schopenhauer regards the 'intuition' present in art as superior to concepts. 'Intuition' is contemplation which apprehends the object without first seeing it in terms of defining concepts. Schopenhauer explains intuition further via the example of literary meta- 
phors, similes, parables and allegories. Although poetry employs concepts, these are not what make something poetry. Poetry results instead from the manner in which the concepts are made into non-discursive images. The argument is similar to one we encountered in Schleiermacher. A poem for Schopenhauer achieves its sense of completeness via its rhythm and rhyme. It thereby becomes a 'sort of music' and appears to be there for its own sake, not 'as a simple means, as the sign of a signified [Zeichen eines Bezeichneten], namely the sense of the words' (II p. 550). Once this priority has been established, the meaning of the words simply appears as 'an unexpected bonus, like words to music' (II p. 550).

It should now be easier to understand Schopenhauer's investment in what he himself terms the 'metaphysics of music'. Music is the crucial component in a metaphysical conception of aesthetic autonomy. The reason for Schopenhauer's elevation of music is that, unlike other forms of art, music is non-representational and thus is least bound to the world of appearance that can be grasped in concepts. Music has the status of the 'true general language': it 'does not talk of things, but rather of nothing but well-being and woe, which are the sole realities for the Will' (V p. 507). Words take one into the realm of concepts and abstractions; absolute music, on the other hand, combines direct access to the world of feelings with a basis in the pure forms of mathematics. It is this combination which is so powerful, as we saw in Chapter 7, because it seems to bring together the two most distant and opposed aspects of modernity, the individual feeling subject, and the condition of possibility of the modern sciences.

The Will is 'divided in itself'. This fact is most obviously manifested in us through desire, which entails an inherent incompleteness and a need on the part of that which desires to move beyond itself. In Hegel desire is only an early, undeveloped stage in the subject's self-constitution. The culmination of this self-constitution takes place when the subject becomes capable of an intersubjective recognition of the other that transcends the subject's desire to consume or destroy the other. Because music expresses mere subjective inwardness, it fails, like the primitive manifestations of desire, to engage with the other in a developed manner. For Schopenhauer, in contrast, music is the source of the deepest insight into the nature of reality because it speaks directly of desire. Music is, though, grounded at the same time in 'completely determinate rules which are expressed in mathematics, which it cannot deviate from without completely ceasing to be music' (I p. 358). It therefore crosses the Kantian divide between the sensuous and the intelligible, much as Hamann claimed that language does. The mathematical proportions in music are sensuously heard as intelligible connections. If the 'maths' is wrong because of the use of a note that does not take adequate account of the proportions on which the intervals are based, the music also sounds wrong. The point is that one does not have to knom explicitly the mathematical way of expressing dissonance to be able to hear it, because we hear dissonance primarily via the way in which it impinges on our feelings. Music 'directly affects the Will, i.e. the feelings, passions and emotions of the hearer' (II p. 574). The language of music is universal in the way that 
geometrical figures, the a priori forms of intuition, are universal: 'the world of appearances or nature, and music' (I p. 365) are, therefore, two different expressions of what is ultimately the same.

Schopenhauer goes so far as to maintain that, because music is the direct image (Abbild) of the Will, it is 'the metaphysical to everything physical in the world, the thing in itself to every appearance. One could accordingly just as well call the world embodied music as embodied Will' (I p. 366). Like the early Schlegel, he therefore thinks music and philosophy are inseparable from each other. If one succeeded in giving a complete conceptual explanation of music one would have 'the true philosophy' (I p. 369), in which sensuous and intelligible are united. The point is, of course, that such an explanation is impossible in purely discursive terms. What is to be explained matters precisely because it articulates what words cannot. Music is thus regarded as an indication of the limits of philosophy. This, however, leads to an instructive problem.

If what music says is inaccessible to discursive language, assertions about music's philosophical significance cannot claim to be true, and the question is what status they therefore have. One productive way of approaching this problem is to acknowledge that music can show what cannot be said, so that any attempt to say what music shows will inherently involve Romantic irony. This irony entails, as we saw, the negation of any assertion, but not in favour of a determinate contrary assertion, because all determinate assertions are relative to other assertions. No assertion could be definitive, unless one were able finally to grasp the totality of interlinked assertions. Our finitude renders this impossible, so irony is the inevitable result of attempts to articulate the highest things. This conception led the early Romantics to the idea that it is only via a constant interplay between different forms of articulation that we can come to terms with the limitations of each form. However, Schopenhauer does not deal with the question of music and philosophy in this manner. The way he does tell us something important about the relationship between conceptions of philosophical aesthetics and cultural politics.

Schopenhauer's claim is that music best represents the unconscious forces which are the productive ground, not just of our representations, but also of the rest of the world: 'the composer reveals the innermost essence of the world and pronounces the deepest wisdom, in a language which his reason does not understand' (I p. 363). The key here is the notion of rationality, which Schopenhauer simply equates with what is conceptually determinable, thereby rendering music extra-rational, and creating a divide between reason and its other. The difficulty is that claims about this other rely upon there being a clear division between it and reason, but the division cannot be established in rational terms. In consequence, any claims must rely on 'intuition'. This creates a rigid division between the rational/conceptual, and the irrational/intuitive. What is required can only be directly experienced, not established via a theoretical claim about intuition. The claim must therefore be resistant to any discursive articulation at all. This is not only a questionable position in epistemological terms, but also 
excludes the possibility that music, rather than offering an escape from reality, might actually be able to contribute to a notion of reason which helps us come to better terms with reality by revealing it and constituting it in new ways.

For Schopenhauer music, $q u a$ aesthetic experience, temporarily redeems one from the fundamental suffering in which life consists, but it does so whilst expressing precisely what makes life a torment. In Odo Marquard's terms, aesthetics moves towards 'anaesthetics'. Music, as The Birth of Tragedy will argue, thus plays a role analogous to tragedy, which presents the worst human events in the form of aesthetic appearance whose purpose is to render the unbearable bearable. The problem with this position becomes apparent in the fact that Schopenhauer argues wholly within the specific Western musical tradition which develops with Viennese classicism: the resolution of tension within sonata form is the best example of the sort of music he is referring to. What he says - and something similar will apply to Nietzsche's early view of Wagner is therefore predicated upon the development of specifically modern music. The music in question moves away from the more static contrapuntal music of the past and opens up the new harmonically-based dynamism and possibilities for subjective expression encountered in Beethoven. How, in that case, is it that such historically developed music expresses a metaphysical truth which transcends history?

Schopenhauer does state that his argument about the metaphysical status of music cannot be proven, 'because it assumes and establishes a relationship of music as a representation [Vorstellung] to that which essentially never can be a representation' (I p. 358). He argues that the basis of what we term reason is the drive for self-preservation, and for the propagation of the species. Selfpreservation is the 'basic endeavour of the Will in all its appearances' (II p. 386). Such claims, as we shall see again in Nietzsche, are faced with the problem that what they seek to establish undermines the very possibility of establishing anything determinate. Discursive argument must, for Schopenhauer, always be motivated by the Will, as the intuitively accessible ground of all appearances, including, of course, the appearance of the argument itself. The ground of the argument is therefore self-preservation, but this means we have no reason to accept its truth, and should, even in his own terms, seek instead to establish why Schopenhauer thinks the argument contributes to self-preservation. What would enable us to move from Schopenhauer's claims to a valid account of a metaphysical principle like the Will? The answer is that nothing could, except an act of intuitive faith which could never be cashed in as a discursive claim. Schopenhauer is here clearly involved in a performative contradiction. This cannot be circumvented in the manner Nietzsche sometimes achieves, because Schopenhauer claims to be telling us the ultimate truth about things, while at the same time saying that this cannot be done. Freud is faced, as we have seen, with a structurally similar problem with regard to the grounding role of the id. He echoes Schopenhauer's view when he insists that a 'drive can never be an object of consciousness, only the idea $[$ Vorstellung] that represents it'. Freud 
then has the problem of how to show that the representation is of a drive at all. The difficulty this involves is evident in the way that his accounts rely heavily on metaphorical resources that preclude a definitive statement of the theoretical point. The shared structure of the problem here is in fact a classic problem for metaphysics. How is one discursively to articulate a non-discursive condition of possibility of discursivity?

These arguments might seem pretty conclusive, but it is clear from the influence of Schopenhauer, not least upon Wagner, Nietzsche and Freud, that he articulates something vital in the experience of modernity. The limits of Schopenhauer's ability to make a philosophical case for his metaphysical proposals are pretty evident, but one of the key features of the aesthetic is the way in which it keeps open the idea of the 'unsayable'. In this respect, even though Schopenhauer's account falsely ontologises the significance of music, its insistence on the combination of music's non-representational character with music's ambivalent relationship to conceptuality can begin to suggest why music may be more apt than other forms of art to the modern experience of the I that is not, in Freud's phrase, 'lord in its own house'. Furthermore, music makes limitations in discursive thought apparent in ways which, as Schlegel and Schleiermacher showed, can then be translated into the claim that other forms of art cannot be understood in merely discursive terms. The links of music to the theoretical articulation of the notion of aesthetic autonomy are crucial here, but Schopenhauer himself does not deal adequately with this theme.

His problem is the absolute status which art must have if his argument is to work. This status is most easily conferred on music because music is least connected to empirical representation, to the relative world of transient objects. The argument is, though, suspiciously circular. Schopenhauer's claim is that art reveals the essential truth about existence. The claim relies, however, on an essence of art with which one must already be familiar if one is to apprehend something as an object of aesthetic contemplation at all. The essence of art consists in its making possible a temporary escape from subordination to the Will. How, then, do those who cannot attain denial of the Will gain access to this essence? Schoenberg criticises 'Schopenhauer's demand that the evaluation of works of art can only be based on authority. Unfortunately he does not say who bestows authority nor how one can acquire it' (Schoenberg 1975 p. 136). Even beyond the questionable circularity of Schopenhauer's argument, there is something arid about its implications. It leads to a monolithic notion of art as the source of the temporary negation of contingent subjectivity, and so omits any sense of the complex developments in the history of subjectivity that are required for modern notions and forms of art to emerge in the first place. A metaphysics of art of this kind ultimately reduces all art to the same significance. By sustaining a sphere of complete philosophical autonomy for art Schopenhauer displaces it from the role of actively enlightening us about the nature and limits of our capacity for cognitive rationality. The intensity of Schopenhauer's antipathy to anything like a theological consolation for the nature of existence means that his 
alternative has to be equally radical in the other direction. However, the result of this is a debasement of the finite, feeling subject which could actually be shared by certain kinds of theological conception.

Many of the theories we have examined so far have been concerned with the idea of consciousness' ground in an other which is not accessible to conceptual thought. This concern has often led the theories to be equated as all equally part of the history of modern irrationalism. It is therefore important to differentiate responses to the idea of the limits of conceptual thought. If the ground of consciousness becomes, as it does for Schopenhauer, an ontological principle, the Will, there is little more to be said about it, apart from revealing that it is what is at work in all areas of life which might have previously been regarded as being based on reason, altruism, individual creativity, and so on. The resulting pessimism about the subject's essential nature has, though, been generated by reflection upon what motivates what we call reason. The problem with Schopenhauer's account of music as the most effective means of access to the motivational ground is that he tends to reduce music to one significance which, despite all claims to the contrary, he then articulates in the general language of theory. Schoenberg says of Schopenhauer:

he loses himself ... when he tries to translate details of this language which the reason does not understand into our terms. It must, however, be clear to him that in this translation into the terms of human language, which is abstraction, reduction to the recognizable, the essential, the language of the world, which ought perhaps to remain incomprehensible and only perceptible, is lost. (Schoenberg 1975 p. 141)

Whether music is really better regarded as 'incomprehensible' is itself questionable. If music reveals the limits of what can be said, it is not therefore per se incomprehensible, as mere random noise might be. Music must be understood to reveal the limits of the sayable, even it generally cannot be said to mean anything semantically determinate. In contrast to Schopenhauer, the Romantic views encountered in earlier chapters avoided the kind of link between music and philosophy in which music becomes merely the means of sustaining an otherwise unwarrantable philosophical thesis. The Romantic conception can thus incorporate both the new autonomy which makes music into a greater resource for exploring the unsayable, and the manner in which music is connected to other ways of interpreting and articulating the world.

Schopenhauer is, then, on the one hand oriented towards the future, by his anti-metaphysical rejection of a redemptive view of both internal and external nature, and the past, because his aesthetics becomes subordinated to a static Platonic metaphysics. For aesthetic thinkers after Schopenhauer the task can be characterised in terms of Peter Dews's phrase, the 'limits of disenchantment' (see Dews 1995). If Platonising options are rejected, how can a conception of art be developed which comes to terms with modern, non-redemptive temporality, without eventually undermining the notion of art altogether? It is Nietzsche who probably radicalises the question of the significance of art and 
beauty more than anyone in the nineteenth century, and the extent to which he may invalidate some of the Romantic ideas we have examined becomes a vital question. In order better to understand Nietzsche's radicalism we need, though, first to look at a famous example of Marx's engagement with art.

\section{Marx, mythology and art}

Some of the main questions that set the agenda for aesthetic theory in the twentieth century appear in a passage from Marx's Introduction to the Grundrisse, written in 1857, in which Marx ponders the relationship of art to the general development of society (Marx 1974 pp. 30-1. All quotations from Marx are from this passage). Why is it that societies, like ancient Greece, whose capacity for controlling nature is not highly developed, can produce great works of art? The way this question highlights aesthetic concerns in the second half of the nineteenth century is evident in the fact that Nietzsche will ask closely related questions about Greek art in The Birth of Tragedy (BT) (1872), linking them to music. Echoing Idealist and Romantic philosophy, Marx sees Greek art as based on mythology, which he characterises, in the manner of the later Schelling, as a collective 'unconsciously artistic processing [Verarbeitung] of nature'. Mythology makes sense of natural forces via the imagination, by telling stories about them and making them into repeatable images. These images give a feeling of control over what is otherwise alien. What, though, happens to art, whose basis is the mythological overcoming of the forces of nature in the imagination, when humankind can really control natural forces through technology? Marx asks: 'Is the contemplation [Anschaunng] of nature and of social relations which is the basis of Greek fantasy and thus of Greek mythology possible with self-actors [i.e. automatic machinery] and railways and locomotives and electric telegraphs?' Modern art requires the artist to have a 'fantasy independent of mythology', because modern science 'excludes every mythological relationship to nature', but what space does that really leave for art? The questions Marx is asking here are, of course, a more praxis-oriented version of what Hegel argues in the Aesthetics about the role of art in modernity, where the essential truth is located in the sciences and in other universalising modes of thinking.

The new investment in the non-representational and non-conceptual medium of music in the nineteenth century becomes easier to understand in the light of Marx's assumptions about the need for a 'fantasy independent of mythology'. The rise of the idea of music as the highest form of art, which is part of what makes possible the emergence of aesthetic autonomy, is also an indication of a coming crisis in art that is clearly connected to the growing success of the sciences. Mythology is tied to the particular, and this diminishes its capacity to illuminate the world in the more general terms demanded by modernity. For art to be sustained as a source of truth and meaning, fantasy must therefore employ forms of articulation which establish an independence from the demands involved in the sciences, at the same time as not losing the 
immediate particularity essential to art. Fantasy may, as a consequence, move away from the attempt to represent the world in images and stories towards the attempt to say what other ways of articulating the world cannot. This move leads not least to a new kind of relationship to the irredeemable aspects of human temporality and transience, as the changed relationship between music and tragedy in the nineteenth century that is the theme of The Birth of Tragedy suggests. Human transience combines a profoundly individual moment, relating to the modern subject's feelings in relation to its fragility, with a universal sense of the new temporal character of a post-redemptive world. Marx's comments highlight one side of the changes in the relationship between subject and object which occur in modernity when nature comes to be seen more and more in terms of instrumental reason's capacity to control it. The increase in the ability to control nature should, in his terms, lead to the disappearance of myth. However, Marx underestimates those dimensions of the modern subject, suggested by the changes in existential time just referred to, which are not provided for by such control. The importance of the early Nietzsche lies in his attention to the reasons why the increased ability to control nature fails to achieve a new integration of humanity and nature of the kind which the later Kant, and the post-Kantian Idealists and Romantics had hoped for. ${ }^{6}$

One manifestation of the issue Marx discusses becomes apparent in the art of the second half of the nineteenth century. Zola, for instance, sees his novels as actually having quasi-scientific status. However, he will, in La bête humaine, make a locomotive into a mythical object, giving it a status equivalent to anthropomorphic nature in mythology. His novels also combine an attention to the most dominant technological and commercial aspects of modernity with a remythologising of nature itself, for example, in La terre. Zola's mythologising of 'second nature', human society and its products, recurs throughout the Rougon-Macquart cycle, such as in the links of the mine to Tartarus in Germinal. His work exemplifies the tendency of nineteenth-century art to adopt mythical patterns as a way of sustaining itself in the face of a society whose essential processes are no longer visible in the actions of individuals (see Adorno 1974, Bowie 1979). What gave rise to the Idealist demand for a 'new mythology' which would make abstractions sensuously available to society as a whole is evident in the way that the art of Zola's period tends to invoke old mythologies as a way of trying to come to terms with modern society. The importance of such art is not least as an indication of the difficulties involved in Marx's analysis.

This re-emergence of mythology can be understood in terms of Max Weber's suspicion that the competing value-positions of modern societies might well end up looking to many people like the multiplicity of competing natural powers represented in mythology in traditional societies. In this interpretation art may, as Zola's work suggests, attempt to organise into images and stories those aspects of modern social life whose functioning seems like the workings of nature do to societies lacking the real means to control it. The risk is, of course, 
that what are actually soluble problems of human organisation therefore come to be seen as natural fate. ${ }^{7}$ The Introduction to the Grundrisse itself does not offer any plausible explanation of why it might be that in modern societies neither art nor mythology cease to play a vital role in people's lives, despite those societies' increasing control over nature. Indeed, in the case of Wagner's Ring, mythology, in conjunction with music, will form a new, compelling, but highly problematic, response to capitalist modernity. In Wagner, as Marx's argument suggests would be the case, the mythology on its own is no longer believable. However, the power of the myths is transferred into the music in a manner which is a key indication of the inadequacy of accounts of myth and modernity like that of Marx. The persistence of Wagner's capacity to exercise this power, even despite the fact that his work becomes linked to the most evil political movement in modern history, is an essential factor in the attempt to understand modern culture.

Marx also reflects on Greek art in specifically aesthetic manner. Although it is not surprising that Greek art and epic have their foundations in 'certain forms of social development', the 'difficulty is that they still give us aesthetic pleasure and in certain respects are valid as a standard and unattainable model'. This claim is clearly in tension with his developmental view of history as the process of elimination of imaginary control over nature in favour of real control, suggesting that the relationship between the mythical and the aesthetic is more complex than he assumes. It is precisely this complexity which will become evident in the early Nietzsche's work on music and mythology. Marx tries to overcome the problem of Greek art's continuing generation of aesthetic pleasure by suggesting that the Greeks are the children of the human race. They possess a naivety that is now inaccessible to us, but which we value in children and try to 'reproduce on a higher plane'. The charm of Greek art lies in the fact that its basis - 'unripe social conditions' - can, like childhood, never return. This exercise in nostalgia is, as has often been noted, inadequate to account for the perception, throughout Marx's century and beyond, that Greek art has an enduring power which is not diminished by the development of modernity.

At the same time, the attempt to suggest against Marx that Greek art is 'eternal' in its appeal merely regresses to a pre-modern position. What grounds are there for making such a claim, which requires an appeal to a contingent future for its validity? Similarly, the related idea that Greek art's appeal results from the universality of its themes can be used to argue for the immortalisation of 'soap operas', which also deal with families and power. The real question here is not answered by humanist generalisations, and Marx is at least aware of part of the difficulty. His initial puzzlement at the continuing power of Greek tragedy is in many ways an appropriate response to this phenomenon. Greek tragedies' capacity for generating new meaning in differing social contexts can never be definitively explained, and that is what makes them so aesthetically significant. ${ }^{8}$ Marx's problem lies, therefore, in his failure to distinguish the function of myth in a traditional culture from the significances myth can help to gen- 
erate in a modern art work. His direct contributions to aesthetic theory are not in fact that substantial, drawing mainly upon existing views from German Idealism and Romanticism. The real potential of his work for aesthetics can be derived rather from the ways he offers of understanding the conditions and possibilities of artistic production in relation to a general theory of social labour. Little that he himself says is, though, able to account for the specific power of aesthetic products, which he in many respects simply presupposes. This presupposition will become increasingly hard to make in relation to aesthetic modernism, where the very status of 'art' is put into question.

In the light of the problems in Marx's position it seems questionable to assert, as Terry Eagleton and others do, that a properly Marxist aesthetics should be regarded primarily as part of a theory of ideology, or that philosophical aesthetics itself is just part of the ideology of the emancipated bourgeoisie. One of the reasons music is so significant in modernity is precisely because it shows the limits of the notion of ideology when it is applied to art. While music can clearly be employed to reinforce dominant cultural and political assumptions, it need not just be reducible to these assumptions, in the same way as subjects need not be reducible to the ideological assumptions and practices they both reflectively and unreflectively adopt. The most insightful Marx-oriented aesthetic theory, of Bakhtin, Lukács, Bloch, Benjamin and Adorno, which emerges after Marx in the twentieth century, by no means adopts the view of art as inherently ideological, and is often concerned instead with the resistance of art to ideological appropriation, a resistance which music exemplifies in key respects.

The decisive aspect of Marx's thought in subsequent Marxist aesthetic theory is actually the theory of the commodity, and many of the most significant developments of this theory rely on structures of thought derived from German Idealism. In the Foundation of the Metaphysics of Morals Kant had stressed the importance of that which has no price in his concept of 'dignity'. He did so as a way of claiming that rational beings possessed a value which transcended the form of value which was now dominating his world. The commodity is defined precisely by its price, which takes priority over the value of the thing as an individual use-value: the amount of money the thing is worth makes it in one sense equivalent to any other thing worth that sum. The development of modernity is not least a result of the commodity form offering a means of exchanging anything for anything else. This possibility speeds social and material interchange and facilitates social innovations. Critiques of modernity often concentrate on the cultural consequences of this development, suggesting that it destroys the intrinsic value, the 'dignity', of things. This criticism arises from a structure of thought that is germane to nearly all the post-Kantian thinkers. Commodity form is in fact a form of 'reflection', in the sense we explored in Idealism: what an object is qua commodity becomes wholly defined by its negative relationship to other objects within a differential system. Marx regards this system as a kind of metaphysics, that obscures the reality of things in the name of an abstraction. 
Similar ideas will later be advanced in Adorno's view of the commodification of culture and in Heidegger's ideas concerning metaphysics as the subjectification of being.

The difficulty for such positions is that it is no longer clear in a post-theological world whether there $i$ s any intrinsic value that does not derive from a thing's relations to something else. Kant argues that rational beings are 'ends in themselves', so that they cannot be merely the means to others' ends, but it is arguable that in any society one is inescapably likely - and often willing - to be a means for others' ends in many of one's roles. If Kant fails to show that rational beings have access, via their capacity for self-determination, to an intelligible realm of freedom which is not subject to the laws of the appearing world, the notion of the absolute intrinsic value of the rational being becomes open to question. The structure of this problem is another version of the clash between immediacy, this time in the form of non-relational value, and mediation, which renders all values relational.

In the aesthetic theory that follows Kant the ideas of the irreducible particularity of the object and of the subject's disinterested relationship to the artwork are often invoked as a counter to the idea that the modern world is constituted wholly in terms of conceptual, economic and linguistic 'mediation'. However, theoretical claims involving immediacy inherently give rise to the problem of mediating what is supposed in one crucial respect to be immediate. The difficulty lies in the need to appeal to an immediate ground which is only intuitively accessible. At the same time, this kind of immediacy seems in some respect to be germane to the very possibility of art. If art cannot reveal things in ways which cannot be discursively mediated it can be dissolved into other forms of discourse. It may be, though, that one can only appeal to art's status in this respect as a kind of regulative idea. This idea generates the ongoing social demand to legitimate the claims of the artwork, even though there can never be a decisive way of doing so, of the kind which may be possible for claims in the sciences. Without such a conception art becomes merely, as Hegel's position might be seen as implying, a form of mythological expression which is no longer essential to the truths that determine modernity. The ensuing question here is whether art's problematic status is therefore to be understood as an index of a fundamental crisis in modern culture that is occasioned by the dominance of the commodity form. Adorno's insistence on aesthetic autonomy derives from his conviction that the dominance of the exchange principle is so universal that only something which is able to counter that principle can offer insights into an otherwise deluded reality. Unsurprisingly, a stance of this kind, which seeks to base a Marxist account of aesthetics on the autonomy of the artwork, is still highly controversial.

The most significant Marxist aesthetic theory emerges from the tensions between modern art's autonomy and its status as 'fait social'. The suspicion that the sustaining of aesthetic autonomy will lead to art's loss of any real sociopolitical role gives support to conceptions of art which regard it as a means 
towards achieving pressing extra-aesthetic, historico-political tasks. However, in contrast to the demand for engagement, autonomous art can be regarded as the location in which otherwise unarticulated possibilities for the transformation of human existence may be preserved, including possibilities which previously received their expression in mythology. These tensions closely echo those between the beautiful and the sublime, between the new mythology and aesthetic autonomy. In the twentieth century what is at issue in these tensions leads to debates which cost lives and determine cultural politics in often very destructive ways. It is Nietzsche's radicalisation of questions concerning modern culture which begin to suggest why this is the case.

In contrast to Hegel, Nietzsche ceases to regard philosophy's continuous selfundermining, which is so characteristic of modernity, as constituting a progression that incorporates the refuted views of the world into a higher synthesis. The destruction of a metaphysically grounded notion of history tends to work in two key directions, seen in Marx and Nietzsche. Marx aims at a historical realisation of the aims of philosophy which would make it superfluous, as mythology is supposedly made superfluous by insight into its origins in an underdeveloped capacity to control natural forces by technology. He is therefore one of the first to talk of the 'end of philosophy', in the sense of the abolition of the need for philosophy by the achievement in practice of the goals that Idealism formulated in theory. Nietzsche, in contrast, questions the legitimation of any higher collective historical goals on the basis of a Schopenhauer-influenced interpretation of the link between subjectivity and its motive force, which he terms the 'will to power'. Much of Nietzsche's most important work originates, then, in an attempt to transform the philosophies we have considered so far by revealing their failure to overcome the illusions of the metaphysical past. Music plays a vital role in this attempt, for reasons we shall now consider.

\section{Art, myth and music in 'The Birth of Tragedy'}

Nietzsche's first major work, The Birth of Tragedy From the Spirit of Music (BT), published in 1872, addresses the relationship between mythology, art and science examined in the Introduction to the Grundrisse. The $B T$ can be read as a manifestation of what happens to the ideas on art and mythology encountered in the System Programme (SP) and Friedrich Schlegel's Discourse on Mythology in the wake of Schopenhauer's philosophy and the growing separation, both in practice and in theory, of aesthetic, moral and scientific concerns in the second half of the nineteenth century. It is important to establish just how much the $B T$ derives from the traditions we have considered so far, in order to be able to assess whether Nietzsche's later attempted break with these traditions broaches wholly new ground.

In the $S P$ art was regarded as the means by which freedom could be communicated to all strata of society by sensuous means, rather than as an abstract idea. The teleology of human action and the teleology of nature were linked in art, 
so that abstract ideas became available through perceptible images. The $S P$ concluded with the demand for a 'polytheism of the imagination and of art', and a 'mythology of reason', which would integrate into the whole of society the new potential released by science, art and critical philosophy, in the way that myths integrated nature and society in traditional cultures. However, the Discourse on Mythology already began to question whether what was required was just a 'mythology of reason'. Schlegel's stress on the productive potential of the 'original chaos of human nature' suggested that the dominant conceptions of reason might also be an obstacle to what was needed in a post-theological modernity. The early Idealist and Romantic conceptions were often linked to the revival of the idea of Dionysus, the God who combines creation with destruction, who is the 'other of himself'. Dionysus can be understood in two differing ways. Whereas Hegel thought it possible to adopt what was inherent in the Dionysian identity of opposites for a teleological conception of unified reason which emerges from the division embodied in Dionysus, Schopenhauer's Will, which is also divided against itself, was conceived of in a non-teleological manner and was, of course, linked to non-representational music. It should therefore be no surprise that Nietzsche presents Schopenhauer's Will as Dionysus in the $B T$, or that he also links Dionysus to music.

Nietzsche belongs here to an established Romantic tradition. As we saw, the Romantic idea of 'infinite reflection' in art was associated with music because of its freedom from representational determinacy. The connection of Dionysus to music encountered in the $B T$ is already present in Schelling's Die Weltalter (Ages of the World $(A W)$ ) of 1811. In the $A W$ Schelling attempts to show how consciousness arises out of unconscious nature. The story is not far from the model of consciousness which leads Freud to the dictum that 'where It $[E s]$ was, $\mathrm{I}[\mathrm{Ich}]$ should become'. Schelling suggests the emergence of rational thought is far more of a struggle than it was in the $S T I$ or his identity philosophy, and uses music as a means of understanding it. 'Divine madness', the state associated with Dionysian intoxication, is a result of the battle between that aspect of nature whose essence is to remain part of the inherently unconscious primeval One - which is a force of contraction, in contrast to the absolute ' $I$ ' of the STI, which had to limit its infinite expansion if it was to become conscious of itself - and that aspect which strives beyond the One towards consciousness, which is a force of expansion:

As long as the contracting force maintains a predominance over the expanding force it is stimulated on the inside in a still dull manner into a blind unconscious activity [Wirken] by the beginning battle; mighty, violent ... products arise, like those which arise from the play of forces in dreams when the reasonable soul does not intervene and the forces work for themselves. (Schelling 1946 p. 43)

The development of consciousness consists in the painful liberation of a potential which is always threatened by the force of contraction and which relies on that force to be able to become something determinate. 
Schelling goes on to suggest that music expresses the conflict between consciousness and its ground. Dionysus's wagon, which is pulled by wild animals, is accompanied by music: 'For, because sound and tone only seem to arise in ... that battle between spirituality and physicality, only music can be an image of that primal nature and its movement, for also its whole essence consists in circulation, as it, beginning from a tonic [Grundton], always finally returns to the beginning, however many variations it may go through' (p. 40). Music manifests the ground of diversity in unity by its combination of the need for differentiation with a basis in unity: the note which is constituted by vibration between the presence and absence of the vibrating body is a unification of these opposites. This unity is not, though, the kind we saw in Hegel's account of music: rather than being an image of teleological time it is an image of the circular time of mythology. In a later version of the $A W$ music is talked about in terms which parallel Schopenhauer's view of the Will and music. The relationship between the notes in a piece of music is, Schelling claims, like the 'original movement' of attraction and repulsion characteristic of nature's chaotic preconscious productivity. Schelling's overall concern, unlike Schopenhauer's, is still with how reason and freedom can emerge from chaos. In the later Philosophy of Revelation (1841-2), where Schelling will give an account which makes reason's relationship to brute facticity even more problematic, he still attempts to sustain a conception of reason. In the $B T$, on the other hand, Nietzsche, much in the manner of Schopenhauer, uses almost exactly the same scheme as is apparent in the $A W$ to argue that 'reason' is merely a human invention which we impose on a primal chaos.

It is important to make a key distinction with regard to such claims. To say that reason is 'merely human' can mean at least two different things. The claim is consistent with positions which cannot accept the metaphysical realist idea that there is a ready-made world whose truth exists wholly independently of anything we say or think about it. The problem with this position is that it inherently leads to scepticism, because nothing that we say could ever confirm that what we say corresponds to the world as it is independently of what we say. In one sense, positions which think of truth in terms other than correspondence might be said to regard truth as an invention of human beings. This is unexceptionable in so far as it need have little or no effect on the contingency of what is true, which is still independent of what anyone wishes to be or consciously tries to make true, without this meaning that the idea of the world of the metaphysical realist is therefore intelligible. The crucial difference between this position and the other kind of theories of truth as the merely human is that in the latter theories a further assumption is made, namely that what truth really is can be stated in a theoretical claim about the nature of the illusion in which truth has until now consisted. Instead of correspondence, truth then becomes, for example, that which best enables an organism to survive which has developed with certain kinds of needs.

At this point one is justified in making the objection that the claim involves 
the performative contradiction we observed above. The result is as follows: Evidence against the idea that truth is really just generated by $x$ cannot be cited because it will, in the terms of this theory, necessarily be a result of a mistaken view of what gives rise to such evidence, which must always be $x$ itself. The same problem arises in psychoanalysis, in relation to the question of 'resistance' to the analyst's claims about what the analysand is repressing. In both cases it may actually be true that counterclaims against the theory, or the analyst, are based on self-deception. However, given that the argument about truth as self-deception may itself also be false, any account of truth necessarily entails a dimension which transcends this argument and thus renders the argument impossible to propose without contradiction. Nietzsche is often anything but clear about the difference between these two kinds of theories, using the empirical fact that large amounts of communication may indeed be rhetorical, strategic and selfdeceiving to make transcendental claims based on that contingent empirical fact. The underlying issue here is the one we have repeatedly encountered, namely the relationship between the 'mediated' world of knowledge and the ground of that world, and this is the theme of the $B T$.

In his own later self-criticism Nietzsche says of the $B T$ : 'I sought laboriously to express strange and new evaluations with Kantian and Schopenhauerian formulations' (Nietzsche 19801 p. 19). The novelty of the book consisted, he asserts, in the way that it set itself the task of 'seeing science from the viempoint of the artist, but art from the viempoint of life' $(1 \mathrm{p}$. 14). Much of the force of the book lies in its account of the limits of natural science as a means of rendering the world meaningful. By this stage of the nineteenth century, in which the positivist belief in science as the only locus of reliable truth is increasingly the norm, this is a provocative position. However, it should be clear from Schelling's and the early Romantics' views of the limits of the understanding and the need to integrate its products into a 'new mythology' that this cannot really constitute the novelty of the $B T$. The $B T$ 's novelty actually lies more in its separation of aesthetics and morality, developing Schlegel's hints which we examined in Chapter 2. The provocative nature of the $B T$ for classical scholars of the time (and since) lay in its contention that the art which was the source of the ideals of a classical education, the ideals of the good, the true and the beautiful, arose out of an insuperable violent and meaningless division within being. ${ }^{9}$ Because the ground of being is so terrible it can only be justified to us in the form of beautiful Schein ('appearance', but also 'illusion'). As such, art cannot be an indication of a potentially new organic relationship to nature, and is independent of an ethics oriented in a Kantian manner towards the ideas of reason. The terrible ground of being, Dionysus, is above all revealed in Rausch, intoxication, loss of self, as well as in music. The redeeming realm of appearance, which includes dreams as well as mythology, science and plastic and literary art, is Apollo. At the same time great art, initially in the form of Greek tragedy, requires both Dionysus and Apollo.

Once again, Schelling, whom Nietzsche almost certainly read (see Frank 
1988 pp. 55-7), anticipates Nietzsche: 'Not at different moments but at the same moment to be simultaneously drunk and sober is the secret of true poetry [Poesie]. This distinguishes the Apollonian enthusiasm from the simply Dionysian enthusiasm' (Schelling II/4 p. 25). In the manner of Schelling's Philosophy of Art, Nietzsche regards Apollo and Dionysus as being Greek culture's sensuous expression of what we now express in abstract concepts. Nietzsche himself converts Schopenhauer's metaphysics of art into a story about Greek mythology and its relation to modern culture. The essential difference of the $B T$ from Schopenhauer is that, instead of relating aesthetic contemplation to a 'buddhistic negation of the Will' (Nietzsche 19801 p. 56), it regards art as 'the completion and culmination of existence which tempts one into living on' (1 p. 36). Art is already, then, as he will later put it, a 'stimulus to life'. Nietzsche derives important parts of his argument, via Schopenhauer, from Fichte's and the early Schelling's metaphysics. For the latter the world of objects is the manifestation of the absolute I's attempt to 'intuit' itself, and the result in Nietzsche is a similar view of art to that of the STI: 'In the Greeks the "Will" wanted to intuit itself [sich anschauen] in the transfiguration of genius and the world of art' (1 pp. 36-7).

This 'intuition' excludes, as it did for Schopenhauer, any involvement of the individual subject: 'we demand in every type and every level of art above all and first of all the conquering of the subjective, redemption from the "I"' (1 p. 43). The artist's aesthetic contemplation must be devoid of 'interest' and desire: 'To the extent to which the subject is an artist it is already redeemed from its individual will and has become like a medium through which the one truly existent subject celebrates its redemption in appearance' (1 p. 47). The individual subject is merely a mouthpiece for the 'true subject', and the argument is at this point just another version of Schopenhauer's Platonic metaphysics of art. Like Schopenhauer, Nietzsche excludes the side of art which addresses us as sensuous subjects from the truth because it would subject us to the inherent lack generated by the Will. Given the shared misogyny of Schopenhauer and Nietzsche, this suggests a line of enquiry linking aesthetics to the philosophical concerns of feminism. Antagonism to the senses and antagonism to the female go hand in hand in parts of the Western philosophical tradition (see, for example, 1984). Nietzsche will admittedly later revalue the senses, but he does not change his mind with regard to women.

Nietzsche's vision retains the faults of Schopenhauer's in its assumption that the 'one truly existent subject', which could also be called Nature, Life, Will, is intuitively accessible as the other of rationality. Fichte's and Schelling's struggles with how one can grasp what transcends reflection seem forgotten, as does their investment in freedom as that which could make sense of this transcendence. Nietzsche sees no problem in suggesting we have access to what is effectively the absolute by surrendering to the higher power. Significantly, the argument is couched in theological vocabulary. The point of art is not essentially anything to do with ourselves and instead is really for the pleasure of the 'true 
creator'. Indeed, we ourselves are 'images and projections' for this creator. The real direction of the argument becomes apparent when Nietzsche asserts that we 'have our highest dignity in the significance of works of art - for only as aesthetic phenomenon is existence and the world eternally justified' (Nietzsche 1980 1 p. 47). Art enables us to contemplate a world with no telos, without this leading to the desire for self-annihilation born of despair at the transience and destructiveness of the world.

Nietzsche goes on to employ a questionable version of Schelling's argument that the genius combines conscious and unconscious production to back up his own position. In the act of creation the genius merges with 'that primal artist of the world' (1 p. 48) and thus transcends reflection by being both subject and object. Where Nietzsche's view of the cultural importance of art can lead is apparent, however, in a passage, written in early 1871 for the $B T$, which was not included in the book but which is consistent with the structure of its argument. Nietzsche reflects upon the role of slavery as the condition of possibility of Greek art and proposes that in modern culture 'the misery of the laboriously living masses must be further intensified in order to enable a number of olympic people to produce the world of art' (7 p. 339). Ethical imperatives take second place in the face of the need to produce great art as the justification of an otherwise meaningless existence. The separation of ethics and aesthetics thus leads to a perverted pseudo-ethical imperative. This imperative results from the assumption that the only answer to questions of meaning in a post-theological world lies in the overcoming of everyday human existence in the name of a world of art. The production of this world can justifiably ignore the rights of individual subjects in the name of superior beings who create new meanings. A related position later recurs, minus the aesthetic imperative, when, in Beyond Good and $E v i l$, he says that a 'good and healthy aristocracy' will 'accept with a good conscience the sacrifice of a host $(U n z a h l)$ of people who will have to be repressed and diminished to incomplete people, to slaves, to tools for its sake' (5 p. 206).

The Idealists and the early Romantics thought that the flowering of modern culture would depend upon the creation of a new public sphere and of universal free communication. Art was consequently inextricably linked to politics and ethics, and to the realisation of the freedom of the subject. Nietzsche's petitbourgeois, elitist vision, in contrast, translates easily into the fascist 'aestheticisation of politics' described by Walter Benjamin in the 1930s. The power of ideas like Nietzsche's to influence politics in the modern world can be seen as deriving from the failure of what was intended by the project of a 'mythology of reason'. A socially sanctioned collective renunciation of critical thinking clearly has great seductive force in times of crisis. Aesthetically manipulated events and experiences dictated from above which offer a feeling of unification with a larger entity often have greater appeal than critical politics of the kind demanded by the democratic Left. Nietzsche's brutal remarks may be understood as simply making an invalid projection of the conditions of possibility of great art in the pre-modern past onto the modern present (which means, of 
course, that he is concerned with the same issue as Marx), but he nevertheless reveals what can happen when the ethical wholly gives way to the aesthetic, instead of there being an interplay between the two. Even then, however, the issue Nietzsche points to is not wholly obviated by these kinds of objection.

This becomes apparent if we relate Nietzsche's diagnosis of the state of the culture of his time to our contemporary situation. In the $B T$ the essence of modernity is regarded, as it was in Schlegel's Discourse, as the lack of a 'centre'. Nietzsche's formulations could, despite their refusal to celebrate it, be seen as an account of what has now come to be seen as postmodernity:

think of a culture which has no firm and holy original abode [Ursitz], but is condemned to exhaust all possibilities and to nourish itself meagrely from all cultures ... What do the massive historical need of dissatisfied modern culture, the gathering around itself of countless other cultures, the consuming desire to know, point to, if not to the loss of myth ...? (1 p. 146)

Worries about the consequences of the decentring of culture are inseparable from the tradition of aesthetic theory. Is diversity actually a cultural gain, or does it, by destroying unified social aspirations, lead to an undermining of what can render life collectively meaningful? The problems involved in any answer to this question are inseparable from modernity. While the acceptance of the need to acknowledge cultural diversity is one of the great advances initiated by thinkers like Herder and the Romantics, it is far from clear that diversity is of its own accord a positive value. Culture thrives on criticism, and criticism needs exemplary models which can reveal the deficiencies of inferior cultural production. However, Nietzsche has little to offer here. Unlike Schlegel and the Idealists, who wished to synthesise a new mythology out of cosmopolitan diversity, Nietzsche at this time sees the answer to the decentred state of culture in terms of a 're-birth of German myth' (1 p. 147), a solution which will have disastrous echoes in the twentieth century. He will, one should remember, later move to a - in some respects - cosmopolitan, anti-nationalist position, but he never gives up the idea that healthy culture is the product of superior beings, rather than of education, cultural opportunity and democratic debate. In the $B T$ Nietzsche invokes a 'splendid, internally healthy, age-old power' (1 p. 146) which is hidden under the surface of a decadent culture, and which can reawaken. Such a reawakening must, of course, happen spontaneously and unexpectedly because the people who bring it about have no conscious say in the workings of this power.

The flaw in this position is simple, and we have encountered other versions of it already. If the essence of modernity is the demand for self-legislation, the appeal to a grounding power which transcends the existing social world and can transform it has no prior claims to legitimacy, otherwise that power is playing the same pre-modern role as 'dogmatism' and illegitimate feudal traditions. It is, then, no surprise to see Nietzsche toying with inhuman ideas like the producers of art being sustained by the suffering of the masses. Who, though, 
decides who these producers are, if not people in society who must try to justify their superiority to the rest of society? Nietzsche's appeal to the primal force goes along with his insistence on the separation of the aesthetic from the ethical. The Dionysian force which gives rise to tragedy, and which tragedy and music enable us to contemplate, is prior to any ethical considerations, as the nature of his appeal to it would suggest. Nietzsche attacks those accounts which use ethical categories to explain Greek tragedy, insisting that tragedy has nothing to do with a Schillerian 'arousing of moral-religious powers' ( 1 p. 143). Tragedy is rather what enables the aesthetic contemplation of the horror which is the ground of existence, a horror which, once revealed, would otherwise render life intolerable. Only a culture unified by mythology is able to find ways of enabling such contemplation to have a positive role in rendering life justifiable.

Once again, nothing discursive can justify this argument. Our access to the creative forces Nietzsche sees as present in tragedy is always as historically situated subjects. Any ontological claim concerning primal forces, of the kind on which the $B T$ relies, must identify these forces as the ground of our own thinking, and this raises yet again all the problems of reflection seen in Fichte and Schelling, which recur in Schopenhauer and Freud. How do we know that it is the primal force which is acting? If it is to act as primal force it cannot be available to knowledge, which is inherently secondary and derived. The answer has to be in terms of a prior 'intuition' of an essence which escapes discursive thinking. In the context of the idea of a re-birth of German myth the notion of such intuitive access is incipiently racist: presumably only the members of the German nation will be able to attain it.

However, as we have seen, the significance of the aesthetic in some respects derives precisely from its extension of our understanding of the world in nondiscursive, 'intuitive' ways. This is why the issues raised in aesthetics are necessarily uncomfortable ones. The choice seems to be either to subsume art in a Hegelian manner into the realm of intersubjective cultural legitimation, and thereby run the risk of losing the essence of aesthetic experience by subordinating it to discursive articulation and validation, or, by suggesting that art offers something resistant to wholesale mediation, to run the risk of the kind of intuitionist irrationalism present in the worst aspects of the $B T$. The choice between these abstract alternatives cannot be made on methodological grounds alone: what is at stake here has differing significances at different times. It is, of course, in music that the resistance to mediation is most obvious, and this is why Nietzsche, like Schopenhauer, makes it his main focus.

In the $B T$ Nietzsche is concerned with the fact that everything in modernity, and particularly the natural sciences, fails to offer a serious counter to what Max Weber will term the 'disenchantment' of the modern world, a disenchantment which points inexorably to our irredeemable transience and fragility, despite modern technical advances which can further self-preservation. Nietzsche thinks the only possible response to disenchantment lies in our capacity to create illusions that sustain life. The fact that these are illusions follows for him from 
intuitive insight into the Dionysian nature of the real. The $B T$ radicalises Kant's and Idealism's ideas about the limits of scientific knowledge as the means of articulating the truth about our existence into a view that all human cognitive and imaginative activity, be it scientific or aesthetic, is essentially 'artistic', creating significance which the world otherwise lacks. The question is then, of course, which significances are most appropriate for successful life, and this question will form the centre of much of Nietzsche's later work. Nietzsche pursues the consequences of the restricted nature of the understanding that concerned the German Idealists and early Romantics, retaining the idea of human cognitive and other activity as 'artistic', in varying versions throughout his philosophical career.

The capacity for the creation of appearances, which Nietzsche terms 'art', including both science and religion in the category, is itself grounded in the Dionysian, the noumenal force that engenders the phenomenal world. Like Schopenhauer, Nietzsche tries to overcome the difficulty of explaining our access to this Dionysian ontological principle by referring to music: 'The worldsymbolism of music cannot in any way be exhaustively grasped with language, because it is symbolically related to the primal contradiction and the primal pain in the heart of the primal One, and thus symbolises a sphere which is superior to all appearance and prior to all appearance' (1 p. 51). Only music can really say what the 'justification of the world as an aesthetic phenomenon' means: 'The pleasure which the tragic myth creates has the same home as the pleasurable feeling [Empfindung] of dissonance in music. The Dionysian, with its primal pleasure [Urlust], which is even perceived in pain, is the common womb of music and of the tragic myth' (1 p. 152). Dissonance in music reveals to us the transience and incompleteness of individuated existence by giving rise at the same time to a striving for the infinite, a striving for harmonic overcoming of all differences, which is in these terms a return to the unconscious primal One.

The $B T$ offers, however inadequately, a way of understanding some of the appeal of certain kinds of music in modernity by linking music to the temporality of myth. Mythological time can be regarded as a way of escaping the abstract sequential divisions and unidirectionality characteristic of the dominant forms of modern, rationalised temporality. In The Ram and the Cooked Lévi-Strauss claims myth and music are both 'languages which, in their different ways, transcend articulate expression, while at the same time . . requiring a temporal dimension in which to unfold. But this relation to time is of a rather special nature: it is as if music and mythology needed time only in order to deny it. Both indeed are instruments for the obliteration of time' (Lévi-Strauss $1975 \mathrm{pp}$. 15-16). In the more metaphysical terms of the $B T$, tragedy therefore affirms eternal life, and the death of the hero is only the destruction of one form of appearance of the Will: "“We believe in eternal life", tragedy shouts; while music is the immediate Idea of this life' (Nietzsche 19801 p. 108). A rebirth of myth is possible through music because music is the counter to 'Socratic optimism', the belief in the progress and perfectibility of mankind by science. 
Science is, in this view, ultimately just another means of trying to conceal the real horror at the ground of being, not the revelation of the real nature of things.

Art, in the form of music, is a more apt response to the nature of existence because it does not require concepts. Concepts necessarily take one into the regress of causal explanations which lead to the 'abyss'. ${ }^{10}$ At the same time music expresses the creative principle of existence in a manner which makes existence's destructive aspect tolerable. Although, by unfolding in time, music relies on the divided nature of all phenomenal existence, it also conveys an affective overcoming of that existence, albeit at the price of the loss of reflective self-awareness. It is at this point that one comes up once again against Schopenhauer's problem, namely that music seems therefore to be reduced to a single metaphysical significance. This view is simply inadequate to the sheer diversity of ways in which music can signify in a secular society. Nietzsche's argument is itself a historical one, concerning the need for modern Germany to rediscover myth, but this rediscovery is then seen as the return to something originary and mythical, rather than as a new revelation of the kind hoped for by the Romantics. Nietzsche's vision is undeniably powerful, affecting many subsequent artists and thinkers, but he himself came to be dissatisfied with it. The question is: if music is not an answer to metaphysical questions about the transient nature of human existence, what is its significance? Answers to this question offer a way into the further development of Nietzsche's philosophy.

\section{Myth, music and language}

It is in relation to language and art, particularly music, that one can best see the differences - and the continuities - between the early and the later Nietzsche. Nietzsche's legacy for subsequent aesthetic theory in many ways depends on how he rejects or transforms many of the ideas of the $B T$. The relationship of myth, music and language is, for example, central to Nietzsche's view of Wagner in the Untimely Meditations, (1876), entitled 'Richard Wagner in Bayreuth'. Here Nietzsche equates the poetic with the mythical in a manner derived from aspects of Schelling and the Romantics. Myth is a thinking in 'processes that can be seen and felt . . Myth does not have a thought for its basis, as the children of an artificial [verkünstelt] culture think, but it is itself a form of thinking ... The Ring of the Nibelungen is a massive thought system without the conceptual form of thought.' Wagner 'forces language into an original state where it thinks virtually nothing in concepts, where it is still itself poetry, image and feeling' (Nietzsche 19801 pp. 485-6). This idea is connected to the assumption that myth functions as the locus of meaning in cultures which do not have the conceptual apparatus of modernity. The foundation of the idea is therefore an 'original state' of language which we have lost, and which music will help to restore.

Unlike Schleiermacher and the early Romantics, who thought music and lan- 
guage were complexly interrelated in ways that can point to new potential in both, Nietzsche here suggests that conceptual thought is a 'fall' from something higher. When Schelling talked of language as 'faded mythology' he was aware that the notion of a recovery of the lively particularity of myth that had been lost was in contradiction with the universalising necessities which determine the nature of language in the modern world. Nietzsche, in contrast, claims that the fallen condition of language in modernity can be immediately overcome. The structure of thought involved here is, once again, highly questionable. It recurs, for example, when Heidegger talks of the 'language of metaphysics' which obscures the question of being and which it is his task to circumvent. The underlying problem lies in disentangling the significance from early modernity onwards of the revaluation of music as a non-conceptual language that changes the perception of poetic and other language, from the kind of philosophical claims made by Heidegger and Nietzsche. The problem is that these claims are subject to the logically impossible demand of circumscribing the totality of the language they see as questionable while avoiding using that language and so falling prey to what they are questioning. How can one say when one has really got in touch with the original state of language, or that one has avoided the language of metaphysics? Discursive claims to this effect involve the performative contradiction of using concepts to make assertions about what is dependent on the non-conceptual. As we have seen, the only valid approach here is to appeal to what music or a poem can show that cannot be said, but this sits ill with the broader philosophical claims about the nature of an era that Nietzsche infers from his ideas about the state of language.

The specific claims Nietzsche makes here are in many respects based on a Romantic cliché, not on the real substance of early Romantic thought. Language has fallen ill because it has become too closely linked to conceptual thinking, and has lost its connection to feeling, and thus to 'nature'. It therefore works solely by convention, like a machine, and only music, now seen as a 'return to nature' ( 1 p. 456), as well as a 'purification of nature' (the two hardly seem compatible), is the 'language of true feelings' ( 1 p. 458). This version of what can be a valid topos of cultural criticism - a critique of the dehumanising effects of bureaucratic, racist and other kinds of language is eminently possible in terms of the counter-example of poetic language - is simply too schematic and universalising. The implied language of 'false feelings' (whatever that might mean) must presumably be conceptual language, but the borderline between this and other kinds of language need not at all be the same borderline as that between language whose effects are cruel and stultifying, and language which is liberating and creative. In such passages Nietzsche falls below the level of thinking about music which enabled the Romantics to formulate notions of aesthetic and literary autonomy that were based on music's non-representational character and on the reciprocal and changing dependence of language and the 'musical'.

What is really wrong with Nietzsche's position does not, though, lie in the claim that music tells us something important about the functioning of language 
in modernity. It clearly does, though it does not tell us what Nietzsche says it does. The problem is that those who may suffer from the narrowing of language into mere instrumentality, individual subjects who have feelings here and now, seem not to count in his argument. The liberation of the aesthetic from wholesale subordination to the ethical, which can, in some circumstances, open up new resources for ethical and existential exploration, can also lead to a dangerous aestheticism when the idea of music is linked to the return to amoral, mythical forces. Nietzsche's ideas about a return to mythical origins actually result from a particular way of understanding the most technically advanced music of Western culture. The great achievement of Wagner's music, whatever Wagner may have thought he was doing, is to articulate specifically modern experience, not a lost, primordial experience. The sheer complexity and ambiguity of the affective dimensions of Tristan und Isolde far transcend anything deriving from merely mythical traditions. These dimensions of the work have to do with the modern awareness of the subject's failure to grasp its ultimate nature, not with some kind of ultimate insight into the ground of being. Otherwise the continuing power of such works for very different audiences becomes incomprehensible.

Nietzsche is anything but clear what he thinks about language in the earlier 1870s. In The Dionysian Weltanschauung (1870), for example, he still regards conceptual language as lacking something essential and as therefore needing to return to something lost. Those forms of communication that Nietzsche also sees as 'languages', such as dance and song, which offer a more immediate access to the world of feeling, are superior to verbal language because they are 'thoroughly instinctive, without consciousness' (1 p. 572). Wagner, he claims, combines these 'languages' into a higher unity in his operas, mixing gestural and musical languages, the realm of the image and the realm of sound. There is nothing here of particular philosophical significance. The 1871 fragment on music and language is, however, much more interesting.

Nietzsche here begins to become aware of the problems in Schopenhauer's ontology of the Will, because he advances a more sophisticated view of language than he does elsewhere in this period. Words, he argues, in the manner of Saussure, are only symbols, not of things in themselves, but of representations (Vorstellungen). In consequence, even the notion of the 'life of drives' (Triebleben), the fundamental reality for Schopenhauer, is itself only a representation, and the "Will" is nothing but the most general form of appearance of something which is wholly undecipherable to us' (7 p. 361). We saw a similar point in Freud's claim that we only have access to drives via the representations that are attached to them. The problem is, of course, that the 'something' sounds rather like Kant's thing in itself, and we still seem to be moving in the direction of an appeal to a non-discursive intuition if the something that is wholly undecipherable is to be accessible at all. Following the Kant of the Critique of Fudgement and Schopenhauer, the main forms of appearance of this primal basis are the sensations of pleasure and unpleasure, which are not subject 
to rule-based cognition - as suggested in Cascardi's description of the individual as 'an irreducibly particular centre of affectivity' (Cascardi 1999 p. 48) - and thus are in possible tension with their articulation via general signifiers. These sensations are symbolised in language by the 'tone of the speaker'. They contrast with the consonants and vowels, which constitute 'gesture symbolism', being conventionalised gestures of the lips, tongue, and so on, rather than the articulations of a conceptual truth 'behind' language. The tonal, 'musical' basis of language - rhythm, pitch, dynamics, tone, etc. - is seen as 'comprehensible beyond the difference of languages' (Nietzsche 19807 p. 361). Conceptual language, on the other hand, is reduced to the conventional, collectively repeated repetition of differentially constituted verbal gestures in a society. These enable what Schleiermacher saw as 'construction', the creation of identity of cognitions by a habitual consensus about usage within a particular language. Thinking of language in this way begins to move in the direction of an anti-metaphysical conception, for which language is primarily a form of social action. At the same time, the vital dimension for Nietzsche is access to the essential underlying reality, which happens in music, not in the conceptual dimension of language which reduces difference to identity. As we shall see, this suspicion of identification will be central to most of Nietzsche's thought.

Nietzsche suggests in the fragment that, although music may give rise to a multiplicity of images, it is impossible for images or concepts themselves to produce music, because they are of a radically different order from each other. He consequently offers a new way of attempting to understand the significance of the aesthetic autonomy of music. Regarding music as a language of feelings is mistaken, he argues: 'What we call feelings are . . . already penetrated and saturated with conscious and unconscious representations and thus not directly the object of music, let alone able to produce music out of themselves' (7 p. 364). Feelings have a history in which the growing differentiation of what may initially be largely inchoate tensions, of the kind inherent in the idea of the Will, is part of what feelings are. The genesis of music is consequently not explicable in terms of already constituted feelings that are simply represented or expressed in music. 'Music' itself makes articulated feeling possible: think of the way in which rhythm and gesture can give a shape to otherwise inarticulate feelings, or of the way music can give rise to feelings one previously did not have. Nietzsche here echoes ideas we encountered in Schlegel's remark about the Orphic period in Greece, where 'rhythm in this childhood of the human race is the only means of fixing thoughts and disseminating them' (Schlegel 19882 p. 16), and in Novalis's question: 'Might musical relations be the source of all pleasure and unpleasure?' (Novalis 1978 p. 772). ${ }^{11}$ Feelings are, then, in a sense only symbols of music, as the ground of possibility of articulated feelings. Nietzsche here opposes the commonplace in some Romantic thinking (and in his own other work at this time) that music is the language, in the sense of the 'representation' of feelings. ${ }^{12}$ The shifting relationship of musical to linguistic articulation means that determinate articulation of the world is reliant upon chains of 
differentiation which concepts, as themselves articulated in language and thus as reliant on the same kinds of differentiation, could never wholly grasp. By arguing in this way Nietzsche begins to deconstruct the idea of an immediate intuition of the ground of representations which gave rise to some of the problems in his other work at this time.

However, not all the issues involved in questions of intuition are obviated by these arguments. The specific Romantic concept of 'feeling' - pre-propositional immediate self-consciousness that 'cannot feel itself' - without which particular feelings would have no locus of significance, suggests an intuitive dimension without which differentiation could not function as significant differentiation, and would instead be mere dispersal. Nietzsche, like De Man, gives no account of the subject which hears music as music. The medium of articulation may be a necessary condition of the differential constitution of feelings, but it is not a sufficient one. The constitution of music as music, as opposed to as mere noise, requires a self-conscious ground of connection between the phenomena which can give rise to something non-semantically intelligible. Nietzsche has little interest either here or elsewhere in a subjectivity which might be more than the epiphenomenal result of an ontological basis such as the Will, or, later, the 'will to power'. It should be apparent from what I have said so far that removing the subject from a decisive role in aesthetics removes the point of art. The question is how to arrive at an adequate account of that subject and its relationship to its means of articulation. Nietzsche has little to offer here, despite his undoubted insights into the deficiencies of some models of the subject.

Nietzsche's argument in the 1871 fragment is most interesting as a statement about absolute music that goes beyond the more reductive arguments seen in Schopenhauer. His refusal to give the cognitive dimension of language priority results from his claim that this dimension is itself grounded in the 'tonal basis', the musical in language. The musical is generally comprehensible in ways that particular verbal languages are not, even though it is non-semantic. Nietzsche's conception here actually points in the direction of the later Heidegger's approach to language. We get a sense of the essence of language, Heidegger maintains, when we cannot find the right word, rather than vice versa: 'Then we leave what we mean in the unspoken, and by doing so, without really thinking it, we experience moments in which language itself distantly and fleetingly brushes us with its essence' (Heidegger 1959 p. 161). Heidegger's pursuit of a wholly new kind of 'thinking' does not lead him explicitly to make music part of what he is seeking. However, what both he and Nietzsche point to is in fact closely related to the idea of Romantic irony. This was Schlegel's response to his insight into the limitations of the sayable. Romantic irony derives precisely from the realisation that one cannot say what matters most and can therefore only point to it via the acknowledgement, even as one says it, of the ultimate failure of what one says. It is through music's simultaneous demand for, and resistance to semantic determination that this conception can best be understood. Without the changes in music in the early modern period this whole con- 
ception seems unlikely to have developed. Despite the other shifts in his philosophical position that we are about to consider, Nietzsche will often keep trying to avoid reducing music to what can be said about it, or to a general philosophical theory.

\section{The illusion of truth}

The essay 'On Truth and Lie in the Extra-Moral Sense' (1873) contains the seeds of Nietzsche's later philosophy, which he will develop after the split with Wagner. At the same time, it is a distillation of many themes which we have seen as originating in aesthetic theory, which Nietzsche now attempts to mobilise for philosophy as a whole. The problem of truth arises, he maintains, from the attempt to make what are inherently particular human perceptions universally valid. He therefore contests the possibility of an Idealist harmony between the subjective and the objective: 'Between two absolutely different spheres like that between subject and object there is no causality, no rightness, no expression, but at the most an aesthetic relation, I mean a suggestive transmission, a stammering translation into a completely strange language' (Nietzsche 19801 p. 884). The argument is Kantian in some respects, except that the synthesising activity of the transcendental subject now consists in an essentially arbitrary historical production of metaphors: 'Truth is a moving army of metaphors, metonyms, anthropomorphisms, in short a sum of human relations, which were poetically and rhetorically intensified, transmitted, elaborated, and which, after long use, seem canonical and binding to a people: truths are illusions which one has forgotten are illusions' (1 pp. $880-1)$. Nietzsche is characteristically silent on how to avoid the obvious performative contradiction his assertion entails: how can he identify the illusion, if he wishes truly to assert that all truths are illusions? What, in that case, is the difference between truth and illusion?

Samuel Wheeler says of Nietzsche's argument: 'How could metaphors . . . fail to live up to the literal without the possibility of something to live up to. That is, without the possibility of Platonic or Cartesian spirit tokening to be the full-fledged "literal"?' (Wheeler 2000 p. 119). If one thinks, as the Romantics already began to suspect, that the correspondence theory of truth is unintelligible, it is, as Wheeler suggests, a strange form of nostalgia then to make its unintelligibility into a drama in which truth is supposedly not in touch with the real, and is therefore an illusion. If, as Nietzsche himself claims, there cannot be said to be bits of the world which make our sentences true, what we say cannot be merely illusory, as that would entail there still being a true nature of things that we are missing, a claim which is clearly contradictory. As we saw above, it is perfectly possible to claim that truth or reason is in one sense 'merely human' because words and things cannot be said to correspond, as long as one does not make the further positive claim which Nietzsche makes.

Nietzsche's argument really relies on the kind of anthropological stance which became very popular in the wake of Darwin. The intellect is a means for 
the self-preservation of the individual, and is consequently most adept at deception, 'truth' not necessarily being the most effective means of self-preservation. As such, the aesthetic creation of 'truth' is merely one aspect of self-preservation, which has no inherent advantage over aesthetically created deception. Nietzsche's divorce of aesthetics and ethics is now even more radical, and he shows no awareness, either of a normative dimension to communication that is not simply based on power over the other, of the kind present in Schleiermacher, or of the fact that deception itself relies upon knowing what one thinks to be true. Like many contemporary evolutionary theorists Nietzsche obliterates the difference between the natural and the cultural.

This generalised attempt to undermine truth relies on the contentious idea that language is essentially based on a specific conception of convention. ${ }^{13}$ The argument is in fact not far from Schleiermacher's account of one aspect of language as the creation of identity from difference by schematism: 'overlooking the individual and the real gives us the concept' (Nietzsche 19801 p. 880). However, Schleiermacher did not prioritise convention, regarding all communication instead as 'a constant test as to whether all people construct identically', and he was able to account for linguistic innovation in terms of an individuality that could gain general intersubjective acceptance. Inherent in Schleiermacher's position was therefore the search for social consensus based on mutual recognition of individuality, not on mere reduction of difference to identity. Nietzsche, in contrast, thinks the truth constituted in language is in fact a socially instituted compulsion to construct identically. He thus prefigures psychoanalytic theories which regard insertion into the 'symbolic order' as a form of primary repression. Such theories fail to see that language also makes possible the redeeming articulation of what may otherwise remain inchoate suffering or tension. In the $A W$ Schelling offers a far superior account of the tension in language between its ability to articulate and express in novel and individual ways, and the possibility that it will, as he puts it, 'congeal' into static schematisation. Nietzsche only sees one side of what is a more complex picture, and paints himself into a metaphysical corner which fails to do justice to the tension in the nature of language between repression and expressivity.

For Nietzsche, language just converts the world into something reductively anthropomorphic, into the 'endlessly broken echo of an original sound' (1 p. 883 ), so that the world becomes merely the reflex of humankind. Mistaking the result of the reflection of ourselves in the world for objective truth results, he asserts, from the fact that 'man forgets himself as subject and, indeed, as artistically creating subject' (1 p. 883). This forgetting allows man to live with a sense of security, which would be destroyed were he to understand the real nature of his belief in objective truth, namely that it is a creation with no external, or even - as it had in Kant - internal, foundation. All human production is therefore 'aesthetic', including the production of truth. Though it is actually unclear how any questioning of truth could arise at all in this conception, Nietzsche's interrogation depends on the idea that truth is a repressive reduction to identity of 
something which inherently resists our identifications. 'Truth' is the domination of one controlling power, the need for self-preservation based on identity, over the resistance of a world which is infinitely particular and differentiated. The world in question still sounds, though, like the true Platonic world which Wheeler suggests is lurking in Nietzsche's argument, rather than one which we are aware is always open to redescription and re-evaluation, because there is no way finally to match up the divisions within a language and 'a natural segmentation of the world' (Wheeler 2000 p. 120).

The historical dimension of Nietzsche's text offers rather more possibilities, once one moves away from a general philosophical claim about language to the justified concern that certain kinds of use of language might be damaging to our being in the world. Modernity is associated by Nietzsche, as it was in aesthetic theory from Hamann, Schelling and the Romantics onwards, with the repression of 'intuition' (Anschaunng), in the sense of the particular, immediate relation to the world which concepts cannot capture. While this view can, as it surreptitiously does for Nietzsche, involve a reversion to a myth of the given, such that the truth about the world is contained in this original intuition, we have seen that the tradition of aesthetics offers other ways of considering immediacy. Despite the confusions Nietzsche creates in relation to this issue, his concern to defend intuition against its being swallowed by abstraction will inform his more interesting reflections on music. The reasons for this defence form the basis of his later work, and also explain his persistent attention to aesthetics in his critiques of metaphysics.

Nietzsche's suspicion of the modern devaluing of intuition is a function both of the critique of language as the creator of repressive identity and, when he develops his ideas in more interesting directions, of the theory of 'nihilism'. It was Jacobi who brought the term nihilism into wider currency at the end of the eighteenth century, referring to philosophies which reduced the world to the functioning of endless chains of deterministic laws and so failed to show why the world was intelligible to us at all. Nihilism for Nietzsche, in contrast, is inextricably linked to the history of philosophy since Plato: 'The need for a metaphysical morld is the consequence of being unable to derive any meaning, any mhat for? from the world at hand. "Consequently", it was decided, "this world can only be apparent"' (Nietzsche 198012 p. 374). 'Psychological nihilism' is a result of three factors in modernity. The first is the failure to find any teleological meaning in existence, such as a movement towards a moral world order, so that 'becoming' ceases to have a goal and is just arbitrary change; the second is the realisation that there is no unity in the multiplicity of existence which would enable one to believe in one's own value as part of something greater; the third is the loss of the belief in a supersensuous world, which is accompanied by the realisation that one cannot bear this world without that other world (cf. Nietzsche 198013 pp. 47-8). Nihilism is therefore a result of metaphysical beliefs which have turned out to be illusory. Because meaning was sought in a world which does not actually exist, the world which does exist appears 
meaningless. Schopenhauer's philosophy devalued the transient sensuous world in the name of art as the means of temporary access to the non-transient world, in order to come to terms with irredeemable facticity. Nietzsche, having still been attached to ultimately Platonic notions even in the essay 'On Truth and Lie', now has to take a very different course, because there is only one world.

It would be misleading to claim that his response to this situation is a logical development from his awareness of the failure of the metaphysical theories of previous Western philosophy. An effective critique of metaphysics cannot just be a critique of the illusory idea of a supersensuous true world apart from the transient world of the senses. Nietzsche (presumably approvingly) cites Feuerbach's attack on Hegel, for example: " "healthy and fresh sensuousness" ... against "the abstract philosophy"' (12 p. 261). This revaluation of the sensuous cannot, however, make the sensuous into a new basis for philosophy. Its very status as the sensuous depends upon its counterpart, which has now been put into question, so that the nature of the line between the two must also be contested. Nietzsche will have a tendency in the face of such difficulties to opt out by either simply asserting his attachment to the opposite principle or by dogmatically positing the intuitively available 'real' basis of the illusions of previous philosophy in the form of the 'will to power'. Like Schopenhauer's Will, the will to power is the principle of all change, which is now the result of the victory of one quantum of will to power over another. The will to power therefore becomes the condition of possibility of any kind of differentiation, which gives it the same role as the absolute, as the One which makes possible the many as the other of itself. Much of the confusion of Nietzsche's later philosophy is a result of the dilemmas generated by this ultimately metaphysical principle. At his best, Nietzsche will suggest more interesting perspectives than those offered by the will to power.

\section{Music and metaphysics}

Just how radically Nietzsche can change his mind is easily demonstrated by two passages from Human, All Too Human (1878), which endorse the opposite view of music to that of the $B T$. The passages mark the beginning of a complex series of new reflections on the significance of music which form part of Nietzsche's attempt to overcome metaphysics and to respond to nihilism. The first passage is a reflection on religion and art which could be used to summarise Hegel's Aesthetics, and which involves the issue Marx pondered in the Grundrisse: 'One must have loved religion and art like mother and nurse - otherwise one cannot become wise. But one must see beyond them, be able to grow out of them; if one stays in their spell one does not understand them' (Nietzsche 19802 p. 236). There is no suggestion here of the need for a Romantic synthesis, of the kind still present in the $B T$, which would transcend divisions between art and science. Indeed, Nietzsche tends here to see science as self-legitimating, rather than as leading to the abyss that gives rise to the need for a new mythology. The 
second passage is a reflection on 'absolute music', which argues that it is either a very primitive form of articulation, or the result of the historical mixing of music with complex concepts and feelings: 'In itself no music is deep and significant, it does not speak of the "Will", of the "thing in itself"; the intellect could only think something like that in an age which had conquered the whole extent of inner life for musical symbolism. The intellect itself first of all read this significance into the sound' ( 2 p. 175). The historical point is clearly valid: the 'idea' of absolute music is a result of particular historical developments. However, the passage neglects the Romantic reflections on music, philosophy and language we have looked at so far. It assumes, rather as Hegel does, that all there is to be said about music depends upon concepts, and that what it offers is symbolism of 'inner life', which has now been conquered by the intellect. The idea we have encountered in Novalis and Schlegel, and in Nietzsche's fragment on music and language, that what constitutes music is itself essential to the constitution of thought now seems forgotten. Nietzsche, perhaps rightly, wishes to say farewell to the Schopenhauerian link of music to ontology, but here he does so in too one-sided a manner.

The direction of Nietzsche's ideas at this time is most apparent when he also claims that music is, precisely, just a remainder of metaphysics: 'the highest effects of art can easily produce a resonance of the metaphysical string which has long been silent, indeed has broken' ( 2 p. 145), referring as an example to part of the last movement of Beethoven's Ninth. Elsewhere he is insistent that 'Music is precisely not a general, supra-temporal language', arguing that it is a 'late-comer of every culture', and that contemporary German music may soon be incomprehensible to anyone else (2 p. 450). Nietzsche is, of course, spectacularly wrong about the German music of his time, which may not have supratemporal significance, but which has a culture-transcending power which he provocatively refuses to countenance. Nietzsche the performative thinker now begins to come to the fore. The inconsistencies that emerge in the texts we are now considering both suggest new ways of looking at the issue of music and undermine any sense that one might arrive at a decisive characterisation of the significance of music. There is here another connection between Nietzsche's varying approaches to music and Romantic irony. In the same way as music cannot be said to state anything semantically determinate, attempts to grasp music themselves fail to reach ultimate adequacy to what they seek to grasp and so are undermined, even as they may also offer important insights into music. The question is, what happens if the notion that music, like Romantic irony, is a way of pointing to the absolute, can no longer be sustained? Is the alternative the assumption that all a post-metaphysical modernity can permit is the idea of a mere arbitrary collection of competing forms of articulation which are inherently never fully determinate, and none of which has any privileged role over the others? Questions like this go to the heart of aesthetics: what, then, are the limits of the disenchantment of art, and what role does music play in that disenchantment? 
Nietzsche's deflationary view of music in texts like Human, All Too Human is essentially a result of his suspicion that music could, by becoming the new locus of feelings of transcendence, take the place of the metaphysics he now wishes to overcome. At the same time he remains aware that music does pose important questions for post-metaphysical philosophy that are not exhausted by his deflationary view. The fact is that the borderline between the metaphysical and the non-metaphysical becomes notoriously elusive in relation to the meaningfulness of music. Music in the broadest sense - which can just be rhythm (Schelling, remember, sees rhythm as the 'music in music') - is constituted both by the 'positive' materiality of the notes, as sensuous phenomena and, also, like language, by the 'negative' gaps between the notes, by differentiality. The differences are 'nothing', but not in the sense of absolute non-being: the difference between $\mathrm{B}$ flat and $\mathrm{C}$ is not the same difference as that between $\mathrm{B}$ and C. As we saw in Chapter 7, the subject, which is not reducible to its articulation by the signifier, can be situated in terms of its role in making the differences between the signifiers significant. Musical articulation would not be possible without the differences via which acoustic elements become determinate and meaningful. A further consequence of this is that the silences in great music can be as important as the articulated sounds: the 'pause' in the second movement of Schubert's great C major symphony is, for example, not the same silence as any other silence in another piece, even though all silences are in one sense identical. Silence thus seems to be the non-articulable ground of musical and linguistic articulation. The point is that this can only be so because there is a subject which is continuous between the moments of a piece: silence is the absence of sound, and absence is absence to somebody or something, not absolute non-existence.

When he moves away from Schopenhauer, Nietzsche argues that the mistake is to see music as an expression of something else, the Will, rather than as being irreducible to other things. This view should seem more convincing in the light of the above. Music is therefore not the expression of an intuitively known principle, and is better understood in terms of its ever-differing revelations of the consequences of the fact that the ground of articulation cannot itself be articulated. Music can, then, be both a temptation to metaphysics, if it is supposed to announce some pre-existing truth beyond itself, and can be used to oppose metaphysics, because it is non-representational and discloses aspects of the world which would not exist as such without music. Because music resists wholesale appropriation in scientific terms - acoustic frequencies or any other quantifiable, conceptualisable aspect of music are necessary, but not sufficient, for something to be music - it keeps alive issues which are germane to the role of aesthetics in modern philosophy. Nietzsche's main objection to Wagner - and this is one of the few constants in the philosophy of the later Nietzsche - will be precisely that Wagner attempts to turn music into something determinate.

Nietzsche's own contradictory interpretations of what music is themselves become an indication of the possible nature of a post-metaphysical aesthetics. 
Instead of being the locus of a positive answer to metaphysical questions, art is seen as offering something that is lacking in cognitive and ethical responses to essential questions about modern existence. However, what art offers need not be, as Habermas and others claim it is, merely an expressive dimension otherwise missing in those responses. For thinkers like Adorno the resistance to discursive articulation, particularly of the paradigmatic modern art of music, can make accessible what the other spheres of modern culture may repress. Music plays a special role in this respect because of the way it links both affective and structural dimensions of modern existence, at the same time as being a nonrepresentational form. The difficulty for a post-metaphysical aesthetics is that, qua theoretical discipline, its success must in one sense also be its failure. A discipline which is concerned with the individuality of the work of art is faced with the paradox that an adequate theoretical characterisation of that individuality would ultimately dissolve what it sought to reveal. Unlike disciplines such as the psychology of music, music history, or musicology, aesthetics cannot succeed in terms of its ability to bring its object under the appropriate concept, which leaves it open to the constant threat of redundancy. At the same time, aesthetics is a reminder to these disciplines that they rely on pre-interpretations which cannot be definitively grounded. What will make a post-metaphysical approach to aesthetics successful lies, then, in what is revealed by showing the limitations of any particular perspective on a work or aesthetic event. This more negative function of aesthetics, which already lay behind some of the Romantic (but not the Hegelian) conceptions we have examined, is developed in Nietzsche's best work. What is missing in that work, though, is an adequate account of the role of the subject in a conception of art which can no longer rely on the seeking of metaphysical goals. His reasons for this desideratum have to do with the reasons for his radical rejection of metaphysics, which he expressly connects to his accounts of music.

In contrast to the $B T$, Nietzsche comes to be suspicious of music which intoxicates the listener, associating this with the effects of religion: 'The dangerousness of the Christian ideal lies in its value feelings, in that which can do without conceptual expression: my fight against latent Christianity (e.g. in music, in socialism)' (12 p. 453). Certain kinds of music thus become part of the tradition of morality which Nietzsche wishes to overcome. Christian morality loses its metaphysical ground once God is dead and is therefore part of the history of nihilism. The music which has the effect of religion similarly gives the illusion of a higher purpose to life, in which one can lose oneself. When this purpose is revealed as illusory, vital creative energy will turn out to have been directed towards nothing. Nietzsche often relates music's lack of conceptual articulation to the attempt to replace theology: it encourages one to indulge in indeterminacy and so prevents attention being paid to the real concerns of daily life that become central once the greater goals are revealed as illusions.

At the same time, however, conceptual articulation still involves for the later Nietzsche, as it did in 'On Truth and Lie', the reduction of irreducible intuition 
to 'truth'. The positions are simply incompatible: insistence on the concept, construed as that which imposes identity, militates against individuality and indeterminable uniqueness. Nietzsche never resolves this tension. He tends to think in a way which prevents mediation between extremes, preferring what may be interpreted as a 'performative inconsistency' to the kind of unclosable dialectic proposed, for example, by Schleiermacher. Hegel suggested that philosophy is 'its age grasped in thought'. Nietzsche's stance highlights the tension in the age of modern capitalism between the production of collective - potentially repressive - identity, and the production of isolated individuality, but he offers little which might help move beyond that tension.

However, a philosophy which claims productively to overcome the metaphysical past ought to have more to offer in relation to this tension than Nietzsche does. Nietzsche is often seen as a counter to dialectical thinking because he rejects the idea that the resolving of difference leads to a higher identity. Too often, though, the rejection of the metaphysical version of dialectic leads merely to a static juxtaposition of contradictions which leaves no alternative but the performative stance described above. There is no simple way of understanding the consequences this has in relation to Nietzsche's thought as a whole: indeed, it can be argued that regarding his thought 'as a whole' is itself mistaken. In what follows I shall, therefore, pursue the ramifications of just one strand of Nietzsche's thought, though it will soon become apparent that this rapidly takes one into general questions that affect the whole of his philosophy.

Nietzsche's reflections on music and language take on a particular intensity in relation to Wagner. However much his polemical stance may have been generated by his personal break with Wagner, these reflections obviously transcend their immediate occasion. Nietzsche claims that in Wagner's music the lack of articulation leads to 'swimming', instead of 'dancing' ( 2 p. 434, 6 p. 422), because the music loses all sense of balanced proportion and therefore threatens to dissolve as 'music'. This claim shows what Nietzsche now thinks music really is: Wagner is 'chaos instead of rhythm' (6 p. 422). Music is rhythm, in the sense it had for Schelling, the 'transformation of a succession which in itself is meaningless into one which is meaningful ... the institution of unity into multiplicity' (Schelling I/5 pp. 493-4). Nietzsche adheres in his later work to a 'classical aesthetics', an aesthetics of proportionality and unity. However, his classicism does not explain the real difficulties which he tries to confront when he talks about music.

In his influential work on Nietzsche, Heidegger concurs with his objections to Wagner, but misunderstands them in a way that has important consequences. Like the Nietzsche of the passages from Human, All Too Human, Heidegger is a Hegelian in relation to music. In fact, he simply borrows part of the argument from Hegel's Aesthetics, giving little evidence in his writings of being explicitly aware of music in a way which would bear seriously on the rest of his thought. When Heidegger discusses the 'Gesamtkunstwerk' he seems to be following Nietzsche, but is actually following Hegel, when he maintains: 'According to the 
intention the music should be a means of showing the drama to its best advantage; but in reality music becomes the real art in the form of the opera ... Poetry [Dichtung] and language remain without the essential and decisive forming power of real knowledge. The dominance of art as music is artificial and thus the dominance of the pure state of feeling' (Heidegger $1961 \mathrm{pp.} \mathrm{102-3).} \mathrm{This}$ supposed 'dominance' of feeling is the culmination of what Gadamer will term the 'subjectification of aesthetics', the location of the significance of art in the feelings of a subject, which he associates with Kant and the Romantics. Aesthetics, the 'logic of sensuousness', is therefore the historical complement of the end of great art, because it separates art from truth by making it 'subjective'. The fact that music takes on the status of the highest art 'already has its basis in the increasingly aesthetic attitude to art as a whole; it is the conception and evaluation of art from out of the naked state of feeling, and the increasing barbarisation of the state of feeling itself into the naked seething and surging of feeling which has been left to itself' (p. 105). This is a peculiarly crass judgement on the development of music in the modern period, and Heidegger's attitude towards feelings in music has more than a hint of repression about it. Moreover, if one can show that the change in the status of music does not entail the triumph of the subjective in aesthetics, then the argument that the rise of aesthetics is just part of the subjectification of being ceases to be tenable. This is why Nietzsche's responses to music have more than just local significance for aesthetics, and go to the heart of the debate about aesthetics and modernity.

Unlike Heidegger, Nietzsche does not regard music as just an expression of feeling. (As we saw, Heidegger's own later discussions of language may be more reliant on music than he realises.) Heidegger's argument about Wagner in fact misses most of the point both of Nietzsche's variable view of music, and of his critique of Wagner. Nietzsche is actually not exclusively concerned with music's domination of the verbal arts. At the beginning of The Case of Wagner Nietzsche says: 'Have people noticed that music liberates the mind? gives the thought wings, that one becomes the more a philosopher the more one becomes a musician? - It is as though lightning flashes through the grey sky of abstraction' (Nietzsche 19806 p. 14). The point here is that musical articulation can escape the repression of the sensuous by abstraction, at the same time as sustaining creative thought. Nietzsche's real concern is with the fact that Wagner's music is 'theatrical', and so lacks an essential attribute of the aesthetic, the absence of an ulterior purpose. The crucial passage is the following, which, for once, is consistent with many others:

Wagner was not a musician by instinct. He proved this by giving up all lawfulness and, more exactly, all style in music, in order to make of it what he needed, a theatrerhetoric, a means of expression, of the amplification of gestures, of suggestion, of the psychologically-picturesque . . he increased the linguistic capacity of music into the unmeasurable - he is the Victor Hugo of music as language. Always provided that one first of all considers it valid that music should be allowed in certain circumstances not to be music, but language, but a tool, but ancilla dramaturgica. (6 p. 30) 
As in the fragment on music and language, 'real' music should be autonomous, not reducible to language, to representation, or even to being the expression of feelings. By language in this context Nietzsche clearly means rhetoric, pragmatic utterance. Given the performative nature of his thought, the demand that music avoid rhetoric has special significance. Far from underestimating modern music, or just regarding it as the culmination of the decline of art into subjective feeling, Nietzsche is aware of how important music is for many of his arguments, precisely because it is not directly performative and is not just an expression of the feelings and desires of a subject.

Heidegger, then, misses an important point of the kind he himself makes about language in poetry. Nietzsche suggests that music itself can bring about new - aesthetic - feelings in the subject, of a kind which could only be occasioned by such a non-representational medium. Consequently, instead of merely expressing existent feelings, music can have world-disclosive power, just as much as poetry. Indeed, music need not merely function at the level of aesthetic feeling in the narrower sense implied by Nietzsche. It can even render aspects of the objective world accessible: for example, music can affect what one sees in a film, or give significance to a landscape which it would otherwise not possess. The earlier Heidegger saw this kind of world-disclosure in terms of moods which were not simply 'subjective', but the later Heidegger, whose concern is precisely with non-instrumental forms of language, fails to appreciate how far this concern is inherently linked to music. It seems clear from these points that the relationship of aesthetics and subjectivity has to be re-examined in a way which invalidates quite a lot of the rhetoric directed against the subject in the traditions influenced by Heidegger. This becomes particularly apparent with regard to art's relationship to sensuousness.

It is, of course, odd that Heidegger, who wishes to bring metaphysics to an end, should adopt the antagonism of a Plato to the world of the senses in his view of music. One has, though, to be careful to specify what is really in question here. Part of the point of both Romantic and Idealist thinking was to escape the sensuous/intelligible divide and the problems to which it gives rise. The difficulty is that this escape can end, as it does for Hegel, with the self-negation of the transient sensuous world being seen as the route to philosophical insight into the intelligible absolute, an insight which devalues the sensuous as the 'merely transient'. Art, particularly music, disrupts this metaphysical idea by its reliance both on time and on sensuous particularity. A Bruckner Adagio can be heard as conveying something like 'noble sadness', but the banality of such a verbal description reveals what makes the shape and development of the particular movement significant. This particular development is unlike any other, and is not captured by the general terms used to characterise it. Without the sensuous particularity of the music, which is manifested in contrasting degrees of acoustic intensity and shifts of mood and temporality, a whole dimension of insight into the possibilities of temporal existence is lost. Given Nietzsche's desire to revalue the sensuous, by denying the possibility of a Hegelian 
Aufhebung of the particular into the conceptually universal, how does he regard music's relationship to metaphysical questions?

The fifth book of The Gay Science most clearly spells out the questions raised by music for the later Nietzsche. In Section 370 Nietzsche outlines his critique of Romanticism, by which he largely means German music, Schopenhauer and Wagner. Art is regarded here as a means of dealing with the contradictions of 'growing, struggling life'. Life necessarily involves suffering: if the suffering is a result of an excess of life, then a 'Dionysian' art is required, which involves acknowledgement of the tragic unavoidability of destruction if life is to remain creative. Romanticism is the result of the needs of those who suffer from the 'impoverishment of life, who seek peace, quiet, a calm sea, redemption from themselves by art and knowledge, or also intoxication, convulsion, numbing, madness' ( 3 p. 620 , cf. 6 p. 425). Romanticism therefore shares the attributes of metaphysics which lead one away from the immediacy of sensuous life here and now. How this sensuous immediacy is construed is the decisive issue. In Section 372, entitled 'Why we are not Idealists', Nietzsche considers the traditional antagonism of philosophy to the senses in a manner not so far from the $B T$ 's critical view of Socrates: "Wax in the ears" was at that time almost a condition of philosophising; a real philosopher did not hear life any more, in so far as life is music, he denied the music of life, - it is an old philosophical superstition that all music is music of the sirens' (3 pp. 623-4). In a characteristic reversal Nietzsche ponders whether the best thing to do is to acknowledge that 'Ideas' may be more dangerously seductive than that which appeals to the senses. An unwritten section of the projected but abandoned Will to Power is entitled, echoing Novalis, 'Value of "transience"' (13 p. 210), suggesting Nietzsche's opposition to Schopenhauer's metaphysics of art, which sees the only value in the escape from transience.

The later Nietzsche's positive investment in certain, usually Italian, music derives, therefore, from its freedom from the metaphysical attitude of Schopenhauer and Wagner, which he terms the 'religious need masked as music' (13 p. 210). His concern is with music which creates diversity rather than unity. In Section 373 of The Gay Science, "'Science" as Prejudice', Nietzsche attacks the notion that natural science's mathematisation of the cosmos is the only possible interpretation of the world, indeed he suggests that it may turn out to be 'one of the most stupid, that is, the poorest in meaning [sinnärmsten] of all possible world-interpretations'. He cites as evidence the fact that a scientific evaluation of music which relies upon 'how much of it can be counted, calculated, brought into formulae' would be absurd: 'What would one have grasped, understood, recognised of it! Nothing, almost nothing, of that which is really "music" in it! ...' (3 p. 626). Significantly, he leaves open the question of what 'music' is in such a context, rather than seeking to determine its essential nature.

The problem is now that if Nietzsche is to avoid the trap he thinks scientific accounts of music fall into, he has to find ways of circumventing the conceptual reduction which threatens any philosophical determination of the nature of music. This would seem to be one of the reasons why Nietzsche mixes a 
bewildering number of different ways of talking about music in the later work. In a note of 1884, for example, Nietzsche describes music as 'an echo of states whose conceptual expression was mysticism - feeling of transfiguration of the individual. Or: the reconciliation of inner contradictions into something new, birth of the third' (11 p. 75) - what might sound like a Hegelian dialectical reconciliation actually lies, though, outside conceptuality altogether. Elsewhere, in the notes of autumn 1887, Nietzsche can still claim, in line with his critique of the concept, that 'In relation to music all communication via words is brazen; the word depersonalises; the word makes the uncommon common' (12 p. 493). Music becomes a corrective to the view of language which begins with 'On Truth and Lie'. Such statements presuppose music's ability to say more than other forms of articulation, rather than being merely another form of articulation which, like verbal articulation, does what it can. Nietzsche's assumption in this passage would seem to point in a metaphysical direction of the kind suggested by Hoffmann and others. As we have seen, Nietzsche is fairly consistent in his demand that music should remain free of a metaphysics which would create the illusion of taking one beyond the sensuous world, rather than investing it with a new value. How can these differing views be sustained? The ambiguities here take one to the heart of his thinking.

\section{Aesthetics, 'interpretation' and subjectivity}

We need now to look more closely at what Nietzsche says about subjectivity and art in the later philosophy. A major tension in these writings derives from their shifting conceptions of the status of aesthetics. Most notoriously, Nietzsche claims aesthetics is 'nothing but an applied physiology' (6 p. 418), rejecting Wagner's music for its detrimental physical effects: 'And so I ask myself: what does my whole body really mant from music at all? For there is no soul ... I think it wants its relief: as though all animal functions should be speeded up by light, bold, lively, self-confident rhythms' (6 p. 419). The best music is that most suited to the organism, and 'aesthetics is indissolubly bound to . . biological preconditions' (6 p. 50). In such passages Nietzsche falls squarely into the same trap as many critiques of metaphysics. If the problem with metaphysical accounts of what there is derives from the attempt to provide an ultimate ground of explanation, it is no good seeking to find a further ground which will replace metaphysics by playing the same role in the same manner, because the results will be just as circular. In the passage cited Nietzsche grounds the explication of aesthetic ways of articulating the world on another way of articulating the world, biology. However, one can equally do the opposite, by arguing that the science of biology itself is really founded in 'aesthetic' production, as he himself argued in 'On Truth and Lie'. Just as nothing in a piece of music can explain the physiology of the organism which produces the music, nothing in biology can account for a piece of music qua aesthetically particular phenomenon. It is not that biology and music have nothing to do with each other, but neither can be the 
ground of the understanding of the other, without the resulting explanation simply failing to grasp what matters about what is being explained.

The vital issue here is rhythm, whose importance in some of the best writings on music, language and philosophy has been apparent at various stages of our investigation. Rhythm, Schelling's 'music in music', has the kind of inbetween status which keeps recurring in aesthetically decisive phenomena. There is, on the one hand, a sense in which nature can be said to function rhythmically, from cyclical phenomena, like day and night, to the heartbeat of some kinds of living organisms, but the idea that these recurrences can be apprehended as rhythm is not 'natural' in the same sense. The role of rhythm in the very intelligibility of the world of nature is the key question here. Schelling insisted that rhythm converted a non-rhythmic, meaningless succession into a meaningful one, and this points to a crucial constellation. Rhythm is both linked to cognition and to somatic pleasure, and is therefore a further instance of something which does not fit on one side of the sensuous/intelligible divide.

Self-consciousness and rhythm are, then, inseparably connected: natural rhythm cannot be such without what connects the moments of which it consists, and what can connect moments into rhythms cannot make something into something determinate without having something repeated to organise. ${ }^{14}$ This pre-conceptual identification of something as the same through the connection of its different moments is 'rhythmic' in Schelling's sense, because it makes arbitrary succession into something meaningful. The establishing of 'meaning' also relies, as we have seen, upon the kind of existential continuity of the self which the Romantics termed 'feeling'. The consequence is that it may not be abstruse to claim that language is derived from more fundamental rhythmic forms of identification, of the kind which are necessary at some minimal level for what we term music. Such an account already takes one well beyond mere biology, and is at the same time based on anthropologically plausible assumptions. The more germane question in the present context actually concerns the point at which music becomes a specifically aesthetic phenomenon, which, of course, takes one even further from the biological. Although one might think of music as Darwin and Edward Gurney do, as originating as a means of attracting a sexual partner, this does little to explain why one organism should want to create a means of stimulation for another in situations which are no longer immediately - or even potentially - sexual. This question leads to questions of communication and intersubjectivity, not to a supposedly biological reduction of the issue of the kind Nietzsche too often engages in. The genesis of something does not determine what it can become in new contexts.

At his best Nietzsche is aware of these objections. The section, 'Our new "Infinite"' of The Gay Science, which follows the passage mocking the pretensions of science to grasp music, relates to the refusal to reduce music to any evaluation in terms of something else. Nietzsche here presents a version of the Romantic notion of 'infinite interpretation'. The 'perspectival character of existence', the result of the loss of an absolute conception, Nietzsche argues, 
applies also to the human intellect itself, which can only see itself in terms of its own perspectives. This returns us to the questions of reflection which we have found in Fichte, Schelling and the Romantics. A perspective entails a splitting of two aspects - the 'view' from which something is surveyed, and the something itself. The structure involved here is the same as gives rise to the problems both of the correspondence theory of truth and of self-identification. What guarantees that a perspective is a correct one, unless there is an 'absolute perspective' which ensures the correspondence of the perspective to the reality of the scene, or an immediate identity between the two aspects of the self, of the kind Fichte sought in 'intellectual intuition', with all the resultant problems? An absolute perspective is not a perspective in the normal sense of the word at all - as Nietzsche himself will argue - and the Romantics reject Fichte's epistemological notion in the name of an ontological conception of the self which is always more than it knows, and which can therefore never achieve a final - cognitive or reflexive - perspective on itself. The overall result of this situation for Nietzsche is the anti-reductionist assertion that the world 'has again become "infinite" for us: to the extent that we cannot reject the possibility that it contains infinite interpretations in itself' (3 p. 627). In later texts Nietzsche claims that this infinity of interpretations is dependent upon the radical meaninglessness of existence, to which meaning must be given. Significantly, he still sees the idea initially in relation to music: 'That is the way it is with notes [Tönen] but also with the destinies of peoples: they are capable of the most various interpretation ...'(12 p. 359). This contention is evidently incompatible with the idea that aesthetics can be reduced to physiology, which is just one aspect of our perspectival relationship to the world and ourselves. The problem is then whether these perspectives are any more than essentially 'Fichtean' projections onto a world which therefore itself has no determinate conceptual effect upon what we hold to be the case. ${ }^{15}$

Nietzsche's ambiguous relationship to music is, then, a result of his veering between a biological reductionist view of aesthetics, a related desire to rid aesthetics of any trace of metaphysics, a residual 'Fichtean' projectionism, and an attachment to what are in fact anti-reductionist ideas from Romantic aesthetics. Any approach to Nietzsche must attempt to disentangle the commitments involved in these conflicting approaches and see how they might relate. Infinite interpretation, which Nietzsche often relates to music, is central to the later writings, and it is striking how often in the notes from 1885 onwards Nietzsche also echoes Idealist and Romantic views of subjectivity. One side of his efforts is directed towards the destruction of the notion of the unified subject in the name of a subject constituted in terms of the conflictual nature of the 'will to power', and this leads to his claims that a world which is stable or unified is another Idealist illusion. The subject is to be dislodged from a privileged legislative role as the location of intelligibility by the revelation of its inherent dependence upon an ungraspable Other, which is yet also itself. In many respects Nietzsche says little that is fundamentally new on the question of subjectivity: 
much that we observed in Schopenhauer recurs, though usually in affirmative, rather than negative terms. He does, however, take certain questions we have looked at a stage further.

In common with his epigones, Nietzsche identifies all philosophies of the subject with the metaphysics he wishes to overcome: 'What separates me most thoroughly from the metaphysicians is this: I do not concede to them that the "I" is that which thinks: instead I take the I itself as a construction of thought, of the same status as "matter" "thing" "substance" "individual" "purpose" "number": thus as a regulative fiction' (11 p. 526). Nietzsche's assertion echoes both Lichtenberg's claim that 'It thinks, one ought to say, as one says: it's thundering (es blitzt, literally 'it's flashing (lightning)'). To say cogito is already too much as soon as one translates it as I think. To assume the $I$, to postulate it, is a practical need' (Lichtenberg 1994 II p. 412), and Schelling's remark against Descartes (circa 1833-4) that 'It thinks in me, thinking goes on in me, is the pure fact, in the same way as I can say with equal justification: "I dreamed", and "It dreamed in me"' (Schelling 1994 p. 48), as well as echoing aspects of the critique of Idealism common to Feuerbach and Marx. This subversion of the reflexive subject is not, though, sufficient to obviate all the issues we have encountered concerning subjectivity: regarding the 'I think' as a moment of merely illusory self-grounding knowledge, for example, does not evade the problems of reflexivity. In Nietzsche's passage just cited, 'thought' must synthesise itself into a reflexively aware ' $\mathrm{I}$ ', otherwise there would be nothing to subvert, but it could only do so if it were already conscious in some nonreflexive sense: Schelling's 'It' is obviously not an inert object. Nietzsche's further claim in this connection is that language itself perpetuates the delusions of the metaphysics of subjectivity via the necessities of the grammar of subject and predicate. This idea is another version of his claims about the perspectival nature of existence: we think we see a world made up of Strawsonian 'sentenceshaped items', among which we include ourselves as the 'I that thinks', but this is because our language supposedly gives us no alternative.

Nietzsche contends that both the synthesising of that which thinks into the I, and the synthesising of effects from 'outside' into the object are really aspects of the will to power acting upon each other. Kant's 'transcendental unity of apperception', the I which 'must be able to accompany all my representations', therefore consists of a series of warring aspects, whose unity is a deception:

We need unities in order to calculate: there is no reason to assume for this reason that such unities exist. We borrowed the concept of unity from our concept of ' $\mathrm{I}$ ' . . . If we did not consider ourselves unities we would have never formed the concept of a 'thing'. Now, fairly late, we are thoroughly convinced that our conception of the concept of I does not guarantee any real unity. Nietzsche 1980 (13 p. 258)

Thought is therefore 'just a certain behaviour of drives in relation to each other' (3 p. 558), and "What we call "consciousness" and "spirit" is only a means and a tool by which not a subject but a struggle mishes to preserve itself' (12 p. 40). 
Self-consciousness is reduced to the self-preservation of quanta of the will to power which are opposed to each other (though why they should remain in this self-preserving relation of opposition is unclear).

The problem here is the one indicated earlier by Wheeler: Nietzsche has to rely on what he opposes, in order to dramatise his opposition to it. The 'real unity' whose existence he denies has to be the unity of a metaphysically conceived substance. For any unity to 'exist' in Nietzsche's terms means for it to be as real as what is, so to speak, 'really real'. In such passages he makes it very clear what is 'really real', namely the opposing 'drives' that constitute both thought and the I. All this means is that he proposes a Heraclitean ontology of difference, in opposition to a Parmenidean ontology of sameness. But what allows him to choose between what is 'really' a unity, and what is 'really' endless difference, without just re-running all the arguments about the Same and the Different from Plato to German Idealism and Romanticism? At this point Nietzscheans have a tendency to volunteer the argument from performativity, but this seems of little help. These remarks of Nietzsche cannot be construed as rhetorical strategies to undermine or circumvent the traps of metaphysics, because the assertions are themselves so patently metaphysical. They are remarks about the real ground of explanation of phenomena in the world. Unmasking conceptions of the I in postCartesian philosophy is a favourite contemporary pastime, but if the result is a theory which cannot begin to deal with inescapable questions about selfconsciousness - that require some kind of answer if such issues as self-deception, moral conflicts, aesthetic pleasure are even to be intelligible - all the unmasking achieves is a repression of the real issue, not a brave new approach.

Nietzsche's more reductive approaches suggest the power of the conceptions we considered in Romanticism, which do not consider the I to be an absolute ground. Schleiermacher's account of the I, for example, does not lead to a unified, self-transparent subject, but rather one which strives to attain a unity that is thwarted by the fact that what it is transcends what it can know. The subject's inherent lack of unity forces it into self-interpretation, which is impossible to complete, but which is not simple dispersal. If it were merely dispersal, basic facts of conscious life would become inexplicable. Nietzsche's justifiable denial that subjectivity could be a secure philosophical foundation for truth is questionably combined with his attempt to account for a subjectivity without which the point of his claims to undermine the notion of the I would dissolve.

His basic strategy is to posit an underlying true subject whose divided nature renders it inherently self-deceptive: the will to power. However, no consistent line is discernible in his approaches to this issue. At times, for instance, he gets oddly close to Fichte, though he wants to avoid Fichte's Idealism, in favour of a voluntarism of the subject, which also seems to echo aspects of Leibniz's Monadology: “"Thingness” is only created by us. The question is . . whether that which "posits things" alone is real ... The subject alone is provable: hypothesis, that there are only subjects - that "object" is only a kind of effect of subject on subject ... a modus of the subject' (12 p. 396). His attacks on subjectivity as 
a ground of cognitive certainty often echo both Fichte, and Schelling and Romanticism: 'One would like to know what things in themselves are like: but look, there are no things in themselves! But even supposing there were an in itself, an absolute [Unbedingtes], then it could not be known for precisely that reason! Something absolute cannot be known: otherwise it would precisely not be absolute!' (12 p. 141). ${ }^{16}$ Nietzsche also echoes the notion of the infinite activity which limits itself that Schelling develops from Fichte in the System of Transcendental Idealism: the idea of an object is 'the sum of the limitations [Hemmungen] experienced of which we have become conscious' (12 p. 98). However, he rejects any teleological development in this self-limitation of the will to power. The ascending genealogy of consciousness of the STI now becomes instead a genealogy of repression, the story of the growing obstruction of creative potential by science and morality because of their reduction of particular intuitions to general terms. At one point Nietzsche even adopts a central contention of the STI: "visible organic life and the invisible creative spiritual working and thinking contain a parallelism: via the "work of art" one can demonstrate these two sides most clearly as parallel' (12 p. 139). In true Romantic fashion, he also sees the world 'as a work of art which gives birth to itself' (12 p. 119). It may be that these remarks are just Nietzsche's working through of others' positions in his notes, but the question then is what he has to offer as an alternative.

In the wake of Kant's Critique of Judgement, Schelling presented aesthetic production as a way of overcoming the limits of the understanding and thereby opening up a relationship to nature that is lacking when nature is seen solely in terms of objectifying empirical science. Nietzsche, in contrast, starts from the premise of the radical meaninglessness of existence, of the kind familiar from the materialist science of his day. However, the way he uses this premise again falls prey to Wheeler's objection of nostalgically presupposing a metaphysical world in order to confront one with the consequences of its absence, rather than accepting that this whole picture is based on the bewailing of a deficiency that is a necessary deficiency in every case, and so a deficiency only relative to an impossible dream' (Wheeler 2000 p. 118).

In The Gay Science Nietzsche formulates a defiant challenge: the universe 'is neither complete, nor beautiful, nor noble, and does not wish to become any of these, it absolutely does not strive to imitate mankind! It is not touched at all by any of our aesthetic and moral judgements' (Nietzsche 19803 p. 468). Such judgements are, he thinks, still tinged with theology, and he asks, echoing the young, Feuerbach-influenced Marx: 'When will we be allowed to begin naturalising ourselves with the pure, newly found, newly redeemed nature' (3 p. 469). However, the real question is whether it makes sense to invest so much in the idea that nature is not 'per se' beautiful, apart from in terms of countering a theological approach to nature. As we have seen, the beauty of nature is clearly affected by history, but the fact that nature can be beautiful in differing ways at different times suggests there is more at issue than this radical subjective/objective split. 
New encounters with nature can shake us out of rigid conceptions or lifeless ways of being. This need not simply be a result of our projections, even if there may be no strong metaphysical arguments available which would allow one to invoke Kant's 'code through which nature talks to us figuratively in its beautiful forms'.

In the light of the growing ecological crisis, it seems clear, moreover, that an aesthetic failure on the part of humankind does indeed touch nature. It does so, for example, when no account is taken of the effects of human action upon the natural world, of the kind observable in the wake of Chernobyl or of other manmade environmental catastrophes, which distort any kind of ecological integration in ways of which nature on its own seems to be incapable. Schelling's point was always that we are ourselves part of nature, and that the question is therefore how to conceive of the subjective, the locus of our judgements, as not totally separate from nature. Frank has made the main structure of his argument clear: 'Instead of saying that nature is mind (which would be absurd), one must rather say: there is an $\mathrm{X} \ldots$ and this $\mathrm{X}$ is on the one hand nature and on the other hand mind (these would be the predicates of $\mathrm{X}$ ); but that does not mean that mind therefore is as such nature, or nature is as such and in the same respect mind' (Frank 1991 pp. 143-4). Nietzsche simply concentrates on attacking a representational notion of knowledge and beauty which his Romantic predecessors already suggested was inadequate to these issues. Once the representational notion has been put into question, appealing to it in order repeatedly to show its failure is both nostalgic and actually likely to obscure new ways of thinking about our place within nature.

It is perhaps important to add that none of these objections to Nietzsche involves a denial of the essential indifference of a nature which will destroy the species humankind when the sun explodes and which will in all likelihood decline in the end into entropy-generated stasis. However, if Nietzsche is serious about the 'value of transience' his real problem ought to be the consequences of failing to see what nature has to offer us, not nature's now pretty firmly established ultimate futility as far as the human race is concerned. Once again the question is Dews's 'limits of disenchantment'. The point of the aesthetic tradition prior to Nietzsche was that it sought resources in new relationships between 'internal' and 'external' nature. The Idealist version of this search can, it is true, entail indefensible claims to commensurate mind and nature. Does this mean, though, that there is no longer any possibility of rethinking the consequences of living in a deterministic nature which, not least because we are part of it, is not fully grasped by exploration of its law-bound aspect?

By the time of the work on The Will to Power Nietzsche's view of art's relationship to nature becomes more cynical, but the significance of the aesthetic has actually become even greater. Art has come, in the manner of the $B T$, to include science and philosophy of every kind, but not in a higher synthesis which would integrate us into nature, as proposed by the System Programme or 
the Discourse on Mythology. Instead: 'man must by nature be a liar, he must more than anything else still be an artist ... And he is too: metaphysics, morality, religion, science - All inventions of his will to art, to lie, to flight from the "truth", to the denial of the "truth"' (Nietzsche 198013 p. 193). Art is still the stimulus to life in the face of a meaningless existence. The problem, of course, is what the 'truth' is that is being denied. At such points it seems clear that Nietzsche is actually revelling in the performative contradiction of making claims about something his argument precludes, but if that is the case, one still needs to understand what the performative effect might be. Two strands of Nietzsche's thought emerge here. One is 'deconstructive', attempting to overcome metaphysical claims by undermining the possibility that they could ever be an absolute foundation for our truth claims beyond our own practices of validation. The other, the concern with the will to power, is metaphysical, even though the aim may be deconstructive: this becomes apparent in what Nietzsche says about 'interpretation'.

It is worth remembering here that Nietzsche's view of language has a deeply questionable element which is apparent in claims like the following. In Beyond Good and Evil, for example, Nietzsche maintains that 'the spell of certain grammatical functions is in the last analysis (im letzten Grunde) the spell of physiological value-judgements and racial conditions': as such, 'unconscious domination ... by the same grammatical functions' determines what can be thought by a thinker of a particular race. It is therefore no surprise that Nietzsche's 'interpretation' is founded on an agonistic view of nature, of the kind we encountered in Schopenhauer. 'Interpretation' is, as it must be, given Nietzsche's ontological assumptions, a power struggle over identity, which takes place both in the natural and the cultural world: 'The will to power interprets: in the constitution of an organ it is a question of an interpretation' (12 p. 139). 'Interpretation' depends upon power differentials, in which one power subordinates another. The plant subordinates the materials it needs to constitute its identity, mankind constitutes nature as the object of its fight for self-preservation, and the successful artist, scientist, politician, race (?) constitutes the chaos of a meaningless world in forms according to their will. Crucially, Nietzsche separates 'interpretation' from subjectivity by again suggesting that it is grounded in something that transcends it, which he, of course, is therefore able to identify: 'One may not ask: " $w h o$ is interpreting then?" rather interpretation itself, as a form of the will to power has existence (but not as a 'Being', rather as a process, a becoming) as an affect' (12 p. 140). Interpretation is, then, an activity of the will to power, and the truth from which we fly when we invoke 'metaphysics, morality, religion, science' is the underlying nature of being as will to power.

However, Nietzsche himself suggests a problem in this conception of interpretation as a series of power differentials: 'Simple differences in power could not feel themselves as such: there must be a something there which wishes to grow, which interprets every other something that wishes to grow in terms of its value' (12 p. 140). The characterisation here must, then, be of a kind of subject. 
It is something which must be able to feel itself as limited and as able to develop. This drive for greater power only makes sense if that which wishes to grow has, however minimally, a self-conscious identity beyond its mere difference from the other, otherwise the growth in power could not be registered as a growth in its power and would have no motivating force. Furthermore, the associated idea of a 'transvaluation of all values' that will result from the destruction of metaphysical goals and the acknowledgement of being as will to power also requires something for which a value is a value, rather than a stimulus for a natural instinct. Value cannot, as Nietzsche himself makes clear, be interpreted merely in terms of relations between differing quanta of the will to power, of the kind which might be used to describe the lion's relationship to the value of the lamb. Unfortunately, he has little useful to say about what a value might be that is not subject to that kind of reduction. We saw in Chapter 4 that a Hegelian response to the development of ethical consciousness via the growing acknowledgement of the autonomy of the other is faced with the problem of how one could be aware of the freedom of another at all without one already being pre-reflexively familiar with freedom oneself. Schelling's middle work in particular tries to come to terms with the contingency of the development of freedom, which cannot be grounded by reflection in the other, but this does not lead him into the kind of reductive physicalism present in many of Nietzsche's remarks.

Now some of this might seem unfair to the Nietzsche who has been enthusiastically adopted by those, like Rorty, who regard him as a resource for pragmatism. The simple answer to this worry is that the deconstructive Nietzsche of the theory of 'infinite interpretation', who comes closest to the anti-essentialist strand of the Romantics, too often seems to rely on the Nietzsche of the will to power, who advances the metaphysical argument that the ground of the understanding of the manifest world are different quanta of will to power. What else can one infer from the remarks on 'interpretation'? Rorty sees Nietzsche as part of the crucial idea, directed against the metaphysical tradition, that we 'must give up the idea that we are answerable to anything other than ourselves'. We saw one possible objection to this in Chapter 5, with regard to the idea that natural beauty can be understood as an indication of a responsibility to something beyond ourselves, rather than merely as a moment of 'private transcendence'. Although not being answerable to anything beyond ourselves can be construed in terms of a defensible attempt to evade commitment to the pursuit of the Platonic goals of much of Western philosophy, it can also involve the danger of a narcissistic relationship to things which the best of the aesthetic tradition, from Hölderlin to Adorno, regards as one of the main threats in modernity. Nietzsche's idea of the 'intoxication' of the creative artist, 'the increased feeling of power; the inner compulsion to make of things a reflex of his own fullness and completion' (13 p. 356), contrasts with what motivated artists such as Hölderlin and Beethoven. They were fired, not by a narcissistic relation to things and people, but by a desire to create new possibilities of non-repressive community by transcending existing means of articulation. 
Nietzsche's conception actually makes aesthetics highly dependent upon the subject: the idea of transforming things into a reflex of oneself is, of course, an echo of a familiar (if unjustified) construal of Fichteanism. Nietzsche's attachment to the autonomy of music, which is directed against a narcissistic view is, of course, at odds with the reduction of aesthetics to 'intoxication'. Just how arbitrary his mixing of conceptions can become is evident when he returns elsewhere to reductionist claims: 'Keep in mind that every art which has physiology against it is a refuted art ... One can refute Wagner's music physiologically' (13 p. 471). However, if the value of music is assessed physiologically, it is no longer autonomous, but rather a means towards the well-being of the organism. Aesthetic pleasure becomes just pleasure, and art becomes no different from a tasty soufflé. Nietzsche's essential objection to Wagner's music was that it was in the service of something else; now the objection is that it fails to serve the body in a healthy manner. He just does not like the effect Wagner has on him any more, and aesthetic judgement is a question of personal (physiological) preference - if Wagner makes you feel more powerful and in control, he can presumably be 'proven', rather than refuted. Nietzsche, by this time, happens to feel better listening to Bizet.

Despite their mutual inconsistency it is important to remember that all these differing assessments of music can actually play a role in how people relate to music and how it affects them. To this extent Nietzsche's refusal to remain with a particular approach may be said to be in line with a pragmatism which sees different ways of talking about something in terms of making it a tool for differing purposes. Whether this is an adequate response to the major questions of aesthetics can, though, be seriously doubted.

On the one hand, Nietzsche seems to sustain an aesthetic concern with art's autonomy as a vital aspect of modern culture, and this is in line with his consistent attention to music. On the other hand, this concern would at the same time appear to be something which, in the terms he proposes elsewhere in these texts, is susceptible to unmasking via the idea of the will to power. Any judgement about aesthetics must be referred to the condition of possibility of differentiation, the will to power. A positive aesthetic judgement will increase the quantum of power, a negative judgement will diminish it. This actually tells one nothing at all about the aesthetic judgement qua specifically aesthetic judgement, because the same kind of differentials constitute the way we apprehend or deal with the rest of reality, as the conception of 'interpretation' suggested. The metaphysical foundation therefore drops out of the equation in dealing with actual differences between our 'interpretative' practices, because its implication is the same whatever happens. Any attempt to sustain this foundation would draw one back into the familiar circle in which the judgement that the will to power is the correct explanation of the phenomena is itself determined by a greater quantum of will to power than the alternative. If one accepts that the metaphysical part of Nietzsche's conception is redundant, it seems possible then to adopt a pragmatist approach which seeks to assess the different kinds of 
purpose served by aesthetic production and reception in relation to other kinds of relationship to the world. Nietzsche can often be very profitably read in this latter manner, though one wonders whether the more than questionable aspects of his work one must ignore in doing so should not give his supporters more pause for thought than they usually do.

The problem with this short-cut to a post-metaphysical account of aesthetics is that it fails to deal adequately with a crucial issue which has emerged at various points in this book. The two terms aesthetics and subjectivity already suggest what is in question here: one of the connotations of 'aesthetics' is of a publicly accountable philosophical discipline; 'subjectivity' often has the connotation of something private. Rorty wishes to separate 'public' discourses of social cooperation from the 'private' search for transcendence, not least because he justifiably thinks the attempts of thinkers like Heidegger to make the latter into the former can be disastrous. At the same time, the culture and philosophy of modernity is characterised precisely by attempts to combine the 'public' and the 'private' in some kind of new synthesis. In the light of the story told in this book, Rorty's judgement on this issue seems too schematic, as I try to show further in the Conclusion. While some of the key insights in modernity have undoubtedly been into the ways in which the public, in the form of the symbolic orders into which we are socialised, constitutes what we are 'privately', the desire to transcend the forming impact of language, history and socialisation, in order to attain an irreducibly individual identity, is often regarded as vital to being human and as the source of the imaginative resources which sustain a post-theological culture. The further need for this identity not to become merely a way of repressing the cultural other has also become a decisive part of the debate, as it had already begun to be for thinkers like Herder, Schleiermacher and the early Romantics. Tensions between these aspects of modernity have been a major factor in the aesthetic reflections of the thinkers we have considered so far. In the Conclusion I consider these issues in relation to contemporary theoretical concerns, some of which have been sparked by the renewed attention to Nietzsche's questions concerning a post-metaphysical culture.

Notes

1 The pieces by Holub are the following: 'Nietzsche and the Jewish Question', New German Critique 22.3 (1995): 94-121; ‘Nietzsche and the Women's Question', German Quarterly 68.1 (1995): 67-71; 'Reading Nietzsche as Postmodernist: Rhetoric, Genealogy, Perspectivism in Ahistorical Context', Why Literature Matters: Theories and Functions of Literature (eds) Rüdiger Ahrens and Laurenz Volkmann. Heidelberg: Carl Winter (1996): 248-63; 'Nietzsche's Colonialist Imagination: Nueva Germania, Good Europeanism, and Great Politics', The Imperialist Imagination: German Colonialism and Its Legacy (eds) Sara Friedrichsmeyer, Sara Lennox and Susanne Zantop. Ann Arbor: The University of Michigan Press (1998): 33-49; 'The Birth of Psychoanalysis from the Spirit of Enmity: Nietzsche and Psychology in the Nineteenth Century', Nietzsche and Depth Psychology 
(eds) Jacob Golomb, Weaver Santaniello and Ronald Lehrer. Albany: SUNY Press (1999): 149-169; 'Understanding Perspectivism: Nietzsche's Dialogue with His Contemporaries', (to appear in a Festschrift for the 100th birthday of Hans-Georg Gadamer, edited by Ulrich Arnswald and Jens Kertscher in MIT Press); 'The Pen Is Mightier Than the Whip: Nietzsche's Confrontation with the Women's Question', (to appear in German Quarterly); 'The Elisabeth Legend or Sibling Scapegoating: The Cleansing of Friedrich Nietzsche and the Sullying of His Sister', (to be published in Nietzsche: Godfather of Fascism?: On the Uses and Abuses of Philosophy (eds) Jacob Golomb and Robert S. Wistrich (Princeton: Princeton University Press)).

2 This problem admittedly can apply to any text, but which is more central in relation to Nietzsche than many authors because of the history of his reception.

3 Schopenhauer seems to have been most influenced by Schelling's 1809 essay on human freedom, which claims that 'Willing is primal being' (see Bowie 1993 chapter 5).

4 It is worth remembering that Schopenhauer was at the same time a misogynist and an animal lover. The reason why his thought caught on in the wake of Darwin is also apparent here.

5 It is arguable that this view of the sensuous world corresponds to one aspect of Hegel's conception of philosophy, if one takes the links of Hegel to Plato seriously.

6 A combination of Marx's conception with that of Nietzsche will be the basis of Horkheimer and Adorno's Dialectic of Enlightenment.

7 This is one of the key themes of Adorno's Wagner book.

8 This does not mean that they are somehow mysterious: there are plenty of significant interpretations which account for tragedy's importance in certain historical situations. The problem is how they continue to appeal in differing contexts.

9 See Wolfgang Lange (1983): 'Tod ist bei Göttern immer nur ein Vorteil. Zum Komplex des Mythos bei Nietzsche' in (ed.) Bohrer, pp. 111-13.

10 Nietzsche's idea of how science leads to a regress of explanations seems to derive from Jacobi's idea of the nihilistic world of 'conditioned conditions' which requires a further principle of coherence to be intelligible (see Bowie 1997).

11 Cf. also the remarks on Kant and schematism's relationship to rhythm in Chapter 1.

12 As we saw in Chapter 7, if anything, Romantic philosophy is a reaction against the idea of music as the representation of feelings.

13 Davidson has argues that this gets things the wrong way round, conventions only being possible on the basis of language.

14 Kant's insistence that concepts require intuitions if they are not to be empty, and intuitions concepts if they are not to be blind would, in this conception, be a subsequent development of this more fundamental idea. I think this is what Schlegel and others were suggesting in the remarks on rhythm cited in earlier chapters. See also Bowie 2001.

15 This is a more than questionable reading of what Fichte means, and just refers to the most extreme Idealist formulations of the 'positing' of the I. When Rorty says we are tied to the world causally but not representationally he moves in this direction, as his attachment to Nietzsche would suggest, though he is in certain respects closer to the Romantics.

16 Cf. my remarks on perspectivism above. 


\section{CONCLUSION}

The difficulties involved in giving an account of the contemporary significance of the 'aesthetic tradition' from Kant to Nietzsche become apparent when one considers phenomena such as the following. ${ }^{1}$ It might, for example, seem surprising that many of the thinkers who enthusiastically pursue a postNietzschean undermining of the illusions and repressions they associate with 'Western metaphysics' still have a considerable investment in art and in philosophical reflection on art. A radically anti-metaphysical view of art is in some respects more congruent with the idea that art itself is now something whose very existence has been put in doubt by various manifestations of the avantgarde from Duchamp onwards, and by the anti-essentialist, radically historicised temper of much recent thinking. The fact is, though, that questioning of art as, say, a form of ideology is present more in aspects of Cultural Materialism and New Historicism than in many philosophically oriented approaches to the end of metaphysics. It is therefore not surprising that, despite his desire to circumvent metaphysics, Rorty has, in Romantic philosophical vein, criticised such recent theory as 'part of the latest attempt by knowing philosophers to gain supremacy over inspired poets' (Rorty 1998a p. 138). ${ }^{2}$ Or as Schlegel once put it: 'If the chemist thinks a thing is not a whole because he can dissect it, that is just the same as what bad critics do to literature. - Didn't the world emerge from slime?' (Schlegel 19885 p. 48). A further complication here is apparent in the way that suspicion of the notion of art is accompanied, in the wider culture of capitalist modernity, by large-scale economic and cultural investment in many forms of art, including, of course, avant-garde 'anti-art' itself.

If all this seems rather confusing, now consider the following very different aspect of contemporary thought's relationship to questions of subjectivity, of the kind which I have tried to show are inextricably linked to aesthetics. Philosophers who regard aesthetic and other culture as part of the realm of 'folk psychology' - by which they mean our everyday ways of thinking about ourselves and our minds that cognitive science is supposed eventually to replace with a physicalist explanatory theory - share a rejection of any major philosophical role for the subject with Nietzsche-influenced, post-Heideggerian antimetaphysicians, such as Lyotard. However, these latter thinkers are, at the same time, precisely the ones who regard art as a crucial counter to the 'subjectification of being' characteristic of the 'Western metaphysics' which Heidegger came to equate with the modern technologically-oriented natural sciences upon which reductive physicalists base their assumptions about folk psychology (see Bowie 1999a, 2000).

On the one hand, then, philosophers who wish to deconstruct any grounding role for the subject, as part of a general move away from the 'history of metaphysics', often still see in art something decisive which is not accessible to 
philosophy or science; on the other, physicalists concerned to overcome folk psychology tend to regard the sciences, which increasingly obviate many questions that were formerly thought of in metaphysical terms, as making accessible the true world that is inherently inaccessible to art. The recent revival of interest in the Idealist and Romantic philosophical traditions presented in the present book is in part a result of the perception that both the Heideggerian and the physicalist ways of thinking about the subject are inadequate as interpretations of the subject's role in accounts of modernity. Ernst Tugendhat has claimed that 'The right path for modern philosophy between romanticism and positivism is yet to be found' (Tugendhat 1992 p. 432), and the contrasts just described make this very apparent, even as they reveal the bizarre alliances which can result between the extremes of these differing paths. The seeds of the contrast between 'romanticism' and 'positivism' are, of course, already sown in the period we have been considering. Fears about the nihilistic consequences of Spinozist determinism in the wake of the Pantheism controversy from the 1780s onwards (see Beiser 1987, Bowie 1997) are in many respects analogous to fears about the nihilistic consequences of contemporary scientism, and the aesthetic responses of Schelling and the Romantics to Spinozism are echoed in aspects of Heideggerian and post-structuralist views of art as the counter to the dominating nature of modern science and technology, the key manifestations of 'metaphysics'. ${ }^{3}$ The changing role of music from the period of the Pantheism controversy onwards also begins to raise questions about the primacy of representational thinking and representational conceptions of language which have become part of mainstream contemporary philosophy in the form of ideas about language as social practice and as the basis of the world's intelligibility.

What is really at issue here, and was in many respects already at issue for Kant and his successors, are the kinds of philosophical response to modernity which can steer a course between the increasingly powerful claims of science, and the cultural needs of the life-world. Karl Ameriks has talked in this respect of the contemporary failure to 'bridge the gap between private idealistic visions and an analytically rigorous but narrow focus on the latest scientific developments' (Ameriks 2000 p. 268). Why bridging this gap matters can begin to be made clear by the following, and will concern us in the rest of the Conclusion. One of the consequences to be drawn from the Romantic heritage is that claims that there will eventually be a scientific explanation of the most fundamental and puzzling aspects of human existence can still be met with the following rejoinder. Even if cognitive science, for example, were to come up with a widely accepted exhaustive law-based explanation of the nature of self-consciousness, this would, as Dieter Henrich has argued, still leave open unavoidable questions about how to integrate this explanation into the forms of our self-understanding which cannot be reduced to this kind of explanation. Such forms are, of course, encountered not least in the aesthetic domain. In the same way as the ways of thinking which lead from myth to art are not wholly obviated by scientific discoveries that explain in law-bound terms what was previously 
explained in stories and images, forms of self-understanding that do not and could not have scientific status remain vital to one's ability to make sense of and inhabit one's own world. To this extent, one can also question theories in the philosophy of mind which aim to provide a definitive account of the structure and nature of self-consciousness. The link between aesthetics and subjectivity can be used to ask whether such theories might be bought at the expense of obscuring aspects of self-consciousness, which, for instance, only become comprehensible through the experience of musical production and reception.

The reductionist assumptions that lead to the idea of folk psychology themselves involve serious methodological problems which are shown up by arguments from the aesthetic tradition. As we saw, Schleiermacher first made the hermeneutic point that the everyday language that we need to begin to do science cannot itself be reduced to a subsequent grounding scientific explanation. An explanation of this kind will itself always already rely on a prior understanding of everyday language that is not simply based on learnable rules. If the explanation were supposed to be solely rule-based, it would be faced with another regress of rules for rules. This argument logically precludes the kind of wholesale explanation of language proposed in the more extreme forms of reductionism. It does so, of course, in terms of an idea which is essential to thinking about art, and which led Schleiermacher to term interpretation an 'art', namely that creation and understanding of art cannot be achieved solely by following rules. Similarly, ideas about the role and nature of self-consciousness from Kant to the Romantics suggest that attempts to explicate subjectivity in the terms used to explain objective nature will themselves fall prey to the problems of reflection. The dimension of the self required to make even scientific claims intelligible, that Kant tries to capture in the transcendental deduction, cannot be reduced to a further explanatory substrate of the kind demanded by those who employ terms like folk psychology to conjure away questions of self-consciousness. This is because, as Frank Farrell insists, in line with the Kantian tradition: 'intentional directedness toward the world is not something that can be added on after we have given a causal account of intentional states nonintentionally described' (Farrell 1996 p. 62).

It will already be apparent that such discussions very rapidly take one into a whole series of specialised debates engaged in by very different branches of contemporary philosophy. What began as, at least in some respects, a unified series of questions has, in the manner of the development of the natural sciences, splintered into specialised disciplines which too rarely communicate with each other. Questions arising from Kant's transcendental deduction are now dealt with in increasingly sophisticated ways in the philosophy of mind, and debates about language in contemporary semantics offer ever more complex reflections on the possibilities of using language to analyse itself and its relations to the world. In the light of such work, where answers to questions, for example, about the temporal continuity and self-knowledge of the I become the source of endless renewed controversy, reference to the historical sources and consequences of the 
issue, and to links between the issues, often may seem irrelevant to the philosophical problem at hand. However, this very specialisation and concentration is not self-legitimating. The tendency in analytical philosophy to regard philosophical problems as being amenable to answers achieved by rigorous circumscription of the specifics of an issue is in many ways a result of the influence of the natural sciences on modern philosophy, which may not always be of advantage to philosophy. If one looks at the actual history of many such problems, the internal rigour of argument about a problem has often been bought at the expense of omitting perspectives on the problem which might preclude such rigour.

This seems pretty clearly to have been the case in a variety of areas, such as the following. The analytical philosophy of language excluded the holistic hermeneutic insights of the nineteenth century for a long time, only for them to return with a vengeance in the wake of Quine, Davidson and others (see Chapter 6 above, Bowie 1997a, Schleiermacher 1998, Wheeler 2000). The exclusion of Kant from mainstream analytical philosophy in America for many years has now been answered by the remarkable resurgence of interest both in Kant and his most impressive defender in the analytical tradition, Wilfrid Sellars. This interest is based in part on the kind of arguments which Fichte and others employed against attempts to explain the self in the terms used for the world of objects, which thinkers like McDowell now employ against 'bald naturalism' (see Bowie 1996). Analytical aesthetics, which until very recently spent much of its time narrowly obsessed with things like the ontological status of 'the work of art', has been undermined by the demonstration, in the light of the work of Wittgenstein, Adorno and other Romantic-influenced thinkers, that the notion of 'work' in question is a recent historical product whose ontological status is inseparable from considerations of its changing social status. In this perspective, merely reviving the detail, context and effects of some of the questions from the aesthetic tradition may actually be a means of avoiding the kind of narrowing of philosophical focus in which amnesia has too often been mistaken for rigour. In the last two hundred years, there has clearly been a descent from the higher ambitions of the thinking about art encountered in Hölderlin, Schelling or the early Nietzsche. Whether this descent need only take the form of the growing specialisation described above is, though, far from clear. These remarks are not intended to diminish the philosophical importance of, for example, the growth of specialised attention to the Kantian and post-Kantian traditions, which has produced some outstanding work. They are intended instead to open up questions about the role and effects of philosophy in contemporary society in relation to issues raised by the aesthetic tradition.

The aims of Kant, Hölderlin, Schelling and others were social and political, as well as philosophical, and this posed the question we encountered in Chapter 2 , of how philosophical ideas - 'ideas of reason' - could be communicated within society as a whole. ${ }^{4}$ Aesthetic thinking in this tradition seeks ways of integrating different kinds of experience, thereby revealing new ways to relate to a 
no longer 'dogmatically' conceived nature, and this is why it inevitably leads to questions about the subject. The crucial aspect of the subject here is the fact that what it knows of itself and what it is can never be said fully to coincide. Some recent theorists, particularly post-structuralists influenced by Nietzsche, have tended to regard any attempt at a unified understanding of the subject as somehow nostalgic, as though the kind of attempt to come to terms with the divided nature of self-consciousness encountered in Hölderlin, for example, were a repression of some other more authentic possibility, or were politically questionable. However, as we have seen, questions about the 'decentred' subject can only emerge at all in relation to what makes the divisions in the self significant, and this entails some kind of unity, of the kind suggested in Schleiermacher's arguments concerning the existential continuity of the I. A subject that lived simply for ever new moments of intensity would not, for instance, have access to a new mode of existence by being freed from repressive forms of identification: new experience and differing degrees of intensity of experience require something continuous between their moments for them to register as new, or more or less intense at all. Aesthetic experience requires both immediacy and mediation, and it is the relationship between the two in accounts of the subject that gives rise to some of the most interesting and difficult theoretical problems, as the contrast between Hegel and the Romantics made clear.

How these questions affect contemporary political and social dilemmas has rarely been adequately addressed, because too many recent theories of contemporary culture have relied on a model in which power or the symbolic order is the ultimate determining factor in the constitution of modern subjectivity. Although insertion into language does undermine the notion of a purely spontaneous, 'immediate' self, the mediation of the self by language does not per se, as Schleiermacher shows, obviate the possibility of linguistic innovation and selfdetermination within - and possibly beyond - that language. This possibility cannot be circumscribed by a prior theoretical description, because the description would itself have to be given in terms of already existing linguistic resources, thus missing what is most essential about the possibility itself, namely that it involves more than merely random innovation. Justified philosophical suspicions of a strong Cartesian construal of the $I$ as the ultimate foundation of knowledge should, then, not become a licence, as it has seemed to in some recent theory, to regard all conceptions of self-conscious identity and spontaneity as, for example, part of 'metaphysics' as the history of the 'subjectification of being' ${ }^{5}$ This view of self-consciousness may be a possible response to some interpretations of the Idealist tradition from Fichte to Hegel, and to its effects on foundationalist projects in modern philosophy (see Ameriks 2000). However, this response fails to address the counter-tradition in Romanticism which does not see the I in strong foundationalist terms, even though the Romantic I still has an ineliminable role in accounting for the nature of cognitive, moral and aesthetic experience.

Part of the problem here lies in the conflicting interpretations of the importance of philosophy in modernity. The Heidegger-Gadamer tradition, 
for example, links the rise of aesthetics, as one aspect of the history of 'subjectification', to the dominance of the 'method' of the natural sciences as another, in order to try to show how a different kind of truth happens in art which cannot be reduced to an account in terms of the role of the subject. The argument relies precisely on the assumption that the totalising, strong Idealist, construal of subjectivity, of the kind they see as present in modern technology, is the essence of this period of Western philosophy, and thence of modernity. The consequence is an equally totalising counter position which ignores conceptions in which the subject is neither a principle of domination nor the locus of merely arbitrary feelings. The Heideggerian position could only work as the essential account of subjectivity in modernity if Heidegger's philosophical story could be shown to be the real ground of the historical development of modernity. Although the increasing demand for control of the social and natural Other surely is one necessary basis for understanding the links between modern technology and the global expansion of modern capitalism, it is not sufficient to establish the kind of links between philosophy and history that Heidegger wishes to construct via his interpretation of the moves from Descartes to Nietzsche and beyond. It may be a valid enterprise to try to establish that such links are decisive, but work on looking at how one might translate between philosophical stories and the concrete development of history does not seem to be a notable feature of Heideggerianism. ${ }^{6}$

The problem is that there are very differing levels involved in any investigation of modernity and the subject. On the one hand, these levels require careful separation, so that epistemological, historical, political, psychological and other questions are not simply conflated. On the other, we need ways of translating between the differing levels, in order that the questions do not become so specialised and disconnected that they can have no serious practical impact on our self-understanding. The sheer difficulty of agreeing what the crucial aspect of questions about subjectivity and modernity really is should, though, make one wary of the hasty desire to 'subvert' the notion of the subject, as if any conception of the possible integrity of the subject, for instance in ethical matters, were inherently repressive. What is clear is that these debates do still have an important socio-political aspect which can only be articulated in an effective manner if one keeps in mind the danger of a splintering of the issues into specialised philosophical questions. Rorty is right in this respect to suggest that the novel may, for example, sometimes be a better place for the investigation of and response to these issues than professional philosophy.

My insistence on the significance of music has a similar source. At a time when at least the quantitative role of music in cultural life has probably never been greater, ${ }^{7}$ music rarely plays even a minor role in most contemporary philosophy. Questions can therefore be asked about such philosophy's failure to engage with imaginative resources that must be inseparable from its own aims. Beethoven's heroic middle period works may, as Bahro suggests (see Chapter 7), offer many people a better way of understanding some of the changes in the 
notion of the subject in Beethoven's time than an abstract philosophical account of the nature of the I. Clearly a verbal framework is required for such understanding, but the investment of that framework with semantic force that can change people's whole relationship to the issue leads in the direction of affective and other dimensions of communication, which are not merely propositional, and which may therefore be better articulated by music. The danger here is that in seeking to be reflexive about philosophy's connection to social and cultural life, the attempt to pursue issues with analytical rigour suffers; at the same time, however, in seeking analytical rigour, the endless task which tends then to open up offers too little substantive insight into what matters to people about the issue in question. This dilemma is, of course, one of the reasons why aesthetics has become an essential part of some recent attempts to re-think the direction of philosophy. Art, as it did for the Romantics, becomes a reminder of what dominant forms of philosophy may obscure.

What seems necessary now is to seek a balance between the internally rigorous exploration of a specific issue and the attempt to see how this issue fits into a broader picture of the nature and effects of our self-descriptions. In the preceding chapters I have, while outlining some often neglected approaches to philosophy and art, tried to suggest the strengths of some of the more technical arguments about the nature of self-consciousness that emerge in Kant, and as a response to Kant, in Romanticism. Any such presentation gives rise to problems, either of a lacking analytical rigour occasioned by the need to expound ignored positions in some detail, or a lack of historical perspective occasioned by the concentration on argument at the expense of context. The most important arguments are those which reject reductionist, objectifying accounts of subjectivity, and these have proved to be the most durable legacy of the Idealist tradition. At the same time, the Romantic arguments we have considered do not, in the main, fall prey to the Idealist tendency to make self-consciousness into the new foundation of philosophy. These arguments lead to the difficult task of defending that strand of thinking, already beginning with Rousseau and Kant, which keeps a role for the irreducible 'feeling' of the subject as that which is not merely subsumable into cognitive or ethical articulation, but which cannot be ignored if crucial questions about the self, of the kind revealed by music, are not to be overlooked. This is the most problematic aspect of my account of aesthetics and subjectivity, and the arguments I have presented do no more than begin to sketch a route to a more adequate account than is present in some forms of contemporary philosophy. I hope, though, that by attempting to mix argument and contextualisation I have offered an example of how conflicting interests may yet give rise to useful insights for future research.

I want in a moment to return to the issue of the relationship between public and private, with which Chapter 8 concluded. This issue can connect many of the problems of the aesthetic tradition which have returned to haunt contemporary philosophy. Before doing so, the following perhaps rather obvious point needs making, which has so far been dealt with somewhat too indirectly. The 
tradition we have examined establishes conceptual models whose durability has been proved by their again becoming the focus of contemporary debate. However, the world in which these models originated, and our world, are separated by such massive social, historical and aesthetic changes that any continuity of the substantive content of the issues seems threatened. Whereas art has, for example - at least until the beginnings of modernism with Baudelaire and others in the middle of the nineteenth century - a predominantly affirmative status for much of eighteenth- and nineteenth-century philosophy, some of the most important debate in the twentieth century is concerned with the idea that the art which matters most can no longer function affirmatively, and may therefore be forced into the position of undermining its own aesthetic status. By reconciling people to the horrors of the world as it is, affirmative art risks reinforcing the social realities which have led to appalling suffering. The extermination camp commandant who goes home to play Schubert has to be a central figure in aesthetic reflection after the Holocaust, both as a reminder of the limits of art's capacity to humanise, and as a reminder of the constant need to sustain the truth of major works of art that transcends their contingent reception. At a different level, the kind of connections between art and society characteristic of Schelling and the System Programme were made in a small, in many ways still feudal, society which lacked many of the possibilities and problems occasioned by industrialisation, mass communication and other aspects of modern technology. The power of Hegel's conception lies in this respect precisely in its prescience with regard to how modern forms of thought and their application limit the possibilities of a renewal of society via aesthetic culture. It is here that building a bridge to the contemporary world becomes possible, despite the radical difference of the worlds in question.

The bridge results from differing construals of the importance attached to art in modernity, which we have encountered in the paradigmatic opposition between Romantic philosophy and Hegel. Despite all the historical and aesthetic changes just suggested, versions of the dilemmas which emerged in the period from Kant to Nietzsche still tend to define the agenda of contemporary discussion. The contemporary versions of these dilemmas can be suggested in the following kinds of question. Is art the source of a kind of truth inaccessible to other forms of articulation, or is it merely a means of rendering a post-theological world more tolerable for those with the resources to have access to art at all? Indeed, are these two perspectives necessarily separate: might art's ability to disclose temporalised post-theological meaning be precisely the kind of truth which only it can offer? If, on the other hand, art is merely a name for certain kinds of cultural practice, and no longer has any claim to be superior to other cultural practices, do criticisms of the ideological nature of those practices (including art) therefore themselves occupy a higher position in our selfdescriptions? If that is the case, what is the aim of such criticisms, if they offer none of the kind of utopian possibilities which have been a characteristic of art in modernity? In Rorty's terms, where is the 'knowingness' of such positions to 
lead that is preferable to the world-disclosing possibilities of what they criticise. ${ }^{8}$ In short, are theoretically based critical perspectives a superior resource for meaning-creation than art, and how is the line between the two to be established anyway?

These questions are closely linked to the interpretation of the crises which affect all the arts in the twentieth century, from the 'emancipation of dissonance' in music, to the dissolution of any sense of binding forms in literature, to the challenge of the avant-garde undermining of visual art, to the emergence of the culture industry and the tension between the 'torn halves' (Adorno) of 'popular' and 'high' culture. In their extreme form these crises result in such problematic (and not always wholly separable) alternatives as those between: (1) an art that tries inauthentically to please in the manner of the art of earlier periods by merely reproducing what constituted the appeal of that art (this can take place in the realm of 'popular' or 'high' culture); (2) a functionalisation of art in the name of extra-aesthetic goals, for example in advertising, but also in attempts to bring aesthetic resources to bear on areas where they may have been neglected, such as in new approaches to design - the functionalisation can, of course, also take the form of the mobilisation of art for political goals; (3) the related dissolution of art into a series of cultural practices in which aesthetic questions of beauty and truth play no role, the essential aspect being how means of articulation that were previously regarded as aesthetically autonomous can improve everyday life; and (4) the move towards artworks increasingly refusing to fulfil dominant cultural and aesthetic expectations, in the name of sustaining the world-disclosive and critical possibilities of aesthetic innovation when so much has already been done in the history of art and so much of this has been incorporated into other cultural practices. These directions are echoed in theoretical alternatives for the understanding of modern culture, so that (very schematically): (1) can be the domain of conservative cultural critics, who, by mixing causes and effects, regard aesthetic modernism as a contributing factor to a perceived general decline in public values in modernity; (2) can be the preserve both of the kind of postmodern ironists who see no point any more in a defence of the pursuit of the goals of high culture as a means of self-transcendence, selfdiscovery and self-criticism, and of politically committed critics who regard high culture as a distraction from real political change; (3) can be the domain of cultural materialists and other related thinkers who adopt the 'knowing' attitude to the art which those attached to an aesthetic attitude or to Heidegger regard as world-disclosive, in order to reveal its role, for example, in the history of patriarchy, or of racial and cultural oppression ((2) and (3) often go together); (4) is linked most obviously to the heritage of Adorno and to the attempt to show that autonomous art is a unique resource for seeing through the deceptions of modern culture. In many respects both the aesthetic and theoretical manifestations described here (my list does not claim to be exhaustive) involve a version of the tension between the new mythology and aesthetic autonomy which emerged with Idealism and Romanticism. The tension between the desire for 
art to facilitate new versions of existing forms of communication, and the idea that modernity leads to the need to find forms of articulation that circumvent the dominant modes of human exchange in the name of radically new possibilities is a perennial aspect of modern culture.

Decisions on the significance of these positions and practices will only be of value if they contextualise the issues appropriately. One of the dangers in the traditions leading from Hegel to Heidegger and Adorno has been a tendency to over-essentialise accounts of art and philosophy. This is particularly the case if a strong, exclusive connection is made between art and truth, so that, as in Heidegger, only art which is inseparable from its grounding context discloses being in an essential way, or, as in Adorno, only the most technically advanced art is regarded as expressing the truth of the historical situation. What may be a regressive stance in one socio-political situation may, though, be progressive in another. A defence of the Western classical tradition in music can, for example, be appropriate in relation to the production of music by the culture industry, where what can be learned from tradition is ignored or denigrated for the sake of commercial imperatives; the same stance can be regressive if it is used against the attempt to explore new musical possibilities, as though Western tonality were some kind of natural given.

What, though, is the connecting thread that would allow an examination of these issues to suggest future possibilities for the exploration of the relationships between art and philosophy, an exploration which, despite the concern of aesthetics with particularity and individuality, inevitably leads to more universal kinds of judgement? Even concentration, in the manner of some post-structuralists, or of Karl-Heinz Bohrer, on aesthetic events as some kind of 'sublime', 'immediate' singularity, for example, does not offer a plausible way of locating aesthetic issues as the radical 'other' of metaphysics and philosophy. If the immediacy of singularity is to attain theoretical significance, as a means of questioning the reduction of difference to identity in modernity, it must already be located in the realm of mediation. The irreducible aesthetic event which escapes being grasped conceptually can indeed be the source of insight denied to other, conceptually mediated forms, but the significance of such an event will still depend on some account of what it disrupts and relocates, thus on an account of a subject that is more than the sum of its linguistic and social determinations. It will also at some stage depend upon open-ended public debate as to its significance. The relationship between immediacy and mediation is not adequately dealt with by the attempt to subordinate one wholly to the other: what matters are the results of the interaction between the two.

In Chapter 8 I suggested that certain uses of the terms 'aesthetics' and 'subjectivity' can point to the tensions between public and private realms in modernity, and it is these tensions, above all, which can reveal a connecting thread between some of the questions examined above. As we have seen, Rorty characterises the development of modernity in terms of how the 'public', problemsolving resources of natural science and 'projects of social cooperation' become 
separate from 'private' projects of self-development, in which he includes 'romantic art' and, possibly, religion. His characterisation is, in fact, a version of Hegel's ideas about the end of art. What is 'public' depends on the intersubjective cashing-in of discursive commitments; the 'private' is up to individuals, so long as they do no harm to others. ${ }^{9}$ Rorty sees this division as important as a way of ensuring that private dreams of transcendence, of the kind he thinks constitute the real value of Nietzsche's and Heidegger's writings, do not get used in the democratic public sphere for political aims. ${ }^{10} \mathrm{He}$ is concerned, for example, with the blanket suspicion of technology which some contemporary thinkers derive from Heidegger's thought. The reasons for Rorty's investment in the distinction are clear, particularly in the light of German history. However, given the new ways in which the aesthetic tradition sought to combine the public and private, the question is how much of this tradition therefore needs to be consigned to the past. Decisions on this question depend precisely upon the story one tells about the relationship between subjectivity, art and truth. This can be shown by considering some of Adorno's contentions in relation to Rorty's account of public and private.

Adorno is an apt figure to invoke in this context because, in the wake of Nietzsche, he thinks, as does Heidegger, that the ills of modernity are rooted in the attempt by the subject to dominate the world of objects. However, despite his interpretation of the subject as the instance of domination of the other, he also sees the potential of what Rorty characterises in terms of the 'private' selfdevelopment manifest in modern art as one of the few remaining counters to the often catastrophic effects of the 'public' aspect of modernity. What makes Adorno's admittedly very problematic view still worth considering is his working out of the claim, in the light of the nightmare of much of twentiethcentury history, that art which resists the dominant forms of communication in society can offer perspectives beyond those forms. This claim is perhaps rather more persuasive than is sometimes thought. Even if the particular art which Adorno invokes in this context seems not to achieve what he claims, this does not per se invalidate what he is trying to do. It may be that other forms of art, or other aesthetic practices actually do offer a counter to dominant forms of communication.

Adorno, then, extends what is intended by Rorty's notion of the public to the aesthetic, in opposition to the 'Hegelian' conception, and so deconstructs any simple public/private divide. ${ }^{11}$ He does so because he thinks that many established problem-solving forms of social cooperation can be linked to the kinds of coercion that reach their extreme form with Nazism. The source of this coercion is, he claims, the following: 'the limitedness, to which people are compelled by the course of the world and which makes them see their interests and only their interests, is precisely the same power which turns against people and thereby asserts itself as the fate which is blind to them and almost inescapable' (Adorno 2001 p. 42). The pressure of what people produce collectively without their being aware of it undermines their capacity for critical reflection. Public 
pressure for conformity, which seems to come from outside, is in fact also produced from inside by the combined effects of people's own ways of understanding and pursuing their interests.

Adorno's conception entails an - in certain respects Hegelian - attempt to re-think the relationship between social determination and individual freedom, so that the two cannot be conceived of as being simply opposed. This conception also, therefore, affects the understanding of art: many of the theories we have looked at were precisely concerned with art's combination of the bound and the free. Actions and production which are supposed to derive from freedom could, in Adorno's view, be expressions of an unconsciously enforced conformity, though they need not be. ${ }^{12}$ The question is how this pressure for conformity could be opposed, and at this point it is noticeable that Adorno sometimes still appeals to the individual subject: 'In the face of the totalitarian unity which the eradication of difference directly proclaims as meaning, it may even be that temporarily something of the liberating social force has contracted into the sphere of the individual' (Adorno 1951 p. 11). It is important to remember the time at which such reflections are written: the point of orientation for Adorno is the barbarism perpetrated very recently by culturally advanced, industrial societies. How far, though, are Adorno's concerns at the perennial possibility of the repetition of barbarism in modern societies to determine judgements about the contemporary role and status of art and philosophy as means of exploring and articulating human possibilities for non-coercive freedom? The tension between conformity to general rules and individual initiative is inherent in modern art, but how much does this tension reveal about the social realm, and what role can philosophy play in relating art and society with regard to such issues?

It would evidently be foolish to give any kind of general answer to these questions, not least because of the ever-changing nature of aesthetic production and reception. Does this mean, though, that any suggestion of a more universal perspective is now invalid, as much recent theory concerned to defend cultural diversity against ethnocentrism tends to suggest? One way of indicating why such a perspective may not be as questionable as is often claimed is apparent in the following argument, from Aesthetic Theory, against the 'private' relativisation of aesthetic judgement:

Shoulder-shrugging aesthetic relativism is for its part a piece of reified consciousness; [it is] less melancholic scepticism against one's own insufficiency than resentment against the claim to truth of art which alone would legitimate that greatness of artworks without whose fetish [i.e. the fetish of greatness] the relativists rarely get by. Their behaviour is reified as behaviour which accepts from the outside, consumes, which does not participate in the movement of the artworks in which questions of their truth become conclusive. Relativism is the isolated self-reflection of the mere subject which is indifferent to the objective nature of the work (Sache). (Adorno 1973 p. 419) 
This might seem like just the complaint of an over-sensitive aesthete at admittedly irritating attitudes to art all too often encountered in modern societies, of the kind summed up in the proffering of the 'judgement', 'Well, it's what I like', when a person's prejudice is questioned. The deeper point of Adorno's position emerges, though, when he says: 'That people incessantly get involved in aesthetic disputes, no matter what position they take up with regard to aesthetics, proves more against relativism than its philosophical refutations: the idea of aesthetic truth gets its justification despite its problematic nature and in its problematic nature' (p. 419). Aesthetic truth is thus linked, as it already was in Kant, to the question of judgement, and this cannot be simply divided into a public and a private aspect: 'Contemplation of beauty is a judgement, and not a pleasure' (Kant 1996 p. 109). Involvement in aesthetic disputes is in this sense as public as involvement in any other kind of disagreement. ${ }^{13}$ Although the substantive idea of a sensus communis may be metaphysical, the regulative idea or focus imaginarius of cultural consensus generated by the universal concern about aesthetic matters indicated by Adorno is something Rorty himself is loath wholly to renounce.

However, Rorty is also insistent on distinguishing ('Hegelian') public 'arguments', in which 'the same vocabulary' must 'be used in premises and conclusions', as part of the same 'language game', and ('Romantic') private 'suggestions about how to speak differently' (Rorty 1991 p. 125). All the latter can do is 'fluidize old vocabularies' (p. 126). Some heuristic distinction of this kind does play a role in how we work in differing areas of human practice, though the truth-determinate and the world-disclosive constantly shift their boundaries. The question is how we might, in the light of the aesthetic tradition, and of Adorno's questions, draw this sort of distinction in relation to contemporary culture, where the idea of private transcendence is increasingly in tune with a commodity world that fragments individual taste into niche markets, at the same time as diminishing the level of cultural discrimination. Despite Rorty's rejection of 'mindless and stupid cultural relativism ... the idea that any fool thing that calls itself culture is worthy of respect' (Rorty $1999 \mathrm{p}$. 276), his idea of private transcendence can still slide into a version of what Adorno is opposing. The possibility of a search for public critical consensus on issues which do not have any means of empirical validation is excluded from private transcendence by the division between argument and suggestions about how to speak differently. In Chapter 7 I cited Stanley Cavell's remark that: 'It is essential in making an aesthetic judgement that at some point we be prepared to say in its support: don't you see, don't you hear, don't you dig? . . . Because if you do not see something, without explanation, then there is nothing further to discuss' (Cavell 1976 p. 93). The ground of aesthetic judgements is, in these terms, not something immediately accessible to public discursive argument. It is this fact that connects it to the analysis of the implications of immediate selfconsciousness we examined in the preceding chapters.

However, Cavell's point, like Adorno's, is that aesthetic judgement is still a 
'public' matter. Rorty would, in contrast, seem to think at times in similar terms to Lyotard's idea of the 'differend' (see Chapter 6) with regard to disagreements regarding how to speak differently, of the kind that arise most obviously in relation to aesthetic questions. In aesthetic judgements there generally are no shareable argumentative grounds of the kind provided by using the same vocabulary in premises and conclusions, precisely because the point of art is to 'fluidize vocabularies'. Rorty's conception, though, risks missing the point that, as the arguments about 'feeling' suggested, our understanding of art and of art's effects on us cannot be fully articulable in propositions. This does not, however, relegate our understanding to merely private transcendence, or to arbitrary 'speaking differently'. The reasons why are apparent both in Schleiermacher's Aesthetics, and in the best of Adorno's accounts of the development of modern art in terms of the tension between historically developed conventions - such as rules of harmony, rules of perspective - necessary for the existence of art as a 'public' concern, and individual, 'private' expression that transcends those rules (see Bowie 1999).

Cavell thinks the critic must 'start training and instructing you and preaching at you', even though the reasons she could offer must 'come to an end' (Cavell 1976 p. 93) because of the lack of argumentative grounds. If it is translated into the wider public sphere, Cavell's stance still involves demands for legitimation of the kind required for other discursive commitments, and this must affect political and institutional attitudes to 'private' transcendence. The power of Adorno's claim about aesthetic relativism lies, then, in its reminder that what is involved in Cavell's stance is a part of cultural and political life which must not be surrendered to the kind of false liberal tolerance that all too easily leads to an indifference to culture, rather than a new openness to the challenges to self-understanding that it can present. Even though he allows for transitions between the private and the public, Rorty at times underestimates the extent to which private self-development can become part of the public resources for problem-solving in societies where effective public debate about culture provides a bastion against the culture industry. The undoubted difficulty here, which is a major source of Rorty's scepticism about the political import of philosophical reflection on aesthetic issues, lies in the fact that, as he puts it (in Hegelian fashion): 'philosophy is responsive to changes in amount of social hope, rather than conversely' (Rorty 1999 p. 229). These changes undoubtedly have more to do with economics and power than anything else. Adorno remains important here because he does not underestimate the impact of economics on modern culture, and yet tries to make art a source of social hope, linking it closely to philosophy. To what extent, then, do objective circumstances render new resources for social hope redundant, and what role does philosophical understanding of art play in sustaining such hope after the demise of the Idealist and Romantic projects in their emphatic versions?

The difficulties Adorno faces in maintaining his position result from the fact that he relies on a 'Hegelian' conception of an integrated social totality, which 
is dominated by the exchange principle and by instrumental reason, even as he invokes art in 'Romantic' fashion to oppose that conception. In consequence, he must attempt the balancing act of insisting on the singularity of authentic art while attempting to arrive at a general 'public' story about the singularity of art in relation to other forms of truth in modernity. One part of his diagnosis relies on the kind of totalising philosophical claim made by Hegel, while the other wishes to abjure this kind of philosophy because it is an obstacle to insight into modern reality. In this respect Adorno puts too much weight on the (in a very broad sense) cognitive significance of art by forcing it into a pattern of development which he analogises to the development of modern philosophy.

This position can be questioned via the example of Beethoven, whom Adorno criticises (see Chapter 5), in much the same way as he criticises Hegel, for his attempts to achieve a totalising reconciliation of difference into identity. Such a reconciliation can, for Adorno, only be ideological, because it is bought at the price of ignoring the unreconciled state of modern societies. Adorno focuses in this case on the historical development of artistic material in a manner which suggests that it is subject to the same 'logic of disintegration' as philosophy. He admittedly has very persuasive things to say about why there is no longer any point in writing music like that of Beethoven - without the historical content absorbed by Beethoven and then transmitted by his work through differing historical circumstances, music of this kind loses its disclosive power. His story is too monolithic, even with regard to the demands of aesthetic production, because of the way he links it to the idea of subjectivity as domination. Adorno takes art, in this respect at least, too much as a public 'project of social cooperation' that reveals the truth about the delusions determining modernity. Art's continuing capacity to offer something other than truth-content of this kind is consequently underestimated. The radical temporalisation of art suggested by Adorno tells us too little about the idea of the persistence of art that is, for example, so significant in Gadamer. It also excludes too many of the ways in which, in differing contexts, problematic works can have serious significance, and even second-rate works or underdeveloped artistic activity can have vital affective and ethical significance for individuals in a manner which has little or nothing to do with Adorno's large-scale judgements on contemporary culture and society.

To this extent, Rorty's idea of private transcendence can be justified in certain respects: in a post-theological world, symbolic resources which can render life meaningful are, if they do not harm others, in one sense self-legitimating. The problem is, however, that the decision on their lack of harm is not as straightforward as Rorty's argument needs it to be. The symbolic resources in modern societies are increasingly controlled by a commodity market which manipulates judgement in a manner that evidently distorts cultural communication, and this returns the issue to the public side of the dichotomy. Rorty's way of making the public/private distinction, then, can privatise art to too great an extent and thus ignore some of more productive resources in Adorno's con- 
ception. It is not that Rorty would deny that so much contemporary culture really is industrially produced trash, but his manner of making the distinction can lead, by giving up any idea that aesthetic judgement might still sustain the aim of universality, to a de facto version of an aesthetic relativism that he himself elsewhere rejects. In relation to the 'inspirational value of great works', for example, Rorty actually sides with the 'party of hope', against those who think that they have nothing to learn from these works, because their theories can show how they contribute to the discourses of sexism, racism, class and so on. His espousal of the party of hope seems at odds, though, with his view that art should essentially be about private transcendence. Cavell's point is that the aim of transcendence manifest in art's irreducibility to the sayable and arguable is not something which can simply be left in the personal and private sphere, and the increasingly impoverished nature of much contemporary globalised culture backs up his claim.

The risk involved in a strong defence of the public status of the aesthetic can be what Walter Benjamin termed the 'aestheticisation of politics', in which the aim of transcendence is then inflated in a distorted manner into a public project, with no attempt at democratic legitimation of that project. Despite his justified insistence, in the wake of Marx, that the key issues of political justice ultimately depend on the economic sphere far more than on the politics of cultural difference, Rorty's conception of the political is too restricted in a media-dominated era where cultural communication is increasingly linked by the new forms of information exchange to the political sphere. This link is, I suspect, one of the reasons for the renewal of interest in texts like the System Programme, which were precisely about understanding the role of the aesthetic in the modern public sphere. Public debate and criticism are inseparable from the kinds of private transcendence which are often their motivation. However, the fact is that we still do not have adequate tools for understanding the effects of issues that were central to the aesthetic tradition in relation to the contemporary, image-dominated cultural world. The commodity world leads to increasingly deluded projection on the part of significant numbers of people, of the kind that results from the failure of what was intended in the idea of a rational new mythology. The result is often investment in the immediate satisfactions offered by the culture industry, rather than in the complexities and difficulties of real cultural involvement. ${ }^{14}$ How, then, might the possibilities of imaginative renewal relate to the commodity world in a manner which does not reinforce the projections to which that world gives rise?

One response to this is, of course, Adorno's radicality, which severs any attachment to the idea that art could now be simply pleasing. In a world where beauty is manipulated for commercial and other instrumental ends and is likely to conspire with the 'context of delusion' responsible for the barbarism he regards as a constant possibility in modernity, art must be critical. The problem is that art which fulfils Adorno's stringent demands is unlikely to have any significant effect on society as a whole, being a possible esoteric source of critical 
meaning for those prepared (and able) to engage with it, but being little else. Great art from the tradition can itself only be said to be 'critical' to the extent that it offers symbolic coherence and dynamism which may be lacking in the everyday world, but this is not what Adorno means, because he seems so sure such art can reconcile people to the given. However, the borderline between this art's reconciling people to the given, and its offering something which transcends the given and therefore makes them able to live in a more effective - and potentially critical - manner is too often ignored by Adorno. His failure to take account of this difference is one reason why he is forced to link avant-garde art so strongly to a philosophical account of the state of society, in order to reveal what he sees as its significance in the contemporary world. Adorno's radicality with regard to aesthetics derives from an extension of the arguments concerning coercion and self-coercion in modern societies that we considered (pp. 322-3). In order not to conspire with the totality, artworks have to be closed off from the dominant aspects of consumerist society by following the immanent logic of artistic production, rather than the demands of the market; at the same time, the resultant refusal to communicate is supposed to be precisely what allows them to reveal things which instrumental reason and the culture industry cannot: 'The aesthetic totality is the antithesis of the untrue totality' (Adorno 1973 p. 429); 'While the unity of the artworks derives from the violence reason does to things, at the same time it institutes the reconciliation of their moments in the works of art' (p. 454). Once again, this position relies too strongly on the totalising story about modern reality, which restricts art's significance to what can be construed via its link to Adorno's philosophical story.

This conception does, however, have a justifiable aspect, which is apparent in its refusal to regard art as offering an escape from what is wrong in modernity by just providing positive images of how the world ought to be. The culture industry relies in many respects on pretending to do precisely this, which often results in a culture of uncritical projection. Art is too inextricably linked to the reality it emerges from for it to play a wholly positive role in presenting alternatives to that reality - this is one way of understanding why modern music is so often difficult and not immediately accessible in the manner of music from earlier periods. Adorno's argument, though, still does not give enough space to the ways in which the aesthetic transcendence offered by works from the tradition of great art may make people aware of new critical possibilities. The other plausible side of Adorno's approach is apparent in the deeply ambiguous development and role of art in the contemporary Western world. Art's very status as art is indeed continually in question, even though the potential for the major traditions of art to make non-coercive sense persists even in the face of the culture industry. The problem with Adorno's theory is really that it does not allow sufficient space for the role of individual subjects, who, both as producers and receivers of art, can resist subsumption into the dominant forms of communication and articulation. Adorno himself seems unclear in this respect. On 
the one hand, he insists on the overwhelming pressure of the - subjectively produced - consciousness-forming objective conditions that led to fascism, which he believes still characterise advanced capitalist societies; on the other, he can also suggest in the same context that 'Critical incorporation (Aufarbeitung) of the past as enlightenment is essentially . . . a turn to the subject, a reinforcement of its self-awareness/self-confidence (Selbstbemusstsein) and thus also of its self' (Adorno 1970 p. 27).

This tension is crucial in trying to think about the respective roles of art and philosophy today. To what extent can the objective conditions which increasingly demand conformity from the subjects of contemporary societies (a conformity which the culture industry presents, for example, as the freedom of the consumer) be opposed in the name of autonomous judgement? This is ultimately not a question of yet another philosophical theory which could decisively establish that there is a human capacity for autonomy, but rather a question concerning the creation of the kind of cultural resources, both theoretical and aesthetic, which might enable subjects to become more self-reflective and selfcritical. Questions about the social role of philosophy are inescapable here. In this respect theories devoted to demonstrating how subjects are just the product of symbolic systems and power structures, for which self-determining autonomy is therefore a metaphysical illusion, may themselves be more a symptom of the pressure of objective conditions than the source of radical new insight. It is evidently foolish to deny the extent of the objective pressure exercised on subjects in modern societies. The aesthetic tradition, though, often aims to keep open the possibility that symbolic and other constraints may, within limits that are never wholly transparent to us, be transcended.

As the preceding chapters have shown, aesthetic theory in modernity is constantly faced with versions of the tension between positions, like that of Hegel, which insist that 'immediacy' only reaches its truth by conceptual mediation, and positions which insist that immediacy is essential to the very possibility of aesthetic creation and experience. Adherents of the first position emphasise the need for critical, social and historical analysis in all serious engagement with the aesthetic; adherents of the second argue that there would be no significant art if art were not resistant to attempts definitively to explain it in concepts. The first position stresses the 'public', and the primacy of the universal over the individual; the second tries to locate the 'private' in a new way, and insists on the possibility that individual subjects may say more than the existing general resources of articulation seem to permit. That so much theoretical attention has been in the direction of the former, rather than of the latter, is not surprising. The development of twentieth-century modernist art towards increasing specialisation, to the point where the category of art itself is attacked in the avant-garde gives credence to the idea on the part of the first position that modernity can only be understood via something like the theory of rationalisation which goes from Hegel to Weber, which Adorno himself relies upon for significant parts of his understanding of the situation of modern art. Art reaches a crisis because it can only sustain its 
capacity to generate new insight by itself becoming conceptually more and more reflexive - by ever greater mediation - so that, for example, the expressive dimension which Beethoven's music opened up may no longer be available to later composers, who have to take a much more complex route to expression. The importance of the second position lies in its ability to argue that Beethoven's music still possesses world-disclosing potential. It does so because it cannot be reduced to the ways in which it is conceptually mediated - including its being historically mediated by thinkers like Adorno - and can be played and heard by differing individuals in new contexts in novel ways. Great art's ability to continue to generate new meaning remains one of the few utopian unifying possibilities left to an increasingly secular society. It would be mistaken to underestimate the power of such art in the name of a knowing approach that seems to think it can itself, by its critical unmasking of the ideological aspects of art, offer more than the temporalised transcendence which takes the greatest art beyond ideology.

However, the problem now, as Adorno's arguments suggest, is that for contemporary art to continue in a decisive world-disclosive and critical role, it would need not to fall below a level which commands as much attention as the most advanced cognitive production. ${ }^{15}$ It could well be that we are in a situation where the kind of great art which has been the main point of orientation for the aesthetic tradition, is no longer possible in a world of increasing cultural diversity, and of new and increasingly rapid forms of communication. The kind of universal significance Adorno attributes to the Western classical music tradition may be the product of a very specific era with specific forms of communication and social exchange. Whether this means that we are left with nothing but the alternative between the culture industry and minority esoteric art is open to question. The possibilities of aesthetic transcendence may shift their location in contemporary society, but the need for the imaginative resources they open up will not go away. Adorno's totalising story about the objective pressure that subjects create for themselves is, as we saw, in tension with his realisation that the possibility of individual autonomy is a source of continuing cultural hope. In the latter case there is more space for the potential of a now more decentred aesthetic production still to have both critical and meaning-creating effects, even if it is no longer possible to regard art as the essential key to interpreting largescale aspects of history and society which are obscured by dominant forms of cognition and technical control.

One of the most important Romantic ideas resulting from the idea that the absolute is not accessible to reflection was that there should be an interplay of cognitive, ethical and aesthetic modes of articulation. The implications of this idea are well conveyed in Cavell's claim that knowing things is not the only way of relating to them. The inaccessibility of the absolute is not just a cognitive matter, but also leads to questions about how we can evaluate the differing aspects of both our own existence and of external nature without being able to invoke ultimate grounds for that evaluation. The vital factor therefore becomes the need for differentiated judgements about the relative contribution to human 
well-being, and to the integrity of the objective world, of different ways of relating to things, such that the cognitive-instrumental does not have inherent priority. ${ }^{16}$ It is the aesthetic tradition above all which sustained the possibility of thinking in these terms because of its ambiguous location between the differing areas addressed by modern philosophy. To this extent contemporary pragmatist attention to the idea that normativity is inseparable from each of the notional cognitive, the moral and the aesthetic realms is, ${ }^{17}$ I would contend, an essential factor in the contemporary revival of the desire to extend the scope of philosophical reflection towards a concern with the relations between our differing practices which has been characteristic of aesthetic thinking.

In Chapter 5 I cited Danto's remark that 'when art attains the level of selfconsciousness it has come to attain in our era, the distinction between art and philosophy becomes as problematic as the distinction between reality and art. And the degree to which the appreciation of art becomes a matter of applied philosophy can hardly be overestimated.' (Danto 1983 pp. 1-2). Danto captures something important about the nature of contemporary art, but his position is one-sidedly Hegelian (as well as being over-influenced by developments in the visual arts). One of the vital factors in philosophical reflection in the wake of the Romantic tradition, which has again come to the fore in post-structuralism, and in Rorty's and others' neo-pragmatism, is that it is not just the case that art may need philosophical resources. Art and aesthetic experience can themselves provoke philosophical reorientation by, for example, revealing limits of theoretical models of the self, suggesting new ways of questioning scientism, provoking new kinds of reading of texts, revealing new understandings of the background knowledge which informs even the most rigorously scientific practices, or, above all, enabling a wider picture to be grasped that is being obscured by dominant specialised theories. The real question here is how the line is drawn between art, philosophy and science if these are, as Rorty argues, not natural kinds. This is not, in the terms at issue here, some kind of definitional enterprise, but rather an occasion for reflecting on how we attend to the things we most need and value, and on how that attention can both contribute to and damage our world.

The demise of Hegel's emphatic claims for philosophy, and the suspicions cast by modernist aesthetics and artistic practice on the notion of the autonomous work of art can be read as simply part of a wider narrative of the disenchantment inseparable from modernity. They can also point in the direction of a more fluid and mutually critical relationship between differing kinds of articulation than is allowed for in some contemporary philosophical thinking. It may be that the history of aesthetic theory and of art in modernity is most important at present for what it can show about the shifting relationships between differing human practices and the kinds of meanings those practices convey. Clearly the scientistic assumptions that inform significant parts of contemporary philosophy in the analytical tradition, and the exaggerated suspicions of science and technology which play a role in some aesthetic thinking in 
the European tradition, both fail to capture what was perhaps most important about the aesthetic tradition. The failure of the project of a definitive grounding of philosophy need not necessarily result either in the rejection of all nonscientific accounts of our place within things, or in the claim that the principle of subjectivity is really the principle of domination of the other that is manifest in modern technology's forgetfulness of being. ${ }^{18}$ The subject that appears in the best of the aesthetic tradition is evidently aware of the dangers of repression of the other that can result from its self-assertion: this was one source of the suspicions of Fichte's foundationalism. At the same time this subject is aware of the possibilities both of transcendence and of destructive self-deception that result from its failure to be the ground of itself. Such a view of the precarious status of the subject seems to me most plausible in the light of the experience of modernity. The acceptance of the resultant fallibilism and of the need to negotiate intersubjective norms without repressing individuality remains the crucial challenge for any contemporary philosophy that seeks both to understand and to contribute to contemporary culture. If this is so, the tendency in some recent theory to regard questions about subjectivity as part of a metaphysical legacy that must be overcome is now proving to be a symptomatic mistake, rather than the path to new insight.

\section{Notes}

1 In what follows the term 'aesthetic tradition' is shorthand for the tradition examined in the present book.

2 One of the most acute responses to the difficult status of art in modernity is Kafka's final story, 'Josefine the Singer', which plays with the idea that art objects and the production of art are in one sense really just the same as any other kind of object or production. The text in which this idea is explored is, characteristically, itself a great modernist work of art.

3 On the ambiguities of the term metaphysics see Bowie 2000.

4 On the issue of transcendental philosophy's relationship to everyday understanding, see Ameriks 2000.

5 Cf. the remarks on de Man in Chapter 7.

6 In some respects the same problems apply to the Dialectic of Enlightenment, though some of the story told there is connected to historical investigation of phenomena like antiSemitism.

7 I leave to one side for the moment the question of the quality of the music in question.

8 I do not mean by this that it is inappropriate to reveal ideological aspects of works of art. The question is whether this revelation may not sometimes obscure more than it reveals, by limiting the perspective on the work in question to what fits a preconceived position.

9 Aspects of this position echo Habermas's account of modernity.

10 Nietzsche's reliance on 'intuition' (see Chapter 8) can suggest one reason why Rorty puts him on the 'private' side of the divide. The problem of intuition is precisely that attempts to validate it contradict its very nature as something immediate. However, as I suggest (pp. 329-30), the issue is more complex than this.

11 It should be added that because Rorty rejects the systematic side of Hegel in favour of a 
(Romantic) open-ended dialectic, he would not make the distinction between the public and the private in exactly the way Hegel does. For example, he regards the Phenomenology in much the same way as I do, that is, as in some respects being more significant as a 'private' text. My difference from Rorty is described below.

12 In some respects this is reminiscent of Kant's idea, in the Foundation of the Metaphysics of Morals, that we can never finally know if we act morally, as there may be a degree of heteronomy even in our apparently most autonomous acts.

13 Clearly the kinds of agreement and what kinds of evidence can be valid differ, depending on the aims of a particular practice, but transitions between the demands of cognitive, ethical and aesthetic judgement can also be a vital source of new insight.

14 I do not mean that other people are free of the mechanism of projection, just that the manipulation of this mechanism is now perhaps the dominant factor in much of Western popular culture.

15 It seems no coincidence that many critics now think the best popular writing about science is aesthetically superior to most contemporary fiction. Indeed, some of the best aesthetic writing seems increasingly to eschew extended fiction in the name of the attempt to use the results of lived experience and historical and other research to offer new worlddisclosing perspectives. I am thinking, for example, of a book like W.G. Sebald's Die Ringe des Saturn.

16 If this sounds too abstract, what I mean is exemplified, for example, in the ways in which government-imposed evaluations of humanistic disciplines in academia are increasingly carried out in terms of the conveying of information. These evaluations fail to see how background culture which develops slowly over time and cannot be methodologically controlled, as though it just consisted of information, is indispensable for such disciplines.

17 As I have suggested in previous chapters, I do not think these 'realms' can be separated in any more than a heuristic sense.

18 The derivatives of such an account, in Levinas, Lyotard and others, all entail some version of the idea that being a subject inherently represses alterity. 


\section{APPENDIX: THE SO-CALLED \\ 'OLDEST SYSTEM PROGRAMME OF GERMAN IDEALISM' (1796)}

recto

An Ethics. As the whole of metaphysics will in future come under Morality - of which Kant only gave an example with his two practical postulates and exhausted nothing, this ethics will be nothing but a complete system of all Ideas, or, which is the same, of all practical postulates. The first Idea is naturally the notion of my self as an absolutely free being. With the free self-conscious being [Wesen] a whole world emerges at the same time - out of nothing - the only true and thinkable creation from nothing - Here I will descend to the fields of physics; the question is this: how must a world be for a moral being? I should like to give wings again to our physics which is progressing slowly and laboriously via experiments.

Thus - if philosophy gives the Ideas and experience the data we can finally achieve the grand physics which I expect from later epochs. It does not appear that our present physics could satisfy a creative spirit which is like ours, or like ours should be.

From nature I come to human activity [Menschenmerk]. Putting the Idea of humanity first - I want to show that there is no Idea of the State because the state is something mechanical, just as little as there is an Idea of a machine.

Only that which is an object of freedom is called an Idea. We must, then, also go beyond the state! - For every state must treat free people as a piece of machinery; and it should not do this; thus it must come to an end.

You can see yourselves that here all the Ideas, of eternal peace etc. are only subordinate Ideas of a higher Idea. At the same time I want here to establish the principles for a History of Mankind and to completely expose the whole miserable human creation of state, constitution, government, legislature. Finally come the Ideas of a moral world, divinity, immortality - the upturning of all superstition, the pursuit of the priesthood, which has recently been feigning reason, by reason itself. - Absolute freedom of all spirits who bear the intelligible [intellektuelle] world in themselves, and may not seek either God or immortality outside themselves.

Finally the Idea which unites all, the Idea of beauty, the word taken in the higher platonic sense. I am now convinced that the highest act of reason, which embraces all Ideas, is an aesthetic act, and that truth and goodness are brothers only in beauty - The philosopher must possess just as much aesthetic power

\section{verso}

as the poet [Dichter]. People without aesthetic sense are our pedantic philosophers [BuchstabenPhilosophen]. The philosophy of spirit is an aesthetic philosophy. One cannot be spiritual [geistreich] in anything, one cannot even reason spiritually about history - without aesthetic sense. It should here become apparent what it is that people lack who understand no Ideas - and admit faithfully enough that everything is a mystery to them as soon as it goes beyond charts and registers.

Poetry thereby gains a higher dignity, at the end it again becomes what it was at the beginning - teacher of (History) Mankind; for there is no philosophy, no history any more, poetry alone will survive all the remaining sciences and arts. 
At the same time we hear so often that the masses should have a sensuous religion. Not only the masses but also the philosopher needs monotheism of reason of the heart, polytheism of imagination [Einbildungskraft] and of art, this is what we need!

First of all I shall speak here of an Idea which, as far as I know, has never occurred to anyone - we must have a new mythology, but this mythology must be in the service of the Ideas, it must become a mythology of reason.

Before we make the Ideas aesthetic i.e. mythological, they are of no interest to the people and on the other hand before mythology is reasonable the philosopher must be ashamed of it. Thus enlightened and unenlightened must finally shake hands, mythology must become philosophical and the people reasonable, and philosophy must become mythological in order to make the philosophers sensuous. Then eternal unity will reign among us. Never the despising gaze, never the blind trembling of the people before its wise men and priests. Only then can we expect the same development of all powers, of the individual as well as all individuals. No power will be suppressed any more, then general freedom and equality of spirits will reign! - A higher spirit sent from heaven must found this new religion among us, it will be the last, greatest work of mankind.

(Trans. Andrew Bowie) 


\section{REFERENCES}

Adorno, T. W. (1951) Minima Moralia (Frankfurt: Suhrkamp)

Adorno, T. W. (1970) Erziehung zur Mündigkeit (Frankfurt: Suhrkamp)

Adorno, T. W. (1973) Ästhetische Theorie (Frankfurt: Suhrkamp)

Adorno, T. W. (1974) Versuch über Wagner (Frankfurt: Suhrkamp)

Adorno, T. W. (1975) Negative Dialektik (Frankfurt: Suhrkamp)

Adorno, T. W. (1996) Probleme der Moralphilosophie (Frankfurt: Suhrkamp)

Adorno, T. W. (2001) Zur Lehre von der Geschichte und von der Freiheit (Frankfurt: Suhrkamp)

Ameriks, Karl (2000) Kant and the Fate of Autonomy (Cambridge: Cambridge University Press)

Ameriks, Karl (ed.) (2000) The Cambridge Companion to German Idealism (Cambridge: Cambridge University Press)

Bahro, Rudolf (1979) . . . die nicht mit den Wolfen heulen (Cologne, Frankfurt: EVA)

Bauer, Michael and Dahlstrom, Daniel O. (eds) (1999) The Emergence of German Idealism (Washington D.C.: Catholic University of America Press)

Baumgarten, Alexander (1988) Theoretische Ästhetik (Hamburg: Meiner)

Beiser, Frederick C. (1987) The Fate of Reason: German Philosophy from Kant to Fichte (Cambridge, Mass. and London: Harvard University Press)

Benjamin, Walter (1980) Gesammelte Schriften (Frankfurt: Suhrkamp)

Bernstein, J. M. (1992) The Fate of Art: Aesthetic Alienation from Kant to Derrida and Adorno (Cambridge: Polity Press)

Bohrer, Karl Heinz (1983) Mythos und Moderne. Begriff und Bild einer Rekonstruktion (Frankfurt: Suhrkamp)

Bowie, Andrew (1979) Problems of Historical Understanding in the Modern Novel, unpublished $\mathrm{PhD}$ dissertation, University of East Anglia

Bowie, Andrew (1993) Schelling and Modern European Philosophy (London: Routledge)

Bowie, Andrew (1996) 'John McDowell's Mind and World, and Early Romantic Epistemology', Revue internationale de philosophie, Vol. 50, No. 197, 3/1996

Bowie, Andrew (1996a) 'Rethinking the History of the Subject: Jacobi, Schelling and Heidegger', in (eds) Simon Critchley and Peter Dews, Deconstructive Subjectivities (Albany: SUNY Press) pp. 105-26

Bowie, Andrew (1997) From Romanticism to Critical Theory (London, New York: Routledge)

Bowie, Andrew (1997a) 'The Meaning of the Hermeneutic Tradition in Contemporary Philosophy', in (ed.) Anthony O'Hear, 'Verstehen' and Humane Understanding, Royal Institute of Philosophy Lectures (Cambridge: Cambridge University Press)

Bowie, Andrew (1999) 'Adorno, Heidegger and the Meaning of Music', Thesis 11, Nr. 56, $2 / 1999$

Bowie, Andrew (1999a) 'German Philosophy Today: Between Idealism, Romanticism, and Pragmatism, in (ed.) Anthony O’Hear, German Philosophy Since Kant, Royal Institute of Philosophy Lectures (Cambridge: Cambridge University Press)

Bowie, Andrew (2000) 'The Romantic Connection: Neurath, the Frankfurt School, and Heidegger', in British Fournal for the History of Philosophy, Part One, 8 (2) 2000; Part Two, 8 (3) 2000 
Bowie, Andrew (2001) 'Music and the Rise of Aesthetics', in (ed.) Jim Samson, Cambridge History of Nineteenth Century Music (Cambridge: Cambridge University Press)

Bowie, Andrew (2002) 'Schleiermacher and Post-Metaphysical Thinking' (forthcoming)

Brandom, Robert (1994) Making it Explicit (Cambridge Mass. and London: Harvard University Press)

Brandom, Robert (2000) Articulating Reasons (Cambridge Mass. and London: Harvard University Press)

Cascardi, Anthony J. (1999) Consequences of Enlightenment (Cambridge: Cambridge University Press)

Cavell, Stanley (1976) Must we Mean what we Say? (Cambridge: Cambridge University Press)

Dahlhaus, Carl (1978) Die Idee der absoluten Musik (Munich and Kassel: dtv)

Dahlhaus, Carl and Zimmermann, Michael (1978) Musik zur Sprache gebracht (Munich and Kassel: dtv)

Danto, Arthur C. (1983) 'Art, Philosophy, and the Philosophy of Art', Humanities, Vol. 4, No. $1(2 / 1983)$

Davidson, Donald (1984) Inquiries into Truth and Interpretation, Oxford: Oxford University Press

De Man, Paul (1983) Blindness and Insight. Essays in the Rhetoric of Contemporary Criticism, second edition revised (London: Routledge)

Derrida, Jacques (1967) La voix et le phénomène (Paris: Presses Universitaires de France)

Derrida, Jacques (1972) Marges de la Philosophie (Paris: Seuil)

Derrida, Jacques (1972a) Positions (Paris: Minuit)

Dews, Peter (1987) Logics of Disintegration: Post-structuralist Thought and the Claims of Critical Theory (London and New York: Verso)

Dews, Peter (1995) The Limits of Disenchantment (London and New York: Verso)

Eagleton, Terry (1990) The Ideology of the Aesthetic (Oxford: Blackwell)

Farrell, Frank B. (1996) Subjectivity, Realism and Postmodernism (Cambridge: Cambridge University Press)

Fichte, J. G. (1971) Werke I, Werke II (Berlin: de Gruyter)

Förster, E. (1995) 'To Lend Wings to Physics Once Again.' Hölderlin and the "Oldest System-Programme of German Idealism"', European Fournal of Philosophy, Vol. 3. No. 2. pp. $174-8$

Foucault, Michel (1970) The Order of Things (London: Tavistock)

Foucault, Michel (1988) Politics Philosophy Culture (ed.) Lawrence D. Kritzman (New York and London: Routledge)

Frank, Manfred (1977) Das Individuelle-Allgemeine. Textstrukturierung und interpretation nach Schleiermacher (Frankfurt am Main: Suhrkamp)

Frank, Manfred $(1979,1995)$ Die unendliche Fahrt. Ein Motiv und sein Text (Frankfurt am Main: Suhrkamp. Second substantially extended and revised edition, Leipzig: Reclam)

Frank, Manfred (1982) Der kommende Gott. Vorlesung über die neue Mythologie I (Frankfurt: Suhrkamp)

Frank, Manfred (1984) Was Ist Neo-Strukturalismus? (Frankfurt: Suhrkamp)

Frank, Manfred (1985) Eine Einführung in Schellings Philosophie (Frankfurt: Suhrkamp)

Frank, Manfred (1986) Die Unhintergehbarkeit von Individualität. Reflexionen über Subjekt, Person und Individuum aus Anlaß ihrer 'postmodernen' Toterklärung (Frankfurt am Main: Suhrkamp) 
Frank, Manfred (1988) Grenzen der Verständigung (Frankfurt: Suhrkamp)

Frank, Manfred (1991) Selbstbemußtsein und Selbsterkenntnis (Stuttgart: Reclam)

Frank, Manfred (1997) 'Unendliche Annäherung'. Die Anfänge der philosophischen Frühromantik (Frankfurt: Suhrkamp)

Frank, Manfred and Kurz, Gerhard (eds) (1975) Materialien zu Schellings philosophischen Anfängen (Frankfurt: Suhrkamp)

Freud, Sigmund (1982) Studienausgabe (eds) Alexander Mitscherlich, Angela Richards, James Strachey (Frankfurt: Fischer)

Fulda, Hans Friedrich and Henrich, Dieter (eds) (1973) Materialien zu Hegel's 'Phänomenologie des Geistes' (Frankfurt: Suhrkamp)

Gadamer, Hans-Georg (1986) Hermeneutik: Wahrheit und Methode 2 Ergänzungen, Register, Tübingen: J. C. B. Mohr

Gadamer, Hans-Georg and Boehm, Gottfried (1976) Seminar: Philosophische Hermeneutik (Frankfurt: Suhrkamp)

Habermas, Jürgen (1974) 'Arbeit und Interaktion. Bemerkungen zu Hegels' “Jenenser Philosophie des Geistes"', in (ed.) Gerhard Göhler, Georg Wilhelm Friedrich Hegel Frühe politische Systeme (Frankfurt, Berlin, Vienna: Ullstein)

Habermas, Jürgen (1983) Moralbemußtsein und kommunikatives Handeln (Frankfurt: Suhrkamp)

Habermas Jürgen (1988) Nachmetaphysisches Denken (Frankfurt: Suhrkamp)

Habermas Jürgen (1999) Wahrheit und Rechtfertigung (Frankfurt: Suhrkamp)

Hamann, J. G. (1967) Schriften zur Sprache (ed.) Josef Simon (Frankfurt: Suhrkamp)

Hegel, G. W. F. (1959) Enzyklopädie der philosophischen Wissenschaften (eds) Friedhelm Nicolin, Otto Pöggeler (Hamburg: Meiner)

Hegel, G. W. F. (1961) Philosophie der Geschichte (Stuttgart: Reclam)

Hegel, G. W. F. (1965) Ästhetik Vols I and II, ed. F. Bassenge (Berlin, Weimar: Aufbau)

Hegel, G. W. F. (1969) Wissenschaft der Logik I, II, Vols 5, 6 Werkausgabe (Frankfurt: Suhrkamp)

Hegel, G. W. F. (1970) Phänomenologie des Geistes (PG), Vol. 3 Werkausgabe (Frankfurt: Suhrkamp)

Hegel, G. W. F. (1971) Vorlesungen über die Geschichte der Philosophie Vols 18, 19, 20 Werkausgabe (Frankfurt: Suhrkamp)

Heidegger, Martin (1959) Untermegs zur Sprache (Pfullingen: Neske)

Heidegger, Martin (1960) Ursprung des Kunstmerks (Stuttgart: Reclam)

Heidegger, Martin (1983) Die Grundbegriffe der Metaphysik (Frankfurt: Klostermann)

Heidegger, Martin (1986) Untermegs zur Sprache (Pfullingen: Neske)

Heidegger, Martin (1988) Zur Sache des Denkens (Tübingen: Niemeyer)

Henrich, Dieter (1982) Selbstverhältnisse (Stuttgart: Reclam)

Henrich, Dieter (1987) Konzepte (Frankfurt: Suhrkamp)

Henrich, Dieter (2001) Versuch über Kunst und Leben. Subjektivität-Weltverstehen-Kunst (Munich and Vienna: Hanser)

Hoffmann, E. T. A (1988) Schriften zur Musik. Singspiele (Berlin, Weimar: Aufbau Verlag)

Hogrebe, Wolfram (1989) Prädikation und Genesis. Metaphysik als Fundamentalheuristik im Ausgang von Schellings 'Die Weltalter' (Frankfurt: Suhrkamp)

Hölderlin, Friedrich (1963) Werke Briefe Dokumente (Munich: Winkler)

Humboldt, Wilhelm von (1973) Schriften zur Sprache (Stuttgart: Reclam)

Jähnig, Dieter (1966) Schelling. Die Kunst in der Philosophie, Vol. 1, Schellings Begründung von Natur und Geschichte (Pfullingen: Neske) 
Kant, Immanuel (1974) Kritik der praktischen Vernunft, Grundlegung der Metaphysik der Sitten, Werkausgabe VII (Frankfurt: Suhrkamp)

Kant, Immanuel (1968) Kritik der reinen Vernunft (CPR), Werkausgabe III and IV (Frankfurt: Suhrkamp)

Kant, Immanuel (1968) Kritik der Urteilskraft (C7), Werkausgabe X (Frankfurt: Suhrkamp)

Kant, Immanuel (1989) Prolegomena zu einer jeden Metaphysik, die als Wissenschaft wird auftreten können (Stuttgart: Reclam)

Kant, Immanuel (1996) Schriften zur Ästhetik und Naturphilosophie (eds) Manfred Frank and Véronique Zanetti (Frankfurt am Main: Deutscher Klassiker Verlag)

Kroner, Richard (1924) Von Kant zu Hegel, 2 Vols (Tübingen: Paul Siebeck)

Lepore, Ernest (ed.) (1986) Truth and Interpretation (Oxford: Blackwell)

Lévi-Strauss, Claude (1975) The Ram and the Cooked (New York, Hagerstown, San Francisco and London: Harper Row)

Lewis, Charles (1985) 'Kant and E. T. A. Hoffmann: "The Sandman”', Ideas and Production 3, pp. 28-43

Lichtenberg, Georg Christoph (1994) Schriften und Briefe (Frankfurt: Zweitausendeins)

Lloyd, Genevieve (1984) The Man of Reason. 'Male' and 'Female' in Western Philosophy (London: Methuen)

Lukács, Georg (1971) Soul and Form, trans. Anna Bostock (London: Merlin)

Lukács, Georg (1971a) Die Theorie des Romans (Neuwied und Berlin: Luchterhand)

Lypp, Bernhard (1972) Asthetischer Absolutismus und politische Vernunft. Zum Widerstreit von Reflexion und Sittlichkeit im deutschen Idealismus (Frankfurt: Suhrkamp)

Makkreel, Rudolf A. (1990) Imagination and Interpretation in Kant. The Hermeneutical Import of the 'Critique of Fudgement' (Chicago and London: Chicago University Press)

Marx, Karl (1974) Grundrisse der Kritik der politischen Ökonomie (Berlin: Dietz)

McDowell, John (1994) Mind and World (Oxford: Oxford University Press)

McDowell, John (1998) Mind, Value, and Reality (Cambridge, Mass. and London: Harvard University Press)

Menninghaus, Winfried (1987) Unendliche Verdopplung. Die frühromantische Grundlegung der Kunsttheorie im Begriff absoluter Selbstreflexion (Frankfurt: Suhrkamp)

Nagel, Thomas (1986) The Viem from Nowhere (Oxford: Oxford University Press)

Nagel, Thomas (1995) The Last Word (Oxford: Oxford University Press)

Neubauer, John (1986) The Emancipation of Music from Language. Departure from Mimesis in Eighteenth-Century Aesthetics (New Haven and London: Yale University Press)

Nietzsche, Friedrich (1980) Sämtliche Werke. Kritische Studienausgabe in 15 Bänden, (eds) Giorgio Colli and Mazzino Montinari (Munich, Berlin and New York; de Gruyter)

Novalis (1968) Schriften. Die Werke Friedrich von Hardenbergs Vol. 3: Das philosophische Werk (eds) Paul Kluckhohn and Richard Samuel (Stuttgart: Kohlhammer)

Novalis (1978) Band 2 Das philosophisch-theoretische Werk (ed.) Hans-Joachim Mähl (Munich Vienna: Hanser)

Novalis (1981) Werke (Munich: Beck)

Pinkard, Terry (1996) Hegel's Phenomenology. The Sociality of Reason (Cambridge: Cambridge University Press)

Pippin, Robert (1999) Modernism as a Philosophical Problem (Oxford: Blackwell)

Proust, Marcel (1981) The Captive. Remembrance of Things Past, Vol. 3 (Harmondsworth: Penguin)

Putnam, Hilary (1981) Reason, Truth and History (Cambridge: Cambridge University Press) 
Putnam, Hilary (1999) The Threefold Cord: Mind, Body, and World (New York: Columbia University Press)

Rorty, Richard (1989) Contingency, Irony and Solidarity (Cambridge: Cambridge University Press)

Rorty, Richard (1991) Essays on Heidegger and Others. Philosophical Papers Volume Two (Cambridge: Cambridge University Press)

Rorty, Richard (1998) Truth and Progress. Philosophical Papers Volume Three (Cambridge: Cambridge University Press)

Rorty, Richard (1998a) Achieving Our Country (Cambridge, Mass. and London: Harvard University Press)

Rorty, Richard (1999) Philosophy and Social Hope (Harmondsworth: Penguin)

Rössler, Beate (1990) Die Theorie des Verstehens in Sprachanalyse und Hermeneutik (Berlin: Dunker and Humblot)

Rousseau, Jean-Jacques (1776) Pygmalion (London: Kearby)

Sacks, Oliver (1986) The Man who Mistook His Wife For a Hat (London: Picador)

Safranski, Rüdiger (1998) Ein Meister aus Deutschland. Heidegger und seine Zeit (Frankfurt: Fischer)

Sartre, Jean-Paul (1943) L'être et le néant (Paris: Gallimard)

Scheible, Hartmut (1984) Wahrheit und Subjekt Ästhetik im bürgerlichen Zeitalter (Frankfurt: Fischer)

Schelling, F. W. J. (1856-61) Sämmtliche Werke, ed. K. F. A. Schelling, I Abtheilung Vols 1-10, II Abtheilung Bde. 1-4 (Stuttgart: Cotta)

Schelling, F. W. J. (1946) Die Weltalter, ed. Manfred Schröter (Munich: Biederstein and Leibniz)

Schelling, F. W. J. (1977) Philosophie der Offenbarung (1841-22), ed. Manfred Frank (Frankfurt: Suhrkamp)

Schelling, F. W. J. (1994) On the History of Modern Philosophy, ed. and trans. Andrew Bowie (Cambridge: Cambridge University Press)

Schlaffer, Heinz (1981) Faust Zweiter Teil: die Allegorie des 19. Fahrhunderts (Stuttgart: Metzler)

Schlegel, Friedrich (1963) Philosophische Lehrjahre (1796-1828) (Kritische Friedrich Schlegel Ausgabe Volume 18) (Munich, Paderborn, Vienna: Ferdinand Schöningh)

Schlegel, Friedrich (1964) Philosophische Vorlesungen (1800-1807) (Kritische Friedrich Schlegel Ausgabe Volume 12) (Munich, Paderborn, Vienna: Ferdinand Schöningh)

Schlegel, Friedrich (1971) Philosophische Lehrjahre II (1796-1828) (Kritische Friedrich Schlegel Ausgabe Volume 19) (Munich, Paderborn, Vienna: Ferdinand Schöningh)

Schlegel, Friedrich (1980) Literarische Notizen, 1797-1807, ed. Hans Eichner (Frankfurt, Berlin and Vienna: Ullstein)

Schlegel, Friedrich (1988) Kritische Schriften und Fragmente, Studienausgabe Vols. 1-6, ed. Ernst Behler and Hans Eichner (Paderborn, Munich, Vienna and Zürich: Schöningh)

Schlegel, Friedrich (1991) Transcendentalphilosophie, ed. Michael Elsässer (Hamburg: Meiner)

Schleiermacher, F. D. E. (1839) Dialektik, ed. L. Jonas (Berlin: Reimer)

Schleiermacher, F. D. E. (1842) Vorlesungen über die Ästhetik. Aus Schleiermacher's handschriftlichem Nachlasse und aus nachgeschriebenen Heften, ed. C. Lommatzsch (Berlin and Leipzig: de Gruyter)

Schleiermacher, F. D. E. (1942) Friedrich Schleiermachers Dialektik, ed. Rudolf Odebrecht (Leipzig: Hinrichs) 
Schleiermacher, F. D. E. (1977) Hermeneutik und Kritik (Frankfurt: Suhrkamp)

Schleiermacher, F. D. E. (1984) Ästhetik (1819/25) über den Begriff der Kunst, ed. Thomas Lehnerer (Hamburg: Meiner)

Schleiermacher, F. D. E. (1986) Dialektik (1811) (Hamburg: Meiner)

Schleiermacher, F. D. E. (1988) Dialektik (1814-15). Einleitung zur Dialektik (1833) (Hamburg: Meiner)

Schleiermacher, Friedrich (1990) Ethik (1812/13) (Hamburg: Meiner)

Schleiermacher, F. D. E. (1998) 'Hermeneutics and Criticism' and Other Texts, ed. and trans. Andrew Bowie (Cambridge: Cambridge University Press)

Schleiermacher, F. D. E. (no date) Über die Religion. Reden an die Gebildeten unter ihren Verächtern (Berlin: Deutsche Bibliothek)

Schoenberg, Arnold (1975) Style and Idea (London and Boston: Faber)

Schopenhauer, Arthur (1986) Die Welt als Wille und Vorstellung, in Sämtliche Werke I-V, ed. Wolfgang Frhr. von Löhneysen (Frankfurt: Suhrkamp)

Schulz, Walter (ed.) (1968) Fichte-Schelling Briefmechsel (Frankfurt: Suhrkamp)

Schweizer, Hans Rudolf (1973) Ästhetik als Philosophie der sinnlichen Erkenntnis. Eine Interpretation der 'Aesthetica' A.G. Baumgartens mit teilweiser Wiedergabe des lateinischen Textes und deutscher Übersetzung (Basel and Stuttgart: Schwabe)

Sellars, Wilfrid (1997) Empiricism and the Philosophy of Mind (Cambridge, Mass. and London: Harvard University Press)

Strunk, Oliver (ed.); rev. edn Leo Treitler (1998) Source Readings in Music History (New York: W.W. Norton)

Szondi, Peter (1974) Poetik und Geschichtsphilosophie. Vol. 1, ed. Senta Metz, Hans-Hagen Hildebrandt (Frankfurt: Suhrkamp)

Taylor, Charles (1985) Human Agency and Language (Cambridge: Cambridge University Press)

Tugendhat, Ernst (1979) Selbstbemußtsein und Selbstbestimmung (Frankfurt am Main: Suhrkamp)

Tugendhat, Ernst (1992) Philosophische Aufsätze (Frankfurt: Suhrkamp)

Welsch, Wolfgang (1996) Vernunft. Die zeitgenössische Vernunftkritik und das Konzept der transversalen Vernunft (Frankfurt: Suhrkamp)

Wheeler, Samuel C. III (2000) Deconstruction as Analytic Philosophy (Stanford: Stanford University Press)

Wittgenstein, Ludwig (1980) Culture and Value (Oxford: Blackwell)

Wittgenstein, Ludwig (1982) Philosophische Untersuchungen (Oxford: Blackwell)

Zizek, S. (1996) The Indivisible Remainder. An Essay on Schelling and Related Matters (London, New York: Verso)

Zöller, Günter (1998) Fichte's Transcendental Philosophy. The Original Duplicity of Intelligence and Will (Cambridge: Cambridge University Press) 


\section{INDEX}

absolute 50-2, 75, 80, 226, 292

as unrepresentable $52,95,330$

Adorno, Theodor W. 94, 103, 137-8, 146, $153,168,169,215,257 \mathrm{n} 21,258$, 274, 295, 321, 322-31

on aesthetic autonomy 124, 274, 320 on genius 120-2

on Hegel 107, 141, 168, 179

on subjectivity $179,240,322-3,328-9$

aesthetic autonomy 2, 47, 120, 212, 216, $240,245,265,274-5,309,320$

Ameriks, Karl 313

Ast, Friedrich 200-1

Bahro, Rudolph 255

Bakhtin, Mikhail 175

Baumgarten, Alexander 5-6, 18-19

Beethoven, Ludwig van 99, 121-3, 153, 155-6, 223, 232-3, 251-2, 255, 267, $308,317-18,326$

Benjamin, Walter 67, 247-50, 254

Bloch, Ernst 67-8, 124

Bohrer, Karl-Heinz 63, 321

Borges, Jorge Luis 250

Brandom, Robert 140, 191-2, 203, 205, $230-1,261$

Bruckner, Anton 156, 298

Cascardi, Anthony 35, 159

Cavell, Stanley 53, 231, 324-5, 327

Dahlhaus, Carl 222, 227

Danto, Arthur 164, 169-70, 331

Davidson, Donald 135-6, 184, 187, 190, 193, 208, 258

De Man, Paul 238-45

Derrida, Jacques 7, 59, 96, 100n15, 171, 184, 204-5, 215, 235, 238, 241-2, 244,258 and Romanticism 248-50

and Schelling 135-7, 145-6, 234

Descartes, René 1-2, 9, 16-17, 21

Dews, Peter 136, 269

Dilthey, Wilhelm 207

Dionysus 65-7, 276-9, 282-3, 299

Eagleton, Terry 273

Farrell, Frank 314

Faurisson, Robert 190-2

'feeling' 29-31, 35, 36, 77, 91, 92, 148, 160-1, 196-200, 226, 230-1, 233, $235,243,247-8,288,318,325$

Feuerbach, Ludwig 292

Fichte, Johann Gottlieb 12, 27, 56, 70-82, 94-5, 97, 105, 141, 247, 254, 262-3, 304

on the absolute 5

and Freud 71-3, 81

and Hölderlin 81, 82-4

on intellectual intuition 77-80, 302

critique of 'reflection model' of consciousness $73-8$

and Schelling 102-3, 108, 109

Foucault, Michel 13-14, 216, 223-5, 228-9, 252

Frank, Manfred 13, 22, 53, 56, 57, 66, 76, $79,84,88-9,92,98,99,113,136$, $162,191,199,242,243,306$

freedom 13, 18, 23-4, 34, 56, 92, 323 and self-consciousness $75,116-17$

Freud, Sigmund 85-6, 171-2, 225-6, 286 relation to Fichte 71-3, 81

and Schelling 105, 112

and Schopenhauer 267-8

Gadamer, Hans-Georg 9, 29, 39, 149, 260, 316-17, 326 
German Idealism 9, 49-51, 137, 145, 250 and art 107, 119, 146

Goethe, Johann Wolfgang von 176, 223

Gramsci, Antonio 62

Habermas, Jürgen 8, 59, 62, 115, 125-6, 141-2, 147, 170, 185, 258, 295

Hamann, Johann Georg 5-8, 11, 48n17, 59, $65,66,186-7,225,248$

Hawking, Stephen 60

Haydn, Joseph 227

Hegel, Georg Wilhelm Friedrich 40, 51, 53, 140-82, 158, 319

on the absolute $141,142-3,144-5,176$

and Beethoven 155-6, 229

on the 'end of art' $46,125,140,150,164$, 169-75, 274, 298, 322

and Fichte 246

on intellectual intuition 137, 146

and Kant 144, 149, 171

and language 148, 160-3, 165-6, 235-6

and music 155, 228, 233

Logic 146, 151-2, 152-60, 169, 173

and music 153-60

on music 158-60, 174, 227-38, 246-7, 255,296

on the novel 175-9

on Romanticism 229

and Schelling 110, 112, 116-17, 129-30, 137-8 146, 152, 153, 157, 229

Heidegger, Martin 8-12, 168, 210, 321

and Hamann 7

on language 130-1, 285

and music 296-7

on modernity and 'subjectification'

$10-11,28,31,103,130,176,218$,

$224,240,251,274,312,316-7$

and Nietzsche 285, 288, 296-8

and Schelling 136-8

Henrich, Dieter 16, 70, 147, 149, 313

on 'reflection theory' 73-8

Herder, Johann Gottfried 187

Hobbes, Thomas 25, 263

Hoffmann, E.T.A. 232-3, 242
Hogrebe Wolfram 106

Hölderlin, Friedrich 82-8, 89, 126, 165

on the absolute $82-4$

on art and politics $123-4,308$

on Fichte 81, 82-4

on intellectual intuition 82-4

on 'reflection' 84-8

Holub, Robert 258-9, 310n1

Humboldt, Wilhelm von 205, 219n3

Hume, David 17, 70

imagination 78, 109, 110

Jacobi, Friedrich Heinrich 27, 50-1, 291

Jähnig, Dieter 111

Joyce, James 178-9

Kafka, Franz 332n2

Kant, Immanuel 2, 16-48, 51, 167, 189, 243,274

on genius $39-40,42-3$

and German Idealism 57-63

Hamann on 11, 186

imagination 20, 33-4, 59-60

intuition 17, 18, 19-20, 22

intellectual intuition 22-4

judgement 17, 26-7, 29-31, 36, 61

objectivity in 17,50

on the sublime 43-7, 58-9, 64, 65, 153

'transcendental unity of apperception'

$$
20-4,71,72,74,84,196,303
$$

Lacan, Jacques 85, 86, 111, 136n3, 198

language 116, 160, 163-6, 184-5, 192, 194, 203-4

and the I $87-8,90,161-2,166,198,235$, 242-3, 245

and music 34, 37, 95-6, 265

and representation 160, 181n11, 221-7, 230,234

La Rochefoucauld 25

Leibniz, Gottfried Wilhelm 195

Lévi-Strauss, Claude 65, 224, 283

Lichtenberg, Georg Christoph 69-70, 303 
Lloyd, Genevieve 172

Lukács, Georg 177-8, 237

Lyotard, Jean-François 8, 11, 190-3, 240, 261, 325

Lypp, Bernhard 57

McDowell, John 47n6, 107, 140, 315

Mahler, Gustav 181

Maimon, Salomon 50

Mallarmé, Stéphane 223-4

Marquard, Odo 99

Marx, Karl 55, 120, 270-5

on commodity, and aesthetics 4, 61,

$$
\text { 273-4 }
$$

and Greek art 43, 270-2

and mythology 270-2

Menninghaus, Winfried 249-50

modernity $3-4,8,124-5,128,130,253-4$, 273-5, 281, 283, 291, 310, 317, 321

and postmodernity $8-14,281$

music 3, 9-10, 35-6, 38, 40, 45-6, 95-7,

123, 153-60, 174, 199-200, 221-57,

264-9, 270-1, 273, 277, 282-4,

292-300, 317-18, 321

and language $6,10,34-5,37,95-6$,

211-19, 221-2, 224, 245, 265-6

as non-representational $40,67,97,174$, 213-14, 216, 225, 226-7, 252, 265, $268,298,313$

Nagel, Thomas 12, 15n.5

nature $3,6,17-18,25,27-8,31-3,38-9$, 41-2, 55-7, 102-4, 168-9, 232, 261, $305-6$

Negt, Oskar 218

'new mythology' 47, 62, 63-8, 120, 124-7, $171,271,276,278,320,327$

Nietzsche, Friedrich 42, 63, 64, 94, 258-311

and Heidegger 285, 288, 296-8

and 'infinite interpretation' 301-2, 308

on language $284-8,290-1,307$

and music $283-8,293$

on music 282-4, 287, 293-300, 300-1 on nihilism 291-2

and Schelling 106, 276, 278-80, 290, 296, 303, 305, 306, 308

and Schopenhauer 275, 276, 286, 292, 299

on subjectivity $279-80,286,295,300-10$

on Wagner 284, 286, 294, 296-8, 309

Novalis (Hardenberg, Friedrich von) 22, 59, 88-99, 197

on the absolute $91-4$

on language $90-1,92-3,95-6,244,253$

on music $94-7,246,253$

on reflection $89-93$

'Oldest System Programme of German Idealism' $(S P)$ 55-63, 86-7, 275-6, $319,327,334-5$

Pinkard, Terry 142, 145, 147

Pippin, Robert 142

Plato 264

post-structuralism 7, 53-4, 190-2, 316

connection to music $238-45$

and German Idealism and Romanticism $54,245,246,254$

Putnam, Hilary 52, 148

Quine, W.V.O. 184, 185

'reflection' 72-4, 147-9, 242, 247, 273, 302 'infinite reflection' 67, 247-9 and music 67

Romanticism (early) 51-5, 81, 98-9, 119, $143-4,162,253-4$

and 'infinite reflection' $248-50,254,255$

on music 119

and natural science 60

and Nietzsche 254, 259, 304

and post-structuralism 52, 245, 246

and the sublime 52

Rorty, Richard 52, 54, 60, 77, 168, 175, 181n11, 192, 193, 222-3, 234, 238, $258,259,308,310,312,317,321-31$

Rousseau, Jean-Jacques 7, 23, 69, 238-9 
Sacks, Oliver 164-5, 212, 217, 243-4

Sartre, Jean-Paul 90, 163, 198

Saussure, Ferdinand de 217, 233

Scheible, Hartmut 6, 32, 40, 58, 112, 164

Schelling, Friedrich Wilhelm Joseph 4, 32, $38,39,49,65,80,102-39,141,204$

on the absolute $110,113,128$

and Derrida 135-7, 145-6, 234

and Fichte 102-3, 108, 109

and Freud 105, 112

on genius $120-3,280$

and Hegel 110, 112, 116-17, 129-30,

137-8 146, 152, 153, 157, 229

identity philosophy $133-7,152$

on intellectual intuition 110-12, 114-15, 134,136

and art $80,118-19,127$

on language $116,128,130-3$

on music 133, 256, 277, 301

'Naturphilosophie' 104-7, 114, 306

and Nietzsche 106, 276, 278-80, 290, 296, 303, 305, 306, 308

on 'reflection' 109, 116, 127

and Schopenhauer 105, 106, 262-3, 277

Schiller, Friedrich 14, 36, 47, 56

Schlegel, Friedrich 52-3, 63-8, 143-4,

$162-3,217,236-7,260,275-6,312$

and 'endless reflection' $65,67,249$

Hegel on 245-6

on language 37

on literature $236,251-3$

on music 37, 236, 246, 251, 287

Schleiermacher, Friedrich Daniel Ernst 11, 173, 180, 183-220, 290, 314

Aesthetics 187, 195, 203

on argument 188-93

on 'divination' 207-8, 218

and Gadamer 183, 206

and Habermas 185, 193, 206

on individuality $194-5,202,203-4,210$, 213 and the 'linguistic turn' 183-7, 200

and literature 200, 209-19

and the 'musical' 209-19, 235

on music 199-200, 232

on self-consciousness 196-200, 211, 215, $234,235,242,304$

and aesthetics 199, 202, 211

and Wittgenstein 189, 217

Schoenberg, Arnold 23-4, 46, 268, 269

Schopenhauer, Arthur 29, 261-70

and Kant 262-4

and music 66, 264-9

and language 265

Nietzsche and 275, 276, 286, 292, 299

and Schelling 105, 106, 262-3, 277

on intellectual intuition 262-3

the Will 262-7

Schubert, Franz 294

Schumann, Robert 234

Sebald, W.G. 333

Sellars, Wilfrid 151, 184, 230-1, 315

Spinoza, Benedict 51, 104, 152

Szondi, Peter 49

Taylor, Charles 160, 181n11

Tugendhat, Ernst 161, 313

Velasquez, 'Las Meninas' 91

Wagner, Richard 69, 139, 256

music and mythology $67,127,272$

Nietzsche on 256, 284, 286, 294, 296-8, 309

Weber, Max 12, 166-7, 271

Wheeler, Samuel 184, 199, 289, 305

Wittgenstein, Ludwig 112-13, 161, 184, 225

Zizek, Slavoj 138

Zöller, Günter 71, 77, 78, 108

Zola, Emile 271 